\title{
USING GEOMETER'S SKETCHPAD TO ENHANCE \\ NON-ACCELERATED MIDDLE SCHOOL STUDENTS' \\ UNDERSTANDING OF NEGATIVE NUMBERS AND EQUALITY
}

\author{
A Dissertation \\ Presented to \\ The Faculty of the Curry School of Education \\ University of Virginia \\ In Partial Fulfillment \\ of the Requirements for the Degree \\ Doctor of Philosophy \\ by
}

Nicole Renée Juersivich

B.S., Salisbury University, 2001

M.S., Virginia Tech, 2005

August, 2009 
(C) Copyright by Nicole Juersivich All Rights Reserved August, 2009 
Department of Curriculum, Instruction, and Special Education

Curry School of Education

University of Virginia

Charlottesville, Virginia

\section{APPROVAL OF THE DISSERTATION}

This dissertation, “USING GEOMETER'S SKETCHPAD TO ENHANCE NONACCELERATED MIDDLE SCHOOL STUDENTS' UNDERSTANDING OF NEGATIVE NUMBERS AND EQUALITY", has been approved by the Graduate Faculty of the Curry School of Education in partial fulfillment of the requirements for the degree of Doctor of Philosophy.
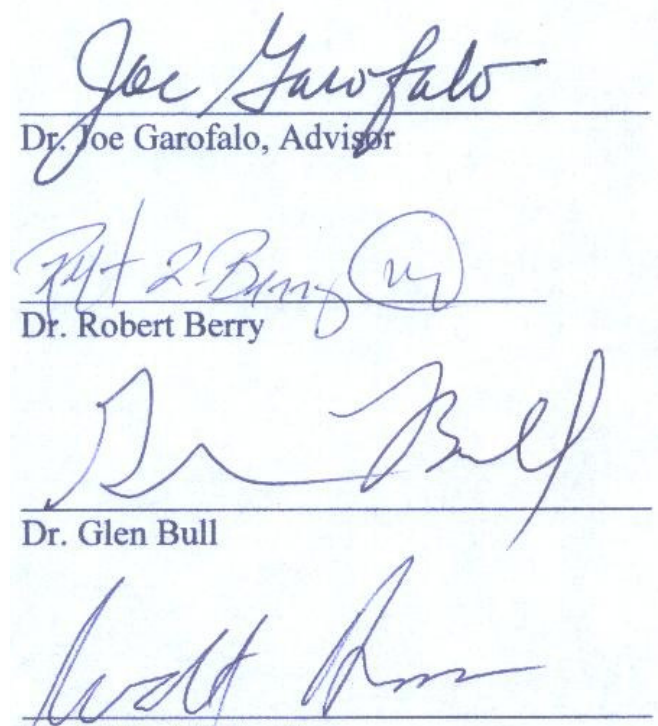

Dr. Walter Heinecke

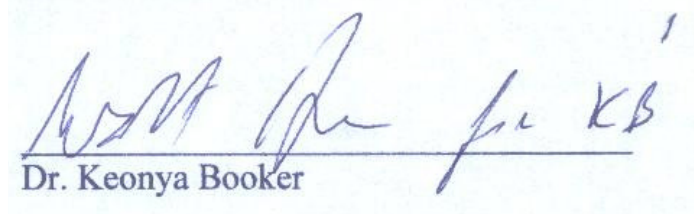




\begin{abstract}
The purposes of this study were to (1) investigate non-accelerated middle schools students' understanding of pre-algebraic concepts, in particular, addition and subtraction of integers and the notion of equality; (2) describe and analyze how non-accelerated middle school students interact with The Geometer's Sketchpad (GSP) applets depicting pre-algebraic concepts in order to discover how these applets can elucidate or mask these concepts and hence facilitate or hinder understanding; (3) to chronicle how students' conceptions of these pre-algebraic concepts change during and after instructional use of the GSP applets; (4) to receive feedback from students to help develop the applets in order to improve the value of such applets in the algebra classroom. The participants in this study were eight middle school students taking part in the after-school math-tutoring program. All participants had had some algebra instruction and needed remediation in addition and subtraction of integers and solving equations. These eight students consisted of five females, three males, three sixth graders, three seventh graders, and two eighth graders. Each participant met with me for at least two sessions, totaling of at least two hours and fifteen minutes.
\end{abstract}

The study included three self-contained modules consisting of two sections: an interview section without technology and an instructional section with technology. The interview section was audio recorded, and the instructional section was video and audio recoded. During the interview section, participants were asked to complete a variety of tasks based on literature findings of pre-algebraic and algebraic concepts in order to elicit participant conceptions. I used the task-based interview section of each module to assess the students' knowledge of the module's topic. If during this section of the module I saw that the student could not solve the task or articulate conceptual reasoning for his answer, I used that task to segue into the accompanying instructional section for that module. Thus, I met the student where he was in terms of understanding and guided him with technology and questions to answer and understand the mathematical idea. However, if the student could solve and satisfactorily explain the correct reasoning behind the answer in the task-based interview section, I moved to the next question in the task-based interview section of the same module. If a student had difficulty with one question in the task-based interview section, he could have not fully finished this section, for he would have been segued into the accompanying instructional section of the module. If a student could correctly solve and reason through every task-based interview 
question in one module, I continued to the next module's task-based interview section.

After data collection, data will be transcribed and reviewed for coding an analysis. Results will be presented in case studies and a cross case analysis. Each case study will illustrate (1) a participant's initial understanding of operations on integers, the notion of equality, and solving equations, (2) the interactions with the dynamic technology applets GSP, and (3) the conceptual changes regarding the pre-algebraic and algebraic concepts. The findings of this study will have implications for mathematics teachers as they prepare students' for learning algebra. These findings will also inform mathematics researchers on the conceptions of these topics for sixth and seventh-grade students and possible future research avenues in algebra. 
Dedicated with love to my grandfather,

\section{Carl Coulter,}

who always asked when I'd be done with school

but didn't get to see me finish. 


\section{ACKNOWLEDGEMENTS}

This dissertation has led me on quite an educational and emotional journey. I have laughed, cried, and stressed over the excitement, frustration, and rewards this project has brought me. I wish to express many thanks to faculty, friends, and family members who have helped me along this journey and complete this manuscript. The following pages hold much more than the study and culmination of my degree work, for they reflect the relationships with many inspiring people I've met along the way.

I am indebted to my UVa professors, for they have provided me with a remarkable graduate education. Through their teaching and stimulating coursework, they have taught me how to think, teach, and teach others to think. Special thanks goes to my advisor and committee members.

I am especially appreciative of my advisor, Joe Garofalo, for his guidance and provision of research and teaching opportunities and economic support. Thank you for consistently being supportive throughout my graduate studies, introducing me to prominent people within the discipline, showing faith in my work, and being a strong advocate for me. Throughout our discussions during the editing phase, I have been amazed at your ability to easily wade through the information and make wonderful suggestions for improvement.

I would like to thank my committee members, Robert Berry, Glen Bull, Walt Heinecke, and Keonya Booker for their valuable contributions to this dissertation. Thank you for your expert feedback!

To Jessica de la Cruz, Beth Lloyd, Christine Trinter, and Virginia Fraser, thank you for your friendship and critiquing my analyses, writing, and presentations. More importantly, thank you for giving me an outlet where I could let off steam during stressful times. I could not have survived the process without you!

On a personal level, I owe thanks to several other people: my parents, my mathematics professors at Salisbury University, and my husband.

To my parents, Jon and Sharon Miller, thank you for your love, faith, and support during the long years of my education. I would not be here today, if it weren't for you both.

To my SU professors, thank you for your inspiration and encouragement to apply to graduate school.

Finally, to my dear husband, Adam, whose abiding love and consolation enabled me to get through the years. Thank you for your understanding and faith in my ability to rise to the occasion and do the necessary work. 


\section{TABLE OF CONTENTS}

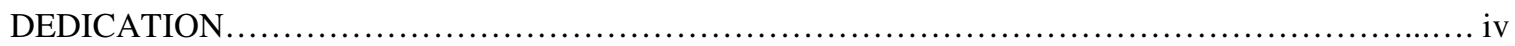

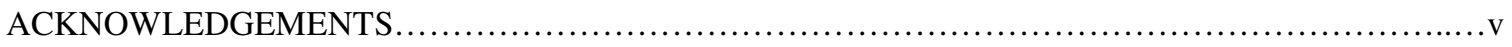

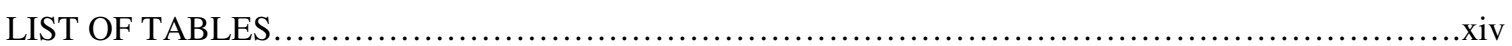

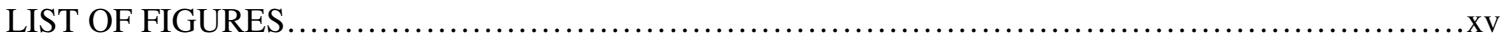

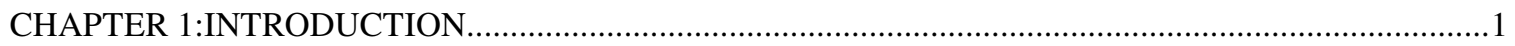

Reason for Studying Middle School Students' Understanding of Pre-algebra ....................................6

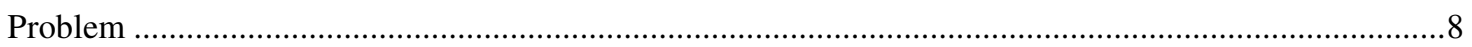

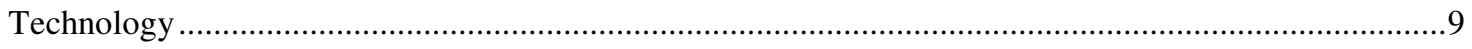

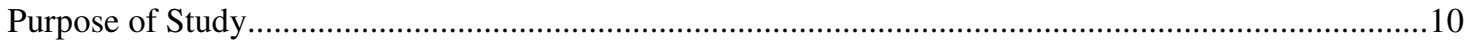

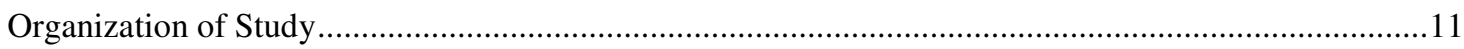

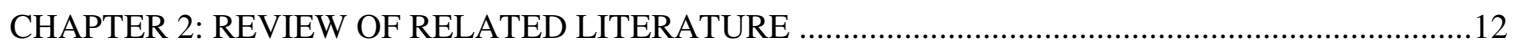

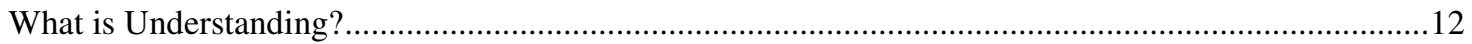

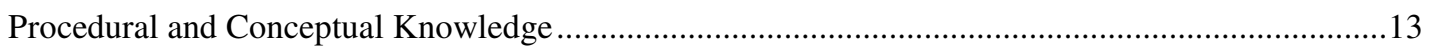

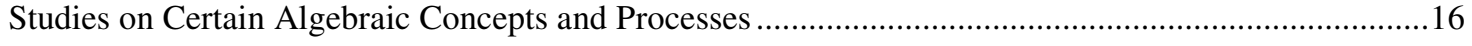

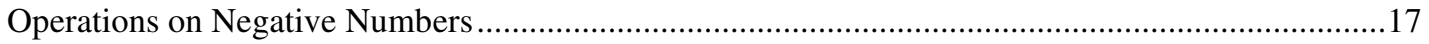

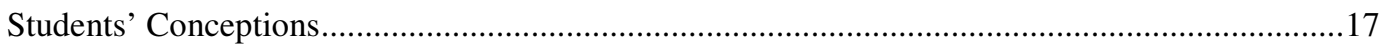

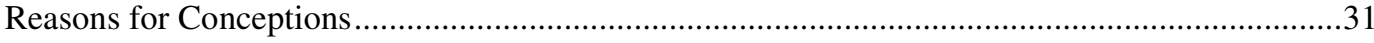

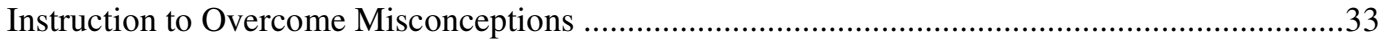

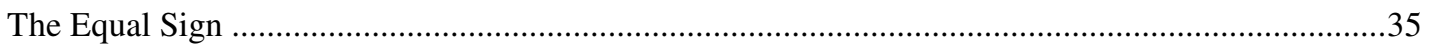

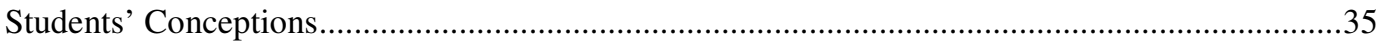

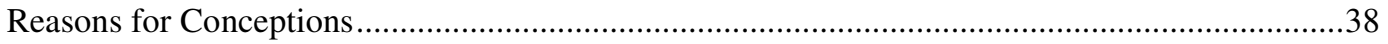

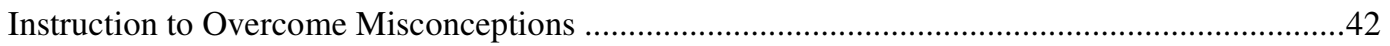

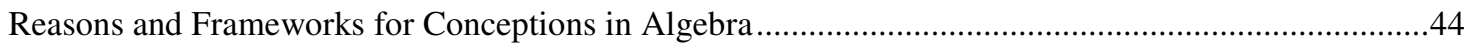

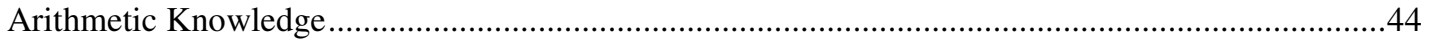

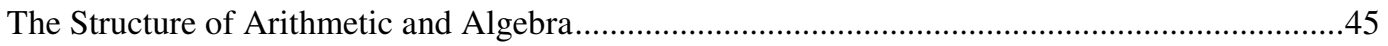




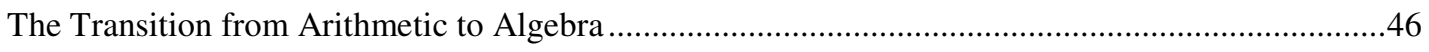

Similarities and Differences of Arithmetic and Algebraic Procedures and Symbolism...................46

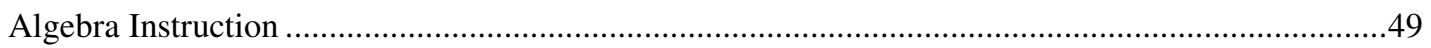

Generational, Transformational, and Global/Meta-level Activities ..............................................49

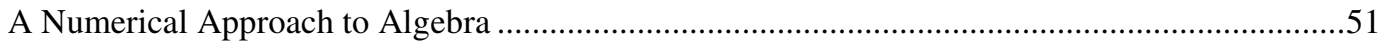

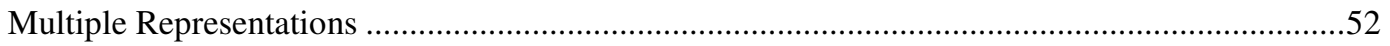

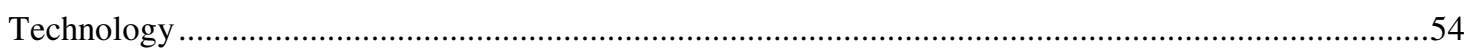

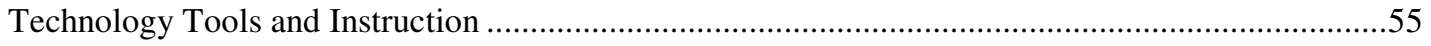

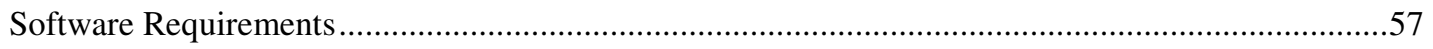

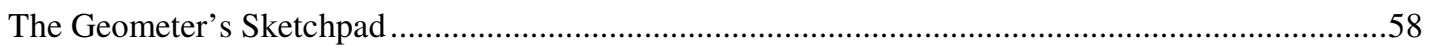

Using Technology to Understand Pre-Algebraic and Algebraic Concepts ...........................................59

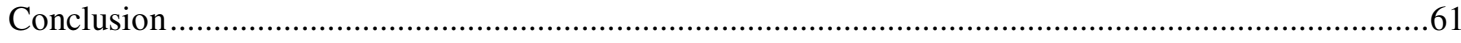

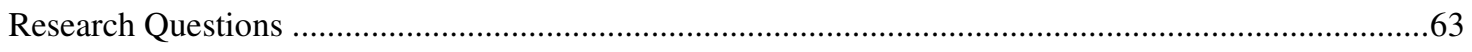

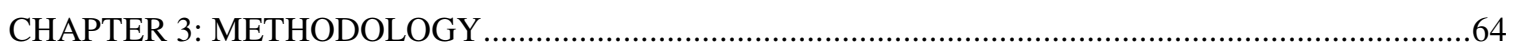

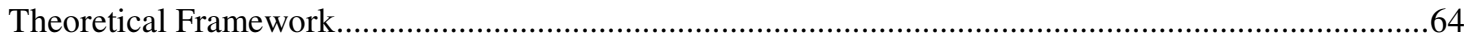

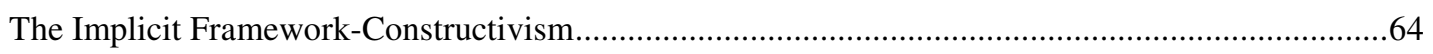

The Explicit Framework- Kieran's Four Methods of Meaning Making ..............................................66

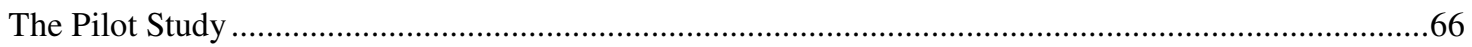

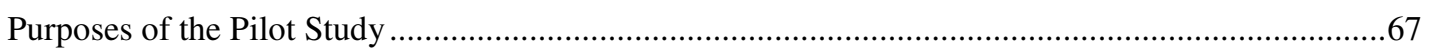

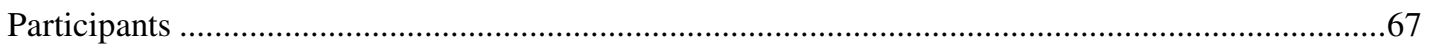

Creation and Adjustment of Tasks and Technology Applets..............................................................68

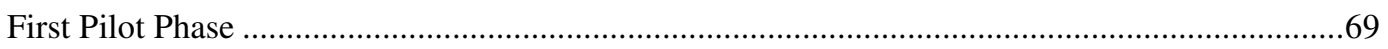

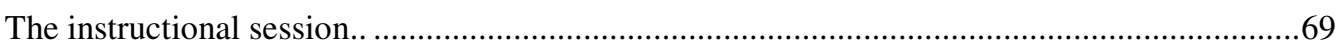

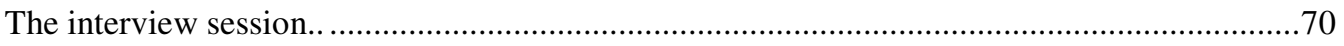

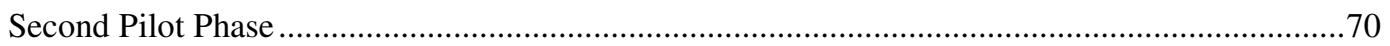

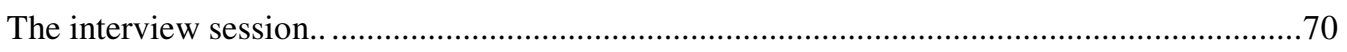

The instructional session......................................................................................................

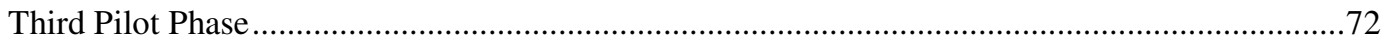

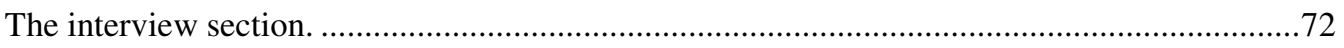

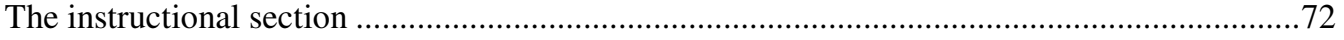




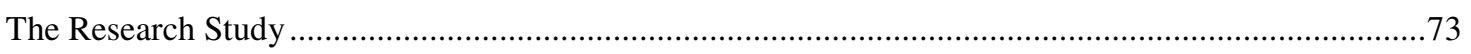

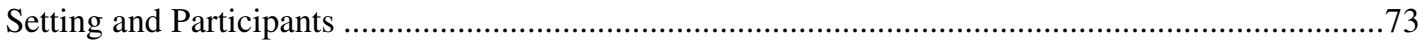

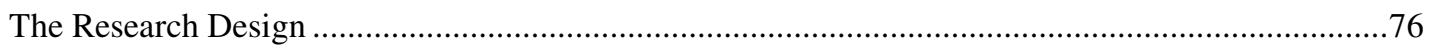

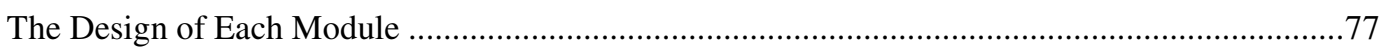

The interview section. …………………...............................................................................

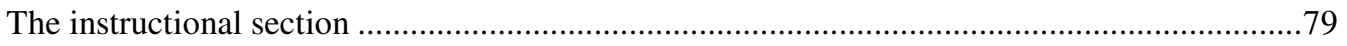

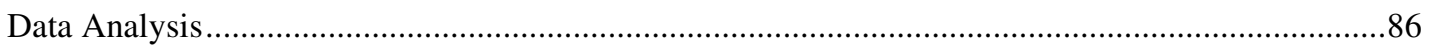

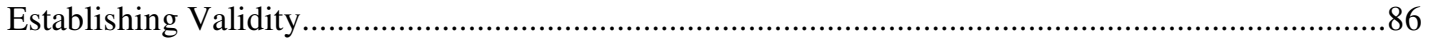

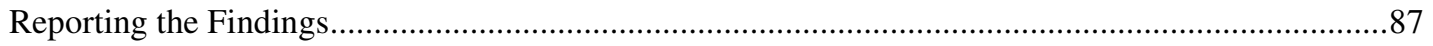

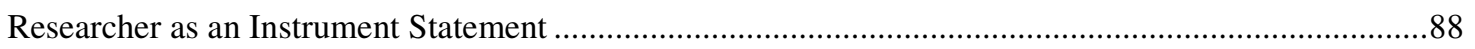

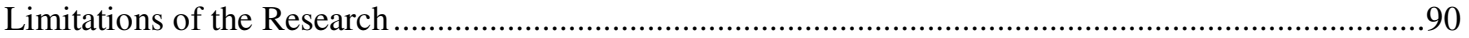

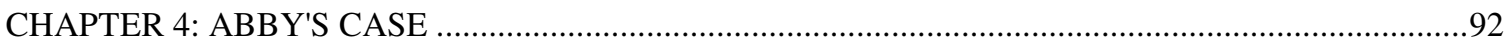

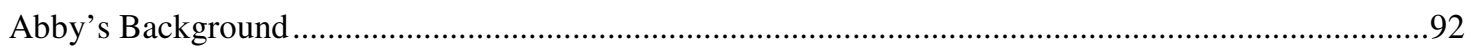

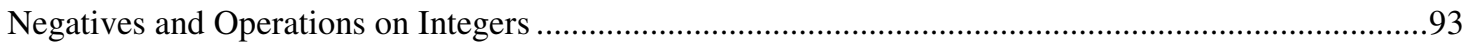

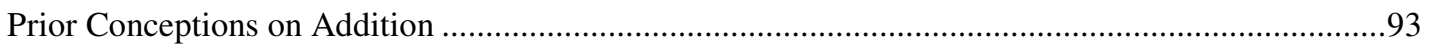

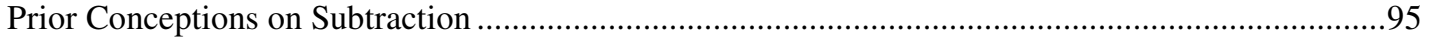

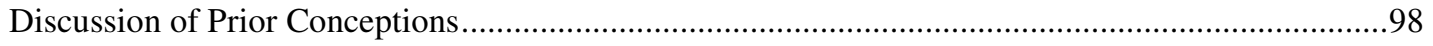

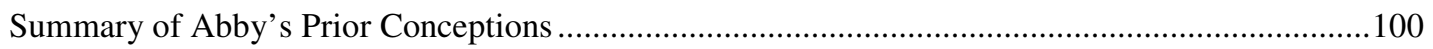

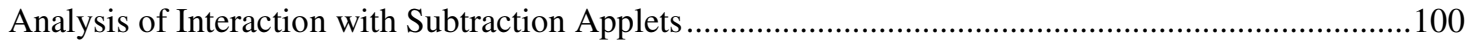

Abby's Interaction and Understanding with Technology ................................................................100

Summary of Abby's Interaction and Understanding with Technology ............................................111

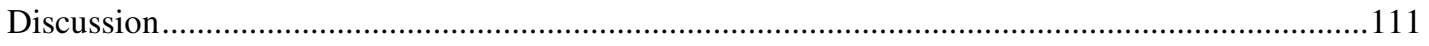

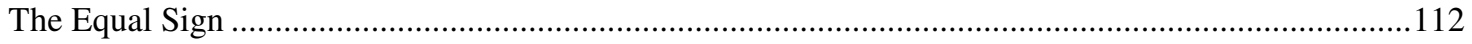

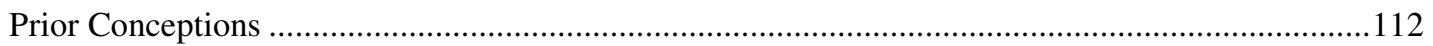

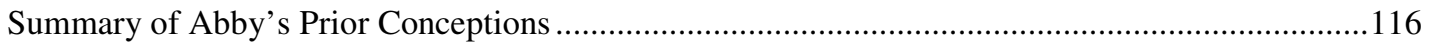

Analysis of Interaction with the Equality Applet ……......................................................................116

Abby's Interaction and Understanding with Technology ............................................................... 116

Summary of Interaction and Understanding with Technology .......................................................122

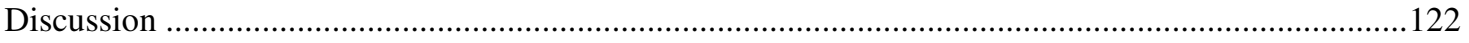


Jim's Background

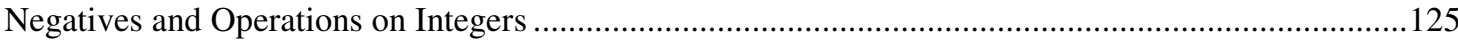

Prior Conceptions on Addition ......................................................................................... 125

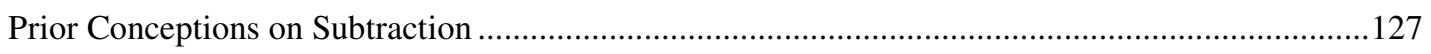

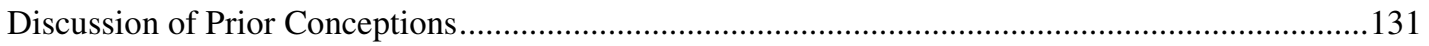

Summary of Jim's Prior Conceptions .................................................................................. 133

Analysis of Interaction with Subtraction Applets ........................................................................ 133

Jim's Interaction and Understanding with Technology ...................................................133

Summary of Jim's Interactions and Understanding with Technology .....................................140

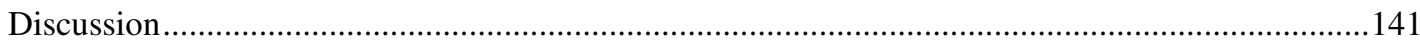

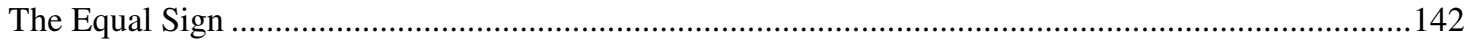

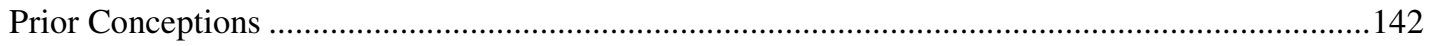

Summary of Jim's Prior Conceptions ................................................................................ 144

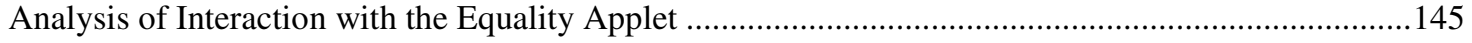

Jim's Interaction and Understanding with Technology .................................................... 145

Summary of Interaction and Understanding with Technology ................................................153

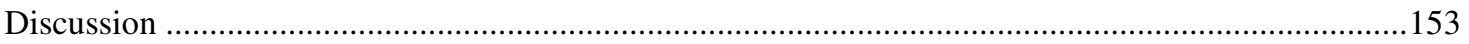

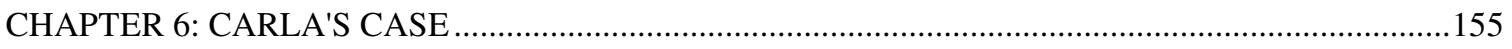

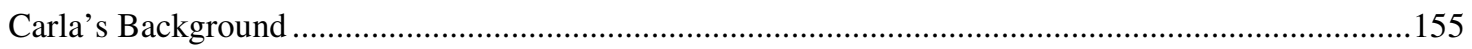

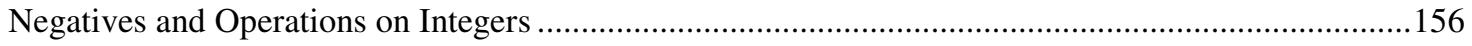

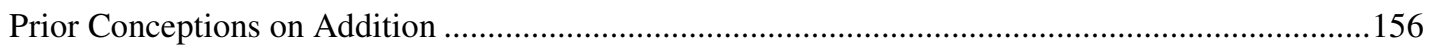

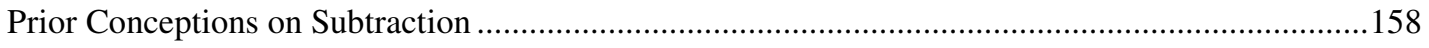

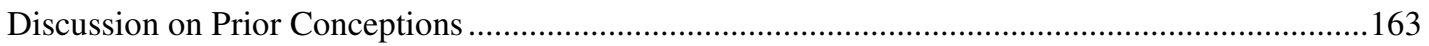

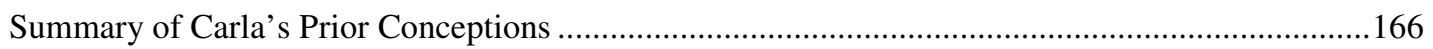

Analysis of Interaction with Subtraction Applets ............................................................... 166

Carla's Interaction and Understanding with Technology .................................................... 166

Summary of Carla's Interactions and Understanding with Technology ......................................175

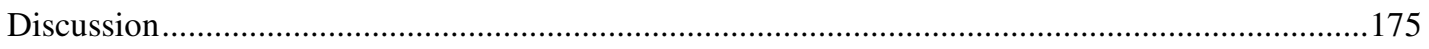

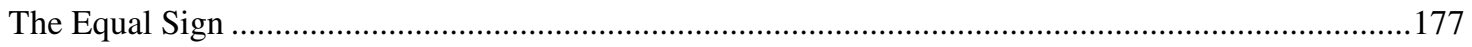




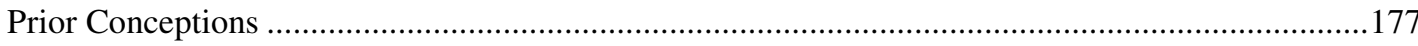

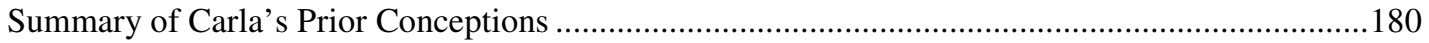

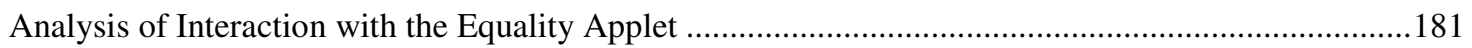

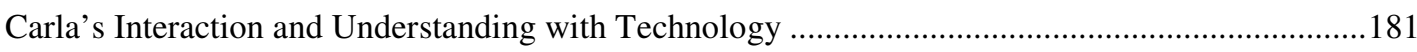

Summary of Interaction and Understanding with Technology .........................................................185

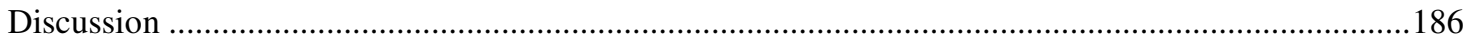

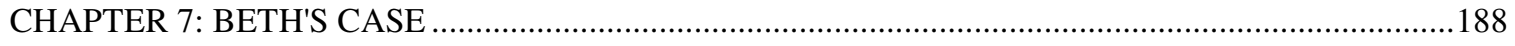

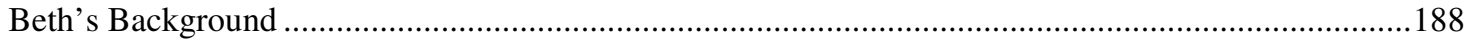

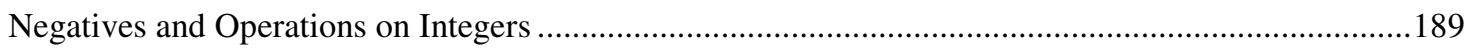

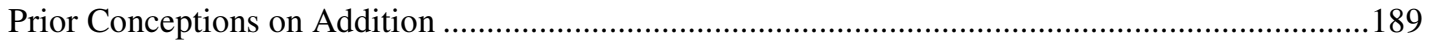

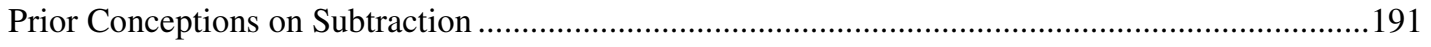

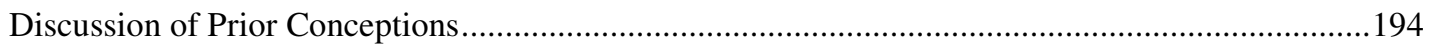

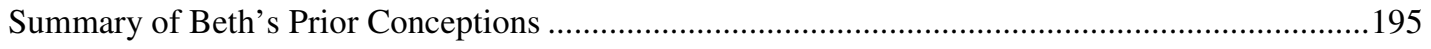

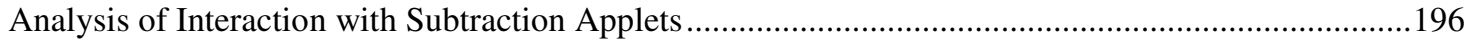

Beth's Interaction and Understanding with Technology................................................................. 196

Summary of Beth's Interactions and Understanding with Technology ............................................208

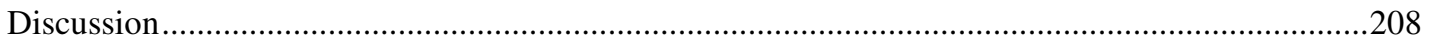

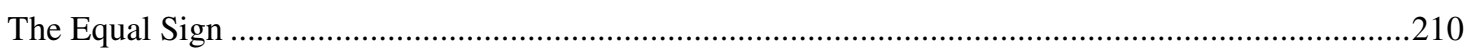

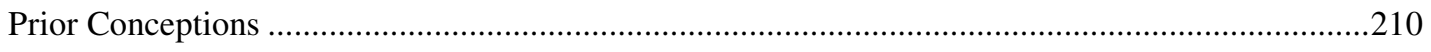

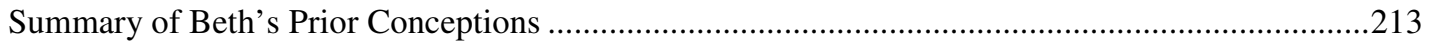

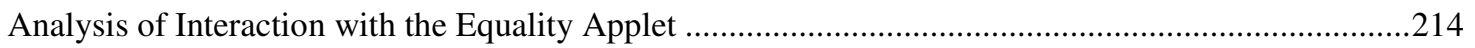

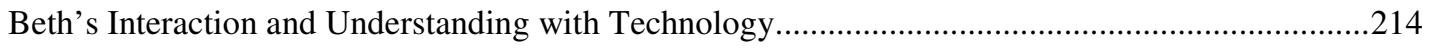

Summary of Interaction and Understanding with Technology .........................................................218

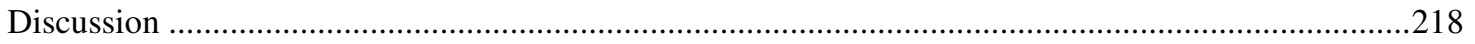

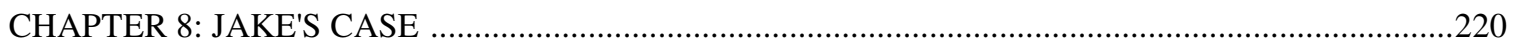

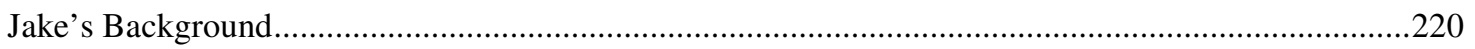

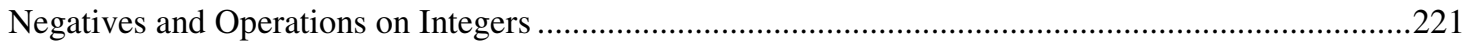

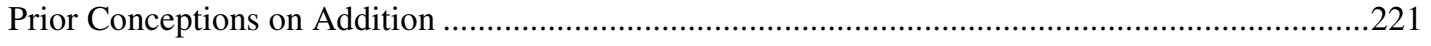

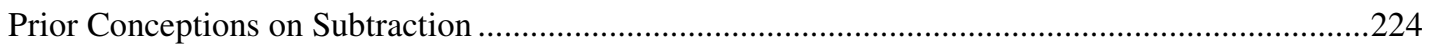




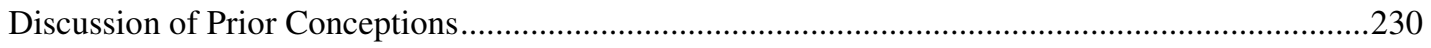

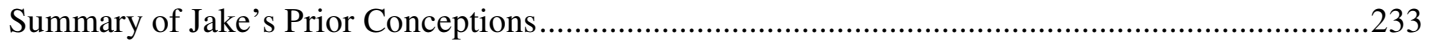

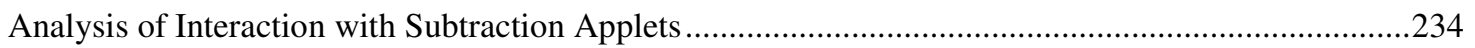

Jake's Interaction and Understanding with Technology ................................................................23

Summary of Jake's Interactions and Understanding with Technology .............................................245

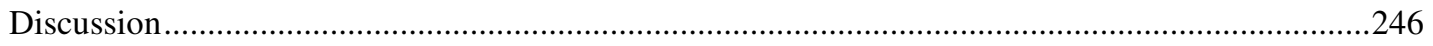

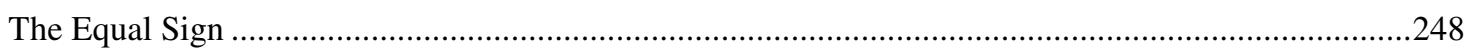

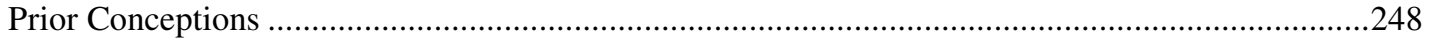

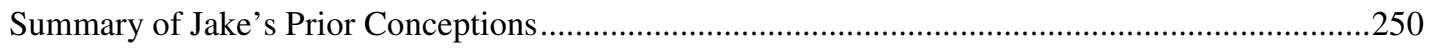

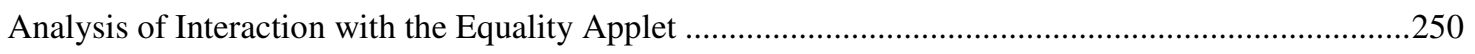

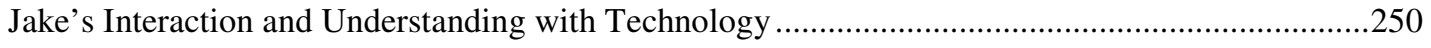

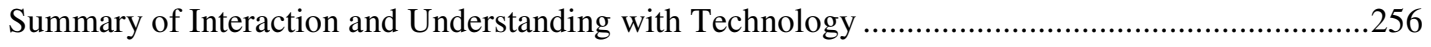

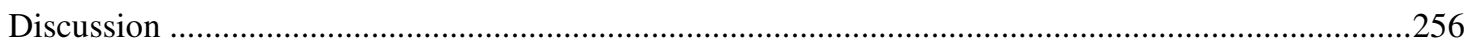

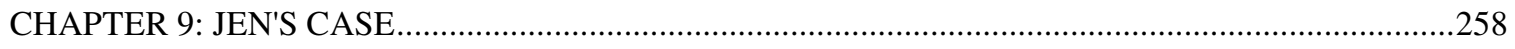

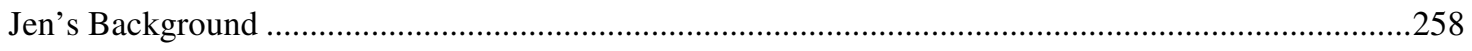

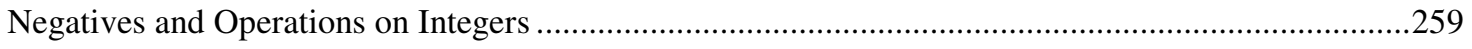

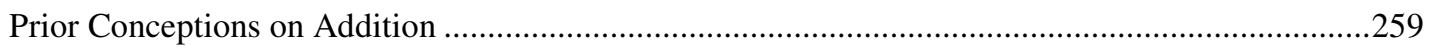

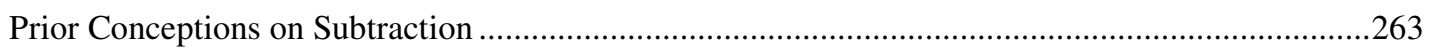

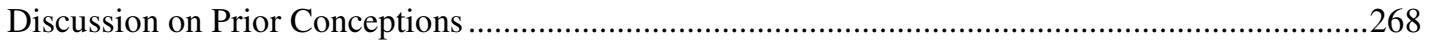

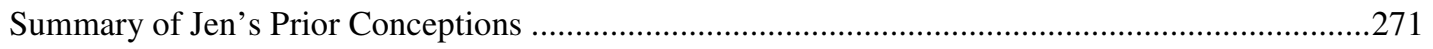

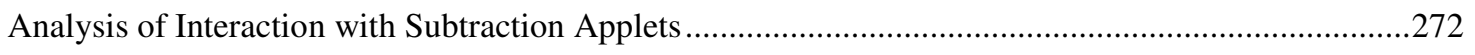

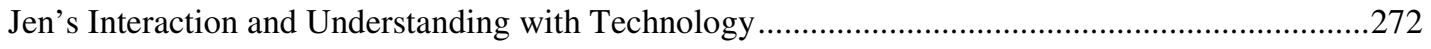

Summary of Jen's Interactions and Understanding with Technology ................................................281

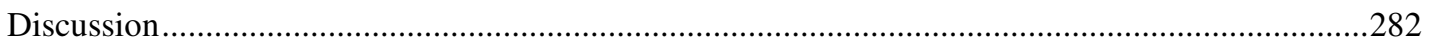

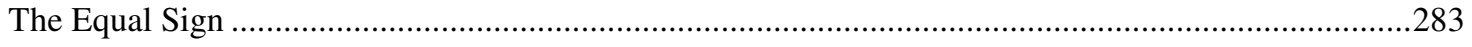

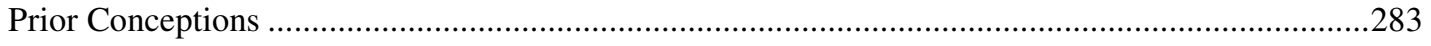

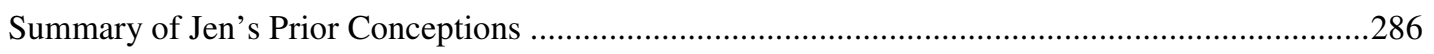

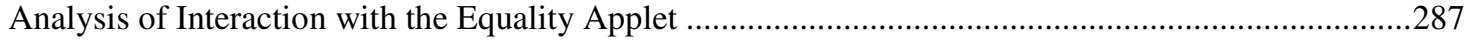

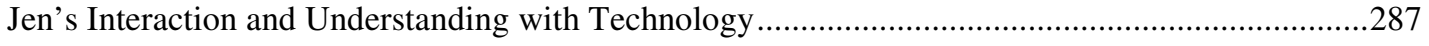

Summary of Interaction and Understanding with Technology ……..............................................29 
Discussion

CHAPTER 10: HOPE'S CASE.

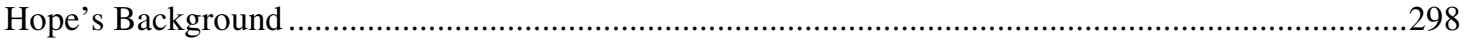

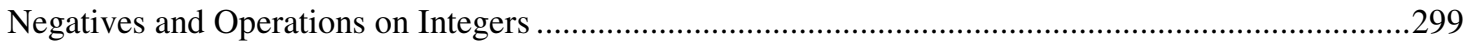

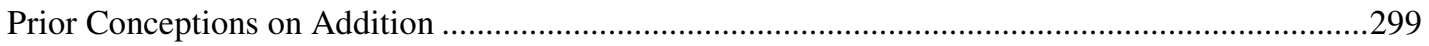

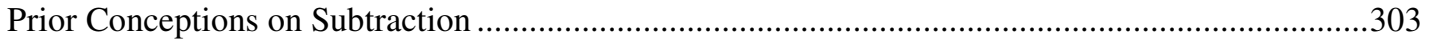

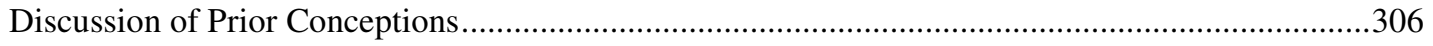

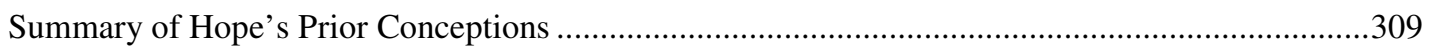

Analysis of Interaction with Subtraction Applets ..............................................................

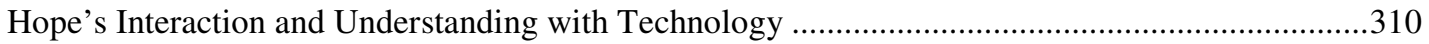

Summary of Hope's Interactions and Understanding with Technology ........................................325

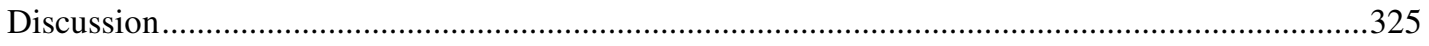

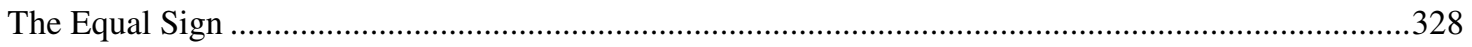

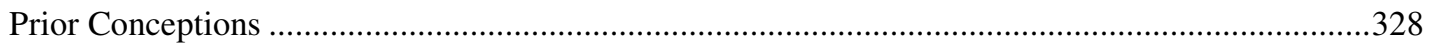

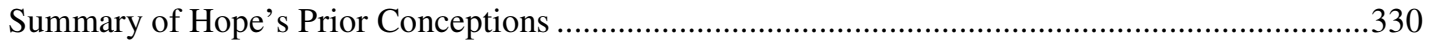

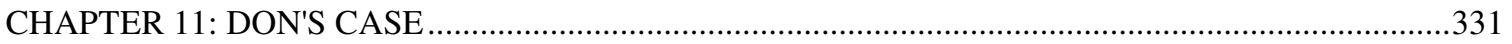

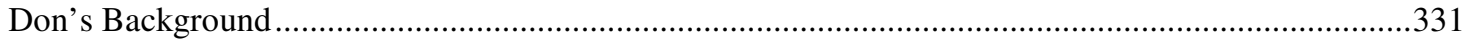

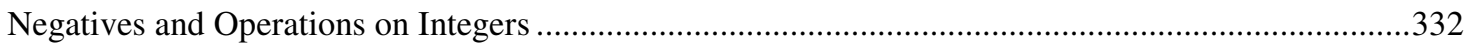

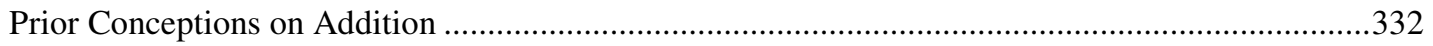

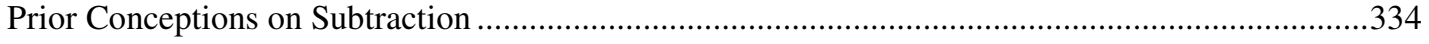

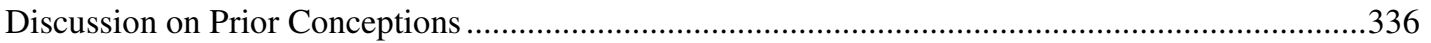

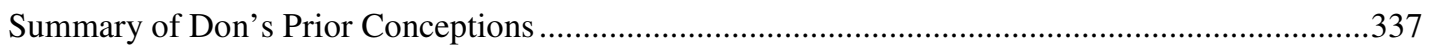

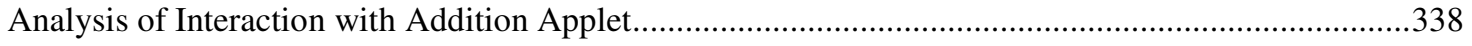

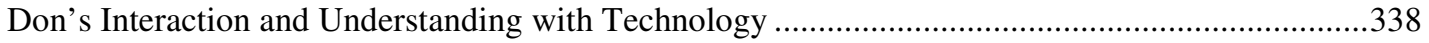

Summary of Don's Interactions and Understanding with Technology ........................................348

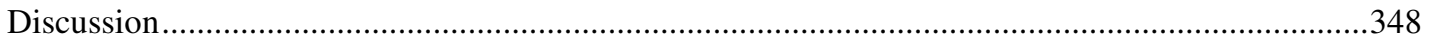

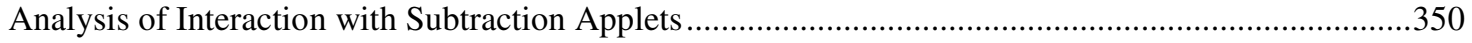

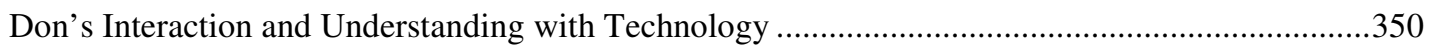

Summary of Don's Interactions and Understanding with Technology ......................................364

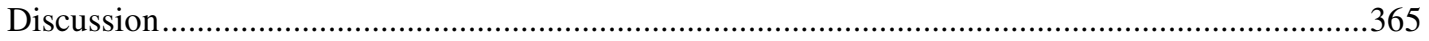




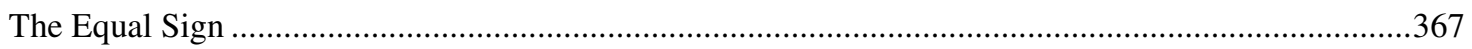

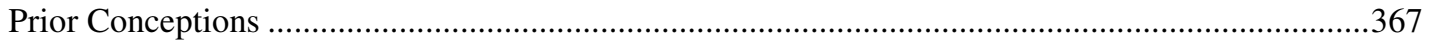

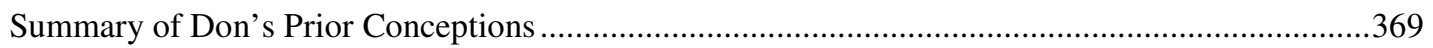

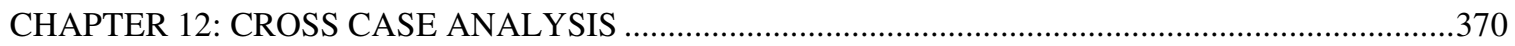

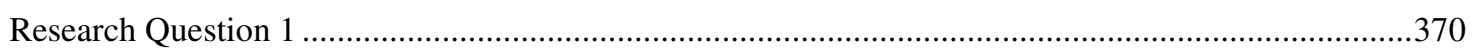

Participants' Initial Understanding of Addition and Subtraction of Integers .........................................371

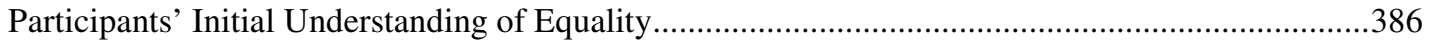

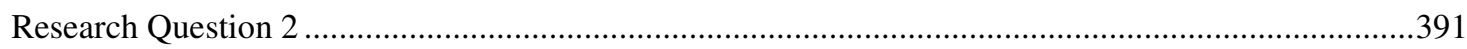

Participants' Final Understanding of Addition and Subtraction of Integers .......................................391

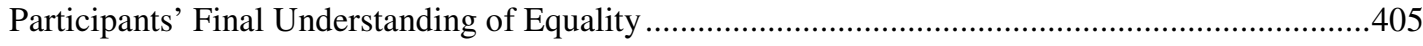

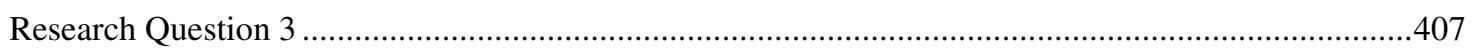

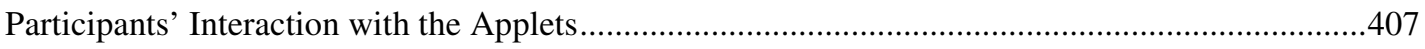

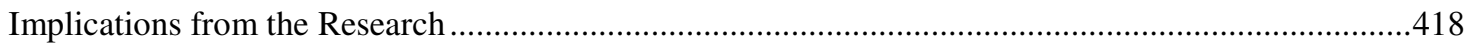

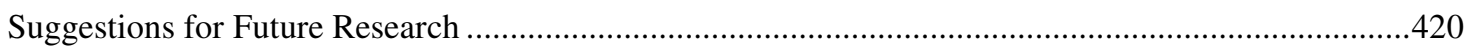

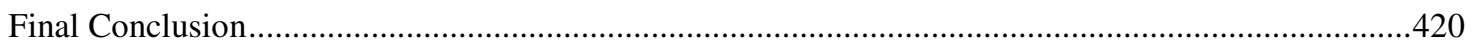

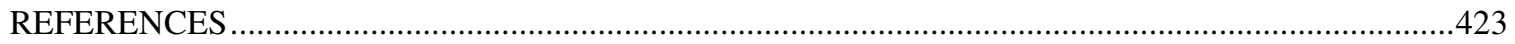

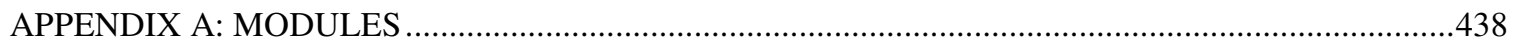




\section{LIST OF TABLES}

Table 2.1. Success Rates on Operating on Negative Numbers 18

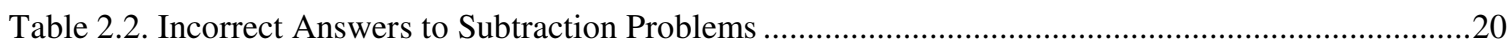

Table 2.3. Codes and Characteristics of Student Responses ...........................................................................

Table 2.4. Interview Contexts and Questions .......................................................................................... 41

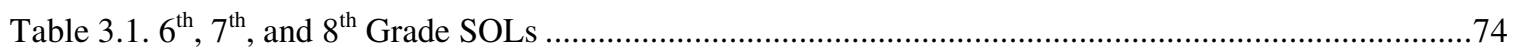

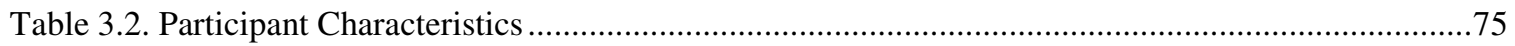

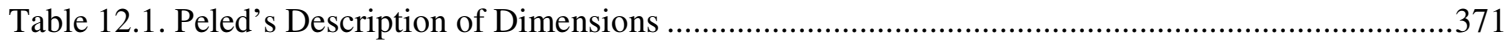

Table 12.2. Initial Participant Levels in Quantitative and Number Line Dimensions ..................................372

Table 12.3. Initial Dimension Levels and How Students Operated for Addition Tasks................................374

Table 12.4. Using Memorized Facts and a Number Line for Addition Task Justification ............................375

Table 12.5. How Participants Initially Operated on Specific Forms of Addition Tasks ...............................377

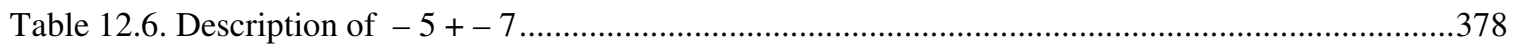

Table 12.7. Participants' Reasoning of Addition with a Negative Addend ................................................379

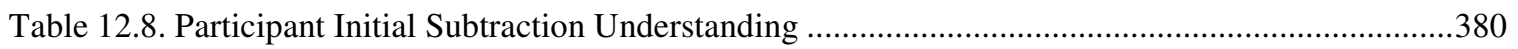

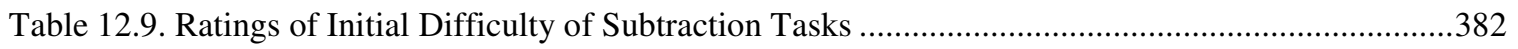

Table 12.10. Participant Addition and Subtraction Initial Understanding ....................................................384

Table 12.11. Participants' Responses to Task 1, Module 3 ...........................................................................387

Tabe 12.12. Six Participants' Initial Understanding of Solving Equations ..................................................38

Table 12.13. Responses to Finding the Value of a Variable in an Equation ..................................................390

Table 12.14. How Participants, Who Had Three Sessions, Operated After Using Subtraction Applets ......392

Table 12.15. How Participants, Who Had Two Sessions, Operated After Using Subtraction Applets ........393

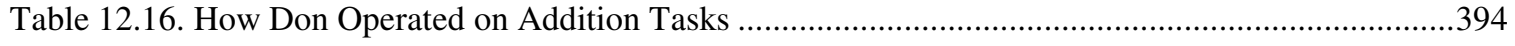

Table 12.17. Participant QD and NLD Levels Before and After Using Subtraction Applets .....................395

Table 12.18. How Participants Operated Beginning of First Interview and End of Last Session ................399

Table 12.19. Type of Representations Used By Participants ...................................................................403

Table 12.20. Six Participants' Initial Understanding of Solving Equations ...............................................406

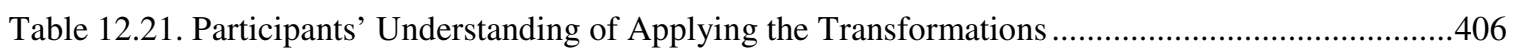

Table 12.22. Participants' Understanding of Order of Operations to Solve Equations ................................406

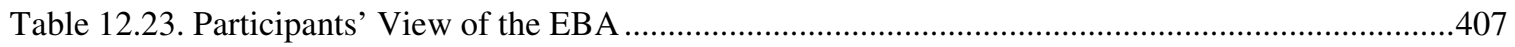

Table 12.24. Participants' Movement Translation on the EBA to Symbolic Operations .............................407 


\section{LIST OF FIGURES}

Figure 2.1. Three-digit subtraction problem with answers based on inappropriate application of subtraction

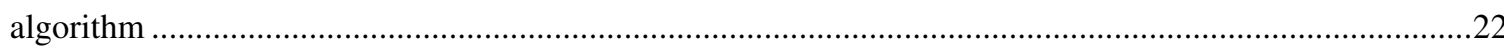

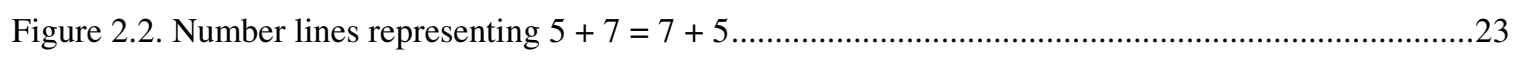

Figure 2.3. Example questions utilized to interpret student transference between dimensions....................28

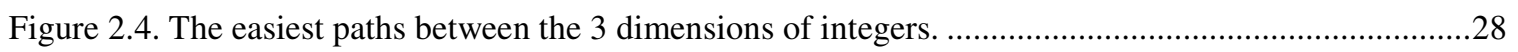

Figure 2.5. Adding integers on the addition arrow applet (AAA) .......................................................59

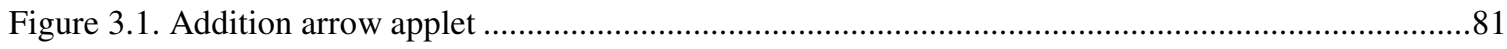

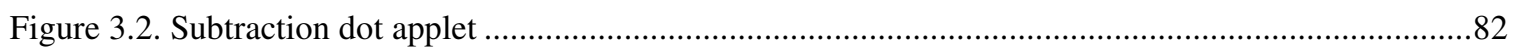

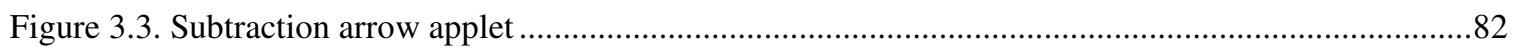

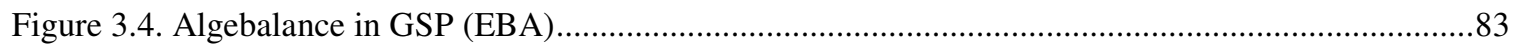

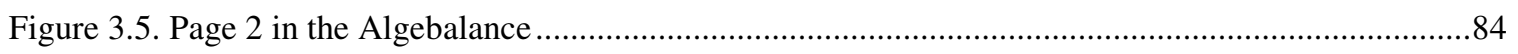

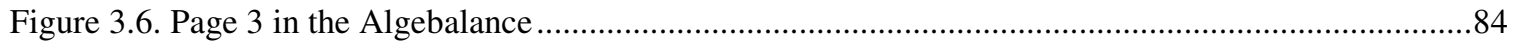

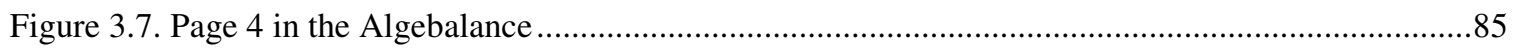

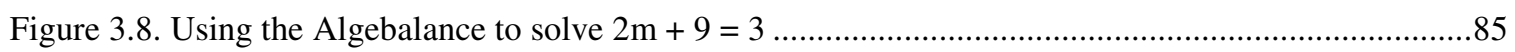

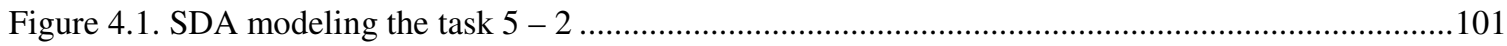

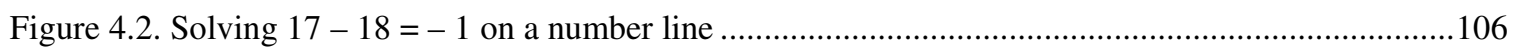

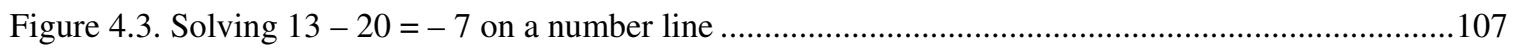

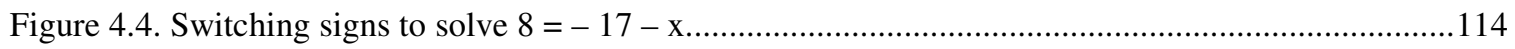

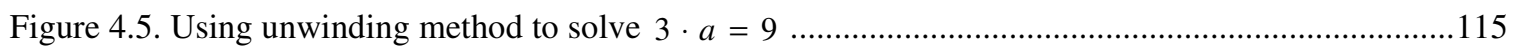

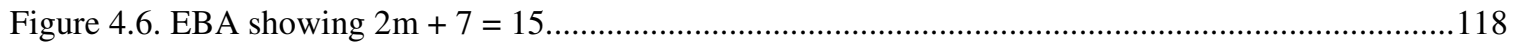

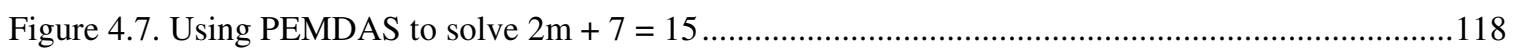

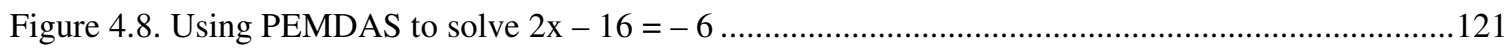

Figure 4.9. Solving $2 \mathrm{x}-16=-6$ using the balance method................................................................121

Figure 5.1. Jim switching absolute values of minuend and subtrahend...................................................129

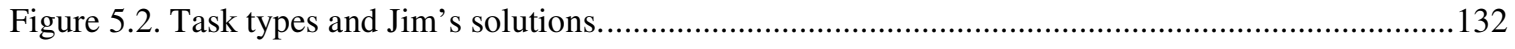

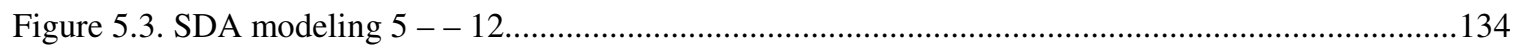

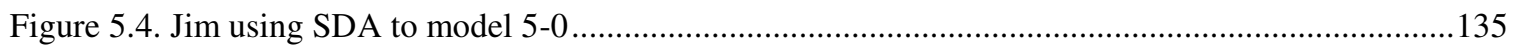

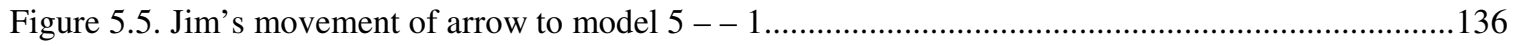

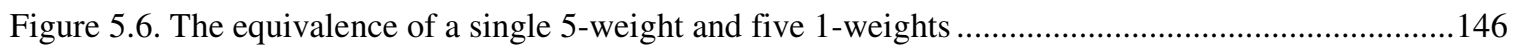


Figure 5.7. EBA illustrating $2 \mathrm{~m}+9=3$

Figure 5.8. Jim trying to add $\mathrm{a}-\mathrm{m}$ balloon to the left and right pan................................................... 147

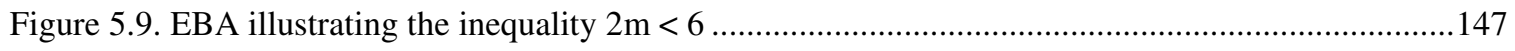

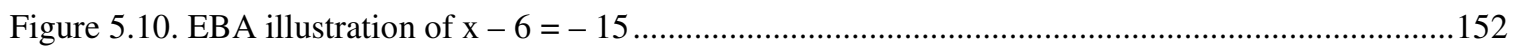

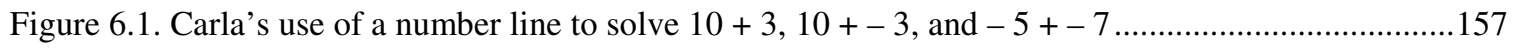

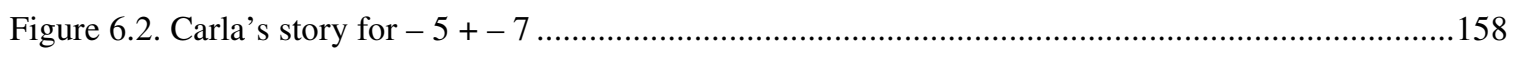

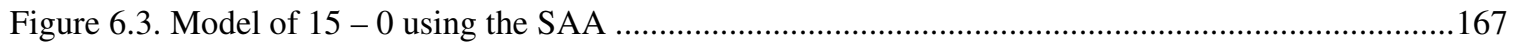

Figure 6.4. Carla trying to model $-4--1$ but instead modeling $-4-1$ on the SAA ...........................168

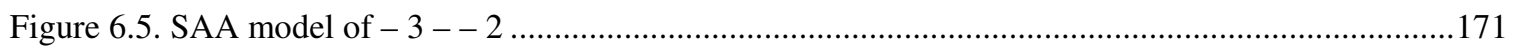

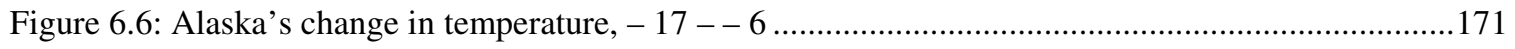

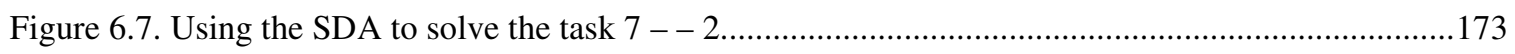

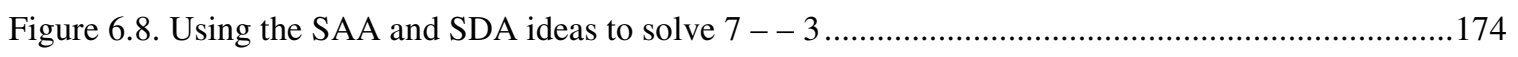

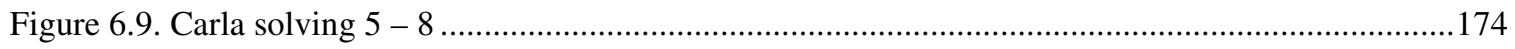

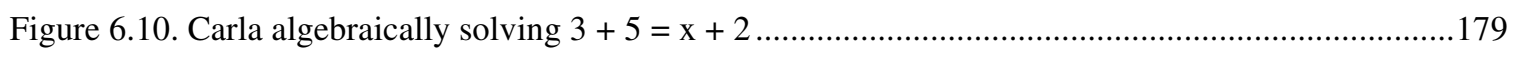

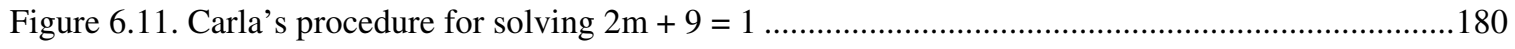

Figure 6.12. Pans containing a 5-weight on the left and a -5 and -1 balloons on the right ....................181

Figure 6.13. Matching the balloons and weights of opposite value....................................................181

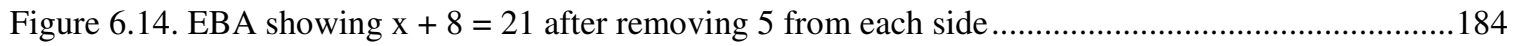

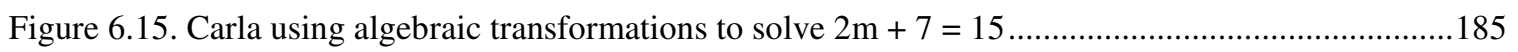

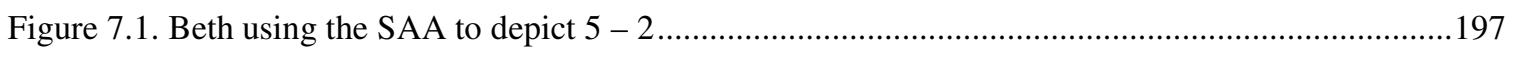

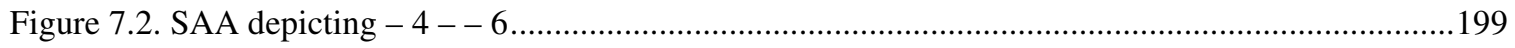

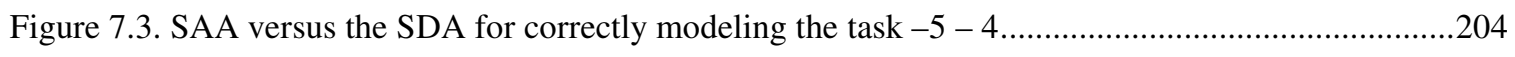

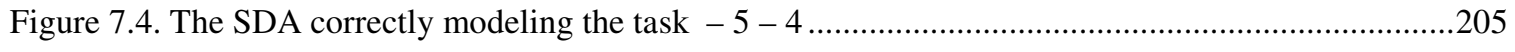

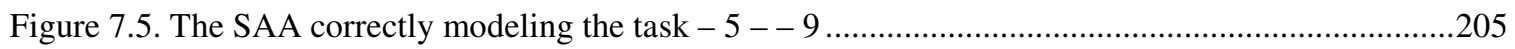

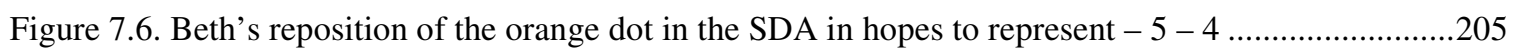

Figure 7.7. Beth's illustration of the task $-4--1$ on a number line

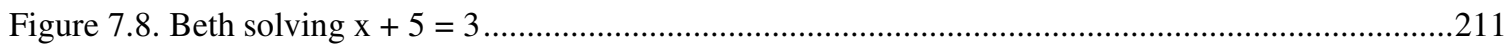

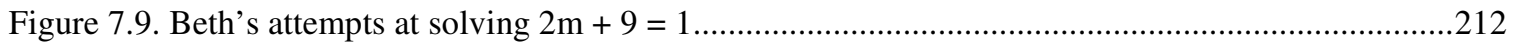

Figure 7.10. Exchanged one 5-weight for five 1-weights to balance left pan. ...................................214

Figure 7.11. The EBA illustration of $3+5=?+2$, and the EBA after Beth removed 2 from the left pan.215

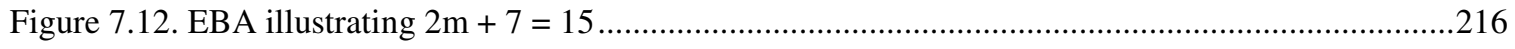


Figure 7.13. Replacing the three 1-weights to the wrong side

Figure 7.14. Correlating manipulations on EBA to solving equations algebraically

Figure 8.1. Jake moving to the right by one to see decrement in arrow value. .235

Figure 8.2. Jake using the SAA and using the take-away method to solve 5-2 .................................236

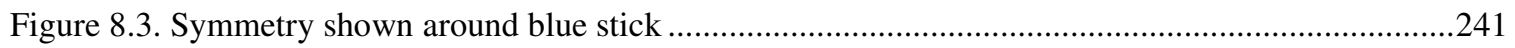

Figure 8.4. Using a number line to emulate the SAA ....................................................................242

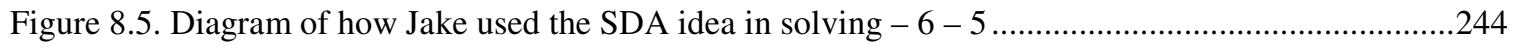

Figure 8.6. The exchange of weights that maintained the balance of the pans......................................250

Figure 8.7. EBA showing $x+8=21$ after removing 6 from each side...........................................252

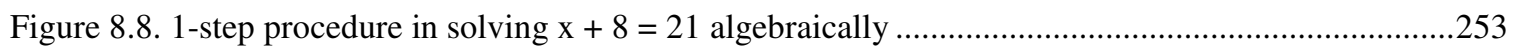

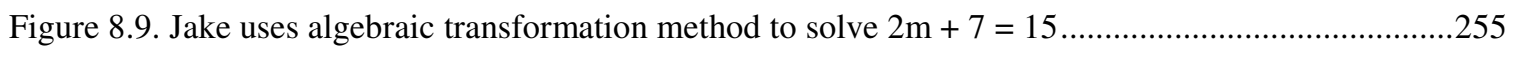

Figure 9.1. Moving from a number line to arithmetic representation................................................261

Figure 9.2. Solving a temperature task on a number line and writing the corresponding equation............268

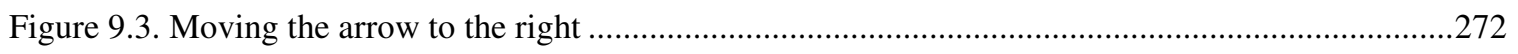

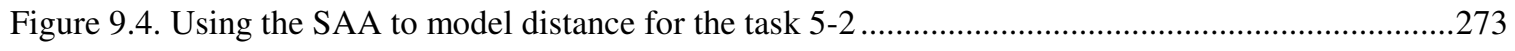

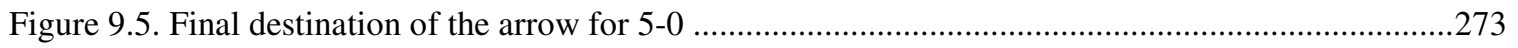

Figure 9.6. Jen illustrating 5-5 using distance method on the SAA .................................................273

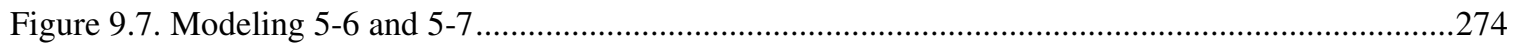

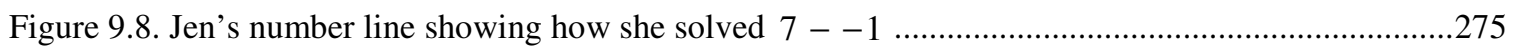

Figure 9.9. Visualizing a second number line above the original blue number line.............................2276

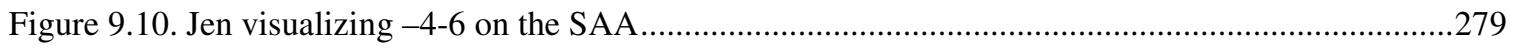

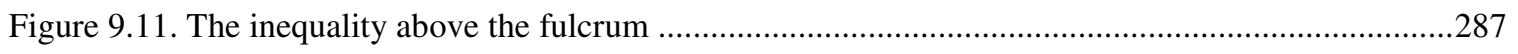

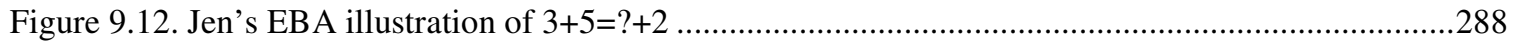

Figure 9.13. After removing two 1-weights from both sides..........................................................290

Figure 9.14. Moving a -5 balloon from the right pan to the storage unit ...........................................291

Figure 9.15. After subtracting -10 from both sides in the equation $x-20=-13 \ldots \ldots \ldots \ldots \ldots \ldots \ldots \ldots \ldots \ldots . . . \ldots \ldots 1$

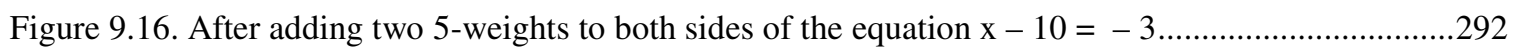

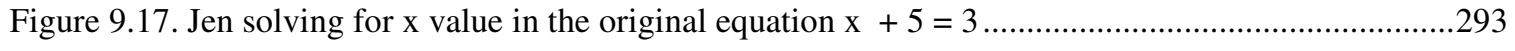

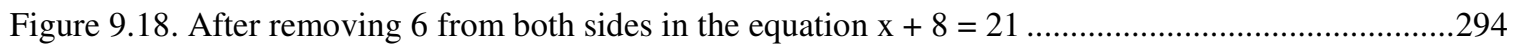

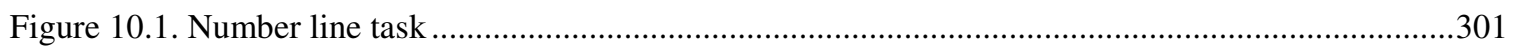

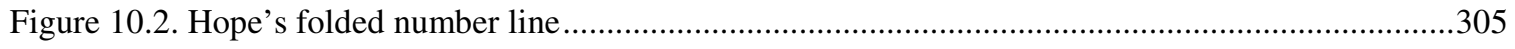




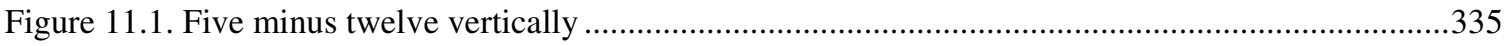

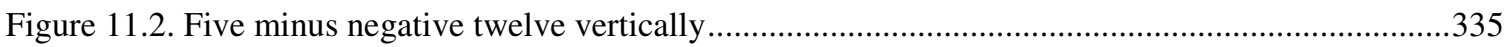

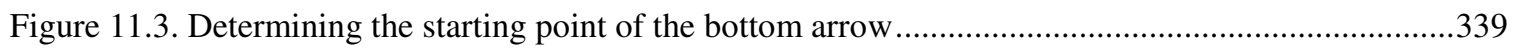

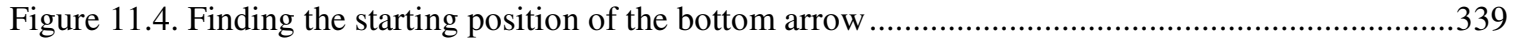

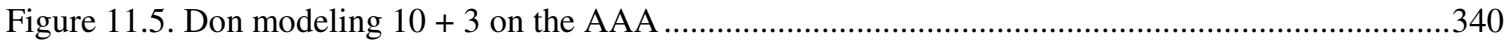

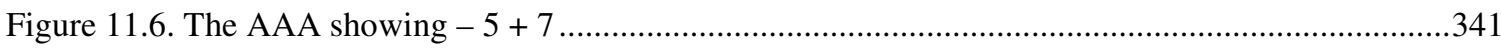

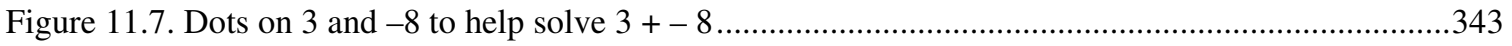

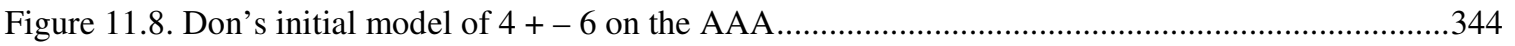

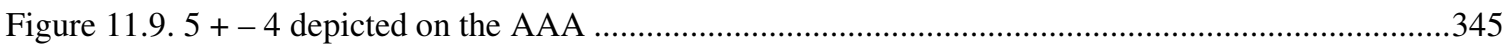

Figure 11.10. Don's drawing of how he solved $-8+-2$ on the number line .............................................347

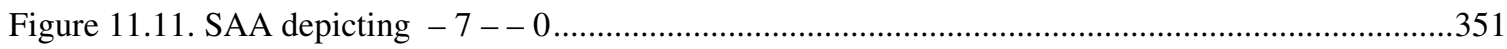

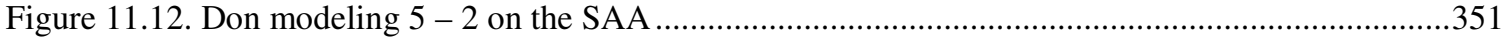

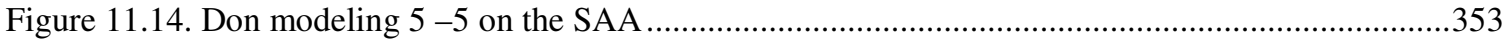

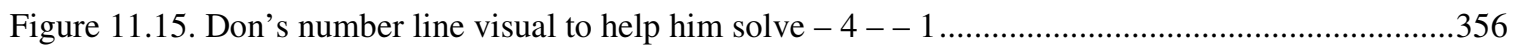

Figure 11.16. Don's drawing of how he solved $8-3$ using a number line …..............................................357

Figure 11.17. Don's drawing of how he solved $8--2$ using a number line .............................................358

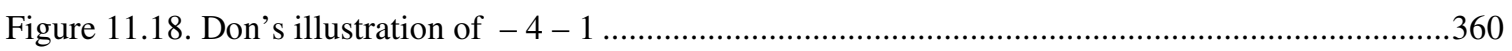

Figure 12.1. After adding two 5 -weights to both sides of the equation $\mathrm{x}-10=-3 \ldots \ldots \ldots \ldots \ldots \ldots \ldots \ldots \ldots \ldots \ldots \ldots \ldots . . . . .110$

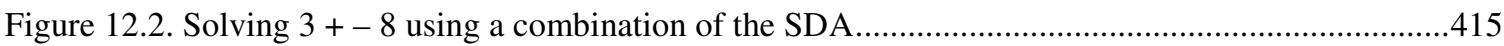




\section{CHAPTER 1}

\section{INTRODUCTION}

Algebra is frequently referred to as a "gatekeeper" that either grants or denies access to many professional and personal opportunities (Kronholz, 1998; Moses \& Cobb, 2001; Payne \& Watson, 1997; Piccioto \& Wah, 1993). If one gains access, he can expect great prospects in the professional world in terms of higher education and business as well as in personal and societal dealings.

Parents, teachers, and counselors use Algebra I scores to determine the course sequence students will take. Algebra is one of the courses that "tracks the students who take it onto a college-bound curriculum" (Payne \& Watson, 1997). Students who do well in Algebra I before or during the first year of high school can take more advanced mathematics. Students, who do poorly in Algebra I, will most likely be placed on a path that will not rigorously prepare them for college admission. Furthermore, both tests for college admission, the SAT and ACT, are heavily weighted with algebra questions.

Akerhielm, Berger, Hooker, and Wise (1998) showed that students who took more advanced mathematics courses in high school had a higher likelihood of attending college. Eighty-seven percent of students who took at least Algebra II in high school attended college, whereas only $50 \%$ of those students who did not take Algebra II went to college. Ninety-seven percent of students who took calculus continued on to college. While the correlation between advanced mathematics courses and college attendance is strong for all students, it is particularly stronger for low-income students. The differences between students attending college who took Algebra II and who did not take Algebra II was larger for lower income students $(75.9 \%$ versus $31.9 \%)$ than for middle-income students $(85.5 \%$ versus $54.9 \%)$ or higher income students $(94.7 \%$ versus $75.3 \%)$. There was a greater gap between those who attended college and who did not attend college depending on the family's income status if the student took or did not take calculus in high school. The difference between students attending college who took calculus and those who did not take calculus for the lower income students was almost twice as large as for the middle income students and almost 3.5 times as large as the higher income students. While more individuals with a higher income took more advanced mathematics courses, there was a smaller discrepancy between income and attending college given that one took more advanced mathematics courses. Ninety-two percent of students in the lower family income group who took pre-calculus attended college compared to $94.9 \%$ of students in 
middle income group and $98.2 \%$ of students in the higher income group. Ninety-four point eight percent of students in the lower family income group who took calculus attended college compared to $94.5 \%$ of students in middle-income group and $99.4 \%$ of students in the higher income group. Since Algebra I must be taken prior to any of these advanced mathematics courses, taking algebra improves the probability of a student going to college.

Furthermore, a student who takes Algebra I sooner can take more advanced courses (i.e. calculus) in high school, and when he does so the income barriers of going to college lessen. In fact, students who took Algebra I in eighth grade were more likely to attend college than those who did not (Atanda, 1999), and this finding held across income groups (Akerhielm et al, 1998). When eighth graders, who took algebra and who did not take algebra, were grouped according to low, medium, or high family income, there was a stronger relationship between taking Algebra I in eighth grade and attending college (Akerhielm et al, 1998). There was a 21 percentage point difference between the percentages of lower income students who attended college and took Algebra I in eighth grade (61.3\%) and those who attended college and did not take Algebra I in eighth grade (39.6\%). This can be compared to a 15-percentage point difference for middle-income students (78.4\% versus $63.5 \%)$ and a 10 -percentage point difference for higher income students $(90.7 \%$ versus $80.9 \%)$.

The above statistics came from the analysis of the National Longitudinal Survey (NELS: 88) with follow-up surveys in 1990,1992, and 1994 as well as the 1993 National Postsecondary Student Aid Study (NPSAS) data. More recent data on high school graduates have been obtained from Educational Longitudinal Study of 2002 first follow-up in 2004 (Dalton, Ingels, Downing, \& Bozick, 2007). However, this data only compared high school mathematics classes taken and educational expectations rather than compared high school mathematics courses taken and college attendance. Nonetheless, Akerhielm and colleagues (1998) showed that there was a high correlation between educational expectations and college attendance: "the higher one's educational expectations, the more likely one is to attend postsecondary education" (p. 32).

With the understanding that there can be a positive correlation between one's college attendance and one's expectation of going to college, one can interpret the data presented by Dalton and colleagues (2007). They found that the students who expected a higher degree were students who completed higher mathematics courses in high school in 2004. In 2004, $53 \%$ of students who expected to earn a graduate degree and $33 \%$ of students who expected to earn a baccalaureate degree completed pre-calculus or calculus, compared to only $7.7 \%$ of students who expected to earn some postsecondary degree and $2.2 \%$ of 
students who expected to earn a high school degree, GED, or less. In contrast, $9 \%$ of students who expected to earn a graduate degree and $17 \%$ of students who expected to earn a baccalaureate degree completed Algebra I or lower as their highest mathematics course, compared to $47 \%$ of students expecting to earn some postsecondary degree and $65 \%$ expecting to earn a high school degree, GED, or less. Considering the positive correlation between college attendance and expectations, one can deduce that students who went to college completed a higher mathematics course.

Once in college, many majors require intermediate algebraic knowledge especially mathematics, science, technology, engineering, economics, electronics, medicine, and business. These algebra dependent majors feed directly into scientific, technological, and professional careers, which are the some of the fastest growing jobs (National Council of Supervisors on Mathematics, 1989; National Research Council, 1991).

Even careers that do not require higher education still call for the thinking and communication skills acquired in basic algebra, for "over $75 \%$ of jobs require proficiency in fundamental algebraic concepts" (Mathematical Sciences Education Board, Board on Mathematical Sciences, Committee on the Mathematical Sciences in the Year 2000, \& National Research Council, 1989, p. 4). When one experiences algebra, one can learn how to understand problems, model those understandings in a symbolic fashion, reason on those symbols, and communicate the solution and the reasoning behind it. This critical thinking requires one to be flexible and efficient in strategies, to work with incomplete information, and to recognize flawed logic.

Algebra skills not only influence one's profession, but they also influence private lives and society as well. Since the study of algebra can enhance critical thinking (Ballew, 1965), people are able to decipher and interpret quantitative information in the form of business, financial, and health issues that require informed decisions. Whether these issues come in the form of bank interest, stock market changes, retirement options, or health statistics, they impact the common citizen. Individual decisions affect one's microenvironment but also affect one's macro environment; thus, making it beneficial for a society to consist of members who are able to take in, comprehend, analyze, and make appropriate decisions based on information, which is rapidly changing. Fey (1989) referred to algebra courses as a "training ground in careful rule-governed reasoning" (p. 207), for it allows people to look beyond the presented information and get to the heart of the matter. The ability to fully understand a problem allows society to make informed decisions and properly question authority on unclear information as well as recognize when information is withheld. 
Research has shown that the students in the United States lag behind their international economic counterparts in mathematics achievement (Center for Public Education, 2005; Mathematical Sciences Education Board \& National Research Council, 1990; Erbas, 2004; Mullis, Martin, Gonzalez, \& Chrostowski, 2004; Stevenson, Lee, \& Stigler, 1986; Zuckerbrod, 2007) while the mathematical demands of employment are increasing because "technology has mathematized the workplace" (Mathematical Sciences Education Boards, Board on Mathematical Sciences, Committee on the Mathematical Sciences in the Year 2000, \& National Research Council, 1989). It is noted in America 2000 (U. S. Department of Education, 1991, p.9) that "employers cannot hire qualified workers" and either spent significant amounts of time on remedial training on subjects, one of which is algebra, or exported skilled work to other countries. Nussbaum (1988) gave a perfect example of this occurring with the IBM corporation: "IBM Corp. discovered after installing millions of dollars worth of fancy computers in its Burlington (VT) factories that it had to teach high-school algebra to thousands of workers before they could run them" (p. 100). He accused the United States of "scrimping on human capital" and called for a change in education.

What has been done by researchers, educators, and the government to help students to better understand algebra so that companies can employ knowledgeable workers and America can catch up to other countries? In the 1986, the Research Advisory Committee of the National Council of Teachers of Mathematics (NCTM) received funding from the National Science Foundation to study four areas of mathematics, one of which was the teaching and learning of algebra. In May of 1987, a conference was held consisting of national and international mathematicians, mathematics educators, psychologists, technologists, researchers, practitioners and curriculum developers discussing the following themes: "(1) What is algebra and what should it become, in light of continuing technological advances?; (2) What has research told us about the teaching and learning of algebra?; (3) What is algebraic thinking and how does it relate to general mathematical thinking?; (4) What is the role of representations in the learning of algebra?" (Sowder, 1989, pp. viii-ix). Presented papers focused on possible solutions to observed algebraic difficulties. The following were mentioned as a few difficult topics for students: variables, writing of expressions and equations, and solving equations. Many suggestions to these problems centered on new instructional approaches. These new approaches advocated the use of technology to facilitate numerical discovery of properties over the traditional axiomatic approach. By focusing on discovery through numbers, students can develop conceptual knowledge about properties rather than strictly learning how to manipulate symbols.

In May of 1997, the NCTM, National Academy of Science, and the Mathematical Sciences 
Education Board (MSEB) joined together to hold a national symposium on The Nature and Role of Algebra in the $K$-14 Curriculum to discuss the following questions: "What do we mean by algebra and algebraic thinking?" and "What do American students really need to know about and be able to do with algebra?" (Alberts, 1998, p. xi). These groups suggested using technology and multiple representations to transform past symbol-pushing algebra instruction into instruction that allows students opportunities to ask "what if", "how come", and "what happened" (Zbiek, 1998, p. 36), so that students could create their own conceptual knowledge. Furthermore, these groups advocated that all students are "algebraically educable" in that all students are capable of using and understanding symbols, generalizing rules and procedures from patterns, noticing relational changes between values, and modeling relationships and situations. These capable students are not restricted to high school students, but also include middle school and elementary school students. During the conference, Kleiman (1998) presented his model of teaching middle school algebra to illustrate that appropriate instruction can develop middle school students' algebraic knowledge. He used contextual situations and mathematical representations to teach topics such as signed number operations, equivalence, and variable.

Yet, even now students' misunderstanding of algebra persists. The 2007 NAEP results show that $29 \%$ of our national eighth graders are below basic performance, $39 \%$ of eighth graders are at basic performance, $25 \%$ of eighth graders are at proficient performance, and only $7 \%$ of eighth graders are at advanced performance in mathematics. Students functioning at basic level are able to solve algebra problems with the help of "diagrams, charts and graphs" but have "limited skill in communicating mathematically" (Lee, Grigg, \& Dion, 2007, p. 36). Students operating on the proficient level can relate arithmetic ideas to algebra topics as well as choose appropriate equations to solve practical problems. Students operating on the advanced level are able to think abstractly and "explain the reasoning processes underlying their conclusions" (p. 36). What is disturbing is that the $90^{\text {th }}$ percentile score in 2007 was 327 , which while it lies in the proficient level, the type of algebra problem associated with the scale score of 329 asks students to "convert a temperature from Fahrenheit to Celsius" (p.36). Thus, ninety percent of the nation's eighth-grade students cannot answer questions similar to questions that ask one to convert temperatures to different scales.

Many researchers believe this poor understanding of algebra is due to the continued instruction that disseminates rules, formulas, and worked-out problems (Kieran, 2007; Mitchell, Hawkins, Jakwerth, Stancavage, \& Dossey, 1999). Educators (French, 2002; NCTM, 2000; Swan, 2000) believe that algebraic instruction should be appropriately enhanced by technology and the use of multiple representations to allow 
students to think about, construct, and reflect on ideas learned through problem solving.

For the past twenty years, students continue to have difficulty with translating word problems into algebraic equations (Hubbard, 2003; Lochhead \& Mestre, 1988; Philipp, 1992; Wolman, 1983;). However, this is not surprising, since students also have had problems with foundational topics such as operations on integers (Gallardo, 2002; Kieran, 2007; Murray, 1985; Silver \& Kenney, 2000), variable (Philipp, 1992; Rosnick, 1981; Schoenfeld \& Arcavi, 1988; Stacey \& MacGregor, 1997b; Usiskin, 1988), and equivalence (Baroody \& Ginsberg, 1983; Behr, Erlwanger, \& Nichols, 1980; Falkner, Levi, \& Carpenter, 1999; Stacey \& MacGregor, 1997a), which provide a basis for translating word problems into algebraic equations. When one considers how these basic concepts influence later concepts in algebra, it is no wonder that students have not been successful in algebra.

Reason for Studying Middle School Students' Understanding of Pre-algebra

Ensuring students begin learning pre-algebra and algebra in middle school increases the probability that they will open the doors to many opportunities in their educational, professional, and personal lives. One concern about students engaging in pre-algebra and algebra at too early of an age is that they have not fully transitioned to formal thought; therefore, they will not comprehend it consequently do poorly in algebra (Piaget, 1964). Yet researchers (Davis, 1998; de Vise, 2005; Morris, 1980; Silva \& Moses, 1990; Sorrentino \& Curcio, 1986) have shown that middle school students have succeeded in learning algebra concepts. Many researchers have advocated the push for algebra for all students in the middle grades (Lawson, 1990 ; NCTM, 2000). NCTM has specifically stated that "students in middle grades should learn algebra both as a set of concepts and competencies tied to representation of quantitative relationships and as a style of mathematical thinking for formalizing patterns, functions, and generalizations" (p. 223). In doing so, middle school students should understand the meaning behind the fundamentals of algebra including arithmetic operations with integers and the understanding of the equal sign. Three reasons provide the basis for this viewpoint.

First, if students take algebra in middle school, they will be able to take the higher level of mathematics sequence in high school. According to NELS (Atanda, 1999) approximately $60 \%$ of the students who took calculus had taken algebra in $8^{\text {th }}$ grade. Moreover, students with conceptual algebraic understanding will enter calculus at any age with a better foundation and be more likely to succeed. This is precisely one of the long-range goals of The Algebra Project. One of The Algebra Project founders' fundamental beliefs is that sixth graders who take algebra will enter college mathematics preparatory course and do well in them (Silva \& Moses, 1990). 
Not only will taking algebra in middle school open doors to advanced mathematics in high school, but it will also allow students to take advanced science courses in high school as well. Mark Johnston, the assistant superintendent of the Arlington school district in Virginia, stated, "[A]lgebra is the gatekeeper for more advanced math, as well as for science courses" (de Vise, 2005). In Arlington, a quarter of middle school students took algebra in 1999-2000, where as in 2004-2005, nearly half the population did so. By taking algebra in middle school, students had more space in their schedule to take advanced mathematics courses. Furthermore, if students have succeeded in their algebra course, they will most likely do better in future educational endeavors and in their careers than students who have not (Evers \& Clopton, 2007; Usiskin, 1995).

Second, taking algebra in eighth grade allows one to pursue advanced mathematics courses in high school, which increases the likelihood of attending college. Not only does taking Algebra I earlier than high school decrease income barriers between students going to college, it also lessens the gap between minorities and non-minorities attending college. Pelavin and Kane (1988) found that the college attendance difference between minorities and non-minorities was less between students who took college preparatory mathematics courses than those students who took general mathematics.

Since taking algebra in eighth grade provides an opportunity to higher education and career opportunities regardless of income and race, it is necessary to have all students take algebra at a middle school level. Yet, the 1996 NAEP data showed that minority students reported taking algebra in eighth grade less than non-minority students (Department of Education, 1997). Not only did middle school aged minorities not take algebra as often as their counterparts; they also were more likely to drop mathematics courses sooner than their counterparts. A study from the National Action Council for Minorities in Engineering (NACME) found that $61 \%$ of minority students in Grades 5-8 reported that they were going to stop taking mathematics courses as soon as they fulfilled their requirements (Department of Education, 1997). If one can implement algebra for all students at the middle school level, many opportunities will become available for all students; therefore, lessening the achievement gap.

One final reason for implementing algebra at the middle school level is to minimize the distance between international competitors in mathematics. Instruction in the middle grades in the United States primarily focus on arithmetic, whereas other countries place a major focus on algebraic and geometric concepts (Department of Education, 1997). In 1997, the United States had $40 \%$ of its 8th grade mathematics curriculum made up of arithmetic, Germany had 13\%, and Japan contained none. Mathematics content taught at the $8^{\text {th }}$ grade level in the United States is comparable to the $7^{\text {th }}$ grade level 
for at least 40 other nations. This is one reason, why the United States lags behind other countries in mathematics. By starting algebra in middle school, the United States may be able to compete internationally on the same level in mathematics.

\section{Problem}

Despite the reasons for middle school students to take algebra and understand the basic principles, research has shown that algebra students often struggle to understand fundamental pre-algebraic concepts. Reasons for these difficulties include a poor knowledge base in arithmetic, poor transitioning of ideas from arithmetic to algebra, and sub-par instructional methods.

Skemp (1987) noted the necessity of understanding arithmetic prior to understanding algebra. He said, "to understand algebra without ever having really understood arithmetic is an impossibility, for much of the algebra we learn at school is generalized arithmetic" (p. 20). His insight is supported by the many studies trying to predict algebra achievement that found a high correlation between students' success in arithmetic and their success in algebra (Barnes \& Asher, 1962; Belli \& Gatewood, 1987; Flexer, 1983; Mogull \& Rosengarten, 1972; Sargent, 1985). Some arithmetic topics that carry over to algebra are operations on integers and the idea of equivalence. Based on Wright's (1991) findings of what arithmetic topics influence success in algebra, she suggested that students needed to develop a sense of number and operations rather than strict computational skills. If students develop a misconstrued number sense and operation sense, their misconceptions can flow over into their study of algebra and make their transition difficult.

One viewpoint of algebra is to see it as generalized arithmetic. From this standpoint, students' problems in algebra may stem from their problems in arithmetic, or their inability to distinguish differences between the procedures and notations of arithmetic and algebra. Booth (1988) recognized these causes of algebraic difficulties:

[A]lgebra is not separate from arithmetic; indeed it is in many respects 'generalize arithmetic.' And herein lies the source of other difficulties. To appreciate the generalization of arithmetical relationships and procedures requires first that those relationship and procedures apprehended within the arithmetical content. If they are not recognized or if students have misconceptions concerning them, then this may well affect the students' performance in algebra. (p. 29)

These types of issues manifest themselves in the understanding of equality, variables, and solving equations (Hersovics, 1989; Kieran, 1989).

The last reason researchers feel that misconceptions develop in algebra is due to the way algebra has been mainly taught: as symbolic manipulation. Traditional approaches to algebra instruction are 
characterized in teaching skills first, and only afterwards applying skills to problems. Yet this method of instruction emphasizes procedural knowledge over conceptual knowledge, which can lead to poor understanding of algebraic ideas. Many educators advocate algebraic teaching techniques that engage students in discussion and construction of conceptual and procedural knowledge (French, 2002; NCTM, 2000; Swan, 2000 ). These techniques include providing context to problems in order for students to relate to real world examples and providing contextual reasons for their procedures. Contextual reasoning for procedures includes using multiple representations for students to reason on and connect their knowledge, and using technology to enhance learning by allowing students to focus on conceptual understanding rather than symbolic manipulation.

Technology

Due to the need of number and operation sense, better transitioning from arithmetic to algebra with the focus on procedures and notation, and context-based instruction in order to relieve students' algebraic misconceptions, it is only natural to consider the affordances of technology to accomplish these goals.

By incorporating appropriate technology into instruction, students are able to manipulate numbers and operations to generalize about phenomena. Technology can generate many examples of a problem in forms such as graphs, tables, and figures in a short amount of time. By doing this, technology quickly provides students with many instances upon which to generalize; thus it gives more time to the student to create a greater conceptualization of the topic (NCTM, 2000). Not only does technology provide more examples in short amount of time, it can also present these examples in static and dynamic visual formats, which can aid students in understanding procedures and ideas. For example, students can come to a better understanding of what it means to add and subtract integers by being able to witness a pattern in a table of many numbers. They can also discover the idea of what it means to add and subtract integers by seeing directional arrows on a number line with a corresponding numerical statement. Building on the concept of generalizing through the visualization of many numerical examples, technology also allows for multiple representations from which to reason and integrate.

Two instructional recommendations provided by Wright (1991) to improve algebra learning are to "provide opportunities for constructing relationships between quantities" and "concrete and pictorial models to construct meaning" (p. 219). By providing multiple representations and connections among them, one can cut down on the misconceptions between arithmetic and algebraic procedures and notations. Technology can create dynamic relationships among symbolic notations, graphical, and tabular models 
(Kaput, 1989ab), which can potentially rectify notational misconceptions. Furthermore, it allows students to act upon these representations as objects and provide feedback instantaneously on their actions. This feedback can supply students with information on the processes of algebra that can inform them of the underlying algebraic structure.

Technology can facilitate a numerical and contextual based approach to algebra teaching (Heid, 1997; Rojano, 1996; Wilson, Ainley, \& Bills, 2004). It can elucidate critical relationships between quantities and ideas by allowing students to dynamically interact with a multitude of different numbers and notice the variant and invariant outcomes. If technological software is programmed appropriately, it can also depict algebra in a real-world context; thus, making algebra instrumental.

Studies have shown that the use of technology, specifically spreadsheets, have aided in students understanding of pre-algebraic concepts including operations on integers (Battista \& Van Auken Borrow, 1998; Sutherland \& Rojano, 1993; Wilson et al, 2004), the notion of variable (Battista \& Van Auken Borrow, 1998; Boers-van Oosterum, 1990; Sutherland \& Rojano, 1993), the notion of equality (Sutherland \& Rojano, 1993), and translating words to equations (Ainley, Bills, \& Wilson, 2005). One will notice in all previously mentioned spreadsheet studies, researchers have used the spreadsheets as an array of cells where one puts numbers in a cell, creates a function referencing that cell, and outputs the corresponding value (Battista \& Van Auken Borrow, 1998; Masalski, 1999; Sutherland \& Rojano, 1993). While operations on integers and the notion of equality have been studied with the use of spreadsheets, the author has not found any research on the use of a dynamic geometry package used to aid in the understanding of any of these basic concepts. Despite the fact that no research has been found using a dynamic geometry software package, I feel that its use can aid students' understanding of these pre-algebraic concepts.

\section{Purpose of Study}

The purposes of this study are to: (1) investigate non-accelerated middle schools students' understanding of basic algebraic concepts, in particular, addition and subtraction of integers, the notion of equality; (2) to describe and analyze how middle school students interact with GSP applets depicting addition and subtraction of integers and the notion of equality in order to discover how these applets can elucidate or mask these concepts and hence facilitate or hinder understanding; (3) to chronicle how students' conceptions of addition and subtraction of integers and the notion of equality change during and after instructional use of the GSP applets; (4) receive feedback from students to help develop the applets in order to improve the value of such applets in an algebra classroom. 


\section{Organization of Study}

This chapter has discussed the importance of taking and passing algebra, the reasons for middle school students to understand basic algebraic concepts, the statement of the problem, the reason for introducing technology, and the purpose of the study. The next chapter opens with the notion of what it means to understand. This discussion is followed by an in-depth literature review of students' conceptions of addition and subtraction of integers and the notion of equivalence, after which, educators and researchers' beliefs about why this occurs are presented, and what has been and can be done to mediate students' misconceptions. 


\section{CHAPTER 2}

\section{REVIEW OF RELATED LITERATURE}

Operations on integers, the notion of variable, the notion of equality, and writing equations are necessary to understand to meaningfully complete an Algebra I course. Since Algebra I is the gateway to future opportunities, mastery of these concepts will benefit students. However, research has shown that students have inadequate understanding revolving around these topics for many reasons: arithmetic misconceptions and difficulty transitioning from arithmetic to algebraic in terms of processes and notation too often because of instructional methods that emphasize procedural knowledge without conceptual knowledge.

\section{What is Understanding?}

This section will attempt to define what understanding means from different perspectives. By identifying these differing denotations of understanding, one can use them as a framework to recognize and comprehend students' conceptions of various algebraic topics.

The definition of understanding has taken on various forms by many researchers (Carpenter \& Lehrer, 1996; Davis, 1992; Minksy \& Papert, 1972; Pirie \& Kieran, 1994). The definition for this study will be similar to that of Hiebert, Carpenter, Heibert, Fennema, Fuson, Wearne, Murray, Olivier, and Human (1997), Hiebert and Carpenter (1992), and Vinner (1997), who suggested that understanding occurs when ideas are integrated into a large network of mental constructs, so that they are easily called upon when activated. Information is structured in such a way that when one supplies connections to it, the more one understands. Thus, mathematical principles, facts, and processes are better understood when students are able to establish mental relationships among them. These relationships, according to Hiebert and colleagues (1997), occur through two processes: reflection and communication.

Students reflect when they are mindful about their actions. They meta-cogitate by mentally asking themselves what they are doing and why they are doing it. By asking themselves these questions, students are calling upon prior knowledge and relationships and connecting them to the new information; thus, they create new associations. Unlike reflection, communication is shared among people. Communication allows students and teachers to socially interact in order to share ideas in hopes to make one fully explain and clarify to others what one is thinking. Communication embodies the old saying that one does not fully 
understand something until they have taught it to someone else. Through social interactions, a student's conception might be reinforced, or he might go through a conceptual change. Chiu, Kessel, Moschkovich, and Muñoz-Nuñez (2001) described a conceptual change as "a constructive process that involves a dialectical relation between individual and social aspects of knowing and learning" (p. 222). A conceptual change can come about by the establishment of new networks to an old conception, by the modification of the initial conception, or by the building onto the initial conception.

\section{Procedural and Conceptual Knowledge}

Knowledge can be broken down into two types: procedural and conceptual. According to Hiebert and Lefever (1986), procedural knowledge is comprised of two distinct parts: awareness of conventional notation, and familiarity of prescriptive procedures to complete a task. For students to be aware of conventional notation means that they can identify surface structure (also known as syntax) of mathematics, but can not always understand the underlying meaning of the structures. For example, students who are aware of notation can understand that 3-5 is an acceptable way of writing a subtraction statement, but that 35 - is not (yet this is how one enters the same statement to be evaluated on a Hewlett-Packard calculator). The second part of procedural knowledge deals with rules, algorithms, and sequential procedures. Students are able to perform these tasks, but might not know why they are doing so. Students sometimes follow rules by rote for operating on integers, dealing with the equal sign, dealing with variable, and translating from a word problem to an algebraic equation. For example, they know to place a negative sign onto the answer when evaluating additive operations, such as $a+b$ and $b+a$ when $b<0<a$ and $b<-a$ or when $b<a<0$, as well as subtraction, such as $b-a$ when $|a|<|b|$ and $b<0$ or $a-b$ when $|a|<|b|$ and $b>0$; however, they do not understand why the end value is negative.

Another example of students using procedural knowledge in arithmetic is when students view the meaning of the equal sign as a do something symbol (Behr, Erlwanger, \& Nichols, 1980; Falkner, Levi, \& Carpenter, 1999; Kieran, 1992). They are able to put the answer to the right side of the sign and obtain the correct answer. However, some students do not see that the equal sign denotes an equivalence relation connecting the expression on the left side of the sign and the right side of the sign.

Students are able to manipulate a variable in order to solve equations, but they do not always understand that the reason for a variable is to symbolize a relationship between numbers or represent any number (Davis, 1975; Matz, 1982; Wagner, 1983). When solving an equation, students can apply inverse operational procedures to set the variable equal to something, but they do not know what it means for the variable to have a value or many values. They do not see a need of representing ideas succinctly so that one 
can find the structure and manipulate it efficiently in order to find values for which on cannot directly measure or obtain.

Partnered with procedural knowledge is conceptual knowledge, which consists of pieces of information that are connected and "rich in relationships" (Hiebert \& Lefever, 1986, p. 3). For instance, in the last example of procedural knowledge, students who had constructed relationships among the meaning of operations and the semantics of the problem would have recognized that even though the problem used the words "less than", the operation used to solve the problem is addition. There are at least two ways to foster conceptual knowledge. The first way is by developing more networks between existing pieces of information. For instance, if one links multiple representations of the same mathematical concept, the linkage can provide a better conceptual understanding. The other way is to create new linkages between existing and new knowledge. One could use multiple representations to create new linkages between existing and new knowledge of a concept by providing at least two representations, one with which the student is familiar, and one that they are not, and creating a link between the two.

Some people believe that procedural and conceptual knowledge are distinct entities and would rather emphasize conceptual knowledge over procedural knowledge. However, these two types of knowledge can be coupled to benefit learning, for conceptual and procedural knowledge can accentuate the other in certain circumstances.

In studying procedural errors, researchers have found that they are usually linked to poor conceptual understanding (Brownell, 1935; Byers \& Herscovics, 1977; Skemp, 1978), especially in arithmetic. Without conceptual knowledge, procedural knowledge of when and how to apply computational steps is unclear, for "one of the purposes of conceptual knowledge is to form a support system for procedural knowledge" (Silver, 1986). Thus conceptual knowledge provides a base for procedural knowledge. Yet, in some instances, students are able to work proficiently with procedures without conceptual understanding. Therefore, conceptual knowledge does not provide a necessary or sufficient condition for procedural knowledge.

Similarly, procedural knowledge does not provide a necessary or sufficient condition for procedural knowledge, but it can provide a base for conceptual knowledge. When students create constructions in geometry, they can use the constructive procedures to develop their ideas about the figures. Silver (1986) noted that by measuring sides and angles and computing perimeter and area of triangles, students learned to differentiate between types of triangles. However, procedural knowledge does not necessarily have to be available to build conceptual knowledge. Silver (1979) found that students were able 
to categorize correctly word problems based on their underlying mathematical structure without having procedural knowledge of how to solve them. Procedural and conceptual knowledge can be linked, and the development of one can result in the improvement of the other.

It is not always feasible to split knowledge into strictly procedural and conceptual understanding in order to analyze students' conceptions, for problem solving usually requires the interactive use of both. Realizing this, Kilpatrick, Swafford, and Findell (2001) integrated the two and called it mathematical proficiency, which has five components: conceptual understanding, procedural fluency, strategic competence, adaptive reasoning, and productive disposition (p. 116). These componential strands are chock full of developing relationships among mathematical skills, concepts, and procedures. The first strand, conceptual understanding, deals with "comprehension of mathematical concepts, operations and relations" while the second strand, procedural fluency deals with "skill in carrying out procedures flexibly, accurately, efficiently, and appropriately" (p.116). The third, fourth, and fifth strands depict student abilities if they successfully create both conceptual and procedural knowledge. The third strand is strategic competence, which allows one to "formulate, represent, and solve mathematical problems" (p. 116). The fourth strand, adaptive reasoning, provides students with "the capacity to think logically about the relationships about concepts and situations" (p. 116), which includes both formal proof and informal intuition. The fifth strand, productive disposition, allows students "to see mathematics as sensible, useful, worthwhile, coupled with a belief in diligence and one's own efficacy" (p. 116). These components work together to produce a framework to situate mathematical learning.

While the previous paragraphs have presented a general notion of mathematical understanding, other researchers have postulated from whence algebraic meaning comes. Kieran (2007) presented four ideas on which students generate algebraic meaning: “(a) meaning from algebraic structure itself, involving the letter-symbolic form; (b) meaning from other mathematical representations, including multiple representations; (c) meaning from the problem context; and (d) meaning derived from that which is exterior to the mathematics/problem context" (p. 711). Algebraic structure deals with the ability to see the underlying mathematical structure by understanding the symbolic representations of the numerical values. The linkage between numbers and representation as well as the operations on those numbers, epitomize algebraic structure. NCTM (1989) recognized the importance of algebraic structure, for it "provides a means of operating with concepts at an abstract level and then applying them, a process that often fosters generalizations and insights beyond the original context" (p.150).

Not only can algebraic meaning be generated by the connection between symbols and numbers, 
but it can also be generated if there are linkages between other representations. By incorporating numerical, graphical, symbolic, verbal, and kinesthetic representations (Murdock, 2007), students can construct multiple meanings of and integrate concepts and ideas into one body of knowledge. Kaput (1989a) highly recommended the use of multiple representations as well as providing a connection among them in algebra, for two main difficulties for students in algebra are "dealing with highly concise and implicit syntax of formal algebraic symbols and the lack of linkages to other representations that might provide informative feedback on the appropriateness of actions taken" (p. 168). Thus, by integrating other representations in instruction and providing connections of those representations to algebraic symbols, students may be able to create meaning.

Students also receive information within the problem context. In fact, many researchers believe that presenting algebra in a problem-solving context can promote algebraic reasoning (Bednarz \& Janvier, 1996; Bell, 1995,1996; French, 2002). Providing students with a contextual problem engages and motivates students by creating relevance and purpose of algebraic ideas. It also encourages students to construct a mathematical model of the situation; thereby having students connect multiple representations in the problem.

Not only does context require students to model and create linkages among representations, but it also stimulates students' non-mathematical knowledge that can be applied to the problem. The type of meaning making where students' apply non-mathematical knowledge to help solve problems is what Kieran (2007) described as "meaning derived from that which is exterior to the mathematics problem context" (p. 711). Students use an intuitive understanding of algebra based on their language, metaphors, gestures, experience, and images, as a foundation on which to build algebraic concepts. Exterior meanings may be compared to Vinner and Dreyfus' (1989) notion of concept image. Students use the "set of all the mental pictures associated in [their] mind with the concept name, together with all the properties characterizing them" (p. 356) to try and move toward an algebraic representation of the problem.

Based on these four types of meaning making sources, Kieran (2007) described a three-pronged approach model to analyze algebraic instructional activities. These three approaches are called generational, transformational, and global/meta level. This model, known as the GTG model, will be discussed in the instruction section.

Studies on Certain Algebraic Concepts and Processes

Recognition of the origins of misunderstanding is necessary for the improvement of the teaching of algebra. Types of misconceptions addressed here will be of arithmetic, pre-algebraic, and algebraic in 
nature, for if students can understand the pre-requisite concepts of algebra, they will be able to transition and understand more formal algebra (Demana \& Leitzel, 1988). Misconceptions that will be described will deal with operations on negative numbers and the notion of equivalence. These types of misconceptions were first identified back in 1935 when Wren (1935) conducted a survey of research in teaching algebra. In 2000, Boulton-Lewis, Cooper, Pillay, and Wilss investigated the relevant knowledge for transitioning from arithmetic to algebra which included “(a) symbols, numbers, and variables (b) basic computations, (c) arithmetic law for individual operations and sequences of operations (d) meaning of equals, (e) operations on variables" (p. 89). They found that algebraic learning is complex and there is a need to concentrate on prerequisite knowledge in arithmetic in order to prepare a student for algebraic symbolism.

\section{Operations on Negative Numbers}

Understanding and operating with negative numbers is a necessity for students' algebra foundation, since algebra encompasses all real numbers and their properties (Stacey \& MacGregor, 1997a). For example, in algebra one must be able to find the value of a negative number raised to an odd or even power, as well as be able to solve an equation, with or without negative coefficients, and obtain a negative number as a solution and know what it means in that particular situation. Furthermore, Vlassis (as cited in Kieran, 2007) found that the negative sign caused difficulties with algebraic expressions and equations. Students had three categorical distinctions of the negative sign: binary, unary, and symmetric. Students who operated under the binary condition saw the negative sign in two ways: as an operation for subtraction and as a direction attached to a magnitude. However, these ways could not be linked as in the unary sense. Students who had a unary interpretation of the negative sign could flexibly maneuver between the subtraction of a number and the negative value of a number; therefore, they viewed "subtract 3" as also meaning the addition of "negative 3". By viewing the negative sign through a symmetric lens, students used the position of oppositeness such that the negative sign signified the opposite of the plus sign; thus, -3 is the opposite of 3. Vlassis found that because there were a few different constructed meanings for the negative sign, it presented a conceptual obstacle in constructing meaning for algebraic symbols for and processes on negative numbers.

\section{Students' Conceptions}

The 1996 NAEP findings (Silver \& Kenney, 2000) showed that only $22 \%$ of students in $8^{\text {th }}$ grade produced the correct work and answer to the following question: "The lowest point of the St. Lawrence River is 294 feet below sea level. The top of Mt. Jacques Cartier is 1,277 feet above sea level. How many 
feet higher is the top of Mt. Jacques Cartier than the lowest point of the St. Lawrence River?” (p. 284). Three percent showed that the operation needed was addition, but they produced the incorrect answer. Seventy-percent of students gave an incorrect response.

Students' difficulties with negative numbers are not new. Back in 1921, Breslich (1921) stated that the first problematic process in algebra was the meaning of and operation on positive and negative numbers. He believed that one reason that students had difficulty with addition of negative numbers was due to addition produce a result that is larger than the two numbers with which they began.

Murray (1985) tested eighth grade students, who only had 20 minutes of introduction on the idea of negative numbers and on subtraction of a larger natural number from a smaller natural number illustrated by using temperatures below zero on a thermometer, and ninth-grade students, who had previously had formal instruction on integer arithmetic. He tested students on addition, subtraction, and multiplication problems located in Table 1. Based on the success rates, Murray found that despite no formal instruction, eighth graders could intuitively solve some addition and subtraction problems, such as $-7+-5$ and $-12--3$. Moreover, some problems remained the most difficult despite instruction such as $8--3$ or improved very little after receiving instruction such as $-12--3$. Overall, students in both grades tended to do better on addition problems than on subtraction problems with negative numbers. There is a $23 \%$ point difference between $-12--3$ and $-5--12$ for eighth graders, which is interesting because if the 5 in the second problem was a 3 , then the second problem would be the first problem with the minuend and subtrahend switched.

Table 1

Success Rates on Operating on Negative Numbers

Problem Success Rate

Eighth Grade Ninth Grade

\begin{tabular}{ccc}
$-8+3$ & $52 \%$ & $78 \%$ \\
$-4+7$ & $48 \%$ & $77 \%$ \\
$10+-3$ & $51 \%$ & $75 \%$ \\
$-7+-5$ & $61 \%$ & $74 \%$ \\
$3-8$ & $48 \%$ & $69 \%$ \\
$-12--3$ & $57 \%$ & $63 \%$ \\
$-5--12$ & $34 \%$ & $55 \%$ \\
$-7-4$ & $21 \%$ & $50 \%$ \\
$8--3$ & $17 \%$ & $46 \%$ \\
\hline
\end{tabular}

Note. From "Children's informal conceptions of integer arithmetic," by J. C. Murray, 1985, Proceedings of the Annual Conference of the International Group for the Psychology of Mathematics Education, 1, p. 147. Based on the results of the written test, Murray decided to interview 52 nine through thirteen year 
olds, who had only been given an introduction to negative numbers using a thermometer, to analyze their conceptions of negative numbers, their computation strategies, and their misconceptions. During these interviews, he found that younger students did not have any more trouble understanding the concept of negative numbers than older students. Students across all ages stated that one "cannot subtract a larger number from a smaller number" (p. 148) and many students realized that negative numbers came "before" or fell "below" zero. When students saw unfamiliar notation such as in problems $10+-3$ or $5--8$, they would transform it into $10+3-3$ and $5-8$, respectively.

Even though students were introduced to this topic using a thermometer, students used the following strategies to compute expressions: a vertical number line, analogies to operations on positive numbers, and logic. One student, Jan, used the vertical number line to answer the question $7+-7=0$ by reasoning that if one started at 7 , and then went down by 7, one ended up at zero. An example of a student who used an analogy to correctly reason through the problem $-5--2=-3$ is that he acknowledged that if one removed the negative signs from 5 and 2 and computed the operation, one received 3. Thus, if one replaced the negative signs on both the numbers, one received -3. Murray also categorized another student, Marcel, who used analogies when he solved the similar expression -7--7. Marcel stated that the solution was zero "because it is minus 7 take away minus 7 and that is nothing" (p.149). Even though Murray clumped these two examples together, Marcel seemed to have a better understanding of the operation of subtraction as an idea of removal. The third type of strategy required the use of logic when finding differences between positive and negative numbers. Two of Murray's three examples of students who used logic based their value of an expression on the value of a similar expression. For example, Ron used the fact that since $5+-3$ was subtraction, then $5--3$ was addition, and therefore the answer was 8 . Human and Murray (1987) later coined this type of strategy oppositeness. The other student couched his answer in linguistic terms such that "minus minus is the same as not not: if I am not not ill, I am ill" (Murray, p. 150).

One misconception found by Murray (1985) was that students over-generalized commutivity from addition to subtraction. Students used this reasoning on the problem 2-5. They reasoned that since $5-2=3$ and $5-2=2-5$ then $2-5$ should be 3 . This reasoning might point to why students were more successful in addition of negative numbers rather than subtraction, since addition is commutative.

Based on Murray's analysis of the previous students, he took another sample of students to see if the results with addition and subtraction of negative numbers could be reproduced. He gave a similarly written test to 514 ninth graders and 332 tenth graders. He found that the four most difficult computations were of questions like $-9--4,-5--9,-7-3$, and $7--5$ with the prominent incorrect answer being 
$-13,-14,-4$, and 2 for each question, respectively. Table 2 shows the percentage of incorrect responses and the percentage of students who gave the prominent incorrect answer.

Table 2

Incorrect Answers to Subtraction Problems

\begin{tabular}{cccccc}
\hline & \multicolumn{2}{c}{ Incorrect Response } & \multicolumn{3}{c}{ Prominent Error } \\
Expression & Ninth Graders & Tenth Graders & Error & Ninth Graders & Tenth Graders \\
\hline$-9--4$ & $42 \%$ & $37 \%$ & -13 & $26 \%$ & $30 \%$ \\
$-5--9$ & $60 \%$ & $52 \%$ & -14 & $34 \%$ & $34 \%$ \\
$-7-3$ & $64 \%$ & $53 \%$ & -4 & $52 \%$ & $45 \%$ \\
$7--5$ & $73 \%$ & $62 \%$ & 2 & $57 \%$ & $52 \%$ \\
\hline
\end{tabular}

Note. From "Children's informal conceptions of integer arithmetic," by J. C. Murray, 1985, Proceedings of the Annual Conference of the International Group for the Psychology of Mathematics Education, 1, p. 151.

He interviewed 30 of these ninth graders, who were above average, to elicit their ideas about these calculations. He found that most of the wrong answers occurred because students misapplied a rule given to them by their teacher. Furthermore, they could not explain what the rule meant but only parrot it. One student used the multiplication rule that a plus and a minus always make a minus for an addition problem. This student evaluated $5+-2$ and got -3 as his answer. Another student used the rule that when one subtracts a negative, one changes the sign on the subtrahend. However, he applied it to $-5--9$ in such a way such that he changed all the signs in the problem, so he obtained 14 as his answer. In evaluating $-7-3$ a student obtained the answer of -4 and reasoned that it was so "because the value of the positive number is greater than that of the negative number, but smaller than the number"; thus, "the answer is therefore a negative number but less in value" (p. 151). She used the fact that 3 is greater than -7 and 3 is smaller compared to 7, so the answer was positive and smaller than 7. This would be correct if the operation was addition and not subtraction.

Under the guidance of Human and Murray (1987), Hugo (as cited in Human and Murray, 1987) interviewed 97 seventh graders to test Murray's results of success on integer addition and subtraction of nine- to thirteen-year-old students and his observations of their reasoning strategies. Hugo also provided a brief introduction of negative numbers using temperatures prior to interviews with questions similar to "It is now 3 degrees $C$, where will the mercury on the thermometer be if it gets 10 degrees colder?" (p. 438).

The success rates for Hugo's study were comparable to the success rates in Murray's study; yet, Hugo's children used more strategies to obtain their answers. Human and Murray completely analyzed the strategies students used in the cases of $-12--4$ and $8+-5$. They found that $36 \%$ of students were able to reason correctly for $-12--4$, while only $13.4 \%$ were able to do so for $8+-5$. Furthermore, students tended to use analogical reasoning rather than using embodiment reasoning, i.e. reasoning from ideas that 
embody the concept, to explain their answer even though Hugo had suggested using temperature during the interview.

Human and Murray found two more strategies used by students to evaluate addition and subtraction of integers. These strategies included simply placing a negative sign in front of the answer for no meaningful reason given and interpreting the negative sign as a subtraction sign. In total, Murray recognized five categories of strategies for operating with negatives numbers: using a number line, using analogies based on operations with positive numbers, using idiosyncratic rules, using embodiment reasoning, and using the negative sign as a subtraction sign. Yet, when comparing the two prominent of these six categories: analogical and embodiment reasoning, students preferred using analogical reasoning over any type of embodiment reasoning.

These studies suggest that some students have an intuitive notion of how to correctly operate on integers by using number lines, analogies, embodiment reasoning, oppositeness, logic, and commutivity (with regards to multiplication and addition). Yet, other students seemed to have no conceptual notion of a negative number; therefore, they resorted to using inappropriate strategies such as idiosyncratic rules, symbol manipulation, and commutivity (with regards to subtraction). Despite having an introduction to negative numbers using temperatures as a medium, these students preferred to use analogies of their understanding of whole numbers rather than embodiment reasoning to solve problems containing operations on negative numbers. Both studies showed that all students, with or without formal instruction, had a more difficult time with subtraction than addition with negative numbers.

Bell (1982) reported similar student strategies surrounding addition and subtraction of negative numbers such as reasoning with a number line, using analogies based on operations on positive number, ignoring the signs, and applying idiosyncratic rules; however, he provided a greater detail of these idiosyncratic rules. He interviewed 25 fifteen-year olds about symbolic addition and subtraction with integers, generalizations about these operations on integers, and applications using these operations on integers. He noted two instances of uncertainty of what subtraction of a larger number from a smaller number meant. The first student stated her confusion with the problem $3-10$ in that she did not know if it meant that 3 was taken away from 10 or if it meant that 10 was taken away from 3 . When Bell explained that it was the latter, she responded with the answer of -7 . The second instance of determining what it meant for a larger number subtracted from a smaller number was in the problem $234-589$. Many students wrote it vertically and tried to use the subtraction algorithm they had learned for a three-digit smaller number subtracted from a three-digit larger number to obtain -645 or -445 . Figure 1 shows how students 
who wrote the expression vertically possibly thought about these answers.

\begin{tabular}{|cc|}
\hline $113^{12}$ & $13^{12}$ \\
$23^{1} 4$ \\
-589 & -589 \\
\hline-645 & -445 \\
\hline
\end{tabular}

Figure 1. Three-digit subtraction problem with answers based on inappropriate application of subtraction algorithm

Both the methods illustrated in Figure 1 use the regular subtraction algorithm except for the hundreds place digit. The left example shows that the student borrowed from an empty thousandths place in order to have a larger number from which to subtract 5. The right example shows the work of a student who realized he could not borrow from an empty thousandths place and therefore subtracted 5 from 1 and received -4 . This showed that students utilized a rule that they did not fully understand.

Students also tended to make up their own rules in evaluating other expressions. In some instances, students ignored the signs attached to both numbers or just the second number and then used the operation between the magnitudes to find the answer. Another method that students used in evaluating an expression similar to $-11-6$ was to link the two numbers between the subtraction sign, evaluate that new expression, and place the negative sign on to the answer. Thus, one worked it out in this fashion: $-11-6=-(11-6)=-5$. Bell noted that subtraction problems caused the most difficulty for these students, for $80 \%$ of the students correctly answered addition problems whereas only $40 \%$ correctly answered subtraction problems. One misapplied rule was that two negatives required a change in a sign. Unfortunately, one student reasoned using his version of this rule to find that $-9--2=-11$ and $7--2=5$. Because there were two negative signs preceding the 2 , he changed the operation sign to a plus sign, but left the other sign alone. Thus, $-9--2$ became $-9+-2=-11$ and $7--2$ became $7+-2=5$.

Another student used a number line, but he used addition rules rather than subtraction rules for the number line to find the answer. He used the first number as his starting position and counted to the right if the sign on the second number was positive or to the left if the sign for the second number was negative. For the problem $-9--2$, he located -9 on the number line and proceeded to count 2 steps to the left to obtain the answer of -11 . When asked what determined the direction of his counting, he indicated that it was the sign of the second number.

Also in his study, Bell asked students to generalize their answers to see if $a+b=b+a$ and $a-b=-(b-a)$ were true for all integers. He showed them that this held true for all natural numbers. For example, he showed $5+7$ was the same as $7+5$ and asked them why it was always true. If a student had 
trouble explaining why this was true, he offered them the following diagram on which to show the equivalent result:

\begin{tabular}{|c|}
\hline$\left|, 1^{5},\right|, 1,1,1+$ \\
\hline${ }^{7}, \perp,|, 5, \ldots|$, \\
\hline
\end{tabular}

Figure 2. Number lines representing $5+7=7+5$

Note. From "Looking at Children and Directed Numbers," by A. Bell, 1982, Mathematics Teaching, 100, p. 70

In Figure 2, the top number line shows the expression $5+7$ and the bottom number line shows the expression $7+5$.

Afterwards, he asked them if one could do the same thing with signed numbers by asking if $-11+3=3+(-11)$ ? He illustrated only two students' ideas concerning this question. One student used separate cases and eventually saw that both operations gave the difference of 11 and 3; therefore, she stated that the equivalence was true. The second student believed it was false since she could not model both those operations because "it moves around too much" (p. 71).

In regards to the second generalization, Bell showed students that with counting numbers big - small $=$ positive and small - big $=$ negative. To illustrate this, he gave the following examples: $7-4=3$ and $4-7=-3$. He asked if those two statements were true for all integers. Following this question, he asked if $a-b=-(b-a)$ was true for all integers. He did not describe students' interpretations to these questions. He believed that the first question required too much of the student because it made the students choose numbers that fit the conditions, calculate correctly, and understand the language of what big and small meant in the context of positive and negative numbers. He did not say anything about the follow-up question.

Like Bell, Peled, Nukhopadhyay, and Resnick (1989) found some similar tendencies for students to apply idiosyncratic rules when they worked with addition and subtraction on integers. They tested first, third, fifth, seventh, and ninth graders on their knowledge of negative numbers. They posited that both age and instruction affected students' conceptions on the test. The majority of first graders did not believe that negative numbers existed; thus, when given a problem such as $4-8$, they either switched the numbers to obtain $8-4$ or answered with a positive number, or they stated that it equaled zero because they did not think there were any such things as negative numbers. If students were given the problem $-4+8$, an expression with a negative number plus a positive number, they ignored the sign of the first number and answered with 12. Most third and fifth graders believed in the existence of negative numbers; however, they obtained incorrect answers based on applying incorrect rules. For example, when given the problem 
$-5+8$, they focused on the operation between the numbers, and applied the negative sign afterwards such that $-5+8$ became $-(5+8)=-13$. This is similar to the findings of Bell (1982).

After analyzing the test results, researchers interviewed six students from each grade to probe for students' justifications to their problem solving. They found that students experienced a progression of understanding negative numbers in which negative numbers did not exist to existing on a "mental number line with a symmetrical organization of numbers around zero" (p. 107). Students passed through three levels to obtain the highest level of understanding: the symmetric notion of negative numbers on a number line. If a child did not believe that negative numbers existed and was asked to write them on a number line, they put the negative numbers beside their corresponding positive numbers, but viewed them as all having the value of zero. At the next level, students placed negative numbers "on the other side" of zero, but in doing so, they did not understand the symmetry around zero. By third grade, most students reached the third level in which one represented both the appropriate ordering and symmetry around zero.

Based on both the test results and interviews, Peled and colleagues believed that children had two notions of a number line: continuous and divided. The continuous number line (CNL) model is what mathematicians use as a number line: a continuum on which numbers increase in value from left to right beginning with negative numbers. Peled and colleagues said that even though some students mentioned a division at zero, they categorized their responses using the number line as continuous if students were able to "count across zero" such that one counted right for addition and left for subtraction. Unlike the CNL, some students believed that the number line was divided. The divided number line (DNL) was used by students who had the conception that a number line consisted of two separate rays: one for negative numbers starting a little left of zero and extending left, and one for positive numbers starting a little right of zero and extending right; thus, it had a split at zero. Students who used the DNL model had to have special rules to deal with operations and numbers that required one to cross over zero. For instance, in the problem 4 - 7 students partitioned the subtrahend into 4 and 3 so that they could return to zero, and then proceeded to move three to the left on the negative side.

While researchers revealed two conceptions of the number line from student interviews, they also found errors due to certain notations. Students described the number line model that they conceptualized, but they could not always use it to model certain problems because of the problem's notation. For instance, the problem $4--2$ was unable to be modeled by students using either form of the number line because of the consecutive negative signs in the expression. To evaluate this expression, students either ignored the negative sign and changed the expression to $4-2$ and obtained the answer of 2 , or they moved one of the 
negative signs to the minuend changing the expression to $-4-2$ and obtained the answer of -6 . The subtraction of a negative minuend is one of the hardest operations for students' to understand because without context it is difficult to make sense of taking away a negative number of objects. However, according to McAuley (1990) by linguistically interpreting the signs and operations, students can utilize their number line to solve the problem. Students can linguistically interpret the subtraction sign as "and then do exactly the opposite to the next instruction," on the number line interpret the positive sign as "go to the right," and the negative sign as "go to the left" on the number line (p. 46). Thus, the problem $4--2$ becomes 'go to the right four steps from zero, and then do the opposite of going to the left two steps.'

These studies suggest that subtraction of a larger number from a smaller number is difficult for students. Furthermore, students have a harder time with subtraction with negative numbers than they do with addition with negative numbers. When students encounter problems such as these, they tend to make up their own procedural rules about how to solve them, which usually have no conceptual basis. When researchers probed these students about their ideas of negative numbers and their rules of operating on them, researchers were able to produce a progression of levels that students pass through as well as two notions of the number line.

Using this preliminary model of children's views of negative numbers and number lines, Peled outlined a framework consisting of two dimensions. There are two dimensions of integral addition and subtraction knowledge: a Quantitative Dimension (QD) and Number Line Dimension (NLD). Each dimension has four levels that are hierarchical, with the exception of QD Level 1 and QD Level 2 can be reversed. Students can possess different ideas about how to operate on different problems; thus, a student can exhibit characteristics at different levels depending upon the problem.

A student operating at the QD views both positive and negative numbers as the amount of things that exist. At Level 1, negativity is looked upon as an opposite quality of the positive values such as debt versus earnings. Due to this notion of the negative numbers, children view negative numbers as a reflection of the positive counterparts so that the larger the amount, the smaller the value.

The second level enables students to subtract a larger natural number from a smaller natural number. The answer is situated into a context of a quality characteristic of deficiency. Peled noted that Level 1 and Level 2 might be reversed.

A child operating at the third level can deal with addition and subtraction of the numbers having the same sign. However, he can only subtract a smaller negative number from a larger negative number, for he can only take away negative items that are already present. It is not until Level 4 that a child can subtract 
a negative smaller amount from a negative larger amount. Also at Level 4, a child can add and subtract two different signed numbers, and he can notice that the operation and the sign of the second number determine the sign in the answer.

Level 1 in the NLD is similar to the QD Level 1 in that it only describes how the student acknowledges positive and negative numbers without regards to an operation. At this level, students understand the number line as either continuous or divided and realize that the farther right that one moves, the greater number one will obtain.

Level 2 introduces the idea of what it means to add and subtract on a number line disregarding the sign of the numbers. Addition means to step right and subtraction means to step left on a number line even if a larger number is subtracted from a smaller number.

At Level 3, students are able to recognize that addition and subtraction are opposite in direction and extend this knowledge to work with the same-signed numbers. Not until Level 4 can they work with integers with different signs and generalize the fact that one needs to pay attention to the operation and the sign of the second addend or subtrahend to determine how one should move on the number line.

Using this two-dimensional framework, Peled (1991) interviewed 20 sixth graders who had instruction on how to add and subtract integers the previous year. In this group there were 10 of the best and 10 of the weakest students in the sixth grade. She did not say how these students were determined to be the best and weakest. She found that even the good students did poorly, and some did not even remember studying the topic. She analyzed three students: 1 strong student and 2 weak students. The strong student used the number line to help solve the problems by moving left and right, but only attained Level 3 because he was unable to deal with numbers of different signs. The two weaker students remained at Level 1 in the NLD and QD. One of the students correctly drew a number line and labeled the negative numbers. However, when he was asked to solve 3 - 7 using his number line, he said that it was impossible since he could not subtract a larger number from a smaller number. A peer of his did not draw an appropriate number line, for his order in which the negative numbers were placed was incorrect. However, this student had Level 1 knowledge in the QD, because he believed 3 - 7 was solvable. In solving this problem, he changed the 3 to a 30 , and obtained the answer of 23 .

While not all of the data have been fully analyzed by the time her paper was published, she postulated that future results would reveal that most of the students were in the first two number line levels and the first three quantity levels, and a few students were in the third number line level. She believed that this framework needed more testing with older children to determine their levels of integral knowledge 
with addition and subtraction.

Based on the previous research on students' understanding of negative numbers in regards to addition and subtraction (Human \& Murray, 1987; Murray, 1985; Peled 1991), Bruno and Martinon (1996) believed that there was a two part division in Peled's QD of operative integral knowledge: the abstract dimension and the contextual dimension. The abstract dimension describes how a student works with abstract symbolism of numbers by applying rules for addition and subtraction. Students working in the contextual realm will apply embodiment reasoning (Human \& Murray, 1987) to numerical arithmetic statements. In their research, Bruno and Martinon used the following six contexts: earnings/debt, temperature, sea level, road directions, time passage, and elevator movement. They did not discuss how the inclusion of this division affected the levels of the QD. However since Level 1 is described as using a quality characteristic to interpret a negative sign (Peled, 1991), it must be located within the contextual realm. By including both the abstract and context in the QD, this inclusion resulted in having three total dimensions of integers: contextual, abstract, and number line.

Bruno and Martinon used their altered Peled framework to devise curricular materials for three groups of 12 and 13-year olds, who would use the materials for 4 to 5 hours a week for two months. At the end of the two months, students were tested. Based on their test responses, 11 students were clinically interviewed and asked 18-20 questions that each required them to transfer between two of the three dimensions. Examples of the types of questions are located in Figure 3.

After analyzing the interview data for the 11 students, Bruno and Martinon found that students had a harder time moving from an abstract representation to a contextual representation rather than vice versa. Furthermore, this transference was the most difficult among all the transferences between two dimensions. All of the students had an easier time moving from contextual to the number line versus the abstract to the number line. They also had an easier time moving from the number line versus the abstract to the contextual. 


\begin{tabular}{|c|c|}
\hline $\begin{array}{l}\text { bstract } \rightarrow \text { number line } \\
\text { Represent on the number line the operation: } \\
-4+10=6\end{array}$ & $\begin{array}{l}\text { number line } \rightarrow \text { abstract } \\
\text { Tell me an operation that might be represented } \\
\text { on the number line in the following way: }\end{array}$ \\
\hline $\begin{array}{l}\text { abstract } \rightarrow \text { contextual } \\
\text { Tell me a situation that might be solved using the } \\
\text { following operation: }-4+7=3\end{array}$ & $\begin{array}{l}\text { contextual } \rightarrow \text { abstract } \\
\text { Tell me an operation that might solve the } \\
\text { following situation: } \\
\text { The temperature in Madrid is } 9 \text { degrees above } \\
\text { zero. In Paris it is } 12 \text { degrees less than in } \\
\text { Madrid. What is the temperature in temperatura } \\
\text { in l'aris? }\end{array}$ \\
\hline $\begin{array}{l}\text { contextual } \rightarrow \text { number line } \\
\text { Represent the following siluation on the number } \\
\text { ine } \\
\text { An elevator was on the floor } 5 \text { of the basement } \\
\text { and went up } 7 \text { floors. After going up in this way, } \\
\text { what floor was the elevetor on? }\end{array}$ & $\begin{array}{l}\text { number line } \rightarrow \text { contextual } \\
\text { Tell me a situation which might be represented in } \\
\text { the following way: }\end{array}$ \\
\hline
\end{tabular}

Figure 3. Example questions utilized to interpret student transference between dimensions Note: From "Beginning Learning Negative Numbers" by A. Bruno and A. Martinon, Proceedings of the 20th Conference of the International Group for the Psychology of Mathematics Education, 1996, p.164.

Only two out of the 11 students had a more difficult time moving from the contextual versus the number line to the abstract. Figure 4 provides a graphic of the easiest path from one dimension to another. Since not all of the students believed that one dimension was the easiest to go from to the abstract dimension, the numbers represent how many students found that path the easiest to travel.

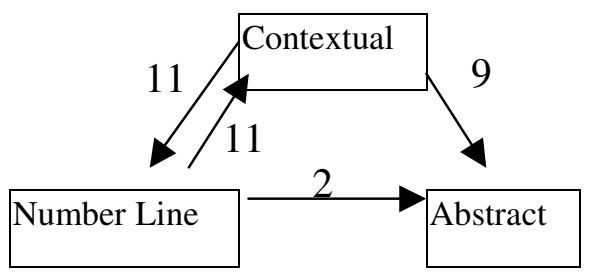

Figure 4. The easiest paths between the 3 dimensions of integers.

Researchers also found that students had specific issues when moving from both the context and abstract dimension to the number line dimension. When students had to represent initial states and variations on a number line, they had difficulty placing the endpoint of the ray and which direction the ray should face. When they moved from the abstract dimension to the number line, some students placed three isolated points on the number line (two addends and the sum, or the minuend, subtrahend and difference) without any indication of the operative relationships among the points.

When students moved to a contextual representation, they used specific ideas based on the initial representation. When the initial representation was a number line, they frequently used elevator and road directions, whereas if the initial representation was abstract, they used credit-debit context. Temperature was the only context in which they felt comfortable representing both the number line and abstract 
dimension.

Most of the movement between the contextual the abstract dimensions produced six frequently used strategies: "looking for operations that match the results on the number line, falsifying results of operations, having erroneous operational rules, changing the structure of the problem, interpreting results incorrectly, and following the order of the signs as the data in the text" (pp. 166-167).

When students moved from contextual problems to the abstract, they often used the number line as an intermediate strategy to solve the problem. This resulted in using the first three strategies. Students solved the problem using a number line and then continually tried to create an operation that achieved their number line result; thus, they did not directly link the operation with the context of the problem. If students did not find the appropriate operation, they wrote down the inappropriate operation with the number line answer rather than the answer produced by the incorrect operation; again, the operation had no contextual linkage. Similarly, knowing the correct answer from the number line, students also formulated the wrong operations for the contextual problem but that produced the correct answer to the problem.

Sometimes students jumped straight to the abstract dimension from the contextual dimension, but in doing so, changed the structure of the problem so they understood it easier. For example, the researchers presented this problem: "A child begins a game with 6 pesetas and finishes the game owing 5 pesetas. What has happened during the game?" (p. 167). A student answered correctly that the child lost 11 pesetas, however, he used the logic that one needed to add 5 and 6 to get 11 instead of subtracting 11 from 6 to get the end state of owing 5 pesetas. This showed that students did not need to deal with negative numbers to obtain the correct answer to the problem, for they could change the structure of the problem and rely on context to navigate through the operations to obtain an answer (see also Gallardo, 1996).

Other students also changed the structure of the problem, but in doing so, obtained an incorrect answer because they used the attached signs instead of converting them. For instance in the problem "A person was born in the year 15 before Christ and died in the year 7 before Christ. How many years did he live?" (p. 167), students again wrote the magnitudes in the order that they appeared in the question. However, in this instance, they did not change the sign of the second addend and wrote $-15-7=-22$.

Students used the last strategy when they moved from the contextual to the abstract and back to the contextual dimension. Students gave incorrect contextual interpretations from their abstract solutions. For instance, when they wrote an abstract equation to the contextual problem that resulted in an answer for the age of a person who lived before Christ, students justified their negative answer as the person "lived before Christ" (p. 167). For example, if a student's calculation produced the number of 20, but the person 
lived before Christ, they would interpret the age to be -20 .

Bruno and Martinon concluded that students had a great difficulty moving from the abstract dimension to the contextual dimension rather than vice versa. Furthermore, students found it easier to move between the number line and context rather than between the number line and abstract or between context and abstract. The results showed it is easier to use the number line in contextual situations to solve problems instead of trying to formulate operations in the abstract setting.

Recent research concerning negative numbers has been done by Aurora Gallardo (2000, 2002). She applied a historical and epistemological analysis on interpreting and operating on negative numbers. She examined Chinese, Greek, Hindu, Medieval, and French historical texts and explored languages, methods, operations, and interpretations of negative numbers. From her analysis of these items, four evolutionary strategies of negative numbers emerged: subtrahend, relative number, isolated number, and formal negative number. Subtrahend means students look strictly at the magnitude of the number and disregard the direction, or sign, of the number and if the operation is subtraction, it places the smaller number in the subtrahend. This was seen in first graders who reversed the minuend and subtrahend in order to solve the subtraction problem with a negative number having the greater magnitude in Peled's study (1989). Relative number is similar to Murray's (1985) idea of oppositeness and related to Peled's QD Levels 1 and 2. Students operating under this level used opposite quantities in regards to some quality characteristic. For example, if one represented traveling north by five miles as +5 , then -5 is interpreted as traveling south, or opposite of north by five miles. She described isolated number as "the result of an operation or as a solution to a [word] problem or equation" (2000, p. 128). The final level, formal negative number, resulted when students integrated both positive and negative numbers into one larger set, i.e. integers.

Gallardo (2002) used the past as a lens in which to view 12 and 13-year olds' answers to the word problem first posed by D'Alembert: “A person has a certain amount of money and receives $\$ 100.00$. If he now has $\$ 50.00$, how much money did he have initially?" (p. 182). She delineated how students solved the following word problem using the four levels. Students working on the subtrahend level did not produce the correct answer, for they were unable to grasp the notion that one can write an arithmetic statement with a negative number in the first position. These students only worked with natural numbers, did not use language such as owe or debt, and only used a negative number as a subtrahend despite the requirements of the problem. Students operating on the relative number level used language such as "owe", "have", and "spent", and were able to write $-50+100=50$. One student used a number line to show the process of 
"spending" and "having money" to justify his answer. None of the students obtained a negative value; therefore, no one exhibited the isolated number level. If the problem were such that one would obtain a negative answer, then the isolated number level could be discussed. She concluded that the first three levels are context dependent and in no hierarchical order, and the fourth level is not usually attained by 12 and 13year olds.

This section described how students viewed and operated with negative numbers. Many researchers put forth strategies that children used to solve abstract addition, and subtraction tasks with negative numbers. Based on these strategies and accompanying answers to abstract tasks, researchers have provided two frameworks to view children's interpretation of operations with negative numbers: Peled's (1991) framework and a historical framework provided by Gallardo (2000, 2002). Furthermore, this section showed how Bruno and Martinon (1996) modified and used Peled's framework and illustrated the difficulties for students to move among three representations: contextual, number line, and abstract.

\section{Reasons for Conceptions}

In light of the research from the last section, four main strategies that children used to operate on negative numbers emerged. These strategies are using a number line, using analogies by relating the structure of a problem you know how to solve to a problem one does not initially know how to solve, using the idea of oppositeness of whole numbers and negative numbers, and using a misapplication of "stated" rules. Analyzing these strategies, one can see that the first three strategies allowed students to obtain the correct answer to problems involving negative numbers; however, students who used the last strategy to compute expressions usually ended up with the incorrect answer. This is logical given that the first three strategies require students to create and understand connections between operations, numbers, and sometimes different representations of both operations and numbers. Thus, students using the first three strategies had to rely on their conceptual and procedural knowledge. Yet, students who utilized the last strategy did not necessarily rely on any conceptual or procedural knowledge, rather they parroted a rule that someone stated for them. For example, Murray (1985) and Human and Murray (1987) showed how students, who correctly answered addition and subtraction with negative numbers, did so by using a number line or applying logic to addition facts as well as properties of whole numbers. These two types of strategies are connected to a concept image of negative numbers. Students were able to make the connection between operating with positive and negative numbers using the model of a number line. By using previously known addition facts, students were using their procedural knowledge to influence their conceptual knowledge about operations on negative numbers. For example, they recalled that adding a 
positive and negative number is the same as subtraction; therefore, they reasoned that subtracting a negative number from a positive number must be addition.

Also in this study as well as others, students' used rules that were inappropriate for the type of problem with which they were presented, for they were unable to connect the problem type with the procedure. Moreover, students, who parroted these rules, did not use number sense to help them state why they had to apply the rules; rather they mentioned the surface structure of the problem. For example, when given the problem $5+-2$, a student responded with the answer of -3 "because a plus and a minus remain a minus" (Murray, 1985, p.151). In solving the problem $-9--2$, one student obtained the answer of -11 because he admitted that he was "doing the signs" or applying the rule for signs by changing one of them because of the two negative signs next to each other. However, he misapplied this rule because he only changed the operation sign rather than both the operation sign and the directed sign of the 2 . These misconceptions stem from poorly constructed relationship regarding operational properties and directed numbers. Because students do not conceptually understand what an operation or a negative number means, they rely on remotely memorized quips to solve the expression.

By using a financial context, Gallardo showed how students who could correctly reason through the problem had understood what a negative number meant in the context of the question. In this case, a negative number was regarded as an amount owed to someone else. While six students came to this conclusion, one of those six not only connected the number to a context, but she also modeled the problem using a number line. She connected many facts about negative numbers. First she related -50 to falling 50 units left on the number line, and second she related -50 as owing 50 dollars to someone. Thus, her zero on the number line represented breaking even. This type of connecting numbers to models and context demonstrated her conceptual knowledge of negative numbers. Furthermore, by modeling the operations and numbers on her number line, she demonstrated her knowledge of the structural aspects of the problem.

Students who could not answer the problem correctly focused on superficial structures of the arithmetic statement versus the meaning behind the statement. These students worked on the subtrahend level and believed that negative numbers could not go in the first position of the arithmetic statement, despite the meaning of the question. If they had utilized the context of the question and focused on the semantic meaning of the problem versus the structure of the abstract numerical statement, they could have obtained the correct answer, for they would have been operating on the relative number level and using their conceptual knowledge of negative numbers and concepts such as debt and owe.

By modifying and testing Peled's framework, Bruno and Martinon (1996) showed how students 
maneuvered between problem representations: contextual, abstract, and number line. Out of the six possible pathways to travel from one representation to the other, they found that students had the hardest time moving from the abstract representation to the contextual representation. In fact, movement from the abstract to any other representation was difficult for students. This is not surprising if students do not have many connections to what the numbers and operations mean. When the original format of the problem is a number line or contextual representation, students can see linkages between numbers and actions on those numbers. When students are presented with a number line problem, they can see the initial point, the ending point, and the arrow describing how one moved to get from the initial point to ending point; thus, students can trace the path and physically enact the problem. When students are presented with a contextual problem, they can picture or draw the scenario. In both instances, students are presented with a format that allows them to associate pieces of knowledge with the numbers. However, if one is presented with an abstract representation, he does not have addition information to relate to the symbols and must rely on his knowledge of the meaning behind the symbols. If these meanings are not fully established, i.e. part of his procedural and conceptual knowledge, he will resort to applying "rules" or meaningless procedures, which may result in computing incorrect answers.

\section{Instruction to Overcome Misconceptions}

In a second project conducted by Human and Murray $(1987), 4^{\text {th }}, 5^{\text {th }}, 6^{\text {th }}$, and $7^{\text {th }}$ graders received one week of instruction that emphasized using a vertical number line or thermometer (they called using these models as using embodiment reasoning) to answer questions, but no computational or rule-based strategies were explicitly taught. Two of these instructional sessions were given, one in April and one in October. Prior to instruction, students took a pre-test on addition and subtraction of integers and took a similar test following instruction. During the period between instructional sessions, students decreased their use of embodiment reasoning and instead used analogical reasoning. Analogical reasoning was used when numbers had the same sign. Students stripped the negative sign from the numbers, applied the operation on positive numbers, and then put a negative sign in front of the answer. For example, students using analogical reasoning said that $-5--2=-3$ "because $5-2=3$ and these are minuses... it is an ordinary subtraction sum" (p. 437). After the instructional session in October, students increased their use of embodiment reasoning. This usage implied that students, when encouraged, used contextual applications. However, when left to their own devices, they recalled procedural facts and reasoned from them. This may have stemmed from students' inabilities to connect their conceptual knowledge with their procedural knowledge. 
Other educators have also advocated the use of contextual models to introduce operations on integers (Breslich, 1921; Janvier, 1988; Kohn, 1978; Wren, 1935). Breslich provided a whole list of how to illustrate the meaning of negative numbers and told teachers to make a good selection since in all likelihood they "will have to return to these devices later when the laws for operating upon signed numbers are to be rationalized" (pp.119-120). A few contextual ideas on the list were: deposits and withdrawals, credit and debit, north and south, walking steps upstairs and downstairs, years hence and years ago. He also advocated the use of a number line for operations as a continuation of the "scale of positive numbers" (p. 120).

Janvier (1988) categorized two types of modeling for teaching students, ages 13-14, how to operate on integers. One model related negative and positive numbers to a number line, and the other model related negative and positive numbers to objects that are opposite in nature (e.g., black and white counters). Janvier termed both the number line and colored counter models as symbolic, for no contextual meaning was given to subtraction and addition. He created a new model, termed the hot-air balloon model, or an image model, which illustrated a hot-air balloon onto which one could attach balloons or bags of sand to raise and lower the hot air balloon, respectively. Thus, adding meant attaching balloons or sacks onto the hot-air balloon, and subtraction meant detaching these items.

Two groups of students were formed: one for each model. Each group received instruction using their model and afterwards was given two tests. The first test was purely arithmetic notation, and the second test asked the same types of questions, only framing the question in terms of the model to which the students were exposed. Janvier found that students in the image model group performed significantly better on the addition problems on both of the tests over the symbolic model group. However, there was no significant difference on the subtraction items on both tests in both groups. After reviewing these results, Janvier interviewed students and found that students in the image model group were able to successfully use the hot-air balloon model to answer questions on the arithmetic test, whereas the other group could not use their symbolic models to answer questions on the arithmetic test. Furthermore, interviews showed that students in the image model group were able to correct their mistakes on the test by going back to the hotair balloon model, where the other group of students would only repeat their mistakes using their models. Janvier concluded that the balloon model was advantageous because it provided a mental image that related concrete objects and directionality to operation with integers.

By compiling the research for teaching operations on integers, many suggest the use of representations, context, and linguistic meaning for symbols when introducing students to operations with negative numbers. 


\section{The Equal Sign}

Understanding the equal sign in arithmetic is crucial for understanding equality in algebra (Horne, 1999; Kieran, 1981), because equality presents itself in solving and writing equations. Instruction needs to help move students from an operational view of the equal sign in arithmetic to a relational view in algebra to conceptually understand the reason behind using variables and solving equations. Literature provides at least four denotations of the equal sign: an association, an operation, an equivalence relation, and a procedural encoder.

\section{Students' Conceptions}

One of the first ways students encounter the equal sign is through an association. They see posters saying "Math=Fun" or "Success = Hard Work" in elementary school or in daily life (Stacey \& MacGregor, 1997a). These depictions do not enforce an equivalence relation, but rather a causation or transitive relation. To say that "Success=Hard Work" implies the use of conditional statements beginning with hard work, supplying intermediate statements, and ending at obtaining success. By logically ordering these statements, you can associate hard work with success through an application of the transitive property.

Another way students encounter the equal sign in elementary school is as a "do something signal" (Baroody \& Ginsberg, 1983; Behr et al, 1980; Falkner et al, 1999; Ginsberg, 1977; Hughes, 1986; Kieran, 1990). Students see the equal sign as an indicator to place the answer to the right of the sign, which separates the question from the answer. Moreover, students believe that the expression must be in the form $a+b=c$ where $a+b$ is the question, and $c$ is the answer. In Behr's study (1980), when students encountered arithmetic sentences in the form $c=a+b$, six-year olds responded that it was "backwards" and proceeded to rewrite it in the form $a+b=c$. In fact, when one student was questioned about why he was rewriting the equation, he asked the interviewer "Do you read backwards?" (p. 14). Thus, the understanding of the equal sign as an operator can lead to the rejection of unfamiliar representations of equations.

Misconceptions surrounding the equal sign also interfere with students' ability to grasp number properties such as the commutative and distributive properties. When asked whether $2+3$ was equal to $3+2$, six-year olds seemed to understand that they were "the same question" but rewrote them so that they were able to show the answer on the right-hand side of the equal sign: $2+3=5$ and $3+2=5$ (Behr et al, 1980). Thus, it seems that students saw these expressions as sets of the same number of objects, since both "questions" elicit "answers" of 5, but did not see the relationship between the expressions. 
Students used the operational notion of the equal sign when they used the equal sign to imply a procedure. Kieran (1979) found that when students wrote arithmetic identities, they wrote down the operations according to the ones that they used to calculate to find the answer and used the equal sign as a symbol to keep a running total, which resulted in a false equality. For example, students wrote $1063+217=1280-425$ in an attempt to solve for the number of trees in the following problem: "In an existing forest 425 new trees were planted. A few years later, the 217 oldest trees were cut. The forest then contains 1063 trees. How many trees were there before the new trees were planted?" (p. 320). The equal sign is used as a break after a performed operation, for which the final usage of the equal sign elicits a running total. This usage resembles how one uses the compute or calculator to evaluate an expression. One would first enter 1063, hit the plus sign button, type in 217 , and then hit the equal sign button. Students would then take the resulting number, 1280 , hit the subtraction button, enter 425 , and hit the equal sign button again to produce the end result. This type of reasoning can spill over into solving algebraic equations. Stacey and MacGregor (1997a, p. 255) saw this phenomenon when students tried to solve algebraic equations such as $2 x+6=17$. Their students' work followed as:

$$
\begin{gathered}
2 x+6=17 \\
=2 x=11 \\
=x=5.5
\end{gathered}
$$

This type of operational reasoning with the equal sign does not remain in elementary school but carries over into middle school and high school. Cooper and colleagues (1997) interviewed 51 seventhgraders asked students to tell them what the equal sign meant in both $\frac{28}{7}+20=$ and $\frac{28}{7}+20=60-36$. For the first question, all but one student, who said something to the effect that the equal sign sometimes meant that the expression was equivalent to something else, said that $\frac{28}{7}+20=$ was looking for an answer. For the second question, half of the students could not explain what the equal sign meant in this context. Of the students who tried to explain the equal sign, nine said something to the effect that 60 - 36 was the "answer" to the left side, and 17 said statements similar to that both sides of the equal sign had the same value. These researchers (Boulton-Lewis et al, 2000) wanted to know what happened as these students went through prealgebra instruction in $8^{\text {th }}$ grade and algebra instruction in $9^{\text {th }}$ grade in terms of their view of the equal sign. Researchers believed that they would see a transition among students moving from an arithmetic response to a pre-algebraic response to finally an algebraic response. They tested these students not only on what the equal sign meant, but also on the commutative law, distributive law, inverse operations, order of operations, and the solution of an equation in one and two variables. Researchers coded the answers to 
students' understanding into four categories: inappropriate, arithmetic, pre-algebraic, and algebraic. Table 3 depicts the characteristics of these codes.

Table 3

Codes and Characteristics of Student Responses

Code Characteristics of Students' Answers

Inappropriate Students lack knowledge required for the task; equal sign means obtaining the answer.

Arithmetic Students recognize and use arithmetical operations, laws, and numerical answers. Each side of the equal sign has the same value.

Pre-algebraic

Students recognize and use unknowns, variables, concatenation, and inverse procedures to find an unknown. Each side of the equal sign has the same value.

Algebraic

Students recognize and use relationships expressed in simplified forms. The use algebraic processes, such as the balance approach, to solve an equation by operating on or with the unknown. They view the equal sign as denoting equivalence.

Note: From "Readiness for Algebra.” by G. M. Boulton-Lewis, T.J. Cooper, B. Atweh, H. Pillay, and L. Wilss, 2000, Proceedings of the 24th Conference of the International Group for the Psychology of Mathematics Education, p.92.

They followed 33 of the original 51 seventh graders with the task of determining what the equal sign meant in the equation $\frac{28}{7}+20=60-36$. In seventh grade, these 2 students had responded that it was the "answer" (an inappropriate answer), 19 students answered that the same value occurred on either side of the equal sign (an arithmetic answer), 9 students said that the expressions on each side were the same value (a pre-algebraic answer), and 3 students made a comment about how each side was balanced (an algebraic answer). After finishing the $8^{\text {th }}$ grade, only one student still held an inappropriate view of the equal sign, 12 students held an arithmetic view, 8 students held a pre-algebraic view, and 12 students possessed an algebraic view. After receiving instruction in linear equations and solving linear equations by balancing procedures, $9^{\text {th }}$ graders were interviewed. This time, no one saw the equal sign as providing the answer. Five students still saw it arithmetically, 9 students viewed it through a pre-algebraic lens, and 19 students saw it as an equivalence relation. While the notion of the equal sign progressed over the years, students did not fully grasp the concept of equivalence. Kieran (1981) indicated that without this notion of the equal sign, students would not be able to operate algebraically. She was later proven correct by Knuth, Stephens, McNeil, and Alibali (2006).

Based on the all the misconceptions surrounding the equal sign, Knuth and colleagues (2006) hypothesized that "there is a relation between students understanding of the equal sign and their success with symbolic expression and algebraic equations" (p. 299) based on the fact that if one does not understand the notion of equivalence, they also will not understand transformations performed on an 
equation. Researchers asked 177 sixth, seventh, an eighth graders for their best interpretation of the equal sign in the statement $3+4=7$ and asked what values of $m$ made each of the number sentences $4 m+10=70$ and $3 m+7=25$ true. If students provided an answer based on an operational standpoint, such as it means to do something, to obtain an answer, or to add the numbers, researchers coded the response as operational. However, if students expressed the idea of equivalence with responses expressing that the two statements are "the same as", the question was coded as relational. Fifty-three, thirty-six, and fifty-two students provided an operational definition in $6^{\text {th }}, 7^{\text {th }}$, and $8^{\text {th }}$ grade respectively, while 32,43 , and 31 gave a relational definition. The rest of the students gave an answer that did not fall into those categories $(15,21$, and 17 according to grade level). Researchers found that as grade level increased, students' relational understanding of the equal sign did not. Thus, it is not maturity that provides understanding of the equal sign.

When analyzing how students solved the two equations for $m$, researchers categorized students' methods as algebraic, answer only, no response, guess and test, unwind, or other. The guess and test method was considered an arithmetic strategy, while the unwinding method was considered to be a prealgebraic strategy. The algebraic strategy required students to perform the same transformations to each side of the equation in order to obtain a value for $m$. Researchers found that $6^{\text {th }}$ graders preferred the guess and test method, $7^{\text {th }}$ graders preferred the unwind method, and $8^{\text {th }}$ graders preferred an algebraic method. They found that students who viewed equality as an equivalence relation also used an algebraic method to find the solutions to the equations. This held true even when students who were enrolled in algebra were not considered. Thus, researchers concluded that there is a "strong relation between the equal sign understanding and success in solving equations" (p. 308).

The previous studies depicted many student interpretations of what the equal sign meant in different contexts. Some studies found that the equal sign implied a conditional statement when two ideas were set equal to each other. In some instances, students misused the equal sign as a procedural encoder when they solved problems. Most of the research that has been done on students' conceptions of the equal sign discussed the differences and implications of students understanding of the equals sign operationally or relationally. Those students who believed the equal sign meant to do something usually operated in arithmetical manner, while those who believed the equal sign denoted equivalence usually operated in an algebraic manner.

\section{Reasons for Conceptions}

Students in elementary, middle, and high school have exhibited different conceptions about the 
equal sign. Two prominent conceptions of the equal sign are that it signifies an operation or an equivalence relation. Since one wants students to realize that the equal sign symbolizes an equivalence relation, one must investigate why some students view it as a "do something" signal. Many researchers have hypothesized why students' view the equal sign as an operator sign. Some believe that it is due to the way equivalence is represented in school, daily life, and technology.

Students encounter equality as early as preschool, where they must count two sets of items and see if the sets are of the same cardinality; if so, then the sets are termed "equal" (Gelman \& Gallistel, 1978). This is a comparative notion of equality, where students compare two groups of items and determine if they have the same number of objects. After acquiring this notion, preschoolers are then asked to combine the two sets and count the final amount. This second notion of equality leads students to an operator idea, where the equal sign denotes the result of an operation. Furthermore, once students operate on the sets, they are unable to symbolically show the comparison of the sets, which enforces the idea of operationality. For example, once students gather the set of objects, the objects are not split into the original sets.

Falkner and colleagues (1999) noticed that kindergarteners could correctly model equivalent sets, but could not express relationships in the correct symbols. For instance, a teacher gave her students the number sentence $4+5=\square+6$ and asked them what number went in the box. All of her children said the box should contain the number 9. She asked them to model this number sentence with Unifix cubes. All of the students noticed that one set, represented by the expression to the left of the equal sign, was 9 and the other set, represented by the expression to the right of the equal sign, was 6 . Furthermore, they stated that the two sets were not the same size, yet they still insisted that 9 went into the box. This convinced Falkner and her colleagues that students understood equivalence relations between sets, but they did not know how to connect the models of these sets of objects to their symbolic representations.

This disconnect between modeling and symbolic representation is precisely why Baroody and Ginsburg (1983) stated that students viewed the equal sign based on their informal arithmetic knowledge. Since students were exposed first to counting, or operating on items, they naturally tended to view the equal sign as a signal to do something. In fact, Seo \& Ginsburg (2003) gave an example of a child's first addition problem: "If you have three points and get two more points how many points do you have now?" (p. 166). This question prompts students to obtain a "change-add-to" notion of the equal sign because they attach their actions to the idea of equality (Baroody \& Coslick, 1998). Children are asked to change the first number by adding the second number to it, and then write down to what number it is equal. This operator notion is reinforced by future mathematical instruction. 
Seo and Ginsburg (2003) avowed that students were normally introduced to the equal sign by arithmetic statements in the canonical format $a+b=$ ?. Students rarely see formats such as 1 foot $=12$ inches and $5+3=9-$ ? Both textbooks and teachers tend to encourage these ideas through instruction. Two different studies (Denmark, Barco, \& Voran, 1976; Seo \& Ginsburg, 2003) researched elementary textbooks to see how equality was established. Both found that the equivalence relation was not cultivated, for textbooks mainly focused on relating the equal sign with addition and subtraction problems that required students to act on numbers using these operations.

Seo and Ginsburg (2003) also examined how one teacher, who thought she provided enough varied instruction to let her students notice the equivalence relationship represented by the equal sign, taught the equal sign and into what context and learning activities she situated it. They found that the teacher taught in six different contexts: number comparison, addition and subtraction, many names for a number, measurement, equivalent-coin, and everyday. The number comparison context consisted of students modeling the number of objects in a set, comparing those numbers, and then using an equality or inequality sign to relate those numbers. The addition and subtraction context incorporated word problems about finding total and difference amounts to which students wrote arithmetic statements. The renaming of numbers context involved students writing arithmetic expressions that were the same as a specific number. For example, in renaming the number 5 , students wrote $1+4=5,2+3=5$, and $7-2=5$. The measurement context activity used Cuisenaire rods to compare lengths of different colored rods. For instance, students answered questions like 1 orange $\operatorname{rod}=2$ yellows rods $=5$ red rods. The equivalent coins activities were similar in that they required students to state how much one coin was worth (e.g., $1 \mathrm{n}=5 \varnothing$ where $\mathrm{n}$ represented the word nickels and not the number of nickels). In the everyday-life context, students used words to describe different ideas, times, and items in the classroom in terms of equality or inequality. For instance, the teacher said, "We have an equal number of people who have five letters and six letters in their first names" (p. 172).

Six months after the initial instruction, 16 of her 29 second-graders underwent 5 different interviews to gather information about how they viewed the equal sign without context, in canonical context, in non-canonical context, in equivalent-length context, and money-equivalent context. Table 4 depicts the types of questions that were asked in each interview, and how many of the 16 students viewed the equal sign as operational or relational. 
Table 4

Interview Contexts and Questions

\begin{tabular}{|l|l|l|l|}
\hline Interview & Question & Operational & Relational \\
\hline 1. No Context & What does equal mean? & 14 & 2 \\
\hline 2. Canonical Context & $\begin{array}{l}\text { What does "=" mean in } \\
a+b=? \text { or } a-b=?\end{array}$ & 13 & 3 \\
\hline 3. Non-canonical Context & $\begin{array}{l}\text { What does "=" mean in } \\
?=a+b \text { or } a=a\end{array}$ & 13 & 3 \\
\hline 4. Equivalent-Rods & $\begin{array}{l}\text { What does "=" mean in } \\
2 \text { white rods }=1 \text { red rod }\end{array}$ & 4 & 12 \\
\hline 5. Money-Equivalent Context & $\begin{array}{l}\text { What does "=" mean in } \\
1 \text { dollar }=100 \text { pennies? }\end{array}$ & 3 & 13 \\
\hline
\end{tabular}

Seo \& Ginsburg (2003) found that students primarily had an operational point of view of the equal sign, however, in some context they saw it as a relation. When the equal sign was used in the context of performing operations, students viewed it as operational. When the equal sign was used to compare items or which no arithmetic operations were performed, students viewed the equal sign as relational. This study was one of the first to show that students can possess a relational aspect of equality. The main problem was that students' views of the equal sign were not well integrated. Students did not have a thorough conceptual understanding of the equal sign because relationships between operational equivalence and relational equivalence are not well connected. Seo and Ginsburg also conceded that both the teacher and textbook emphasized an operational view of the equal sign in some contexts, but the teacher emphasized the relational aspect in other activities such as the Cuisenaire rods and coin activities. Furthermore, they posited that parents and society perpetuate the operational notion of the equal sign, for using mathematics entails "getting the right answer as quickly as possible" (p. 181). When students problem solve, they are encouraged to find the answer and write it down, usually to the right of the equal sign. Moreover, this usage of the equal sign is not only encouraged, but it is also a legitimate way of operating. Even if students retain the idea of equivalence, students must check or perform some type of operation to make sure that each set is equivalent. It is almost a prerequisite that in order to determine an equivalence relation, one must operate on the expressions first. Technology can advance this operational notion, for when people use calculators and spreadsheets to determine if two arithmetic expressions are equal, they first must enter in numbers and operations and then hit equals (or enter) to see if the values are the same.

Seo and Ginsburg (2003) showed that students were able to adhere to two different ideas of equality, but they were unable to connect these two ideas. One reason for this disconnect may stem from the overwhelming cognitive load that presents itself when students try to think about two explanations of 
the equal sign simultaneously. The cognitive load is intensified by the fact that students have to calculate both sides, remember the answers, and then determine if the expressions are equal. Collis (as cited in Kieran, 1981) noticed that students between the ages of 6 and 10 had to replace the values on each side prior to determining if two expressions were equal. For example, in order to see whether $4+5$ was equal to $3+6$, students wrote $4+5=9$ and $3+6=9$ and then determined if 9 was equivalent to 9 . Collis found that it was around the age of 13 that students were able to determine equality without making these replacements.

Students' conceptions of the equal sign occur because of students' prior and present experiences with the idea and symbol. Students first experience the idea of equality and the word "equal" through the comparison of objects, and afterwards they relate the notion of operating with the term "equal". By the time students are introduced to the equal sign, students are in the midst of learning about other operator symbols such as the plus, minus, multiplication, and division signs. Furthermore, they are not exposed to formats that call for a relational aspect of the equal sign such as 1 foot $=12$ inches, $3=3$, and $5+3=9-?$. Despite these experiences, two studies (Falkner et al, 1999; Seo \& Ginsburg, 2003) showed that students understood the relational aspect of the equivalence when given certain contexts. Students' view the equal sign as relational when the equal sign relates two objects, but they resort to the operational view when the equal sign is placed amidst numbers and operator symbols. Furthermore, these two views are not integrated, but perhaps could be if students were given instruction to relate them.

\section{Instruction to Overcome Misconceptions}

Based the reasons for why students have difficulty with the equal sign, many researchers advocated for new instruction. The first researchers to implement teaching strategies to mediate students' conceptions were Anderson (1976) and Denmark, Barco, and Voran (1976). Anderson instructed second graders in the relational aspect of the equal sign and found that those who received the teaching were more apt to accept atypical, non-canonical arithmetic equation forms. Denmark and colleagues examined first graders' ideas of the equal sign and utilized a scale balance to expose them to the notion of equivalence. They also showed students accepted non-canonical forms using the equal sign. The researchers found that these children assumed the operator notion of equals less often and accepted non-canonical forms more often.

Studies have shown that other methods of instruction have also provided students with ideas of an equivalence relation for the equal sign. Falkner and colleagues (1999) depicted a teacher who used declaration, discussion, modeling, examples, and reflection to aid first and second graders in a relational 
understanding the equal sign. She explicitly told them what the equal sign meant, led class discussions on the truth value of certain arithmetic sentences, used Unifix cubes to model these arithmetic sentences, presented multiple arithmetic sentences with the unknown in varying positions, and had students reflect on the meaning of the equal sign by providing them with true/false questions about which to think. After a year and a half of these kinds of activities, she found that students began to understand the relational aspect of equality, and 14 out of her 16 students could answer that 7 should go into the box for the statement $8+4=\square+5$.

Kieran $(1979,1980)$ tried to foster the relational idea of the equal sign in hopes to lay a foundation for solving equations. She clinically interviewed 12 to 14 -year old students to determine their interpretation of the equal sign. Her interviews showed that most students had an operational idea of the equal sign. Therefore, she had them write equality statements using the same and different operations on each side of an equal sign. For example, she had students write $2 \times 6=4 \times 3$ and eventually $2 \times 6=10+2$. She found that students would justify these statements by saying that both sides were of equal value. Therefore, students were able to see a more relational notion of the equal sign because expressions on the left side had to have the same value as expressions on the right side. Students were able to have two notions of equality, but instruction cultivated these notions and explicitly connected them.

Besides instruction showing different formats of arithmetic statements, discussion and concrete modeling of these statements, and constructing arithmetic identities using different operations on both sides of arithmetic statements, researchers suggested other methods to delineate relationships with the equal sign. Baroody and Ginsburg (1983) advocated writing the answer to an addition problem above the plus sign like so:

$$
\begin{gathered}
3 \\
2+1
\end{gathered}
$$

They believed that this would move students from seeing the equal sign as an operator and as more of a relation between equivalent values. Writing the answer above the plus sign instead of below the plus sign might also help students to not view the equal sign as a procedural encoder that Stacey and MacGregor (1997a) witnessed from their students. Stacey and MacGregor suggested that students should be encouraged to use a guess and check strategy by replacing the $\square$ in non-canonical forms (e.g., $7+\square+1=10+2$ ) with a number to see if it makes the statement true.

Seo and Ginsburg (2003) made numerous recommendations to move students from an operational view to a relational view of the equal sign. They believed that teachers should promote different meanings 
of the equal sign using composition, decomposition, renaming, and equivalent sets. By composition, they meant that teachers should display and situate equality in terms of adding together two items, or numbers, to create a new number (e.g., $2+3=5$ ). Decomposition referred to the breaking down the new number into its parts, such as $5=2+3$. Students should know when each of these types of written statements would apply given a specific problem context. Seo and Ginsburg believed that teachers, like the one they observed, should provide renaming activities. Students should be instructed to take a number and think of different ways to rename that number using operations. This may be facilitated through the use of models and equivalent sets. For instance, students should compare two collections of items, a collection of items and a number, and two numbers. Students should feel comfortable seeing that $\square \square \square=\square \square \square, \square \square \square=3$, and 3=3. Explicit discussions of an equivalence relation should be given to students. Students should see reflexivity:5 = 5, symmetry: if $\square \square \square \square \square=5$ then $5=\square \square \square \square \square$, and transitivity: if $5=2+3$ and $5=1 \times 5$ then $2+3=1 \times 5$ of the equal sign. This idea can also be noticed by using different symbols for the equal sign to enable students to become comfortable with the multiple meanings of the equal sign.

Many researchers put forth ideas about how to instruct students on the relational meaning of the equal sign. Most of the researchers and educators gave students atypically represented formats using the equal sign and guided students through modeling, discussion, and reflection on why these representations were reasonable. After teaching students in this manner, students felt more comfortable dealing with all formats and viewed the equal sign as relational more than they had done so previously.

\section{Reasons and Frameworks for Conceptions in Algebra}

Researchers have hypothesized why students possess incorrect conceptions with preliminary algebraic concepts. Since students' conceptions and ideas are based on their meaning-making processes, I will use Kieran's (2007) four sources of algebraic meaning-making to situate misconceptions. She posits that students gain algebraic meaning from algebraic structures, multiple mathematical representations, problem context, and exterior problem information. Gaining meaning from algebraic structures rests on both arithmetic knowledge and the ability to convert between arithmetic and algebraic language and symbols. The last three sources deal with instructional matters dealing with approach methods and types of tasks and tools.

\section{Arithmetic Knowledge}

Some students' algebraic misconceptions have their roots planted in arithmetic. Booth (1989) noted that 
A major part of students' difficulties in algebra stems precisely from their lack of understanding arithmetical relations. The ability to work meaningfully in algebra, and thereby handle the notational conventions with ease, requires that students first develop a semantic understanding of arithmetic. (p. 58)

Many students can produce correct answers to arithmetic problems, but they do not always see the mathematical structure behind the numbers. Students are able to operate on numbers, but not always witness the relationships between them. Thus, they do not see the intricate patterns that generalize for numbers, which in essence produces the description of algebra as generalized arithmetic. If students can meaningfully understand arithmetical relations, they should have no problem generalizing that knowledge and working meaningfully in algebra. But in order for students to conceptualize algebraic meaning by generalizing arithmetic, students must procedurally and conceptually understand the structure of arithmetic.

\section{The Structure of Arithmetic and Algebra}

Much of arithmetic is focused on getting the answer, as can be seen in students' view of the equal sign as "do something" signal. Students are taught to remember the rules for operating on numbers, and some students can do this well, for they are aware of the surface structure of arithmetic expressions. According to Kieran (1989), surface structure consists of the arrangements of the numbers and operations. For example, students who are aware of surface structure will be able to compute $9-8 \times 7$ correctly because they will know how to use the order of operations. Operating in this way is purely procedural. When students notice the systemic structure of an arithmetic expression, it allows them to conceptualize the meaning behind the numbers and operations. Systemic structure of an expression is more than operations and numbers, for it includes the properties inherent to the expression. For instance, students who are aware of systemic structure notice that $9-8 \times 7$ is equivalent to $9-56$, and they will see that it can be written $9-7 \times 8$ because of multiplicative commutivity. Students who notice systemic structure will recognize properties of operations including commutivity, associativity, identity, and equivalence as well as relationships between operations such as distributivity.

Students who had no structural sense of arithmetic found difficulties with algebraic structure. Linchevski \& Livneh (1999) interviewed 53 sixth-graders to assess whether their algebraic problems stemmed from arithmetical structural problems. Linchveski \& Livneh tested students on order of operations and other operative properties. They found that students consistently encountered the same difficulties in numerical contexts as algebraic contexts. What is interesting to note is that within certain types of structures, students changed operative strategies. Researchers found that there existed competition between structure of the expression and the numbers that were used in that expression. When students were given 
$217-17+69$ and $267-30+30$, students would operate left to right on the first expression, but detached the 30s in the last expression, added them, and then subtracted the sum from 267. Students acted similarly on algebraic expressions of these types. Therefore, Linchveski and Livneh suggested that there might be competing attention for certain numerical combinations. In fact, they posited that students might intuitively see $267-30+30$ as $267-2(30)$. They advocated that teachers should enable students' structural sense by connecting arithmetic structure to algebraic structure, for students need to obtain a "deep understanding of arithmetic" which "requires mathematical generalizations that are algebraic in nature" (Carraher \& Schliemann, 2007, p. 671).

Herscovics and Linchevski (1994) tried to determine the boundary between arithmetic and algebra. In order to find the borderline, they interviewed 22 seventh graders, who had not had algebra instruction, to elicit a cognitive gap. Where that cognitive gap occurred marked the dividing line between arithmetic and algebra. The first test focused on the operation properties, order of operations, arithmetic statements, and equality. The second test focused on equations with one unknown. Researchers found similar misconceptions in regards to operational number properties and equality as the previously mentioned studies, and noted that the cognitive gap appeared when students had to operate on an unknown. They deemed that students needed to have conceptual understanding of operational properties, order of operations, and equality in order to operate algebraically.

\section{The Transition from Arithmetic to Algebra}

To understand students' conceptions of algebraic structures, one should analyze the adjustments transpiring during the path from arithmetic to algebra (Matz, 1980). When a change from arithmetical thinking to algebraic thinking does not occur, either procedurally or representatively, students will employ prior knowledge inappropriately to new situations. These errors can then be analyzed to determine students' prior conceptions as well as how students generalized these conceptions to apply to a new setting. Most misconceptions in algebra deal with the two critical differences between arithmetic and algebra: "the use of letters to represent numbers, and explicit awareness of the mathematical method being symbolized by the use of both numbers and letters" (Kieran \& Chalouh, 1993, p. 179).

\section{Similarities and Differences of Arithmetic and Algebraic Procedures and Symbolism}

A way of analyzing students' ideas of algebra is to examine the contemporary similarities and differences between arithmetical and algebraic procedures and symbolism. Booth (1988) believed that many of students' misconceptions could be traced to the following four aspects: 
(1) The difficulties in arithmetic understanding

(2) The differences between arithmetic and algebraic activities

(3) The use of conventional notation in algebra

(4) The understanding behind letters and variables (p. 21)

Students must have a strong conceptual and structural understanding of arithmetical operations and relationships in order to appreciate algebraic ones. Stacey and MacGregor (2002) believed that all students are able to do computations, but they do not necessarily know how to describe what they are doing or how to verbalize why they are doing it. Students need "to recognize and articulate the processes of arithmetic and the structures of the relationships between numbers" before they can possibly understand algebraic structures (p.148). One of the major difficulties that students must overcome in order to think algebraically is to realize the difference between strictly performing procedures and valuing the structures that allow them to perform those procedures. To overcome this difficulty, students must progress in a more structural approach toward mathematics and learn how to operate with integers, variables, and operations as mathematical objects. Nunn (1919) referred to this change as acquiring a different "attitude" towards the same mathematics, since arithmetic is primarily concerned with calculations, and algebra is primarily concerned with the processes involved in those calculations. In order to be able to operate structurally, Sfard (1991) found that people must first acquire proficiency at operating procedurally. She felt that there was a vast gap between functioning operationally and functioning structurally that will not be bridge quickly. This slowness in bridging this gap can be explained by the immense cognitive load that students must accept regarding procedures and notations in algebra (Wagner \& Kieran, 1989).

In arithmetic, students operate on numbers to find an answer. They usually do not delve into what the numbers, operations, and final result represent. In algebra students must acknowledge that numbers can represent objects or parts of procedures, letters can represent relationships or act as an object itself, and operations represent a procedure that must be performed or a relationship between two values.

Furthermore, students do not only have to acknowledge these different representations, but they also have to perform different types of operations on these represented mathematical objects including simplifying, solving, factoring, and rationalizing. In the transition from arithmetic to algebra, notation that represents different structures seems to change in their meanings. The equal sign becomes representative of equality rather than a "do something signal," and basic operations do not necessarily need to be performed but rather become representative of relationships between numbers and variables. Thus, students see the same mathematical symbols, but come to two different meanings about the structure depending on whether they 
are viewing it through an arithmetic attitude or viewing it through an algebraic attitude. For example, Hilbert (as cited in Steiner, 1975) gave the following statement: $a+1=1+a$. Hilbert said that this statement could be viewed in two different ways: as a "hypothetical judgment that comes to assert something when a numeral is given" or as a general statement postulating the commutivity of all counting numbers with the number 1 (p.144). In arithmetic, students would see that $a+1$ means to add 1 to a specific number, $1+a$ means add $a$ to 1 , and the fact that they are equal means that after the addition takes place, the values are the same. So if one were to evaluate addition with $a$ and 1, they would find that the outcomes would be equal. Whereas, if one were viewing this statement algebraically, one would notice the structure of the statement and see that it is generalizing an arithmetic statement, for it is describing the relationship between all numbers and 1 through the process of addition. Thus, students need to recognize that $a+1$ represents both an instruction to perform the operation as well as the result of performing the operation (Booth, 1988). She felt teachers can stress this dual meaning by verbalizing the expression as "add $a$ and 1 " and "the number that is 1 more than $a$ " (p. 25).

Students must use their arithmetic knowledge of basic operations as a foundation on which to build knowledge of how to use letters and symbols to symbolize mathematical structures and processes (Kieran \& Chalouh, 1993). Yet, students have a difficult time doing this because their "interpretations of algebraic symbolism are based on other experiences that are not helpful" (Stacey \& MacGregor, 1997b, p.110).

Due to the inabilities for some students to understand algebraic symbolism, Skemp (1982) produced a model for symbolic understanding. Symbolic understanding requires "assimilation between a symbol system and an appropriate conceptual structure" (p.60). To obtain this assimilation, students must have many relationships connecting what the symbols look like to a symbol system, which provides the meaning behind the symbol. Thus, when one encounters a symbol through written or verbal communication it will be sent to the symbol system structure. In order for a person to conceptually understand this symbol, many connections must be provided linking it to a conceptually formed structure. Skemp added that conceptual structures must be formed early and strengthened, or symbols will be attracted to the structures that are used for spoken language, or some other previously developed knowledge. He advocated that students need to use their own individual symbolic system prior to being introduced to the conventional algebraic symbolic system. He ended with a great quote epitomizing the reason mathematicians invented symbolic systems and the reason why they are difficult to understand: "Symbols are magnificent servants, but bad masters, because by themselves they do not understand what they are doing" (p. 61). 


\section{Algebra Instruction}

Misconceptions often develop from an incomplete treatment of a topic (Matz, 1980). Students will compensate for their incomplete knowledge by over-generalizing. Some of the incorrect knowledge might be unveiled and students will correct it, but other parts may not be unveiled until that knowledge is called upon in a novel situation. Thus, students who do not encounter a novel situation may easily slip through the cracks because they can perform well on routine problems.

Researchers believe that misconceptions in algebra result from inadequate conceptual understanding due to a formal axiomatic approach to algebra instruction (Thorpe, 1989; Leitzel, 1989). Students come away from their first course in algebra thinking that it was about symbolic manipulation and solving for the letter $x$ instead of understanding it was about the use of logic to solve problems with the help of abstract structures and procedures (NCTM, 2000). If students are provided with situations that exhibit algebraic symbols and structures and are asked to only manipulate variables, it is no wonder that students have a limited view of algebra. These kinds of instructional activities do not allow students to gain deep algebraic meaning.

\section{Generational, Transformational, and Global/Meta-level Activities}

As noted in a prior section, there are four means through which to obtain algebraic meaning: algebraic structure, problem context, peripheral information, and multiple mathematical representations. Students who solely deal with manipulation of symbols barely even scratch the surface of learning about

algebraic structure, let alone touch any of the other three meaning making methods. To help educators and researchers integrate algebraic meaning into algebraic instruction, Kieran $(1996,2007)$ created the $G T G$ model, by partitioning algebraic activities into three categories: Generational, Transformational, and Global/Meta-level. Generational activities involve forming algebraic structures including expressions and equations. In order for students to build algebraic structures, they must have substantial knowledge concerning variables, unknowns, equality, and the notion of a solution to an equation. While generational activities require mainly conceptual knowledge, transformational activities require more procedural knowledge. Transformational, or rule-based, activities deal with manipulative processes based on algebraic properties such as equivalence and other axioms. This type of activity includes collecting like terms, simplifying, factoring, expanding, and so on. Most of today's algebraic instruction can be classified as transformational. Transformational activities include conceptual knowledge such as why one can apply certain procedural knowledge to expression and equations. However, some students in school algebra 
usually do not witness or retain this conceptual knowledge and choose to only focus on the procedures. The third type of algebraic activities is global/meta-level. It is so named because this type of activity is not strictly related to algebra, but can be applied to any type of mathematics. It includes activities such as problem solving, modeling, looking for patterns, making conjectures and predictions, and justifying. Since most algebraic activities are already transformational, and since I only want to include relevant literature to my study, I am going to focus specifically on generational activities in the global/-meta-level context.

Much of initial meaning making of algebra is situated in generational activities in a global/metalevel environment (Kieran, 2004). A global/meta-level environment can be considered constructivist in nature, for problem solving, conjecturing, predicting, observing, and justifying are actions that enable students to construct their own knowledge about algebraic structures. In fact, many researchers advocate for a constructivist teaching sequence that provides contextual, non-routine, applicable problems, creates motivation for the usage of symbols, generates discussion, and builds on students' informal prior knowledge (Booth, 1988; Carraher \& Schliemann, 2007; Confrey, 1998; French, 2002; Herscovics, 1989; Lodholz, 1990; NCTM, 2000; Wagner \& Parker, 1993). Students' misconceptions develop due to decontextualized algebraic manipulation that is based on rote memorization of rules. Without contextual meaning and seeing the need for symbolic representation, students have nothing to which to connect the procedures; therefore, they inappropriately over-generalize and apply incorrect procedures because of lack of conceptual understanding. Both Davis (1998) and Bell (1995) showed that the constructivist method is more effective in the learning of algebra. Davis (1998) recommended the usage of communication and reflection in the classroom, concrete and abstract modeling of mathematical phenomena, and word problems. Communication and reflection allow students' thought processes to be known to themselves as well as to their teacher. This acknowledgement provides opportunities for students to discuss different conceptions, which can invoke cognitive conflict. By invoking cognitive conflict, students must actively overcome and confront mental inconsistencies and by doing so will be less inclined to cling to previous misconceptions (Bell, 1995, Mestre, 1987). Concrete and abstract modeling encourages discovery and generalization that elicits the opportunity for connection to symbolic representation. By giving students word problems, one enables them to construct mental models based on previous and informal knowledge and to translate those models into mathematical language.

Another way to build on previous and informal knowledge is by drawing on students' arithmetic knowledge in generalizational activities. Generational activities are surrounded by two frameworks: functions and generalized arithmetic. A functions approach situates all algebra topics within a functional 
context. For example, it views first-degree equations as two functions that are represented by linear graphs. The solution for an equation is the value of $x$ that makes both functions elicit the same value. This approach centers on the idea of variable as an object in and of itself rather than an unknown value. The numerical approach, also known as treating algebra as generalized arithmetic, focuses on generating and interpreting algebraic structures. It views these structures as representations of numerical processes and considers variables as unknown values. Research has pointed to the fact that algebraic misconceptions result from both poor arithmetic knowledge and the inability to transition from arithmetic to algebra. Furthermore, supporters of constructivism advocate building on students' prior knowledge, and in this case it is arithmetic. Therefore, one will adhere to the generalized arithmetic framework, and hence apply the numerical approach to instruction for mediating misconceptions.

\section{A Numerical Approach to Algebra}

Arithmetic and numbers are inherent in algebra. According to Bass (1998), the sum and substance of algebra is "the basic number systems, the arithmetic operations on these number systems, the study of algebraic equations that arise naturally in these systems, and the linear ordering and resulting geometric structure defined on the real line" (p. 9, his emphasis). One particular number system is the field of real numbers. Using real numbers, students can intuitively come to an understanding of field properties such as commutivity, associativity, distributivity, identity, and inverses through simple patterned calculations before formally symbolizing these properties in terms of variables. Carpenter, Franke, and Levi (2003) used number sentences such as $3+5=5+3,9+5=14+0$, and $9+5=0+14$ to encourage students to think algebraically in expressing generalizations about numeric structure. Other studies have also pointed to students being able to algebraically think and work with numerical instantiations of arithmetic properties (Bastable \& Schifter, 2007; Schifter 1999). While students may be able to describe these relationships algebraically, they have not yet been able to write these notions using algebraic symbols.

Fujii (2003) and Fujii and Stephens (2001) used arithmetic sentences to emphasize structural properties of arithmetic to students. They used arithmetic sentences that hold true no matter what numbers were substituted into the original numbers. They called the original numbers in these sentences quasivariables. For example, given the sentence $54-36+36=54$, one can replace 36 with any number and 54 with any number and still have a correct statement, for the algebraic structure is the identity $a-b+b=a$. They tried to get students to recognize the type of number sentence and gain insight into the structure of the number system, instead of requiring students to write the equation using variables.

Herscovics and Kieran (1980) also used number sentences, or in their case, arithmetic identities to 
establish equality, order of operations, the use of bracketing, the defining of equations, and the notion of variable. They used students' ideas about basic operations and re-presenting the same number to ascertain equality between two expressions. After guiding students to the correct representation of operating order, they had students cover up one of the numbers to indicate a "hidden number": intuitively guiding them to the notion of variable. While this method of teaching equations is somewhat backward to the norm, Hercovics and Kieran anchored students' knowledge in arithmetic, which provided meaning for mathematical form and symbolism.

Algebraic properties can be enhanced through use of arithmetic operations and numerical instantiations of the properties. In order to fully generalize these properties, students must have a sound idea about signed numbers, specifically negative numbers and operations on them. Negative numbers, especially subtraction of negative numbers, are abstract ideas for children. One way to ground these abstract ideas is by using quantities and direction on the real number line in the context of temperature, time periods, traveling, and sea level. Bass (1998) advocated the incorporation of the number lines in the learning of algebra to facilitate movement to and understanding of algebraic representation. He states, “([i]t is both natural and advantageous to give an early emphasis to the geometric real line model of the real numbers, in which basic arithmetic operations are interpreted geometrically and developed alongside the more algorithmic development" (p. 15). Klein and colleagues (1998) also found that using a number line could be a powerful display for operating and representing numbers and operations with second graders. Carraher, Brizuela, and Earnest (2001) combined the use of variable and number lines in what they called variable number lines, to show number structures of operations. Instead of using numbers on their number line, their number lines contained variables and numbers such as $N-3, N-2, N-1$ on which students explored the concept of differences between numbers.

All of these studies emphasized how naturally it is to extend the numerical properties of arithmetic to introduce algebraic structure. Historically, the purpose of algebra was to make arithmetic easier as well as to attempt to find solutions to problems that arithmetic was unable to solve. Students should see these aspects during instruction.

\section{Multiple Representations}

Numbers are not the only way to facilitate algebraic discovery and ease the transition from arithmetic to algebra, for students who used and understood the relationships among multiple representations were able to conceptualize algebraic topics better than those who did not (Moseley \& Brenner, 1997). Twenty-seven junior high school students taking pre-algebra participated in Moseley and 
Brenner's study. Prior to instruction, all students took a pretest asking them to write an equation for two word problems. For twenty days afterwards, fifteen of the students received instruction on word problems using tables, graphs, words, and algebraic notation, while the other twelve students received direct instruction in only words and algebraic notation. After instruction, students were asked to generate two equations for two different word problems during an interview. Students who were instructed using multiple representations showed significant improvement from pretest to posttest, whereas the other group did not. Furthermore, the non-multiple representation group attacked the word problems in more of an arithmetic manner than an algebraic manner. Therefore, researchers concluded that multiple representations made "substantial differences in the ways that students conceptualize variables and their notation" (p. 17). Instruction including multiple representations even allowed fourth graders to perform significantly better than sixth and seventh graders who received traditional instruction in representing and solving word problems containing first-order equations (Bodanskii, 1991). These differences can be expected since students will have more methods of modeling problems and answering questions based on the problems. Multiple representations, for the purposes of this dissertation, are those that exist outside the learner's internal cognitive structure. Within mathematics, there are four representations: graphs, tables, equations, and words (Ainsworth, 1999; Brenner, Mayer, Mosely, Brar, Durán, Reed, \& Webb, 1997; Goldin, 2002; NCTM, 2000). Each of these representations has special characteristics that emphasize certain aspects of a concept. Graphical representations show a consolidated, larger, and continuous view of a concept's structure and highlight the directionality and magnitude of the information, whereas tabular representations present an explicit, smaller, discrete view of the information that can be read easily to find the numerical value changes between the variables (Kaput, 1989a, p. 172). Algebraic representations express the succinct, symbolic relationship between variables to demonstrate how a change in one variable will affect a change in another. Verbal representations can express not only the denotation of mathematical phenomena encoded in other representations, but also allows people to bring connotations to the mathematical objects, which become part of their concept image. Because of the advantages of each representation, they are important for students to learn in their own right.

Representation is essential for student learning and understanding, for it provides students the opportunity to communicate and support their mathematical reasoning processes through mathematical modeling of problem situations (NCTM, 2000, p. 67, 360). Through modeling, students "select, apply, and translate among mathematical representations to solve problems" (NCTM, 2000, p. 67), which illustrates three main benefits of multiple representations mentioned by Ainsworth and colleagues (1997). The first 
benefit is that they provide supplemental information that helps students to reason more effectively. The second benefit is that students can recognize what they might not understand, which might normally lead to a misconception if they were provided with only one representation from which to reason. By having at least two representations, one representation can assist in the interpretation of the other. During the process of connecting the two representations, it is more likely that students will be confronted with irreconcilable ideas, which will compel them to adjust their conceptual notions. The third benefit of using multiple representations is that they "promote deeper understanding of the domain" (p. 94). By this, Ainsworth means that multiple representations collectively help elicit the underlying mathematical structure, which in turn encourages generalization and abstraction of the concept.

Therefore, when students learn a concept through the use of multiple representations and how to translate between them, they are able to better comprehend the algebraic concept, communicate, and apply that knowledge (Arcavi, 1995). Through this comprehension and communication, they can maneuver among the representations to capitalize on the advantages of each. Being able to maneuver among representations permits students to choose the best representation during problem solving (Ainsworth, 1999; Ainsworth, Bibby, \& Wood, 2002; Lesh, Post, \& Behr, 1987; Piez \& Voxman, 1997). For students to be successful in transitioning from arithmetic to algebra, they need to use generalized arithmetic, contextual algebraic problems, and the opportunities to connect multiple representations (Warren, 2004).

\section{Technology}

Besides the introduction of the graphing calculator, algebra has not changed in its instruction in the past 50 years even though technology has opened the door to many different educational opportunities (Thorpe, 1989; Leitzel, 1989). Algebra has been taught primarily as rule-based procedures for manipulating symbols. With the advancement and widespread usage of technology, students now have access to many different forms of hardware and software including graphing calculators, spreadsheets, computer algebra systems, and dynamic geometry systems. These technologies can facilitate algebraic instruction to be constructivist in nature, to focus on generalizing arithmetic, and to incorporate multiple representations. All three of these instructional attributes contribute to students' conceptual understanding of a topic.

There are two types of mathematical activity: technical and conceptual (Zbiek, Heid, Blume, \& Dick, 2007). Technical activities are activities that involve "mathematical actions on mathematical objects or on representations", while conceptual activities include "understanding, communicating, and using mathematical connections, structures, and relationships" (p. 1170). These two types of activities are similar to procedural and conceptual knowledge in that technical activities require mainly procedural knowledge to 
carry out mechanical actions, and conceptual activities mainly require conceptual knowledge to perform analytical and reflective actions. Researchers debate whether technology fosters the synergistic relationship between technical and conceptual activities. Some researchers (Borwein, 2005; Hoyles, Noss, \& Kent, 2004; Lagrange, 1999) believe that technology encourages a combination of the two, with conceptual reasoning directly impacting mechanical actions, whereas others (Artigue, 2002; Kaput, 1992) view that technology consolidates technical activity to allow students to engage in more conceptual pursuits.

\section{Technology Tools and Instruction}

Technology allows students to act like mathematicians by encouraging actions in which mathematicians engage (Heid, 1997). These actions are stimulated by five different types of technological tools: conceptual fluency tools, mathematical exploration tools, learning how to learn tools, problemsolving method tools, and representational tools (Pea, 1986). Conceptual fluency tools are tools that perform routine processes that allow students to analyze and problem-solve. These types of tools free students from procedural routines so that they can focus more on the interpretation of mathematical objects (Ruthven \& Hennessy, 2002) so students are able to learn concepts and applications prior to knowing procedural skills.

Mathematical exploration tools are tools that emphasize discovery. Students who are engaged in discovery guide their own learning so that instruction becomes student centered (Dugdale, Thompson, Harvey, Demana, Waits, Kieran, McConnell, \& Christmas, 1995; Jenson \& Williams, 1993). Students participate in the decision-making of actions upon mathematical objects, which allows them to construct many connections between their prior knowledge and new knowledge. Mathematical exploration tools are linked to learning how one learns, in that once students start actively doing mathematics, they are able to reflect on their actions. This type of tool allows students to generalize about specific examples. By using technology, students can generate many examples from which to reason (Jensen \& Williams, 1993; Ruthven \& Hennessy, 2002). Students use these examples to hypothesize, test, reflect, and change their notions about the mathematical phenomenon. Through immediate feedback, technology facilitates students' experience in a mathematical environment.

Problem-solving method tools facilitate students' heuristic knowledge of a problem, which helps them problem solve. These tools include drawings, annotations of diagrams, and other scaffolding ideas so that the students see the path to the solution. These tools show the reasoning strategies that students should incorporate into their own problem solving skills.

The last type of cognitive technology tools is representational tools. Many researchers (Dickson, 
1985; Dugdale et al, 1995; Foley, 1997; Heid, 1997; Kaput, 1989, 1992; Pea, 1986) have heralded the benefit that technology easily provides linkages among different representations of mathematical concepts, relationships, and processes. NCTM encouraged the use of technology as a delivery medium of multiple representations because it "affords access to visual models that are powerful but that many students are unable or unwilling to generate independently" (2000, p. 25). Jiang and McClintock petitioned mathematics educators to "make the best use of multiple representations, especially those enhanced by the use of technology, encourage and help our students to apply multiple approaches to mathematical problem solving and engage them in creative thinking" (2000, p.19). Since many students have difficulty linking and moving among multiple representations (Goldenberg, 1988; Leinhardt, Zaslavsky, \& Stein, 1990), appropriate use of technology can be used to improve their ability to see the connections and structure of the mathematical concepts.

With specific regards to algebra, technology can provide an easy transition to algebra through multiple representations. Researchers have shown that when relationships between quantities are emphasized in arithmetic, students can start to represent and notate them in an algebraic manner (Lesh, 1987; Noss, 1988; Sutherland, 1989; Thompson, 1992). Technology can lessen the computation needed by students, and allow students to focus on, reason on, and analyze the relationships between quantities. For example, in Excel, one can easily extend a numerical table without computation, which allows students to focus on how two quantities relate without being burdened with the individual computations. Geometer's Sketchpad can also create tables based on functions so that when students are on a point of a graph, they can capture the values in a table so they can simultaneously reason graphically and numerically. Both of these types of technologies allow students to access many numerical examples in a short amount of time; thus, students are able to analyze and generalize on these examples to become aware of the underlying algebraic structure (Gluck, 2000; Koedinger, 2001; Koedinger \& Anderson, 1998). Moreover, these technologies have the ability to provide a dynamic linking, or a hot link, between two or more representations (Kaput, 1992). When two representations are hot linked, students are able to act on one representation and see an immediate reaction in all connected representations. This type of dynamic linking and immediate feedback can aid in students' understanding of symbolic notation through the usage of numbers. If the symbolic notation is displayed and connected to other representations such as tables and graphs, students will be able to access meaning of both the mathematical concept and the notation of it. Students who are symbolically weak can acquire access to algebraic knowledge through the other representations. In fact, when given technology, some students, who were weak in symbolic manipulation 
skills, were able to outperform their peers, who were strong in symbolic manipulation, in tasks involving creation and interpretation of algebraic situations (Huntley, Rasmussen, Villarubi, Sangton, \& Fey, 2000). Technology provided the access to algebra to students who were unable to attain comprehension in the traditional symbolic manner.

\section{Software Requirements}

While technology can improve the quality of algebra instruction, it does not do so by itself. To reap the benefits of technology, one must also incorporate appropriate content delivered with appropriate content pedagogy, which can only be implemented by a qualified instructor. Researchers agree that mathematics teachers are the vital element in the movement towards proper incorporation of technology into the mathematics classroom (Kaput, 1992; NCTM, 1991, 2000). The instructor must design and implement software files suitably, in order for constructivist learning to take place. There are many opinions about how technology should be designed and implemented to follow the NCTM Standards. Flanders (1988) specified five software requirements. The software should not require any specialized skills other than basic computer skills to run. Even with those basic skills, instruction should prepare students to use the software (Heid, 1990). The second requirement is that the "software should be truly interactive" (p.149). Software should not simply present information, but rather allow students to make decisions and manipulate objects so that they are part of the process in constructing their own knowledge. Software "should be robust" in that it must be "difficult to crash" or corrupt through the user's entries (p. 149). If the user enters an incorrect input, the software should simply prompt for a new input or generate a corresponding incorrect, mathematical output. The fourth and fifth requirements demand that software is syntactically and computationally mathematically appropriate. This means that the notation of the software should mirror the correct mathematical notation. Furthermore, when values are entered, software should provide the correct mathematical computation and present an appropriate output. These last two requirements correspond to what Dick (2007) termed mathematical fidelity. The mathematical fidelity of a technology tool indicates how accurate the technology portrays the underlying mathematical properties of technologically generated representations. If the mathematical fidelity of a tool is high, then actions on a representation will truthfully imitate resulting behaviors on the representations. By following these requirements, technology enhanced algebra instruction will become laboratory-like in that students will be able to explore, investigate, and analyze mathematical ideas based on their inputs and resulting computer generated outputs (Drier, 2001; Heid, 1990). Computers allow students to conjecture, act on their conjecture through manipulation, and then alter their conjecture based on the immediate feedback from 
their manipulation, without requiring the students to become bogged down in calculations or symbols. Thus, technology allows students to ask "What if" questions, act on their questions, and reason through the outcomes to develop their conceptual knowledge about the mathematics behind the situation (House, 1988).

Technology allows students to model situations in two ways: by "constructing a set of computergenerated representations of an abstract mathematical structure or concept which enables one to understand underlying relationships among the elements of this structure or concept," and by "constructing an abstract mathematical model through the decontextualization of computer-generated manipulatives which portray mathematical concepts on a concrete, intuitive, action-oriented level" (Abramovich \& Brantlinger, 1998, p. 29). These two ways differ about on what is being operated. In the first modeling structure, one of the representations (i.e. graphical, algebraic, or numerical) is being manipulated, whereas in the last model, actual objects are being manipulated. These two models do not necessarily need to be separate, for one can manipulate objects which are hot linked to a representation. This way, students can see connections between the manipulation of concrete objects and the impact on graphical, algebraic, and numerical representations. The Geometer's Sketchpad (GSP) accommodates this type of modeling.

\section{The Geometer's Sketchpad}

GSP is an example of general software, for it can be used for different aged students and mathematical topics (Kurz, Middleton, \& Yanik, 2005). It elicits all the advantages of general software including manipulation, investigation, and immediate feedback. GSP allows students to manipulate both objects on the screen and representations (see Figure 7). Furthermore, it allows one to program linkages between representations, which enables students to discover and hypothesize about underlying mathematical structure. Studies have shown that technologies can facilitate algebraic thinking when they provide dynamic links among symbolic, tabular, and graphical representations (Balacheff \& Kaput, 1996; Friedlander \& Tabach, 2001; Ruthven, 1996). 


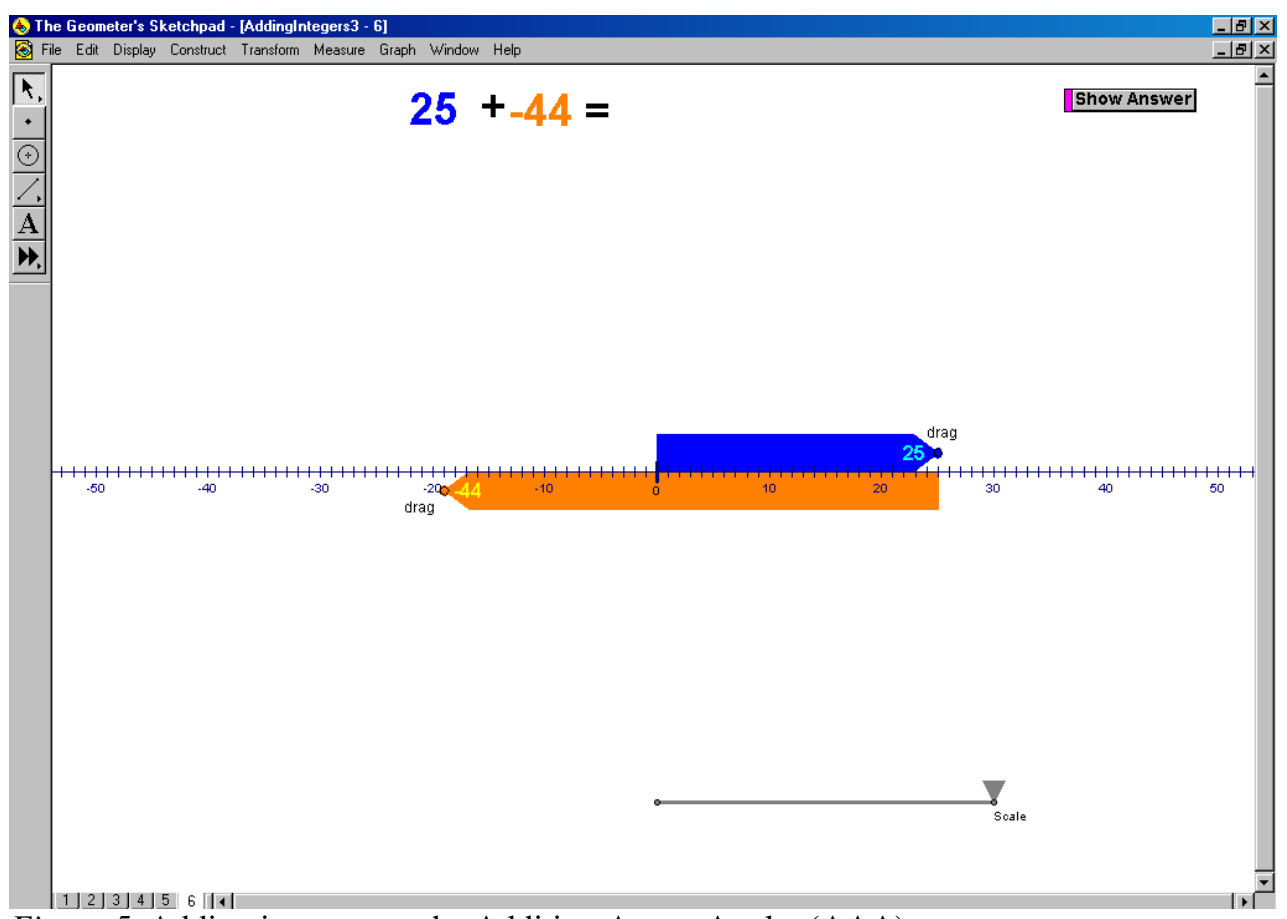

Figure 5. Adding integers on the Addition Arrow Applet (AAA)

\section{Using Technology to Understand Pre-Algebraic and Algebraic Concepts}

The author has not found any research using a dynamic geometry software package to aid in prealgebraic or algebraic understanding. However, there have been many studies using spreadsheets to foster algebraic thinking. They primarily deal with understanding the notion of variable. There are a few that describe how students interact with Excel to transition from arithmetical thinking (operational) to algebraic thinking (structural) (Filloy, Rojano, \& Rubio, 2001). Sutherland and Rojano (1993) instructed 16 students of ages 10 and 11, who had not had any formal algebra, in the usage of spreadsheets to solve algebra story problems. Prior to instruction, students were interviewed about their understanding of function and operations. During instruction, researchers emphasized representing unknown values in a spreadsheet cell, using the unknown to represent relationships, and finding a value for the unknown. Students were interviewed after instruction to see if their views changed from the beginning of solving word problems with a spreadsheet to the end of the instructional session. Researchers noted that most students shifted from an arithmetic way of viewing expressions by carrying out operations on numbers to an algebraic view by verbalizing an expression in terms of the unknown cell address. Sutherland described this mental activity as a "shift from thinking with a specific object to thinking with a general object" (p. 375). They concluded that the spreadsheet was able to help students "explore, express, and formalize their informal ideas" about algebra (p. 380). 
Ainley (1996) showed that spreadsheets, due to their algebraic-like notational conventions, fostered students' symbolic notation. She believed that students' difficulties with formal algebraic language were due to the lack of seeing a need for such notation. Since spreadsheet language is similar to algebraic language, she believed that a natural way to show purpose for algebraic notation was through the usage of a spreadsheet. She described interviews with two 11-year olds on an algebraic word problem asking for the dimensions of a fence with perimeter 30 meters enclosing a maximum amount of area. They were able to generalize values in a table, generate more data in the table, and graph the resulting points to determine the formal relationship between width and area. She concluded that spreadsheets allowed students to use a computer environment to formalize their informal problem-solving procedures.

Thomas and Tall (1986) also tested whether a computer program could improve students’ understanding of variable. The computer program consisted of boxes labeled with the variable names $a, b$, and $c$, where one could put in different values in the labeled boxes for those variables. The computer program also had two boxes labeled with different functions comprising of those variables, inside which would go the values of those functions. When students altered the values for the variables, the function values would automatically update. While this program was not in a spreadsheet, one could easily have used a spreadsheet for the dynamic linking of variable and corresponding expression values. Thomas and Tall split a group of 42 12-year olds into a control group, which received normal mathematics instruction, and an experimental group, which received twelve hours of instruction with the program instead of their normal mathematics instruction. The experimental group was guided to use different values for $a, b$, and $c$, and notice the values for expressions that illustrated the properties of commutivity, distributivity, and finding solutions to equivalent and non-equivalent expressions. Students in each group took a post-test and a delayed post-test examining how they used variables in each of the four levels described by Küchmann (1981). Students in the experimental group were significantly better at the .01 level on the post-test and at the .05 level on the delayed post-test at using variable as a specific unknown, generalized number, and variable than the control group. Furthermore, researchers found that when a comparison was possible, the students in the experimental group outperformed students who were three years older receiving traditional instruction. Therefore, researchers concluded that students were able to grasp a better understanding of variable through the use of the program.

While Thomas and Tall used their computer program to allow students to see equivalent and nonequivalent values of expressions, Kieran and Saldanha (2005) studied the usage of technology, Computer Algebra Systems (CAS), to promote equivalence reasoning. This study found that students were not able to 
recognize equivalent forms of expressions when using CAS. Kieran and Saldanha found that the majority of $10^{\text {th }}$ graders, who had learned how to factor and solve linear and quadratic equations, were able to explain what it meant for an equation to have a solution and were able to show that only certain solutions existed to an equation by using a CAS. Sixty percent of students were not able to interpret what it meant for two expressions making up an equation to be equivalent or not equivalent. Based on the students' incorrect answers, researchers based students' misconceptions on the inability to understand the CAS environment. For a specific equation, the CAS would return either "true" if the expressions were equivalent, or the original equation if the expressions were equal for only certain values. Students thought that if the original equation was returned, since it contained an equal sign, that it meant that the equation comprised of these expressions were equivalent. When students were asked to expand or solve these non-equivalent expressions, they were able to see whether some solutions were possible. From this activity, students rationalized that there was a mysterious exception to some non-equivalent expressions. Researchers then introduced the SOLVE command to build on the notion that some expressions are equivalent and have all possible solution values, non-equivalent but are equal for specific values, or non-equivalent and never equal. The usage of the SOLVE command compelled students to lump all equations that had any kind of solution as containing two equivalent expressions. Students who used this type of reasoning and understood common forms of an expression (either by factoring or expanding) were able to recognize equivalent expressions. Based on both studies, Kieran \& Saldanha suggested that students needed to understand and know how to interpret the CAS tests as well as know what it meant for expressions to be equivalent.

The author could find no other studies using technology to overcome students' misconceptions dealing with addition and subtraction of integers or equivalence. Furthermore, she has not encountered any study that uses Geometer's Sketchpad to aid in conceptual understanding of these algebraic ideas.

In the regards to future usage of computer software and activities in the algebra curriculum, Dugdale and colleagues (1995) recommended five goals that software activities should meet. Software and activities should be student centered and guide the students to meaningful discovery, and in the process of doing so, should strengthen students' investigative skills. The discovery process should include valuable examples that "enable students to apply, generalize, integrate, and extend their knowledge" all the while being easy to use (p. 343).

\section{Conclusion}

A large body of literature was explicated concerning students' misconceptions on pre-algebraic and algebraic topics such as operations on integers and equality. Researchers have proposed three major 
reasons for students' difficulties: poor arithmetic knowledge, poor transition from arithmetic to algebra, and poor instruction. In fact, most of the researchers have suggested better instruction to rectify students' conceptions. If students are able to gain conceptual understanding of arithmetic on integers, they might be able to grasp the unary sense of operation and signs (Kieran, 2004, 2007). This understanding can provide a foundation for algebra to see both the operation and structure behind the numbers. If students gain a deeper understanding of arithmetic, different usages of the same symbol will not be so alien to them. For instance, if students see the equal sign as describing an equivalence relation between the left and right side of an arithmetic statement, they are more apt to see the structure behind the numbers in the expression. By seeing the structure of expressions, students are better able to deal with the usage of variable as a generalized number and hopefully build on that notion to include describing relationships between variables. If students have a solid understanding concerning the equal sign and variable, then they should not have a difficult time translating word problems into equations.

Using the knowledge of these cognitive obstacles, some researchers have tried to utilize technology in instruction to overcome these conceptions. The most success has come from using spreadsheets to aid in understanding of variable (Thomas \&Tall, 1986; Ainley, 1996; Ainley et al, 2004, 2005). This success has been linked to the benefits of the spreadsheet in relation to algebraic language. Furthermore, none of these studies used GSP.

Studies done in hopes to improve students' notion of equivalence, have not been very successful. They have dealt with moving students from an operational to relational viewpoint of the equal sign. Only one study was found that used technology to do this, and it was done by using a graphing calculator. Furthermore, there was no context applied to the situation of determining the equivalence of the expressions.

While there have been limited suggestions for and usage of technology for instruction to help students with operations on negative numbers (Perks, 1995; Demana \& Leitzel, 1988; Sutherland \& Rojano, 1993) no studies have been carried out using these suggestions.

There has been a lot of work focusing on students' difficulties with pre-algebraic and algebraic notions and the transition from arithmetic to algebra; thus, new work needs to focus on algebraic understanding obtained from dynamic environments using multiple representations, understanding about properties obtained from manipulative activities, understanding obtained the usage of spreadsheets and graphing technology in middle school and lower level high school (Kieran, 2007). Kieran recommended that future research needs to attend to "how students can be assisted in (a) becoming aware of structure and 
in using symbols to express the structure, (b) seeing relations between graphical representations and lettersymbolic forms, and (c) making connections between verbal problem-solving activities and generating equations" (p. 729).

\section{Research Questions}

Many researchers have described and analyzed students' pre-algebraic and algebraic misconceptions, and have also provided instruction suggestions with and without technology to help

mediate these conceptions. However, no one has attempted to use GSP to instruct students on the topics of operations on integers and equivalence. By using GSP to make a compilation of technology applets to address these pre-algebraic and algebraic concepts, one will be attempting to undertake Kieran's new algebraic research focuses. Thus, the research questions are:

1. How do non-accelerated middle school students initially understand pre-algebraic concepts such as addition and subtraction of integers and the notion of equality?

2. How do non-accelerated middle school students, after receiving technologically enhanced prealgebraic instruction, understand addition and subtraction of integers and the notion of equality?

3. How do non-accelerated middle school students, who received technologically enhanced prealgebraic instruction, interact with the dynamic applets created in GSP, specifically in regards to analyzing, generalizing, communicating, and justifying their responses to algebraic tasks? 
CHAPTER 3

\section{METHODOLOGY}

The preceding chapter explains students' misconceptions concerning specific pre-algebraic and algebraic concepts, why they occur, and suggestions for appropriate mediating instruction. This study builds on previous research and broadens this research base by focusing on middle school students' understanding of pre-algebraic concepts obtained through interactions with dynamic software, such as Geometer's Sketchpad, that utilize multiple representations. This study will also answer the call of future research needs in algebra by addressing "how students can be assisted in (a) becoming aware of structure and in using symbols to express the structure, (b) seeing relations between graphical representations and letter-symbolic forms, and (c) making connections between verbal problem-solving activities and generating equations" (Kieran, 2007, p. 729).

\section{Theoretical Framework}

\section{The Implicit Framework-Constructivism}

The design of the technology applets in GSP and the related activities that use these applets are based on constructivism. Constructivist learning differs from the traditional view of mathematics learning. Traditional instruction centers on the teacher who acts as the authority to dispense already established facts and skills, which students must passively absorb, or "learn". In contrast, proponents of constructivism believe that this type of learning results in students creating weak sense-making constructions, for students are simply mimicking the teachers' actions; yet, appearing to understand the procedures.

The ontological position of constructivism is that there is no one true reality to be discovered; however, there are many individual realities that are made up from one's experience and interactions with others (Simon, 1995). Based on this premise, people must actively create their mathematical knowledge through physical, mental, and social actions (Clement \& Battista, 1990). Since knowledge is individually constructed, this knowledge will also be filtered through experience and prior knowledge. With regards to

prior knowledge, I do not simply mean memorized school facts, but also existing sense-make schemes that allow students to interpret their past knowledge and predict their interpretation of new knowledge. Since students rely on their past experiences, new mathematical ideas are only valued as true if they are 
compatible with other experiences of the learner.

Given this constructivist perspective, Clements and Battista (1990) felt there are two major goals of mathematics instruction: it should aid students' development of more powerful mathematical structures and procedures than they presently have, and it should allow students to become self-sufficient and selfmotivated in mathematics (p. 35). In order to achieve these goals, Clements and Battista recommended that teachers present appropriate tasks that advance students from a concrete introduction of a topic to an abstract, symbolic representation of the topic. These tasks should allow students to use their own problemsolving methods. Furthermore, teachers should act as guides to facilitate discussion and reflection on the activities, rather than stripping away students' decision-making power. The role of the mathematics teacher is to plan instruction so that students are able to explore, reflect, and actively construct knowledge.

Richards (1991) stated that the role of the teacher is to design these kinds of instructional activities, and he noted that by having these types of activities, students would construct their knowledge accordingly rather than by simple absorption of the material.

It is necessary to provide a structure and a set of plans that support the development of informed exploration and reflective inquiry without taking initiative or control away from the student. The teacher must design tasks and projects that stimulate students to ask questions, pose problems, and set goals. Students will not become active learners by accident, but by design, through the use of the plans that we structure to guide exploration and inquiry (p. 38, emphasis his).

While teachers can design material and lessons so that students can actively engage learning, students only learn, or change their conceptions, when they can "comprehend and accept ideas because they are seen as intelligible and rational" (Posner, Strike, Hewson, Gertzog, 1982, p. 212). Based on this view of learning, or conceptual change theory, Posner and colleagues call upon two phases of conceptual change: assimilation and accommodation. While these phases have the same names and connotations as the two learning principles of Piaget (1964), it must be noted that Posner and colleagues did not "intend any commitment to his theories" (p. 212). Assimilation is used when students rely on their previous knowledge and try to apply it to new ideas. Yet, this is not always successful since new ideas might go against some of this previous knowledge. Thus, students must accommodate, or reorganize and replace, the ideas that do not conform to the new knowledge or create new ideas. In order for accommodation to occur, four conditions must be met: learners must be dissatisfied with existing conceptions, the new conception must be intelligible to the learner, the new conception must seem plausible to the learner, or seem consistent with some of the learner's prior knowledge, and the new concept should seem worthwhile to learn in that it will 
open up opportunities for new inquiry (Posner et al, 1982, p. 214).

Consistent with constructivist teaching and learning position, I created and modified pre-existing technology applets and accompanying activities that allow students to manipulate, inquire, reflect, and actively take part in one's learning through the dynamic interaction with the software. While working with the software, I hope that the students who exhibit some of the misconceptions in pre-algebra, notated in the last section, will satisfy some of the conditions for accommodation. I hypothesize that by accommodating their conceptions, students will be able to both procedurally and conceptually understand addition and subtraction on integers and the notion of equality through the use of technology.

In working with students and trying to grasp their understanding, I realize that I will be anticipating their understanding based on my past experience and understanding of past literature findings. I will be trying to comprehend students' constructs of the material, and in doing so, I will be involved in the construction process. Therefore, I am aware that I may play a role in students' understanding.

\section{The Explicit Framework- Kieran's Four Methods of Meaning Making}

While constructivism is an implicit framework for viewing the technology applets and activities, I will also be using the explicit algebraic meaning-making framework of Kieran (2007) for both interview activities and accompanying technology activities. As stated in the last chapter, this framework consists of four ways that students are able to make sense of algebra. These four ways of creating algebraic meaning are from:

1. Algebraic structure itself, involving the letter-symbolic form

2. Other mathematical representations, including multiple representations

3. The problem context

4. That which is exterior to the mathematics/problem context (p. 711).

By utilizing this meaning-making framework, Kieran categorized all algebraic activities into Generational, Transformational, and Global/Meta-levels groups. All the tasks for this dissertation are Generational and Global/Meta activities, for they involve understanding and forming algebraic structures as well as require problem solving, analysis, and justification of answers.

\section{The Pilot Study}

I conducted an informal three-phased pilot study prior to collecting data for the research study. The first phase of the informal pilot study consisted of an instructional session and an interview session with one child in fifth grade. The second phase consisted of an interview session and an instructional session 
with a seventh grade student. The third phase consisted of instruction and interviews with two eighth grade students in the sample population. The interview session allowed me to assess the student's conceptions of the topics and tasks presented, while the instructional session built upon those conceptions and corrected any misconceptions. These interview and instructional sessions took place to help me develop and refine my tasks, software, and questioning techniques.

\section{Purposes of the Pilot Study}

The interview session was conducted for two purposes. One of the purposes was to make adjustments to the tasks and wording of the tasks so that students could better understand what was asked of them. The second purpose was to improve my interviewing skills. The instructional session was conducted for three purposes: the same purposes as the interview session as well as to make any adjustments to the technology applets in regards to both user friendliness and inconsistencies.

By conducting this pilot study, I noted instances of students' confusion due to ambiguous questions and poor sequencing of the questions. I also noted when students' used my language to answer questions, which could imply that I influenced them too much with a leading question. After reviewing my notes, I noticed that I should have asked some follow-up questions to make sure that my interpretation of what the students had said was accurate.

\section{Participants}

There were four participants between the ages of 10 and 14 years old who participated in the pilot study. One participant did not have any algebra instruction, while the other participants had some algebra instruction. For my pilot study, I had created multiple technology applets in both Excel and Geometer's Sketchpad (GSP) to cover pre-algebraic and algebraic conceptions in the field of operations on integers, the notion of equality, the notion of variable, and translating words to equations.

The first participant, Ruth, was chosen out of convenience. She was readily available on short notice, in the top section in her mathematics class in fifth grade (she was actually learning sixth grade mathematics), and vocal in her feedback. She met with me for a 90-minute instructional session and completed eight tasks and an initial set of software products. Based on her interactions with the activities, my questions, and the software, I made adjustments accordingly by discarding, modifying, or creating new activities. Two weeks later, my advisor interviewed Ruth for one hour on the initial interview tasks. Based on her answers and responses to probing questions, we made adjustments accordingly.

The second participant, Dick, was also chosen out of convenience and because he fit the profile of 
the targeted student. Dick was in Dick grade standard level mathematics, yet recommended for advanced level mathematics for $8^{\text {th }}$ grade. Since I was looking for the average mathematics student in middle school, he fit this profile quite nicely. Dick met with me for 1 hour to work through interview tasks and 3 hours to work through the technology instructional tasks. Based on his responses and interactions with the tasks, my questions, and the software, I made adjustments accordingly by discarding and modifying activities. For instance, the original Excel applets were eliminated because their content was subsumed by the GSP applets. Not only were tasks and applets modified and eliminated, but the study was also reformatted. Originally, the study was structured so that students received an initial interview, an instructional session, and a final interview; however, after working with Dick it changed to five self-contained modules consisting of 2 parts each: an interview section and an instructional section.

The third and fourth participants, Nina and Deb, were chosen from the sample population as the population for the study, in order to prepare for the targeted participants. Both Nina and Deb were in eighth grade Standard Mathematics and attending Wilson Middle School. Nina and Deb met with me twice for 1 hour each time. They worked on as many Modules as they could in that time frame. Based on their responses and interactions with the technology applets, I made adjustments accordingly by eliminating 2 of the modules, eliminating the Excel applets, and creating one more GSP applet for subtraction.

\section{Creation and Adjustment of Tasks and Technology Applets}

Dugdale and colleagues (1995) recommended five requirements for future usage of software activities. They must

1. focus student attention by suggestions, questions, possible avenues of inquiry, and work strategies

2. build students' inquiry skills

3. suggest provocative examples that illuminate important cases to explore

4. enable students to apply, generalize, integrate and extend their knowledge

5. be relative easy to use (p. 343)

The technology applets and activities that were used in the instructional sections satisfy each of these requirements. Students were presented with activities that encouraged them to use the technology applets as they saw fit, constrained by some software features, to answer questions concerning operations on integers and the notion of equivalence. Students were able to construct many examples and were asked to generalize, conjecture, and test hypotheses concerning these examples, which built their inquiry skills and encouraged them to test unique cases. These applets were easily used due to features already existing in 
GSP as well as features, such as buttons and sliders, which I programmed and placed into these applets.

The technology applets and activities used in the instructional session also met the five requirements of Flanders (1988):

1. No knowledge of the computer should be required to use the software other than the basics: how to insert a disk, turn it on, and type in a few letters.

2. The software should be truly interactive, with emphasis on active - require user-generated input to produce any output.

3. Instructional software should be robust-extremely difficult to crash.

4. The syntax for input should be as close to the way mathematics is written as possible.

5. Input of real numbers should allow expressions that evaluate to reals (ditto for rationals or for integers), so that the computer does the calculations. (pp. 149-150)

The participants did not need any prior knowledge of GSP to interact with these applets. Furthermore, participants were easily able to drag, slide, and input numbers to obtain corresponding outputs. This allowed participants to observe, conjecture, and adjust their ideas of a concept. In essence, it allowed the participants to be in control of their own learning and actively think and interact with the computer applets.

GSP is a reputable program that does not crash easily. Moreover, the work that participants did in this program did not require intensive amount of RAM to function, so the likelihood of the program crashing is minimal.

In regards to the fourth and fifth requirements, GSP allows users to enter in and receive real values in mathematical form. Furthermore, the syntax of entering and evaluating mathematical expressions reinforce the usage of the order of operations and brackets.

By having the technology applets and activities adhere to these requirements, the participants were able to construct pre-algebraic conceptual and procedural knowledge in an interactive fashion.

\section{First Pilot Phase}

The instructional session. In the instructional session, I used nine technology applets with accompanying tasks concerning the topics of addition and subtraction on integers, the distributive property, order of operations, and translating word problems into equations. The distributive property and order of operations were first used because they illustrated multiplication and division of integers. However, based on the first phase, these five applets were eliminated because I realized I could gather this type of information by adjusting my integer applets and tasks; furthermore, I did not want to test distributivity and 
order of operations. One applet concerning subtraction and addition of integers using directionality of an object to travel the number line was eliminated because the two other applets concerning addition and subtraction on integers represented the same information only in a clearer format. Thus, the author felt the elimination of these applets was justified because these concepts could be brought out in other technology applets and tasks and were unnecessarily time consuming. Furthermore, these tasks contained some procedures and questions that were confusing.

Of the last three remaining applets, two technology applets, which concerned addition and subtraction of integers, were modified for three reasons. The first reason was to let students have more control over their learning by creating more options in the software. The second reason was to lessen the cognitive load having to mentally remember some information while observing others; therefore, more multiple representations were included. The third reason was to make the applets user-friendlier by adding more details to the applets as well as larger items to manipulate. The last applet, which concerned translating word problems into an equation, remained the same.

After this first phase, I reflected on both the literature and interactions with Ruth. Based on this reflection, I created two technology applets concerning variable and translating of words to equations and modified three existing technology concerning equivalence and variable to be used in the second phase. I felt that these topics were not adequately addressed in the first phase; thus, I needed to include them to make the study complete.

The interview session. This interview session consisted of 40 questions concerning operations on integers, the notion of the equal sign, the notion of variable, and translating a word problem into an equation. After my advisor interviewed Ruth, we discussed Ruth's answers to the 40 questions. Due to the length of the interview, confusion on some of the questions, and redundancy of elicited answers to different questions, we eliminated and modified some of the questions. After this modification and elimination, we had 30 questions to use in the second phase.

\section{Second Pilot Phase}

The interview session. The interview session consisted of 30 questions concerning addition and subtraction on integers, the notion of the equal sign, the notion of variable, and translating a word problem into an equation. This interview took approximately 1 hour to complete. After interviewing Dick, I reflected on his answers. Due to the length of the interview, confusion of the purpose and wording of a question and subsequent aggravation, and the redundancy of information obtained from the answers, I 
eliminated and modified some of the questions.

The instructional session. In this session, I tested nine technology applets and accompanying tasks. There were three applets for operations on integers and two applets for each of the following topics: the notion of equivalence, the notion of variable, and translating word problems into equations. Operations on integers had two GSP applets, one for addition and one for subtraction, and an Excel applet. For this set, there were 8 tasks. Both the notion of variable and writing equations topics each had a GSP and Excel applet accompanied with 10 and 8 tasks respectively. The notion of equivalence topic had two GSP applets and a total of 19 tasks. This instructional session took a little over three hours to complete. Based on the answers and length of the instructional session, tasks and applets were modified and eliminated, and the structure was reorganized.

Four technology applets, one from subtraction, equivalence, variable, and writing equations three Excel and one GSP, were eliminated from the study because I realized that having multiple technology applets for each topic was redundant and time consuming. Since the GSP applets were more comprehensive and more versatile than the Excel applets for these topics, I easily made slight adjustments to these applets that allowed them to include the same information as the Excel applets. Similarly for the topic of equivalence, one of the GSP applets was more comprehensive, so I chose that applet to remain and adjusted it to include some tasks and manipulation features characterized by the eliminated GSP applet.

Not only were applets adjusted and eliminated because of the time factor and instructional redundancy, but the instructional and interview sessions were also restructured. As I reflected on the interactions with Dick and the goal of my study, I re-organized the study from a two-sectioned structure that contained one interview session and one instructional session to a five self-contained module structure each consisting of two parts. The five modules were addition of integers, subtraction of integers, the notion of equivalence, the notion of variable, and translating words to equations.

The main goal of the study was to help students who struggled with each of these five topics by providing them with technology-enhanced instruction. However, with the original structure, an initial, large amount of time was spent diagnosing a student's conception on each of the topics, only after which, he would receive instruction. Instead of having the student complete 24 tasks prior to receiving any instruction to overcome his misconceptions, I wanted to begin instruction as soon as a misconception was uncovered. This way, the student received immediate instruction where and when he needed it. The proximity of the instruction and diagnosis of a student's misconception on a topic was beneficial for two reasons. The first reason was that if a student took a large amount of time to complete both the interview and instructional 
tasks, such that the time to complete all of the instructional tasks was cut short, the student might be left with misconceptions on some of the topics. For instance, if a student had misconceptions in all topics, but time expired while he was working to rectify his variable misconceptions, he would not have had time to tackle his writing equation misconceptions. The second reason that this new structure benefited the student was that the student could remember his answer to the problem as well as his justification for his answer. For instance, when I worked with Dick, he had had misconceptions with subtracting negative numbers. Yet, after an hour of working through 30 tasks concerning integers, equality, variable, and writing equations, he could not remember his answers or why he gave certain answers to a particular problem when we returned to the problem to begin working to overcome his misconceptions. This meant he would have to resolve and re-justify how he solved the problem. By repeating this process, it lengthened the interview. In resolving the problem, sometimes Dick would solve it a different way or provide an alternative justification. When he did this, I would remind him of what he said before and asked him how that related to his new answer or method of solving. During these occurrences he sometimes became confused or mistrusted what I had told him that he had previously said. This confusion, mistrust, and lengthening of the session was eliminated by restructuring the format of the sessions.

\section{Third Pilot Phase}

The third pilot phase consisted of 5 self-contained modules that included an interview section and instructional section. The first module dealt with addition of integers; the second module dealt with subtraction of integers; the third module dealt with equality; the fourth module dealt with the notion of variable; the fifth module dealt with translating words to equations.

The interview section. The interview sections of each module consisted of the following number of tasks: Module 1 had five tasks, Module 2 had four tasks, Module 3 had six tasks, Module 4 had eight tasks, and Module 5 had five tasks.

The instructional section. Five applets were ready to be used in the instructional sections of the five modules ( 1 for each module). All of the applets were programmed in GSP. For Module 1, there were four accompanying tasks; for Module 2 there were six accompanying tasks; for Module 3 there were 14 accompanying tasks; for Module 4 there were 11 accompanying tasks; for Module 5 there were four accompanying tasks.

Both Nina and Deb did not have enough time to do Modules 3, 4, and 5. Nina correctly answered all the addition interview tasks, but struggled a lot with the subtraction tasks in the interview section. Thus, 
she worked with the subtraction applet in Module 2, but went no further. Deb did not correctly answer addition tasks in the Module 1 interview section, so she worked with the addition applet. After working with the addition applet, she moved on to the interview section of Module 2 but did not get to work with the subtraction applet.

Based on participants' responses, I eliminated Modules 4 and 5, and created one more subtraction applet so that participants could see subtraction as both take away and distance.

\section{The Research Study}

\section{Setting and Participants}

The study took place in a central Virginian town, Carlsburg, with a population of about 40,000. Participants were selected from Wilson Middle School. Wilson Middle School (WMS) is a public middle school teaching grades sixth through eighth with a population of 607 students. The number of students to a teacher in this middle school falls between 12 and 13, and the percentage of free and reduced lunches is $31 \%$.

The sampling procedure was purposeful and based upon the following criterion sampling. Students had to fulfill the following criteria:

1. They must have already had some instruction on these topics

2. They were in need of remediation on these topics

3. They had seen technology use in mathematics

The necessity of fulfilling these criteria stemmed from the fact that my overarching goal of the study was to help students who struggled with a topic by providing them technology-enhanced instruction. According to the Virginia SOLs, these topics are typically taught in sixth grade and expanded upon through seventh and eighth grade. The Grade 6 SOLs state that students will use basic operations on positive real numbers, and they will be introduced to integers, algebraic terms, and solving algebraic equations in one variable. The introduction of integers includes identifying, representing, ordering, and comparing integers. Algebraic terms that will be introduced are variable, coefficient, term, and equation. Students will use concrete manipulatives to solve algebraic equations in only one variable with positive rational solutions. According to the seventh grade SOLs, students should be proficient with the basic operations on integers and be able to analyze and generalize algebraic relationships obtained from tables, graphs, and symbols. Furthermore, these students should be able to write and solve linear equations from verbal sentences. The eighth grade SOLs state that students will review and extend their knowledge from previous grades. Students will continue to gain proficiency in computation of integers and analyze different representations in the forms of 
graphs, tables, and rules. They will extend their knowledge of solving 2-step equations to solving 2-step inequalities. These SOL requirements are listed in Table 2.

Table 1

$6^{\text {th }}, 7^{\text {th }}$, and $8^{\text {th }}$ Grade SOLs

\begin{tabular}{|l|l|}
\hline \multicolumn{2}{|l|}{ Standard of Learning } \\
\hline 6.5 & The student will identify, represent, order, and compare integers. \\
\hline $6.6 \mathrm{a}$ & $\begin{array}{l}\text { The student will solve problems that involve addition, subtraction, } \\
\text { multiplication, and/or division with fractions and mixed numbers, with } \\
\text { and without regrouping, that include like and unlike denominators of } 12 \\
\text { or less, and express their answers in simplest form. }\end{array}$ \\
\hline $6.23 \mathrm{a}$ & $\begin{array}{l}\text { The student will model and solve algebraic equations, using concrete } \\
\text { materials. }\end{array}$ \\
\hline $6.23 \mathrm{c}$ & $\begin{array}{l}\text { The student will use the following algebraic terms appropriately: } \\
\text { variable, coefficient, term, and equation. }\end{array}$ \\
\hline 7.5 & $\begin{array}{l}\text { The student will formulate rules for and solve practical problems } \\
\text { involving basic operations (addition, subtraction, multiplication, and } \\
\text { division) with integers. }\end{array}$ \\
\hline 7.19 & $\begin{array}{l}\text { The student will represent, analyze, and generalize a variety of patterns, } \\
\text { including arithmetic sequences and geometric sequences, with tables, } \\
\text { graphs, rules, and words in order to investigate and describe functional } \\
\text { relationships. }\end{array}$ \\
\hline 7.2 & $\begin{array}{l}\text { The student will write verbal expressions as algebraic expressions and } \\
\text { sentences as equations. }\end{array}$ \\
\hline $8.22 \mathrm{a}$ & $\begin{array}{l}\text { The student will solve one-step linear equations and inequalities in one } \\
\text { variable with strategies involving inverse operations and integers, using } \\
\text { concrete materials, pictorial representations, and paper and pencil. }\end{array}$ \\
\hline $8.14 \mathrm{~b}$ & $\begin{array}{l}\text { The student will relate and compare tables, graphs, and rules as } \\
\text { different forms of representation for relationships }\end{array}$ \\
\hline 8.15 & $\begin{array}{l}\text { The student will solve two-step equations and inequalities in one } \\
\text { variable, using concrete materials, pictorial representations, and paper } \\
\text { and pencil }\end{array}$ \\
\hline $\begin{array}{l}\text { The student will: simplify numerical expressions involving positive } \\
\text { exponents, using rational numbers, order of operations, and properties } \\
\text { of operations with real numbers (subset being integers) }\end{array}$ \\
\hline
\end{tabular}

Since I wanted to help students, I needed to be able to determine which students were struggling with these topics. I also wanted to make sure that students had seen technology used in teaching mathematics. I did not want them to be overwhelmed by the features and visuals of the technology that could possibly distract them from being able to see the structure of the mathematics afforded by the technology. All of these criteria were satisfied by selecting participants from Wilson Middle School's before and after school tutoring program.

Students who attended the mathematics-tutoring program offered at WMS had already been 
identified by their teacher and parents in needing remediation in mathematics. Students were identified as needing remediation based on their midterm assessment scores and teacher recommendations. Letters were sent home to the parents of these students stating that the school was offering supplemental mathematics tutoring to these children because of these factors. Students returned a parent-signed form indicating that they were voluntarily attending these tutoring sessions. While certain students were recognized to take part in these tutoring sessions, they were available to the whole student population. The objective of this tutoring program was to provide extra mathematical activities, most of which were enhanced with technology, to students who were in need of assistance. During these tutoring sessions, the mathematics specialist and other teachers used digital manipulatives to assist students who had difficulties with particular concepts.

The mathematics specialist at WMS met with my advisor and myself to determine which students from the tutoring program would benefit the most from working with these topical technology applets. The mathematics specialist identified 10 students from this population who also would be willing to work with me and would be vocal in their answers and justification. Two of these students participated in the pilot study and the other eight participated in the study. These eight students consisted of five females, three males, three sixth graders, three seventh graders, and two eighth graders. At WMS there were two levels of mathematics classes: Standard and Advanced. If a student was in Advanced Mathematics, it usually meant that he or she was learning the material at a higher level, but this was not necessarily true all the time.

Three of my participants were in the Advanced Math Level, while the rest were in Standard. Table 3 depicts the characteristics of the eight students who participated, along with the length of the interview, and the parts of each module that was completed.

Table 2

Participant Characteristics

\begin{tabular}{|c|c|c|c|c|c|c|c|c|c|}
\hline \multirow[b]{2}{*}{ Student } & \multirow[b]{2}{*}{ Grade } & \multirow[b]{2}{*}{ Level } & \multirow[b]{2}{*}{ Hours } & \multicolumn{2}{|c|}{ Module 1} & \multicolumn{2}{|c|}{ Module 2} & \multicolumn{2}{|c|}{ Module 3} \\
\hline & & & & $\begin{array}{l}\text { Interview } \\
\text { Section }\end{array}$ & $\begin{array}{l}\text { Instructional } \\
\text { Section }\end{array}$ & $\begin{array}{l}\text { Interview } \\
\text { Section }\end{array}$ & $\begin{array}{l}\text { Instructional } \\
\text { Section }\end{array}$ & $\begin{array}{l}\text { Interview } \\
\text { Section }\end{array}$ & $\begin{array}{l}\text { Instructional } \\
\text { Section }\end{array}$ \\
\hline Abby & 6 & Advanced & $3: 30$ & $\mathrm{x}$ & & $\mathrm{x}$ & $\mathrm{x}$ & $\mathrm{x}$ & $\mathrm{x}$ \\
\hline Hope & 6 & Advanced & $2: 15$ & $\mathrm{x}$ & & $\mathrm{x}$ & $\mathrm{x}$ & $\mathrm{x}$ & \\
\hline Don & 6 & Standard & $2: 30$ & $\mathrm{x}$ & $\mathrm{x}$ & $\mathrm{x}$ & $\mathrm{x}$ & $\mathrm{x}$ & \\
\hline Jim & 7 & Standard & $3: 00$ & $\mathrm{x}$ & & $\mathrm{x}$ & $\mathrm{x}$ & $\mathrm{x}$ & $\mathrm{x}$ \\
\hline Jen & 7 & Standard & $2: 20$ & $\mathrm{x}$ & & $\mathrm{x}$ & $\mathrm{x}$ & $\mathrm{x}$ & $\mathrm{x}$ \\
\hline Jake & 7 & Advanced & $2: 30$ & $\mathrm{x}$ & & $\mathrm{x}$ & $\mathrm{x}$ & $\mathrm{x}$ & $\mathrm{x}$ \\
\hline Carla & 8 & Standard & $2: 30$ & $\mathrm{x}$ & & $\mathrm{x}$ & $\mathrm{x}$ & $\mathrm{x}$ & $\mathrm{x}$ \\
\hline Beth & 8 & Standard & $2: 15$ & $\mathrm{x}$ & & $\mathrm{x}$ & $\mathrm{x}$ & $\mathrm{x}$ & $\mathrm{x}$ \\
\hline
\end{tabular}

Only Don required instruction on the addition of integers. Every participant completed subtraction 
instruction.

The parents of these eight students received a letter, approved by the principals and district, providing them with a description of this study and the choice to withdraw their child from the study. Students who participated in this study received a gift certificate to a local ice cream shop.

By interpreting middle school students' conceptions and their interactions with technology applets and tasks, I was able to answer three questions:

1. What type of conceptions occurred prior to and after pre-algebraic and algebraic remediation instruction?

2. How did students who have had pre-algebraic and algebraic instruction interact with the technology applets and tasks?

3. Based on the answers to the first two questions, how did these technology applets and tasks best support the remediation of these concepts?

\section{The Research Design}

Because the nature of the research questions dealt with understanding non-accelerated middle school students' ideas of pre-algebraic concepts with and without the aid of technology, I needed to use qualitative methods to obtain detailed data about students' interactions with these tasks and applets. "Qualitative methods facilitate study of issues in depth and detail" by providing freedom from predetermined choices (Patton, 2002, p. 14). While predetermined categories enable quantitative approaches to survey a vast amount of people in order to generalize findings, qualitative methods allow researchers to "produce a wealth of detailed information about a much smaller number of people and cases" (p. 14). By having this wealth of information on a limited number of cases, researchers are able to look for themes between and among cases, which provide a detailed description of occurrences that is constrained by circumstances surrounding the situation.

Patton (2002) provided three ways to collect qualitative data: in-depth, open-ended interviews, direct observation, and written documents (p. 4). Each of the four modules contained a mixture of interview, direct observation, and written document data.

However, during the interview section of each module, I primarily collected both interview and written data based on students' understanding of pre-algebraic and algebraic concepts. During the instructional section of each module, I primarily collected direct observation data and written data. 


\section{The Design of Each Module}

This study had three self-contained modules. The first module dealt with integer addition, the second module dealt with integer subtraction, and the third module dealt with equality.

Each module consisted of two parts. The first part of each module was a task-based interview section without technology and instruction founded on the review of literature. The second part of each module was an instructional section with technology. The instructional sections also used tasks founded on the literature.

I used the task-based interview section of each module to assess the students' knowledge of the module's topic. If during this section of the module I saw that the student could not solve the task or articulate conceptual reasoning for his answer, I used that task to segue into the accompanying instructional section for that module. Thus, I met the student where he was in terms of understanding and guided him with technology and questions to answer and understand the mathematical idea. However, if the student could solve and satisfactorily explain the correct reasoning behind the answer in the task-based interview section, I moved to the next question in the task-based interview section of the same module. If a student had difficulty with one question in the task-based interview section, he could have not fully finished this section, for he would have been segued into the accompanying instructional section of the module. If a student could correctly solve and reason through every task-based interview question in one module, I continued to the next module's task-based interview section

Depending upon which tasks a student could solve and explicate with correct understanding, determined how fast he moved along the continuum of tasks in the task-based interview section and the accompanying instructional sections in each module. Thus, the time requirement for each instructional section varied depending upon the ability and prior knowledge of the student. I wanted to work with students for at least 2 hours. Thus, the minimum time spent with a student was 2 hours, however, some students were able to meet with me for a longer time due to the flexibility of their schedules.

The interview section. Each of the interview sections was task-based and provided the researcher with information on students' understanding of each of the three topics. During these interviews, the researcher observed participants' actions and probed for verbal justifications for their actions. Probing during the interview was used to give a more accurate and detailed picture of the participants' knowledge on these topics. The probing focused on asking participants to explain what they did on the tasks, why they did it that way, and if there was another way to do the same task. These task-based interviews provided flexibility to the researcher in asking questions based on students' actions, which in turn, increased the 
richness of data collected. Furthermore, they provided insight into students' thought processes and conceptions of these topics.

Patton (2002) listed four primary strengths of open-ended interviews:

1. The exact instrument used in the evaluation is available for inspection by those who will use the findings of the study.

2. Variation among interviewers can be minimized when a number of different interviewers must be used.

3. The interview is highly focused so the interviewee time is used efficiently.

4. Analysis is facilitated by making responses easy to find and compare (p. 346).

In order for future researchers to access my data as well as for my own analysis benefit, I audiorecorded the interviews and wrote out the tasks that were used (see Appendix A). These audio recordings were stored on my home computer that was secured with a password that only I knew.

Interviews took place in a private classroom at a round table where the interviewee sat beside of the interviewer. I explained to the participant that I was solely interested in his ideas, and not on him obtaining the correct answer; therefore, I asked him to speak aloud as he thought about the problem. I presented each task on a separate sheet of paper to the student. I also provided the participant with blank paper, graph paper, and paper that contained a number line, and I encouraged him to write and draw anything that could help him solve the problem. Depending on his reasoning and work, I made inquiries and asked him to "help me understand" what he was thinking and asked follow-up questions to his responses. I took field notes and audio record these interviews.

The types of tasks that were asked of the students were similar to the ones asked that elicited misconceptions observed by other researchers. On Module 1, Tasks 1 and 2 were asked of students to see what kind of conception (unary, binary, or symmetric) they had of the negative sign. Tasks 3 of Module 1 and Task 1 of Module 2 were used to see if students could evaluate symbolically represented problems that contain operations on negative numbers. Tasks 4 and 5 from Module 1 and Tasks 2, 3 and 4 from Module 2 were asked to see if students can translate between representations (abstract, number line, and contextual).

In Module 3, Tasks 1-4, deals directly with students' conceptions about the equal sign. Many of these tasks have been taken from or modeled after literature and were used to determine if and when students' had an operational or a relational sense of the equal sign. For example, similar tasks to 1 and 4 have appeared in Cooper and colleagues' study (1997). Task 3 is similar to tasks seen in the work of many researchers (Boulton-Lewis et al, 2000; Falkner et al, 1999; Kieran, 1979, 1980; Stacey \& MacGregor, 
1997a). Task 2 was modeled after tasks in Seo and Ginsburg (2003). Tasks 5 and 6 were based on Knuth and colleagues' study (2006).

All of these tasks are constructivist in nature, for they required the student to explore and reflect on what they believe is plausible for the answer. Furthermore, these activities utilize all four mathematical meaning-making ideas (Kieran, 2007). These tasks encompass all four mathematical representations: symbolic/abstract, graphical, numerical, and verbal. Moreover, some of the tasks require students to move between two representations. By working on the tasks, maneuvering between representations, using the problem context and other ideas they may have about these concepts, students should have been able to see the structure behind the symbolic algebra and come to understand operations on numbers and variables.

The instructional section. Each module had an instructional section to be completed if the student needed remediation on this topic. The instructional section incorporated technology applets and activities concerning addition and subtraction on integers, the notion of equivalence, the notion of variable, and translating word problems into equations. Direct observation of the students interacting with the technology applets provided data concerning students understanding of these topics. It also allowed me to observe whether and how the technology applets impacted participants' conceptions regarding these topics.

Patton's (2002) listed six benefits for the researcher in using direct observation:

1. He is better able to understand and capture the context within which people interact.

2. He is able to have first-hand experience with a setting and people in a setting, which requires less of a need to rely on prior conceptions.

3. He has the opportunity to see things that may escape awareness from people within the setting because these things are common occurrences.

4. He can gain more information because people might be more willing to discuss information in a familiar setting rather than in an interview setting.

5. He can gain a more holistic idea of information by correlating both interview and observation data, for interviewees provide information filtered through their own perceptions.

6. He can draw on impressions and feelings received from the field to interpret the data (pp. 262264).

During the instructional sections, I directed the participants on how to use the technology applets to complete the tasks. Participants used a computer located in a private classroom. I positioned a video recorder such that it viewed the student working with the technology applets on each task. In order to gain full understanding of the students' thought processes and conceptions, I asked structured questions during 
these sessions (see Appendices A). Moreover, I uniformly and non-uniformly probed students for their reasoning based on their interactions with the computer. Uniform probes were asked of all participants and required students to explain and clarify their actions with the technology applets in answering the tasks. Non-uniform probes were used and based on a student's initial conception given during the interview section as well as his specific actions and answers given during the instructional section. These probes were useful in clarifying ambiguous answers and actions to discover specific details that may be important.

Based on the video recordings, I typed field notes documenting questions asked by the participant and myself, responses of the participants, types of illustrations written down and described by the participants, and any other events that were noteworthy.

Through direct observation during these instructional sections, I was able to better understand how students conceptualized pre-algebraic concepts with regards to the technology applets; thus, I did not have to solely rely on the literature to describe participants' conceptions concerning these topics. I also was able to better interpret these conceptions by triangulating all data collection methods.

The instructional sections in Module 1 used 1 applet I created in GSP that focused on addition of integers using an interactive number line. Similarly, Module 2 used two applets I created in GSP focusing on subtraction of integers using an interactive number line. Both these applets were used with some contextual questions that required the student to add and subtract with positive and negative integers. Both of these applets illustrated multiple representations, one of which was the symbolic representation. The goal was to have students generalize symbolic rules of addition and subtraction on integers.

Students used the GSP addition applet (Figure 1) to complete tasks in the interview section (see Appendix A). This applet compares the numerical statement of values to the initial and final values on the number line. Furthermore, the applet shows the embodiment of vector addition on the number line. To use this number line, students first placed their mouse pointer of the blue slider and dragged the dot to the position of their first addend. The number to which the blue slider points is nestled in the arrow in light blue color (in Figure 1 this value is 16). This action controlled the value for the first addend in the numerical statement. Students then clicked Show Orange Arrow button and placed their mouse pointer over the orange pointer and dragged it until the green value nestled inside the orange arrow said the second addend (in Figure 1 this value is -22 ). The sum, in black, is seen where the orange arrow ends. There are other buttons on this applet: Show Answer and Show Expression. Students were able to see the expression, without the answer. This allowed students to predict the answer without seeing it first. However, by allowing the answer to show with the expression, students were able to see the relationship between the 
addends and sum both within the algebraic equation and with the number line. It is important to note that each button available on this GSP applet toggles between show and hide. Since all the buttons were pressed to show the objects in Figure 1, that is why they now all say hide. This allowed students to control what features they wanted to see at what time they wanted to see them. This applet also has the feature of adjusting the scale of the number line. If students wanted to try large values for the addends, they could simply slide the scale to the right, which was a dilation of the scale factor that created the number line. The sliding of the scale triangle, would increase or decrease the amount of numbers shown on the number line that fit into the screen's perceptual field.

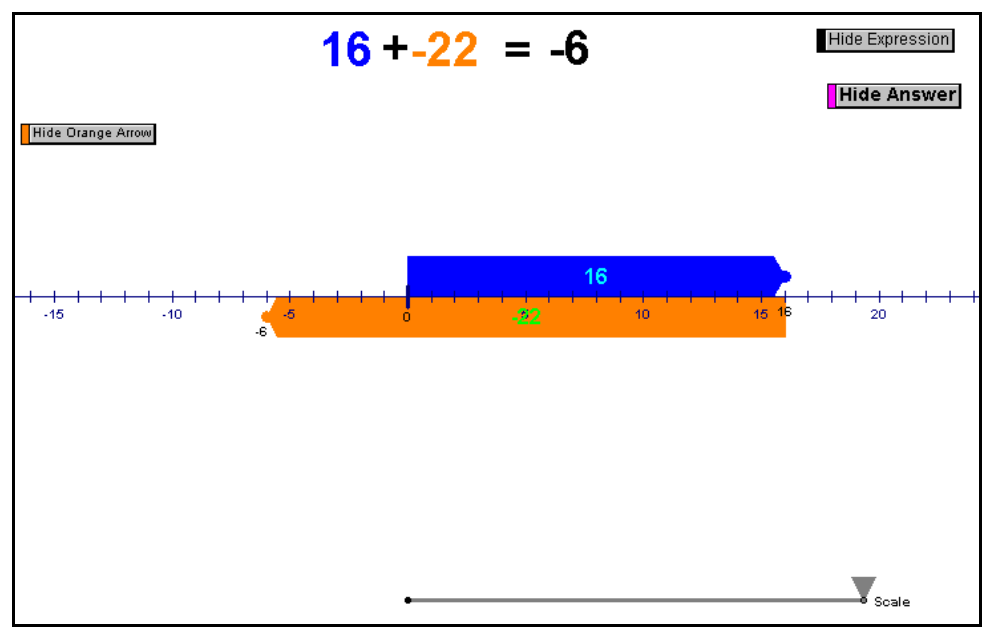

Figure 1. Addition arrow applet

The first subtraction applet showed subtraction as a distance between the minuend and subtrahend and the second applet showed subtraction as a distance between the minuend and difference, also known as the take-away notion of subtraction. Two applets were needed because literature has shown that students have two, not necessarily connected, conceptions of subtraction. By using both of these applets, some students were able to link these ideas to have a better understanding of what subtraction means. Students used both of these applets to complete tasks in Module 2, interview section. Both of these applets compared the numerical statement of values to the position and direction traveled on the number line.

The first applet, Subtraction Dot Applet (SDA) showed subtraction as a distance (see Figure 2). To use the SDA, students moved the blue dot to the number that represented the minuend. Next, they clicked Show Second Dot, and subsequently moved the orange dot to the number that represented the subtrahend. After this movement, they predicted how this motion affected the difference. To observe the actual motion, they clicked Show Arrow. This allowed them to see which direction they moved from the minuend to get to the subtrahend. If they wanted to see the absolute value between the minuend and subtrahend, they clicked Show Arrow Value. This applet also provided the option for the student to see the hot-linked expression 
with or without the answer. These features allowed the students to progress at their own pace as well as discover relationships between the numbers and representations.

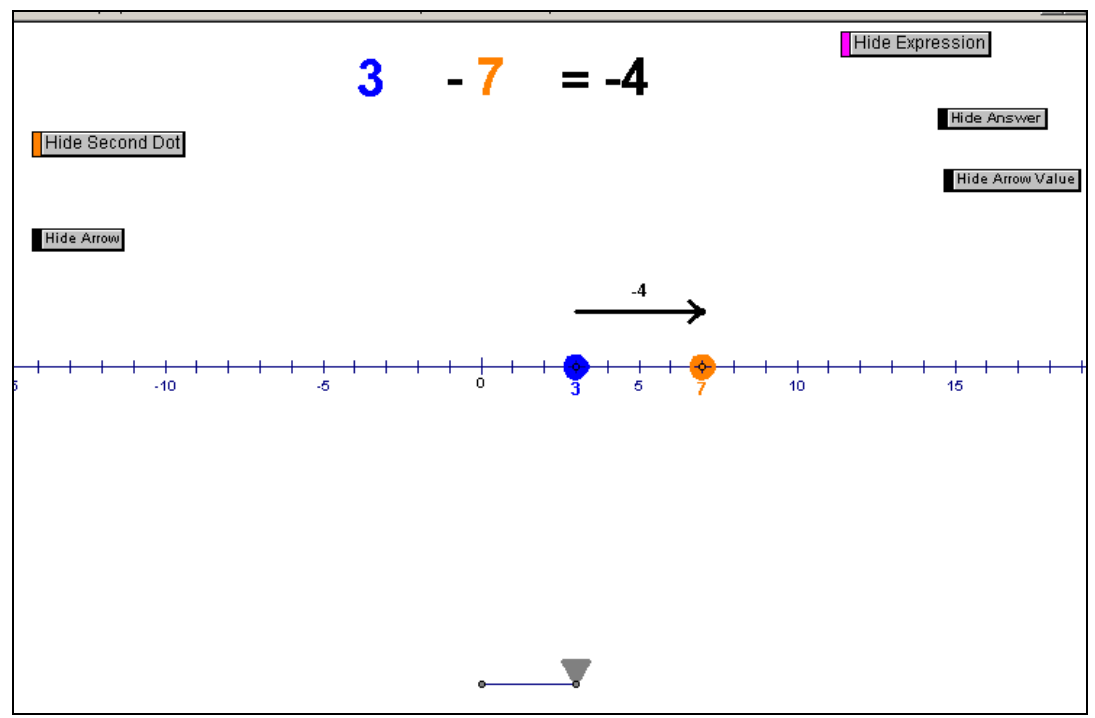

Figure 2. Subtraction dot applet

The second applet, Subtraction Arrow Applet (SAA), worked similarly to the first. To use the second number line, students moved the blue stick to a number representing the subtrahend (see Figure 2). They clicked Show Arrow, which caused an arrow slider to appear that projected from the blue stick. Students moved this arrow by dragging its tip the amount of spaces designated by the subtrahend. In order to make sure they moved the correct amount of spaces, they could Show Arrow Value, which showed the distance one traveled from the minuend (in Figure 3 this is -12 ). Where the arrow ends is the value of the difference. Similar to the other subtraction applet, this applet also has the features that allowed students to show and hide the arrow value, the expression, and the answer. It also allowed students to control the scale of the number line.

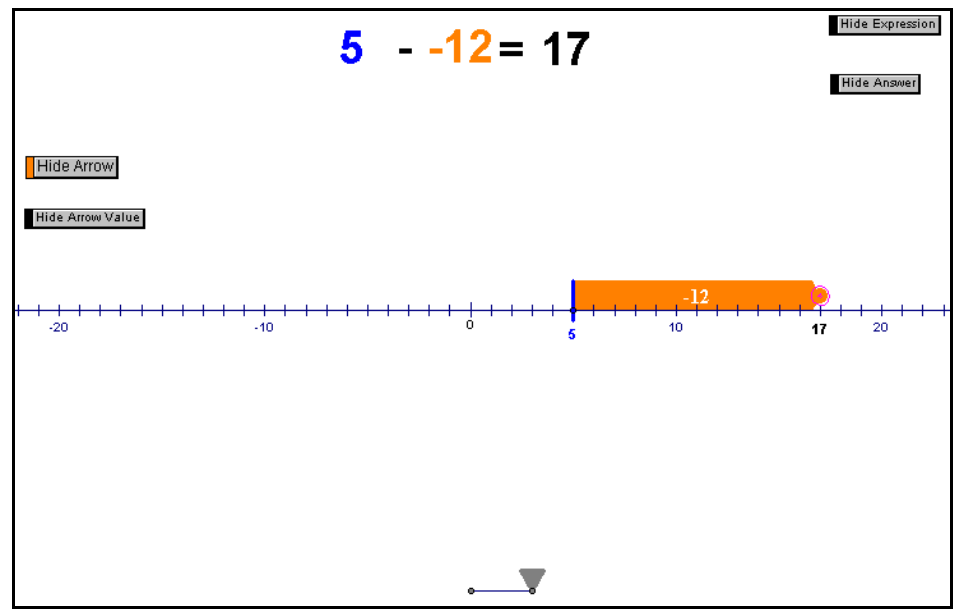

Figure 3. Subtraction arrow applet 
There were two reasons for the addition and subtraction dynamic number lines. The actions on these number lines allowed students to visualize the connection between the problem context, graphical representation of the problem, and the numerical statement as well as to generalize on what it meant to add and subtract negative numbers.

The equal sign and the notion of equality were addressed in the third module. I used a pre-existing GSP applet that I obtained from Key Curriculum Press for Exploring Algebra 1 with Geometer's Sketchpad (2006) and modified it to suit the tasks.

The GSP applet contained 12 pages in it, eight of which were used to solve specific equations in one variable. I used this applet to help students rename numbers, show equivalence between the number of objects and numbers, and apply equivalent transformations to find the value of a variable that makes an equation true. The first page (Figure 4) was used for students in a guided exploration activity so that they became comfortable using the software containing the Algebalance. This exploration activity included Tasks 1-5, which was taken from the activities proposed to be used with this applet from Key Curriculum Press for Exploring Algebra 1 with Geometer's Sketchpad (2006). Students experimented using the Algebalance by clicking on and dragging objects from the storage area (left of the vertical line) to the left or right balance pan. By dragging these objects, the pan tilts according to the amount placed on the pan. For example, if there were three -1 balloons on the left pan, the balance will tip downward to the right and will not become balanced until a value of -3 is on the right pan. The usage of balloons and weights on this balance scale is similar to the hot-air balloon model proposed by Janvier (1988).

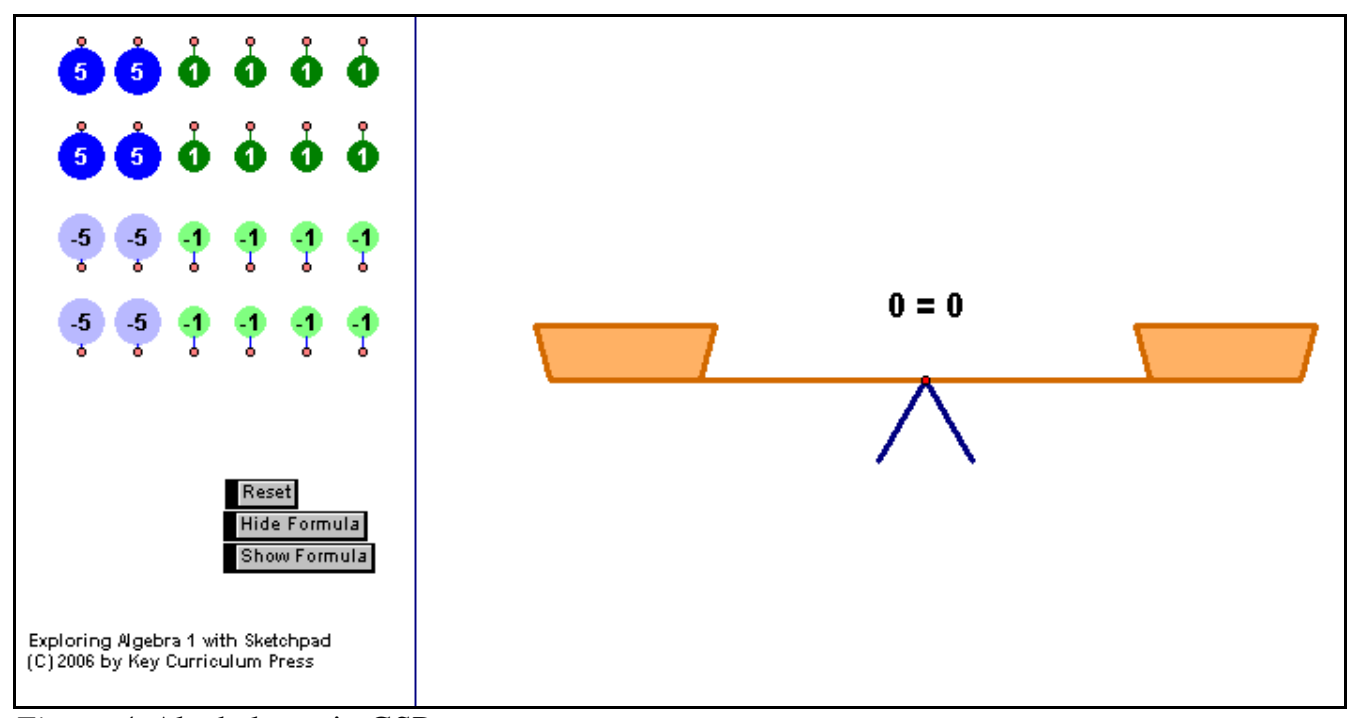

Figure 4. Algebalance in GSP

If students had difficulty answering Task $2 \mathrm{a}$ and $\mathrm{b}$ in the Interview Section, Page 1 was used. If 
students had difficulty answering Task $2 \mathrm{c}$ and d, Pages 2 and 3 were used (see Figure 4 and 5). All subsequent pages were created based on modifications of Page 1. Pages 1-3 were used to help students understand the relational aspect of equals as well as renaming and understanding the transitive property of equality.

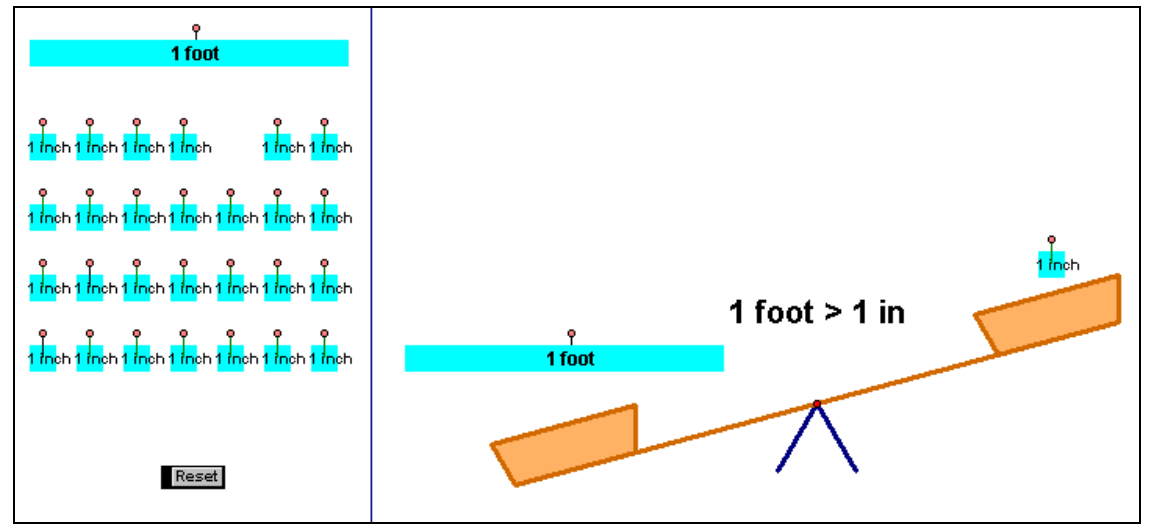

Figure 5. Page 2 in the Algebalance

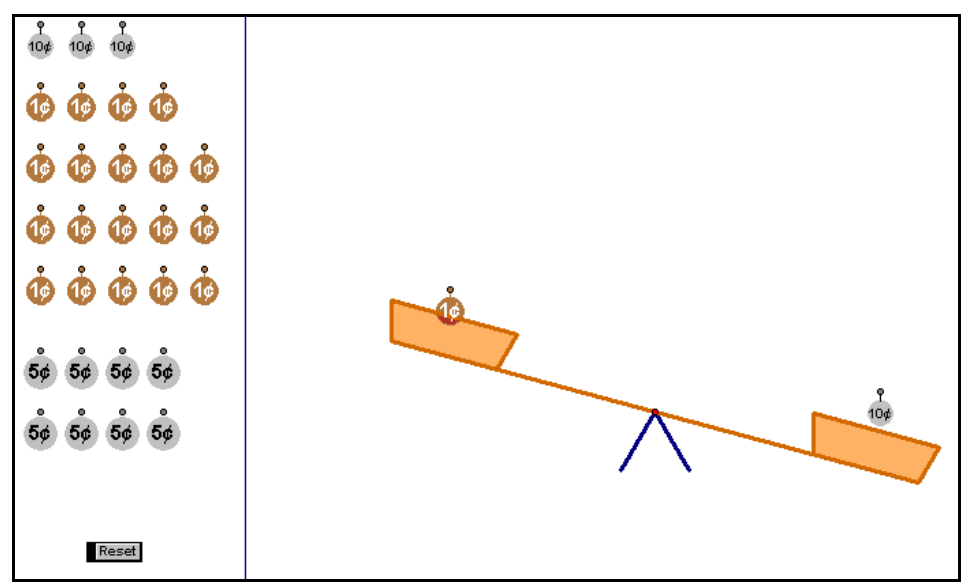

Figure 6. Page 3 in the Algebalance

Page 4 (Figure 6) used the balance to show the value of the question mark. Students could drag the amount from the storage unit to represent the problem. After representing the problem, they removed similar objects from both pans to figure out what the value of the question mark had to be. This applet corresponded with Task 6 in the instructional section. 


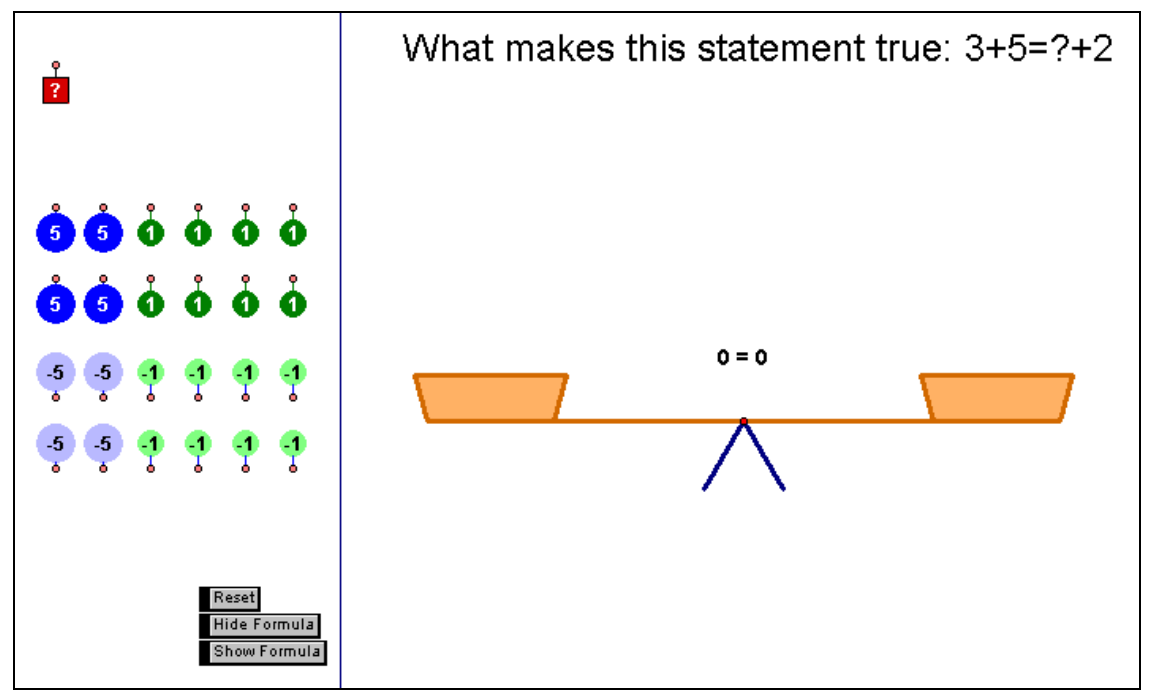

Figure 7. Page 4 in the Algebalance

Similar to the last page, Pages 8-12 showed an equation with the corresponding amount of objects placed on the pans. These applets corresponded with the Tasks 7-14. Based on the exploration and rules that students learned to keep the Algebalance balanced, students dragged the appropriate objects to the pan to solve $2 m+9=3$ (see Figure 7). This applet allowed students to experiment with many different strategies to solve equations. It also allowed them to reset to the original equation, show the formula, and hide the formula with a click a button.

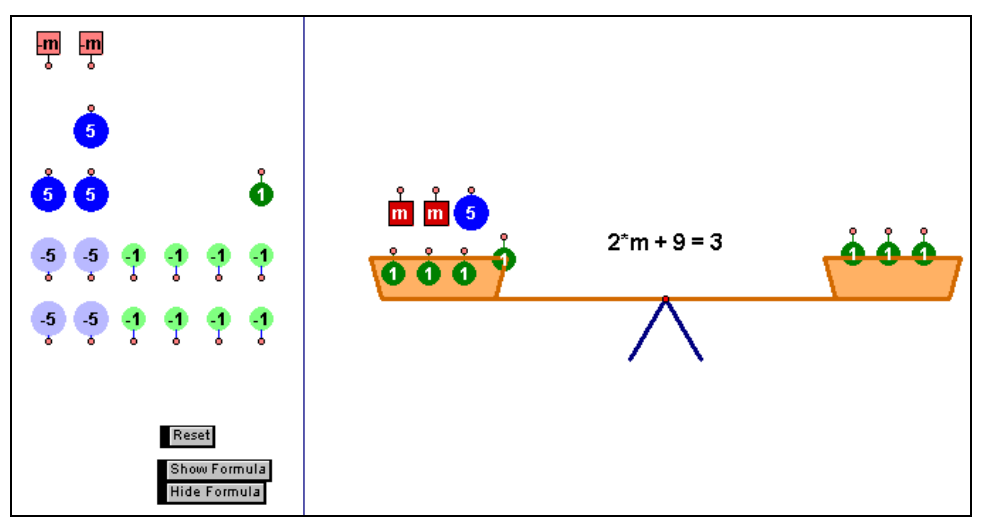

Figure 8. Using the Algebalance to solve $2 \mathrm{~m}+9=3$

Written documents. The last method of data collection used in this study was the collection of written documents. Patton (2002) believed that documents were useful for two reasons: they contain more information for analysis, and that information can be used to stimulate paths of inquiry for follow up questions (p. 294). The written documents in this study was participants' written work on tasks, GSP applets, and any notes students scrawled on paper during the instructional and interview sections.

By collecting students' written work, viewing and listening to the audio and video taped initial, final, and intervention sessions, I was able to scrutinize and analyze the data to compare within and between 
student cases in order to obtain a thorough description of students' understanding of pre-algebra and algebra topics.

\section{Data Analysis}

Since the overarching goals of my research were to improve educational practices by providing plausible reasons for the kinds of interactions with technology and the understanding of pre-algebraic concepts as well as to provide insight into what could positively influence children's understanding, I used Erikson's (1986) method of analytic induction to search for patterns of action and meaning making from students' who engaged in constructing their knowledge of pre-algebraic and algebraic concepts.

Erikson's (1986) method of data analysis consists of two phases. During the first phase, the researcher creates tentative assertions by searching through the entire corpus of data. After these tentative assertions are made, the second phase requires the researcher to find evidentiary warrant for each assertion, which is done through "a systematic search of the entire data corpus, looking for disconfirming and confirming evidence, keeping in mind the need to reframe the assertions as the analysis proceeds" (p. 146).

After each data collection session, I analyzed my data for each participant by reading the whole account and creating a reflective report. From the interviews and instructional sections, I noted each student's descriptions, interpretations, and thoughts of the pre-algebraic concepts as well as the strategies they used to solve tasks with these concepts. The information I gathered noted both conceptual and procedural understanding, any resemblance to errors that have been mentioned in previous literature, and interactions with the technology applets. I reviewed my reflective accounts, field notes, and transcripts of the audio recordings and video recordings. Prior to any coding, I analyzed these descriptions and interpretations and make tentative assertions about each student's understanding.

After generating tentative assertions for each participant, I used my reflective accounts and transcriptions to test these assertions. I highlighted and annotated each relevant piece of information. I used these highlights and annotations to create a summary for each task. The summary included significant comments, themes that appeared across tasks, methods used to obtain the answer, and mistakes made. By highlighting and annotating, I was able to piece traces of evidence together to compare the detailed cases, find particulars, and stay close to the concrete evidence. This allowed me to test assertions to make sure they were plausible within and between participants.

\section{Establishing Validity}

In qualitative research, Erikson (1986) believes there are five threats to validity: (1) inadequate 
amounts of evidence to warrant assertions, (2) inadequate variety of data, (3) faulty interpretations of data due to inadequate time period of the study, (4) inadequate amount of disconfirming evidence, and (5) inadequate amount of discrepant case analysis.

The first and second threats to validity will be addressed by collecting data from a variety of sources: interviews, observations, field notes, reflective accounts, and students' written artifacts. By utilizing many types of data, I was able to provide many instantiations of confirming and disconfirming evidence for assertions by triangulating students' descriptions of their understanding of pre-algebraic and algebraic concepts.

While the third threat to validity was usually eliminated by staying in the field for an extended amount of time to truly be able to interpret participants' meanings behind their actions, this study dealt with the third threat to validity differently. I have designed the task-based interviews to focus on "key aspects" that elicit the "complexity of action or of meaning perspectives held by the actors in the setting" (Erikson, 1986, p.140). Furthermore, I focused on the complexity of behaviors, comprised of both actions and meanings behind those actions, surrounding participants' understanding of pre-algebraic and algebraic concepts by the structuring of my interviews. By interviewing prior to the instructional section, I was able to understand students' changes in conceptual knowledge and describe their interactions with the technology applets.

The fourth and fifth threats to validity involve lacking amounts of disconfirming evidence. I addressed these issues by gathering data in a variety of ways including interviews, observations, artifacts, and reflective journaling. By frequently reviewing all documentation, highlighting and annotating relevant pieces of data, analyzing, and actively writing about the data, I assessed the instantiations of disconfirming evidence through triangulation. Furthermore, I engaged in discussions about the data with peer debriefers (Lincoln \& Guba, 1985), each of who was either a recent graduate of a mathematics education program, or currently in a mathematics education program.

\section{Reporting the Findings}

The data is reported as both a series of descriptive cases and through assertions based on crosscase analysis. Thus, data analysis takes on both particular description, where one focuses on specific cases, and general description, where one surveys the patterns among the cases (Erikson, 1986). Both types of descriptions are framed by interpretive commentary with quotes from field notes and interviews according to the interactions among data collection, analysis, and interpretation described by Erikson.

There were three main reasons why I chose to employ a case study method of reporting. The first 
reason was that each person could be considered both a micro unit of analysis and a macro unit of analysis. Within each person, there were many interactions with the technology applets and myself. Each interaction can be considered a unit of analysis. However, one can use the individual person as a conglomerate of each of those interactions; thus, making the person a unit of analysis. The second reason for using case studies was that it provided a detailed description for both the reader and myself on which to make meaningful interpretations (Patton, 2002). Providing a rich, detailed account of a specific case, facilitates analysis for the researcher. It also enables the reader to understand the situation, judge whether my biasness infiltrated the data, and decide whether there were any internal inconsistencies (Lincoln \& Guba, 1985). The third reason was that by presenting the data in a case study fashion, I was able to provide the specific details of the interactions of students' with the technology applets on these topics, which has never been done. This detailed description is grounded in a contextual setting. By using the descriptions and setting, the readers are able to hypothesize about the repeatability and transferability of the study as well as see the evolution of students' thinking.

Researcher as an Instrument Statement

Since the researcher is the instrument in a qualitative study, both Erikson (1986) and Patton (2002) agree that the researcher must be self-aware of her interpretive frames. Though the act of reflection, the researcher can appropriately judge her "training, preparation, field work procedures, and analytical processes" (Patton, 2002, p. 64) in order to add credibility to her assertions. By asking myself two questions "What do I know?" and "How do I know it?" (Patton, 2002, p. 64), I furnished four factors prejudicing my perceptions of algebra and technology.

First, my experience as a student teacher has shaped by ideas about students' understanding of algebra. During student teaching, I was assigned to teach a lower level ninth-grade algebra class; however, I never taught this class because my cooperating teacher could barely keep the students up to the level that they needed to be in order to pass a test similar to the algebra Standards Of Learning. Since I was not teaching this class and since she had one student who severely misunderstood the content, I was assigned to be this student's tutor. I would cover basic ideas of algebra with her including operations with integers and solving equations which required the ability to use basic operations, the idea of equivalence, and the notion of variable. My cooperating teacher encouraged me to make flash cards, outline the procedures needed to manipulate numbers and variables, and verbally quiz the student on number sentences and these procedures. At the end of student teaching, I did not see much improvement in my students' conceptual knowledge or procedural knowledge if any at all. I was disheartened and felt that there needed to be some 
other way for students who struggled with algebra to grasp these concepts.

Second, the experience I received at Virginia Polytechnic Institute and State University as a graduate assistant has influenced my beliefs about teaching mathematics and learning mathematics with technology. As I was earning my master's degree, I worked as a Calculus I and II instructor for the university. I enjoyed learning and teaching calculus in a traditional manner (not constructivist) because of the many creative ways that I could represent a concept. For instance, while the notion of the secant line becoming the tangent line may be hard to grasp using algebraic notation, it lends itself quite nicely to a graphical representation. When I taught this concept, I would use multiple pictures and make a flipbook on the overhead projector to show the limiting notion of the secant line becoming the tangent line to help explain the idea of a limit of a slope of a line. Similarly, the idea of finding the volume of a figure by taking cross sections lends itself quite nicely to concrete manipulatives, even though visualizing, drawing, and relating the algebra to the drawing is somewhat taxing. For instance, I would bring in Play-Doh-shaped figures (e.g. a pyramid) and take a piece of floss to make cross sections of the shape. I would then stick the Play-Doh to the board where my axes were drawn. Students would remark how they could "see" how the algebra related to finding the volume of the figure.

Despite using these representations, I never used any technology to teach calculus. I was not fond of technology in the classroom because I had seen students in high school and college who could not perform simple procedures, like adding and subtracting integers, rely solely on their calculator to do the computations for them. I felt that by endorsing the use of technology, I was depriving my students of learning mathematics. Moreover, this view of technology hindering mathematics education was affirmed by spending time as a Math Emporium employee at the University.

The Math Emporium was an empty department store that housed 500 computers. Undergraduate students would go there to take online mathematics courses such as linear algebra, business calculus, and trigonometry. Once they logged in to the computer system, they completed tutorial modules by reading and going through the examples. After working through the modules, they would take online multiple-choice quizzes and tests. In some courses, they could retake the quizzes and tests as often as needed until the deadline. Since there was a finite database of examples, many instructors would encourage students to take many quizzes because they would eventually encounter the same problem. As a graduate student, my job was to walk around the emporium and answer any questions that students had. There were many questions, some of which were simple and some of which were complex. I saw many students who were frustrated and confused and asked if there were any class that "had a real instructor" that they could take instead. 
While some educators thought this method of teaching mathematics with technology was wonderful, it deepened my antipathy for technology in mathematics education.

My views of teaching mathematics in a traditional manner and without technology drastically changed upon receiving a graduate education at the University of Virginia. Based on my student teaching experience and during the time I obtained my master's degree in pure mathematics, I considered the possibility that some students could not learn mathematics as easily as others, if at all. Upon reading articles and texts (Clements \& Battista, 1990; NCTM, 2000; Simon, 1995), discussing ideas, and working with professors, who are proponents of constructivism, my outlook has drastically changed. I believe that all students are able to learn mathematics, yet they might not do so by receiving traditional instruction, for student learning is based on the content and methods for actualizing that content that are provided by the teacher. I now believe that for students to truly learn and understand, they must actively take part in their own learning, which can be facilitated through projects encouraging inquiry, analysis, and synthesis.

The third influential factor is due to my position at the Center for Technology and Teacher Education (CTTE). Considering my position on student learning, it is only natural to acknowledge how the appropriate use of technology can facilitate that type of atmosphere for student learning. At the CTTE, I have generated, examined, researched, and presented types of technology and corresponding tasks with which to use technology to pre-service and in-service teachers. Many of the technologies I have worked with and presented are programmed in Excel and GSP. I believe that instruction containing appropriate content and content pedagogy can make a difference in the teaching and learning of mathematics.

\section{Limitations of the Research}

The limitations of this study deal with two factors: generalizability and validity. Generalizability refers to what one can do with the results of the study. If the study employs a large random sample, the results can apply to any student provided certain conditions are met. However, with qualitative research, one sacrifices generalizability for rich descriptions that are situated within a certain context and cannot be easily removed from this context and still remain true. By qualitatively researching only 8 participants, I could not make all-inclusive statements about how all middle school students view these pre-algebraic and algebraic concepts or their interactions with the technology. Yet, by using qualitative methods, teachers and researchers can glean some information about how some of their present and future students might view these concepts and work within a technological setting. Using the insight gained from this study, educators can change their instruction to benefit their students' understanding of algebra, and researchers can test or build on these findings. 
The other limitation has to do with the validity of the study. Erikson (1986) advises researchers to stay in the field for an ample amount of time to be able to fully come to terms with participants' meanings attached to their actions. However, in my study, I could only work with some students for 2 hours due to schedule restrictions. The length of these sessions may not been long enough to grasp an adequate understanding on students' understanding of these concepts and interactions with the technology applets, or allow the students to fully realize their own understanding of these concepts. Furthermore, two hours was not enough time to move some students through each of the 3 modules. 


\section{CHAPTER 4}

\section{CASE STUDY: ABBY}

Abby's Background

Abby is in sixth grade Advanced Math. Advanced Math covers mainly sixth grade material and then some seventh-grade material at the end of the school year. A student in Advanced Math in sixth grade will most like take pre-algebra in seventh grade and algebra in eighth grade. At the time of the study, data collection was during the end of the year, so Abby was covering seventh-grade material in her math class. She feels confident in her math ability and bases this on her experiences. Abby revealed that her teacher told the class that they "were ready for ... seventh grade material" and subsequently started working on specific seventh-grade concepts such as solving 2-step equations. Also during the interview, she frequently told me that the tasks I had given her were easy and reminded me that she was in Advanced Math not in the lower level Standard Math. In response to my asking how she solved a subtraction task she said, "It's just easy. Of course, I'm at a higher level math than Standard." When I asked her to explain her thought processes in solving some of the tasks, she occasionally replied, "I didn't think about it" or "It's just a simple math task that we probably learned in kindergarten." While discussing variables, Abby told me how she could quickly do all her math homework even when there were lots of tasks.

A: We have like 20 worksheets full of it [tasks with variables] for homework. Like I can show you it...I did my homework last night in 20 minutes.

$\mathrm{N}$ : So you're good with variables then?

A: Yes.

During the interview, she also provided some insight into her perspective of learning mathematics. As I tried to probe for justification about an answer for which she did not provide a reason, I asked her to tell me how she would explain the concept to her little brother.

I would just tell him what the answer is and tell him [her voices goes up in pitch when she say that following] 'This is what the answer is and get it stuck in your head,' cause that's what I did. Cause my mom... when she taught me things she'd just say 'Here's the answer, get it stuck in your head cause I'm going to ask you later' and I had to learn it.

Abby was home-schooled when she was younger, and that is why she refers to her mom requiring her to memorize answers to math tasks. Not only did she think that mathematics required memorization, she also believed that students must follow rules given by their teacher. After solving 2-step equations (e.g. 
$2 m+7=15$ ), I asked her why she divided first and she responded, "That's what you're supposed to do [in whiny voice]! You take your multiplying and division first." For example, she divided the term $2 m$ by 2 , and then wanted to divide either the 7 or 15 , but not both numbers, but was unsure which one to divide by 2. Even after she saw that applying this procedure to both the 7 and 15 gave her the wrong answer, she maintained that this was the correct way of solving it because, "Well that's how Mrs. G. told me to get it."

Abby's math experiences include memorization, following rules given to her by an authority figure, and using a calculator on mainly computational tasks. She relies on calculators to such a degree that she said, "I use calculators a lot in math class so I don't depend on my brain. I depend on the calculator a lot. [pause] For very simple math questions." I asked her if she had used anything else in math class to help her solve tasks, such as number lines. While she had seen number lines before, and used them a few times, she had not used them during $6^{\text {th }}$ grade, when they learned adding and subtracting integers.

We don't use a number line in math class. Well, she [the teacher] used to have one on the wall, but it's not up there any more because we had the SOLS and stuff like that. We're not supposed to have anything up that has to do with SOL stuff. They have to take down all the math signs, because someone cheating might look up there and take the answers. And we don't want anyone cheating.

It is interesting to observe the words that Abby used to describe the purposes of a calculator and number line. Abby believed that the calculator was a tool to help a student solve easy math exercises so that one did not need to think about it, while a number line was a tool that a student might use on an exam to obtain the answer if the teacher left it in plain sight. This attitude may be due to the fact that Abby used calculators frequently in math class, and therefore they seemed to be an acceptable tool. Because she did not use number lines in class and because the Standards of Learning tests required teachers to remove them during testing, she viewed them as giving a hint of how to solve the task, which made the task easier for students.

\section{Negatives and Operations on Integers}

\section{Prior Conceptions on Addition}

I gave Abby tasks to complete before using the technology to assess her initial understanding of negative numbers and operations on negative numbers. While completing these tasks, I did not allow her to use a calculator even though she specifically stated, "We usually use a calculator" to solve addition and subtraction of integers. I wanted to see how she conceptualized these processes. Abby's notions of negative numbers and how she operated on them do not firmly fit into one category according to past literature (Bell, 1982; Kieran, 2007; Murray, 1985; Peled, 1991). Abby’s approach to computational tasks is to pay little attention to the operation and focus on the sign of the integer. Whether the task involved addition or 
subtraction was of no consequence, for Abby solved it as if it were implicitly addition. (e.g. She would solve $5--12$ as $5+-12$ ). She used the signs of the numbers to indicate which direction to move on the number line. Thus, Abby was able to solve correctly addition tasks involving negatives and could justify the procedure using number lines.

While Abby used the signs of the numbers with a number line to solve addition tasks, she had a unary (Kieran, 2007) conception of addition tasks with different signed addends and a binary (Kieran, 2007) conception of addition tasks with two negative addends. For instance, her unary conception is demonstrated by how she describes that addition of a negative number is the same as subtraction of a positive number in the task $10+-3$.

$\mathrm{N}$ : Ok, and how did you get 7 for that task?

A: Cause you have a negative and a positive number, and so even though it says you add them, you subtract them. ... You always get a lower number when you add a positive and negative number.

$\mathrm{N}$ : Why do you get that?

A: Because if you are adding 10 to -3 , then you will have 3 left of zero so you have 7 left to add.

$\mathrm{N}$ : Do you want to show me that on a number line?

A: Add 3 here to get to zero and then you have 7 left out of 10 , so you get 7 .

When using the number line to justify her answer, she showed me that you needed to start at -3

because it was on the negative side of the number line (three left of zero). Then she had to move to the right 10 spaces because 10 was positive, and the positive portion of the number line lies to the right of zero. Similar to this task, Abby justified her solution to $-5+-7$ on a number line by using the signs to determine which way she will move. However, unlike the previous task, she did not view- $5+-7$ as subtracting 7 from -5 . Because she did not have this unary view of addition tasks containing two negative addends, she relied on a rule of signs to find the answer.

A: -12

$\mathrm{N}$ : How did you get that?

A: I added 5 plus 7 and they are both negative so I got a negative number, negative 12.

$\mathrm{N}$ : So you're adding 5 and 7.

A: Yes, and I got $12 \ldots$ when they are both negative, I put the negative sign.

$\mathrm{N}$ : Could you show me that on the number line?

A: You go, -5 plus 7, and you go one, two, three, four five, and you're at negative 12.

She justified her solution by a rule that one adds the absolute value of the numbers and then tacks on the negative sign because both addends are negative. When she explained her solution using a number line, she started at one of the addends, -7 in this instance, and moved toward the left 5 spaces because the other addend was -5 . In response to why she went left on the number line she responded, "Because they are both negative and this is the negative number side." Thus for addition, she believed that the sign of the number 
determines which way you move on the number line. If the number was positive, you moved right because the positive side is situated to the right of zero, but if the number was negative, you moved left because the negative side is situated to the left of zero. This idea that the number line has two "sides" is similar to the notion of the divided number line proposed by Peled (1991). According to Peled, children who have this notion make up rules to deal with operations that require the student to cross over zero, much like Abby did with $10+-3$, since she added 3 of the 10 to get back to zero, and then proceeded with the 7 left over from 10.

\section{Prior Conceptions on Subtraction}

Even though Abby talked frequently about the number line having two different sides, she was still able to cross over zero as if she had a continuous number line view, as seen when she was asked to write an equation for a number line that had a dot at -6 and a dot at 5 .

A: Um, well, I guess you would take 5 and subtract 10 so 5 minus 10 would get you into the negative numbers, because there are 10 in between here, I' $m$ sure. [she counts] Yeah, there's 10 in between.

$\mathrm{N}$ : Could you write numbers for me?

A: So you would have 5 minus 11 , because this is the $11^{\text {th }}$ spot from the 5 over here...There is 10 in between, but this is the $11^{\text {th }}$ space, because if you did 5 plus 6 you'd get 11 .

$\mathrm{N}$ : And where did you get the -6 in the task?

A: It would be at the end over here, equals -6 [pointing to the dot on -6 ]

In her rationale to her written equation, Abby talked about the 10 tick marks between the 5 and -6 on number line. She knew that if there were 10 tick marks, then there were 11 spaces between 5 and -6 ; therefore, she wrote $5-11=-6$. What is interesting about the way she solved this task on the number line is the fact that she viewed subtraction of the subtrahend as taking away spaces from the minuend, rather than interpreting subtraction as a distance between the minuend and subtrahend. If she had viewed subtraction as the distance between the minuend and subtrahend, she would have written the equation $5--6=11$. She again can be seen using this idea of subtraction as taking away items when she solved $5-12$.

A: You're going to get a negative number if you subtract 12 away from 5 because you don't have enough.

$\mathrm{N}$ : You don't have enough?

A: You don't have enough from 5. If it were the other way you'd have enough and you would get a positive 7 .

The fact that she used the word "enough" implied that she thought about subtraction in terms of possessing a certain number of items and the removal of the number of items in the subtrahend. Since the subtrahend was greater than the minuend, she did not have an adequate quantity from which to take away; thus, she 
finished with a negative number of items. While she gave this "not enough" verbal justification for tasks of the form $a-b$ where $b>a$, she did not give any verbal justification for tasks in the form $-a-b$. For instance, when she solved the task $-5-12$, she told me, "You'd get -17 because, well I don't know, but if the negative number is first then I don't know." Her uncertainty in providing a verbal explanation may stem from the fact that there were a negative amount of objects from which she needed to subtract a positive amount. Given that she could interpret not having enough items from which to subtract, subtracting a positive number of items from an already non-existent negative amount of items is much harder to explain. Yet, she showed me how to use a number line to find the answers to both $5-12$ and $-5-12$.

What is common in the last three examples $(5-11=-6,5-12$, and $-5-12)$ is that they all have a positive subtrahend that is larger than the absolute value of the minuend. Because of this, Abby was able to obtain the correct answer to these tasks by looking at the signs of the numbers and using her number line. To solve $5-12$, she began at 5 and went 12 spaces towards the negative side of number line, and ended at 7. For the task $-5-12$, she began at -5 and went 12 spaces towards the negative side of the number line and ended at -17. There are two possible explanations for why she did this. One explanation is that she used her unary notion of negative numbers in that she knew that subtraction of a positive number is the same as the addition of a negative number. Thus, she mentally transformed these tasks into $5+-12$ and $-5+-12$, respectively, and used the number line as she did for addition tasks. The second explanation is that she did not attend to the operation and interpreted the subtraction sign as a negative sign for the subtrahend and simply followed her addition rules for navigating on the number line by starting at one number and moving left if the sign of the other number was negative and moving right if the sign of the other number was positive. Based on her answers and procedures for solving tasks with a positive subtrahend, the second explanation is more plausible.

\section{Prior Conceptions on Subtraction}

When Abby solved subtraction tasks with a negative subtrahend, she consistently obtained incorrect answers. Her answer was based on the fact that she only paid attention to the signs of the numbers and not the sign of the operation, as can be seen in her reasoning for the following tasks.

$\mathrm{N}$ : And what about the next one $-4--6$

A: -10 , because they are both negative, so it's negative. [pause] Because you're going farther back here because they are both negative [she points to the -10 on the number line]. Positives you want to go closer to the positive side. And that one [task $-4--1$ ] would be -5

$\mathrm{N}$ : And why is that?

A: Because you're going farther back into the negative side because they are both negatives. 
Abby chose one of the numbers and began at that point on the number line and then moved according to the sign of the other number. Because both numbers were negative in this case, she would always move to the left. This is similar to Bell's (1982) finding as one of his interviewees said that he used the second number's sign to determine which direction to move on the number line. However, in Abby's case, she did not necessarily have to use the sign in the second number, for she could start with the second number, and then use the first number's sign to determine the direction to move.

Even though Abby used addition procedures on subtraction tasks, she was aware that these tasks were subtraction and not addition, but she solved them in exactly the same manner. Moreover, she was cognizant that she solved them in the same way and because of this awareness, she believed that addition and subtraction tasks containing the same numbers elicited the same answer.

N: How about the next one? $5--12$

A: That, [pause], -7

$\mathrm{N}$ : How did you get that?

A: Cause you're adding because one is negative and one is positive.

$\mathrm{N}$ : So you're adding 5 and -12 ?

A: Yes. Because the bigger number is negative, so it's a negative number. Then you have to go up because the other one is positive and positive numbers they go up and negatives makes it go backwards.

Abby said that she was "adding" 5 and -12 , even though the task displayed subtraction. It is important to note that prior to doing this task, she placed parentheses around the -12 "because it separates the negative from the subtraction," so I did not feel that she overlooked the operation and mistakenly took it as addition. To make sure of this, $\mathrm{I}$ had her show me how she would solve $5+-12$ on a number line, and she solved it using the same strategy she used for solving 5--12. I then had her compare these two tasks.

A: Well, even if I subtracted -12 from 5 I still get -7

$\mathrm{N}$ : So how does this task $5+-12$ compare to this task $5--12$ ?

A: One is addition and one is subtraction, but they are both the same answer.

Abby had a unique way of operating with negative numbers. While she discerned the written difference between addition and subtraction tasks, she solved them in the same manner by disregarding the operation and using the signs of the numbers to determine the direction to move on a number line. Using this method, she obtained the correct answer for all addition tasks. She also correctly solved subtraction tasks in the form $a-b$ and $-a-b$ because she used the subtraction sign as a negative sign for the subtrahend. However, because she disregarded the operation sign, this method does not work for subtraction tasks in the forms $a--b$ and $-a--b$. 


\section{Discussion of Prior Conceptions}

Abby's procedure for solving addition and subtraction tasks with negative numbers has some associations with past literature. As stated before, Abby had a unary idea of negative numbers regarding adding a positive number and a negative number. However, she exhibited a binary notion when she encountered addition of two negative numbers and the subtraction of negative numbers, for she could not connect the fact that subtracting a negative is the same as addition or that adding a negative number to a negative number is the same as subtracting it from the negative minuend. In fact, when it came to subtracting a negative, she could not make a connection between the operation and sign; thus she relied on the sign of the numbers to tell her the direction to move on a number line.

Abby's additive procedure for solving tasks with negative numbers allowed her to obtain the correct answer for all addition tasks and for subtraction tasks where the subtrahend was positive; however, it did not allow her to get the correct answer when the subtrahend was negative. The fact that addition tasks were easier than subtraction tasks for Abby agrees with Murray's (1985) findings. Yet, it deviates in the fact that Murray showed that students were able to solve tasks with a negative subtrahend more easily than tasks with a positive subtrahend.

There are two other interesting parallels between Abby's procedure and Murray's research: overgeneralized commutivity and transformations of tasks. If one simply looked at Abby's work for subtraction tasks, one might not think she is over-generalizing commutivity. She would give these answers and reasons for the following tasks.

$$
\begin{aligned}
& 5-7 \neq 7-5 \text { because }-2 \neq 2 \\
& -5-7 \neq 7--5 \text { because }-12 \neq 2 \\
& 5--12 \neq-12-5 \text { because }-7 \neq-17 \\
& -5--12=-12--5 \text { because }-17=-17
\end{aligned}
$$

One would think that because of the first three answers, Abby knew that commutivity did not generalize to subtraction, and that the last answer was a mistake. However, if one would explain to her that commutivity was exchanging the positions of the numbers but applying the same operation, and then asked her if these tasks exhibited commutivity, she would answer no for the first three. For her, commutivity for the first three tasks would look like:

$5-7=-7+5$ because the sign of 7 is negative and the sign of 5 is positive.

$-5-7=-7-5$ because the sign of 7 and 5 are both negative.

$5--12=-12+5$ because the sign of 12 is negative and the sign of 5 is positive. 
While she might not be over-generalizing commutivity to subtraction by the true mathematical definition, if one uses her idea of commutivity, she is over-generalizing it inappropriately.

In regards to $-5--12=-12--5$, Abby would obtain the same answer because she would ignore the subtraction sign in both tasks. If the task was $-5-12$ the subtraction symbol would become her negative sign for the 12 , but since the 12 already has a negative sign, the subtraction sign is redundant and useless to her. This is the same for $-12--5$. Because the subtraction sign is of no use, $-5--12$ is equivalent to $-5-12$ in her eyes. Murray (1985) believed that students transformed these equations because they were not familiar with the notation of two negative signs beside each other. However, I do not think this is the case for Abby. I think she only remembers learning addition rules in regards to operating on negative numbers and applied these to her subtraction tasks. In fact, when solving the task $5--12$ she firmly believed it was -7 not only because of the way she solved it on the number line, but also because she used Unifix cubes to incorrectly prove to me that it was, in fact, the answer. She grabbed the Unifix cubes from the shelf and said, "Well, I can show you with blocks. The pink is positive, the green is negative. You use zero pairs and then you have 7 and they are all negatives." She stuck five of the pink cubes to five of the green cubes and then showed me that there were 7 green cubes remaining. When I asked her if she was showing me addition or subtraction she responded, "I forget which one it is, but I remember this from our integer unit. We used this type of cubes."

Abby did not fit cleanly into Peled's (1991) categorization of students' understanding of negative numbers. In fact, I had to separate her understanding in terms of addition and subtraction. Furthermore, she did not fit cleanly into one level. Thus, I adopted my own system of ratings based on Peled's levels. A plus sign following the number level indicates that Abby was able to go beyond that level but could not completely attain the subsequent level. An asterisk in the Quantitative Dimension (QD) indicates that Abby was able to solve higher-level tasks than her number level indicates, but she did not put the answer or task into context. An asterisk in the Number Line Dimension (NLD) indicates that Abby was able to subtract natural numbers, know that plus and minus signs are opposite in direction, but could not do all same-signed subtraction tasks, yet could solve some opposite-signed subtraction tasks.

Because Abby could accurately solve all addition tasks by starting at the first addend and moving to the left or right based on the sign on the second addend, she attained Level 4 in the NLD with respect to addition. Abby knew that positive and negative numbers were opposite in quality characteristic, but she never contextualized her answer or addition task; therefore, she attained Level 1+ in the QD even though she could accurately solve all addition tasks. 
Abby could correctly solve subtraction tasks with a positive subtrahend and negative minuend (criteria of Level 4), but could not solve tasks with a negative minuend and subtrahend (criteria of Level 3). She typically solved subtraction tasks using memorized rules and the number line; therefore, she attained Level $3 *$ in the NLD. Because she did not situate her answers or tasks into context, yet mentioned taking away items when solving them, she attained Level 2* in the QD.

\section{Summary of Abby's Prior Conceptions}

- Abby exhibited a combination of unary and binary understanding of negative numbers. The way she viewed negative numbers depended on the form of the task.

- Abby used the words "not enough" when she talked about subtracting a larger positive number from a smaller positive number, which implies that she thought about subtraction as "taking away". This is more evident in the way she subtracted using a number line. She used the first dot to represent the minuend and the second dot to represent the difference, and the distance between these two dots to represent the subtrahend.

- Abby algebraically solved addition tasks involving negatives and justified the procedure using number lines. She used the same procedure (using the signs of the numbers to tell the direction on the number line) for subtraction tasks. Thus she correctly solved subtraction tasks where the subtrahend was positive, but not when the subtrahend was negative.

- Abby was unable to correctly solve tasks of the form $a--b$ and $-a--b$. She mentally transformed these tasks into $a-b$ and $-a-b$, respectively, and applied her additive procedures to solve the task even though she was aware that the task specified subtraction. She believed that addition and subtraction tasks containing the same numbers elicited the same answer.

- According to the literature, students tend to over-generalize commutivity to subtraction. This is implicitly true in Abby's case. While this never explicitly came up in a task, her rules for solving subtraction tasks would lead her to believe that subtraction was commutative.

- Abby determines the size of a number based on its absolute value instead of its actual value.

- Abby fits unusually into Peled's negative number dimension theory. In some instances she operated on the number line as if it were divided, and in other instances as if it were continuous. She attained Level L1+ in the QD and Level L4 in the NLD with respect to addition. She attained Level L2* in the QD and Level L3* in the NLD with respect to subtraction.

\section{Analysis of Interaction with Subtraction Applets}

Abby worked with the Subtraction Dot Applet (SDA) that showed subtraction as distance because she was able to easily integrate her notion of "take away" with distance.

\section{Abby's Interaction and Understanding with Technology}

Abby's initial idea of subtraction was "take away". Using this notion, she was able to procedurally solve tasks in the form $a-b$. To solve tasks that had a negative number in the minuend and/or subtrahend, she misapplied rules she had learned for solving addition tasks with negative numbers. Thus, she was able 
to obtain the correct answer to tasks of the form $a-b$ and $-a-b$, however, she would get the incorrect answer for $a--b$ and $-a--b$. Her reasoning was supported by using the number line as she would for solving addition tasks: focusing on the sign of the number and not attending to the operation.

I wanted to build upon Abby's understanding of tasks using $a-b$ to explore how the SDA worked. Before we began, I asked her to solve 5-2, to which she answered 7. When I asked her how she solved it, she told me "it's just a simple math problem". Even after verbally repeating it twice more, she twice answered 7. I knew she knew how to subtract whole number by evidence of other tasks, yet she was not focusing on this task. However, once I had the SDA opened and asked her to place a dot on the value for the minuend she immediately changed her answer even before I asked her to manipulate anything else (see Figure 1).

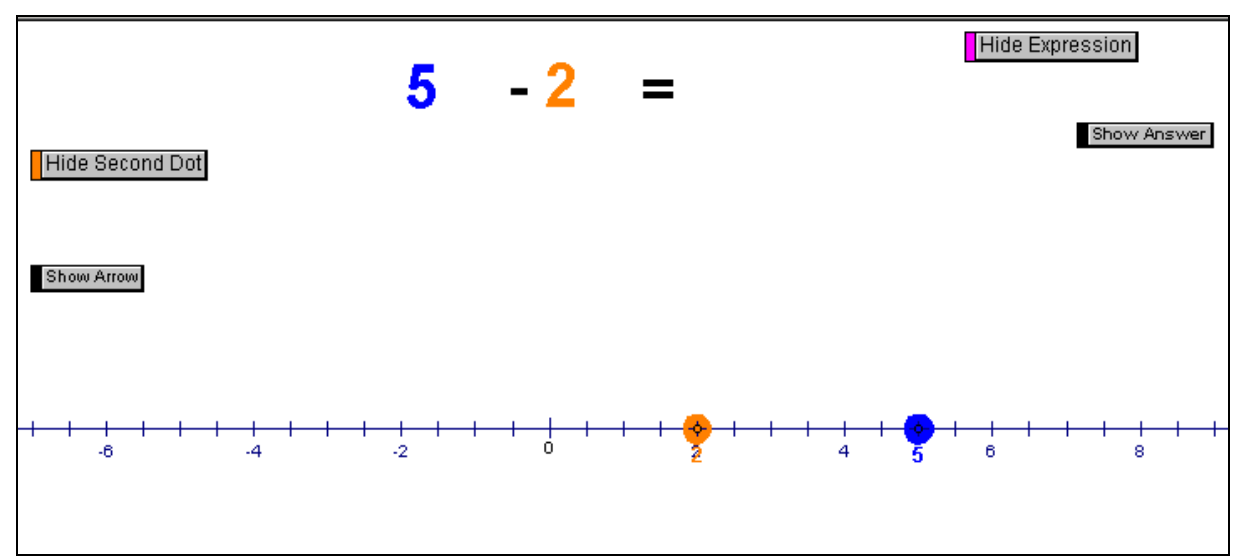

Figure 1. SDA modeling the task $5-2$

The SDA allowed her to focus on the numbers and the positions on the number line. She automatically realized that she had been adding the numbers instead of subtracting them.

Since she thought of subtraction as taking away the subtrahend, I wanted to see if she could interpret the idea of subtraction as a distance using the SDA. I had her manipulate the SDA to place a dot on the minuend and a dot on the subtrahend to determine the difference for a few tasks to which she already knew the answer and branch out to include tasks she had not been able to solve. In doing this, she solved tasks having the same minuend and different subtrahends. For example, we started with 5-2, 5-0, $5--1$ and so forth. During this time, she saw that even when you subtract a negative number, you could obtain a larger difference.

A: It's getting bigger.

$\mathrm{N}$ : Why is it getting bigger?

A: Because it's going farther and farther, farther, farther...because it's going farther and farther away

$\mathrm{N}$ : Farther away from what?

A: Five. And five is the main number...like in these two [tasks] it's stayed five. 
$\mathrm{N}$ : So what's it doing?

A: It's getting larger [by] one because I move it over one dot.

To get a new subtrahend to display in the corresponding algebraic expression on the SDA, she had to move the dot representing the subtrahend over by one. With each movement, she said "farther" to show that the arrow measuring the distance (and the difference in the equation) was getting larger as one moved away from the minuend. She called the minuend the "main number" because she had been using 5 to delineate the pattern of distance between the minuend and subtrahend. Furthermore, she noticed that as she decremented the subtrahend by one, the difference incremented by one because she moved it farther away from the minuend (in this instance). After working on a few more tasks, she could predict answers for tasks in the form $a--b$ before manipulating the SDA. Yet even with this new idea of subtraction as traveling away from the minuend, she refused to believe that $5--12=17$ was true. I asked her to model the task on the SDA and to explain why these numbers acted differently than the others.

$\mathrm{N}$ : Which way did you move it?

A: To the left?

$\mathrm{N}$ : Is that the way we should go?

A: Yes because you have to go to -12 to do the problem we are doing

$\mathrm{N}$ : So if we started at 5 and moved the whole way to -12 , how many have we gone?

A: 17 [pause] it says

N: Do you think it's 17 ?

A: [She counts] I agree

$\mathrm{N}$ : So do you think the answer is 17 or -7 ?

A: I'm not sure, but I'm pretty sure it's -7 .

Based on this response, I thought that she did not believe the answer showing on the SDA was correct because she counted the number of spaces in between the 5 and -12 . So I asked her if she agreed with the answer and the method of obtaining an answer to the other tasks. She agreed in the other instances, but in this case it should be done differently because "you're trying to get closer to 5." She explained that in the SDA she brought " 5 all the way to -12 " but now she had to bring the dot up to the -7 so "it would be 5 difference." She then proceeded to tell me how she solved this task using Unifix cubes, even thought she used an additive procedure instead of subtraction. This particular task caused difficulty, which seemed to be due to the fact that she had already determined that her answer was correct before working with the technology because she could justify her answer in two different ways. She calculated the same answer because she continued to use addition in both instances. At this point, because she firmly believed that $5--12=7$, yet understood how subtracting a negative number elicits a larger number because of the distance between the minuend and subtrahend. She struggled with how to integrate these two notions. She tried to combine these notions by accepting the fact that she manipulated the SDA by using it to determine 
the distance between 5 and -12 , but because she was adamant that the answer was -7 , she said that she needed to move the dot again from -12 up to -7 so that there would be distance of 5 showing on the SDA. It is interesting to note that she was able to see the relationship for why she obtained a larger difference as she moved the subtrahend farther into the negatives. It was only for this particular task that she believed that while the SDA showed 17, the answer was -7 because one should get closer to 5 rather than moving farther away from 5.

Due to her resistance on this task, we started to work on tasks that had a negative minuend and subtrahend (e.g. $-4--1)$. From previous tasks she was able to see that a negative number subtracted from a positive number became bigger as one decreased the negative number. She also began to notice that subtraction of a negative was similar to addition. In fact, she tried to connect this knowledge to her knowledge of solving 1-step equations. After noticing the pattern of numbers in a chart she said, "It's also like if you have an algebraic expression, like if you have two negatives, if you have a subtraction and a negative sign, you switch them to addition and then you subtract the bottom one." While she began to see the relevance, she did not totally connect these ideas. This may be due to the fact that this knowledge was not solidified because of her resistance in accepting $5--12=17$. I hoped to continue her recognitions of patterns through using the SDA and working on tasks that had a negative minuend and negative subtrahend. During this time, she used her new ideas of subtraction as a distance.

$\mathrm{N}$ : Ok, let's try this one $-4--1$ what would you do for this one on the computer?

A: I would put this [the blue dot] on -4 . [She then puts the orange dot on -1] I went the wrong direction, I was adding. It's -3 .

$\mathrm{N}$ : Why do you think it's -3 ?

A: Because you're subtracting more from 4 and you get 3 and they are negative numbers so it would be -3 . And I went the wrong direction... I was adding and not subtracting.

$\mathrm{N}$ : So you would change your answer?

A: Yes.

$\mathrm{N}$ : And what about this one, $-4--6$

A: I think I would make it -2 [her previous answer was -10 ]

N: Why?

A: They are both in the negative numbers, so you're subtracting 6 from 4 and the answer is -2 ...I still think that this one is -7 [referring to the 5- -12]

$\mathrm{N}$ : What if we did $-4--3$ ?

A: I would do... -1 .

$\mathrm{N}$ : Why do you think it's -1 ?

A: Because it's in the negative numbers and there's one difference.

By analyzing two out of the three tasks, Abby seems to understand subtraction as a distance between the minuend and subtrahend. Even though she acknowledged that she was "adding" before and not "subtracting," she did not mean these operations in the normal mathematical sense. Instead she used the words addition and subtraction in the sense that she took the absolute value of the minuend and subtrahend 
and added or subtracted them, and then tacked on the negative sign. Thus, she calculated -5 and -10 for the tasks $-4--1$ and $-4--6$, respectively. Since she knew to look at the distance between the minuend and subtrahend, she changed her answers so that the magnitude of the difference was correct, but the sign was not always correct. She attached a negative sign on the magnitude because both numbers were negative. While her notion of subtracting negative minuends and negative subtrahends evolved to include distance, she was not able to apply the correct sign to the difference. To tackle this obstacle concerning direction, I gave her the task- $4--4$, and asked her to predict the answer without using the SDA. She answered, "Zero, oh wait, -8. I think -8....Because if I were to add -4 and -4 I would get zero so since I'm subtracting, I'd get -8." She resorted back to using her old ways of solving these tasks: ignoring the subtraction sign, and viewing it as -4 and -4 . However, once she began modeling it on the computer she saw that there was no distance between the numbers in this task, and was able to understand how the sign connected to the direction of the movement.

A: It's no difference... See, I'm traveling [she is moving the dot that represents the [subtrahend] so if I had it right here [she positions it on the -2 while the minuend dot is still on the -4] I would say I was traveling 2.

$\mathrm{N}$ : So if we had -4 minus -4 , what do you think that would be?

A: I would travel zero because they are on top of each other.

$\mathrm{N}$ : So if we were to move [the subtrahend] to -5 , what do you think would happen?

A: One.

$\mathrm{N}$ : Why do you think it's 1 ?

A: Because you move this way [to the left]

$\mathrm{N}$ : Why is it positive?

A: I don't know. I guess because they are two negatives and two negatives equal a positive? [pause]...You're going that way [points to the left] and not that way [points to the right]. Since I was going to the left from -4 to -5 , then I would be 1 difference because we are going down...If you go this way [to the right] it would be negative. If I would go this way [points to the left] it would be a positive number.

Since the SDA allowed Abby to manipulate and take action on numbers that embodied the operation of subtraction and received instantaneous feedback, she was able to observe the patterns due to the movement. By manipulating the dots to show the subtraction of the same negative number from itself allowed her to realize the importance of direction in movement of the subtrahend in relation to the minuend. To make sure she fully understood this, I had her return to the task $-4--6$ to which she previously answered as -2 .

A: I got 2.

$\mathrm{N}$ : Ok. Before you thought it was -2 , what happened?

A: I thought it was -2 because they are both negatives, and I wasn't really looking right here to see it.

$\mathrm{N}$ : So if you were looking here, how would you show me, or show someone else?

A: Well, if I'm here and here [she points to -5 with one finger and then again with another finger] there's zero in between -5 and -5 . And so it [this problem] it would be one, two. Two spaces, so it would be positive 2 . 
$\mathrm{N}$ : Why would it be positive?

A: Because if you were going this way on the number line, it would be if you went from here to here it would be positive 3 [she points to here paper that shows 5-2] and from here to here it would be -2 [pointing at the 5 and then going up 2 to get to the 7].

The SDA allowed Abby to see the idea of subtraction as well as the dynamic link between the expression and action on the number line. When she was asked to show someone why it worked, she used her previous knowledge and experiences from working with the SDA. She first described why the magnitude was 2.

Then she described why the sign was positive by relating it to other tasks. She knew that 5-2 was positive 3 , and if she modeled that task on the SDA, she would move the subtrahend to the left from the minuend. She knew that when she moved the dots to model for this task, she went left to get from the minuend to the subtrahend. Thus, both tasks required her to move to the left and in the first task she obtained a positive number. She also knew how to solve 5-7 because she would use her knowledge of not having enough and thus getting a negative value, i.e. -2 . She showed that modeling this would require one to go to the right from the minuend to the subtrahend. Thus, moving to the left means the answer will be positive and moving to the right means that the answer will be negative. I called her attention back to the task $5--12$. She immediately changed her previous answer of -7 to 17 .

I think it's 17 ... because I'm going that way [pointing left] from five. If I subtracted 5 minus -12 then I think I'd get 17. But I use calculators a lot in math class so I don't depend on my brain. I depend on the calculator a lot, for very simple math questions. I'm sure it's going to be positive, because after working with all of these numbers, that um, because I came from here to there and there are two differences [referring to the previous example $-4--6$ ], so it's positive. And there's one, two, three, four [whispers five through 17 as she counts] and there are 17 of these little marks here.

Due to the fact of using the SDA with so many numbers, she was able to see a pattern in subtracting integers. She was unable to get the correct answers to my initial tasks because in math class she had relied too much on a calculator, and subsequently did not think about what she was doing or how she was operating. The technology allowed her to focus, manipulate, and observe her actions that required her to think about what it means to subtract negative numbers.

After she tried other examples with the SDA, I asked her to stop using it to see if she could generate some quick rules to use to solve these tasks algebraically now that she understood subtraction. Since she has previously thought that the sign of the minuend and subtrahend determined the sign in the answer, I asked her what would be the sign in the answer if you subtracted two numbers with different signs. She said, "Here, well, if ...I'm moving to the left with a negative number, then it's positive, but [if] I have to move right it's negative". She pointed such that she was using a positive number as the minuend and a negative number for the subtrahend, but since she was moving left, she knew that, despite the signs of 
the numbers, the answer would be positive. Next, she chose a negative number for the minuend and a positive number for the subtrahend, and since she moved right, the answer was negative. I then asked what would happen if the signs were both positive.

Then it's going to be positive, unless it's a really large number like if you had $8-17$ then I don't have enough, so it would be negative b/c I don't have enough to take away 17. Like if I had 17-18 I would have - 1.

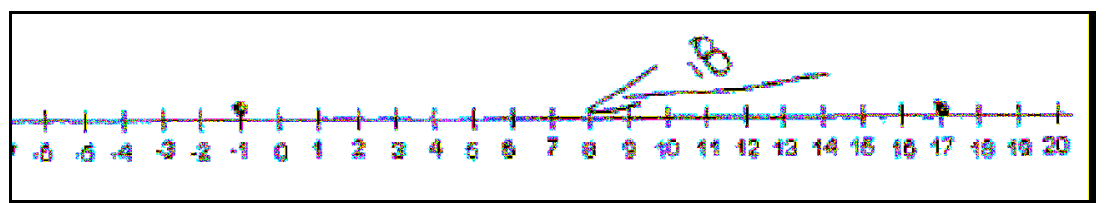

Figure 2. Solving $17-18=-1$ on a number line

She drew this last example on a number line (Figure 2). With the SDA, she would have placed the 18 as the second dot and the -1 would have been above the arrow that moved from 17 to 18 on the number line. I was confused as to why instead she used 18 as the arrow value and the difference of -1 . She told me, "to show that you get a negative number when you subtract a positive number from a greater positive number." This did in fact answer my question, but I wanted to know why she switched the subtrahend and difference in the model. I had asked her if this is how she had been using the SDA to model the equations, to which she responded no and she picked up the mouse and modeled on the computer as she had done before with the dots on the minuend and subtrahend.

A: Then it would be...-1. That's exactly what I said. I just showed it a different way [stated matter-of-factly]. So 17 subtracting 18 is -1 .

$\mathrm{N}$ : So which way, if you're getting the same answer, do you like better?

A: Well, I can do either way, and I like them both!

$\mathrm{N}$ : Why do you like this way? (distance between the minuend and subtrahend)

A: Because if I do it this way then I can see what I'm subtracting from the number. Like there is one whole difference here (pointing to the middle between 17 and 18), and I'm subtracting...I would need 18 to be able to subtract 18 and I don't have enough from 17 to subtract 18 .

Abby explained why the value was -1 when using the distance modeling method. First she stated why the magnitude was 1 , because there was a difference between 17 and 18 of 1 . Then she stated why it was negative by relating it back to the previous task where she had to subtract the same number from itself. She realized that in order to obtain zero, she would need 18 to subtract from 18 . However, she only had 17 with which to begin; thus, her arrow would point right showing a negative value because she did not have enough from 17 to subtract 18. I then asked her about the other way she solved the task, and she said that by using the take away method, the orange dot "is the answer". She knew to put the dot there because she knew the answer ahead of time.

$\mathrm{N}$ : So what if you don't know your answer right away? You had said that you knew that 17-18 
was -1 so you knew to move the orange dot to -1 , but what if you don't know your answer first? How can you figure it out?

A: Cause if I did [not know the answer], I'm just coming up with a random problem. If I were to do this one (13-20, she puts a dot on 13 and 20 and then counts to the right) one, two three,... seven. Negative seven. Or I could do here (puts a dot on the 13 and a dot on the -7 ).

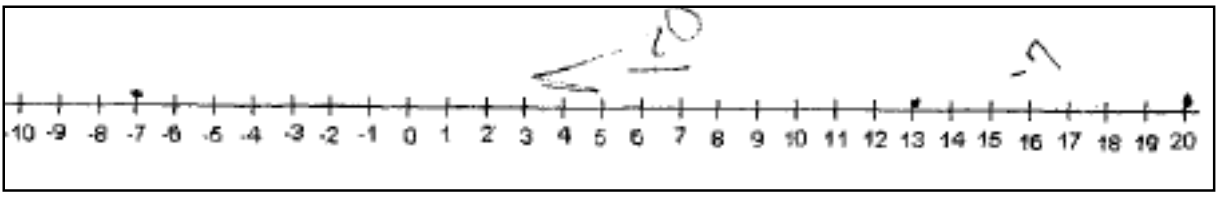

Figure 3. Solving $13-20=-7$ on a number line

She believed that if she did not know the answer, then she could figure it out by putting a dot on the minuend and subtrahend and counting the distance between them. After finding the answer, she then could then create the equivalent task. To show me this, she created the task $13-20$. She used a number line, not the SDA, and drew a dot on the 13 and one on the 20. She then counted to the right 7 spaces and labeled the distance with a -7. After this, she drew a dot on the 13 and then on the -7 and drew and arrow and labeled it with a 20 (see Figure 3). It is important to note that she was not using the SDA, but rather recreating it by using a bare number line. After showing me this on her number line, she then grabbed the mouse and showed me using the SDA.

A: Cause If I did this one, then I subtracted 20, and it's [the answer] negative 7, or I could put it [the orange dot] onto -7 here and that's 20 [she uses the mouse to point to the arrow value]. So sort of it's the same thing, just different.

$\mathrm{N}$ : What's the same?

A: We're subtracting 20 from 13, because this stays the same (she points to the arrow value) that's on my answer right now, but if I were to move it up here (she moves the orange dot to 20) then I would get what my answer was (as the arrow).

Through the manipulation of the SDA, Abby was able to see the relationship between subtraction tasks in the form $a-b=c$ and $a-c=b$ for integers. By simply moving the orange dot on the SDA, Abby was able to swap the subtrahend and difference and observe the equivalent forms. She said, "You can just switch them, but you can't switch your blue number, your blue number has to stay the same." At the end of our first session, she told me how she thought this movement and the ability to choose the position of the orange dot was valuable in determining the answer in a subtraction task.

Well it was helpful on those where like you had a choice on like which way to go... it's harder to decide which way. Sometimes you could move it [the orange dot] to get the answer and sometimes how to get the answer by putting it on both numbers. So sometimes it depends on where you put it [the orange dot].

In our next session, I wanted to check whether she retained the ideas she had discovered in a previous session. I asked her to solve 5 - -12 . 
A: Wouldn't you count the spaces? 17

$\mathrm{N}$ : Is that your answer?

A: I'm pretty sure. But there is something that says you should just add 5 even though you're subtracting, so I guess it would be 17 the more and more that I see it cause it makes sense, because it's the difference.

$\mathrm{N}$ : The difference. Can you say more about that?

A: Well there are 17 differences, cause that's pretty much it.

$\mathrm{N}$ : So what do you mean by 17 differences?

A: Well, there is -12 and then to 5 and there are 17 in the difference.

Since she still thought that there was a rule she had learned that said this task should be -7 , I ask her if there was a difference between addition and subtraction of these numbers. Remember that before she had believed that $5--12$ and $5+-12$, while one task used subtraction and one used addition, elicited the same answer, -7 . She now responded, "Well, if this was a plus sign, then it would give you -7... so I think it will be 17." She hypothesized that if the subtraction sign was a plus sign and the task was $5+-12$ the answer would be -7 , but since it was a subtraction sign, the answer to $5--12$ was different. She understood that there was a difference between subtraction and addition, and that they required different procedures to solve.

Based on Abby's knowledge that she could solve a subtraction task two different ways, distance or take away, and that she now knew the difference between addition and subtraction using the same numbers, she was able to make a new discovery. When solving the task $8--9$, she stated, "Well, I know for adding it would be -1 ." Thus, she compared $8--9$ to $8+-9$. She then saw the relationship between the tasks $8+-9$ to $8-9$ and said, "If you were to subtract 8 and 9 you would get $-1 \ldots$ because if you are adding, you would go [right]." She tapped on the computer screen beginning at -9 and went to the right 8 spaces and landed on -1 . In doing this, she noticed that if you were to use the take away notion to solve $8-9$, you would also land on -1 , however you would be going left. She exclaimed, "So if you're subtracting, you go to the left. I just realized that.” While this is an important process to understand, especially since it showed that she was not operating on a divided number line but rather a continuous number line (Peled, 1991), I wanted to see if she knew that it happened only in certain instances. After doing other tasks with the SDA, she was able to see that a negative number resulted when one moved to the right from the minuend "because you're subtracting, because the blue is your first number, the orange is your second number, and this orange one is greater than your blue one!" By using her previous knowledge that $8-9$ equaled -1 , and by seeing the movement on the SDA that she moved right for tasks that had a greater number for the subtrahend, she was able to connect the fact that moving right gave her a negative value because she was subtracting a larger number from a smaller number. When I asked her when she would receive a positive answer she said, "So to not have a negative the blue number has to be larger than 
the orange number, to get a positive answer." Since the SDA allowed Abby to move blue and orange dots to represent the minuend and subtrahend, respectively, as well as see a link between the expression and this movement, she was able to see that the signs of the minuend and subtrahend do not strictly determine the sign of the difference, rather the sign of the difference is dependent upon whether the minuend is larger or smaller than the subtrahend.

I told Abby that I wanted her to be able to solve tasks without the computer; however, she could use a number line if she wanted in order to solve the tasks. I asked her to solve $4--2$. She looked at a number line on a piece of paper in front of her and said, "Four here -2 here, one, two, three, four, five, six. Positive six." When I asked to explain how she thought about it, she said, "Because there are six in the middle and...it's positive because it's going that way on the number line...[4] the blue dot, to -2 , the orange dot." As she was saying this to me, she did not draw or even touch the number line in front of her. This was all purely in her head in which she was visualizing the blue dot on 4 and the orange dot on -2 and moving to the right and counting 6 in between.

In determining the answer to this previous task, she noticed that in tasks that had a positive minuend subtracting a negative subtrahend, that she could obtain the same answer as if she was adding the minuend and the absolute value of the subtrahend. For $4--2$ she said, "You could do 4 plus 2." But when I asked if this type of reasoning would work for tasks that had a negative number first, she said, "I don't think always, because if you made all of these positives you would get 10". She was referring to the task $-7--3=-4$. I asked her to classify the ones she thought would work as addition and she said, "You only have one negative [sign] and you switch them to positive." She pointed to the tasks that she did that showed a subtracting a negative number from a positive number. As she said the word switch, she pointed to the subtraction sign and negative sign as to say that they were to become positive signs. When I asked her which tasks would not work as addition she told me, "I don't think you can have three negative signs, or a subtraction sign and two negative signs and add." While she could compute tasks that had a negative minus a negative, she did not view this as following her addition rule because according to her, she must add all positive values. Thus to follow this rule, $-7--3=-4$ would have to turn into $7+3=10$. Another obstacle that could have made this more difficult to see was the fact that she had used the word subtraction to describe what happened. By subtraction, she meant that there were 4 units difference between -7 and -4 and this is similar to the subtracting 3 from 7 . While she put these types of tasks in a chart before we finished the session, she was unable to articulate a rule for solving them.

Two weeks after this last session, I met with her again to determine what she remembered. I had 
asked her to solve subtraction tasks in all forms. First, I had her solve $8--3$ and asked her how she solved it.

A: I changed them [signs] to both positive.

$\mathrm{N}$ : How did you know to do that?

A: Because that's what we learned before.

When I asked her if she could show me on a number line, she looked a number line, placed her right pinky finger on 8 and the left pinky finger on -3 and said, "Well, that would be there and that would be here, and it would be... 1,2,3,..11...because that's what we did before and it worked." To clarify that she meant that she learned it while working with me and not in her math class, I asked, "You mean you and me?" and she responded, "Yes, we don't use a number line in math class."

She was also able to solve all tasks algebraically. What is interesting is how she solved $-7--4$ and obtained the answer of -3 . Once again, she separated the subtraction sign from the negative sign by placing parentheses around the -4 . She made the subtraction sign into a plus sign and the negative sign into a positive sign, then looked at a number line and counted to four. I asked her to explain what she was doing, and her response was interesting.

A: We use calculators in math class.

$\mathrm{N}$ : But you did this without a calculator.

A: I know, but some things we use it for.

$\mathrm{N}$ : But you knew this answer, so you didn't need a calculator

A: Yes, but I use the calculator all the time though. I don't know why.

$\mathrm{N}$ : So how did you do that?

A: I don't know

N: Do you think the stuff we were working on before with subtraction helped?

A: I guess.

$\mathrm{N}$ : What do you think helped?

A: I guess getting it into my head.

$\mathrm{N}$ : So do you remember the files on the computer? Like this file [I show her the SDA]

A: Yes, I remember that... I've been thinking about that... I just kind of missed it... it's just something you get used to, and then it's just gone and it sticks in your head. It's just like there... when I wake up sometimes I just think about it.

Remember, that in our prior session, Abby had not been able to articulate a hard and fast rule about switching the signs. Due to using calculators in math class, I think she was able to see a pattern emerging from doing so many tasks similar to this one. Not only did she turn the negative signs into positive signs, she also used the number line to count the distance between the minuend and subtrahend. This showed that she was using the strategy she learned from the SDA. Because of this, I asked her if she thought the SDA helped her solve these tasks. Her response showed that by actually being able to "do" subtraction through the manipulation of the SDA and witnessing the subsequent outcomes, the image became ingrained in her mind that she thought about it outside of our time working together. 


\section{Summary of Abby's Interaction and Understanding with Technology}

- Abby had a unary perspective of the negative sign after working with the SDA and SAA. She was able to see that subtraction of a negative is the same thing as adding a positive. However, at first she was not able to articulate this idea, but could employ it to solve tasks. For instance, after working with the SDA, she was able to solve all subtraction task forms, but she believed tasks in the forms: $-a-b$ and $a--b$ were similar to addition and tasks in the forms $a-b$ and $-a--b$ were similar to subtraction. She believed this because the magnitude of the difference is "bigger" for the first form and "smaller" for the second form.

- Abby integrated two notions of subtraction: take away and distance. In fact, she easily showed how these two notions are equivalent on the SDA by illustrating the equivalence of $a-b=c$ and $a-c=b$. Moreover, she does this without being prompted or asked any questions relevant to finding this pattern.

- After using the technology, Abby was able to tell the difference between addition and subtraction as operations and conclude that an addition and subtraction task consisting of the same numbers will not elicit the same answer.

- Abby was able to solve all types of addition and subtraction tasks and justify her reasoning for her answers using a number line. Yet, she used a number line more often to solve tasks that she was not able to solve prior to technology.

- While working with the SAA and SDA, Abby frequently transformed tasks in the forms $a--b$ and $-a--b$ into $a+b$ and $-a+b$, respectively. As time passed, these instances decreased and eventually became extinct.

- The SDA allowed Abby to view both the algebraic and graphical notion, which helped her focus her attention to operation in the task.

- Because Abby was able to correctly solve all subtraction tasks after working with the SDA, she would not over-generalize commutivity. She now is aware of the operation and the relationship of how it acts on the numbers.

- Abby continued to use the words: big, smaller, larger, and smaller to denote the absolute value of numbers instead of the actual value of the numbers. However, when she used the number line, she knew that when one moves right, the numbers become larger and when one moves left, the numbers are smaller.

- After working with the technology, Abby attained Level 4 in both QD and NLD with respect to subtraction.

\section{Discussion}

Prior to working with the SDA, Abby believed that addition and subtraction tasks containing negative numbers were solved the same. She would use the signs of the numbers to determine which way to move on a number line to answer the task. She would also use rules to explain how she had gotten her answers.

By working with the SDA, Abby was able to see the necessity of applying different procedures on a number line dependent on the operation in the task. She could correctly solve both addition and 
subtraction tasks. By using the SDA, Abby manipulated the minuend, subtrahend, and difference and was able to enact the operation of subtraction. This manipulation helped her to focus on both the operation and the signs of the numbers. It also helped her understand subtraction as both taking away the subtrahend from the minuend and measuring a distance between the minuend and subtrahend.

By moving the subtrahend and minuend, Abby tried many different tasks in a small amount of time because the SDA provided flexibility and was controlled by the user. It also included a dynamic link between the number line and numeric equation. Using this link, Abby observed patterns and connections between task forms. She used these patterns to come up with hypotheses about signs and relationships between numbers that she then tested using the SDA. By testing and producing generalizations, Abby's notion of subtraction evolved. Abby saw the importance of magnitude and direction and the equivalence of the forms $a-b=c$ and $a-c=b$. Because Abby could manipulate and observe the outcomes, she could do and see subtraction, which became fixed in her mind.

At the end of the interview, I asked her what she thought of the SDA. She said, "It was fun... [because] you could move it around." She enjoyed the manipulation aspect of the SDA. She also told me that the SDA helped her because "It made my brain a little smarter... I could do it without a calculator." She also informed me that she liked the SDA because "Now I can subtract all the other ones. When the numbers are integers...not just positives, I knew those, those were obvious, like 9-8." Prior to using the SDA, she was only able to subtract positive numbers, however, the SDA showed her how to easily solve all tasks containing positive and negative numbers.

\section{The Equal Sign}

\section{Prior Conceptions}

Prior to using technology, Abby viewed the equal sign as both operational and relational depending on the task. For tasks that included an equation, she had an operational viewpoint because she believed that the equal sign was an announcement of a result. When I asked her to compare $5+6,5+6=$, and $5+6=11$, she told me that they were all equal to 11 , but "this is just 5 plus 6 , here they have an equal sign, and here they have the answer." Similarly, when asked what the equal sign meant in $8=10-2$, she

told me "your answer, well, next coming is your answer." When I asked her what side her answer was on in this task, she said "This side [left], but usually it's on that side [right], but it's on that side this time." When I asked her if it was ok to write your answer on your left, she said, "Yes....I don't know why. It's ok because it's in my algebra book." Since she does not view the equal sign as showing equivalence, she 
defaults to believing it is ok to write because she has seen it in her math book. Yet, when she was asked about another non-canonical form, $3=3$, she said, "It just means 3 is 3 , pretty much. Yeah, that's right." She seemed to be unsure of this answer.

Her view changes to a relational perspective when I asked her what the equal sign meant in 1 foot $=12$ inches and 1 dime $=10$ pennies. She told me that the equal sign in 1 foot $=12$ inches it meant "the same as" and in 1 dime $=10$ pennies it meant "they both have the same value." What is interesting is that after doing these tasks, she pointed back to $5+6=11,3=3$, and $8=10-2$, and said, "and that's the same as, and that's the same as, and that's the same as." It seems as if this relational view was only applied to the previous tasks in retrospect because she believed that the answers to "what does the equal sign mean here" should be identical. Since she felt more comfortable answering the last tasks that dealt with units than the two tasks containing non-canonical forms, she used her response of "the same as" and applied it to the first tasks. This idea seems plausible especially since she debates whether the value for the question mark should be 8 or 6 in the equation $3+5=?+2$.

A: Eight plus 2. Question mark?

$\mathrm{N}$ : So how did you get 8 ?

A: Because 3 plus 5 is 8 , but it has a question mark.

$\mathrm{N}$ : So are you saying that the value of the question mark is 8 ?

A: Yes, [pause] or 6.

$\mathrm{N}$ : How do you know which one?

A: Um, because that equals 8 [referring to the $3+5$ ] and 6 plus 2 equals 8 too. So they're the same thing.

$\mathrm{N}$ : So do you want to make the question mark, 8 or 6 ?

A: I'm not sure. Cause they are both the same thing, but I guess it would have to be 6 because they're the same. I'm going to say 6 .

$\mathrm{N}$ : So what's the same?

A: Six plus 2 is 8 and 3 plus 5 is 8 .

It is important to note that she had seen 1-step equations at this point, so she knew what a variable was in an equation. Yet, she struggled because the question mark fell immediately to the right after the equal sign. She was unsure if she should place the answer to the expression $3+5$ there or if she should take into account the 2 that must be added to the value of the question mark. Her explanation shows that she was working toward a relational view of the equal sign as she determined the value to be either 8 or 6 . The fact that she used "they're the same" may imply that the preceding tasks directly impacted her final decision to make the value 6 so that both sides of the equal sign have the same value.

While her viewpoint of the equal sign in equations containing all numbers seemed to be relational, her view of the equal sign in equation with a letter as a variable was quite different. When I asked her to tell me what the equal sign meant in $x+5=3$ she responded, "It just means that this [x] is your answer." 
Because she had previously told me that the equal sign announced the answer, which was to the immediate right of the equation, I asked her what the 3 was if $x$ was her "answer". She responded to this by solving the equation using a number line. She started at 3 and went to the left 5 spaces and landed on -2 and said she, "went backwards because you had to add it to $x$ " in order to get 3 . Thus, the equal sign in an equation with a variable meant that the variable was your answer and you had to find the value of it. To see if she changed her view of equations containing all numbers, I asked her what the equal sign meant for $-2+5=3$.

It means what your answer is, but coming up or something, not like what $x$ is. But I never really learned what it means, I've just known that it means that you put it after an equation and then put your answer.

She returned to her original operational view that the equal sign signified that the answer was coming up. Since before she had use the terms "same value" and "same as" in talking about the equal sign, I specifically asked if $-2+5$ was the "same as 3 ", with which she agreed. However, when I asked if $x+5$ had the same value as 3, to which she responded, "No." Thus, when the equal sign was used in an equation containing a variable, she did not believe that it showed equivalence between the two sides, nor did it tell you that one side is your answer, but rather it implied that " $x$ is your answer."

In solving the above task for $x$, Abby used an arithmetic strategy (Boulton-Lewis et al, 2000) because she applied an inverse numerical procedure using the number line. She knew that because one had to add 5 to $x$ to get 3 , she had to go in reverse, or subtract 5 from 3 to find $x$. Yet, she did not always do this when solving equations. During the interview she had created her own algebraic tasks to show me what she had been doing in her math class. She came up with the following tasks: $8=-17+x, 8=-17-x$, and $b-5=-12$. She solved all of these tasks algebraically (Boulton-Lewis et al, 2000) by applying the same transformation on each side. In fact, she told me that she had to do the same inverse operation to both sides because "you have to get $x$ by itself." However, she incorrectly solved the tasks that had both a negative number and subtraction sign.

A: If you have like $8=-17-x$, then you switch that to positive number[s] (the 17 and the $x$ ) then you subtract the 17 from this [8] and subtract the 17 from this [+17] and that would be the $x$ number.

$\mathrm{N}$ : So did you switch the number here [the 8]?

A: No, because you weren't trying to get that one by itself [i.e. there is no $x$ ] but I did get that one by itself and I did get that one by itself [the $\mathrm{x}$ and she crossed out the $17 \mathrm{~s}$ ] b/c I made it positive 17 and then subtracted.

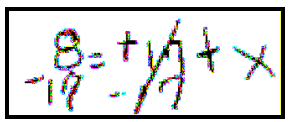

Figure 4. Switching signs to solve $8=-17-\mathrm{x}$ 
She switched the negative sign and the subtraction sign to be both positive (see Figure 4). Then she proceeded to subtract 17 from both sides to solve for $x$. When asked why she switched only the negative and subtraction sign in the task and not the sign of 8 , she said, "You weren't trying to get that one by itself but I did get that one by itself ... because I made it positive 17 and then subtracted." She meant that since 8 was on the opposite side of the equation from the side $x$ was on, she did not need to change the sign. Similar to this task, she switched the subtraction sign and the negative sign in the task $b-5=-12$ so she could then subtract 5 from both sides to get $b$ by itself. She did not justify why she changed the signs here, but rather stated that they needed to be changed. It seems that in order to solve an equation, she had to subtract the constant value on the same side as $x$. In order to do that in these tasks, she had to switch the sign of the constant value on the same side as the variable, and if she had to do this, then she needed to switch all the negative signs to positive signs in the task. By changing only some and not all of the signs of the numbers in the task, she further illustrated that she did not view the equal sign as denoting the equivalence of two expressions.

When presented with a 1-step equation that only used multiplication, she thought she should subtract to solve it. Then she reasoned that she had to perform the inverse operation, so she divided and said, "So if I divide [9 by] 3, I get 3. So 3 times 3 is 9. So $a$ equals 3." Her voiced justification and written work (see Figure 5), show that she used the unwinding method (Knuth et al, 2006). She did not apply the transformation of dividing by 3 on both sides, but only applied it on one side; thus, using an inverse operation to unwind the equation.

$$
\begin{array}{r}
b * a=9 \\
\frac{-3}{3}
\end{array}
$$

Figure 5. Using unwinding method to solve $3 \cdot a=9$

After seeing how she solved 1-step equations, I gave her the equation $2 m+7=15$ to solve. She immediately recognized that it was a 2-step equation and told me that her class had done "this not very long ago because Mrs. G said that we were ready for them.... and I know to get $m$ by itself you have to divide that [m] by 2 and you get the zero." She said that the first thing you had to do was divide $2 m$ by 2 , but when she divided $2 m$, she was unsure what to do next.

A: And then wouldn't you have to divide that one [15] by 2 ?

$\mathrm{N}$ : Why do you think that?

A: Because you do things under the equation, you do a lot of things under the equation But, you would get a decimal, pause for either one. 
At first she thought you might divide the 15 by 2, but then saw that if you did that you would get a decimal. Then she considered dividing 7 by 2 , but noticed that in doing that one would also receive a decimal value. Obtaining a decimal answer to this task made Abby uneasy and question whether she did something incorrectly. She wholeheartedly believed that she needed to divide first because her teacher told her that she needed to solve equations by using PEMDAS. She said, "Cause if you do it by PEMDAS, you're supposed to do multiplication and dividing first." Yet even after voicing this rule that she was supposed to follow, she still did not know how to solve the task.

\section{Summary of Abby's Prior Conceptions}

- Abby exhibited both an operational and relational view of the equals sign for equations that did not include a variable, yet they were not fully integrated.

- Abby was familiar with non-canonical forms, but she believed they were written appropriately because she had seen the forms in her algebra book; not because they made relational sense.

- Since Abby did not have a fully integrated operational and relational view of the equal sign, the ordering of the questions may have influenced Abby's answer regarding the task $3+5=?+2$.

- Abby's view of the equal sign was operational when she encountered a 1-step equation. She believed that the equal sign meant the answer for $x$, and not that the expressions are equivalent.

- Abby solved 1-step equations using arithmetic, unwinding (pre-algebraic), and algebraic strategies.

- In 1-step equations, Abby wanted to subtract the constant on the same side of the variable to solve the equation. Therefore, if the equation was written so that she had to add the constant, she changed all the negative signs and subtraction sign in the task.

- Abby could not solve 2-step equations, for she wanted to multiply or divide prior to adding or subtracting because she believed that she must follow PEMDAS in solving equations.

\section{Analysis of Interaction with the Equality Applet}

\section{Abby's Interaction and Understanding with Technology}

Because Abby already knew how to obtain a correct answer for 1-step equations, she had no need to use the Equal Balance Applet (EBA) as a tool to help her solve for the answer. While she understood how the EBA worked, how adding and removing weights and balloons corresponded to addition and subtraction, and why she had to perform the same operation to both sides to keep the pans balanced and thus the expressions equal, she did not use the EBA as it was intended for solving these tasks. Instead, she used it to check her answers to tasks.

For each 1-step equation, she solved the task and then cleared the equation from the pans and placed an $x$ on one pan and the answer on the other. She used this to determine that her answer was correct 
and said to me, "So it's right...because they are the same." Because she had learned the characteristics of the EBA, she knew that if the pans were balanced for the original equation, then any other equation that made the pans balanced was equivalent to the original.

While Abby did not always manipulate the EBA to solve the equation, she sometimes used it as a visual in solving 1-step equation tasks. Prior to using the EBA, Abby solved tasks that had subtraction and a negative number by switching all the signs. However, she did not do this when she had the visual model of the task in front of her. For the equation $x-6=-15$, the computer showed an $x,-5$ balloon, and a -1 balloon on the left pan and three -5 balloons on the right pan. She said, "You add 6, and then you add 6, so that would be 9 ...negative." She then told me that you add 6 because by adding 6 it gave you zero on the left pan. Thus, by using the visual of the task, Abby knew that she had to add 6 to both sides to keep the pans balanced, even though previously on paper, she had would change all the signs so that she could subtract.

In addition to being able to correctly solve 1-step equations with subtraction and negative numbers by referring to the EBA, she also solved the tasks algebraically instead of using arithmetic or unwinding method. For instance, she solved the equation $x+8=21$ by counting backwards on her fingers from 15 by 8. Yet, when I motioned to her to look at the computer screen showing the visual model of the task in order to explain to me how she solved it she said, "if you want to figure out what $x$ is, then you subtract 8 there, and then you subtract 8 over here, and then you get 13." While she said this, she first pointed to the left pan, and then pointed to the right pan to show from where one needed to subtract 8 . When I asked her how she knew to subtract, she said, "Well that's your only choice because that's the only ones you have over there" meaning that the weights are the only things remaining on the left pan with the $x$ weight. She used the visual characteristic of the EBA to show why she needed to subtract the constant on the same pan with the $x$ in order to solve the equation.

Since she was unsure how to solve 2-step equations, she used the EBA to try different strategies to help her solve 2-step equations, unlike solving 1-step equations. Since she struggled with the task $2 m+7=15$, I created this task using the EBA. When she found out that I had it on the computer she exclaimed, "Oh cool! I didn't know you had this one on here." She told me later in the interview that she liked this EBA because it helped her solve these equations and was "better than paper." I assumed that her excitement was due to the fact that she knew the computer would help her discover how to solve these tasks because she was unsure of what to do.

Due to the flexibility and ease of trying different strategies, Abby did not use the EBA as it was 
intended to solve this task (i.e. by removing 7 weights from both side and then dividing by 2). Instead, she tried to make an equivalent 1-step equation to help her solve this 2-step equation. The EBA showed two $m$ weights, one 5 weight, and two 1 weights on the left pan, and three 5-weights on the right pan (see Figure $6)$.

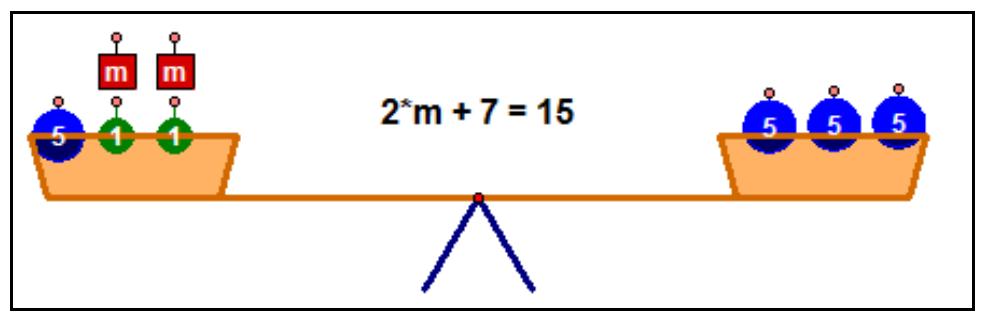

Figure 6. EBA showing $2 \mathrm{~m}+7=15$

She removed all but one $m$ weight on the left pan and one 5-weight on the right pan. She noticed that the right pan was heavier, so she added a 1-weight to the left pan and they became balanced. During this process she said, "I don't want that one $[m]$ on there...I just want one $m$ on there at a time...so there. Ok, so there, that keeps it even. It's $5 . . . m$ is 5." The pans were balanced and the equivalent equation she made was $m+1=5$. I asked her why she thought answer was five, but she did not give a straightforward answer. One reason could be that she did not see the 1 weight on the left pan and therefore assumed the pans were balanced with an $m$ on the left pan and a 5 on the right pan. Another explanation is that she knew that this new 1-step equation could help her solve the 2-step equation since they were equivalent. Yet while she intuitively understood that the solution to the equivalent 1 -step equation satisfied the 2 -step equation, she was unsure how to integrate the two forms. This may explain her response to why she thought five was the answer.

Well, minus 5 here is 2 and minus 5 here is 10 . So, [pause] cause if you do it by PEMDAS, you're supposed to do multiplication and dividing first, so divide, hmm, but who knows a decimal might become...cause you can't do decimals on there so it must not be a decimal.

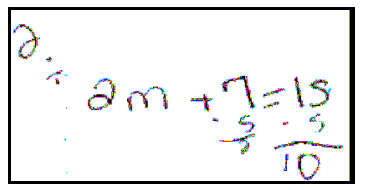

Figure 7. Using PEMDAS to solve $2 \mathrm{~m}+7=15$

She returns to original equation and subtracts 5 from 7 on the left side of the equation and then subtracts 5 from 15 from the right side (Figure 7). She seems to have subtracted 5 because the 1-step equation is set equal to 5. She subtracted first because she remembered removing weights first in the other tasks, which corresponded to subtraction. She also subtracted from both sides because she realized the pans had to stay balanced, and removal of the same amount from both sides accomplishes this. Yet, after she finished this 
subtraction of 5, she was unsure of what to do by the pause in her explanation. She then tried to integrate her knowledge of PEMDAS to what she had already performed. However, when she attempted this, she was left with her previous confusion of what to divide by 2 . This time, because she looked on the computer and saw there were no decimal weights, she believed that the answer must be a whole number but she is just puzzled as how to get there.

I asked her to remember how we solved the 1-step equations using the EBA. She subsequently removed a 5-weight from both sides and continued to remove 2 more weights from both sides and said, "we would subtract 2 from both sides. Oh, and there's another thing I can do, can I take a [pause], I have to figure out how what $m$ is though." As she pauses, she pointed to one $m$ on the left pan with the mouse and looked like she was about to remove it and place it in the storage area, but then changed her mind. I asked her how many she took off total and she said, "7, we subtracted 7." Then she realized that there were $2 \mathrm{~m}$ weights on the left pan and 8 on the right pan and says, "8, so each one equals 4 ." When I asked her how she knew to divide the 8 weights into 2 sets, she said, "because there were two of them [ $m$ weights]" and "well, half of 8 would be 4." When I acknowledged that before she was not sure how to solve this task, but that she did now, she responded, "yes, logicness." The balance allowed her to try many strategies and incorporate her past knowledge in solving 1-step equations. She intuitively knew to subtract first because she needed to get the $m$ weights alone on one pan, and to do this, she had to remove the valued weights in that same pan. Afterwards, she intuitively knew to divide by 2 because there were $2 \mathrm{~m}$ 's remaining on the left pan and they balanced the remaining value on the right pan. She deemed these intuitions on how to solve the equation "logicness."

While she knew how to use the balance to solve a 2-step equation, she ran into some difficulty in solving $2 m+9=3$ with it because there was a great amount to subtract from the left pan than from the right pan. She was not used to adding negative balloons to the left pan in order to get a negative number on the right pan, which needed to be done in this case. She subtracted 2 weights from both sides, but became uneasy when she needed to remove one more because the right pan would become zero. She said, "I can't do no more... because I only have 1 over there and ... it would make it uneven because one is empty and other one isn't." She became stymied based on the visual of the balance. She knew that as long as she took away the same amount from both pans, the pans should remain balanced because they were equal in value. Yet, when she saw a pan that was about to become empty, she began to think of the balance having one pan containing items and another pan not containing items. It seemed that the pan containing items would naturally weight more than the pan without items. While this is a potential characteristic shortcoming of the 
model, she was able to overcome this notion by actually removing the weight from both side and "seeing" that it remained balanced. After she removed the weights she said, "Oh, it didn't make sense to me," but when I asked if it made sense to her now she responded, "Yes, because you are taking off one from each side." At this point, she had the equation $2 m+6=0$, and she could not remove any more weights, but rather needed to put negative balloons onto the pans. She decided to manipulate the objects to create another 1-step equation to help her solve this task. She began by telling me that she was "just trying something" to let me know that she will be trying different ideas than what she had already learned. She constructed the equation $m+6=m+6$ and realized, "so they are balanced [but] I' $m$ going to put this one [back] over here." She realized that this new equation allowed the pans to be balanced, but it did not help her solve the original; therefore, she placed the $m$ weight back onto the left pan. She then used the EBA's feature that showed her which expression is greater or "heavier" than the other to help her determine where she needed to add or subtract weights to make the expressions equal. She stated, "I'm going to add one [more] until it becomes even." She continued to add to the pan until she created $2 m+12=6$. She then looked at the original equation and guessed that $m$ was 6 , but then immediately changed her mind and said "Let me try something really fast...I'm going to try something different...don't ask me why I'm trying this, but it just might work." She tried to construct an equation so that the expression on the left was $2 m+0$ and the value on the right was a positive number. In doing so, she noticed that she could only create an inequality in which the left value was always less than the right value. Eventually she gave up and created the equation $m=m$ and was excited that they equal, but after a few seconds, she knew that although they were equal, they naturally should be and it did not help her solve the task. I then asked why she had not considered negative numbers, and asked if she could use them just as she did the positive numbers. She responded, "I don't know... at least I'm not thinking so." She placed a -1 balloon on both sides and exclaims, "It's equal now!" She then said, "Wait, how did I figure out the last one? Oh wait a second. Can I see something?" She reset the task and referred back to the other tasks. She noticed that in the last task she subtracted 7 because "that's what we had on that side" and generalized that she needed to "subtract 9" for this task. Upon doing that, she got -6 on the right pan and $2 m$ on the left pan; hence, $m$ was equal to -3 . She then said, "I want to try something" and placed an $m$ on the left pan and stated, "The $m$ is not very heavy is it?... I love to mess with things." She then positioned a -5 weight on to the pan and noticed that the right pan was less than the left pan and stated, “Wait, I didn't mean to do that, it doesn't make sense." She then removed the -5 and put $3-1$ balloons and announced, "They're equal." Thus, she once again used the EBA to check her answer. 
Since she was able to solve the 2-step equations on the computer and wrote her steps out algebraically on paper, I wanted to check how she would do it without the visual and manipulation provided by the computer. I asked her to solve $2 x-16=-6$. She immediately told me that she needed to "divide by 2." When I asked if that was how she solved the other equations she referred back to her old ways of trying to solve the 2 step equations.

No, but that's what you're supposed to do on PAPER. That's what you're supposed to do! You take your multiplying and division first....that's how Mrs. G told me to get it...because if you if you do it by this [PEMDAS] you HAVE to do multiplication and division first!

Since she was so adamant that if one solved equations on paper that one must use PEMDAS because that was what her teacher told her to do, I asked her to solve this equation both ways. Using PEMDAS she divided -16 by 2 and obtained 8, and divided -6 by 2 and obtained 3 and said, "Eight and [pause] 3. And then you're done and you get 3." Even though she divided incorrectly and only used the quotient on the right side of the equal sign, she divided the whole equation by 2, unlike previous instances (see Figure 8). This may be because in working with the EBA, she had to do the same to one pan as she did to the other pan. This would require dividing the whole set of weights on one pan by 2 , both the variable and numbered weights, as well as the weights on the other pan.

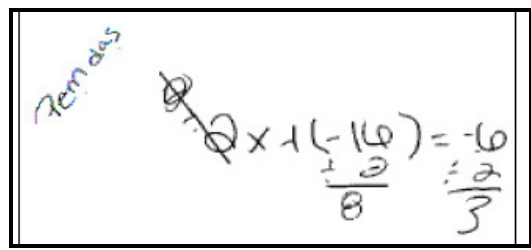

Figure 8. Using PEMDAS to solve $2 \mathrm{x}-16=-6$

When she solved it using the method she learned using the computer, she did not change the negative signs on the task, and solved it correctly (see Figure 9). She also noticed that she solved it by going backwards through PEMDAS. She said, "So we're undoing it...so we did the opposites." In saying that, she emphasized "we" to delineate what she had learned in math class and what she had learned during out sessions together. When I asked her which answer she thought was right, she grabbed the mouse and checked it on the computer by seeing if $x$ balanced with 5 . Afterwards, she also checked the task by plugging the value 5 into the original equation. She determined that the way we solved it on the computer, although contrary to what her teacher told her, was the correct way of solving it "because it's right."

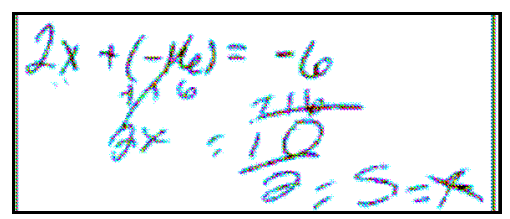

Figure 9. Solving $2 \mathrm{x}-16=-6$ using the balance method 
As I gave her other equations, she solved them correctly by performing the same transformations on both sides. However, much like the one above, she started using the equal sign as a procedural encoder when it came to division. She would show the addition or subtraction on both sides of the equation, but when she had to divide, she showed the division on one side and set it equal to the value of the variable to which she set that equal to the variable.

\section{Summary of Interaction and Understanding with Technology}

- Since Abby knew how to solve 1-step equations without the computer, she did not use the EBA to solve these equations. Instead she used it to check her answers because she knew that when the pans were balanced, the equations produced were equivalent.

- When Abby used the visual representation of the task shown in the EBA to solve 1-step equations that contained subtraction and a negative number, she did not switch the signs. Instead, she added the correct amount to each side of the equation.

- When Abby used the visual representation of the task, she solved the 1-step equation algebraically by applying the same transformations to both sides.

- Abby enjoyed using the manipulation, visual, and instantaneous feedback characteristics of the EBA because it allowed her to try different strategies that helped her solve 2-step equations.

- Abby used the balance feature of the EBA to find equivalent 1-step equations to help her solve a 2-step equation.

- When Abby first solved an equation of the form $a x+b=c$ where $b>c$, the visual and balance component of the EBA created a conflict in Abby's mind. While she knew that adding and subtracting the same amount from both sides maintained the balance of the pans, she also believed that a pan containing objects would be heavier than a pan containing no objects.

- Abby realized, although contrary to her memory of what her teacher taught her, that she should use the order of PEMDAS backwards to solve 2-step equations.

- Abby used the visual aspect of the EBA to intuitively tell her which operation to apply first because she knows that she needed to get the variable weights by themselves.

\section{Discussion}

Prior to working with the EBA, Abby had a segregated view of the equal sign. In instances where the equal sign joined two numbers with units, she saw the equal sign relationally. In instances where the equal sign joined a numeric equation, she at first exhibited an operational view of the equal sign, which later changed into a relational view. In instances that used an equation containing a variable, Abby believed the equal sign meant the answer was the value of the variable and did not mean that both expressions were equal to each other.

According to past literature (Knuth et al, 2006; Boulton-Lewis et al, 2000), students who do not have a 
relational view of the equal sign do not solve equations algebraically. This is somewhat true in Abby's case. Abby did not consistently solve 1-step equations algebraically, for she used arithmetic and unwinding methods. This furthers the notion that she did not have a fully integrated view of the equal sign as operational and relational. Moreover, in 1-step equations that contained subtraction and a negative number, Abby would switch the signs so that she could apply addition to make the expression containing the variable. This again shows that she did not have a relational view of the equal sign. Abby could not solve any 2-step equations because she relied on her memory of a rule that her teacher told her, which was not fully understood.

The dynamic aspect of the EBA allowed Abby to try different strategies to solve equations. She used her ability to manipulate different objects and the immediate feedback she received on how that adjusted the balance and the corresponding equation that showed which side was heavier and lighter. She also was able to easily see how the addition of weights and balloons corresponded to the operation of addition and the removal of weights and balloons corresponded to the operation of subtraction. Thus, now when she solves equations on paper, she applies the same operation to both sides.

Due to the visual aspect of the balancing of the pans, Abby observed the relational aspect of the equal sign. She not only saw that if the pans were balanced that the expressions were equal in that instance, but she also noticed that any other equation that she created that caused the pans to balance was equivalent to the original equation. In fact, she used this knowledge to help her solve equations and check her answers.

The visual aspect of the EBA also allowed Abby to intuitively see what operation needed to be done in the correct order. In 2-step equations, she was able to see that she needed to subtract or remove weights from the pan containing the variable before she divided by the coefficient. Furthermore, once these weights were removed, she intuitively knew to divide by the coefficient because that was the number of each variable she had remaining in the pan; thus, she had to partition the remaining value on the other pan into that many. In fact, when I asked if the EBA had helped her solve these equations she agreed and responded, "it's better than paper, I don't have to use my brain really." When I showed her that she indeed could solve them on paper, she said that she knew how to "cause I knew what to do from that thingy." She had used the ideas she learned from the balance and applied them to her work on paper. 


\section{CHAPTER 5}

\section{CASE STUDY: JIM}

\section{Jim's Background}

Jim is in seventh grade Standard Mathematics. He recently moved here from the Caribbean. While he quickly acclimated to the school, he had a trouble acclimating to the environment of Carlsburg. Upon moving to Carlsburg, he started suffering from extreme allergies, for which he takes medication. This medication leaves him drowsy, impassive, and reticent. During the interview, he would often ask me to repeat questions and tasks and would sometimes have difficulty articulating reasons for his answers, become frustrated, and without giving a plausible justification; he would resort to saying statements such as:

Well, that's how I see it. It's pretty easy. That's how I think.

Because it's just something simple. It's something simple that I knew how to do. I don't know...just some way I figured it out.

Well that's just simple stuff, that's stuff I remembered from elementary school.

He eventually told me that he was not used to giving reasons for his work because he never needed to give reasons before. He learned math by memorizing the procedures performed by his teachers.

I don't know, it's just something I do. I don't really look for reasons; I just put down stuff that I already know what to do. Like if I see a teacher do something, I try to memorize what they did and see how they got it, and I put it down.

By not having reasons and memorizing procedures, Jim came to the conclusion that mathematics was a set of rules. Jim would often make up his own non-absolute (not consistently applied) rules to provide a reason for his answer. Because these rules were not absolute he could claim that they only worked for certain tasks - mainly the one he was solving. For instance, in solving the task $5--12$, he claimed that $5+-12$ was the same as $5--12$ and both equaled -7 , even though one task used addition and one used subtraction. When I asked him if adding the same number was the same as subtracting that same number he responded, "Well, not all the time. Like if it's a problem like this, it would be the same thing." Similarly in another task, $10--3$, he believed the answer was 7 and reasoned that "because two negatives doesn't make it [the subtrahend] a positive." Then he paused and said, "Well, some, well, yes it is. It does make it a positive... well, not on this." 
Since Jim became frustrated with giving reasons for his answers, he subsequently made up rules to fit his answer choice. He may have done this because he felt diffident about his mathematical ability. After answering a task, he would sometimes ask me if his answer was correct before he would give me his reasoning. Furthermore, when I asked him how he solved the task, he would tell me his method, but also noted that there were probably more efficient ways of obtaining the answer. For instance, when I asked him how he solved Task 5 in Module 1, he told me he counted, but also noted, "instead of counting each [tick mark] I should have counted easier, like by doubles."

His lack of confidence in his mathematical ability could have stemmed from obtaining incorrect answers in his math class and the approach his teacher took of remedying the situation. To help Jim obtain correct answers, his teacher urged him to use a calculator and other technology. However, this did not necessarily improve his understanding, but rather negatively impacted his confidence and perspective on mathematics. For instance, when I asked Jim if he ever used a calculator in math class, he told me that he did not because he solved all the tasks in his head, but knew this was against his teacher's wishes. He told me that he solved addition and subtraction tasks "in my head, [but] my teacher tells me not to do it...because sometimes I get it wrong. But at the same time, I get it wrong on the calculator sometimes too."

Since he sometimes needed more assistance on topics, his teacher also had him use Study Island, a web-based program to help prepare students for the state standardized tests. Study Island asked math questions in a multiple-choice format. He talked about it with dislike because he had to repeat each topic and continue to answer questions until he received a perfect score. When I asked if he did not enjoy it he told me, "yeah, well, she makes us do it until we get 100\%, because if you get a 90 score, she makes you have to do it until you get 100."

\section{Negatives and Operations on Integers \\ Prior Conceptions on Addition}

Jim had a good grounding in conceptual and procedural knowledge of addition but not of subtraction. While he used different strategies to operate on computational arithmetic tasks, he used a consistent rule in operating on integers with regards to the number line: positive and addition signs signified that one had to move right on the number line, while negative and subtraction signs signified that one had to move left on the number line.

Jim had a unary conception of the addition of a negative number, because he could see the 
relationship between subtraction and the addition of a negative number. While he knew this relationship, he had a hard time articulating it. For instance, when he was presented with the task $10+-3$, he calculated that the value was 7 and tried to explain his calculation in terms of subtraction.

J: Since it's -3 , I just practically take it away from 10, so 7 plus 3 equals 10 .

$\mathrm{N}$ : Why do you think it's "taking away"?

$\mathrm{J}$ : Because it's negative. And you might, well it doesn't matter if your adding or sub[tracting]..., well it doesn't matter if you're adding if it's a negative number, it's going to be, it's going to be ... um, ok. It's just subtracting if it's plus with a negative.

Because he had a hard time articulating this fact, he also made it known that he could use addition to justify his answer by stating that $7+3=10$. After these comments, he noted that $10+-3$ was the same as $10-3$. When I asked him why he thought they were similar, he responded, “the addition doesn't really matter. The addition sign, there is a positive and negative sign, and you know how to get this number, the higher number, then you figure out the task of how to get it." He meant that the operation was not important in this case because he looked at the signs and determine how to find the answer. He knew that if one number was positive and one was negative, the operation that he implicitly used was subtraction. While this was his mode of operation for addition tasks of different signed numbers, he used a different procedure for samesigned numbers, even though he understood it to be subtraction as well.

$\mathrm{N}$ : Can you solve $-5+-7$ for me?

$\mathrm{J}$ : If there are two negatives, and you add them together, it's just like normal integ[ers]... like normal, yeah, numbers. So as soon as you do it, you just have to put the negative sign on there. $\mathrm{N}$ : How did you know to do that?

$\mathrm{J}$ : Oh, cause you're adding them together and, you know how that's [like] subtracting, and that's [pointing to the 5 and 7 to make 12] practically adding, which makes it go lower. Which is 12 , and then you put a negative.

His procedure to solve this type of task was to strip way the negative signs, add the remaining numbers, and then tack on a negative sign to the resulting sum. He mentioned that this procedure was risky because it was easy to forget to add the negative sign at the end. Jim said, "But sometimes that's a bad idea because they take credit off if you forget that [sign]...I could have had a 100 on a test, but I didn't. I completely forgot." While he used a procedure to solve this task, he recognized that while the task stated addition, and that 12 was the sum of 5 and 7, this task really was similar to subtraction because it decreased in value.

To see if he could move from a computational arithmetic context to a verbal context, I asked him if he could create a word task that went along with his equation $-5+-7=-12$ instead of providing me a word task, he told me that the task "was pretty easy" and that there was no need to write a story about it because he could "just see it the way it is" to solve it. He then reiterated his procedure for how he solved 
the task to show me that he had solved it correctly. Either he did not know how to express the addition of negative numbers as a word task, or he felt that word tasks were only to be used if he already did not know how to solve a task that was written abstractly.

Since I wanted to see if he could move from number line to an arithmetic computational context, I asked him to write an equation for a number line that showed a dot on -6 and a dot on 5 . He counted each space between 5 and -6 beginning at 5 . He explained, "if you count them down, if it's negative, you count down, you count left, if it's positive, then you [count right] like $-6+11=5$." He then showed me this by starting at -6 and counting to the right on the number line and landing on 5 . He believed that the sign in front of the second number indicated which direction he should travel on the number line. It is important to note that on this task, Jim chose to use addition instead of subtraction to model this situation. He did the same when he was presented with a contextual task and asked to write an equation that modeled it.

\section{Prior Conceptions on Subtraction}

Given two temperature tasks that asked him to calculate the change in temperatures, Jim never needed to use subtraction to answer these contextual questions. While he provided three equations for the first task, one using addition and two using subtraction, he did not create any subtraction task for the second contextual question.

$\mathrm{N}$ : On February $14^{\text {th }}$, the high temperature was 32 degrees and the low temperature was 0 degrees. Could you tell me how many degrees it changed?

J: 32

$\mathrm{N}$ : And how did you think about that to get the answer?

$\mathrm{J}$ : 32 take away 0 would be 32 . And 32 take away itself is 0 . I don't know how to answer your questions they just seem right. I mean $32+0$ is 32 and 32 take away 32 is 0.

Jim may have provided three different equations here because he was not cognizant of how he thought about the task. Furthermore, he was not comfortable with providing reasons for his answers, so he shared every equation he could think of that fit the numbers in the scenario. This most likely occurred because he was unsure of what the task was asking, or what kind of answer I was hoping to get, so he simply started spouting off everything that he knew. Unlike his answers to the first task, he only supplied one addition equation for the second temperature task.

$\mathrm{N}$ : If the high temperature in Alaska was -6 degrees and the day's low was -17 degrees, could you tell me how many degrees it changed and could you show me on the number line?

$\mathrm{J}:-11$

$\mathrm{N}$ : And how did you think about that to get the answer?

$\mathrm{J}$ : -6 plus -10 is -16 , so -6 plus -11 is -17 . 
Jim did not show me how he solved this on a number line because he felt that since he got an answer, he did not need to show me. Jim also did not discuss any other methods of solving this task. He may have solely relied on addition to solve the task because he could not accurately solve or conceptually understand subtraction tasks containing two negative numbers. Another possible explanation is that Jim solved the task in his head using subtraction, yet when it came to explaining his reasoning, he couched it in terms of addition.

Jim believed that subtraction signified that one must move left, or when it came to dealing with positive minuends and subtrahends, he viewed subtraction as "take away" rather than distance. Due to these ideas and Jim's unary view of addition of a negative number, Jim accurately solved subtraction tasks containing a positive subtrahend. Jim justified his answers to subtraction tasks with both positive minuend and subtrahend by taking away an amount that left him with remaining items or taking away more than with which one began, so one had negative number of items remaining. For tasks with a positive minuend as well as for tasks that had a negative minuend, he saw the equivalence between the tasks $a-b$ and $a+-b$. Since he believed that both the subtraction sign and negative sign meant to move left, he illustrated how he could obtain the answer using a number line by starting at $a$ and moving left $b$ spaces.

Jim maintained that both the subtraction sign and the negative sign meant take away and move to the left for subtraction tasks involving negative subtrahends. He was unconcerned with the fact that there were two of these signs beside each other. He felt that the signs were redundant in interpreting the task. When I asked him if there was anything special about having a subtraction sign and a negative sign sideby-side he responded, "No, it just means take away...see, uh, negative numbers go this way [pointing to the left] .... and if you take away, if you subtract it goes that way [pointing to the left]." In fact, when I had him compare his methods of solving $5-1$ and $5--1$ he told me he solved them the same way.

N: So how would you solve 5-1?

$\mathrm{J}$ : I'd move one to the left.

$\mathrm{N}$ : So what about $5--1$ what would you do then?

J: Left 1 .

N: So it would be the same thing as 5-1?

$\mathrm{J}$ : Yes.

$\mathrm{N}$ : I thought before you told me that this tells you how far you go [pointing to the subtrahend] J: [chuckles] ok, um, well, I guess I don't. Negative means subtract, and I'm not going to move twice, I'm only going to move once. So like 5 take away -1 and 5 take away 1 is 4 . So if it's -1 it just stays the same. So I mean like, 5 take away -1 is the same as 5 take away 1 it would be 4 .

Jim similarly solved $5--12$, by starting at 5 and moving to the left 12 spaces and landing on -7 . However, in this task, I specifically asked him if subtracting 12 was the same thing as subtracting -12 , to which he said yes. However, when I asked if 12 was the same number as -12 , he responded, "no, ... [but] it 
would be the same answer." Thus, he knew that the subtrahends were different numbers, but he believed that both forms $a-b$ and $a--b$ were equivalent based on the fact that the negative sign and the subtraction sign meant the same thing.

By having Jim work on the task 5 - - 12, I obtained a lot of insight into Jim's thoughts about subtraction and negative numbers. In the next two excerpts, he described some of his thoughts on how to solve this task. These excerpts were difficult to interpret if one strictly looks at the words with which he chose to express himself; thus, it is necessary to supply Jim's actions while he solved these tasks.

$\mathrm{N}$ : So the next one, $5--12$, you said, "oh, it's still take away" what were you thinking about then?

J: Right. 12 take away 5 , is -7 , so you just switch it around.

$\mathrm{N}$ : Why did you switch it around?

$\mathrm{J}$ : Cause it's just easier, if you just switch it around for the bigger numbers, the bigger number's on this side [right] instead of that side [left].

$\mathrm{N}$ : So how would you write that? [He writes $12--5$ ] So that would be 12 minus negative 5 ?

$\mathrm{J}$ : Um, hmm.

N: So those are the same thing?

$\mathrm{J}$ : Um hmm.

$\mathrm{N}$ : And what would this one [pointing to $12--5$ ] equal?

J: Negative 7

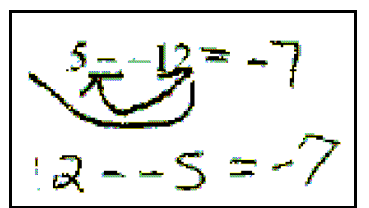

Figure 1. Jim switching absolute values of minuend and subtrahend

This excerpt was difficult to interpret because his words and writing were not congruent. He said, "12 take away 5" which would be interpreted as $12-5$ and not $12--5$, which he wrote. However, from previous examples, he viewed both of these expressions as the same because the negative sign was redundant. Another inconsistency occurred when he said that he switched the numbers to help him solve the task more easily. Switching the numbers meant that he would have gotten the task $-12-5$, which he could accurately solve and obtain -17 . However, what he meant by switching the numbers, which was evident in his writing, was leaving the signs in place and switching the magnitude of the numbers. So he not only believed that $5-12=5--12$, but also he believed that $12--5=5--12$. What is interesting is that while he believed that $5--12=12--5$ he did not believe that $5-12=12-5$, because he knew that the $5-12$ was equal to -7 and $12-5$ was equal to 7 . Thus, he would only switch magnitudes in a task when the task subtracted a negative subtrahend. Another interesting note on this example is his movement on a number line. If he truly believed that negative meant move left and positive meant move right, $12--5$ should not equal $5--12$, because the first one dictated that one started at 12 and moved left 5 spaces and 
hence landed on 7, while the second one dictated that one start at 5 and move left 12 spaces, subsequently lending on -7 . As we were discussed this task further, I learned how he dealt with this conflict.

$\mathrm{N}$ : So how did you take away the -12 from the 5 ?

J: Well, well, you can't take away the -12 so to make it easier you have to switch it around. The -12 is smaller, but -12 plus, take away 5 is -7 . So like if -12 was adding with 5 , or if it was subtracting with 5 , it would still be -7 .

$\mathrm{N}$ : So if you were adding something it would be the same thing as subtracting it?

$\mathrm{J}$ : Well, not all the time. Like if it's a problem like this, it would be the same thing.

$\mathrm{N}$ : And why is that?

$\mathrm{J}$ : Because on the number line it would just go the same way.

The first idea to notice about this excerpt was that he told me that he knew that -12 was smaller in value than 5 , however, because of the magnitude of -12 , he placed it in the minuend position so he could easily see that the value was -7 . What he meant was that even though he knew you could take a smaller number away from a larger number, it was easier for him to calculate the magnitude of the answer, in this case 7 , by switching the numbers.

The second idea to notice in this excerpt was that once again his words did not match his algebraic notation or his movement on the number line. He stated, "-12 plus, take away 5 is -7 ." First of all, this wording is awkward. One would naturally think that he meant the algebraic task $-12+-5=-7$, but what he pointed to when he spoke these words was the expression $12--5$. He was incorporating the old task that had a -12 and thus said negative twelve, but the word "plus" came into play because when he switched the expression to $12--5$, the 12 was now positive. Then he proceeded with take away 5 , because once again, the negative sign and subtraction sign was redundant. The next part of his quote refers to movement on the number line and not the algebraic expressions $-12+5$ and $-12-5$, which was implied by the statement, “-12 was adding with 5 , or if it was subtracting with 5." While he said this, he pointed to -12 and moved up 5 spaces to the -7 , and then started at 5 and moved down 12 spaces. Because he believed that moving up on the number line was addition, he said that the first movement was -12 plus 5 . Since he believed that moving down on the number line was subtraction, he said that the second movement was -12 subtracting 5, even though it technically was the subtraction of 12 from 5 . After he said this and showed me on the number line, I asked him specifically if subtracting the same amount was the same as adding the same amount to see if he would re-word his description, but instead, he told me that in some instances subtraction and addition elicited the same answer, as long as the motion on the number line remained the same.

Subtraction tasks that contained both a negative minuend and subtrahend were not as cumbersome to work with because the signs were both negative, so switching the numbers did not affect the fact that 
both numbers remained negative after the switch. Jim struggled with two conceptions on these types of tasks; in fact, he initially gave two answers for each task. For instance, for the task $-4--1$, he said it was either -3 or -5 , and for task $-4--6$, it was either -2 or -10 . While he solved both tasks similarly, there was one difference between these tasks. For $-4--6$, he rewrote the task as $-6--4$, but he did not rewrite the task $-4--1$ because it had the highest magnitude as the minuend. He wanted the highest magnitude in the minuend due to one of the ways he operated on this task. The first way he solved the tasks to get the values -3 and -2 was to take the absolute value of the minuend and subtrahend, subtract the numbers, and then tack on a negative sign to the answer. This is similar to his procedure with addition of two negative numbers. The other way he solved it was by adding the absolute values of the numbers and tacking on a negative sign. He then decided that this was the correct way of solving the task because he could support it by moving to the left on the number line, for he started at the minuend value and moved left the number of spaces described by the subtrahend. Thus, he obtained the values -5 and -10 .

\section{Discussion of Prior Conceptions}

Jim's way of operating on subtraction tasks is unique and confusing at first glance due to his notation, words, and movement on a number line, especially since he seemed to deal with different cases of subtraction tasks differently. After analyzing his work, I categorized his way of thinking and solving each of the four types of subtraction tasks where both $a$ and $b$ were non-negative integers (see in Figure 2).

Based on the methods of Jim's solutions, Jim was able to correctly solve subtraction tasks with positive subtrahends, but not tasks with negative subtrahends. This deviated from Murray's findings (1985) that students are able to solve subtraction of negative subtrahends more easily than subtraction of positive subtrahends. Another aspect of Murray's findings that does not apply to Jim is the fact that while Jim believed that $-a--b$ was equivalent to $-a-b$, he did not believe this because he was unfamiliar with the notation. Rather he believed that this was the case because the subtraction sign beside the negative sign was redundant.

Jim also exhibited some notions relevant to other literature, namely Peled (1991). In terms of Peled's Quantitative Dimension (QD) with respect to subtraction, Jim was most likely on Level 2. Level 1 required Jim to notice negative values as opposite in quality characteristics from positive numbers; one example being debt. While he did not specifically use these terms, he did note that given two negative numbers, the one with the larger magnitude was in fact smaller in value. The second level, required students to subtract a larger natural number from a smaller natural number, which Jim was able to do. However, he was not able to do this accurately when he encountered a negative subtrahend, thus he did not 
attain Level 3 or Level 4.

\begin{tabular}{|c|c|}
\hline Task Type & Solution \\
\hline$a>b$ & $\begin{array}{l}\text { Take away } b \text { objects from } a \text {; idea } \\
\text { supported by moving left on number line. }\end{array}$ \\
\hline$b>a$ & $\begin{array}{l}\text { Idea of take away meant that value had to } \\
\text { be negative; Idea supported by moving left } \\
\text { on number line }\end{array}$ \\
\hline$a>b$ and $b>a$ & $\begin{array}{c}\text { Mentally transformed into }-a+-b \text { based on } \\
\text { unary perspective, and solved as addition; } \\
\text { Idea supported by moving left on number } \\
\text { line }\end{array}$ \\
\hline \multirow{2}{*}{$a--b$} & $\begin{array}{l}\text { Transformed expression into } a-b ; \\
\text { Supported by redundancy of signs. Then he } \\
\text { supported this by moving left on the } \\
\text { number line once. }\end{array}$ \\
\hline & $\begin{array}{l}\text { First re-wrote expression as } b--a \text {; } \\
\text { however he thinks about it as }-(b-a) \text {; so } \\
\text { while he solved it in a different style, he } \\
\text { still obtained the answer to } a-b\end{array}$ \\
\hline$a>b$ and $b>a$ & $\begin{array}{l}\text { Solved it by }-(a+b) \text {; This was supported } \\
\text { by moving left on the number line. }\end{array}$ \\
\hline
\end{tabular}

Figure 2. Task types and Jim's solutions.

In Peled's Number Line Dimension (NLD), Jim believed that the number line was continuous because he counted across zero, and believed that the farther right one moved, the greater the value of the number. Furthermore, he attained Level 2 because he believed that addition meant to move right and subtraction meant to move left, even if a larger number was subtracted from a smaller number. Jim did not completely attain Levels 3 or 4 in terms of subtraction, because he was unable to subtract negative subtrahends accurately using a number line due to his inability to see that the subtraction of a negative reversed the direction.

Jim did not fit precisely into Peled's (1991) categorization of students' understanding of negative numbers. In fact, I had to separate his understanding in terms of addition and subtraction. Furthermore, he did not fit cleanly into one level. Thus, I adopted my own system of ratings based on Peled's levels. An asterisk in the Quantitative Dimension (QD) indicates that Jim was able to solve higher-level tasks than his number level indicates, but he did not put the answer or task into context. An asterisk in the Number Line 
Dimension (NLD) indicates that Jim was able to subtract natural numbers, know that plus and minus signs are opposite in direction, but could not do all same-signed subtraction tasks, yet could solve some oppositesigned subtraction tasks. Jim attained Level 4 in both the QD and the NLD with respect to addition. He attained Level 2* in the QD and Level 3* in the NLD with respect to subtraction.

\section{Summary of Jim's Prior Conceptions}

- Jim had both conceptual and procedural understanding of addition of negative numbers. He used different procedures to solve addition tasks based on the arithmetic form of the task, but had a unary sense of addition of negative numbers, and thus viewed it similar to subtraction.

- Jim preferred using addition rather than subtraction to create corresponding equations for tasks illustrated by context or on a number line. When he created these equations, he first figured out the answer, and then produced an equation that elicited that answer.

- Due to his unary view of negative numbers, Jim could solve a negative minus a positive number or a positive minus a positive number because he viewed it as addition of a negative number.

- Jim believed that subtraction meant take away rather than distance.

- In working with a number line for both addition and subtraction, Jim believed that the signs of the numbers told him which way to travel. Negative and subtraction both meant to move to the left, and positive and addition both meant to move to the right. When he encountered a task that required him to subtract a negative number, he did not believe that he should move to the left twice (or in reverse), but rather only move once since having two negative signs was redundant.

- Because Jim believed that the subtraction of a negative was redundant, he believed that $a--b=a-b$. Since he knew how to accurately solve $a-b$, he used this answer as the answer to $a--b$. For instance, for the task $5--12$ he believed it was equal to $5-12$, which was equal to -7 .

- Jim believed that you could switch the absolute value of the subtrahend and minuend, leaving the signs of the original numbers in place, and still obtain the same answer except when both subtrahend and minuend were positive.

- Jim believed that algebraic rules were never absolute. He believed that rules tended to be applied sometimes, however, he did not know the appropriate times. This allowed him to conjecture a lot of rules and only apply them when he wanted- without reason.

- Jim attained Level 4 in both the QD and the NLD with respect to addition. He attained Level $2 *$ in the QD and Level 3* in the NLD with respect to subtraction.

\section{Analysis of Interaction with Subtraction Applets}

Jim's Interaction and Understanding with Technology

Due to his responses in the interview, Jim seemed to see subtraction as take-away. I wanted to make sure that was true, so I had him use the distance subtraction applet (SDA) first, to see if he could visualize subtraction as a distance. 
Jim quickly picked up how to manipulate the applet and was able to see how the arrow was calculating how far apart the two dots were. In using the distance applet, Jim used the hot-linked equation to figure out where to place the blue and orange dots and how the arrow related to the answer showing on the screen. He modeled the task 5-- 12 (see Figure 3) and noted that the arrow showed "how far apart the numbers" even though he said this, he did not count the spaces in between, for he just discarded distance altogether. Yet, he looked at the equation and the corresponding number line model quizzically. I asked him if this model illustrated the equation $5--12=17$. He shook his head no and said that instead this model showed that "you can subtract 17, you subtract 17 from 5 to get -12 ". He used the arrow as the subtrahend value that was being taken away instead of as the distance between the 5 and -12 .

I don't think the problem has anything to do with how much it's apart. I just think it's how much it takes away...The problem is 5 take away 12, so the dot should be on 5 and then take away 12 , so one, two, three... which would be -7 still. So that's the answer.

As he said this, he moved the orange dot in increments of one to the left, until it landed on the -7 . At this point, the corresponding equation read $5--7=12$, but he dismissed this because he thought that the equation and the model were not equivalent.

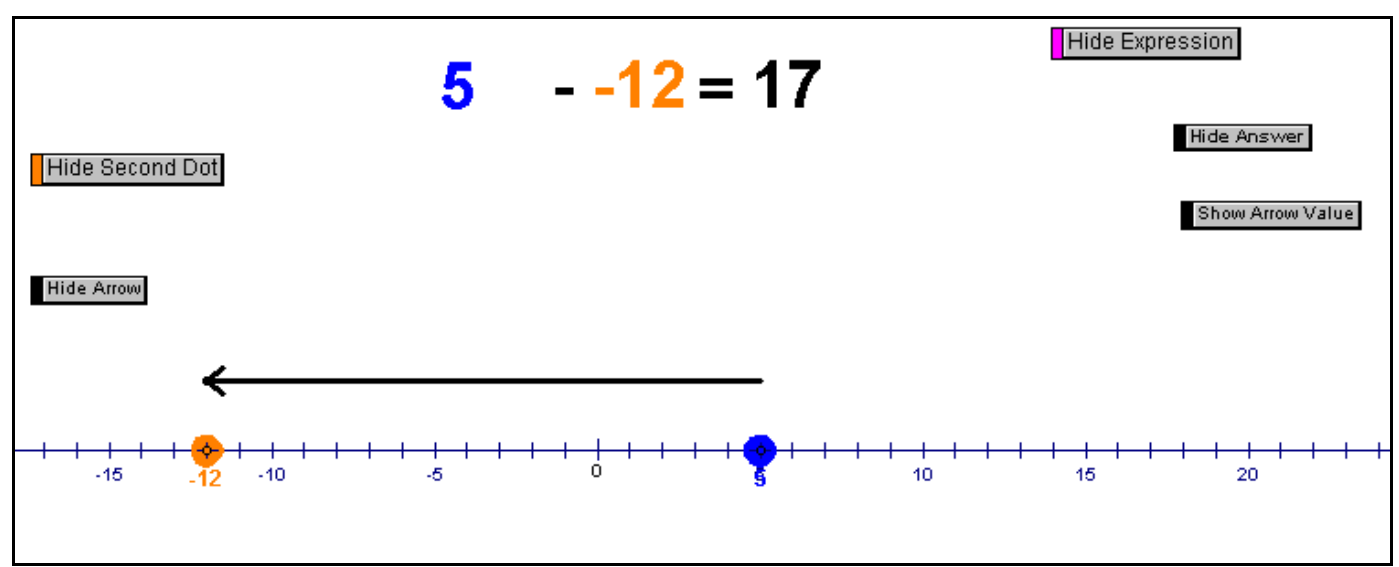

Figure 3. SDA modeling $5--12$

Since he did not believe that the distance between the minuend and subtrahend had anything to do with subtraction and since he could accurately solve subtraction tasks where both the minuend and subtrahend were non-negative and the subtrahend was less than the minuend, I had him use the applet to model them. He acknowledged that the answers to these tasks were the same as the distance between the minuend and subtrahend. Yet, he said, "It doesn't matter how far apart they are... at least not all the time. It depends on the question." He said this after he had modeled a task in which the subtrahend was zero. He acknowledged that the answer was a distance between the numbers, but it was a coincidence because "like 0 , like everybody knows that 0 doesn't do anything to a number, if you add or subtract it." This instance 
made him doubt that the equation on the applet represented the manipulations and visual model on the applet (see Figure 4). Since both addition and subtraction of zero elicited the same answer, but only an equation using subtraction showed on screen, he believed that the applet might not be illustrating subtraction. He thought that since both operations could be used in the model, this model might not be representing one single operation, but many and not necessarily subtraction.

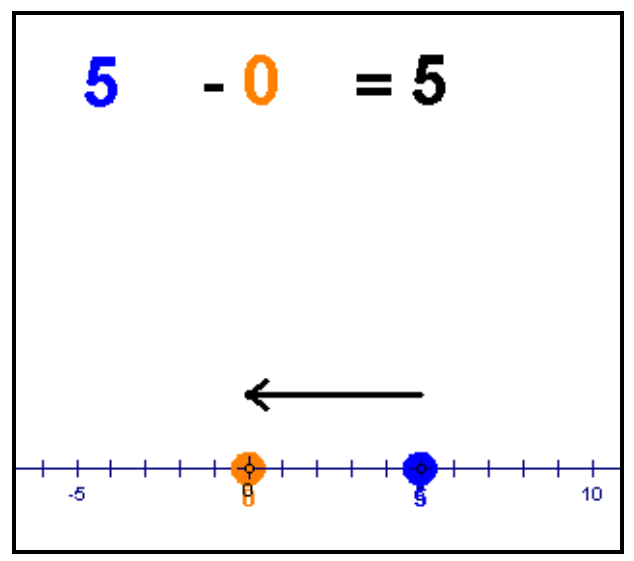

Figure 4. Jim using SDA to model 5-0

To remedy this idea, I had him consecutively model and reason through the tasks $5-3,5-2$, $5-1$, and $5-0$. He noted that in each instance, subtraction gave the distance between the two numbers, but also noted that zero still did not "do anything" to the value of 5. It is interesting to note that he did not show any signs of counting the numbers in between the minuend and subtrahend. I then asked him to predict the answer to $5--1$ without manipulating the applet. He said, " 6 , because 5 is 5 apart from zero, and so one further down is 6." He then moved the orange dot and showed me that it was in fact 6. Similarly he reasoned through $5--2$ and $5--3$. I then asked him what he thought the value of $5--12$ was, to which he answered 17 at first until he shuffled through his papers and found his previous answer of -7 .

Hmm, what did I have before?... I'm getting confused. I had put down -7. [pause] I ...I don't think 17 has anything to do with it... because the question isn't asking you how far apart, it's asking you what if, how much will it be if you take away the second number.

At this point, he firmly believed that the applet was not depicting an accurate representation of subtraction. I switched from the distance applet to the take-away applet (SAA) to see if he could discover patterns that would help him to conceptualize subtraction and solve tasks that had a negative subtrahend.

The SAA fit well with his prior conception of subtraction. While he understood that the arrow showed positive values when it moved left, and showed negative values when it was dragged to the right of the minuend, he did not believe this related to the subtraction of positive subtrahends and negative subtrahends, respectively. Moreover, when he solved a task that had a negative minuend, he continued to 
drag the arrow to the left instead of the right, even when the expression showed a different expression than he was trying to solve. In fact, he switched an earlier answer that he had gotten from using the previous applet, as well as disregarded the pattern he had noticed. For example, I had him model and reason through the same tasks he used with the other applet $(5-3,5-2,5-1$, and $5-0)$. He manipulated the arrow and told me that he moved the arrow to the left "cause anything that's negative or subtraction" went to the left. Furthermore, he told me he knew how many spaces he needed to travel to the left "because the task said what to do." While he said this, he pointed to the value of the subtrahend. He noticed that as he completed the tasks on the applet, each time the arrow became smaller because it was not going as far to the left. In fact, when he answered 5-0, he told me that the arrow "went nowhere." Yet when he answered 5--1, instead of moving to the right one space, he moved it to the "left 1 " because it was the same as $5-1$ (see Figure 5). Even though he had recognized the pattern and even had previously told me that $5--1=6$ by using the other applet, he still moved the arrow to the left. Furthermore, the corresponding equation that was showing on the applet was not $5--1$ but rather $5-1$. Yet, this was not surprising since he seemed to distrust that the expression, answer, and number line model were equivalent representations of the same task.

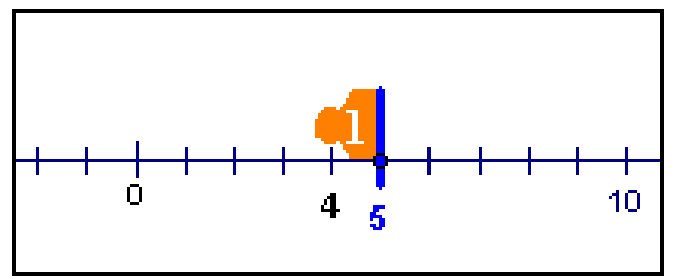

Figure 5. Jim's movement of arrow to model $5--1$

I think his distrust of the SAA and SDA were due to his dislike of both calculators and math computer programs because in the past he had sometimes obtained incorrect answers when he used them. Given his distrust of the applets, I tried to use temperature to contextualize the tasks and had him model them on the number line. However, he used addition to answer them instead of subtraction; therefore, he could not use the applets to show the expression. Since he used addition tasks, we started discussing addition tasks. During this discussion, we returned to the task $10+-3$. Previously he had told me that it was the same as $10-3$ and reasoned that both answers were 7 because $7+3=10$. I tried to build off of this by asking him how he could use the same logic and solve the task $10--3$. I had hoped that since he justified his answer to the subtraction task by adding his difference and the opposite of the subtrahend, he would do the same for $10--3$ and come into a cognitive conflict. For instance, since he believed that the answer was 7, I had hoped he would see that $7+-3=4 \neq 10$ and thus, the answer of 7 was wrong. 
Instead of this, he reasoned that $10--3=10-3$ and $10-3=7$ because $7+3=10$. While this is not what I intended, he did use the "same logic" as before.

At this point, I asked him to use a calculator to check his answer to $10--3$. After entering the expression, he turned to me and said, "See, 7." As it was hard for me to see him hit the buttons, I asked him if he had entered 10, hit the subtraction button, and then entered 3, to which he responded, "No, I hit the subtraction twice, see 7." This time he showed me the exact steps he took. He had, in fact, hit the subtract button two times prior to entering 3. I asked him if he could enter it another way, to which he gave me a puzzled look and shrugged like it would not make a difference if he did. He said, "I could enter it like the problem says, 10 minus negative 3 equals, [pause], 13?" He seemed thoroughly surprised at this answer. This time he had entered 10, subtraction, open parenthesis, negative sign, 3, closed parenthesis and obtained the value of 7. I asked if him if he could enter it another way to make sure he knew which one was correct. He entered 10, subtraction, negative sign, and 3 with no parentheses, and obtained the value of 13. I asked him which way he thought was correct and he said, "That one is right because on the problem, we had to enter parentheses. And when you minus a negative 3 you get a positive 3 , and when you have a positive 3 plus 10 you get 13."

After this revelation that the subtraction of a negative number produced positive value, he could algebraically solve every subtraction task that I gave to him, even tasks with a negative minuend. When he provided justification he said, "when you have two negatives, well, when you subtract a negative, it's almost like you, well both of them together make a positive." He later went on to say that he thought of "it in terms of language arts, cause if you say 'not' all the time, like if you say 'not' twice it's going to be positive." Because he had altered his justification radically and associated the negative sign with the word "not", which has been used to teach subtraction of negative numbers, I felt that checking that one task using the calculator had activated his memory of this rule. Yet, I was not sure that he truly understood the significance of this rule.

The next time we met, he did not remember the rule that subtraction of a negative was the same as addition. In fact, he once again believed that $5--12=-7$ and returned to his original justification. He also used a number line and counted 12 to the left of 5 and landed on -7 . Because I knew that he at least had a memory of two negatives equaling a positive, I showed him the SAA and had him manipulate the arrow prior to solving any tasks. Not only did he notice that as he moved the arrow to the left of the starting number the values of the arrow were positive and as he moved the arrow to the right of the starting number the values of the arrow were negative, but also he noticed that when he moved left, the value of the arrow 
always increased, and when he moved right the value of the arrow always decreased in an orderly fashion. I then asked him to model the sequence of tasks: $5-5,5-4,5-3,5-2,5-1,5-0$. When I asked him to predict the answer to $5--1$, he told me it was 6 and moved the arrow to the right from 5 (unlike last time when he moved the arrow to the left). I asked him why he chose to move right and he said, "If you start with a positive number and you're taking away a negative number, it's going to be a positive number that it equals." By using the arrow on the applet, he showed me that to get a negative subtrahend, he had to go right. If he started with a positive minuend, then he could only end up with a positive difference after he subtracted a negative number because negative numbers were the only numbers that appeared inside the arrow value when the arrow was moved to the right of the original number. He then realized the connection between going to the right and addition. In fact, in the next task he told me that he moved right "because of the two take away signs, that would be just like an addition problem."

While he understood the SAA, I also wanted him to understand subtraction as a distance between the minuend and subtrahend. Since he already knew how to correctly solve subtraction tasks abstractly, and because he had thought that distance had nothing to do with subtraction, I integrated contextual tasks with the SDA to see if that would help him see this new notion of subtraction.

$\mathrm{N}$ : So let's do some temperature tasks. The low temperature of the day was -12 and the high J: -7 temperature of the day was -5 . What was the change in temperature throughout the day?

N: How did you get -7 ?

$\mathrm{J}$ : Because -5 plus -7 and -12 .

Once again he used addition, rather than subtraction, to justify his solution for temperature tasks. I asked him if he could write the equation using subtraction, and he wrote $-12--7=-5$ and said, "- 12 take away -7 equals -5 ", but modeled $-5--12$ on the applet. He said that it was "how far away they are...-7 away from each other." While he expressed the idea of distance verbally and used the applet to model a distance, he still used the word "take away" to represent subtraction. However, he started to see the relevance of distance between the minuend and subtrahend.

Because he had used addition to model the temperature tasks as well as the words take away, I wanted to see if there was another context he could use that would help him understand subtraction as a distance. I asked him if he had ever seen negative numbers in the real world or had ever had them in a word task. He said he had seen them "on number lines and credit card debt." So I asked him to contextualize, and then model, the task $5--12$. He computed the value and told me that it was 17 because "it is how far apart they are." He then contextualized the task by saying "cause if you owe $\$ 5$, well, if you owe $\$ 12$ and you have $\$ 5$, and you have to get rid of your debt, so you take it away." Unlike before, when he thought that the 
negative sign and subtraction sign were redundant, he separated them in his interpretation such that subtraction meant take away while the negative sign in front of the 12 meant that he owed 12 dollars. While he made the connection between distance and subtraction, he still contextualized tasks in terms of take away. As I asked him to solve other abstract tasks, he noted that the answer was the distance between the minuend and subtrahend, but he used the word "take away" when describing subtraction, and also justified his answer by referencing the SAA instead of the SDA even though he was not using any applet to help him solve the task. He referenced it by pointing to the screen where the applet remained open but inactive, and said that he had gotten the answer because he moved to the right or moved to the left dependent upon the sign and the amount of the subtrahend.

When I met with Jim the last time, I asked him to solve addition and subtraction tasks. After an initial trouble with the task $5--12$, he could accurately solve all tasks.

$\mathrm{J}$ : Negative seven, because 5 minus 12 is -7 , it's just 5 take away 12 . I'm pretty sure we did that before, and 5 take way 12 ..it turns into a negative.

$\mathrm{N}$ : So you're saying that you took away 12 . So what if you had 5 minus a negative 12 ? What would that be?

J: It's the same thing.

I then asked him to model both of these on a number line. He showed $5-12$ by starting at 5 and counting to the left 12 spaces, and then paused. He said, "It would be the same answer...[pause] 17." When I asked him why he changed his mind he said, "Because two negatives equal a positive." When I asked how he knew that, he said, "I remembered it." While it is not clear as to what caused Jim to remember this, it did occur after him being asked to draw it on a number line. This could have been due to the fact that it caused him to focus and see that there was a subtraction and a negative sign instead of just a subtraction sign, or it could have been because he remembered moving the opposite direction on the number line for subtraction of negatives. Due to this finding, it is natural to ask if he would have focused on the two negative signs had the negative subtrahend been contained in parentheses. After this task, he could accurately solve every task I gave to him, and for the subtraction of a negative subtrahend, he used the rule that subtraction of a negative was the same as addition. When I asked him if the technology applets helped him remember this, he shook his head yes and said, "It kind of helped me on my SOLS. Some of it I knew, but other stuff...it helped me feel confident in my answers. It reminded me to look at the signs." He also told me that it helped him in math class because he "use[d] it in graphs. We had a graph test, [and] it kind of reminded me of this...like one guy is in front of another and you have to find out where he is...it just helped in determining which way to go." By allowing Jim to position the dots and arrows, and see how it adjusted the corresponding equation, and create patterns with the numbers, Jim was able to determine the direction to 
travel on the number line and how this direction related to addition when he subtracted a negative

subtrahend. The visual also prompted him to look at the signs to determine which direction he should move on the number line to solve the equation. The ability to recall the visual and reasons behind the visual, allowed him to feel self-assured in his answers to the state mandated test.

\section{Summary of Jim's Interactions and Understanding with Technology}

- Jim had both conceptual and procedural understanding of addition and an improved conceptual understanding of subtraction of negative numbers.

- By manipulating multiple sequences of subtraction expressions, noticing patterns, using contextual tasks, and noticing the connection between movement away from the minuend and the corresponding equation, Jim was able to see subtraction as both take-away and a distance. However, he preferred using the word "take away" for subtraction and referenced the SAA in justifying his answers.

- Jim still preferred using addition over subtraction to create equations for tasks illustrated on a on a number line and in contextual tasks. Unlike before, he could create an equation using subtraction to illustrate the tasks when prompted to do so. In creating these equations, he first figured out the answer, and then produced an equation that elicited that answer.

- In working with a number line for both addition and subtraction, Jim believed that the signs of the numbers told him which way to travel: negative and subtraction both meant to move to the left, and positive and addition both meant to move to the right. Jim still believed that addition and positive numbers meant to move to the right on a number line, and subtraction and negative numbers meant to move left. However, he did not believe that the subtraction of a negative was redundant. In fact, because of his belief of movement based on the signs of the numbers, and because of his working with the SAA, Jim was able to see the connection between the subtraction of a negative being addition because had to move right on the number line. Due to seeing this connection, he was able to accurately solve subtraction of negative subtrahends by changing the signs as well as by moving to the right on the number line from the minuend.

- Because Jim knew the correct way of solving all subtraction tasks, he no longer believed that he could switch the absolute value of the numbers, leaving the signs of the original numbers in place in the subtraction task, and still obtain the same answer.

- As Jim worked through the technology applets and tasks, he decreased his frequency of giving rules that only applied in a certain situation as reasons. The pivotal point of this decrease was when Jim checked his answer in the calculator and found that two negatives equaled a positive. He stuck with this notion through the session, yet when he returned for another session he reverted back to his old reasons. Yet, as he worked through the technology applets and saw visual representations and connections between the algebraic equation, movement on a number line, and context, he more easily remembered this rule and applied it thereafter.

- After working with the technology and tasks, Jim successfully attained Level 4- in both Peled's QD and NLD with respect to subtraction. He attained some of Level 4, but could not smoothly and completely attain it. 


\section{Discussion}

Because Jim did not have a clear conceptual understanding of subtraction prior to working with the technology applets, he used a variety of procedures to solve subtraction tasks. He based his decision regarding the type of procedure and subsequent reasoning for the procedure on the type of task, for he did not have a unified view of all subtraction tasks. Since he did not have a unified view of subtraction, he had difficulty articulating how he solved each task and why he solved it that way; he would resort to saying that certain rules applied to a particular task but not to others or that a specific task was the exception to a rule.

His rules and misunderstandings of subtraction mainly affected his ability to solve subtraction of a negative subtrahend. He believed that because both the negative sign and subtraction sign meant to "take away" the subtrahend value or "move left" the subtrahend value of spaces on a number line, that having them beside one another was redundant. This caused him to believe that equations of the form $a--b$ were equivalent to $a-b$ when $a$ and $b$ were both non-negative integers. Furthermore, because of his notion of take away, he believed that the larger absolute value of the numbers should be in the position of the minuend, but the negative signs should be left in the same place. Thus, he believed that when $a$ and $b$ were both non-negative integers $a--b=b--a$.

While he was aware that he often incorrectly calculated subtraction of integers, he also knew that he obtained incorrect answers when he used a calculator. This caused him to distrust values computed with technology and lack confidence in his answers. This lack of confidence most likely perpetuated his belief that some rules worked for only some tasks, and some tasks were exception to a rule even although he could produce no reason for this justification.

Due to his deeply seated conceptions, distrust of technological generated answers, and his ability to bypass using subtraction and instead use addition to answer tasks involving other subtraction representations such as contextual and number line tasks, he had a difficult time overcoming his misconceptions concerning subtraction of negative subtrahends. Even after initially working with both the distance and take-away applet, he could not see subtraction as a distance, and that a subtraction sign followed by a negative sign was the same as adding, or that one had to move right when one subtracted a negative value using the take away applet. It was not until he used a calculator to verify his answer did this notion begin to change.

It was ironic that even though he professed that the calculator gave him incorrect answers as often as he himself computed the wrong answer, the calculator's computation was what allowed him to open up and accept the possibility that subtraction followed by a negative number was not redundant and that 
subtraction related to distance. This may have been influential because he computed the answer using the calculator three different ways instead of just one. Had he only used his initial computation, which was incorrect, he would have continued to operate the same way. Instead, through the combination of seeing that two out of the three answers did not agree with his initial answer and figuring out why the answer was incorrect, activated a past memory that two subsequent negatives signs produced a positive sign.

Yet, the calculator and remembrance alone did not help him to retain this rule. By working with both applets and using some contextual tasks, Jim was able to make multiple connections to this rule through other representations. He was able to use the applet to enact subtraction as both take-away and distance and observe the association of the numerical equation and movement on a number line. Jim observed this association easily because he already viewed addition as moving to the right. Thus, the realization that two negative signs equaled a positive sign accompanied by the visual movement to the right, solidified the idea that subtraction of a negative subtrahend was the same as addition. In fact, he referenced this motion even when he did not use the applet to help him solve the task.

Because he was able to gain a comprehensive understanding of subtraction, he felt more confident in his answers because he could relate them to rules, movement on a number line, and words. Furthermore, Jim now paid close attention to the signs and was able to connect the arithmetic representation with the number line representation. This confidence made him feel self-assured about his performance on tests and on tasks in his math class.

\section{The Equal Sign \\ Prior Conceptions}

Prior to using technology, Jim primarily viewed the equal sign as relational. Only his answer to Task 1 could be classified as operational. He said that while all of the expression/equations were the same because they had the same "numbers being added," they were at different stages. He said, "This one has the answer, this one is going towards the answer, and this one, well, they haven't finished it yet" for the equations and expression $5+6=11,5+6=$, and $5+6$, respectively.

For Tasks 2 and 4, Jim said that the equal sign meant that the one expression was the same as the other side. Jim had seen both non-canonical forms in Task 2 . He told me that he had seen $3=3$ when he had studied the comparison of quantities using the greater than and less than symbols. While he did not tell me where he had seen equations similar to $8=10-2$, he told me that it was written correctly because "The number it adds up with is the same. Eight is just 8 and 10 take away 2 is 8 ." While these responses seemed 
quite normal, Jim gave a unique response to Task 3.

$\mathrm{N}$ : What does the equal sign mean in this task $3+5=?+2$ ?

$\mathrm{J}$ : I don't remember exactly, but I know what that question mark is....3 [pause] no wait 6.

$\mathrm{N}$ : How did you get 6 ?

J: Three plus five equals eight and two plus six equals eight. And that's how they would be equal.

When I had asked him about how he had gotten 3 at first, he simply said he had made a mistake and the answer was 6. I noticed his pencil movements while he was thinking about this task. He hovered over the 2 and the question mark and then moved left to the 5. By my observation, it looked as though he said the answer was 3 at first because he wanted to find out what needed to be added to 2 to get 5 , which was immediately to the left of the equal sign. When he checked this number, which would account for the pause between answers, he realized that 3 did not make the two expressions equal. Past literature has illustrated students performing a similar strategy, only moving from left to right, for they believed that since the question mark fell immediately to the right of the equal sign, it must have the value of 8 because 3 plus 5 is 8. While Jim did not explicitly say what the equal sign meant in this task, he implied that it had to do with the value of the question mark.

Unlike previous relational responses from Jim concerning the notion of the equal sign, he believed the equal sign meant something different when it was used in an equation containing a variable. When I asked Jim what the equal sign meant in equation $x+5=3$ he said, "What it equals. What it is probably. I don't know, just what it is. $x$ is -2." Every time he said the word "is" he emphasized it. There are a couple of interpretations of Jim's response. The first interpretation is that he associated the equal sign with the word "is." This idea is plausible because in translating word tasks into equations, the word "is" signifies the equal sign. Another interpretation is that Jim believed that equal sign had no meaning until the value of $x$ was found, because until that occurred, the two expressions were not equal to each other. Since this was the eighth time I had asked Jim what the equal sign meant, he started to become impatient with me. So another interpretation is that Jim was aggravated due to the repetition of the question, felt that the equal sign's meaning was obvious, and stated that the equal sign showed that two things were equal, because it just is what it is (equal means equal).

When I asked him how he had gotten -2 for his answer to $x+5=3$, he said, "Cause if it was 2 , that would be 7. [So] it's -2 [because] that's pulling down, not to the right, which makes it 3." To solve this equation, as well as other one step equations, he used a method similar to the guess and check method described by Knuth and colleagues (2006). Yet his method, he made some calculations in his head prior to his guess; thus, I will call his strategy the calculated guess and check method. He chose a number that 
would satisfy the equation when he plugged it into the variable. In fact, when I asked him how he solved $a$ $+5=7$ he said, " 2 , because a 5 plus what equals 7 . And 2 plus 5 equals 7 ." While Jim seemed to understand the equal sign as relational, he did not solve 1-step equations algebraically, but rather arithmetically. This is contrary to Knuth and colleagues' finding that students who had a relational understanding the equal sign typically solved equations algebraically by applying the same transformation to both sides of the equation.

Similar to his method of solving 1-step equations, Jim tried to solve 2-step equations using the calculated guess and check strategy, however he was unable to obtain a correct answer.

$\mathrm{N}$ : Have you ever seen a task like this $2 m+9=1$ ?

J: Yeah. 2 times...hmm. How did they get 1? [pause] I don't know. Two times. Would it be negative, to make that part go lower?

$\mathrm{N}$ : To make which part go lower?

$\mathrm{J}$ : The $2 m$ part? ...if you could get 2 , a positive number, multiplies with a negative number, isn't that a negative number?

$\mathrm{N}$ : Oh, I see what you're doing. So if $m$ wasn't a negative number, what would happen?

J: That wouldn't be right.

$\mathrm{N}$ : Why not?

$\mathrm{J}$ : Because it would be a lot more higher than 1 .

$\mathrm{N}$ : So do you think this $2 m+9$ is equal to 1 ?

J: [Shakes head yes] It's just together, somehow, I know that's multiplication ... I think it would be negative, so that would be 2 times -5 [pause] yeah, 2 times -5 that would be -1 . [pause] I don't know.

Jim recognized that the value of $m$ had to be negative in order for both expressions to be equal; yet, he was unable to find a number. He tried the value -5 , but realized that it gave him -1 instead of 1 after he multiplied by 2 and added 9 to the product. Jim solved all equations using an arithmetic strategy even though he seemed to understand the relational aspect of the equal sign.

\section{Summary of Jim's Prior Conceptions}

- Jim exhibited a relational view of the equals sign for numerical equations and the comparison of numbers and units.

- Jim was familiar with non-canonical forms and was able to state why they made sense.

- Jim did not believe that the equal sign showed equivalence of two expressions when one of the expressions contained a variable. He believed that the equal sign meant the value of the variable.

- Jim accurately solved 1-step equations using the calculated guess and check method. He tried to use this method to solve 2-step equations, but was not able to find the correct value for the variable. Yet, he had the right idea of how to solve them and most likely if he had had more time, he could have reasoned through it. 


\section{Analysis of Interaction with the Equality Applet \\ Jim's Interaction and Understanding with Technology}

As Jim worked through the Tasks 1-5 on Module 3, he noticed that the weights pulled the pan down on the Equal Balance Applet (EBA) and justified this because they had positive value while the balloons lifted the pan up and justified this because they had negative value. In manipulating the applet, Jim determined that adding the same set of objects to both pans allowed the pan to remain balanced. He had placed a 5-weight and -5 balloon on both sides of the balance. When I asked him why this caused the pans to be balanced he stated his reason in terms of understanding what the equal sign meant.

Five and negative five is 0 . It makes it even because I put it on both sides, and when both sides are even, they are equal, so they would be balanced. If they are equal, then they are balanced, but if they are not equal, then one will be up or down.

It is important to note that he maintained this view even when he started placing variables on the pans to create equations with one variable. As was noted earlier, Jim did not think that the equal sign in equations containing a variable meant that both sides of the equations, but rather the equal sign meant the value of the variable.

While Jim easily found that by adding and removing the same amount from both pans, the pans would remain balanced, he did not initially believe that if he removed a negative and positive of the same value from one pan it would still equal the values in the other pan. For instance, after he had placed a 5weight and a -5 balloon onto both pans, I asked him if the pans would still balance if he removed a 5 and 5 from only the left pan. He said that it would not and pointed to the right pan and said, "this would be up." I asked him to try it. He removed them and said, "Oh, I get it. I forgot about these two because that's still zero. I forgot about them," as he pointed to the 5 and -5 that he had removed. He had not realized that since 5 and -5 summed to zero, he was actually removing a value of zero from a pan. Thus, the pans remained balanced. He was able to test his conjecture of moving a positive and negative value from a pan with this applet, see the resulting outcome, and figure out why his prediction was wrong and readjust his thinking. He then claimed that there were not only two ways to make the pans balanced but also "lots of ways" and proceeded to drag different valued weights to both sides. He pointed to the different equations that corresponded to the amounts on the pans and noted that they were all equal. He also showed me that he could exchange weights for other valued weights as long as they summed to the same value. For instance, he placed a 5-weight on the left pan and then highlighted and dragged five 1-weights to the right pan and said that they balanced "because you have one 5 here and then there are five 1's to make one five" (see Figure 6). 


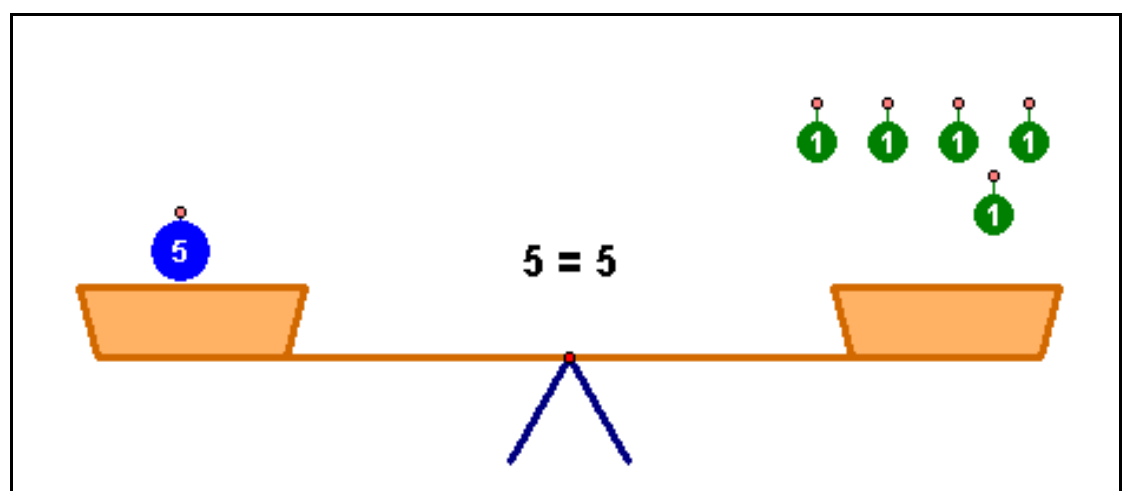

Figure 6. The equivalence of a single 5-weight and five 1-weights

After showing me many different ways to maintain the balance of the pans and noticing how the equation represented the objects in the pan, he created the equation $2 m+9=3$. Previously he had difficulty solving this task, and he wanted to use the applet to model it. He said, "See it equals 3 which is on this side, and 4 plus 5 and the mystery $m$ 's. There are two $m$ s on this side... because you multiply by 2" (see Figure 7).

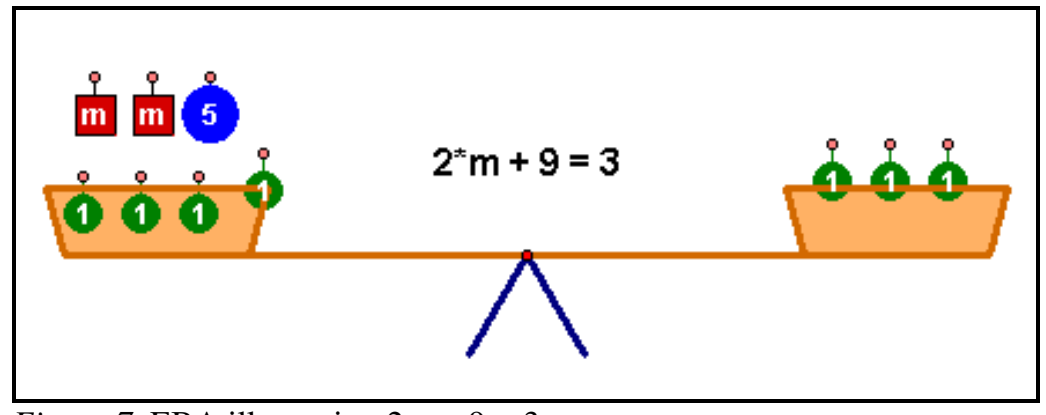

Figure 7. EBA illustrating $2 \mathrm{~m}+9=3$

Because he had not known how to solve this task, I had asked him if he thought this applet might be able to help him solve it, to which he responded, "Yes." Without asking for guidance, he began dragging different objects to and from the pans, that he thought would help him find the "mystery" $m$-value. He started off by dragging a $-m$ balloon from the storage unit to the left pan, and then moved it to the right pan. He might have done this because he knew how to solve equations that had a coefficient of 1 , so he was trying to find an equivalent equation. However, during the process of dragging the $-m$ from one side to the other, he noticed the change in the equation. The inequalities toggled between $m+9>3$ and $2 m+9<-m+3$ if he placed the $-m$ on the left pan or the right pan respectively (see Figure 8 ). He said, "It would be too little on here, so that would be -1 . So that is 1 . Yeah, it's 1 or it should be." He used the balance to see that wherever he placed the $-m$, the opposite pan would go up in the air and become lighter. He also noticed the inequalities and determined the value of $m$ based on the change in the inequalities. When I asked him how he determined the value of $m$ he said, "Cause on here it says. Like if I put this on here, it' $d$ be right there, 
-1." He placed the $-m$ balloon on the right pan and pointed to the corresponding inequality $2 m+9<-m+$ 3. The applet showed the right side as $-1 * m+3$. He had not understood that the asterisk meant multiply, and instead thought that the -1 beside it meant that the value of $-m$ was -1 ; thus the value of $m$ was 1 . After I told him what it meant, he tried other strategies.

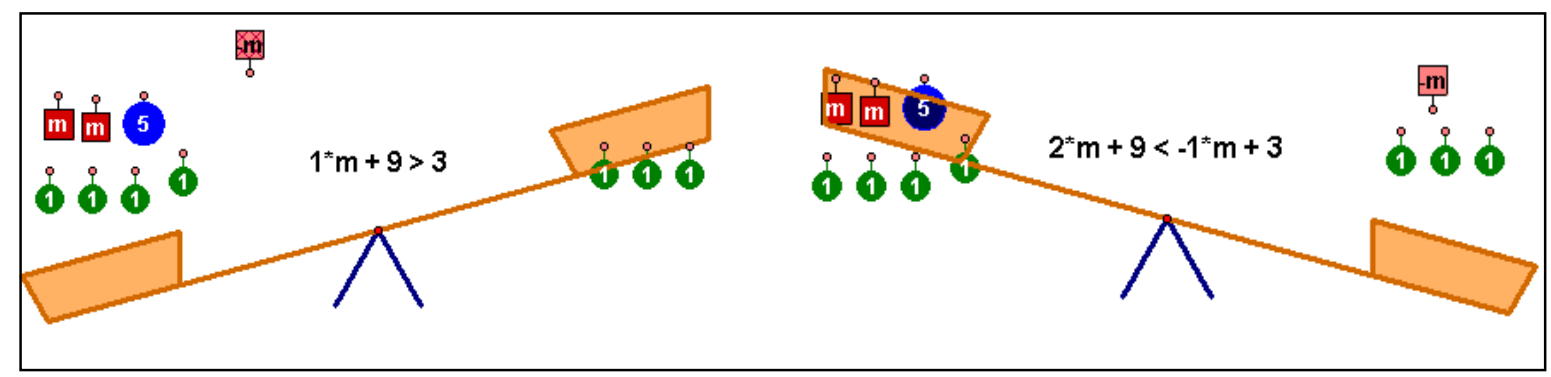

Figure 8. Jim trying to add a $-\mathrm{m}$ balloon to the left and right pan

To help him solve the equation, he replaced the $-m$ to the storage unit and dragged an $m$-weight to both pans to obtain $3 m+9=m+3$. He laughed at this equation because he realized that it was a harder equation to solve and told me that he "was just seeing how it would work." After I reminded him of the rules he discovered of to keep the pans balanced, he decided to take off three 1-weights from both sides, which corresponded to the equation $2 m+6=0$. At this point he said, "Hmm, so it's definitely not 1 ." $\mathrm{He}$ realized that with this equivalent equation, that his previous prediction that $m$ was 1 was not correct because when he plugged in 1 for $m$ and added 6 , he did not get 0 for his answer. He did not move the weights on the screen, but sat there looking at the equation and said, "Oh, I think I know this! Two times 3 , no, -3 ! $m$ is -3 ." He did this calculation in his head by substituting in numbers like he normally did in solving 1-step equations.

When I asked him if he could use the balance to show that $m$ equaled -3 , he said that he could but did not seem to know the procedure. He removed 6 from the left pan and placed it on the right pan because he wanted to try to get $m$ by itself. However, he was left with the resulting inequality $2 m<6$ (see Figure 9).

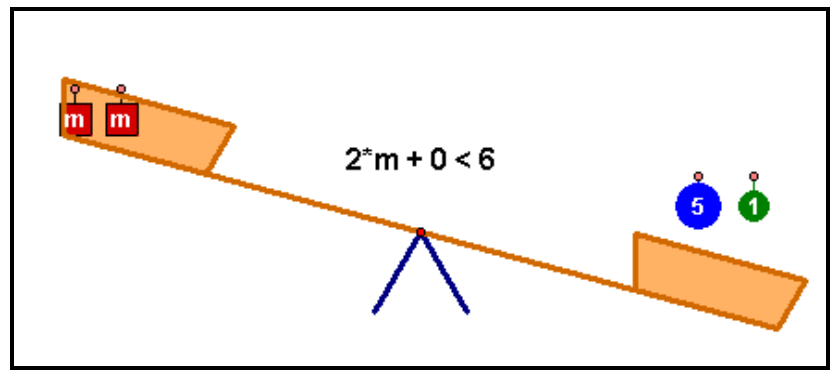

Figure 9. EBA illustrating the inequality $2 \mathrm{~m}<6$

He noticed that since $2 m$ was lighter than 6 , it would not help him find the value of $m$, since he needed the pans to stay balanced to obtain an equivalent equation. He returned the two weights summing to 6 to the 
left pan so that the equation read $2 m+6=0$. I reminded him of the two rules he determined that kept the pans balanced: adding and removing the same value of objects from both sides and removing objects that summed to zero from one side. He determined that he needed to add objects to both sides because he could not remove any more; however, he only considered adding positive values and therefore added a 1-weight to both sides. Even though the pans were balanced after dragging this weight, he looked at the equation and paused, realizing the equation was harder to solve. Therefore, he added a -1 balloon to both sides and obtained the resulting equation $2 m+6=0$, and removed the pairs of 1 and -1 from both sides because "together they make it zero" and thus, did not adjust the equation. He added -1 to both sides and removed the pair of 1 and -1 from the left pan. At this point, his left pan has a 5-weight and his right pan has a -1 balloon. Instead of adding a -5 balloon to both pans like he did with the -1 previously, he removed the 5weight from the left pan and added a -5 balloon to the right pan. When I asked him why he did this, he said, "Cause I took something off each time...well, I put the opposite on, on top of each other." Jim had discerned a pattern: adding a negative balloon from one side was the same as removing a positive weight from the other side.

In doing the previous tasks, he had placed a negative value on both pans, after which, he removed the zero pair (he would usually put the weight and balloon on top of each other) from the one pan, and this still maintained the balance of the pans. He simplified these two steps into one by removing a positive value from one side and adding a negative value to the opposite side. He started to see why the removal of a positive weight was the same as subtraction of a positive, and the addition of a negative balloon was the same as addition of a negative. Since the constant value beside $2 m$ was zero, Jim easily figured out the value of $m$. He said, "Oh, two times, well...that would be negative. The number that you see, and it's multiplying. So if it was negative three times two it would be negative six." While he could easily figure this out by looking at the equation, I wanted him to use the applet to show how $m$ was -3 . He exchanged the -5 balloon for five -1 balloons and then separated them into two groups and said, "- -3 , because half of 6 is -3 ." When I asked him why he had to split them in half he said because "there were $2 \mathrm{ms.}$." While he could easily solve the 1-step equation in his head, he also knew why he had to divide the remaining value by 2 due to the visual in the model.

Before our session ended, I wanted to see how he would use the computer to solve another 2-step equation, $3 m+7=19$. By using his newly found 1-step rule, he removed a 5 from the right pan and added a -5 to the left pan, and saw that they were balanced. However, he said, "Am I doing this backwards? Yep, I am." Even though the resulting equation showed $3 m+2=14$, he believed he was solving it backwards 
because he had collected more items in the left pan and removed items from the right pan. He had hoped to get the $m$ values by themselves in the pan, and this action did not lend itself to that. Thus, he reset the equation, and reversed his action and obtained $3 m+2=14$. He said, "Well, I could put on a -5 or take off a 5." He reset the equation again, and instead of adding a -5 , he removed a 5 from both sides of the equation to again obtain the equation $3 m+2=14$. He had wanted to find the simplest way to get rid of as many objects as possible to help him visualize the task and see the solution. At this point, he removed two -1 balloons from both pans and announced, " $m$ is 4 ...because 3 times 4 plus 0 equals 12." He did not partition the weights on the right pan to obtain this $m$-value, rather he mentally computed value of $m$. This was easy to compute because the constant value on the same side of the equation as the variable was zero. When I asked him if the technology helped him solve these equations he agreed and told me how it allowed him to create an equivalent equation that was easier to solve because the constant value was zero.

Yes, because you find out how, as long as it gets to, if you can get it to zero, it's easier...It shows you what the $m$ is. It was harder to see what $m$ was before. This is an easier way for me to do it.

He pointed to the original equation and noted that it was harder to figure out the value of $m$ because there were so many numbers and operations to take into consideration, but the applet allowed him to see how to remove the other numbers and operations and acquire a 1-step equation involving multiplication, which was easy to solve in his head. When I asked him what the equal sign meant in this equation, he pointed to $3 m=12$ and said, "Cause they are the same. That's already 12, and this is 12 , so that's the same thing....they balance." He saw that the equal sign related two expressions that equaled the same value. In this case, he said that the right side was "already 12" and the left side had to be 12 because the pans were balanced.

At the end of this session, I was left with the impression that Jim used the applet to simplify the equation so that he could use his guess and test method to solve the resulting equation, but he did not thoroughly understand how these actions corresponded to algebraic transformations. When he was introduced to this applet, he was motivated to use this applet to help him solve 2-step equations because he could already find the answer to 1-step equations. However, he could not even solve 1-step equations algebraically, so during the next session, I had him use the applet to solve 1-step equations with the hope that he would discover the connection between his actions and algebraic transformations.

When I met with Jim during the next session, he remembered the technology applet and was able to tell me that the addition of a weight or balloon was the same as addition of the positive or negative number, and the removal of a weight or a balloon was the same as subtraction of the positive or negative number. Furthermore, he immediately remembered one of the rules for keeping the pans balanced: "add 
and subtract the same thing to both sides." However, he needed to manipulate the applet a few minutes to determine the other rule: adding or subtracting the same positive and negative value from a side, yet this came back to him quickly. I then asked him to model the equation $3+5=?+2$ using the applet and use the pans to solve the equation. He modeled the equation and then said, "That's 6. I could just put a 5 and a 1, since that question mark is 6." He then reset the equation and put the question mark on the left pan and a 5weight and a 1-weight onto the right pan, and said, "so put the question mark over here, and put the 5 over here and a 1 to make it even." Rather than using the balance to solve the task algebraically, he had used the balance to show that the question mark's value was 6 . He illustrated this by proving that the pans would balance when he placed a question mark in one pan and a pair of weights that summed to 6 in the other pan. I asked him if he could show how he had determined the value was 6 by balancing the pans and not simply checking his answer. He remodeled the equation, and removed two 1-weights from both sides and said he was "taking away 1" each time, and pointed to the resulting equation $6=$ ?. Not only did he remove the weights this time, but also he noted that it was subtraction that he used to transform the old equation into the new equivalent equation.

Prior to using the balance to solve the equation $x+8=21$, I asked him what he thought the equal sign meant in this task. Before he had explained that in equations that contained a variable, the equal sign meant the value of the variable. This time, he said, "It all equals 21 . This is $21 \ldots$ so that's 21 too." As he said this, he first pointed to the right side and then pointed to $x+8$ as being 21 as well. When I asked him to use the balance to solve this equation, he asked, "Can I figure out what $x$ is first?" Without awaiting a response he said, " $x$ is 16 ." Since he knew how to solve these tasks in his head, he wanted to figure out what he thought $x$ would be before he used the applet to help him find the solution. After predicting the incorrect answer, he removed a 5 and 1 weight from each side. At this point, he was left with the equation $x$ $+2=15$ and an $x$ weight and two 1-weights on the left pan and three 5 weights on the right pan. He muttered, "If I take away a five from here and a 1 from here, it wouldn't be equal." He realized that he would have to exchange five 1-weights for one 5-weight, before he could remove the same amount from both sides. Instead of actually exchanging the weights, he added two -1 balloons to both sides and stated, " $x$ equals 13." When I asked him to talk about how he solved the task, he recounted, "Well, I don't know what $x$ is yet, so. So, it has to be equal 21. So 6 plus 15 [pointing to the right pan] and that would be 8 [pointing to the left pan] and then $x$ is 13 , and $13+8$ is 21 ." He began by saying that because he did not know the value of $x$, he had to find it, and the only information he had been given was that $x+8$ had to equal 21. With the applet he removed 6 from both sides and that gave him the new equation $x+2=15$, 
because if one added 6 to 2 it was 8 , and if one added 6 to 15 , one got 21 . Now, he solved $x+2=15$ by noticing that he had to add two -1 balloons to both sides and got $x=13$. Because $x+2=15$ and $x+8=21$ are equivalent, he plugged in the value of 13 into the original equation and showed that it equaled 21.

While he understood how to solve the task by looking at the visual depiction of the equation, I wanted to make sure he understood what the visual related to with regards to algebraic transformations. When I asked him if he could tell me in terms of addition and subtraction as to how he got the new equation, he said, "Oh, you can just do that off of paper." He then showed me that he subtracted 6 from both sides of the equation, and got $x+2=15$. Even though he stated that he "added two negative ones" he said that was the same as "take away 2" and subtracted 2 more from each side to obtain $x=13$. Notice that instead of subtracting 8 from both sides immediately, he separated it into two steps because of the visual representation of the equation. Since the visual model had only 5-weights on the right side, he only removed one 5-weight from both sides. Then he switched from removing objects, because there were no similar objects to remove from both sides, to adding negative balloons to both sides. He did a similar 2-step process for the next task $x-6=-15$.

Given the option of solving $x-6=-15$ on the computer or on paper, Jim chose the computer (see Figure 10). He instead of adding 6 , he removed a -5 balloon from both sides. He said, "I could take away -5 , that's going to be a -1 and that's going to be a -10. ' I asked him what taking away a -5 was like in terms of an operation and he said it was "subtraction of a -5 " which meant that he added 5 which transformed the task into $x-1=-10$. At this point, he hovered over the -1 balloon with his mouse and said he wanted to "add the same or subtract the same amount." He then dragged a 1 weight to both sides and said, " $x$ equals -9." Again, Jim used two steps to get to the answer instead of adding 6 to both sides. This was due to the visual and dynamic interactions with the technology applet. I drew his attention to both previous tasks $x+8=21$ and $x-6=-15$ and asked him if there was a faster way to solve them. He looked back at his previous work and said, "I could have taken away 8 here" for the task $x+8=21$. Similarly for the task $x-6=-15$, he said, "I get it, I could have just taken away -6 and made it zero. It [would have] made me figure out what -9 was faster." He realized by simply taking away, or adding 6 to both sides of the equation $x-6=-15$, he could have found that $x$ was equal to -9 more quickly. 


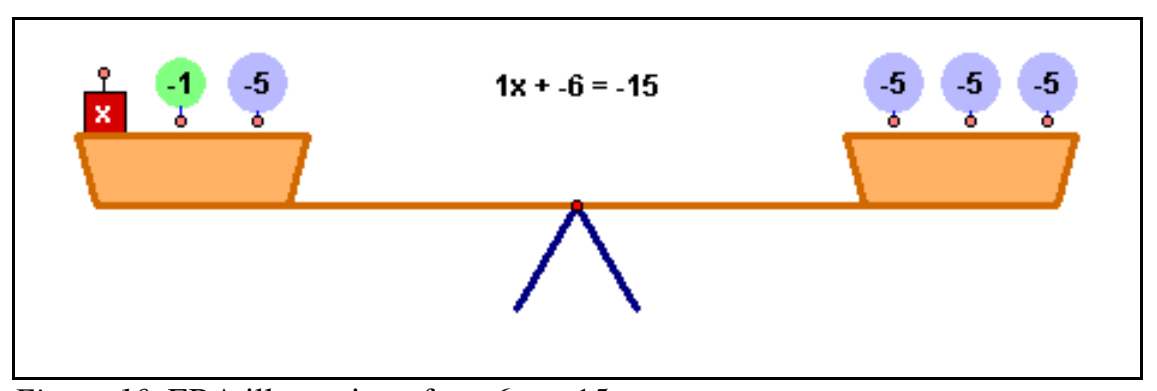

Figure 10. EBA illustration of $\mathrm{x}-6=-15$

The first task he solved without the computer was $x+7=-10$. He algebraically solved this task and would have gotten the correct answer if he had not subtracted incorrectly. At first he said that $x$ equaled 3 , but then realized that $x$ was -17 . When I asked what changed his mind about his answer he said, "Because of what we did before with adding and subtracting negatives. Like where are those examples? Like when we were talking about $5--12$." He had used his previous knowledge of addition and subtraction of integers that he had gained during the second module to be aware of the signs and to check the accurateness of his answer. After this task, he correctly solved all 1-step equations algebraically.

At this point, I asked him to solve the 2 -step equation $2 m+7=15$ to see if he could extend his knowledge of solving the task algebraically to 2-step equations as well as to see if he remembered to subtract before dividing first. He did not use the computer to solve this task. He first subtracted 7 from both sides and reasoned, "Because it makes it easier to get to zero" and pointed to the left side that contained the variable. He knew from the technology applet that he had to eliminate the constant that occupied the same pan as the variable in order to be able to easily solve the equation. He then said, "We have $2 m=8$, so we take away 2. Oh wait, times, oh, I see, $m$ equals 4 ...because 2 times 4 equals 8 and 8 divided by 2 equals 4." At this point, he looked at the computer screen that modeled the equation but did not manipulate the objects. He noted that there were two $m$-weights and not two 1-weights that were on the left pan, so he knew it had to be division that he had to use to find the end value. While he did not write the last transformation step, hence jumping from $2 m=8$ to $m=4$, he divided both sides by two.

Jim algebraically and correctly solved more 2-step equations without the computer applet. He knew to subtract or add before dividing. However, similar to the task above, he never wrote the last step showing the division of both sides. Instead, he wrote the coefficient and variable equal to number and then what the variable equaled. While his written work might not be judged as algebraic, he was applying the transformation to both sides of the equation to find his answer. 


\section{Summary of Interaction and Understanding with Technology}

- Jim exhibited a relational view of the equals sign for numerical equations and the comparison of numbers and units as well as equivalence of two expressions when one of the expressions contained a variable. He no longer believed that the equal sign meant the value of the variable.

- Jim accurately solved 1-step and 2-step equations using the algebraic transformations. However, when he wrote his solutions to the 2-step equations, he did not illustrate division of the coefficient to both sides, rather he simply went from multiplication of a coefficient equaling a value to the equivalent equation of the variable equaling a value.

- Since Jim was able to obtain an answer to 1-step equations without the computer, he tried to initially solve the equation in his head and then modeled the solution procedure on the computer.

- Jim used the manipulation, visual model, and instantaneous feedback to try multiple strategies to solve 2-step equations.

- Jim believed that the visual model and manipulation features allowed him to solve 2-step equations more easily than he was able to do before working with technology because it helped him to find the solution since he could create an equivalent 1-step equation involving only multiplication of a coefficient.

- Due to the visual, manipulation, and feedback characteristics of the applet, Jim noticed that the addition of one value to one side of the equation was the same as the removal of the opposite value from the other side of the equation.

- Jim used the visual aspect of the applet to intuitively tell him which operation to apply because he knew that he needed to get the variable weights by themselves. Even though the technology illustration led Jim to use multiple steps to solve the task instead of one single operation, he recognized this and solved the equations using one step on paper.

\section{Discussion}

Jim had a relational view of the equal sign for all equations without variables and in the comparison of values and units. Furthermore, he was able to justify non-canonical forms using this relational viewpoint. Yet, his relational viewpoint strictly included numerical equations, for when a variable entered the equation, he did not believe that the two expressions separated by the equal sign were the same value, but rather instead believed the equal sign signaled for him to solve the task and referred to the value he obtained for the variable. Thus, the equal sign in the equation meant that the variable equaled a specific value.

Even though Jim had a relational view of the equal sign for numerical equations and comparison of units, he did not algebraically solve 1-step and 2-step equations, unlike the tendency shown in previous literature, rather he solved equations using the calculated guess and check method. He could accurately solve 1-step equations using this method, but could not solve 2-step equations using this method. He was able to make logical inferences about the range values for the variable in 2-step equations and was 
cognizant of the fact that he was unable to solve 2-step equations.

When he began working with the applet, he visually understood that the equal sign in an equation meant that each expression had the same value as the other expression. Furthermore, he discovered many strategies to maintain the balance of the pans and the equality of the expressions. After discovering these strategies by manipulating the objects on the pans, he modeled the 2-step equation that he previously had difficulty solving. Because the applet allowed him to drag different values and see the resulting affect of that manipulation on the pans and corresponding equation, Jim was able to test different intuitive ideas about how to solve 2-step equations.

During the process of working with the technology applet, he discovered the relationship of adding a negative to one side and the removing of a positive value from the other side, the correlation of operations and movements of the applet, and why he needed to subtract or add prior to dividing to solve 2step equations. He also came to realize that there was a faster way to solve equations by looking at the pattern of his written work versus operating solely on the visual model of the equation. Although this method of solving on paper was faster than moving the objects on the applet, he liked working with the applet because it had helped him to see how to make an equivalent, simpler equation to the original equation, so that he could solve it in his head. After discovering the relationships between equality of expressions and the balancing of the pans, and the connection between the manipulation of the objects and solving an equation, Jim was able to consistently solve both 1-step and 2-step equations algebraically. 


\section{CHAPTER 8}

\section{CASE STUDY: CARLA}

\section{Carla's Background}

During the time of the study, Carla was in eighth grade Standard Math. Standard Math mainly covers pre-algebra material. A student in Standard Math in eighth grade will take algebra in ninth grade. Data collection was at the end of the school year; thus, Carla had already covered pre-algebra material, such as creating and solving word problems, using formulas and functions, and solving equations containing one variable.

Carla was a very quiet, hesitant, and thoughtful student. During our sessions, she would find an answer but not provide a justification for her answer immediately. During these times she would look at me and assure me that she was thinking about the task. I provided prompts to get her to talk about what was going through her mind. These prompts usually worked, and on a few occasions, she would open up and state a lengthy reason for her answer. When she did this, the line of reasoning was usually well thought out. Carla also had a nervous laugh that she would do after I asked her to explain her answers. I was concerned that I was making her nervous by asking her to explain her reasoning each time she obtained and answer, so I reiterated that I was only asking for her reasoning to understand how she thought about the task and not because her answer was incorrect. She assured me that she was fine and said, "I always laugh, a lot actually." While it seemed that she laughed at herself because she was unsure of her answers and reasoning, I began to notice that she performed this laugh throughout the interview and even did it when she was selfassured of her answer. However, it is important to note that she also laughed when she realized that she missed something in the task or computed an incorrect answer. When she did get an incorrect answer, you could also hear a hint of embarrassment of getting the wrong answer in her voice.

Like most of the participants, Carla used rules to help her answer addition and subtraction tasks. Prior to working with the computer, she would often justify her answer by telling me that she did a certain procedure because "that's what Mrs. Coffee told us to do" or "we learned to do it that way in Mrs. Coffee's class." While Carla used rules in her math class, she did not seem to have used a number line to help her find the answer. If she did, she did not remember doing so; furthermore, she could not use a number line to 
aid her in solving an addition or subtraction task. When I asked her to use a number line to show me how to obtain an answer, she used her abstract rules to solve for the answer and then plotted the answer on the number line. Yet after using the technology, Carla was able to reference the visual she saw in the subtraction arrow applet (SAA) and subtraction dot applet (SDA). She also was able to use this in conjunction with a number line to help her solve subtraction tasks.

\section{Negatives and Operations on Integers \\ Prior Conceptions on Addition}

Carla had a procedural understanding of addition of integers. While she frequently quoted her teacher in her explanation, she still was able to provide the correct idea behind addition of negative numbers. Sometimes these verbal rules were correct and matched her solution procedure, but other times, she would state a rule that did not apply to the particular task, and it would not correspond to her solution procedure. Thus, she would get the correct answer, but her language did not support it. For instance, when she solved $10+-3$, she correctly explained her procedure and provided a correct, corresponding rule. Yet when she solved $-5+-7$, she obtained the correct answer but her rule did not correspond with her solution.

$\mathrm{N}$ : How did you get the answer of 7 to $10+-3$ ?

C: Mrs. Coffee says that if you are adding a negative to a positive, then you're basically subtracting it. So I subtracted 3.

$\mathrm{N}$ : Good. What about this one? You wrote that $-5+-7$ was equal to -12 .

C: Well, two negatives together would equal a negative. No, well, that's if you're multiplying. Uh... [shakes head and laughs nervously]

$\mathrm{N}$ : Go ahead; tell me what you're thinking about.

C: Like if it's a negative and positive, you're going to go with the positive, I think...so here they are both negatives, so we get a negative.

She remembered that her teacher told her that adding a negative was similar to subtraction of a positive number, yet when it came to solving the addition of two negative numbers, she spouted off a rule that did not fit with her negative answer. Furthermore, she was unsure if that rule applied to addition or multiplication. She decided that the rule applied to only multiplication of negatives, yet did not change her initial answer, which she supposedly had gotten from using the rule, even though this was not possible. Then she tried to notice similarities and differences between the first and second task, and created or adapted a rule to fit the situation. Since she obtained a positive sum when she added a positive and negative together, she believed that maybe when she added two negative addends together it would give her a negative sum. She knew the correct answer, but had difficulty justifying her answer because she tried to rely on her memorization of dispensed rules. 
Because she was unsure in her justification, I asked her if she could explain her answer using a number line. She told me that she had used a number line before to solve these types of tasks and could use one now to show me. After giving her a number line, she looked at her answers to all of the completed tasks and placed a dot on the numbers that represented her answers (see Figure 1).

$\mathrm{N}$ : Ok, so tell me how you used the number line.

C: I used 10 plus 3 for 13 , and then 10 plus -3 for 7 , and then -5 and -7 for -12 .

$\mathrm{N}$ : Ok. Have you ever used a number line to solve just one problem?

C: Maybe. We might have, but I don't remember doing it.

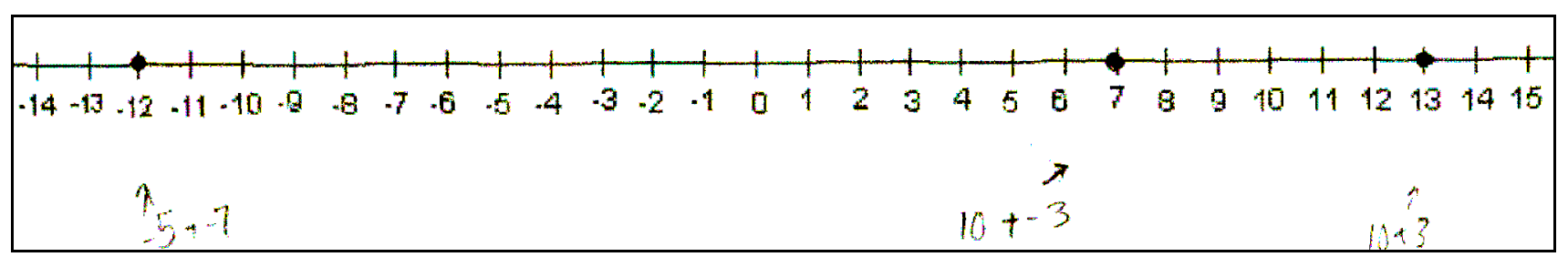

Figure 1. Carla's use of a number line to solve $10+3,10+-3$, and $-5+-7$

Given how Carla used the number line, it seemed that she had not seen a number line used as a tool to represent a mathematical expression, rather she only used it to illustrate a number and, in this case, the answers to each expression.

Carla seemed to have both a unary and binary (Kieran, 2007) understanding of addition of negative numbers depending on the type of expression presented. For addition tasks involving a negative and positive addend, Carla knew that it was similar to subtraction, a unary view. Yet, for addition tasks involving two negative addends, she did not view it as subtraction but rather believed it to be addition with a negative sign tacked on to the answer, a binary view.

Carla was unable to use the number line as a problem-solving tool for abstract addition tasks. I wanted to see if she would be able to use the verbal context to her justify her answer. I asked her if she had ever done word problems before, and she told me that in Mrs. Coffee's class they used the computer to match graphs and verbal descriptions of a task.

We had to write a story matching the numbers and the events of the story... and it would ask like John's dog stopped for 2 hours, and then he went up a hill for 8 minutes, and then stopped to take a rest, and then he went down the hill...then we drew a graph and wrote a story about it.

Carla described how her class moved between the verbal and graphical context of a problem. When I asked if she could write me story about $-5+-7$, she chose to use the context of debt (see Figure 2). Carla seemed to easily move from the abstract to the contextual representation and told me that she had chosen debt as her context because the numbers were negative. 


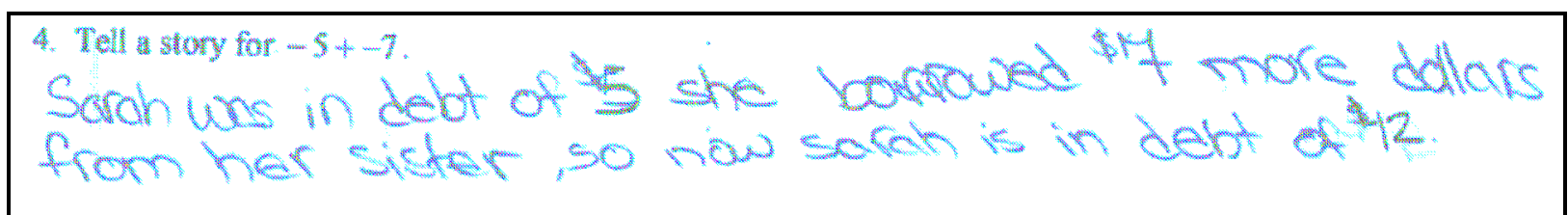

Figure 2. Carla's story for $-5+-7$

Given that Carla easily moved from the abstract to verbal context, but not from the abstract to number line context, I wanted to see if she could transition from a number line to an abstract context. I asked her to write an equation that represented a number line with a dot on the -6 and a dot on the 5 . Instead of constructing equations for the number line visual, she wrote the inequalities, $-6 \leq 5$ and $-6 \geq 5$, in that order beneath the number line.

$\mathrm{N}$ : So explain to me what you wrote.

C: It would be an inequality, like it would be bigger than or less than -6 or 5 .

$\mathrm{N}$ : So what made you think about an inequality?

C: Because I had two dots on -6 and 5, and they are not separated.

$\mathrm{N}$ : Can you talk more about that?

C: They are not connected. There's not a stop point.

$\mathrm{N}$ : Could you show me the $-6 \leq 5$ on this number line?

C: I'd draw a circle colored in [pointed to -6 dot], and going to the left.

At first it looked like Carla tried to relate the values on the number line instead of viewing them as independent points. However, upon closer inspection, she really did not relate -6 and 5 with respect to each other but with respect to a variable. When I asked her to show me where the $-6 \leq 5$ was depicted on this number line, she pointed to the shaded circle on the dot at -6 and then motioned with her pencil to go to the left; thus, she was showing me the representation of $x \leq-6$. This helped explain her use of the word 'endpoint.' She believed that the circles were placed where the variable values started or ended. She needed to include an arrow to show which direction the values were traveling. Since there were two dots, she wanted to include both $x \leq-6$ and $x \geq 5$, yet she wrote these ideas as $-6 \leq 5$ and $-6 \geq 5$. After her explanation of her inequality answers, I asked if she could create another equation using the dots on this number line, to which she responded that she could not. This scenario further supports that Carla was not readily familiar with using the number line as a tool to help her solve subtraction and addition tasks.

\section{Prior Conceptions on Subtraction}

Carla's conceptions of subtraction could not easily be categorized as take-away or a distance because she frequently referred to rules instead of words to describe how she operated on a task.

Furthermore, she was unable to show me using a number line because she had not used a number line to solve addition or subtraction tasks. By working through the tasks, Carla came to understand or remembered 
how she could use the number line to solve subtraction tasks, and I eventually saw that she had a take-away notion of subtraction. Yet, this notion could be due to our interaction together and not from previous experience.

Carla could correctly solve tasks in the form $a-b$ and $-a-b$ because she would mentally transform them into $a+-b$ and $-a+-b$ respectively. She did not explicitly state this, but since she (1) knew how to add negative numbers, (2) did not quote a specific rule, (3) did not use a number line, and (4) said she just "knew" the answer, it seemed that this was what she was doing. While she was able to obtain a correct answer to these types of tasks, she could not accurately solve tasks with a negative subtrahend.

To solve tasks that had a negative subtrahend, she quoted rules that did not match her procedure. For solving tasks in the form $a--b$, she mentally transformed the task into $-a-b$ by redistributing the subtraction sign to the minuend. For solving tasks in the form $-a--b$, she seemed to change the subtraction sign into an addition sign, or she would make an analogous expression by changing all of the signs and getting the expression $a+b$ and then compensating for the change in signs by tacking on a negative sign to the answer. Furthermore, the language that she used to describe her processes seemed unclear and did not always correspond to her procedure. Yet, as I asked her questions about these types of tasks, she became familiar with the number line and eventually started to use it to support her answers. There were three tasks that provided some insight into her thought processes of subtracting a negative subtrahend: $5--12,-4--6$, and $-4--1$.

When she solved the task $5--12$ she got -17 and I asked her how she had thought about the task.

C: I thought you would add -12 and 5 together.

$\mathrm{N}$ : And why did you think you would add them together?

$\mathrm{C}$ : Because it's a negative and you're already subtracting a 5 from the -12 .

$\mathrm{N}$ : So are you saying that since you subtracted a negative there that you would add the numbers?

C: Yeah, well, no. I don't know if I'm right...I just added -12 and 5 together, because -5 , well 5 subtracted from -12 would give you a negative...because -12 is less than 5 , but... .because it was negative and subtracting, and I remember if it's a negative you're like adding it.

Carla's first statement does not fit with her answer of -17 . If she added -12 and 5 together, she would have gotten -7 . Given her solutions to the addition tasks, she would know this to be true. Therefore, she was not expressing herself correctly in the first statement. Her second statement provided a little more detail into what she was thinking, yet still was not totally correct. She was trying to say that since there was a subtraction sign and a negative sign, she had to add the values. However, she expressed it in terms of subtracting a 5 from a -12 , which would result in the expression $-12-5$. Now, given that she knew how to solve these types of expressions and that $-12-5=-17$, this could have been how she thought about the 
expression 5--12. Another possible idea is that she misspoke when she said that she was subtracting the -5 from the -12 , for she could have meant that she was subtracting the -12 from the -5 ; thus the expression - 5 - 12. This is plausible since Murray (1985) showed that for unfamiliar subtraction tasks in the form $a--b$, students have been known to redistribute one of the signs to the minuend and hence believe that $a--b=-a-b$. Since she would have known that $-5-12=-17$, she could have thought about the task in that manner as well.

Carla's last few statements incorporated all her previous ideas that subtraction of negatives is like adding, and adding two negatives elicits a negative number. Using these two ideas, it seems reasonable that she remembered someone telling her that subtraction of a negative number was like adding, so she added the absolute values of the numbers to get 17 . Then since she knew that subtracting a negative was like addition, she believed that she was adding two negative numbers, because she saw two negative signs. When she added two negative numbers, she knew that she had to keep the negative sign on the answer. Therefore, the magnitude of the answer resulted from the addition of the absolute value of the minuend and subtrahend, and the sign resulted from the fact that there were two negative signs (i.e., a negative sign and subtraction sign).

It is important to note that while she was trying to explain to me her answer, she had pointed to a number line in front of her and showed me that -12 was on the negative side of the number line, as was her answer of -17. This is important because of what followed in regards to the number line. Because I had difficulty trying to understand how she was thinking about this task, I asked her to check her answer using a calculator. I had hoped that seeing the correct answer and having her figure out why the correct answer was in fact correct, would give her and myself some insight of how she had incorrectly thought about the task. When she saw that the correct answer was 17, she laughed her nervous laugh and said, "I guess since we're subtracting 12 negative from the 5 , it would have to be a, we went up the number line 12 . Yeah." When she said this, she had pointed to the -17 on the number line and then moved her finger to the positive 17. I think she saw the symmetric relationship of the values and tried to integrate her knowledge of addition when she subtracted a negative with the positive value of the answer she got from her calculator. She said, "So I guess you can start right here, and since you're subtracting a negative from a positive, you have to go up." She placed her pencil on the 5 , did not count up by 12 , but simply moved to the right until she landed on the positive 17. The fact that she did not count up by 12 could indicate that (1) she still did not know how to operate on the number line, and was thinking of the numbers and operations in a pure abstract way; or (2) that she knew where to land on the number line without really using it to get to the ending position. 
When solving the task $-4--6$, she obtained the value of -10 and described her procedure in terms of addition.

C: This was easy because it was two negatives, and you're subtracting, and two negatives being subtracted is like a positive, which is like adding them together.

$\mathrm{N}$ : So if you have two negative numbers, you add them?

C: Yeah, because -4 minus -6 and which -4 and -6 are two negatives, and subtracting them would be like adding them together.

Once again, Carla misapplied a rule that she had learned to help her solve subtraction tasks involving a negative subtrahend. She had heard the rule that subtraction of a negative was like adding, but she did not understand it. Instead of adding 6 to -4 to obtain the answer of 2 , she instead believed that the rule required her to add the absolute value of the numbers and then tack on the negative sign because both numbers were negative. Thus, addition to her meant adding the absolute values of both the subtrahend and minuend instead of adding the absolute value of the subtrahend to the minuend.

After she described how she solved the task- $4--6$, I wrote the task $-4+-6$ directly beneath the previous one and asked her if she noticed a difference between the two tasks and if she could solve the second task for me.

C: The second one is negative te..tw.. [pause] I'm lost. I'm thinking -2 , but I'm not really sure of it.

$\mathrm{N}$ : Why do you think it might be -2 ?

C: I think it still might be -10 .

$\mathrm{N}$ : For which one?

C: $-4+-6$, but I don't know. [laughs] Um, so it would be positive 2 .

$\mathrm{N}$ : I saw you using a number line, what were you doing there?

C: I added -6 to the -4 .

I had asked her this task, because I was trying to get her to compare her answers. Prior to solving subtraction tasks, she could accurately solve addition tasks with negative numbers. Therefore, I thought she would definitely answer that $-4+-6$ was -10 and realize that $-4--6$ was subtraction instead of addition; therefore $-4--6$ could not be -10 . She realized that the tasks differed in their operation, but instead of changing her answer to the subtraction task, she changed her answer to the addition task. To try to justify her answer for the addition task, she grabbed a number line, started at the -4 and moved to the right 6 spaces to land on the 2. This was the first time she used the operation and second number in the expression while operating on the number line. Even though it was interesting that she finally showed me that she could use a number line, she moved the wrong way for adding a negative number. This was not surprising since she did not seem to know how to integrate the operation and second number of a task when using a number line. Because she moved to the right on the number line 6 spaces, I asked her whether she was adding -6 or positive 6 when she moved to the right. She said, "I don't know." Due to her uncertainty, 
I wrote the task $-4+6$ and asked her to solve this task. Carla said, "So you would go up 6, or add -4 to 6 and still get positive 2." Instead of performing the same movement as she did for $-4+-6$ : starting at the 4 and moving to the right 6 , in solving $-4+6$ she started at the 6 and moved to the left 4 spaces and landed on -2 . She most likely thought that because one task added a negative six and the other task added a positive six, she knew she had to move in opposite directions. Instead of starting both times at the -4 , she instead switched her initial position so she could move in opposite directions, but obtain the same answer. I think the sequence of these tasks and her initial answers impacted her movement on the number line. Instead of using the number line consistently, she used it so that she could obtain the same answers she had gotten when she solved the tasks abstractly. After showing me how she got 2 for tasks $-4+6$ and $-4+-6$ using the number line, I asked her if these tasks were different.

C: Not really.

$\mathrm{N}$ : So what are we adding here?

C: Positive 6

$\mathrm{N}$ : And here?

C: Negative 6.

$\mathrm{N}$ : So are they different?

C: No because you're still getting 2 as your answer.

She saw that each task contained a different second addend, but she believed that this did not affect the way she traveled on the number line. Thus both tasks resulted in same answer. It is strange that she could solve addition tasks well prior to this incident. What could have been the cause of her inability to solve these addition tasks correctly was the sequence of questions, cognitive overload, and Carla's attempt to use the number line to justify and figure out her answers. Because she seemed very confused, I asked her to utilize a calculator to check her answers.

C: Oh, yeah, $-4+-6$ is 10 , negative.

$\mathrm{N}$ : What about this one, $-4+6$ ?

C: 2. [sighs heavily with frustration]

$\mathrm{N}$ : So what do you think happens when we add a positive 6 using the number line?

C: We count up,

$\mathrm{N}$ : What do you think happens when we add a -6 ?

C: We're stuck with a negative as your answer, cause you go left. If you add a negative, you move to the left.

To make sure she truly saw the connection between solving addition tasks arithmetically and solving them on a number line, I asked her to solve a few addition tasks with negative numbers. She solved them all arithmetically correctly and solved them accurately using the number line as well. She told me, "When I add a negative number I go to the left, and when I add a positive number, I go to the right." When I asked if this made sense using a number line to solve these tasks, she said, "Yeah!" She said this almost with sense of relief and resignation. Her facial expressions showed that she was happy to have realized how to support 
her arithmetical answer, but it was almost as if she felt that she should have known this already and felt bad that she had not.

At this point, we returned to subtraction. I asked her to explain to me how she had gotten the answer of -5 to the task $-4--1$.

I did -4 subtracted from -1, and I added the two negatives together. So like I just took off the negatives and put a 5 because to make the two negatives into a positive, and make the subtraction sign into a positive, and got 5 and then put the negative sign back on.

Once again she was using the rule that subtraction of a negative number was like addition, only she misapplied the rule to mean that she had to add the absolute values of the numbers and then tack on the negative sign at the end. Similarly, much as she described when solving $5--12$, she interchanged the minuend and subtrahend, for she said that she subtracted the minuend from the subtrahend, in this case -4 from the -1 , even though she did not. It is also interesting in how she described her procedure. Much like Murray (1985) noted, (1) she changed all of the signs, not just the subtraction and negative sign before the 1, to positive, (2) added the numbers, and (3) then believed that if she changed all the signs back to negatives, this meant she also had to change the sign of her answer. Thus, she reasoned that since $4+5=1$, then $-4--1=-5$. Given that we had just done addition, and she was able to do addition of negative numbers accurately using both a number line and arithmetically, I asked her to solve $-4+-1$, which I wrote directly beneath $-4--1$. She solved $-4+-1$ and got -5 and then showed me on the number line that the answer was in fact -5 . Then I asked her if there was anything different between $-4+-1$ and $-4--1$, to which she responded, "No, it would still be -5 ." She saw that one was addition and one was subtraction, but since the tasks elicited the same answer, she believed that they acted the same; hence there were no differences in terms of the answer.

\section{Discussion on Prior Conceptions}

Carla could correctly compute addition tasks involving negative numbers abstractly. She understood that adding a negative to a positive addend was similar to subtraction, but did not view that adding two negative addends was also subtraction; instead, she viewed it as addition of absolute values with the tacking on of a negative sign to the answer. Furthermore, Carla spouted off rules she had been given in order to justify her answers. Rules involving a positive and negative addend matched her answer, but rules involving two negative addends, did not. This parroting of rules is similar to what Bell (1982), Murray (1985), and Human and Murray (1987) described in their studies. They saw that students misapplied rules that they had heard from their teacher. Yet, in these studies, the students actually implemented the misapplied rule to find 
their answer, whereas Carla only verbally conveyed the incorrect rule in her justification, but implemented a different procedure to find the correct answer. There was a disconnect between her procedure and explanation.

Carla may have had to rely on memorized rules for her justification because she could not verbalize how she actually attempted to solve the tasks. She did not seem to have many representations in which to use to explain her answer. In fact, when I had asked her to translate from an abstract representation to a number line representation and vice versa, she could not do it. Instead, she plotted her answers on a number line, which was described by Bruno and Martinon (1996) who saw that students had used the number line to simply plot independent points with no relation to each other that was demonstrated by the expression. When translating from a number line to an abstract expression, Carla used incorrect inequalities. This outcome was not discussed in previous literature. Despite not being able to translate between the number line and abstract contexts, she was successfully able to translate between abstract and verbal context and chose the quality of debt to do so. While Bruno and Martinon (1996) showed that most students chose debt to explain an expression with negative numbers, they also said that moving between a number line and abstract expression was often easier to do than moving between abstract and verbal. However, this was not true for Carla.

As Carla worked through subtraction tasks, she came to understand how to use the number line to help her solve addition tasks. Thus, by the end of our interview, she was able to start at the first addend and know that she had to move right if the sign in the second addend was positive and left if the second addend was negative.

In agreement with Murray's (1985) findings, Carla was able to solve addition tasks more accurately than she was able to solve subtraction tasks. Carla was able to solve subtraction tasks that contained a positive subtrahend arithmetically. While she could not use a number line, and could not verbally explain her reasoning clearly, it seemed that she mentally transformed tasks such as $a-b$ and $-a-b$ into $a+-b$ and $-a+-b$, respectively.

Carla could not accurately solve subtraction tasks containing a negative subtrahend. Given any subtraction expression with a negative subtrahend, she always got the answer as if she added the absolute values of the minuend and subtrahend and then tacked on a negative sign. However, by her verbal explanation, she dealt with tasks for the form $a--b$ differently than those of the form $-a--b$. For tasks with a positive minuend and negative subtrahend, she transformed $a--b$ into $-b-a$ and solved this new task as if it were $-b+-a$. Murray (1985) also saw students move one of the negative signs to the 
minuend and solve the new task as if both expressions were equivalent. Moreover, Carla believed that this had been the correct procedure because she had heard a rule that subtraction of a negative is like addition. While this is a correct rule to follow when subtraction a negative subtrahend, she misinterpreted how to apply it. This is interesting because in previous literature (Bell, 1982, Human \& Murray, 1987; Murray, 1985), most researchers saw students apply a correct rule in the wrong context, which caused the student to obtain the wrong answer. However, in Carla's case, she stated a correct rule in the correct context, but did not apply it correctly; thus she obtained the wrong answer.

For tasks in the form $-a--b$, she changed all of the signs; transforming it into $a+b$, solved this new expression to obtain the answer of $c$, and then believed that $-c$ was the answer to $-a--b$ because she believed that $-(a+b)=-a--b$. Since she was unfamiliar with how to use the number line with subtraction tasks, she could not support her answers except by parroting a rule that subtracting two negative numbers was like addition. The rule, as stated by Carla, is not completely correct. If one has a negative minuend and subtrahend, even though the operation is subtraction, it will be similar to addition in that one will add the absolute value of the subtrahend; however, this is not the same as adding together the minuend and subtrahend. Furthermore, she used the fact that she needed to change the signs when subtracting negative numbers; however, she changed all of the signs instead of only the subtraction and negative sign of the subtrahend. Unlike the correct rule that she supplied for solving $a--b$, she misinterpreted and misapplied a rule for solving tasks in the form $-a--b$.

By the end of the interview, Carla could only use the number line for solving addition tasks after comparing tasks and answers. She knew that she would start at the first addend and move to the right if the second addend was positive, and move to the left if the second addend was negative. She had no issues crossing over zero; therefore, she had a continuous view (Peled et al, 1989) of the number line. Carla achieved Level 4 in the Quantitative Dimension (QD) and Level 1 in the Number Line Dimension (NLD) with respect to addition. However, since she could not use the number line to help her solve subtraction tasks, she attained Level 1 in the NLD with respect to subtraction. Because she could interpret negative numbers as debt, she achieved at least Level 2 in the QD. She did not fit cleanly into Level 3 or 4 in the QD with respect to subtraction because she could not solve subtraction tasks in the form $-a--b$, which was a requirement of Level 3 but could solve tasks in the form $a--b$, which was a requirement of Level 4 but not Level 3. Therefore, she attained Level 2*, as an asterisk represents that Carla was able to solve higherlevel tasks than her number level indicates, but she did not put the answer or task into context. 


\section{Summary of Carla's Prior Conceptions}

- Carla had both a conceptual and procedural understanding of addition of negative numbers. She saw the relationship between the addition of a negative as subtraction. She was also able to move between a contextual representation and abstract representation. She was unable to move between a number line representation and an abstract representation.

- Carla was unable to correctly solve subtraction tasks containing a negative subtrahend. She mentally transformed the tasks $a--b$ into $-a-b$ and solved the latter task. For tasks in the form $-a--b$, she changed all the signs to positive, added the numbers, and then tacked on a negative sign to the answer. Therefore, she believed that $-(a+b)=-a--b$. Carla used memorized rules to justify these answers.

- Initially, Carla did not know how to use the number line to help her solve addition tasks. Once she compared addition tasks and their answers and saw how it correlated with the number line, she believed that the signs of the second number told her which way to travel. Unfortunately, Carla did not apply the idea of movement on the number line with subtraction tasks.

- Carla attained Level 4 in both of Peled's NLD and QD for addition.

- Carla attained Level 1 in Peled's NLD for subtraction. Carla fit unusually into Peled's theory in the QD, for she did not fit cleanly into Level 3 or Level 4; she attained L2* in the QD with respect to subtraction.

- Carla exhibited a combination of unary and binary understanding of negative numbers. The way she viewed negative numbers depended on the form of the task.

\section{Analysis of Interaction with Subtraction Applets}

Because Carla could accurately solve addition tasks with and without a number line, we did not use the addition arrow applet (AAA). However, since she recently had learned how to use the number line to help her solve addition tasks by moving the direction indicated by the sign of the second addend and moving the number of spaces represented by the magnitude of the second addend, I decided to show her the subtraction arrow applet (SAA) prior to the subtraction dot applet (SDA), since the SAA also used the sign and magnitude of the second number, the subtrahend, to determine the direction and how many spaces to travel away from the first number, the minuend.

\section{Carla's Interaction and Understanding with Technology}

When using the SAA, I did not have the hot-linked equation representing the visual on the number line because I wanted to see how the sole illustration of the arrow value appearing inside the arrow impacted her method of solving subtraction tasks. After manipulating the SAA without attempting to solve any tasks, Carla was able tell me the significance of the blue stick, where the arrow began, where the arrow ended, and the portrayed arrow value in terms of subtraction. As she was exploring the SAA, she had moved the blue stick to the 15 , moved the end of the arrow to 3 , pressed the show arrow value button and 
said, "Twelve is in between 15 and 3. It's like subtracting by 3." I had not expressed that the SAA was depicting subtraction, and it was somewhat surprising that she noticed it was subtraction since she could not translate between the number line and abstract representation when dealing with subtraction. Furthermore, she used the number inside the arrow as the subtrahend, much like the SAA was intended to be used; yet she saw the relationship between the minuend and difference as the distance in between them.

After stating her discovery, I asked her to move the arrow to the right by one. She noticed that each time that she did this, the number inside the arrow decremented by one. I asked her what would happen if she moved the arrow so that the end of the arrow landed on 15. She said, "It [the answer] would just be 15. It would be like 15 -0" (see Figure 3).

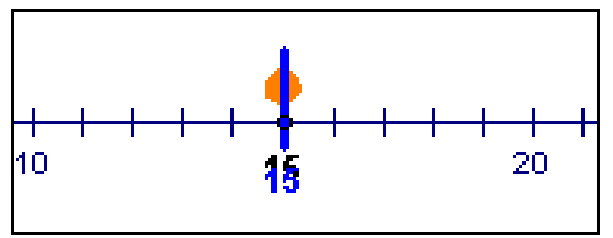

Figure 3. Model of $15-0$ using the SAA

When I asked her to predict what would happen if she moved the arrow to the right one more space she said, "It would be a negative...because we're going to the right." She knew that each time she moved to the right, the number inside the arrow kept getting smaller; thus, since she was positioned where the arrow value was 0 , she knew if she moved one more step to the right, she would get a negative value inside the arrow. To see if she could apply her discoveries about the arrow value and the relationship between the value and the subtrahend of an expression, I asked her to model some abstract subtraction tasks.

When I asked her to model $5-1$ on the SAA, she moved the blue stick to the 5 and moved the orange arrow to the left by 1 . She told me she moved the objects in this fashion "because 5 subtracted by 1 is 4 , and 1 is in the middle between 4 and 5." Not only could she translate from number line to abstract expression, she could also translate from abstract expression to the number line. Furthermore, she was able to see the relationship between the numbers instead of plotting independent points. Moreover, she saw the connection between the amount taken away from the minuend and the distance between the minuend and the answer. She also told me that she knew how many to take away because she could "see it in between" and knew the answer was supposed to be "where the orange arrow ended." It is important to note that the hot-linked expression was still hidden, and she was making these connections to subtraction on her own. To make certain that she consistently knew how to manipulate the SAA to solve subtraction tasks, I asked her to model 5-2 and 5-0, which she did correctly. I then asked her to predict how she would move and the 
answer to the task 5--1. She said, "I'd move it to the $6 \ldots$ because negative is going to the right." She then laughed as if she was surprised at herself for knowing the answer. I asked her why she laughed and she said, "I always laugh."

She easily saw the relationship between the arrow value and the value of the subtrahend in the subtraction task. Because of this, she could now solve subtraction tasks involving a positive minuend and negative subtrahend. In fact, when I asked her to predict the next task, $5--3$, she said, "That's 8 because 5 plus 3 is 8." By using the SAA, she was able to see that when she subtracted a negative number, it was like adding the two numbers together; hence, moving right on the number line. I asked her to explain why she knew it was plus 3, and she said, "Because when you subtract two negatives, well, when you subtract a number from a negative, it's just like adding." While she was able to use the number line to correctly answer the task, she was still getting confused with the verbal description of the reasoning behind it. After she spoke her first couple of words, she realized that she did not have two negative numbers, so she had to adjust her rule. She adjusted it so that she added when she "subtracted a number from a negative." This rule does not make sense for this task, as her minuend was not negative. However, if she had interchanged the words 'number' and 'negative,' the rule would have made sense and would have been correct. To make sure that she understood, I asked her a couple of more subtraction tasks involving a positive minuend and negative subtrahend. Each task she completed accurately and justified it by saying that it was liked addition since she was "subtracting a negative."

Given that she understood how to solve subtraction tasks involving a negative subtrahend and positive minuend, I asked her to use the SAA to solve the task - 4--1, to see if addition of the absolute value of the subtrahend still held when the minuend was negative. Before manipulating the SAA, she guessed, "It's going to be -5 ." I asked her to show me on the SAA, and she moved the blue stick to the -4 and then moved the arrow to the left 1 so that it landed on the -5 . She said, "Yeah it would be -5 " (see Figure 4).

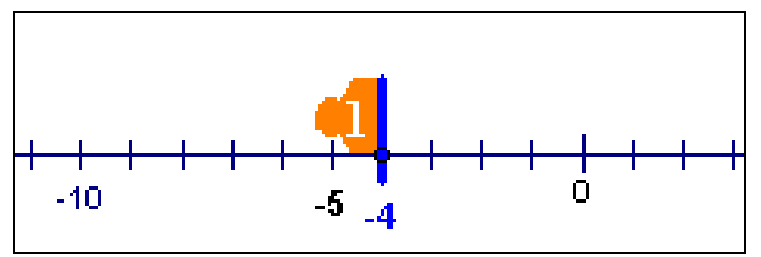

Figure 4. Carla trying to model $-4--1$ but instead modeling $-4-1$ on the SAA

$\mathrm{N}$ : So is this showing minus 1 or minus -1 ?

C: Minus, um, so, it would be -3 .

$\mathrm{N}$ : So why did you change your mind from -5 to -3 ?

C: Because -5 was just a positive 1 and -4 minus -3 equals -1 .

$\mathrm{N}$ : So if we moved the arrow back to the -5 , what would that problem have been? 
C: it would be -4 minus 1 .

Before having Carla display the equation, I wanted to see if she could figure out her mistake; therefore, I asked her if the SAA was showing that she was subtracting 1 or -1 from -4 . She realized that when she had moved the arrow to the left, she had been subtracting a positive 1 instead of a negative 1 . Not only did she change the arrow and move it to the right by one so that it landed on -3 , she also saw the connection between $-4--1=-3$ and $-4--3=-1$ because each of the values were emphasized on the SAA.

To see if she had just mistakenly gone to the left initially for the task $-4--1$, I asked her to solve $-3--2$. First I asked her to predict the answer. She told me that the answer would be -1 . When I asked her how she solved it she said, " 3 minus 2 without the signs is 1 , so put the negative signs back on, is -1 ." Thus, she used the analogous positive minuend and subtrahend, subtracted, and then multiplied the answer by -1 . When I asked her if she could show me how to solve it on the SAA, she moved the blue stick to the -3 and the arrow to the right 2 spaces and landed on the -1 . She exclaimed, "Oh, -1 " like she was surprised they were the same answer. When I asked her how she knew to move the arrow to the right 2 spaces she said, "Because you're subtracting a negative." After solving this task, I had asked her to complete various subtraction tasks involving different signed and same-signed minuends and subtrahends. Each task she completed accurately. Though she did not use the SAA, she justified her answer in terms of moving to the right if the subtrahend was negative, and moving to the left if the sign of the subtrahend was positive. Thus, instead of resorting to rules as she had done previously when solving subtraction tasks, she was using the visual of the number line in her head to solve the tasks and determining which way to move accordingly.

Since Carla could answer all subtraction tasks correctly, I wanted to see if she could recognize any patterns of subtraction of positive and negative numbers besides movement on a number line. Moreover, I wanted her to be able to correctly verbalize and understand the rule of subtraction of a negative number. Carla quickly noticed that when she subtracted a negative subtrahend from a positive minuend, it was as if she was adding the absolute value of the subtrahend to the minuend. I wanted her to build off that intuition to see if this same phenomenon happened when she had a negative minuend. While she could get the correct answers to these types of tasks, she did not seem to make the connection to addition. When I asked if her conjecture held for tasks in the form $-a--b$, she did not say no, but she did not say yes either. Instead she would tell me that she always had to move to the right $b$ spaces to find the answer. This shows that she was influenced by the applet. After having her calculate the answer for tasks in the form $-a--b$ and the equivalent tasks $-a+b$, she finally noticed that the answers were the same; however, it did not seem clear that she understood that they were equivalent tasks. When I asked her which task was easier for 
her to solve, $-a--b$ or $-a+-b$, it was surprising that she said the former, since she had more familiarity of solving addition tasks with negative numbers than subtraction of negative numbers. There may be two reasons for this answer.

One reason is that she did not believe the tasks to be equivalent, so defaulted to the original task. The second reason may be that since she had just worked with the SAA to solve these tasks, the procedural and visual notions were more current in her memory. Thus, she preferred this choice than to switching the signs and remembering her procedure for adding integers. The second reason seems more plausible for when I asked why she believed that $-a--b$ was easier than $-a+b$, she said, "because I know that it's still going to be going right without having to do all that switching signs." She went on to say that she could "see, that the answer was going to be negative" more easily for the task $-4--1$, because she knew she only had to go to the right by 1 . Because she had just worked with the SAA, she started visualizing the number line movement in her head immediately when she saw that the task involved the subtraction of a negative subtrahend. Whereas, the other method, she had to see that it was the subtraction of a negative number, remember which signs to change, change the signs, and then use her procedure for addition. This required more steps than the SAA method. Yet, eventually she was able to become quicker at changing the signs and noticed that in both cases she had to move to the right because subtraction of a negative was the same as addition of a positive number.

N: How would you solve $-6--4$ ?

C: -6 plus $4 \ldots$ because there was a negative and a positive, well, two negatives.

$\mathrm{N}$ : Talk a little more about that.

C: -6 and -4 are the negatives, but we change the -4 into a positive. So it would be -2 .

$\mathrm{N}$ : How did you get -2 ?

C: Because in both ways we go right 4 times from -6 .

Even though she was able to notice that in both cases she had to move to the right on the number line, she had difficulty verbalizing her reasoning in terms of addition and a negative. She did this quite often when solving these types of tasks. She always obtained the correct answer, knew that she traveled to the right in both tasks, and could express the task $-a--b$ in the form $-a+b$, but she would consistently say that she added the negative. For instance, when I asked if $-3--2$ was easier to solve than $-3+2$, after she had told me they were equivalent, she told me their differences and how they related.

They are basically the same...here, you're adding the two negatives, and here you're adding a negative and positive together. Here, you're adding the negatives, so you're switching this subtraction sign to an addition, and the -2 into a positive 2 . Both of them are -1 .

She pointed to the first expression when she said that she was adding two negatives and to the second expression when she said she was adding negative and positive number. I pointed to the first expression 
and said, "So we're adding a negative here?" She responded, "Well, really subtracting a negative, but that's like adding."

This idea of adding a negative could have been attributed to the SAA. Given that she knew that addition of a number required her to travel to the right and the subtraction of a negative number required her to travel to the right, when she visualized the SAA, she saw a negative number inside the arrow. Thus, the SAA gave Carla the visual support she needed in the movements. The appearance of a negative number inside an arrow traveling to the right may have encouraged her to use the language "addition of a negative." For instance, for the task $-3--2$, she would see the blue stick on -3 , the arrow pointed to the right, and a -2 contained inside the arrow (see Figure 5). Thus, it would not be such a far jump to reason that she was adding -2 to the -3 .

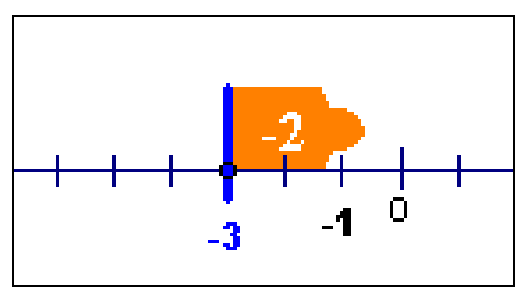

Figure 5. SAA model of $-3--2$

After working through multiple subtraction tasks, with and without the SAA, I wanted to see how Carla would work though a contextual representation of a subtraction task. I asked her a few temperature tasks that included the high and low values of the day and asked the change between them. She always chose subtraction as the operation and solved it by using the SAA method and a number line.

N: Alaska's high temperature for December $16^{\text {th }}$ was -6 degrees Fahrenheit. The low temperature was -17 degrees Fahrenheit. How many degrees did it change?

C: It changed [pause] -17 minus -6 and got -11 .

$\mathrm{N}$ : Why did you decide to use subtraction?

C: Because it said, 'How many degrees did it change.'

$\mathrm{N}$ : So I saw you put a dot at the -17 , count to the right, and put a dot at the -11 .

C: I counted 6 times to the right.

$\mathrm{N}$ : Why did you choose to go to the right?

C: Because two negatives. It's like adding them together. So -11 degrees.

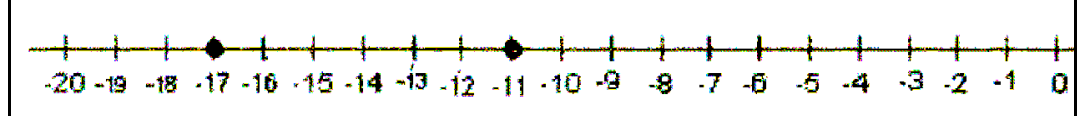

Figure 6: Alaska's change in temperature, $-17--6$

Carla used the SAA method as visual support and showed this by using a number line, placing a dot on the minuend, moving to the right the value of the magnitude of the subtrahend, and placing a dot on the difference (see Figure 6). Even though she stated that she had added the negatives, she really added the absolute value of the subtrahend to the negative minuend. 
After working through subtraction tasks in abstract, contextual, and number line representations, I asked Carla what she thought of the SAA. She told me, "It made it [subtraction] easier...because it's showing me how it's actually working. Like the computer showed the difference between them [the numbers], and which way it's going. Then on the paper, we worked it out." Through the manipulation, visualization, and immediate feedback provided by the SAA, Carla was able to see how subtraction worked using the take-away idea. Furthermore, it helped Carla see the significance of how subtracting a negative number resembled addition, and how it was different than subtracting a positive number. By keeping a paper record of the tasks she solved, she was able to generalize across examples to see how she could switch the subtraction sign and negative sign of the subtrahend and transform the subtraction task into an equivalent addition task.

At our next session, I wanted to see if she remembered what she had learned from the previous day, so I asked her to solve a variety of subtraction tasks. She solved them all accurately and referenced the SAA. She only encountered one minor setback, but it seemed due to a lack of paying attention.

$\mathrm{N}$ : How did you solve $8--1$ ?

C: $8--1$, because you have 8 right here, and you'd have to move, like on the computer, you showed that if it was a negative, it would be going to the right, subtracting how many. So I got 9 for it.

$\mathrm{N}$ : Great. What about $-6-7$ ?

C: That would be 13, positive. I mean, no, -13 .

$\mathrm{N}$ : What made you change your mind?

C: The -6 . I remember when we did it right there [referring to the number line], it was the same, we did the 7 minus -6 , so at first I just switched them around, like $7--6$.

$\mathrm{N}$ : Ok, so how did you solve $7--6$ ?

C: Um, $7--6$ is $13 \ldots$ cause yesterday the way we did it on the computer. We said that if it was negative [the subtrahend], subtraction goes to the right. So we end up at positive 13. If it's [subtrahend] a positive number we go left.

Carla had initially misinterpreted the task $-6-7$ and had transformed it into $7--6$ most likely because she had just solved a task in the form $a--b$. Yet, upon reflection, she quickly realized that the expressions were different because she would have to travel the opposite direction on the number line. She realized this because she had been able to see that when she subtracted a negative, she had to travel to the right on the SAA, and when she subtracted a positive, she had to travel to the left on the SAA.

The SAA got Carla to think in terms of movement on a number line and use that visual support for her answer. Because she was able to answer correctly all the subtraction tasks I had given to her and justified her answers using the SAA, I wanted her to also see the SDA strategy so she could use the distance method as a strategy to solve subtraction tasks. I wanted her to compare these two methods to see which method resonated with her idea of subtraction. Before I showed her the SDA, she said, "I liked the 
one yesterday because it helped me see which way to go." She thought that the SAA helped her figure out which way to travel because she could see the number inside the arrow when she moved it to the left and right. After she manipulated the dot applet, I asked her to model the task $7--2$, which she did correctly according to the SDA. I asked her how she knew how to find the answer and she said, "This shows that we're subtracting to the left, and ending at the -2 , so it's 9" (see Figure 7). She saw that since she had to put the blue dot on 7 , that represented the first number, and the -2 represented the second number, and the arrow showed that since our second number was lower than our first number, we had to travel to the left. I asked her where the 9 came from, and she responded, "It's in between the 7 and -2 ." Therefore, she understood that the distance in between the minuend and subtrahend was the difference.

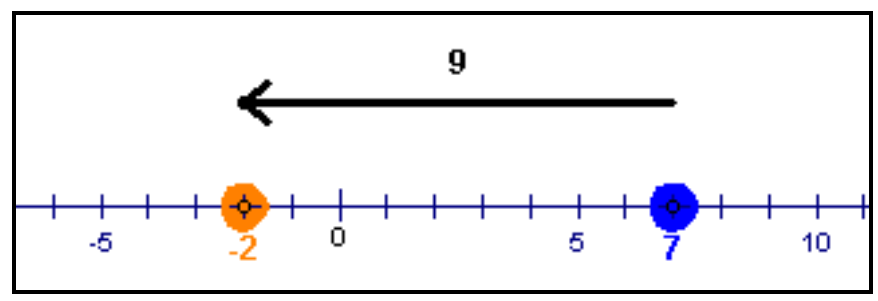

Figure 7. Using the SDA to solve the task $7--2$

After only modeling one example, she was able to predict the answer and tell me how she would move the SDA in order to get the answer to $-4-1$. She said, "I would put the blue dot on the -4 , and the orange dot on the positive 1. It would go right - it would be negative." When I asked her what her answer would be she said, "It's going to be negative and I would count in between, -5." While she saw that placing the orange dot to the right of the blue dot made the arrow value become negative during her exploration of the SDA, she never reasoned that since the second number was larger than the first, that the value of the difference would be negative.

After having her predict answers and justify them using the SDA, I asked her to solve $7--3$ without the SDA. She said, "Seven minus -3 , that would be like adding, so it would be going to the right. Ten, positive." Then she asked for a number line, placed a dot at the -3 and a dot on the 7 and counted the spaces in between the -3 and 7 , by starting at the -3 (see Figure 8). This is not exactly how the SDA or SAA worked, but she tried to integrate her knowledge of both and tried to apply them. She used her knowledge of the SAA in that she saw that she would have to travel to the right since she was subtracting a negative number. However, she used the SDA method to place the dots on the respective minuend and subtrahend to count the value in between to find the difference. 


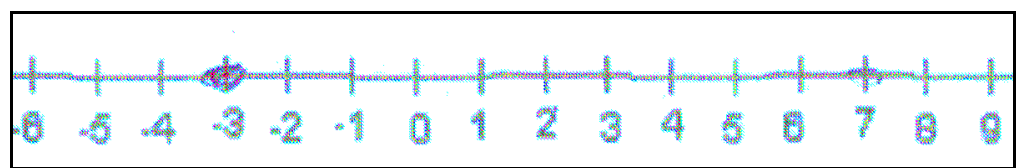

Figure 8. Using the SAA and SDA ideas to solve $7--3$

Given that she had tried to integrate the two applets, I asked Carla whether she liked the SDA or the SAA better. She liked both of the applets for different reasons.

I like them both. Cause on both of them it shows the [difference] value. On this one you have to place the dots, and in between it showed the value of them, and it has 2 dots showing your first endpoint and your second endpoint. The other one you move how far you want to go.

She enjoyed how she could see the visual of the difference in both the arrow value in the SAA, or counting in between the dots on the SDA. The difference between the SDA and the SAA was that the SDA had stationary endpoints that represented the subtrahend and minuend, and the SAA allowed her to move the number of spaces by moving an arrow whose length represented the subtrahend. Even though she liked both the SAA and the SDA, she solely used the idea of the SAA when I asked her more subtraction tasks. She would also refer back to what she learned yesterday when working with the SAA. For example, when I asked her to solve $-6--14$, she said, "you start at the -6 and you go to the left to 14 , well, you to the left 14 times, you would get 8 positive." When I asked her how she knew that she said, "Because yesterday on the computer it showed that when you subtract negatives, you go to the right." Similarly, for the tasks she already knew how to solve prior to the instructional sessions, she used the SAA method and tried to integrate rules that corresponded with similar tasks. For instance, when solving $5-8$, she placed a dot on the 5 and went down 8 spaces and landed on the -3 (see Figure 9). Then she justified her answer by saying "because 8 minus 5 is 3 , and then if you're subtracting 8 from 5 and you know $8-5$ is 3 , then you just know it would be -3 ." She tried to make connections between the visual representation of subtraction on the SAA and the rules that she had learned. In particular, she used the fact the equation $8-5=3$ to help her answer $5-8$.

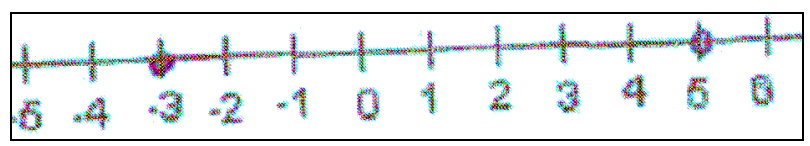

Figure 9. Carla solving $5-8$

As I was asking her to solve subtraction tasks, she also reverted back to using the language of "adding a negative" even though she was adding the absolute value of the subtrahend, which was negative. Although she said this, she consistently obtained the correct answer and moved the correct direction on the number line. Thus, it would seem that Carla had identified more with the SAA than the SDA. 


\section{Summary of Carla's Interactions and Understanding with Technology}

- Carla had both a conceptual and procedural understanding of addition and subtraction of negative numbers.

- Carla could now use the number line to help her solve all subtraction tasks. In fact, she used it more often to solve the tasks than she used her rules. She also did not believe that $a--b=-a-b$.

- Carla was able to tell the difference between the addition of a negative number and the subtraction of a negative number, for she did not believe that $a--b=a+-b$ or that $-a--b=-a+-b$.

- By manipulating multiple sequences of subtraction expressions, noticing patterns, using contextual problems, and noticing the connection between movement away from the minuend and the corresponding equation, Carla was able to see subtraction as both take-away and a distance. However, she preferred using the movement and visual that was gotten from the SAA and referenced it in justifying her answers.

- Carla was able to create correct subtraction equations for tasks represented contextually. In creating these equations, she used the SAA method to justify her answers.

- After learning how to solve addition tasks on the number line, Carla integrated this knowledge with her observation of how subtracting a negative on the SAA moved the arrow to the right. This helped her make the connection between the subtraction of a negative number and addition of a positive number on the number line.

- Carla noticed that the subtraction of a negative number was the same as addition. While she could operate using this idea, she frequently referred to it as "adding a negative" instead of adding the absolute value of the negative subtrahend. She believed that it was easier to solve equations of the form $-a--b$ by directly applying the SAA method rather than rewriting or mentally transforming the equation into $-a+b$ and finding the answer.

- By manipulating the SAA and observing the values inside the arrow, Carla realized why the difference was negative or positive because she could see that she had to travel left of the minuend if the subtrahend was positive, and to the right of the minuend if the subtrahend was negative. She no longer needed to rely on rules to help her solve subtraction tasks, and she no longer referenced rules that did not match her procedure.

- After working with the SAA, SDA, and accompanying tasks, Carla successfully attained Level 4 in both Peled's QD and NLD with respect to subtraction.

\section{Discussion}

Before working with the SAA, Carla did not have a clear conceptual understanding of subtraction.

Carla used memorized rules and procedures to help her justify her answers to subtraction tasks. While some tasks she could not verbally explain, others she would provide verbal explanations that did not corresponded with the procedures she used. For tasks with a positive subtrahend, she would not give any verbal justification but would compute it correctly. Most likely she viewed $a-b$ as $a+-b$ and $-a-b$ as $-a+-b$ since she could correctly perform addition with negative numbers. For tasks with a negative minuend and negative subtrahend, she tried to verbalize rules that she had heard but that did not necessarily 
correspond with her procedures. For tasks in the form $a--b$, such as $5--12$, she claimed that she "added -12 and 5 together", but then adjusted her reasoning and said that she "added -12 and -5 ", which was equivalent to "five subtracted from -12 ." It seemed that she transformed tasks in the form $a--b$ into $-b-a$, which she knew to be equivalent to $-b+-a$, which she could calculate accurately because it was addition. She also stated the rule that subtraction of a negative was like addition; however, she took this to mean that she had to add the negative minuend and negative subtrahend together. She also misapplied this rule to tasks in the form $-a--b$. She believed that the subtraction sign followed by the negative sign indicated that she had to add, so $-a--b$ was equivalent to $-a+-b$. She also provided the justification that she could use an analogous expression involving positive numbers to help her find the answer, namely by switching all the signs to positive, adding the absolute value of $a$ and $b$, and then tacking on a negative sign because the signs were originally negative. Thus, she computed $-a--b$ by solving $-(a+b)$. By using her rules and addition procedures, she was able to answer correctly all subtraction tasks involving a positive subtrahend.

Prior to working with the SAA, Carla either did not know or did not remember how to use the number line to help her solve subtraction tasks. In moving from a number line context to an abstract context, she used dots that were placed on the number line as beginning and ending values for an inequality. In moving from the abstract representation to a number line, she plotted independent points of the answers to an expression rather than using movement on the number line.

After exploring the SAA, Carla realized how the applet embodied subtraction by observing how her manipulation impacted the visual in terms of the beginning, ending, and direction of the arrow and the value in between the blue stick and tip of the arrow. This was surprising given that she had had no recollection of how to use the number line to solve addition or subtraction tasks prior to our session together. She observed how the arrow value changed as she moved the tip of the arrow from left to right. This influenced her understanding of the usefulness of the number line and fostered the connection between (1) the values of the minuend, subtrahend, and difference, and (2) the relationship among the numbers, operations and movement on the number line. In fact, the arrow value seemed to make greatest impact on Carla. She knew which way to move because of the value shown inside the arrow, and this allowed her to see subtraction as take away as well as a distance between numbers. In fact, the SAA made such an impact on her understanding of subtraction, that she was able to accurately predict the correct answer to the first task I gave her that incorporated a negative subtrahend taken away from a positive minuend by using the visual, without manipulating the SAA. She had a little difficulty predicting the subtraction of a negative 
minuend and subtrahend, but after manipulating the SDA, she quickly realized her misconception.

Furthermore, she used the SAA method to help her solve latter subtraction tasks by either (1) using her pencil as a placeholder on the number line or (2) visualizing the number line and movement in her head. She even was able to translate correctly between the abstract and number line representation and justified her translation by using and describing the SAA method.

While she was able to correctly solve all subtraction tasks and justify her answers by using the SAA method, she had some difficulty correctly verbalizing her reasoning, especially when she subtracted a negative subtrahend. Through observation, manipulation, visual feedback, and sequencing of tasks, she was able to see that the subtraction of a negative subtrahend was the same as adding the absolute value of the subtrahend; however, she often referred to this as "adding a negative." Furthermore, even though she said this, she always changed the subtraction sign to a plus sign and the negative sign on the subtrahend to a positive sign. It is interesting that she stated that she preferred to solve $-a--b$ rather than changing the signs and solving $-a+b$ because with the first expression, she was able to envision the movement on the number line right away, whereas with the second expression, she had to switch the signs first and then envision the movement. Even though she knew both expressions required her to move right on the number line, the second expression required an extra step.

During our next session, Carla frequently referred to what she had learned on the computer the day before and used the SAA method to help her solve subtraction tasks. While she learned and understood the SDA, and appreciated the differences and similarities between the SAA and SDA, she felt most comfortable using the SAA, and continued to use this method to justify her answers.

\section{The Equal Sign}

\section{Prior Conceptions}

By her responses on the interview tasks, Carla had primarily a relational (Seo \& Ginsburg, 2003) view of the equal sign. Only her response to Task 1 could be classified as operational. After pointing out that the only differences in the expressions/equations was that some had an equal sign and number following the equal sign, Carla told me what the equal sign meant in $5+6=$ and $5+6=11$. She said, "They both, well, they are all equal to 11. That's already solved for you. Mmm, that's just to solve the problem, and that doesn't have an equal sign." She believed someone had already solved the task to $5+6$ when the 11 was written next to the equal sign, but that she had to solve the expression in order to get 11 when there was no number placed after the equal sign. 
Even though Carla viewed the equal sign Task 1 as operational, her view of the equal sign with regard to other tasks was relational. She was familiar with the non-canonical forms of $3=3$ and $8=10-2$, and told me that she had seen those types of representations in equations in her math class. Specifically, she had seen $3=3$ in "legends on a map and scales" and in "in $3 x$ plus 4." Furthermore, it was accurate to write $3=3$ since it was the same value on each side of the equal sign. Since the value of 8 was on both sides of the equation in $8=10-2$, she believed that it was acceptable to write equal between the 8 and $10-2$. She was consistent in replacing the equal sign with the word "same as" for the rest of Task 2 and Task 4 such that " 10 pennies is the same as 1 dime," "12 inches is the same as 1 foot," and " 2 times 5 is equal to 10 and that's the same as 4 plus 6 that is equal to 10." Similarly, she used the words "the same as" when she talked about the task $3+5=?+2$ and subsequently solved it algebraically. In solving this task, first she decided to change the question mark into the variable $x$, because that was how she was used to seeing a variable represented.

$\mathrm{N}$ : Have you seen any problems like $3+5=?+2$ before?

C: [long pause] Yeah, uh, yeah, the questions mark is like the variable $x$. Can I change it?

$\mathrm{N}$ : Sure. So, if we had this $3+5=x+2$, what do you think that equal sign means?

$\mathrm{C}: 3$ plus 5 is the same as $x$ plus 2 .

$\mathrm{N}$ : Great, and could you solve for $x$ ?

C: It would be $6 \ldots$ because 3 plus 5 is 8 and 8 minus 2 is 6

$\mathrm{N}$ : Why did you subtract 2 ?

C: Because you have to find...it's 8 equals $x+2$ then -2 and -2 , and then they cancel out the 2 , and 8 minus 2 is 6 and that equals $x$.

$\mathrm{N}$ : Good. So I noticed that you wrote a -2 over here and a -2 over there, why did you do it to both of sides?

C: I had to get rid of $2 \ldots$ um, the 2 is right here so I took off the 2 [pointing to the $x+2$ ]

$\mathrm{N}$ : Well, how did you know you had to subtract a 2 over here also [pointing to the 8]?

C: Because the 2s cancel out over here I have to get rid of the stuff over here. You can't just do it to one side.

$\mathrm{N}$ : Why not?

C: Because if you did over here you'd just get 0 , and over here you would get a number.

If one only read Carla's verbal explanation of how she solved the equation, it would look like she used the unwinding strategy (Knuth et al, 2006); yet upon further inspection of her written work, she had actually solved it algebraically (see Figure 10). While Knuth and colleagues differentiate this category from unwinding, they are not entirely different. Even though Carla believed that the equal sign delineated that the expression on the right was the same value as the expression on the left, she did not understand why she had to subtract 2 from both sides of the equation, which called into question whether she really did understand what the equal sign meant, or if she simply had trouble telling me why it was necessary to subtract 2 from both sides. It was also interesting, that Carla was the only participant who justified her answer by applying the subtraction of 2 to both sides instead of using the commonly stated answer that 
'since 3 plus 5 equaled 8 then the question mark had to be equal to 6 because 2 plus 6 equaled 8.'

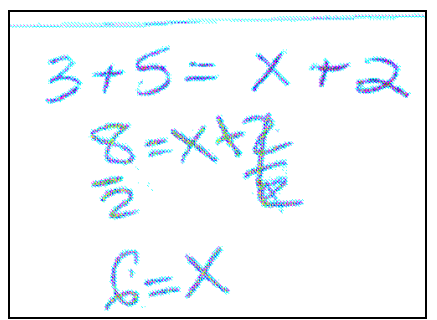

Figure 10. Carla algebraically solving $3+5=\mathrm{x}+2$

Given that Carla could not explain why she had to subtract 2 from both sides, I wondered if she truly had a relational understanding of the equal sign when the equation included a variable. She seemed to have the "same as" notion of the equal sign, but she had a difficult time verbally communicating that when the equation dealt with variables. In fact, after asking her to explain what the equal sign meant in the equation $x+5=3$, it seemed that she did not have this relational understanding, for she said, "it means you need to find what $x$ equals." She then tried to solve for $x$ but did not do so algebraically.

$\mathrm{N}$ : Can you solve for $x$ ?

C: Yeah, but um, wouldn't it be a negative number?

$\mathrm{N}$ : Yeah, and why do you think that?

C: Because 1 plus 5 would equal 4 , and 0 plus 5 would equal just 5 , and -1 plus 5 would equal ... [she grabs a number line]...-1 plus 5 equals, oh, so $x$ would equal -2 .

$\mathrm{N}$ : Great, and how did you think about that?

C: It was a bit difficult.

$\mathrm{N}$ : So what was kind of going through your mind?

C: Well, at first I thought we kind of had to subtract something from one side, which we did have to do, and then I thought we would have to divide something, but we didn't, but we can't because we would have to have a variable beside a number.

Carla did not subtract 5 from both sides of the equation, rather, she reasoned that the value of $x$ would be negative because she had to add it to 5 and obtain a lower number than 5 . When I asked her to tell me why she knew it had to be a negative number, she started noticing a pattern, grabbed a number line, and saw that if she started at the -2 and moved up 5, she landed on the 3 . This type of solution procedure was not categorized by Knuth and colleagues (2006).

This task seemed harder for her to solve than $3+5=?+2$. She solved the two tasks differently, even though she said that she had thought about trying to subtract a value from both sides for the latter, she did not follow through on this, but noticed that she had done so afterward. It is interesting that she solved this task differently because both tasks are 1-step linear equations. The differences between the two equations are that $3+5=?+2$ has an operation on both sides of the equation, whereas $x+5=3$ only has one operation on one side, and the solution of $3+5=?+2$ is a positive number, where the solution of $x+5=3$ is a negative number. The fact that the value of the variable was negative could have impacted her 
solution method.

When I asked Carla if she knew what the equal sign meant in the task $2 m+9=1$, she simply told me that it meant " $2 m$ plus 9 is equal to 1 " and proceeded to solve for $m$.

C: Well ...2 divided by 2 on both sides, and then cross off the 2, and then 9 divided by 2 would give you 4.5 . And then $m$ is equal to 4.5

$\mathrm{N}$ : Ok, so what does this 1 mean there?

C: It's on the other side, so this all has to equal to 1 . So 2 times 4.5 plus 9 has to equal to 1 .

$\mathrm{N}$ : Can we calculate that?

C: Yeah, but it's not equal.

$\mathrm{N}$ : What should we do?

C: We should write greater than or less than.

Through Carla's verbal description, she seemed to understand that the equal sign denoted the equivalence of the expressions; yet, this relational viewpoint did not help her solve the task. Instead of subtracting 9 from both sides, she divided all the terms only on one side by 2 (see Figure 11). This left her with an $m$, and 4.5 on one side of the equation, and as I pointed out to her, a 1 on the other side. She believed that since she was left with an $m$ and 4.5 on one side, this meant that $m$ equaled 4.5. When she substituted this value back into the equation, she realized that the left side of the equation was not equal to one. Instead of changing her solution strategy, she believed she should put an inequality sign between the expression $2 m+9$ and 1 to make the statement truthful.

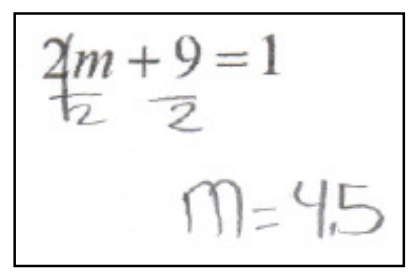

Figure 11. Carla's procedure for solving $2 \mathrm{~m}+9=1$

\section{Summary of Carla's Prior Conceptions}

- Carla exhibited a relational view of the equals sign for numerical equations and the comparison of numbers and units. Furthermore, it seemed that she also had a relational view for 1-step algebraic equations.

- Carla was familiar with non-canonical forms and was able to state why they made sense.

- Carla believed that the equal sign showed the equivalence of two expressions, yet this did not direct her to use an algebraic method to solve equations.

- Carla accurately solved 1-step equations using both an algebraic and her own devised method. Yet, when it came to 2-step equations, Carla was unable to solve them correctly, for she used the wrong operation and did not apply the same operation across the equal sign. 
Analysis of Interaction with the Equality Applet

\section{Carla's Interaction and Understanding with Technology}

As Carla explored the equal balance applet (EBA), she noticed a few of the features that allowed her to understand the two rules that maintained the balance equilibrium. She noticed that when the pans were not balanced, an inequality appeared above the fulcrum of the balance, and the pan that was up in the air contained a lighter amount of objects. For instance, when she dragged a positive 5 to the left pan and a -1 and -5 to the right pan, I asked her what she noticed. She said, "All those negatives are less than a positive number, so it goes up. This says it is not equal." She pointed to the inequality sign between the 5 and -6 (see Figure 12).

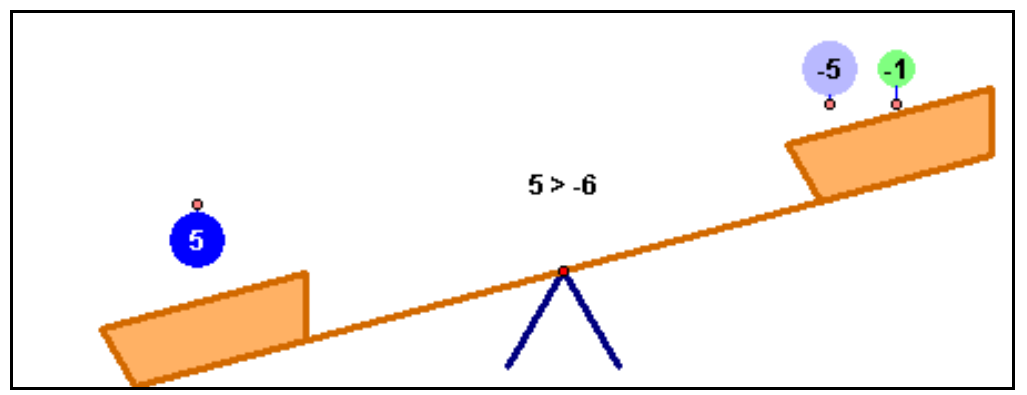

Figure 12. Pans containing a 5-weight on the left and a -5 and -1 balloons on the right

When I asked her how she could balance it, she said, "Use more positives over here." She placed a 5weight and a 1-weight on the right pan, and before she could put another 5-weight on to make it balanced, I asked her why the inequality read $5>0$. At first she was not sure because she could only account for -1 and 1 and 5 in the right pan because the -5 was hidden due to the clutteredness of the pans. After moving the objects around, she saw the -5 balloon and said, "Well, a -1 and a 1 and a -5 and then adding a 5 you're getting zero. So that's where the zero is coming from." She then proceeded to match the -1 and 1 and match the -5 and 5 together by placing the circles together (see Figure 13). She said, "Like you have a 5 and a -5 and that's kind of canceling each other out.“

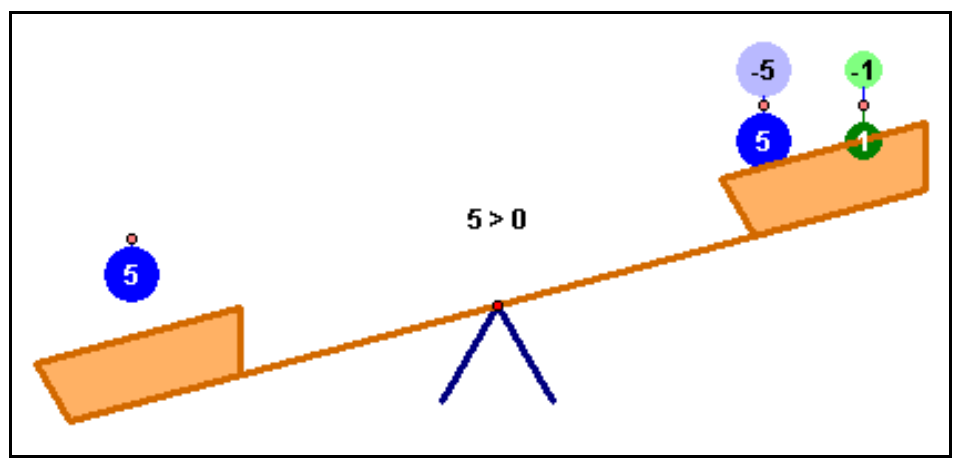

Figure 13. Matching the balloons and weights of opposite value 
Based on her manipulations, Carla was able to see the significance behind the representations of the balloons as negatives and the weights as positives. She noted that they could be matched to produce a value of zero. Furthermore, she removed them as a pair when she dragged them to the storage unit. Moreover, she noted that the applet automatically summed the values in the pan.

I asked her how she could balance the pans, and she said, "I could move the -5 back." She removed the -5 from the right pan and placed it into the storage unit, and the pans balanced. When I asked her what she could do to keep the pans balanced, she moved some objects to and from the pans to test some methods. After exploring how to keep the pans balanced, she said, "you can take the same from both sides" and "you can put the same positives and negatives on there, cause a negative and a positive can equal zero." Thus, she was able to notice that she could remove or add the same amount to both sides or place a positive and negative amount of the same magnitude to one side such that the pans would remain balanced. After discovering how to maintain equilibrium of the pans, Carla worked through the Tasks in Module 3.

Unlike most of the other interviewees, Carla modeled the task $3+5=?+2$ precisely as it was written using the EBA. After placing a 3 and 5 on the left pan and a 2 on the right pan, she did not stop and say that the question mark would have to be equal to 6 . Instead, she added the question mark to the right pan and said, "They're balanced." When I asked her why she told me, "Because they are equal."

$\mathrm{N}$ : How could you figure out the value of the question mark?

C: I could take off a 1 from both sides.

$\mathrm{N}$ : So what would you be left with?

C: Well, I could take off another 1 from both sides.

$\mathrm{N}: \mathrm{Ok}$, so...

C: Five equals question mark, I mean, well, it says 6.

After taking off two 1-weights, she only saw the 5 on the left pan because the 1-weight was behind it. However, she saw the discrepancy between the equation and the visual weights because the equation displayed $6=$ ?. When she moved the 5-weight, she saw the 1-weight behind it. While she was able to notice the discrepancy, the fact that the pans were small and could become overloaded with objects and thus hide some of the values, could impact students' answers.

After realizing that the question mark was the value of 6, I asked her how to describe removing 1weights from the pan in terms of a mathematical operation. She told me it was like subtraction and that she subtracted 2 to get 6 . Much like other interviewees, when I asked her what was left in the pans when she subtracted 2, she said, "Six in the left and zero in the right." However, once I pointed to the question mark she said, "Oh, yeah, and the question mark." This comment could be due to the fact, that Carla did not view a variable as a number, but rather an entity that they had to solve for, whose value would appear on the 
opposite side of the equal sign.

Carla seemed to progress well using the EBA to help her solve 1-step equations. She knew that removing objects was the same as subtraction and adding objects was the same as addition. It was not until she tried to use the EBA to solve the task $x+8=21$ that she deviated from her procedural pattern, which occurred because an exchange of weights was required to solve the task.

After she removed a 5-weight from both the left and right pans, I asked her what remained. She said, "One $x$ plus 3 and a 16." She paused, and I asked what she could do next, to which she responded, "Subtract all the things, all the ones and all the fives." She removed all the objects from the pans except for the $x$-weight. When I asked her what she as doing she said, "I removed them cause they are greater than zero." She then started to remove the 1-weights on the right pan. She removed all the objects to figure out if $x$ was positive or negative. When she saw that the left pan tipped downward when the right pan was empty, she began to add one 1-weights one at a time to see when the pans would balance, for this would tell her the value of $x$. She noticed that $x$ was "heavy" because she ran out of 1-weights before she could balance the pans ( $x$ equaled 13 and she only had four 1-weights in the storage unit). She then asked if she could get back to the original task. By pressing the reset button, she was able to immediately return to the original task and begin again. This time, she removed one 5-weight from each side and stopped. I asked what she would do next. She responded, "I want to remove the 3, but I don't have 3 on the right pan" (see Figure 14). I asked her what she could do if she removed a 5-weight from the right pan so that it would only be like she removed 3. She said, "Hmm, well, I'd have to put 2 back." I told her to go ahead and try that, and she removed the 5-weight from the right pan. She then transferred two of the 1-weights from the left pan to the right pan, and removed the last 1-weight from the left pan to the storage area. This movement was interesting because this action could be the enactive version of the symbolic version of the unwinding method. Unwinding occurs when a student works backwards through the constraints of the task (Knuth et al, 2006). Specifically, students invert operations and perform arithmetic operations instead of algebraic manipulation. Symbolically, one would categorize unwinding for $x+8=21$ if Carla did not show that she subtracted an 8 from the left side of the equation but only subtracted it from the right side. Thus, she was simply moving the 8 from one side of the equation to the other and adding the opposite sign. While this could not occur on the EBA since students would have to choose different objects (since weights are positive and balloons are negative), Carla did in fact move weights from one side to the other because she had to make an exchange in amounts. This could be similar to unwinding, or it could have occurred because Carla wanted to be efficient in her exchange. Instead of removing the weights from the left pan and 
placing them back into the storage area, and then moving them from the storage area to the right pan, she simply skipped the middle step.

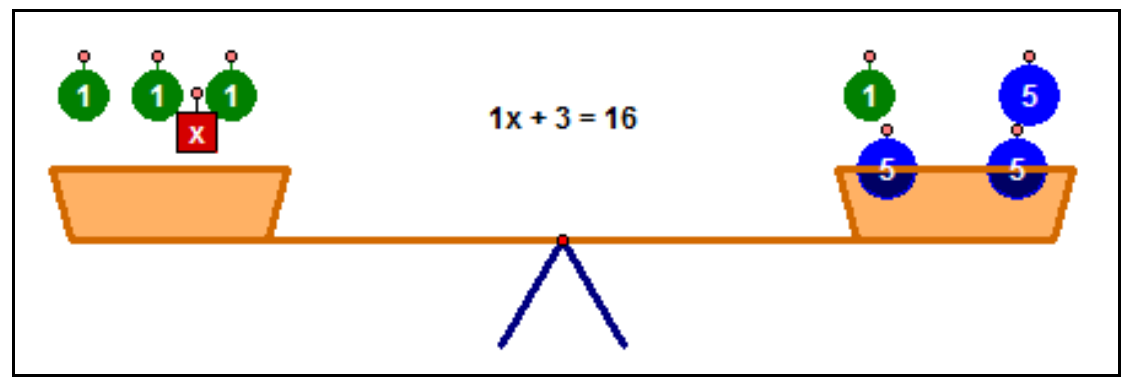

Figure 14. EBA showing $\mathrm{x}+8=21$ after removing 5 from each side

After subtracting 3 from both sides, she said, "One $x$ plus zero equals 13." While she could use the EBA to solve this task in two steps, I wanted to know if she could see the benefit of solving it on paper by using only 1 step. Carla, paused, and said, " 3 and 5, I guess I could have subtracted $8 \ldots$ we would have been left with 21 minus 8 and canceled those out and then 21 minus 8 and would have got 13." She noticed that she could have subtracted 8 right away and still obtained the correct answer. Given that performing this one-step on paper was more efficient than moving the objects on the computer, I asked her whether she thought the EBA was helpful.

Yes, I like it because you had to move the numbers from the balance beam and watch them to see if they be equal to each other... It helped because you got to see it...like it was in your face, and you could see if it was equal or not.

Even though she found the EBA to help her solve 1-step equations, she said, "sometimes I just wanted to know the answer, and sometimes I just like to know the answer before I try it." She was referring to the fact that on paper, she could get the answer more quickly, but she did like that she could try different possible answers by using the EBA to figure out the value of $x$ by removing the objects and testing different values to see if the pan with the $x$ would balance with the pan containing specific amount on the other side.

When I gave her 1-step equations on paper to solve, she did not need the EBA and solved each of the tasks algebraically. One interesting note is that when she added or subtracted amounts from each side, she referred to what she learned in our previous session. For instance, when she solved $x-6=-15$ she said, "you subtract -6 from both sides, so you're really adding it." When I asked her how she knew that, she said, "Because yesterday on the computer, the negative, when you subtracted it, it went to the right." She was referring to the SAA and that when she subtracted a negative subtrahend, she had to move the arrow to the right, which meant that she added the absolute value onto the minuend.

I gave Carla a 2-step equation to solve without the EBA to see how she would solve it. Carla was unable to solve 2-step equations in her interview. However, after working with the EBA for 1-step 
equations, she easily transferred this method to solve 2-step equations and what she had discovered when she was exploring the EBA. When I asked her to solve the first 2-step equation, $2 m+7=15$, she subtracted 7 from both sides, and then divided by 2 . She explained, "We have to make a zero pair, with the 7 . And with the variable, you hold that off for last...you divide, because there is a variable beside the 2 and the opposite of multiplication is division." When I asked her what she meant by a zero pair, she said she had to subtract to get a zero by the variable. While I had not mentioned a zero pair, she must have learned that word somewhere else, however, she correctly applied it because she had noticed that she could add balloons to both sides of the EBA, and match those balloons up with the corresponding weights on the left side, which she had done during her exploration of the EBA. This would give her a variable multiplied by a constant and numbers on the right side of the equation. She then knew to divide because she had to apply the inverse operation. Thus, by manipulating and examining the EBA, she was able to realize a pattern for how to solve 2-step equations algebraically (see Figure 15).

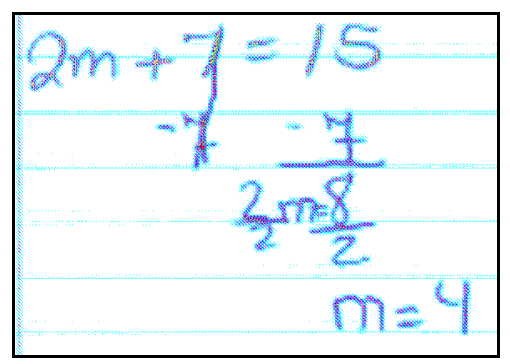

Figure 15. Carla using algebraic transformations to solve $2 \mathrm{~m}+7=15$

I asked her to solve a few more 2-step equations, which she did so correctly without using the EBA. Carla seemed to understand that transformations made the expressions remain equal due to making the pans balance on the EBA. Carla seemed to have developed a relational understanding of the equal sign when an equation involved a variable.

\section{Summary of Interaction and Understanding with Technology}

- Carla exhibited a relational view of the equals sign for numerical equations and the comparison of numbers and units as well as equivalence of two expressions when one of the expressions contained a variable.

- Carla accurately solved 1-step and 2-step equations using the algebraic transformation method.

- Carla believed that the visual model and manipulation features allowed her to solve 1-step equations more easily than she was able to do before working with technology because it helped her to find the solution by intuitively seeing what she had to remove or add to the pans to keep them balanced.

- Carla easily transferred her understanding of solving 1-step equations and to solving 2-step equations. In fact, she was able to do this without manipulating the EBA. 
- Due to the visual, manipulation, and feedback characteristics of the applet, Carla's understanding of zero pairs was reinforced, especially when it came to why she had to perform the same operation on both sides of the equation.

- Unfortunately, the fact that Carla had to exchange some objects for smaller valued objects required more steps to solve the equation using the EBA than it did solving the equation on paper. This fact caused her to use the EBA in a different way - trying to figure out the value of the variable before she started using algebraic transformations to solve the equation.

\section{Discussion}

Carla had a relational view of the equal sign for all equations without variables and in the comparison of values and units. Furthermore, she was able to justify non-canonical forms using this relational viewpoint. Yet, her relational viewpoint wavered when she encountered equations that included a variable. Most of her responses were relational, but she did not always solve these equations using an algebraic method.

The fact that she seemed to view the equal sign as relational, yet solved some equations using nonalgebraic methods is contrary to the findings of Knuth and colleagues (2006). Furthermore, while she was able to obtain the correct answer to 1-step equations, she could not obtain the correct answer to 2-step equations because she applied the incorrect operation first, and applied this operation to the wrong numbers.

When she began working with the EBA, her understanding that the equal sign represented equivalence of two equations was reinforced through the visual picture of the balance. Through exploration of the EBA, she discovered strategies to maintain the balance of the pans and the equality of the expressions.

Furthermore, her understanding of zero pairs was reinforced because she could see how the negative and positive values of the same magnitude matched up and summed to a zero value. After discovering these strategies and reinforcements, she was able to model 1-step equations and solve them using the algebraic transformation strategy by removing or adding the same objects to both the left and right pans. Because the applet allowed her to drag different values and see the resulting effect of that manipulation on the pans and corresponding equation, Carla was able see a visual depiction of the equation and intuitively realize how to find the value of the variable without it algebraically. Furthermore, she was able notice the intuitive correspondence between her manipulations on the EBA and the mathematical operations, which allowed her to easily transform his solution procedure on the EBA to paper.

During the process of working with the technology applet, she discovered the relationship between the expressions by using the EBA; she also came to realize that there was a faster way to solve equations by looking at the pattern of her written work versus operating solely on the visual model of the equation. 
Although this method of solving on paper was faster than moving the objects on the applet, she liked working with the applet because it had helped her to visualize the meaning behind the equal sign and how it applied to solving 1-step and 2-step equations. Furthermore, she was able to consistently solve both types of equations algebraically even though she was unable to solve 2-step equations prior to working with the EBA. 


\section{CHAPTER 7}

\section{CASE STUDY: BETH}

\section{Beth's Background}

During the time of the study, Beth was in eighth grade Standard Math. Standard Math mainly covers pre-algebra concepts. Data collection was at the end of the school year; thus, Beth had already covered pre-algebra material, such as creating and solving word problems, using formulas, and solving equations containing one variable.

Much like Carla, Beth was relatively quiet and shy. While she did not hesitate to give numerical answers, she usually hesitated to give reason for her answers. In fact, sometimes when I would ask her how she thought about the problem, she would sit silently and look straight ahead, almost as if she was hoping that I would move on and not make her give a reason for her answer. When I would ask her again, she would laugh softly and nervously, clear her throat, whisper her answer, and then blush. She did not seem confident in her justification, even if she believed her answer was correct. It appeared that she seldom was asked to provide reasons for her mathematical answers. When she did provide justification, most of it was in the form of procedural description, even if the procedure did not always produce the correct answer. Yet, as we progressed through the interview and instructional sessions, Beth provided her justifications more frequently and decreased in hesitation of her answers.

Like most Standard Math students, Beth had only used the computer in math class to prepare for the Standards of Learning through Study Island; she did not consider this computer usage as helping her to learn new mathematical ideas. Instead, she perceived Study Island as a tool to assess her comprehension ability of mathematical tasks that were taught prior to computer usage.

$\mathrm{N}$ : Have you ever used the computer in math class?

B: Well, not really, but yeah.

$\mathrm{N}$ : What do you mean?

B: We went to, like we only used it for SOLs, like practicing for it.

$\mathrm{N}$ : Oh, was it called Study Island?

B: Yeah. That's pretty much all I remember using it for, just practice.

N: So you didn't use it to figure out stuff, you used it to just to see if you would pass the test?

B: Yeah. Exactly.

Her view of technology as a mechanism to provide instantaneous feedback on the correctness of an answer, perhaps led Beth to not focus on connections between the mathematics and visual ideations produced by 
technology. As Beth experimented with the technology applets, she did not always pay close attention to how the technology helped her find the answer if she already was able to correctly find the answer. It was not until she needed the technology to help her solve tasks that she noticed how her manipulations impacted the visual feedback she received. This helped her to solve both subtraction tasks and 2-step equations.

\section{Negatives and Operations on Integers}

\section{Prior Conceptions on Addition}

Beth had procedural and conceptual understanding of addition of integers. She never mentioned any arithmetic rules that she used to solve tasks, and she did not illustrate that she viewed addition of a negative number like subtraction. Rather, she used a number line. In fact, immediately after I had asked her to solve the first addition task, she began reaching for a number line she saw lying on the table. In using the number line, she believed that the negative sign on the numbers in an addition task indicated that she move left on the number line. This allowed her to obtain the correct answers to addition tasks. She also was able to justify her answer in terms of abstract quality such as debt.

Beth had an interesting procedure for using the number line with addition tasks with negative addends. She would begin on the number line at the first negative number listed in the task reading from left to right. Then she would move to the left if the other addend was negative or move to the right if the other addend was positive. Therefore, for tasks in the forms $a+-b$ and $-b+a$, she would start at $-b$ and move to the right $a$ spaces. For tasks in the form $-a+-b$ she would start at $-a$ and move to the left $b$ spaces. Usually students would start with the first addend, and move to the right or left depending on the sign of the second addend. Yet, Beth noticed that it did not matter if she started with the first or second addend as long as the sign told her which direction to move.

$\mathrm{N}$ : How did you use the number line to figure out that $10+-3$ was 7 ?

B: I started from the -3 and I started counting up 10. Like to the right 10. End at 7.

$\mathrm{N}$ : And what about this one? How did you use the number line to find that $-5+-7=-12$ ?

B: I started at the -5 and I went back negative seven.

$\mathrm{N}$ : How did you know to go back?

B: Because it was a negative number, so I went back.

$\mathrm{N}$ : So, how did you know where to start on the number line? For this task you started at the -3 , and for this task you started at the -5 .

B: Well, I just started here because [long pause] I don't know why I start like that. I guess it doesn't matter. You could start at 10 and go back 3.

Beth was not cognizant of why she always chose to start at the first negative addend she encountered, but she consistently did so. While she realized that it did not matter in addition tasks due to additive commutivity, I believe that she chose this method because it decreased her cognitive load in paying 
attention to all of the negative signs in the task. As past literature has shown, students have difficulty when there are many negative signs present in an expression. Therefore, I think that Beth chose to start with one of the numbers that had a negative sign, so that she could disregard that negative sign when she operated on the number line. This allowed her only to pay attention to the sign of the other addend. While it is not important which number she started with or why she started with it for addition tasks, this method clearly influenced her ideas of subtracting with negative numbers.

Given that Beth did not use a written arithmetic procedure to solve addition tasks, or state that adding a negative was the same as subtraction, she would not be considered to have a unary understanding of addition of negative numbers. Beth solely thought of addition as using the signs of the addends to determine the direction traveled on the number line. Yet, for addition tasks with two positive addends, she used the "counting on" method from the higher valued addend. For example, in solving $10+3$, she said, "I just went 10 plus 3 , so $11,12,13 . "$

Beth also was able to situate her understanding of negative numbers and addition in terms of the quality characteristics of having and owing money. When I asked her to make up a word problem for the task $-5+-7$ and her answer of -12 , she said, "I could say like, I owe 5 dollars, and then I owed 7 because I borrowed another 7...it would mean that I owed 12 dollars in total."

Not only was Beth able to go from an abstract arithmetical task to a number line context and from an abstract arithmetical task to a word problem, she also was able to maneuver from a number line context back to an abstract arithmetical context. However, she could not accurately relate her equation, number line representation, and description. I asked her to write an equation that represented a number line with a dot on the -6 and a dot on the 5 (Task 5 , Module 1). She said, "Umm, five minus negative six." This expression was one of the expressions for which I was looking, since the number line showed a distance between the 5 and -6 . It would indicate finding the difference; hence, the distance between those numbers. Yet, when I asked her what the answer would be, she paused, and said, "I guess you could use the number line to figure it out.” Then she proceeded to count from the 5 back 6 spaces. Thus, she operated such that $5--6=5-6=-1$. This is interesting for two reasons. One reason is that when she only had the expression, she would typically start with the first negative number she encountered; therefore she would normally start at the -6 . In fact, one will see that she operated on subtraction tasks of the form $a--b$ by starting at $-b$ and moving $a$ spaces to the left since the operation was subtraction. However, in this case, she was paying more attention to the visual number line with the dots and used the picture to guide her movements rather than her constructed equation of $5--6$. In particular, after she told me the answer was 
-1 , I asked her, "So five minus -6 is -1 ?" to which she responded, "Yes." This was uncharacteristic of how she solved subtraction tasks in this form. Normally, she told me that $5--6=-6-5=-11$, which will be seen in the next section. The second reason that her procedure was interesting lies in the fact that she believed that she could switch the direction of her movements without adjusting her equation. She said, "Or you could just start at the -6 and go up 5." When I asked if that would be the same expression, she said, "Yeah," and she showed me that it did not matter if she started at the -6 and went up 5 spaces or started at the 5 and went back 6 spaces because she still landed on the -1 . The ability to use the same numbers on the number line and operate by switching the number with which she started, led her to believe that the order of the numbers in her equation did not matter. This most likely occurred because it had always worked for her when she used addition due to its commutivity. Furthermore, even when she started at the -6 and moved up 5 spaces, she believed that she was still subtracting, because it was not until I asked her if she could write another expression did she say, "Well, I guess I could use addition instead of subtraction."

B: You could do like addition, like -6 plus 10.

$\mathrm{N}$ : Ok, so you wrote $-6+10$. Where did the 10 come from?

B: I counted the spaces to get to the 5 .

$\mathrm{N}$ : Ok. So what would the answer to this equation be?

B: Five.

$\mathrm{N}$ : Ok. Is there any other way we could use this number line and those two dots to write a math problem?

B: [whispers] Not that I can think of.

Once again, she was paying more attention to the number line than to the equation that she

created. From the prior questions in the interview, I knew that she could add integers to find the correct value; therefore, she would have known that $-6+10$ was equal to 4 and not to 5 . Yet, she was focused on moving on the number line and incorporating the dots than checking her arithmetical expression. Therefore, Beth appeared to have a more difficult time moving from a number line to an arithmetical expression than moving from an arithmetical expression to a number line.

\section{Prior Conceptions on Subtraction}

Even though Beth was able to understand negative numbers in terms of the quality characteristic of debt, she never used this idea or the idea that subtraction meant, "take away" to solve subtraction tasks. Instead, she solely relied on the use of her number line and directional movements depending on the operation sign. This is unlike her number line method for addition tasks, since her directional movement was based on the addend signs. However, similar to solving addition tasks, she started on the number line at the first negative number in the expression when it was read left to right. Unlike addition, when completing subtraction tasks, she always moved to the left the number of units specified by the absolute value of the 
other number because she believed that subtraction meant to "move to the left." Therefore, she was able to correctly solve all tasks comprised of a positive subtrahend but not tasks with a negative subtrahend.

When it came to solving subtraction tasks in the form $a-b$ where $a>b>0$, Beth could not give a reason for her answer. She would simply sit still, blushing when I asked her how she obtained her answer. Eventually she told me, "I just knew it." Most likely she had done so many of these tasks in elementary school that it became second nature to solve them; therefore, she could not describe her solution method because it was automatic. Yet, if she could have used the same number line strategy she used for other subtraction tasks, she could have gotten the correct answer.

When I asked Beth to solve 5 - - 12, she obtained the answer of -17 and said that she "started at the -12 and went back 5 spaces." Because this task had two adjacent negative signs, I wanted to see (1) what she would do when I asked her to solve $5+-12$, and (2) if she would compare the tasks and their answers.

B: It would be -7 .

$\mathrm{N}$ : Ok, how did you get that?

B: Cause I went to the right. I started at -12 and going to the right. I went right 5 spaces.

$\mathrm{N}$ : So this one $5--12$, you went left, how did you know to go left?

B: Because it's less than, so...

$\mathrm{N}$ : Could you explain that more? What told you to go left?

B: The -12 .

$\mathrm{N}$ : So here we had a -12 and you went to the right, and here we had a -12 and you went to the left.

B: $\mathrm{OH}$, the subtraction sign

Beth did not seem aware of her own reasoning for her solution procedures. She believed that she should travel to the left for the task $5--12$ and to the right for the task $5+-12$ but did not understand why. At first she believed that she should travel to the left because of the negative sign on the -12 , but when I pointed out that both tasks had a -12 in them, she compared the two expressions and realized, in a surprised fashion, that one was addition and one was subtraction. Based on her procedure of (1) beginning at the first negative number she encountered, and (2) moving to the left if the expression contained subtraction and moving to the right if the expression contained addition, it was difficult for me to create an expression having a positive minuend and negative subtrahend that would incite a cognitive conflict while evaluating. There was no expression that I could ask her to solve with a 5 and -12 that would cause her to question her strategy. For instance, with interviewees who thought that $5--12$ was equal to -7 , I could ask them to solve $5+-12$. When they did, they usually obtained the same answer of -7 . Then I would ask them to compare the expressions $5--12$ and $5+-12$, and they would notice that one was addition and one was subtraction. This realization would normally cause them to abandon their strategy and answer because they 
knew that addition was not the same as subtraction. Yet, I could not find an expression that would help me lead Beth to this conclusion because she could easily explain her different answers using the same solution procedure. For example, she would have gotten the answers to the following expressions using the same procedure: $5-12=-7,5--12=-17,5+-12=-7$, and $5+12=17$. Since $5-12=-7$ and $5--12=-17$ were different, there would be no contradiction between subtracting a positive 12 and subtracting a -12 . Since $5--12=-17$ and $5+-12=-7$ were different, there would be no contradiction in subtracting a -12 and adding $\mathrm{a}-12$. Since $5--12=-1$ and $5+12=17$ were different, there would be no contradiction or similarity to subtracting a -12 and adding a positive 12 . Thus, I moved to the next task.

When she solved tasks with a negative minuend and negative subtrahend, Beth would start at the negative minuend on the number line and move left the amount of spaces represented by the magnitude of the subtrahend. Thus, the negative sign on the subtrahend had no meaning with regards to the expression.

$\mathrm{N}$ : Ok, so what about this one? You have -4 minus -6 equals -10 .

B: I started at the -4 and went back 6 spaces, and that got me -10 .

N: So what about $-4+-6$ ?

B: It would be equal.

$\mathrm{N}$ : So here you have -4 minus -6 and here you have -4 plus -6 . If we add the same number, is that the same as if we subtract the same number?

B: No, but [long pause] you move to the left and get -10 .

This excerpt says a lot about Beth's hierarchy of understanding. Due to her ability to add negative numbers, she felt confident in her answer of -10 for the task $-4+-6$. She also felt confident in her solution method of moving on the number line. Therefore, instead of adjusting those ideas, she instead, adjusted her notion of addition and subtraction of a same number. She forewent the idea that subtracting -6 was different than adding -6 , and held on to her belief that since she obtained -10 for both tasks, that in some instances, $-a--b=-a+-b$. Because she had to adjust one of her notions of subtraction of negative numbers, her awareness of her own possible misconceptions was heightened for the next task.

$\mathrm{N}$ : So tell me how you got the answer to the task $-4--1$.

B: I started at -4 and go to the left, and get -5 .

N: Ok, so what if you had -4 minus 1 , how would you do that?

B: It would be equal. Well, like you would go to the right 1, I think. Yeah, I guess. Negative three.

$\mathrm{N}$ : And how did you know that this one was right and this one was left?

B: Because this one was -4 minus -1 and this one was -4 minus $1 \ldots$ so the one without the negative, I went to the right instead of the left.

$\mathrm{N}$ : Oh, ok. So since this one had this extra negative sign, you went to the left for that one?

B: Yeah.

When I asked her what she would do for -4 - 1, I wrote the expression directly underneath the expression $-4--1$. Her answers to these tasks are interesting, because without being questioned, she would have believed that both $-4--1$ and $-4-1$ were equal to -5 because she would have started at the -4 on the 
number line and moved to the left 1 space, since both tasks included subtraction. Perhaps due to the questioning and interactions of the previous task, she started to toy with the idea that these tasks may be different because one was subtracting a -1 while the other was subtracting a positive 1 . What is even more interesting was that before this task, she firmly believed that $-4-1=-5$ and could accurately solve tasks in the form $-a-b$. Moreover, she believed that subtraction meant to go to the left. Yet, here she abandoned these notions and used the sign of the subtrahend to determine her direction she moved. Thus, because she subtracted a positive one, she believed she had to move in the positive direction (to the right), and when she subtracted a negative one, she had to move in the negative direction (to the left). This goes to show that she did not have a unary understanding of addition of a negative number because if she had this understanding, she would have seen that $-4-1=-4+-1=-5$, since she could accurately solve all addition tasks.

\section{Discussion of Prior Conceptions}

Beth could correctly solve addition tasks involving negative numbers by using a number line. The first step in her procedure for using a number line for both addition and subtraction tasks, was to start at the first negative number encountered when she read the expression from left to right and move from that point. Yet, she could not explain why she chose to do it this way. Her movement differed according to the operation in the expression. For addition tasks, she chose to move in the direction according to the sign of the other addend. Thus, if the other addend was positive, she would move to the right, but if the other addend was negative, she would move to the left.

For addition tasks, Beth was able to maneuver from an abstract context to a number line context and from an abstract context to a verbal context, yet she had difficulty moving from a number line context to an abstract context. This is not entirely consistent with past literature. Bruno and Martinon (1996) showed that most students chose debt to explain an expression with negative numbers, as did Beth. However, they also said that students had an easier time moving between a number line and abstract expression than moving between abstract and verbal, but this was not true for Beth.

In agreement with Murray's (1985) findings, Beth was able to solve addition tasks more accurately than she was able to solve subtraction tasks. Beth accurately solved only subtraction tasks that had a positive subtrahend, which was due to her movement procedure on a number line. In using the number line to help her solve subtraction tasks, Beth started at the first negative number she encountered, and usually moved to the left the amount specified by the magnitude of the other number in the expression. This prescription was not described specifically in previous literature. Yet, Peled (1989) did note that most students who solved subtraction tasks tended to go to the left because they believed that subtraction meant 
to "move to the left." Furthermore, when Beth solved a task with both a negative minuend and subtrahend, she paid no attention, or at least attributed no meaning, to the fact that there was both a negative sign and a subtraction sign. She might have considered it redundant, for she would operate in the same manner for tasks in the form $-a-b$ and $-a--b$, by starting at $-a$ and moving $b$ spaces to the left. In fact, she believed these tasks to be equivalent. Beth deviated from this procedure only when I asked to compare the tasks $-4--6$ and $-4+-6$, as well as to compare the tasks $-4--1$ and $-4-1$. After comparing the first two tasks, she abandoned the notion that the subtraction and addition of the same number elicited different answers, and thus believed that $-4--6=-4+-6=-10$. When she compared the second set of tasks, she adjusted her belief that $-4--1$ was equivalent to $-4-1$, and decided that when an expression contained a negative minuend, subtrahend, and subtraction sign, she had to move to the left on the number line, and when the expression had a negative minuend, subtraction sign, and positive minuend, she had to move to the right, since the expression had one less negative sign.

It is interesting that her left movement on the number line due to the negative sign of an addend, and her movement left on the number line due to subtraction, did not allow her to obtain a unary view of negative numbers, in that the addition of a negative number is similar to subtraction. Yet it is not too surprising given that she could not explain why she started with the first negative number she encountered or why subtraction indicated for her to move left on the number line.

Since Beth was able to use a number line to solve addition tasks correctly as well as understand addition of negative numbers in terms of qualitative characteristics, she had achieved Level 4 in both the Quantitative (QD) and Number Line Dimensions (NLD) (Peled et al, 1989) with respect to addition.

Furthermore, she had no difficulty crossing over zero, so she exhibited a continuous view of the number line. While Beth used the number line to help her solve subtraction tasks, she could not accurately solve tasks with a negative subtrahend. Beth attained Level 3* in the NLD and a Level 2* in the QD with respect to subtraction. An asterisk in the Quantitative Dimension (QD) indicates that Beth was able to solve higherlevel tasks than her number level indicates, but she did not put the answer or task into context. An asterisk in the Number Line Dimension (NLD) indicates that Beth was able to subtract natural numbers, know that plus and minus signs are opposite in direction, but could not do all same-signed subtraction tasks, yet could solve some opposite-signed subtraction tasks.

\section{Summary of Beth's Prior Conceptions}

- Beth had both a conceptual and procedural understanding of addition of negative numbers. While she moved correctly on a number line when she encountered the addition of negative numbers, she 
did not state the relationship between the addition of a negative and subtraction. She was also able to move between a contextual representation and abstract representation. While she could translate from an abstract representation to a number line representation, but could not reverse this translation.

- Beth used similar procedures on the number line to evaluate both addition and subtraction tasks. She started at the first negative number she encountered in the expression when she read it from left to right. For addition tasks, she then moved to the left if the other addend was negative and to the right if the other addend was positive. For subtraction tasks, she moved to the left the magnitude of the other number. Therefore, Beth was unable to correctly solve subtraction tasks containing a negative subtrahend.

- Initially, Beth did not attend to the negative sign on the subtrahend if the minuend was negative and believed that the solution to $-a--b$ was the same as the solution to $-a+-b$. By the end of the interview, Beth adjusted some of her conceptions of subtraction of negative numbers. She decided that if the task had a negative minuend and subtrahend, she was to move to the left on the number line and if the task had a negative minuend and positive subtrahend she was to move right.

- Beth attained Level 4 in both of Peled's QD and NLD for addition.

- Beth attained Level 3* in the NLD and a Level 2* in the QD with respect to subtraction.

\section{Analysis of Interaction with Subtraction Applets}

Because Beth could accurately solve addition tasks with and without a number line, we did not use the addition arrow applet (AAA). Since Beth believed that subtraction meant to move left on the number line, and since she moved the number of spaces represented by either the minuend of subtrahend, I decided to have her work on the subtraction arrow applet (SAA) prior to the subtraction distance applet (SDA).

\section{Beth's Interaction and Understanding with Technology}

When Beth used the SAA, I did not have the hot-linked equation representing the visual on the number line revealed because I wanted to see how the sole illustration of the arrow value appearing inside the arrow impacted her method of solving subtraction tasks. After manipulating the SAA without attempting to solve any tasks, Beth noticed that when she dragged the arrow to the left of the blue stick, the value inside the arrow was positive and when she dragged the arrow to the right of the blue stick, the value inside the arrow was negative. Furthermore, she noticed that each time she moved the arrow to the right by one, the value inside the arrow decremented by one. Moreover, she noticed that the value "would go down again" in a positive fashion before she rested the arrow on top of the blue stick, at which point she said, "it would be zero" and then go "into the negatives."

When I asked Beth to model $5-2$ on the SAA, she moved the blue stick to the 5 and moved the orange arrow so that its tip landed on the 3. I said, "Good, so tell me how you knew to go left." She mumbled under her breath, "Because," paused, and looked up at me as if questioning whether that was the 
correct move. I affirmed her answer and said, "That's right, I just want to know how you thought about it." She blushed, sighed, and said, “Because it doesn't have like a negative sign. [pause] I don't know, I just knew it." She seemed to struggle for the right words to express her thinking. Upon prompting, she explained, "the two right there isn't negative." She then pointed to the "2" in the expression 5-2, and subsequently pointed to the screen with the arrow value depicting a positive 2 inside of it (see Figure 1). Most likely she noted this because, prior to using the applet, she would have solved this task similarly using the number line. She would have started at the 5 and moved to the left 2 spaces. However, she would have justified this movement because she was subtracting two and therefore, needed to move to the left. Yet, here, she saw the correspondence between the positive two in the arrow value and the positive two in the subtrahend. Moreover, she explained that the answer was 3 because it was "where the 2 ends, where the other dot is pointing" (see Figure 1). Therefore, she tried to relate the expression and the visual of the SAA even though I did not tell her that this applet depicted subtraction.

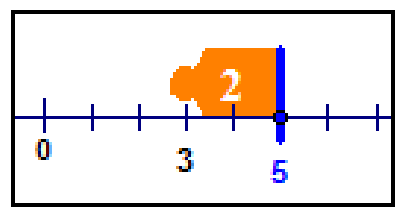

Figure 1. Beth using the SAA to depict $5-2$

Beth modeled the subtraction tasks $5-1$ and $5-0$ correctly. When it came to modeling $5--1$, she moved the arrow to the right one space from the 5 and said, "you go to the right 1 , so 6." Prior to working with the SAA, she would have used her procedure and started at the -1 on the number line, moved to the left 5 spaces, and landed on the -6 . This time, because she had consistently used the blue stick to represent the first number and the arrow value to represent the subtrahend, she knew she saw the pattern of moving to the left the amount represented by the subtrahend if the subtrahend was positive and to the right if the subtrahend was negative. For the first time, she obtained a correct answer for a subtraction task with a negative subtrahend. Similarly, she knew to extend this pattern for the tasks $5--5$ and $5--12$, without modeling the in between expressions.

I called her attention to the fact that she had gotten -17 as the answer to 5--12during the interview, yet obtained 17 to this task now.

B: It's 17 cause I think I went to the left [before] instead of the right.

$\mathrm{N}$ : So why do you think we go to the right?

B: Would we be adding?

$\mathrm{N}$ : Can you explain more of that?

B: I think two negatives make a positive, don't they?

$\mathrm{N}$ : Ok, tell me more about that.

B: Like two negatives make a positive, and um, and I think [sighs and pauses]

$\mathrm{N}$ : That's right, keep going. How did come up with that? 
B: I was looking at this [points to the screen] and the problems above and how it was a positive number.

Beth recognized the difference of her movements on a number line for the task $5--12$ before working with the SAA and her movements with the SAA, and she compared the outcomes. In doing so, she looked back to her movements and answers to tasks with a positive minuend and negative subtrahend, and she saw that she always moved to the right and the answer to these tasks was the same as the answer to an addition task with the same value as the minuend and absolute value of the subtrahend. Because of the movement to the right and the answer resembling an answer to an addition task, Beth remembered that subtraction of a negative number turned both negative signs to positive.

Because Beth was able (1) to solve subtraction tasks with a positive minuend and negative subtrahend accurately without the aid of the SAA, and (2) to justify it in terms of addition due to the two negatives side-by-side, I asked her to predict the solution to $-4--6$. Instead of saying -10 as she would have prior to using the SAA, she said, "It would be 10...because you add like 6 to...oh, negative 4." She thought that since she had added the numbers in the tasks that had a subtraction sign followed by a negative number, she should add the 4 and the 6 together, yet as she said her answer, she realized that she had a -4 and not a positive 4 . She paused and grabbed the mouse to try and use the SAA to model it. After placing the blue stick on the -4 but before turning on the arrow, I asked her to predict which way the arrow would point, to which she responded, "to the left." I asked her why she thought that, but instead of answering, she intensely stared at the screen as if she was hoping I'd ask another question or help her with the answer.

$\mathrm{N}$ : Well, go ahead and drag the arrow and see what happens.

B: Oh, it's a positive number [inside the arrow]

N: Well, go ahead and try to drag it to the right.

B: It would be 2 .

Beth saw that as she dragged the arrow to the left, the number inside was positive. This did not fit with the expression that had a negative subtrahend. Thus, she dragged the arrow to the right 6 spaces so that the arrow landed on 2. She knew this was the correct answer, but her face showed perplexity (see Figure 2). She seemed to be looking at the -6 inside the arrow value and the direction of the arrow because she whispered, "negative six" and then moved the mouse so that it went to the right across the screen as to follow the arrow. It looked as if she wondered why the arrow was going to the right since she typically went to the right when she added a positive 6 , but the visual looked as if she added a -6 (see Figure 2) instead of subtracted a negative 6 . It seemed that she forgot her reasoning for traveling to the right when subtracting negative numbers. Due to her confusion, I asked her if this was similar to addition.

B: Yes. No. [pause] Yes. 
$\mathrm{N}$ : Are you sure?

B: Yeah [blushes and laughs softly]

$\mathrm{N}$ : So tell me what you're thinking.

B: If you go from -4 and go up 6 , you'd get 2 . You go to the right when adding.

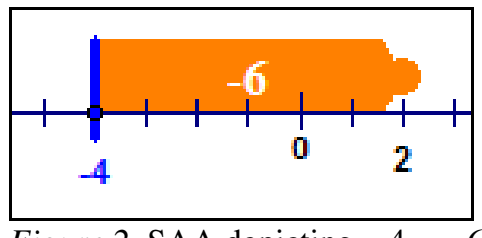

Figure 2. SAA depicting $-4--6$

Beth made the connection between the visual of the applet and adding 6 to -4 , but it seemed as if she thought the applet was depicting the equation $-4+6=2$ instead of $-4--6=2$. Therefore, in hopes that she would make the connection to the subtraction task, I said, "Well, here we are still subtracting" and pointed subtraction sign in the task $-4--6$. She only responded with a "yeah." I asked her if I was confusing her, and she acknowledged that she was a little confused, but thought it was "sinking in."

Due to her uncertainty, I decided to back track and try to figure out what exactly was causing her confusion. I started from the beginning, asking her to predict and then model the tasks $8-3,8-2,8-1$, $8--1$, and $8--2$. She accurately answered and modeled all of these tasks. Then I tried to get her to generalize her movements.

N: How do you know if you are going to move to the right or left?

B: How, like when...like how I know if I am going to go to the right or left?

$\mathrm{N}$ : Um hmm. So like, if you did $8--4$, which way would you move it?

B: To the right.

$\mathrm{N}$ : Why?

B: Because of the -4 .

N: Good. What would you predict your answer would be before you moved it?

B: Negative 12, no, I mean 12.

$\mathrm{N}$ : Good, and what were you thinking about?

B: Like it's the same as 8 plus 4.

Beth knew she had to travel to the right when she subtracted a negative subtrahend, but when asked to predict this answer this time, she seemed to revert back to her first intuition before thinking about incorporating the direction. She immediately stated that the answer to $8--4$ was equal to -12 , which she would have gotten using her previous procedure: starting at the -4 and moving to the left 8 spaces. Once she incorporated the idea of the applet, she remembered that she had to move to the right, which would have been the same as adding 4 to the 8 , which would give her positive 12 . It is not clear if she visualized herself actually moving the arrow to the right from 8 and landing on the 12 or simply added the value of 4 and 8 in her head. It seemed that since she knew that she had to move to the right and moving to the right was the same as addition, she had to add the value of 4 to the value of 8 arithmetically. Thus, it seemed that 
she did not attend to where the arrow tip was actually landing. Instead she remembered that she had to add the values in the expression abstractly.

This time, instead of jumping straight to tasks with a negative minuend and subtrahend, I asked her to model the task $-3-2$. She placed the blue stick at the -3 and dragged the arrow to the left 2 spaces and landed on the -5 .

N: How did you know to go left?

B: Because I'm subtracting 2.

N: So if you had to subtract -2 , or do this problem $-3--2$, which way would you go?

B: To the right, so 5 .

$\mathrm{N}$ : So if you moved the arrow to the right 2 what would you get?

B: Oh, -1 .

Beth knew that she moved to the left and right based on the sign of the subtrahend, but she still resorted to answering immediately without thinking about what she was doing. For instance, when she solved $-3--2$ she combined her initial procedure with starting at the -3 and moving to the left 2 , which would give her the value of -5 . Due to previous experience, she knew she had to travel to the right. Yet, instead of traveling to the right she used her knowledge that moving to the right was adding. She "added" the absolute values, instead of the 2 to the -3 , and she believed that the answer was positive 5 . Once she actually took the time to think about moving the arrow from the -3 up 2 spaces, she was able to visualize the correct answer of -1 . However, I do not think she viewed adding the 2 to the -3 when the expression read $-3--2$, even though she traveled right on the number line from the -3 .

$\mathrm{N}$ : So, before you said when you had a subtraction sign followed by a negative sign, you added. [Pointing to the tasks with a positive minuend and negative subtrahend] Is this true here?

B: No, cause the first number is negative too. Like all the numbers, well, like both the numbers are negative, and you're subtracting both of them.... and here only these two were negative [pointing to the $8--4$ ], well, like this one is negative and you're subtracting it, but here these two number are negative and you're subtracting both of them [pointing to $-3--2$ ]

$\mathrm{N}$ : Oh, so you're saying that's why you get a negative answer for $-3--2$ and positive answer for $8--4$ ?

B: Um hmm.

$\mathrm{N}$ : So here, for $8--4$, did you add?

B: Um hmm, to get 12 .

N: So if you have a -3 and add 2 , what happens then?

B: You get the same thing, -1 [says softly]

$\mathrm{N}$ : So is $-3--2$ similar to $-3+2$ ?

B: Yeah, it's just not positive.

Beth connected the word 'adding' with the idea of always obtaining a positive answer. This is why she believed that $-3--2$ was not like adding because she obtained a negative answer to this expression. One possible reason for this belief about adding is that she associated moving to the right with adding and moving to the right as traveling towards the positive side of the number line. Since she determined the 
answer of the expression by viewing where the arrow landed, typically if she moved to the right, she landed on a positive number. Therefore, she could have begun to associate adding with obtaining a positive answer. When I tried to get her to separate the ideas of adding and getting a positive answer, she understood that adding took on another meaning by her response to saying that $-3+2$ got her the same answer as $-3--2$. She did not seem totally sure about this, and because her answer was negative to a task with both a negative minuend and negative subtrahend, I asked her to solve $-3--7$ without the applet.

B: Um, -4 , oh, um, yeah, -4 .

$\mathrm{N}$ : Ok, so tell me how you got -4 .

B: Because like here, 3 minus 2 equals 1, and there are like negative signs, so I put like 7 minus fff... [going to say four], like 3 minus fff [going to say four], like 4 minus 3, ugh, I'm confused.. [rubs her hand across her forehead]

She had a difficult time trying to verbalize her answer. She justified her strategy by using an analogous task. She knew that $3-2=1$, and she reasoned that as long as she put negative signs on every number, the equality would hold true, so that $-3--2=-1$. Similarly, she tried to use the numbers 7,3 , and 4 , use subtraction, find the answer to that task, and then apply negative signs to every number to show the answer was -4 . What she had hoped to use was the task $7-3=4$ and, consequently, $-7--3=-4$. While this reasoning is true, it does not fit this task, which is the reason she kept adjusting her numbers. She began by trying to use the expression $7-4$ but noticed that would give her an answer of 3 , when she really wanted the answer of 4 . Then she tried the expression 3-4 and 4-3, but knew that neither answer would give a relation to 7. At this point she was thoroughly confused in trying to verbally justify her answer. So I asked if she wanted to use the SAA to help her. She exclaimed, "YES!" and proceeded to move the blue stick to the -3 and the arrow 7 spaces to the right, landed on the 4, and said, "Four...because it's like adding them... because of the two negatives." I followed up by asking why it was not -4 , which was originally what she thought the answer was. She responded, "I was confused, but not any more, at first, but not anymore." Even though she was able to get correct answers with the applet, I did not want her to have to rely on the applet for future tasks. I also wanted to see if she truly understood what was happening, so I asked her some follow up tasks in which she could not use the applet to help her find the answer.

N: How would you solve $-6--1$ ?

B: Um, 5, yeah, 5.

$\mathrm{N}$ : Can you tell me how you got that?

B: I went from -1 up 5 and got 6.

$\mathrm{N}$ : So when you used the applet before, where did you usually start?

$\mathrm{B}$ : The first number.

$\mathrm{N}$ : Then what did you do?

B: You would go up 1, OH, -5.

$\mathrm{N}$ : Right, how did you know that?

B: Because it's a -1 . Cause I think if it was a plus 1 , it would be -7 . 
A few things worth mentioning can be seen from this excerpt. First, Beth did not use her default strategy of starting at the -6 and moving to the left 1 space. Second, Beth did not use her new strategy of comparing analogous tasks. If she had done that, she would have said that because 6 minus 1 is 5 , then -6 minus -1 is -5. Moreover, I had never seen her use this strategy before: starting at the subtrahend, and moving the amount of spaces of the difference. Furthermore, if she had checked her procedure by doing this on a number line, she would have noticed that she landed on the 4 and not the 6 . By simply asking her to remember how she worked with the SAA, by asking her where she put the blue stick, she correctly solved this task even though she did not manipulate the applet. She was able to visualize the movement on the applet, which allowed her to correctly solve the task.

I gave her more tasks to solve without the applet and without me prompting her to use the idea of the applet. She solved each of these tasks correctly by visualizing the applet and telling me that she moved "to the right" if the subtrahend was negative and "to the left" if the subtrahend was positive. She eventually told me, "I don't really like the changing signs, they get me confused...it's better to use the number line." Furthermore, she told me that she liked the applet because "it helped show me which way to go." Even though she disliked changing signs, she used that strategy for tasks with a positive minuend and negative subtrahend, but she justified her answers to tasks with a negative minuend and subtrahend by using the idea of the SAA.

After working through multiple subtraction tasks without the SAA, I wanted to see how Beth would work through a contextual representation of a subtraction task. I asked her a few temperature tasks that included the high and low values of the day and asked the change between them. She always chose subtraction as the operation and solved it by using the SAA method and a number line.

N: Alaska's high temperature for December $16^{\text {th }}$ was -6 degrees Fahrenheit. The low temperature was -17 degrees Fahrenheit. How many degrees did it change?

B: 11

N: So how did you get 11 ?

B: Well, I went from -6 and I went up 17 to 11 .

$\mathrm{N}$ : So how would you write that as a math problem?

B: Like negative six minus negative 17.

$\mathrm{N}$ : So how did you know to use subtraction?

B: Because the temperature dropped down. Like if the temperature would have went up, I would have added. But the temperature dropped down...like -6 to -17 .

Beth correctly solved this task and was able to move from contextual to abstract to number line representations. Because of the movement on the number line and the ideas behind the SAA, she was able to correctly write the equation $-6--17=11$. In fact, our working together seemed to make a lasting impression on how she solved subtraction tasks. 
A week later at our next session, I asked her to solve subtraction tasks. She solved them all accurately and referenced the SAA. Not only did she reference the SAA, but she could also change the signs in the task when she subtracted a negative subtrahend, even if the minuend was negative as well, which she had never done this before.

$\mathrm{N}$ : So can you solve $-6--4$ for me?

B: -2 .

N: How did you do that?

B: Because I started at the -6 and added 4 , instead of subtracting.

$\mathrm{N}$ : And why did you add 4 ?

B: Because it was a minus negative 4.

$\mathrm{N}: \mathrm{Oh}$ ?

B: I remember from last time, I remember that when it was a negative number, we always go to the right, so I added 4, so it's like if you add.

Since the last time, she had worked through her confusion with the changing of signs by visualizing the movement on the number line from the SAA. It was also interesting that in her responses she typically referred to the changing of the signs before mentioning the movement on the number line for tasks with a negative subtrahend. However, with tasks involving a positive subtrahend, she always said that she started at the minuend and moved back the amount of spaces of the subtrahend.

Because she was able to answer correctly all the subtraction tasks I had given to her and justified her answers using the SAA, I wanted her to also see the SDA so she could use the distance method as a strategy to solve subtraction tasks. I wanted her to compare these two applets to see which method resonated with her idea of subtraction.

After manipulating the subtraction distance applet (SDA), I asked Beth to model some tasks. She had a harder time understanding how the placement of the dots and movement of the arrow represented subtraction as a distance than understanding how the SAA represented subtraction. One reason why it may not have made as much sense was because she had no need to use the SDA to help her solve the tasks, since she was able to correctly solve them all arithmetically.

$\mathrm{N}$ : Let's do $9--3$. How would you model this?

B: Move the orange dot to the -3 and the blue dot to the 9 .

$\mathrm{N}$ : And what would your answer be?

B: A positive number.

$\mathrm{N}$ : Why do you think it's positive?

B: Because it's a positive number subtracting a negative number.

$\mathrm{N}$ : And what does the arrow mean?

B: That you go back, or that you go to the left.

$\mathrm{N}$ : So could you tell me how to find your answer?

B: I would add 9 and 3, like just get a positive [second addend] and a positive number [answer], so I kind of like ignored the signs.

$\mathrm{N}$ : Is there a way we can tell the answer by looking at what we did on the computer? What would the arrow value be? 
B: A negative number, no.

$\mathrm{N}$ : Well, let's show the arrow. What does it mean?

B: Where the line is going. Like how many lines there are to get to from 9 to -3 . You would get the same answer.

$\mathrm{N}$ : How did you know that?

B: Because I counted them.

Even though Beth moved the dots to the correct values on the number line, she initially did not use the number line to solve this task. Instead, she used the strategy of switching the signs and adding the values.

When I asked her to predict the arrow value, she mentioned the direction in which it traveled but could not connect the distance in between the minuend and subtrahend to her answer until I allowed her to show the arrow value on the screen. Then she realized that the arrow value was measuring the distance between the numbers, and she confirmed this fact by counting the tick marks. I do not believe that she completely understood this method as a viable strategy for subtraction.

In fact, it seemed that moving from the SAA to the SDA caused some confusion because she wanted to "take away" the amount of the subtrahend and find the answer where the arrow ended rather than use the arrow value to represent the difference between the numbers (see Figure 3).
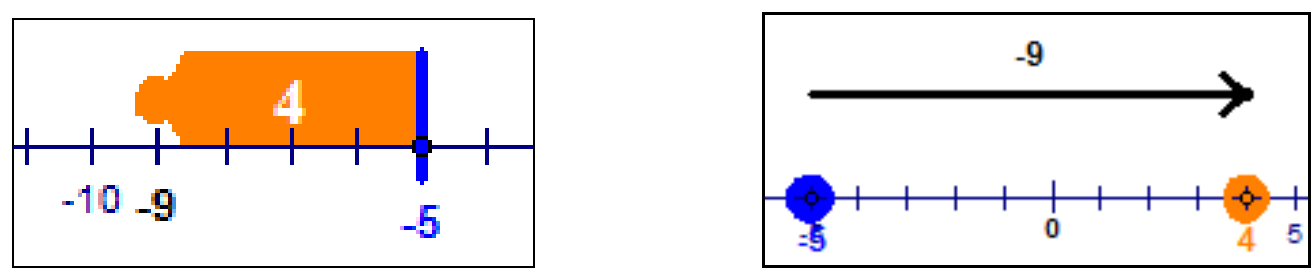

Figure 3. SAA versus the SDA for correctly modeling the task $-5-4$

$\mathrm{N}$ : So what about $-5-4$, how would that look on this applet?

$\mathrm{B}$ : Put the blue dot on the -5 and the orange dot on the -9 . [pause] Oh wait, I put it on the answer!

$\mathrm{N}$ : You did put it [the orange dot] on the answer. So where would you put the orange dot?

B: On the 4.

$\mathrm{N}$ : And how would you find the answer?

B: On those spaces [points to the spaces in between the blue and orange dots]

N: Right. So could you tell me, before you click the Show Arrow Value button, what the answer would be?

B: A positive number because it's going from a negative to a positive, so you're adding like the 9 to the -5 , so you'd get a positive number.

When I asked her to write the expression to which she was referring, she wrote $-5--9=4$. This was not the task with which I had presented her. The SAA had influenced her translation from the number line context to the abstract context. Her model using the SDA (Figure 4) for the task $-5-4$ looked similar to the model using the SAA for the task $-5--9$ (Figure 5). 


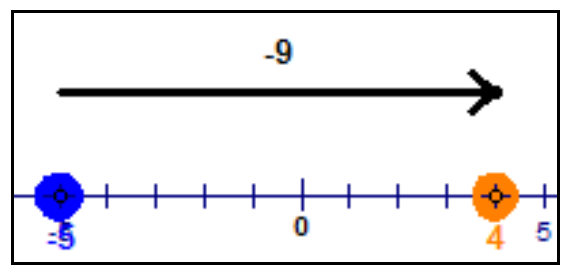

Figure 4 . The SDA correctly modeling the task $-5-4$

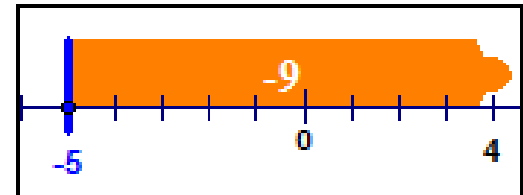

Figure 5. The SAA correctly modeling the task $-5--9$

I drew her attention to the fact that the original task was to solve the expression $-5-4$. She said, "Oh, you would go back then, you would go to the left 4 spaces from the -5 ." She then proceeded to move the orange dot from the 4 to the -9 . Once again, she placed the orange dot on the answer instead of the subtrahend (see Figure 6).

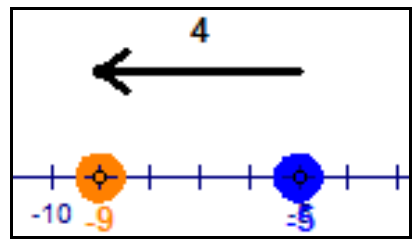

Figure 6. Beth's reposition of the orange dot in the SDA in hopes to represent $-5-4$

$\mathrm{N}$ : Ok, so could we also do it this way, put the orange dot on the 4 and have $-5-4$ equals -9 ?

B: Um, yeah.

$\mathrm{N}$ : Ok, so what would subtraction be here, when the blue dot was on the -5 and orange dot was on the 4 ?

B: You would be subtracting a -5 and a -9 .

$\mathrm{N}$ : So before, you put the blue dot on the 9 and the orange dot on the -3 for the task $9--3$, and did something to find the answer. How did you know the answer?

B: I counted the spaces.

$\mathrm{N}$ : Could you do that here?

B: Yeah. There are 9 spaces.

$\mathrm{N}$ : Now how would you know if that 9 was negative or positive?

B: It's going to be negative because you're subtracting a positive number, and you're going to the right.

Beth knew that she could show subtraction by putting a dot on the -5 and another dot on the 4 , however, she was still using the idea of take away instead of distance between to the two dots, which is evident in her statement that she was subtracting -5 and -9 . She was using the distance between the numbers -5 and 4 as a subtrahend instead of a difference. I tried to call her attention to another task she had illustrated correctly with the SDA, namely $9--3$. She remembered that the answer, or the difference, was the spaces in between the dots. By prompting her to look at this task, she adjusted her way of looking at the SDA to obtain the answer. Yet, I do not think that she understood why the difference was negative. While she 
already knew that subtracting a positive number from a negative number gave her a negative answer from the last time we worked together, and she saw that on the applet, she had moved to the right, she did not connect these ideas. Instead, she simply told me something evident from the abstract expression and something shown on the applet. I was looking for her to make the connection that subtracting a larger number from a smaller number produced a negative number. Even though she never made this connection, she was able to solve and model correctly on the SDA every type of subtraction task I gave her. She justified her answers by saying that she "counted the spaces in between" the minuend and subtrahend and determined its sign by stating whether "the arrow would move" left or right. The arrow was not showing for these tasks, so she had to determine which way the arrow "would move" if it were displayed. If the arrow moved to the right, she knew the difference was negative, and if the arrow moved to the left, she knew the difference was positive. I do not think she felt the need to reason why the signs were positive or negative because the movement and sign were similar to the SAA. Additionally, she knew that the sign in the SAA was based on the fact that when she moved to the left, she subtracted a positive number, and when she moved to the right she subtracted a negative number, because it was opposite in direction.

After having her use both applets to determine her answers to subtraction tasks, I asked her which applet she preferred.

B: The dots. I believe it's easier for me to see.

$\mathrm{N}$ : Oh, and how so?

B: Like you could put the dot on one and the dot on the other one and just count the spaces there. But the other one, you kind of like have to figure out the problem...kind of.

$\mathrm{N}$ : So what do you mean, 'figure out the problem'?

B: Like figure which way to go. I think the negatives, you can just see the answer.

$\mathrm{N}$ : And the other one [SAA]?

B: Like the other one, I'm always thinking that this is the answer, the -2 instead of the 12 .

It was surprising that Beth said that she liked the SDA better than the SAA because of her confusion when she began working with the SAA, for she frequently placed the orange dot on the difference rather than placing it on the subtrahend. Yet, her justification is plausible because rather than having to determine the direction and, subsequently, moving that amount in the subtrahend (i.e. figuring out the problem), she could place the dots on the numbers already specified in the problem and only have to figure out the answer. By her statement, it seemed that the subtraction of negative numbers gave her more difficulty in using the SAA than in the SDA. Her last statement about the -2 versus the 12 stemmed from the last task that I gave to her, $10--2$.

B: I would put the blue dot at the 10 and the orange dot at the -2 .

$\mathrm{N}$ : How would you know the answer?

B: Count the spaces in between, 12.

$\mathrm{N}$ : Would it be positive or negative? 
B: Positive 12 because I'm going to the left.

$\mathrm{N}$ : Could you do another way to solve this problem?

B: Add them, like move 2 up from 10.

She saw the difference between the SDA and SAA, but believed that by using the SDA method, she could easily see that 12 was the answer instead of -2 because the orange dot was already occupying the -2 value, so she knew the number of spaces in between was the answer. After moving the arrow in the SAA, she became confused as to whether the arrow value (-2) was the answer or where the arrow landed (12) was the answer.

Even though she told me that she liked the SDA better than the SAA, I was not convinced, so I asked her to solve more subtraction tasks without the computer to see which strategy she used. As I had anticipated, she always used the SAA method to justify her answers rather than the SDA method.

$\mathrm{N}$ : You can use whatever way you want to solve these tasks. The first one is $-4--1$.

B: I added 1 instead of subtracted.

$\mathrm{N}$ : Ok, and why did you add 1 ?

B: There were two negative signs.

$\mathrm{N}$ : So tell me more about that.

B: Adding, so I did -4 plus 1 ... since you subtract a negative, you go to the right, so adding 1 , and I just put it [a dot] on the -3 (see Figure 7)

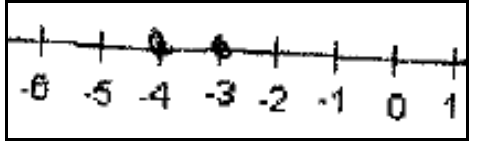

Figure 7. Beth's illustration of the task $-4--1$ on a number line

Beth reverted back to using the SAA method when given the opportunity to solve a subtraction task using any method she saw fit. In fact, for all the types of subtraction tasks I gave to her, she used this method to justify her solutions. While it is not clear as to why she used this method after saying she preferred the SDA method, I would venture a guess that it was because she had made many more connections with the SAA method to her initial understanding of adding and subtracting on a number line. Beth knew how to correctly operate on a number line for addition tasks, and she knew to move the number of spaces represented by the second addend. She would move to the right if the second addend was positive, and to the left if the second addend was negative. Similarly, the SAA allowed her to move the number of spaces represented by the absolute value of the subtrahend. Furthermore, after making the connection that subtraction of a negative number was similar to adding, she knew she had to move to the right. Moreover, since she could solve accurately subtraction tasks with a positive subtrahend by moving to the left, she did not need to adapt this strategy by using the SAA, but had to do so if she used the SDA. 
Summary of Beth's Interactions and Understanding with Technology

- Beth had a better conceptual and procedural understanding of addition and subtraction of negative numbers. In fact, she was able to visualize the applet and tell me the direction she needed to move on the number line dependent upon the sign of the second addend or subtrahend.

- Beth no longer used her previous method of solving subtraction tasks by starting at the first negative number she encountered in the expression when she read it from left to right and moving to the left the magnitude of the other number. In fact, she no longer believed that $a--b=-b-a$ or that $-a--b=-a-b$, and she could consistently solve subtraction tasks with a negative subtrahend accurately.

- By manipulating multiple sequences of subtraction expressions, noticing patterns, using contextual problems, and noticing the connection between movement away from the minuend and the corresponding equation, Beth was able to see subtraction as both take-away and a distance. She stated that she preferred using the idea of the SDA, but referenced the SAA when she justified her answers to computational arithmetical tasks.

- Beth was able to create correct subtraction equations for tasks represented contextually. In creating these equations, she used the SAA method to justify her answers.

- After learning how to solve addition tasks on the number line, Beth integrated this knowledge with her observation of how subtracting a negative on the SAA moved the arrow to the right. This helped her make the connection between the subtraction of a negative number and addition of a positive number on the number line.

- By using the SAA, Beth noticed that the subtraction of a negative number was the same as addition. Even while knowing this, she initially continued to use the movement of SAA for solving tasks with a negative subtrahend instead of changing the subtraction sign to an addition sign and the negative sign of the subtrahend to a positive sign. However, during the second session, Beth seemed to prefer to change the signs and compute the answer, and only when she was prompted to provide reasoning, she justified her answer using the SAA method.

- After working with the SAA, the SDA, and the accompanying tasks, Beth successfully attained Level 4 in both Peled's QD and NLD with respect to subtraction.

\section{Discussion}

Before working with the SAA, Beth did not have a clear conceptual understanding of subtraction.

Beth was unable to provide any justification for tasks in the form $a-b$ where $a>b>0$ and would state that she just "knew the answer." For every other subtraction task type, she started on the number line at the first negative number in the expression when it was read left to right. Starting at this number, she moved to the left by the number of spaces represented by the absolute value of the other number because she believed that subtraction always meant to "move to the left." Therefore, she was able to correctly solve all tasks including a positive subtrahend but not tasks with a negative subtrahend. She would operate in the same manner for the tasks in the form $-a-b$ and $-a--b$, by starting at $-a$ and moving $b$ spaces to the left. In fact, she believed these tasks to be equivalent. Furthermore, it was not until I had her compare the tasks 
$-a--b$ and $-a+-b$ did she believe that they were not equivalent. She believed that since $-a--b$ contained more negative signs than $-a+-b$, she had to move to the left $b$ spaces from $-a$ to solve $-a--b$, and she had to move to the right $\mathrm{b}$ spaces from $-a$ to solve $-a+-b$, even though previously she had accurately solved and justified her movements for addition tasks.

After exploring the SAA, Beth realized how the applet embodied subtraction by observing how her manipulation impacted the visual in terms of the beginning, ending, direction of the arrow, and the value in between the blue stick and tip of the arrow. This was not totally surprising given the fact that Beth used the same method to solve subtraction tasks with a positive minuend and subtrahend. However, when she observed how the arrow value changed as she moved the tip of the arrow from left to right, she saw (1) the connection between the values of the minuend, subtrahend, and difference and (2) the relationship among the numbers, operations and movement on the number line, which helped her understand how and why she needed to move to the right when she subtracted a negative subtrahend. In fact, without any prompting or guiding to attend to this phenomenon, she made this connection and extension of subtracting negative values solely based on the generalizations of the movement of the applet and sequencing of the questions. Because she saw the arrow moving to the right through the visualization of the applet and the answers simulating addition for a positive minuend and negative subtrahend, she remembered that two negatives equaled a positive, or in this case the subtraction of a negative subtrahend was the same as adding the absolute value of the subtrahend. While she had a bit of difficulty extending this understanding to tasks with a negative minuend and subtrahend, due to her confusion of addition versus having a positive answer, she eventually did so with the aid of comparing the movements and visuals of the tasks $-3-2$ and $-3--2$. The SAA made this idea of subtraction clearer because Beth noticed that for one task, she moved to the left and in the other task she moved to the right. This also allowed her to understand that adding meant moving to the right but did not always produce a positive answer. Furthermore, Beth was able to translate correctly between the abstract and number line representation and justified her translation by using and describing the SAA method.

By our next session, Beth not only could solve all subtraction tasks by justifying her answers using the SAA, but she was also able to change the signs to compute the task correctly. Also during our next session, Beth learned, understood, and compared the SDA with the SAA. Due to the strategies Beth learned while using the SAA, she became a little confused when she switched to the SDA. She frequently reverted back to using the method of the SAA on the SDA applet by representing the answer as the orange dot instead of the subtrahend. Eventually she overcame this confusion, and even told me that she preferred 
using the SDA because it allowed her to represent the problem more easily and only think about finding the answer by counting the spaces in between. However, when given the opportunity to use any method to solve subtraction tasks on paper, she reverted back to using the SAA method to justify her answers.

\section{The Equal Sign}

\section{Prior Conceptions}

Based on her responses to the interview tasks, Beth had primarily a relational (Seo \& Ginsburg, 2003) view of the equal sign. Only her response to Task 1 and her initial response to Task 4 could be classified as operational. After stating that each form in Task 1had "all the same problem except that this one has the equal sign, this one doesn't, and this one has the answer and the rest don't," Beth told me that the equal sign "did not meaning anything" for the second form, but the equal sign meant something in the last form in that "it's giving you the answer after it." She believed that if the answer did not follow the equal sign, the equal sign did not mean anything in relation to the expression, as if it was incomplete.

Beth's initial response to Task 4 could be considered operational because she believed the answer to the question mark to be the value of adding the 3 and the 5 , which fell directly to the left of the equal sign.

$\mathrm{N}$ : So what would the equal sign mean in $3+5=?+2$ ?

B: Like 3 plus 5 equals the question mark plus 2 . So it's 3 plus 2 so they are equal.

$\mathrm{N}$ : Oh, so the question mark would be 3 ?

B: Yeah. Wait, no.... it's 6.

$\mathrm{N}$ : Ok, and how did you get 6 ?

B: Because 5 plus 3 is 8 and 6 plus 2 is 8 .

Because the question mark fell immediately to the right of the equal sign, Beth initially thought the question mark symbolized the sum of the values on the left of the equal sign (i.e. problem versus answer). However, upon checking if the value of 3 worked in the equation, she noticed that she had to find a value to the question mark such that when it was added to 2, it gave the same result as the sum of 3 and 5 .

Therefore, she fixed her answer by using a relational view of the equal sign.

Beth's view of the equal sign with regards to the other tasks with only numbers was relational. She was familiar with the non-canonical forms of $3=3$ and $8=10-2$, and told me that she had seen those types of representations in equations in her math class. Specifically, she had seen $3=3$ in "equations when you get $x=3$ and you have to check if it works." When I asked if it would work to write $3=3$ without the $x$, she acknowledged that it would still be true. Since the value of 8 was on both sides of the equation in $8=$ $10-2$, she believed that it was acceptable to write an equal sign between the 8 and $10-2$. She noted that it was "reversed" and that she did "not understand why they do that, why they reverse them." For both of 
these previous tasks, she never mentioned the word "same" as in describing why she felt the equations were correct. Similarly, she never used this word in Task 2, but did for Task 3. For the rest of Task 2, she used the idea that one unit was comprised amount of a smaller unit. For instance, 1 foot $=12$ inches because " 12 inches is in 1 foot," and 10 pennies $=1$ dime because " 10 pennies is in 1 dime." For Task 3 , she said, "2 times 5 equals 4 plus 6 because 2 times 5 is 10 and 4 plus 6 is 10, so they are the same.” This was the first time she used the word "same" in describing the equal sign even though she used the idea of equivalence in solving the Task 4 .

After I presented her with Task 4, I asked her if the equal sign meant that one side still had to equal the other side as she thought for tasks involving an operation on both sides, i.e. Tasks 3 and Task 4 .

B: No, this time the problem has to equal 3.

$\mathrm{N}$ : What is the problem?

B: The $x$ plus 5. So the $x$ has to make sure that adding the 5 is equal to 3 .

$\mathrm{N}$ : So the equal sign means...

B: You have to find the $x$ value, so the answer is 3 .

Even though Beth did not seem to view that this equation had 'sides' that were equivalent, and instead viewed it as a 'problem' on the left and the 'answer' on the right, an operational view, Beth seemed to talk enough about the fact that she had to solve for $x$ so that when she added 5 to it, it would have to equal 3 relational view. The reason she talked about this equation having a problem and answer could be due to the fact that there was no operation on the right side of the equation, as in Tasks 3 and 4; therefore, she did not have to operate on that side because it was already "the answer." She then asked me if this task was "like a 1 or 2 step equation?" When I said that it was, she immediately began to apply a solution procedure to solve for $x$, without being asked to do so (see Figure 8).

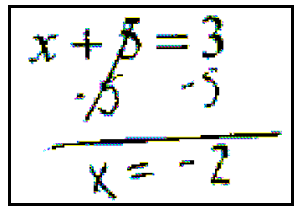

Figure 8. Beth solving $\mathrm{x}+5=3$

$\mathrm{N}$ : Ok, so tell me what you were doing?

B: Well, 5, so you have to cross out the fives so the variable can be by itself, so you have to cross it out. And you have to do the same thing on the other side, so 3 minus 5 is -2 because 2 minus 5 is negative 3 .

$\mathrm{N}$ : Ok, so how did you know you had to do it to both sides?

B: If you go on one side, you have to do it to the other, that's just what we do from class, we have to.

$\mathrm{N}$ : What would happen if you didn't do it to both sides?

B: It wouldn't get you the right answer. Cause if they're like adding on this side, like 8 plus 5 doesn't equal 3.

Beth solved for the variable algebraically (Knuth et al, 2006), yet she did not seem to know why her 
procedure worked, except for the fact that it gave her the correct answer when she substituted the value for the variable. When describing her procedure, she emphasized the word "have" to let me know that she had to follow this specific method because of the way she had been taught to solve these types of tasks. When I asked her what might happen if she deviated from this method, she simply told me that she would not get the correct answer and proceeded to combine the numeric values in another fashion to show me that the answer obtained from this fashion would not fulfill the equation. Instead of subtracting 5 from both sides, she added a 5 to the right side of the expression and told me that if $x$ was equal to 8 , the expression $x+5$ would not equal 3 .

It was evident that she did not understand why she performed these procedures by the way she tried to solve the next task, $2 m+9=1$. She told me that she had solved tasks that looked like these and blushed when she told me that she could not remember exactly what to do. She attempted to do the task two different ways (see Figure 9).
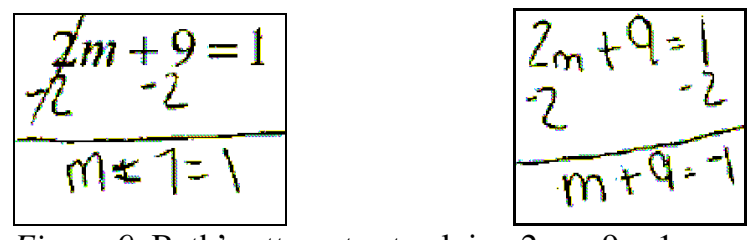

Figure 9. Beth's attempts at solving $2 \mathrm{~m}+9=1$

B: I'm not really sure how to do these actually.

N: Ok. So go ahead and explain to me how much you know.

B: I thought you just subtracted so you could so then you could. I thought you'd subtract these numbers, but I guess you don't.

$\mathrm{N}$ : And why do you think you don't?

B: Because like when I did the problem, 2 times 7 equals 14 plus 9 doesn't equal to 1 .

N: So when you got the answer and you checked it, it didn't work.

B: Yeah.

By looking at the left picture, Beth subtracted 2 from the $2 m$ and a 2 from the 9 . She decided to subtract 2 because she wanted to get the variable by itself, which was the whole reason for her previous procedure in solving 1-step equations. She knew that she had to subtract 2 from two portions of the equation, but she did not understand the idea of equivalence; therefore, she did not subtract it from 1 . What is interesting is that she brought the equal sign and the 1 straight down as if they were in columns, but never considered what the 1 represented with respect to her subtraction of 2 or how it related to the value of $m$. She simply ignored it; thus, believing $m$ was equal to 7 . She abandoned this idea because when she substituted 7 for $m$, she did not get the value of 1 . Once again, she based the appropriateness of her strategy not on the meaning of the equation but on whether it produced the correct answer.

$\mathrm{N}$ : So I see you started another problem over there.

B: I can't get that one either. [blushes] 
$\mathrm{N}$ : So here you took 2 away from the $2 m$ and the 9 , and here you took 2 away from the $2 m$ and the 1. So what made you decide to do it that way? How did you know to try this strategy?

B: Because if I did it on this side [subtracting a 2], it would just be the same, so I tried something different.

$\mathrm{N}$ : Ok, so I see you're left with $m$ plus 9 equals -1 . What could you do now?

B: Wouldn't it be -1 plus 9 equals 1 ? No, I don't understand.

$\mathrm{N}$ : I'm sorry, could you tell me which would be the -1 ?

B: The $m$.

$\mathrm{N}$ : Oh, so you're saying that when you solve this new equation you get $m$ equal to -1 ?

B: Yeah. [like she's been saying this all along]

$\mathrm{N}$ : And how did you know that $m$ was equal to -1 ?

$\mathrm{B}$ : Because 1 minus 2 is -1 .

$\mathrm{N}$ : So what would the $m$ plus the 9 equal to -1 mean? [pointing to last step]

$\mathrm{B}$ : $\mathrm{Um}, m$ is equal to -1 .

$\mathrm{N}$ : So does this mean that $m$ plus 9 equal to -1 , or is $m$ supposed to be equal to -1 ?

$\mathrm{B}: m$ is equal to -1 .

$\mathrm{N}$ : So what's that plus 9 part there? [points to last step] Is it useful?

B: No. You have to add it, unless you do -1 plus 9, but that doesn't equal 1.

$\mathrm{N}$ : So what happens when you put -1 in for $m$ ?

$\mathrm{B}$ : Two times -1 is -2 plus 9 is not equal to 1 .

$\mathrm{N}$ : Is there another way you might be able to solve this?

B: I don't know.

Beth believed that she had to subtract 2 from two terms in the equation, one of the terms had to be the $2 m$ so she could isolate the variable. Because she saw that her first attempt did not produce the correct value, she decided to subtract 2 from the 1 . This gave her the resulting equation $m+9=-1$. Instead of solving this equation for $m$, she decided that $m$ was equal to -1 because she ignored the +9 in the equation. It seemed that she believed that she should have obtained the answer to the variable after 1-step. This was consistent in both procedures. She ignored any values that she did not operate on, in the first attempt the 1 and on the second attempt the 9 , and believed that the resulting $m$ value equaled the value she obtained after operating on the other term. This is why she placed an equal sign between the $m$ and the 7 on the first attempt and simply ignored the +9 in the second attempt. When I called her attention to the 9 in the equation $m+9=-1$, she told me it was useless by showing me that when she plugged in -1 for $m$, it produced 8 and not 1 , the answer to the original equation. Once again, she based her reasoning of a procedure's appropriateness on whether it allowed her to obtain the correct answer. Yet, she did realize that she had not gotten the correct value for $m$ because when she substituted -1 into the entire original equation, she did not obtain 1 .

\section{Summary of Beth's Prior Conceptions}

- Beth exhibited a relational view of the equals sign for numerical equations and the comparison of numbers and units. It is difficult to classify Beth's understanding of the equal sign for equations involving variables, but it seems as if she had a mixed understanding of relational and operational. 
- Beth was familiar with non-canonical forms and was able to state why they made sense, although she admitted as to not knowing why one would write the forms in this manner.

- Beth did not explicitly say that the equal sign showed the equivalence of two expressions, but she alluded to this idea when solving Task 4 and Task 5. However, she did not use this idea in solving 2-step equations.

- Beth accurately solved 1-step equations using an algebraic method, yet she did not understand the reasoning behind her procedures. When it came to 2-step equations, Beth was unable to solve them correctly.

\section{Analysis of Interaction with the Equality Applet \\ Beth's Interaction and Understanding with Technology}

With only a few minutes of exploring the equal balance applet (EBA), Beth was able to easily understand how it worked. She first showed me how she knew one contained weight than the other. She placed a 5-weight on the left pan and a -1 balloon on the right pan.

B: It's tilting left.

$\mathrm{N}$ : Why is that?

B: Because 5 is bigger than -1 .

I then asked her how she could make the pans balance. She asked, "Can I show it?" and removed the -1 balloon from the right pan, and replaced it with a 5-weight, and commented, "Five on both sides." I asked her if there might be another way to keep the pans balanced, to which she asked, "You mean like put more than one?" She proceeded to remove the 5-weight on the right pan, exchange the 5-weight in the storage unit for five 1-weights, and place them on the right pan (see Figure 10). Thus, she noted that the applet automatically summed the values in the pan.

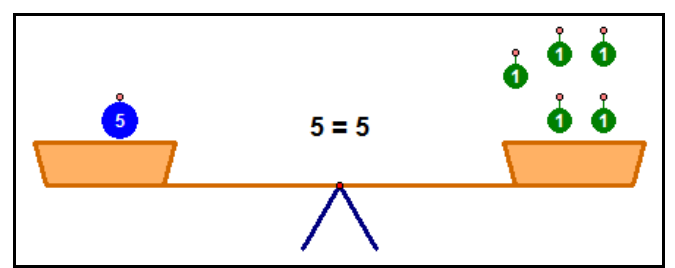

Figure 10. Exchanged one 5-weight for five 1-weights to balance left pan.

Since I wanted to see if she recognized the other method of keeping the pans balanced, I asked pointedly, "Is there a way we could balance the pans by having something on the left pan but nothing on the right pan?" She instantly responded, "Yeah, a 5 and -5 ." She returned the five 1-weights from the right pan to the storage unit, placed a -5 balloon on the left pan, and pointed to the computer screen and looked at me as if to confirm her correct answer. Based on her manipulations, Beth was able to see the significance behind the representations of the balloons as negatives and the weights as positives. She noted that they could be 
matched to produce a value of zero. I asked her to summarize how she could make the pans balance.

You can have a positive number and change it to like a negative. Like right there, you have a positive, so you could just add the same negative number to get it to zero. So if we have a positive 5 , you add a negative 5. Or you can add to the other side like the same amount you added to one side. Like a 5 and a 5.

By manipulating the applet for only a brief amount of time, she was one of the quicker students to discover the rules for the Algebalance.

Given that she discovered the two rules to maintain the equilibrium of the pans, I asked Beth to begin the tasks in the instructional section in Module 3. In modeling the equation $3+5=?+2$, she dragged three single weights and one 5-weight onto the left pan, and paused before completing the right pan. I asked her, "Ok, so what would you do for the right pan?" She dragged two 1-weights onto the right pan but did not utilize the question mark on the screen.

$\mathrm{N}$ : Ok, so how could you tell by looking at this what the question mark was equal to?

B: 8 minus 2 ?

$\mathrm{N}$ : Ok, how did you get that?

B: Because that would give you the answer of what the number plus 2 equals 8 .

$\mathrm{N}$ : So if you were to subtract 2 from this side [left], would it stay balanced?

B: No. [Removes two 1-weights from the left pan]

$\mathrm{N}$ : So what would you have to do?

B: Add 6 ?

Beth knew how to solve this task before using the EBA. Therefore, she knew she had to subtract 2 from both sides. However, when given the visual on the EBA, she focused on removing the two 1-weights from the left pan, but forgot she had to remove them from the right pan. Instead, she looked at the resulting image (see Figure 11) and tried to figure out how to get the value of the question mark. At this point, she believed that she had to add the two 1-weights from the right pan with the amount of 6 on the left pan in order to get the question mark.
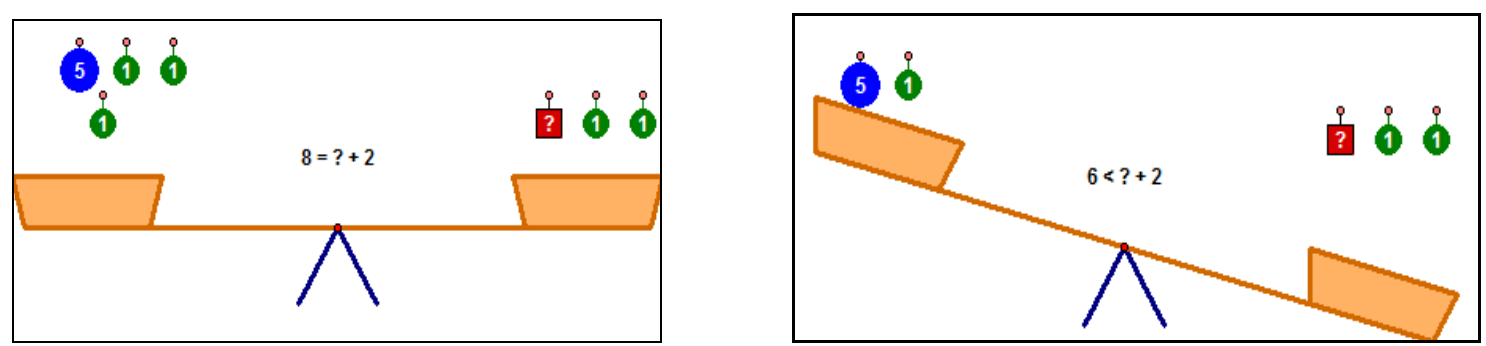

Figure 11. The EBA illustration of $3+5=?+2$, and the EBA after Beth removed 2 from the left pan

It seemed her knowledge of how the balanced worked for numbers did not transfer when she started using variables. I had reminded her of the rules she discovered for the Algebalance, and she said, "Oh, subtract 2 from that side." Then she told me that she had " $x$ equals question mark. I mean 6 equals question mark."

When solving other 1-step equations, Beth never seemed to use the EBA to solve the tasks, for she 
saw no need to do so. She solved all the 1-step equations on paper and applied the correct inverse operation to each side to do so. Because she did not use the applet to help her solve the tasks, I asked her to model $x+8=21$ using the applet and asked her how she solved the task. She removed the 8-amount worth of objects from the left pan, and all of the 21-amount worth of objects from the right pan; then she placed 13 on the right pan.

B: 21 minus 8.

$\mathrm{N}$ : Ok, so how were you thinking about that?

B: Because 8 plus would give you the number. Like 8 plus some number would equal 21 . So if you do 21 minus 8; it would give you that number.

$\mathrm{N}$ : So what did you end up with?

B: $x$ equals 13.

It was obvious that Beth applied her knowledge of solving equations, found the answer for the variable, removed all the objects from the pans except for the variable on the left pan, placed the final amount on the right pan, and then used her understanding of equations to justify her answer. She did not use the attributes of the applet to discover the solution, rather only to confirm it.

Since she was solving 1 -step equations without the EBA, I asked her to try to solve $2 m+7=15$ without it as well. After giving her the equation, she sighed, placed her elbow on the table, and held her head in her hand. I asked her if she could figure out what to do by looking at her other work. She did not say anything at first and finally said, "I want to try it on there!" and pointed to the computer. I was hoping that given the chance to see the need for the applet, she would try and use the applet to help her.

Before moving any objects, I asked her what she thought she might do first. Beth said, "Take off an $m$, [pause], or take off the two ones." I encouraged her to try one of those options. After moving the two 1-weights from the left pan to the storage area, she paused and said, "No, take a 5 off." I had her hit the Reset button and she then removed a 5 from the left pan and a 5 from the right pan. She had noticed that if she removed two 1-weights, there was no 1-weights to remove from the right pan to keep the pans balanced (see Figure 12). Therefore, she decided to start over and remove a 5-weight from both sides first.

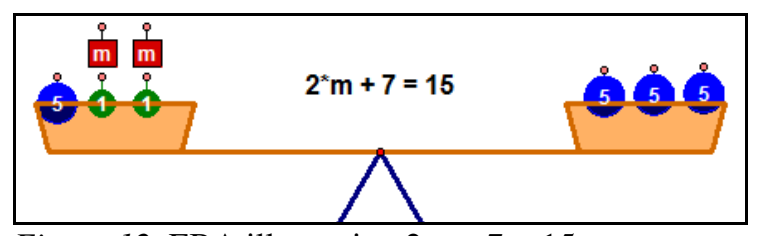

Figure 12. EBA illustrating $2 \mathrm{~m}+7=15$

After removing a 5-weight from both sides, she announced the amount of the remaining objects, "10...and 2 ones and $2 \mathrm{~ms}$ on the other side." Given that she would run into the same issue she had before, I asked her, "So now what can you do?" She must have realized that there was no way to avoid removing the two 
1-weights from the left side in order to get the $m$-weights by themselves because she said, "Take the 2 off." Then I asked what she would do about the other side, and she responded, "Take off the 5. Add back 3." After she removed the 5 from the right pan, she added three 1-weights to the left pan instead of the right pan (see Figure 13). She paused and looked at the applet with a confused facial expression as if she knew the pans should be balanced, but she could not figure out why they were not. I asked her, "Which pan did you remove the 5 from?" She immediately realized what she had done, "Oh, the right." I asked her if she could tell the value of $m$.

B: Four

$\mathrm{N}$ : How did you get 4 ?

B: 8 divided by 2 would be 4 , and 4 times 2 is 8 .

N: How did you know to divide by 2 ?

B: Because there are $2 m$ s over here and 8 over here.

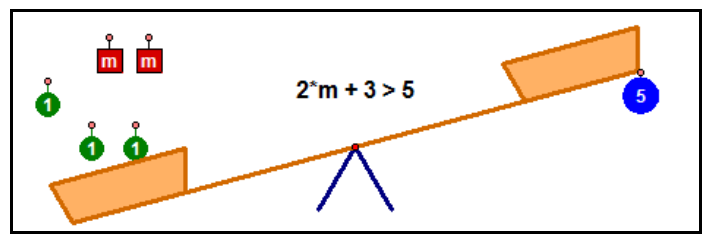

Figure 13. Replacing the three 1-weights to the wrong side

By using the visual of the EBA, Beth intuitively knew she had to divide the amount on the right pan into two portions, so that each $m$ would be of equal value. I then asked Beth to summarize what she did on the pans and we kept a tally of it on paper. I reset the EBA so that she could walk through each of her steps and tell me what they were similar to in terms of arithmetical operations (see Figure 14). After she told me that she subtracted 5 from each side then subtracted 2 from each side, I asked if she could have done the same in an easier way. She responded, "I guess subtract like 7." I asked, "So if you subtracted 7, what would you have left?" She said, " $2 m$ plus 0 equals $8 \ldots m$ equals $4 . "$

$$
\begin{aligned}
2 m+7 & =15 \\
-5 & =-5 \\
2 m+2 & =10 \\
-2 & =-2 \\
2 m+0 & =8 \\
m & =4
\end{aligned}
$$

Figure 14. Correlating manipulations on EBA to solving equations algebraically

By manipulating the EBA, Beth was able to realize a pattern for how to solve 2-step equations

algebraically. By correlating her actions to algebraically solving the 2-step equation on paper, she was able to solve correctly every remaining 2-step equation I gave to her without using the EBA. 
After solving 2-step equations, I asked her if the EBA helped her at all. She said, "Yeah, like it gave you like a visual to know what you had to subtract or add or multiply.” The EBA allowed her to visually see what values she had to subtract in order to get $m$ by itself and the order in which she needed to do it. After doing these manipulations on the EBA, she was able to generalize across written examples and solve these tasks using algebraic transformations. She now understood the significance of performing the same operation on both sides of the equal sign.

\section{Summary of Interaction and Understanding with Technology}

- Beth exhibited a relational view of the equals sign for numerical equations and the comparison of numbers and units as well as equivalence of two expressions when one of the expressions contained a variable.

- Beth accurately solved 1-step and 2-step equations using the algebraic transformation method.

- While Beth could correctly model 1-step equations using the EBA, she preferred to solve the equation using paper and pencil, using the EBA to confirm her answer by removing all the objects from the pans and placing a variable weight on the left pan and the resulting value on the right pan and showing that the pans were balanced.

- Because Beth did not understand conceptually her solution procedure to 1-step equations, she was unable to accurately generalize the procedure to 2-step equations. The EBA allowed her to understand her solution procedure: why she had to apply the same operation to both sides, why she needed to get the variable by itself, and how to get the variable by itself. It allowed her to do so through the visual and manipulations features, which helped her to find the solution by intuitively seeing what she had to remove or add to the pans to keep them balanced.

\section{Discussion}

Beth had a relational view of the equal sign for all equations for numerical equations and the comparison of numbers and units. Furthermore, she was able to justify non-canonical forms using this relational viewpoint. Yet, her relational viewpoint wavered when she encountered equations that included a missing value. While she solved 1-step equations using an algebraic procedure, she did not seem to understand the equivalence of expressions.

The fact that she solved 1-step equations using the algebraic method without having conceptual knowledge was not specifically mentioned in the findings of Knuth and colleagues (2006). They contended that students who understood the meaning behind the equal sign typically used algebraic transformations performed on both sides of the equation to solve for the variable. However, they did not talk about students following this algebraic procedure without having understanding of equivalence. Evidence of Beth not understanding equivalence in this context of expressions manifested itself when she tried to solve 2-step equations and did not know how to extend her procedure from 1-step to 2-step equations. 
When Beth began working with the EBA, her understanding of equivalence with respect to numbers without the idea of variable, was reinforced by the visual picture of the balance. Because she had a grounded understanding of the equal sign in terms of numerical values, she was able to grasp quickly the rules to maintain the equilibrium of the pans.

Even after she developed strategies in how the Algebalance worked, she did not use it to help her solve 1-step equations. Rather she would solve the 1-step equation on paper and, subsequently, remove all objects initially placed on the pans to represent the equation, place the missing variable on the left pan, and objects representing the weight of the answer value on the right pan. Thus, she used the Algebalance to confirm her answer. Since she was unable to solve 2-step equations without the Algebalance, the applet allowed her to model these equations and add or remove objects from the pans that enacted algebraic transformations to each side of the equation. Because the applet allowed her to drag different values and see the resulting effect of that manipulation on the pans and corresponding equation, Beth was able to see a visual depiction of the equation and intuitively realize how to find the value of the variable. Furthermore, she was able notice the intuitive correspondence between her manipulations on the EBA and the mathematical operations, which allowed her to easily transform his solution procedure on the EBA to paper.

During the process of working with the EBA, she discovered the relationship between the expressions, she also came to realize that there was a faster way to solve equations by looking at the pattern of her written work versus operating solely on the visual model of the equation. Although this method of solving on paper was faster than moving the objects on the applet, she liked working with the applet because it had helped her to visualize the meaning behind the equal sign and how it applied to solving 1step and 2-step equations. Furthermore, she was able to solve consistently both types of equations algebraically even though she was unable to solve 2-step equations prior to working with the EBA. 


\section{CHAPTER 8}

\section{CASE STUDY: JAKE}

Jake's Background

During the time of the study, Jake was in seventh grade Advanced Math. Advanced Math mainly covers seventh grade material and then some of eighth grade material at the end of the school year. A student in Advanced Math in seventh grade will most likely take algebra in eighth grade. Data collection was at the end of the school year; thus, Jake was covering eighth grade material, such as creating and solving word problems using formulas and functions and solving inequalities in one variable. Standard Math prepares students to enroll in pre-algebra in eighth grade. Jake was aware of a difference between students' mathematics ability in Standard versus Advanced Math. This was apparent when I asked him whether he had used a computer in math class.

$\mathrm{J}$ : We had to use it to study for the math SOL.

$\mathrm{N}$ : Oh, like Study Island?

$\mathrm{J}$ : We don't use Study Island in Advanced Math. We do games that help us learn like number brain and brain pop. It teaches you while you use the computer.

I had only offered the suggestion of Study Island because I had other interviewees, all enrolled in Standard Math, tell me that they had used the program to help them study for the SOLs. According to Jake, only students who were in Standard Math used this program. Jake emphasized the collective 'we' to refer to students in Advanced Math in order to distinguish between students in Standard Math. Furthermore, he emphasized the word 'learn' to stress the difference in computer programs that were used by Advanced Math students and Standard Math students. Advanced Math students used programs that helped them learn content while Standard Math students used programs that helped them to become quicker at computation of content they already learned. This idea is also supported by Jim's account of Study Island [see page 125].

Not only was Jake aware of a distinction in ability between Advanced Math students and Standard Math students, but he also classified himself within his own level. He said, "I'm in advanced, but I'm one of the dumbest ones." Even though Jake claimed that he was in Advanced Math, he made a point of telling me that he did not feel confident in his math ability. When I asked him why he considered himself to be one of the dumbest students, he told me that he was not good at it and did not like the subject, and preferred reading, because reading "allowed him to see the people in [his] head, like a movie" where math was "just 
numbers." For him, math seemed to be numbers on paper that did not hold any value or interpretation. In fact, as will be seen when Jake worked with the technology applet, the technology affordance that impacted him the most was the visualization of the applet. It allowed him to see the mathematics in action, which in turn allowed him to make sense of the numbers and operations on paper.

Jake tended to use rules for solving math tasks instead of trying to make sense of the operation on the numbers. When he tried to solve $-5+-7$, he summed the absolute value of the addends and obtained 12 and said, "because a negative plus a negative equals a positive or that's what a teacher told me, you just do negative plus negative equals positive." Furthermore, after continually asking him for justification for his answers, he finally exclaimed, “This is hard!... No one's usually questioning me like why things are this way or why I think it's the answer." He was used to just using a rule and obtaining an answer rather than trying to reason why the answer was correct. However, this strategy of asking him why his answers were correct seemed to help him reason through his thought processes, help him remember information, and come to generalizations that he made during instructional sessions with the technology.

\section{Negatives and Operations on Integers}

\section{Prior Conceptions on Addition}

Jake seemed to have procedural and conceptual understanding of addition of integers, and a combination of unary and binary (Kieran, 2007) conceptions of addition of a negative number depending on the type of task presented. He exhibited these conceptions when he solved tasks abstractly. Jake did not abstractly solve all the addition tasks correctly on the first attempt, for he misapplied a rule given to him by a teacher. However, after asking him to solve a task with similar addends, he experienced a cognitive conflict and was able to realize his misapplication of the rule and obtain the correct answer. Furthermore, he could appropriately use the number line to justify his correct answers to the tasks by starting at the first addend and moving to the right the magnitude of the second addend if the second addend was positive, or moving to the left the magnitude of the second addend if the second addend was negative.

Jake easily solved tasks with positive addends, and was able to justify his answer by using the idea of place value, which no other interviewee had done. When he solved $10+3$ and obtained 13, he said, "I just ... dropped the zero and put the three there on the end, because it was 10 plus a 3." When he used a number line, he started at the 10 and moved up 3 spaces to land on the 13 . When asked why he had moved to the right, he said, "because I had to add the 3."

When it came to tasks with different signed addends, he exhibited a unary view of the negative 
sign because he could connect the addition of a negative number to the idea of subtraction of a positive number. For instance, when he solved $10+-3$ he said, "You just take away the 3 part because it's a negative, so that's 7." He had a take-away notion of subtraction; thus believed he had to take away the magnitude of the second addend. In fact, when he solved this task using the number line he started at the 10 and moved to the left 3 spaces and landed on the 7. He said, "Well, you put 10, and then you plus, but since it's negative, you subtract and go backwards." Therefore, he believed that addition usually meant to move to the right, however, if there was a negative addend, he had to move in the opposite direction, or backwards, similar to subtraction to solve the task.

While he had a unary view of different signed addends, he had a binary view of negative addends. When the two addends were both negative, he did not liken his operating method to that of subtraction, instead he viewed it as addition. In fact, when he used the number line to solve this task, he started at the first addend, and traveled to the left simply "because you're adding a negative." Unlike before, he used the word 'add' instead of subtract, yet indicated that he had to move "backwards" on the number line because the second addend was negative. This idea that addition of two negative numbers is like addition instead of subtraction may be due to the fact that he initially answered this task incorrectly by taking the absolute value of both addends and added them together to get the answer. However, when I posed another, similar task and wrote this task directly underneath the first, he recanted his first answer and came up with the correct answer to the original task.

J: Negative five plus negative seven, I just added those which equaled twelve, because a negative plus a negative equals a positive, or that's what a teacher told me, you just do: negative plus negative equals positive.

$\mathrm{N}$ : So is this task like five plus seven?

$\mathrm{J}$ : That would be twelve.

$\mathrm{N}$ : So both of these tasks give the same answer.

$\mathrm{J}$ : Yeah, but [pause; whispering to himself] I think I should change my answer to $-12 \ldots$ because I kind of don't think they are the same thing. I think the person who told me, he was kind of ...maybe he was thinking of something else.

While he seemed to be able to use both abstract ideas of negative numbers and addition, as well as using a number line to solve addition tasks, I wanted see if he could move from an abstract context to a verbal context. I asked him if he could make a word problem that embodied the equation $-5+-7=-12$ to which he responded, "Hmm, I had 5, no, if I have 5 lollipops and then they took 10 away, but yeah, I didn't have 10 so... um." Through this description it can be seen that Jake viewed a negative amount much like he did subtraction - as an amount taken away. At first he used lollipops as his objects that he had, yet, he realized that if he had 5 lollipops, this was not representing the first addend. Thus, he tried to use those 5 lollipops to get the value of -5 by having someone take away 10 of his lollipops. Yet, at this stage he 
realized that this did not make sense because he did not have enough lollipops from which to take away 10 . Because of his view that numbers represented a amount of concrete objects one had, he viewed negative numbers as an amount obtained when someone tried to take away more objects than he had, yet this notion did not lend itself to be used in a verbal context. Since he sat there hemming and hawing at the task, I asked him if he had ever seen negative numbers used somewhere. At first he said that he had not until I asked him about temperature. Then he said, "Oh yeah. Duh, it's negative five degrees outside...then it dropped seven degrees more, so it would be negative twelve." Once I provided the idea of temperature, he could accurately apply this idea to contextualize the equation.

I also wanted to see if he could transition from a number line to an abstract context. I asked him to write an equation that represented a number line with a dot on the -6 and a dot on the 5 . He wrote $-6+11=5$.

$\mathrm{N}$ : Why did you start with the dot on the -6 ?

$\mathrm{J}$ : Because I did the lowest, which is -6 , cause like I want to go farther into the positives. So I did -6 , and I kind of counted the spaces, which was 11 , which would be -6 plus 11 , would equal to the positive number, would be 5 .

$\mathrm{N}$ : Is there any other way you could write an equation?

$\mathrm{J}$ : You could do 5 minus 11 would equal -6 .

N: Ok. How did you know that would work too?

J: Cause I just switched the numbers around, backwards. Like I knew the 5, so then I would start with the 5 , and then choose to add or subtract.

$\mathrm{N}$ : How did you know to subtract?

J: Cause I added to get a positive answer, so you would subtract to get a negative answer. You go that way to positives [points right] and that way for negatives [points left]. Cause, 5, uh, subtract more than 5 will get you into the negatives. And you had to get into the negatives to get to the -6 .

It was interesting that he chose to use addition first rather than subtraction as the operation in the equation, even though subtraction is typically used when there is a distance between two points on a number line.

Furthermore, when he used subtraction, he used the second dot not as the subtrahend, but as the difference. Thus, he exhibited a take-away notion of subtraction. This take-away notion may have been encouraged by the way he used the distance between the numbers in his addition equation. Since he used the second dot as the sum and the first dot as the spaces in between the dots, it was only natural for him to think that the subtrahend should be represented by the number of spaces in between the dots.

It was also interesting that he used the dot on the -6 as the first addend instead of the 5 . Based on other tasks, Jake knew that $5+-11=-6$; therefore, he should have been able to give that equation. Yet when asked to give another possible equation after he had given the equation $5-11=-6$, he could not. One likely reason for this is that he started with the -6 simply because it was the first dot that he came to when he was reading the number line from left to right. Since he saw that the dot on the 5 was located 
farther right, and since he interpreted addition as moving to the right on the number line, he thought of the addition equation $-6+11=5$ first. Then when I prompted him to give another equation, instead of thinking about how the numbers related, he thought about the inverse operation of addition. Given that he used subtraction, he knew he had to reverse the order of the equation; thus, he started at the dot that allowed him to move left to the other dot since subtraction meant moving left on the number line. Therefore, he came up with the equation $5-11=-6$. He believed that the number line only represented these two equations. He did not think about the additive relationship of the first equation, which may be because he could not give an analogous equation in terms of subtraction. While he knew that $5+-11=-6$ and he might have known that this too would have represented the dots on the number line, he was not able to solve the analogous equation $-6--11=5$. Furthermore, these types of equations go against his salient notion that moving right on the number line indicates addition and moving left on the number line indicates subtraction. Thus, when he created an abstract equation that represented the dots on the number line, he used the direction he needed to travel from one dot to the other combined with his idea that subtraction meant moving left and addition meant moving right, even though he knew that addition of a negative was similar to subtraction and required one to move to the left.

\section{Prior Conceptions on Subtraction}

Jake believed that subtraction signified that one must "take-away" the amount in the subtrahend from the minuend instead of signifying a distance between the subtrahend and minuend. Due to this idea and Jake's unary (Kieran, 2007) view of addition of a negative number, Jake accurately solved subtraction tasks containing a positive subtrahend. Because Jake had a "take-away" notion of subtraction, he justified his answers to subtraction tasks with both a positive minuend and subtrahend by taking away the subtrahend that left him with remaining items, or he took away more than with which one began, so he had negative remaining items. For tasks with a positive minuend as well as for tasks that had a negative minuend, he saw the equivalence between the expressions $a-b$ and $a+-b$, where $b$ was non-negative integer and $a$ was an integer. Since he believed that both the subtraction sign and negative sign meant to move left, he illustrated how he could obtain the answer using a number line by starting at $a$ and moving left $b$ spaces.

Since Jake believed that both the subtraction sign and the negative sign meant take away and move to the left, he initially did not attend to both of the signs when he encountered the subtraction of a negative subtrahend. He treated the expression as if it were addition instead of subtraction. Therefore, for tasks such as $5--12$ and $-4--6$, he initially obtained the answer of -7 and -10 respectively. After he solved these 
tasks abstractly, he justified these answers by beginning at the minuend and moving to the left the absolute value amount of the subtrahend. Yet after he solved these types of tasks, I asked him questions that allowed him to realize the difference between $a--b$ and $a+-b$ and between $-a--b$ and $-a-b$ where $a$ and $b$ were non-negative integers. Even though he realized a distinction, however, he did not appropriately solve these types of tasks.

Given that he could accurately solve subtraction tasks involving a positive subtrahend, I asked him how he thought about the specific task $5-2$. He said, "Well, I had to think about it because I'm not good with subtraction. So ...if I get stuck on it, I just...count up from 2, so 2 plus 3 would equal 5." Since he preferred addition instead of subtraction, he used the number line and started at the subtrahend and counted up the number of spaces it took him to reach minuend. Thus, he thought about the subtraction task as 'the subtrahend plus what equals the minuend.' This is interesting because it is an opposite strategy than he used to solve the task where he had to write an equation that represented the dots on a number line. In the number line task, he used his knowledge of $-6+11=5$ to come up with the equation $5-11=-6$. Thus, he used the 5 as the sum and the -6 as his minuend, and the number of spaces to travel as the subtrahend. Even though he worked from addition to subtraction, one can also interpret this as he could have use subtraction to come up with the addition equation since they were equivalent. Thus, he took the difference of -6 plus what equaled the minuend instead of taking the subtrahend value of 11 plus what equaled the minuend.

When I first asked him to solve the task $5--12$ he could not decide between the answer of 7 or - 7 . He said, "Oh, hmm. Five minus negative twelve," and then he whispered to himself, "Is it -7, 7, -7?? I asked him to tell me how he was thinking about the task.

Well, I knew that since 5 was a positive number, it was going to be closer to 0 in the middle, so I just did kind of the same thing as before, I just did 5, counted up 5 to 12, which gets you to 7 , which would be 7 . Then I just added the negative sign, because of -12 .

He used the number line since he could not solve this task abstractly and tried to use his method of counting up to help him solve the task. He started at the 5 on the number line and moved to the right 7 spaces to land on the 12. Since he moved 7 spaces to get from the 5 to the 12 , he believed the answer should have been of magnitude 7 . Since the subtrahend was negative, he believed he had to add a negative sign to this magnitude. This incorrect strategy was not discussed in previous literature.

There are three aspects of his solution procedure for $5--12$ that are interesting. First, even though he said that he solved this task exactly how he solved the subtraction task $5-2$, he did not. What was similar in his solution procedure was that he used the distance between the minuend and absolute value of the subtrahend by moving to the right on the number line to find his answer, but this is where the 
similarities end. In solving $5-2$, he essentially solved the task $2+?=5$ by starting on the number line at 2 and counting how many spaces he needed to move to the right to get to the 5 . In solving the task $5--12$ he used the number line and solved $5+?=12$. While he used the minuend and absolute value of the subtrahend as starting and ending points, moved to the right from one value to the other, and counted the spaces in between, he used his subtrahend in $5-2$ as his first addend and his minuend as the sum. For the task $5--12$, he used the minuend as his first addend and the absolute value of the subtrahend as the sum. He may have done this for a couple of reasons. First he may have simply kept the 5 in the first position in both tasks $5--12$ and $5+?=12$ because he was not attending to numbers. Second, he might have thought that his strategy was commutative, so it did not matter if he used the 5 as the first addend or the second addend. Third, he might have thought that the first addend had to be of lower absolute value than the absolute value of the sum. Since he wanted to use the equation $x+?=y$ as a strategy to help him solve the task $y-x=$ ?, he may have believed that $|x|$ had to be less than or equal to $|y|$ so he could add something to $x$ in order to get $y$. While he could have done this by using the equation $-12+?=5$, I do not think he was as comfortable working with negative numbers as he was with positive numbers.

The second aspect that is interesting about his reasoning occurs when one thinks about it in a pure algebraic sense. Given that he used $5+?=12$ to find the answer to $5--12$, this meant that he in essence believed that $12-5$ or $5-12$ would help him find the answer to $5--12$. Now, suppose that he had used $12-5$ to help him solve this task. He knew that $12-5=7$, but since the 12 had a negative sign attached to it, he believed that the answer would have to be -7 . Algebraically his logic would be represented as $-12-5=-7$. But this does not agree with the fact that he could solve tasks that had a negative minuend and positive subtrahend, for he knew that $-12-5=-17$. Therefore, another way to translate his verbal reasoning into algebraic reasoning would be to say that since he knew that $12-5=7$, and since the 12 was negative, then he had to change all the signs in the task, and therefore, $-12+5=-7$. Jake would agree that this equation was true. If this would be his reason, then he would believe abstractly that $-12-5$ was equivalent to $5--12$.

Alternatively, if he chose 5-12 to help him solve 5 - - 12, he would know that $5-12=-7$ because he could solve subtraction tasks involving a positive minuend and subtrahend. Now, using his words, he acknowledged that the 12 was negative, so he would have gotten $5--12$, which was the original task. Thus, he would have thought that $5-12$ was equivalent to $5--12$. This last possibility seems the most plausible as will be seen in a dialogue later. Although, while he did not specify enough to determine these scenarios at this point in time, they are interesting to consider in order to try to directly correlate his 
verbal reasoning to the algebraic structure of the task, especially considering that students may be thinking about it in a way that they cannot express algebraically or verbally.

Third, Jake reasoned that because one number was negative and the other number was positive, he believed that when he subtracted them, the answer would have to split the difference, i.e. be closer to zero than the -12 . Thus, he thought both 7 and -7 were reasonable guesstimates because they were both closer to zero than the distance between -12 and 0 and 12 and 0 . What is strange is that he used the distance between the largest absolute valued number and zero instead of the distance between -12 and 0 and 5 and 0 . If he had done this, he would have surely believed the answer to be -7 . Although, he came to believe that -7 was his final answer on his own after I tried to clarify his verbal reasoning.

$\mathrm{N}$ : So let me see if I got this right. So you started at the 5 and went up to the positive 12 ? $\mathrm{J}$ : Wait, I think I did that wrong. So you start out with the 5 and move minus negative twelve, so would be $1,2,3 \ldots 12$, yeah, -7 .

$\mathrm{N}$ : So you started out at the 5 and $\mathrm{J}$ : subtracted 12 .

Prior to this dialogue, Jake had said that he had solved this task in the same manner he had solved $5-2$, by starting at the 5 and moving $\underline{\text { to }}$ the 12. In order to make sure I was hearing him correctly, I emphasized the preposition 'to'. However, following my question, he said that he needed to 'move' negative twelve spaces. Thus, he started at the 5 and counted to the left 12 spaces and landed on the -7 . This did not fit with how he previously thought about the task. Yet, the first thing I wanted to address and understand was why he was interchanging 12 and -12 , and if those numbers were different to him.

$\mathrm{N}$ : So are you subtracting 12 or -12 ? I notice you are saying both of them.

J: You subtract...negative, well, I was subtracting 12.

$\mathrm{N}$ : So could you solve $5-12$ for me?

J: It would be 7. Wait [pause] no that would be -7 .

$\mathrm{N}$ : Ok, so are these two tasks the same [pointing to $5-12$ and $5--12$ ]?

$\mathrm{J}$ : Yeah! [pause] wait, I'm confused right now.

$\mathrm{N}$ : What is confusing you?

$\mathrm{J}$ : This, [pause] the different [pause], so I solved for 5 right there and then I subtract -12 , so I go left, yeah.

$\mathrm{N}$ : So tell me if I understand you correctly, you go left if it is subtraction or a negative?

J: Yeah, so I'm confused. We have subtraction and a negative. I don't know how to move.

At first Jake thought that both 5-12 and 5--12 gave the same answer: -7 . Furthermore, he supported this answer by moving on the number line starting at 5 and moving to the left 12 spaces because there was a subtraction or negative sign in each case, and both types of signs indicated that he needed to move left on the number line. Yet, he realized that the first expression subtracted 12 and the second expression subtracted -12 , and 12 and -12 were different; thus, he was not sure about his answer and blamed it on the fact that he was not sure how to move when the expression showed a negative and subtraction sign side-by- 
side.

Given the fact that he was confused and that he had told me that he was not good at subtraction, and when he was in doubt he used his strategy of switching the subtraction task into an addition task and finding the number of spaces he needed to count between the numbers, I encouraged him to remember how he solved $5-2$.

$\mathrm{N}$ : So in the previous, you said you figured it out by starting at the 2 and counting up to 5 .

$\mathrm{J}$ : Yeah.

$\mathrm{N}$ : So could you use that same strategy here?

J: Oh, yeah, I would start at the -12 and count up to 5 . So 1,2,3, 4, 5, yeah, -7 .

$\mathrm{N}$ : So how did you count?

$\mathrm{J}: \underline{\mathrm{Up}} 5$.

$\mathrm{N}$ : Is that what you did before with $5-2$ ?

$\mathrm{J}$ : Yeah.

$\mathrm{N}$ : Oh, can you show me again how you did $5-2$ ?

J: I started at the 2 and went up to $5.1,2,3,3$.

$\mathrm{N}$ : Now how would you use that same strategy with $5--12$ ?

$\mathrm{J}$ : Yeah, so I would start at the -12 and then count up 5, because, I don't know. But that's how you do it. Because you would start off at the -12 because it would be the 5 that isn't negative.

When speaking to him about his previous strategy, I emphasized the word 'to' in hopes to make sure he understood that he counted to the minuend instead of the amount in the minuend. I also pointed on the number line as I said this statement. However, he did not pick up on it, in fact, he interchanged his verbal description of his reasoning, which I noted by underlying the words in the passage. While he would say that he counted up to 5 on occasions, other times he would say that he counted up 5 spaces, yet, he always did the same procedure: started at the -12 and counted 5 spaces to the right and landed on -7 . When I tried to get him to recognize the difference between counting up 5 spaces versus counting to the 5 , he eventually changed his answer.

$\mathrm{N}$ : So here, you started at the 2 and went up to the 5. So for this one, wouldn't you start at the -12 and count up to the 5 as well?

J: No, you'd count up 5.

$\mathrm{N}$ : So did you count up 5 here or did you count up 3 here?

$\mathrm{J}$ : Can you say that again?

$\mathrm{N}$ : So here you started at the 2 and counted 3 to get to the 5 , so you did 1,2,3 and landed on the 5 .

$\mathrm{J}$ : Oh, so here, I would do the same thing. I would start at -12 and then count $T O 5$. So then my answer would be 17 or 16 . One of those.... [counts] It's 17 . So $5--12=17$.

$\mathrm{N}$ : How did you know it was going to be positive 17 instead of -17 ?

$\mathrm{J}$ : [pause] Cause you have to count up to a positive number, so it's gonna pass zero.

As I said the first two sentences, I pointed out the similarities of the expressions and movements. For instance, I pointed to the subtrahend 2 and said we started at the 2 and then pointed to the subtrahend -12 and started at the -12 . Then as I said about going up to the 5 , I put my pencil on the 5 in the minuend and then the 5 on the number line, and did the same for the expression $5--12$. Jake finally understood what I 
was trying to imply: for him to be using the same strategy for the other subtraction task. In fact, he emphasized the 'to' in "then count to 5 " to show me that he was not counting up by 5 anymore. Even though he figured out how to apply his additive strategy to a subtraction task in which the subtrahend was negative, he did not fully understand the sign in the difference. He believed that since he traveled to a positive number, then his answer would be positive, instead of realizing that his answer was positive because the minuend was greater than the subtrahend.

I wanted to see if he could generalize this strategy to subtraction tasks involving both a negative subtrahend and minuend, and thus realize the mistake in his original answer of -10 to the task $-4--6$.

$\mathrm{N}$ : Ok, so tell me how you got -10 for the problem $-4--6$ ?

$\mathrm{J}$ : I just added 6 plus 4 , which is 10 , and they were negative numbers, so it's negative.

$\mathrm{N}$ : Ok, so if I gave you this problem, $-4+-6$, what would that answer be?

$\mathrm{J}$ : This would be -10 . Oh, I was doing addition and not subtraction.

$\mathrm{N}$ : Which is showing you subtraction?

$\mathrm{J}$ : $-4--6$ and this would be -2 , cause you subtract, yeah, subtract 6 from it, and you get -9 . $\mathrm{N}$ : So explain to me how you got -9 ?

$\mathrm{J}$ : Well, it said -4 here, and you subtract -6 so you subtract $6,1,2,3 \ldots$ so it's -10 .

$\mathrm{N}$ : So the answer is -10 ?

J: Yeah. [pause] Wait a second. Yeah, that's right.

Initially, Jake took the absolute values of the minuend and subtrahend, added them together, and then tacked on a negative sign to the answer. Therefore, to find the answer to $-a--b$, where $a, b>0$, he solved the algebraic expression $-|a+b|$ and believed they were equivalent. When I presented him with the similar task $-a+-b$, he realized that the difference between the two tasks was that one had a plus sign, and hence was addition, while the other had a subtraction sign, and hence was subtraction. Thus, he modified his equivalent expression $-|a+b|$ to $-|a-b|$ and obtained -2 for his answer to $-4--6$. He determined that he had added the numbers because he had added the absolute value of the numbers and then tacked on the negative sign, where subtracting would mean that he needed to subtract the absolute value of the numbers and then tack on the negative sign. This meant that he believed that $-4--6=-|4-6|$, although he probably did not think about it in terms of the absolute value of the difference, rather he saw that the 6 was higher than the 4 and therefore, switched the order of the minuend and subtrahend because it was easier to subtract a smaller number from a higher number. Why I say that he found this easier is because based on other examples, Jake could correctly solve tasks where the subtrahend was a larger positive number than the minuend, yet he did not do that here.

Even though he changed his answer based on the operation in the similar task, he returned to his incorrect answer of -10 after trying to support his answer by moving on the number line. Once again he did not attend to the position of a negative number preceded by a subtraction sign. While he recognized that he 
was supposed to subtract a negative six, he instead counted down by 6 and moved from -4 to -10 . It might be that he viewed subtraction of -6 to be the same as 6 because he said, "you subtract -6 " immediately followed by "you subtract 6." Another possibility is that since both the subtraction sign and negative sign indicated that he had to move left on the number line, he simply moved left regardless of how many signs were used. It is also important to note that he used the take away notion of subtraction instead of the discovered distance method we had talked about for the previous task $5--12$. Instead of moving from -4 down to -6 , he moved 6 spaces to the left.

It is interesting how Jake solved the next subtraction task: $-4--1$. Based on the previous responses, one would think that he would say the answer was -5 at first, followed by perhaps -3 if I gave him the task $-4+-1$, then followed by and answer of -5 when he moved on the number line. However, he initially answered that the difference was -3 because he "just subtracted 4 minus 1 , which is 3 , and it was negative, so." Furthermore, when I asked him to show me on the number line, he started at -4 and moved up 3 spaces to the -1 and reasoned that since $4-1=3$, he had to "move 3 to get from the -4 to the -1 ." Based on his algebraic reasoning, he solved the task $-4--1$ by solving what he believed to be the equivalent expression $-|4-1|$. Then he justified this answer by using the fact that he knew $4-1=3$ and made sure he moved 3 spaces away from -4 . This would either get him to -7 or -1 . Since -1 was part of the task, he decided to move to the right. It is unclear as to why he stuck with his algebraic method instead of changing his answer based on the number line movements. It could be due to the fact that in this task, the absolute value of the minuend was greater than the absolute value of the subtrahend.

\section{Discussion of Prior Conceptions}

Jake seemed to have a mixture of unary and binary (Kieran, 2007) understanding of negative numbers in addition tasks. Even though he used the same number line strategy to solve tasks involving mixed addends and two negative addends, he used different abstract strategies. In both types of tasks, Jake started at the first addend on the number line and moved to the right if the sign of the second addend was positive or to the left if the second addend was negative. He traveled the number of spaces represented as the magnitude of the second addend. This type of movement on the number line to solve addition tasks involving negative numbers was described by Bell (1982).

While he used the same number line strategy, Jake only considered the addition of a negative addend like subtraction, a unary (Kieran, 2007) perspective, if the addends had different signs. If the addends were both negative, he obtained the correct answer, but viewed the operation as addition, almost as if he removed the negative signs of the addends, calculated the sum, and put a negative on the sum. He was 
in effect using the distributive property of addition for he solved $-a+-b$ as $-(a+b)$.

In moving from one context to another, Jake was unable to come up with a verbal description of an addition task containing two negative addends until prompted by the researcher. He had difficulty creating a verbal representation of an abstract task because he viewed a negative number as deficient in a concrete quantity; thus, trying to add two deficient concrete quantities did not make sense in a real context, because he could not have a negative amount that was still tangible. It was not until he was given the idea of temperature that he could formulate an appropriate verbal representation of the abstract task. Bruno and Martinon (1996) noticed that when students moved from abstract to contextual, most students prefer to use credit-debit as their context, however Jake did not use this. Furthermore, Bruno and Martinon (1996) noted that despite the initial context, all students felt more comfortable using temperature when required to use the contextual representation, which seems to fit with Jake's case.

As Bruno and Martinon suggested, most students have more difficulty going from an abstract to contextual representation than moving from a number line representation to an abstract representation. Jake was no different, for he had a smoother transition when he moved from a number line representation to an abstract representation. Jake used an equation with addition before he created an equation with subtraction that represented the dots on the number line. In solving this task, he used the first dot he came to when reading from left to right as the first addend, the spaces between the first dot and second dot as the second addend, and the sum represented by the left-most dot. Jake also came up with a correct subtraction equation that modeled the task, although, he derived it based on the addition equation he created. He simply reversed the order of the numbers from left to right; thus, his first addend became his difference, his second addend became his subtrahend, and his sum became his minuend. His decision for why he used this equation may not have been solely based on the number line visual, but rather his understanding that subtraction was the inverse operation of addition. In fact, it is not abnormal for students to have difficulty moving from a number line context to an abstract equation according to Bruno and Martinon (1996).

In agreement with Murray's (1985) findings, Jake was able to solve addition tasks more accurately than he was able to solve subtraction tasks. Jake correctly solved subtraction tasks that contained a positive subtrahend with and without the use of a number line. In solving tasks with both a positive minuend and subtrahend, Jake used his idea of take-away. He knew that he had to remove the amount specified in the subtrahend from the minuend. If the subtrahend was larger than the minuend, his difference would be negative because he had to take more away than was originally provided by the minuend. For tasks with a negative minuend and positive subtrahend, he used the equivalence relation between $-a-b$ and $-a+-b$ 
where $a, b>0$. Furthermore, in using the number line to solve a task with a positive subtrahend, he used the subtraction sign to indicate that he had to move $b$ spaces left of $a$.

Since he believed that both the negative sign and the subtraction sign meant to move left, Jake became confused in solving tasks with a negative subtrahend, because the similar signs were next to each other. Initially, he toyed with the idea of ignoring one of the signs, and he mentally translated $a--b$ and $-a--b$ into $a-b$ and $-a-b$, respectively, and solved the transformed expression. This strategy was used by other students, which was noted in Peled and colleagues' study (1989). The answer Jake obtained from mentally transforming this task was supported by the way he operated on the number line: he started with the minuend and traveled left the amount specified by the magnitude of the subtrahend. Therefore, he did not attend to either the subtraction or the negative sign of the subtrahend.

After presenting him with an analogous expression in the form $a-b$ and asking him to compare this expression with $a--b$, he eventually concluded that he needed to add the absolute value of the subtrahend to obtain the answer. Moreover, he devised a different method of operating on the number line that was not seen in previous literature. He transformed the task $a--b=?$ into $-b+?=a$, started at $-b$ on the number line, and counted up to the value of $a$. The number of spaces he traveled was the answer to the original task. It is not clear why he adapted this strategy for only tasks in the form $a--b$ and not $-a--b$.

For tasks in the form $-a--b$, he differentiated between the forms where $a>b>0$ and $0<a<b$. When $a>b>0$, he solved the task abstractly first by utilizing the distributive property and hence performing $-(a-b)$. He would then use that answer to determine how he should move on the number line. When it came to tasks in the form $-a--b$ where $0<a<b$, he treated this expression as if it were equivalent to $-a-b$. To justify his response on the number line, he started at $-a$ and moved to the left $b$ spaces.

Jake did not fit precisely into Peled's (1991) categorization of students' understanding of negative numbers. In fact, I had to separate his understanding in terms of addition and subtraction. Furthermore, he did not fit cleanly into one level. Thus, I adopted my own system of ratings based on Peled's levels. A minus sign indicates that Jake attained some of that level, but could not smoothly and completely attain it. An asterisk in the Quantitative Dimension (QD) indicates that Jake was able to solve higher-level tasks than his number level indicates, but he did not put the answer or task into context. An asterisk in the Number Line Dimension (NLD) indicates that Jake was able to subtract natural numbers, know that plus and minus signs are opposite in direction, but could not do all same-signed subtraction tasks, yet could 
solve some opposite-signed subtraction tasks. Jake attained Level 4- in the QD and Level 4 in the NLD with respect to addition. He attained Level 3* in the QD and Levels 3*/Level 4- in the NLD with respect to subtraction.

Because Jake understood that addition meant to move right and subtraction meant to move left on a number line even if the larger number was subtracted from a smaller number, and he was able to recognize that addition and subtraction were opposite in direction, Jake reached Number Line Dimension (NLD) Level 2 in both addition and subtraction. He also had no issue crossing over zero; thus indicating he had a continuous view of the number line. However, Jake does not fit into one specific level, for he was able to solve addition tasks in all forms, but only subtraction tasks in the forms $a-b,-a-b, a--b$ where $a, b>0$ and $-a--b$ where $|a|>|b|$. To attain Level 3, Jake would have to be able to solve all subtraction tasks containing same-signed numbers, yet he could not do this for tasks in the form $-a--b$ where $|a|<|b|$. To attain Level 4, Peled (1991) noted that the student had to be able to solve subtraction tasks with different signed numbers, which Jake could do.

Jake did not fit completely into one level in Peled's (1991) Quantitative Dimension (QD). Jake was clearly able to understand negative numbers as counterparts in terms of opposite quality as shown in his ability to create a verbal representation of a negative numbered subtraction expressions; thus, he attained Level 1. Furthermore, Jake was able to subtract a larger natural number from a smaller natural number, which placed him on Level 2. Peled (1991) designated Level 3 for students who were able to solve subtraction tasks involving the same-signed numbers, and Level 4 for students who were able to solve subtraction tasks involving differently signed numbers. With this classification, Jake fit in part of Level 3 and part of Level 4, because he could solve all subtraction tasks correctly except those with a negative minuend that is larger than the negative subtrahend.

\section{Summary of Jake's Prior Conceptions}

- Jake had both a conceptual and procedural understanding of addition of negative numbers. He used different procedures to solve different types of addition tasks. When he used the number line to explain his answer, he started at the number represented by the first addend and moved in the direction and amount of spaces represented by the sign and magnitude of the second addend.

- Jake was unable to correctly solve tasks in the form $-a--b$ where $0<a<b$. He mentally transformed these tasks into $-a-b$ and used the number line to confirm his answer.

- Jake preferred using addition rather than subtraction to create corresponding equations for tasks illustrated on a number line. While he was able to create the correct subtraction task for the number line, he most likely based his equation off of the addition equation he used instead of using the visual represented on the number line. 
- In describing subtraction, Jake used the notion of take away rather than distance. This was evident in the way he reasoned through subtraction tasks with a positive subtrahend. However, when he tried to solve subtraction tasks in the form $a--b$, he used another strategy and came up with the equivalent addition equation $-b+?=a$ and solved for the question mark. Furthermore this take away notion was evident in the way he navigated the number line to reinforce his answers to tasks with a positive subtrahend, for he would travel to the left the number of spaces represented by the magnitude of the subtrahend.

- In working with a number line for both addition and subtraction, Jake believed that the signs of the numbers told him which way to travel, except in two types of subtraction tasks involving a negative subtrahend: if the task had a positive minuend or if the minuend was negative and smaller than the subtrahend.

- In all tasks but the form $-a--b$ where $0<b<a$, negative and subtraction both meant to move to the left, and positive and addition both meant to move to the right on the number line.

- Jake attained Level 4 in both of Peled's QD and NLD for addition.

- Jake fit unusually into Peled's theory for subtraction. In the QD and NLD, he did not fit cleanly into Level 3 or Level 4. He attained Level $2 *$ in the QD and Level 3*/L4- in the NLD with respect to subtraction.

- Jake exhibited a combination of unary and binary understanding of negative numbers. The way he viewed negative numbers depended on the form of the task.

\section{Analysis of Interaction with Subtraction Applets}

I showed Jake the subtraction arrow applet (SAA) without the equation displayed to see how he would use the SAA, given that he seemed to have the notion of take-away for subtraction.

\section{Jake's Interaction and Understanding with Technology}

When using the SAA, I did not have the hot-linked equation representing the visual on the number line because I wanted to see how the sole illustration of the arrow value appearing inside the arrow impacted his method of solve subtraction tasks.

Jake took only a few seconds of manipulating the SAA when he realized that it embodied subtraction. This realization occurred after I asked him to place the blue stick on a specific number and move the arrow around. He noticed that when he set the arrow to the left of the and then incremented it by one to the right, it got " closer to the first number you put it on." Furthermore, he noticed that once he passed the blue stick, the value inside the arrow became negative (see Figure 1). He explained this phenomenon to me.

It's like you've gone past the first number. It's kind of like you subtracted more than you're allowed. I don't know how to explain it. It's like if you have 10 and you subtracted 11, so it would be -1 because you went one off. 

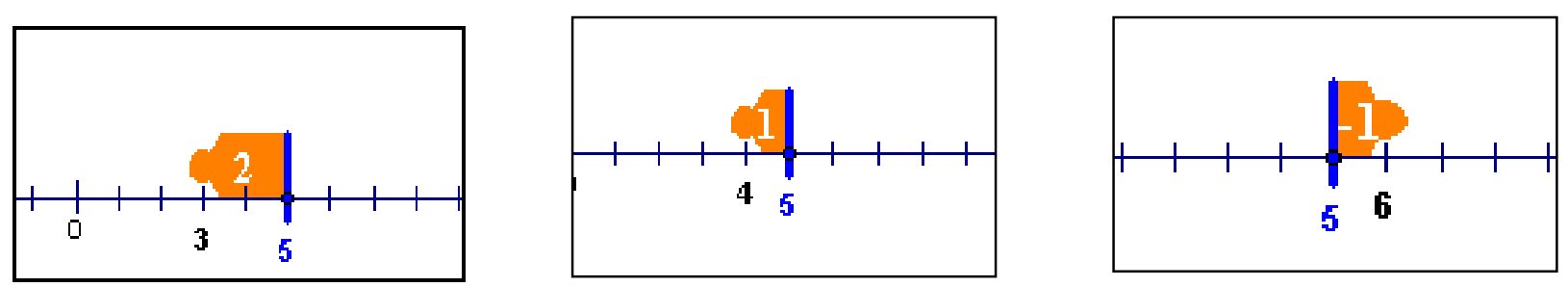

Figure 1. Jake moving to the right by one to see decrement in arrow value.

It is important to note that not only was the equation not displayed, but also I had not specified that the SAA depicted subtraction, for he had made that connection on his own. This connection was made due to the visual depiction of an amount being taken away from the original, and the fact that he could compute some subtraction tasks, which allowed him to notice the visual and abstract relationships among the minuend, subtrahend, and difference. He used the SAA as if it were the SDA because as he described how it worked, he believed that the number shown in the arrow was the difference between the two numbers, not the amount in the subtrahend. This is interesting because it was the opposite of what he had done 1 hour before when he had solved tasks with a positive minuend and subtrahend, for he had taken away the amount in the subtrahend to find the difference. What should also be noted is that he manipulated the blue stick and arrow on the number line before arriving at the abstract equation, and not the other way around. This was contrary to the way he transitioned from the number line context to the abstract equation in Task 5 in Module 1. When Jake transitioned from the number line representation to the abstract equation he used the second dot to represent the answer instead of the amount taken away. Yet, when reading the number line now, he used where the arrow landed as the subtrahend and the difference as the distance between the numbers where the blue stick was positioned and where the arrow landed.

Due to this fact, I wanted to see if he could show me a number line representation by manipulating the SAA when given an expression. I asked him to use the applet to show me10 - 15. Jake placed the blue stick on the 10 and dragged the arrow to the right 5 spaces so the tip of the arrow landed on the 15 . This is how the SDA worked, but could be shown with the SAA as long as the equation was not showing, which would contradict the original expression since it would have shown $10--5=15$. Jake explained to me what he had done.

If you had 10 and you subtracted 15, it would be like, yeah, just here. So you'd have like 5 off so it would be like -5 because you took more than you have away. Kind of like with money, you're in debt. If you have that much, and take away more, kind of like that.

Once again, Jake used the words 'take away' yet used the SAA to show a distance between the minuend and subtrahend. Furthermore, Jake had never used the context of money during the interview. In fact, he 
had tried to use concrete items during our interview session to no avail. Only after I suggested temperature, was he able to contextualize an abstract expression. Yet, here, perhaps due to the visual of taking away and the depiction of the amount between the initial value and subtrahend by the arrow value, Jake was able to make the connection between negative numbers and debt.

I then proceeded to ask him to model the instruction tasks beginning with $5-2$. Since these were both positive numbers, I assumed he was going to model the movement just as he did with the above tasks; yet, this was not exactly how he proceeded. He placed the blue stick on the 5, fiddled with the arrow, and said, "Yeah 5 minus 2 would be [pause] wait, I'm confused as to how this works. Well, 5 minus 2 would be 3, yeah." While he stated this, he toggled back and forth on putting the arrow tip at 2 and 3 . He chose to land on 2; thus using a distance method, but paused for a bit, and moved the arrow tip to 4, then 3 , then 2 and then back to the 5. After this exploration, he settled on landing on the 3; thus, using the take-away method, for the arrow value showed 2, which was the amount in the subtrahend (see Figure 2). When I asked him why he had kept changing it and finally landed on 3 he said, "Yeah, cause I thought, I was looking at the orange instead of down here. The orange is going to be how much you're taking away." This is contradictory to what he had been modeling for the other tasks. Yet, for this task, he had used the SAA as it was intended. When he solved this task in the interview section, he had used the same idea - take away; thus, he may have reverted to this because this method was the most familiar to him. Furthermore, the answer is correct in both instances. Another reason he may have chosen this method because he was fixated on the number inside the arrow value. Since this value was the most prominent, as was the value of the subtrahend, it is only natural to equate the two; therefore, using the take away method (see Figure 2).

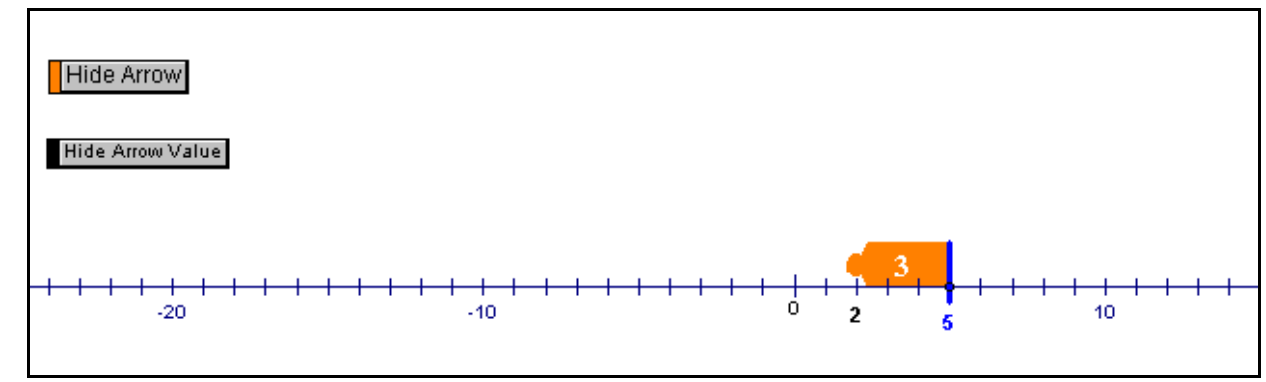

Figure 2. Jake using the SAA and using the take-away method to solve 5-2

After reverting to the SAA method, he consistently used this method for solving the rest of the tasks. For instance, when I asked him to model $5-1$ and $5-0$, he used the orange number within the arrow to represent the subtrahend. Given that he had noticed the decreasing in the number inside the orange arrow when he moved the arrow to the right by one, as well as seeing that understood that this applet represented subtraction, I wanted to see how he would model 5-- 1 . Without any pause, he moved the orange arrow to 
the right so that the orange value showed -1 and he answered, "Five minus negative 1 would be 6 ." He explained how he knew the answer and why he had to travel to the right.

If you take 1 more, like since you already passed zero, and not positive, then if you take one more away, then it will be 6 ...it jus keeps on getting higher. But what you're taking away, it gets higher in negative form. Like if I did [one more] higher, then it would be up to 7 but you're taking away -2 , so like each get one more higher each time...See like in that one, it [the answer] went from 3 to 4 , when we did 2 to 1 , and then -1 to -2 , it [the answer] went from 6 to 7 .

Jake explained that since he moved past his original position of the blue stick, and not in the positive direction past zero but so the arrow value was negative, then his answer would increase by one from the answer he obtained when the arrow was positioned at the blue stick. Thus, he knew that since his answer was five for 5-0, then his answer to 5--1would be 6 . Based on the sequence of tasks and the movement of the arrow and visual corresponding to the amount he moved it, he was able to see the connection between $5--n=m$ and $5--(n+1)=m+1$ where $m$ and $n$ are whole numbers. Given that he saw this connection, I thought he would be able to figure out the answer to $5--12$ using the take-away method. However, this proved to be too difficult for him, even though he was able to tell me the correct answer less than an hour ago by starting at -12 and moving to the 5 on the number line. He said, "Five minus negative twelve? Negative twelve? Hmm. I can't remember what I was doing. I'm kind of confused." This confusion may have due to a few different factors. First, he was unsure of the answer to begin with the last time and had to compare it to his answer to the expression 5-12 and his solution strategy to $5-2$ to eventually find the correct answer. Second, he ended up solving this expression using the distance method rather than the take-away method; thus, trying to use the SAA may have been confusing.

Instead of answering that task, I asked him to predict the answer to $5--3$, given that he had just used the SAA to find 5-2,5-1,5-0,5--1, and 5--2, and had recognized the pattern among the tasks. Yet, he still seemed to be confused from the last question. It was almost as if the last task interrupted his thought patterns just enough to make him forget what he had been doing.

Five minus 8 would be -3 , I mean, wait, 5 minus -3 would be 8 . Wait, 5 minus, 5 minus, uh, 5 minus 8 would be -3 . No! five minus 3 would be -8 . [Heavy sigh] Eight minus 5 ?

While his actions on the SAA were correct, for he moved the arrow to the left 3 spaces from the number 5 , he was unable to definitively provide a corresponding equation. Using this take away method, the equation should have been $5--3=8$. I think he was flustered and trying to balance the visual information with trying to make a connection to the arithmetic of the tasks. Because of this, I asked him some of the fundamental ideas of the SAA in hopes he would be able to figure out the equation on his own.

N: So where did you put the blue stick?

$\mathrm{J}$ : The 5 . 
$\mathrm{N}$ : And how far did you move?

$\mathrm{J}:-3$

$\mathrm{N}$ : So what does this say?

J: It says ...five minus negative 3 equals 8 , yeah!

$\mathrm{N}$ : So how did you know that you were subtracting -3 ?

$\mathrm{J}$ : The number you are subtracting is inside the orange.

$\mathrm{N}$ : And how did you know the answer was 8 ?

$\mathrm{J}$ : Cause it shows up here each time, where it [the arrow] stops.

I think Jake was overwhelmed by the information he was receiving and trying to process it all, for he was clearly able to answer my questions and formulate an equation. My questions about the SAA allowed him to take each piece of information represented on the SAA and integrate them into an equation. At this point, I asked him to click on the "Show Expression" to observe the changes in the equation while he manipulated the SAA.

When I asked him to show me 5- -5, he initially told me, "It would be equal to zero because it canceled each other out." Yet, when I asked him to remind me of what he got for the expressions $5-0$, $5--1,5--2$, and $5--3$. He could answer them correctly, but did not realize how this affected the present task. Therefore, I backed up and asked him how to solve the intermediate task $5--4$ that would bridge $5--3$ and $5--5$. Jake moved the orange arrow so that a negative four was showing inside and said, "Oh, that would be 9." Then I asked him to predict what $5--5$ would be and he said, "Oh, that would be 10. Yeah, and then 5 minus the next one would be 11, and then 12." After he predicted the answer, he moved the arrow over by 1 each time to show me that he was correct. Then I returned to the task $5--12$.

$\mathrm{J}$ : I would have to bring it [the orange arrow] until I get to the 12. Wait, hmm.

$\mathrm{N}$ : So are you looking at the 12 on the bottom where the arrow lands or the 12 in the orange?

$\mathrm{J}: 12$ on the bottom.

It is important to note that while he had some qualms about this answer, he seemed rather firm. His misgivings may have been assuaged by the fact that when he moved the arrow to the 12 , the number inside the arrow was -7 . This was the value he had often referred to as the answer when he used the number line by starting at the 5 and counting down by 12 and landed on the -7 . Thus, the visual in the SAA reinforced his incorrect answer to the task. Once again, I used old tasks that he had solved using the SAA to remind him of what he was looking for when he solved the tasks with the SAA.

J: See when you said -5 , I looked in the orange and it said -5 , so I stopped at 10 .

$\mathrm{N}$ : So what would that mean for this problem?

$\mathrm{J}$ : Oh, I'm going to go to the 17 because it's -12 in the orange. I have to subtract the orange part.

After doing this task, Jake noted that the SAA was becoming "a little clearer" in how it was to be used to help solve subtraction tasks. To make certain that this was the case, I asked him a few more subtraction 
tasks involving a positive minuend and positive or negative subtrahends. He noted that when the subtrahend was negative he had to move to the right and if the subtrahend was positive he had to move to the left.

So if it was negative, you have to go up, [the answer will be] more than what you started with. If you have a negative, then it means it's passed where the first number is...but if it's a positive, that's to the left, [the answer will be] like below it or in the negative numbers.

As he was saying this, he showed me on the applet that if he started at the 4, then when he moved the arrow to the right, the subtrahend was negative, and the answer was positive. He could see this by viewing the equation as he moved the arrow and blue stick on the number line. When he moved it to the left, the number was smaller than 4 and was either positive or negative depending upon how much was subtracted.

Since he had a good idea about how the applet was working and recognizing patterns of subtracting positive and negative numbers, I asked him how to use the SAA to solve the task $-2-1$. Jake placed the blue stick on the -2 and dragged the orange arrow so that it landed at -1 . The value inside the arrow said -1 , and if the expression had been showing on the SAA, it would have read $-2--1$.

$\mathrm{N}$ : so if this subtracting 1 ?

$\mathrm{J}$ : Yeah

$\mathrm{N}$ : so how did you know to travel to the right?

$\mathrm{J}$ : Because you had a negative, and when you plus a positive, you're going to the right, because it gets, cause like [frustrated sigh]. I kinda do it in the money way. Like if you're already in debt, and each time you give up money, you get close and closer to not being in debt.

When I asked him to reveal the expression, it showed -2 - - 1 and he said, "Wait I'm not sure" and fell silent. Since he thought of it as debt, I wanted to continue to use that strategy.

$\mathrm{N}$ : So are you getting in more debt or is your debt being taken away?

$\mathrm{J}$ : Taken away. We're in 2 dollars of debt and we're taking away the debt so you have -1 .

$\mathrm{N}$ : So are you taking away a dollar of debt or are you taking away a dollar we have?

$\mathrm{J}$ : Taking away a dollar of debt.

It's important to note that he knew which expression to which I was referring. I had pointed to the task $-2-1$ as I was speaking, and he pointed to the -2 when he referred to being 2 dollars in debt and the subtraction sign and 1 as he said that he was taking away the debt. I then pointed to the expression showing the SAA and asked him what $-2--1$ meant. He said, "Minus -1 which means you're going more into debt." He started manipulating the applet to show me what he meant. He moved the orange arrow so that it landed on the -3 ; thus the value inside the arrow was 1 . At this point, he mumbled, "Yeah, no wait. I think I may have got it backwards. Hmm." Then he moved the arrow to see the value inside go from 1 to 0 to -1 to -2 and then puts it back to -1 . He was not paying attention to the expression showing at the top of the SAA, but rather trying to make his contextual example fit with the expression and the his idea of movement 
on the number line using the SAA method. The movement on the SAA should have complimented the contextual idea of removal of money because they were both based on the take-away idea of subtraction. Yet, Jake seemed to misinterpret the universal set that positive subtrahends represented money that he had that was being taken away, and negative subtrahends represented debt that was being taken away. He also may have been confused as to who was giving, receiving, and giving up money. For when he referred to 'giving up money' he believed that he went further in debt, which is true, but he was interpreting the task $-2-1$ almost as if someone was trying to give him the $\$ 1$ and he was not taking it so he was "giving it up" and putting it towards his debt.

When I asked him why he thought he did it backwards, he stopped, looked at me, and exclaimed, "This is hard! No one has ever asked me to explain why things are this way." I tried to help him make sense of the signs by questioning him about why he thought the negative sign in front of the 2 meant debt, but the positive sign implicitly shown in front of the 1 also meant debt instead of a credit to the account. It turned out that working with the expression $-2--1$ in terms of money was more helpful because he was able to see the negative signs in front of the 2 and 1 and relate the subtraction sign to the words 'taking away'. His being able to recognize the misinterpretation of the task by using words to describe $-2--1$ could have happened for a couple of reasons. First, the sign of the subtrahend is clearly explicit in $-2--1$ whereas, it is an implicit positive sign in $-2-1$. Therefore, it was easier to attach a word to a sign that is easily seen than to one that is not explicitly expressed. Second, if someone is already in debt $\$ 2$ it does not make sense that someone would be taking away money that the person has, since he has none to begin with. It makes more sense that if someone is in debt a certain amount of money, that someone removes the debt. Not only did Jake understand $-2-1$ after correctly interpreting $-2--1$ in terms of credit and debit, he was also able to model both expressions on the SAA. In fact, he noted that it made "more sense" because "you'd be less in debt" for $-2--1$ because "you'd move to the right 1 and my answer would be where the arrow stops, -1 ." Furthermore, for $-2-1$, he moved the arrow to the left 1 space because "you go back 1 from the -2 , so you get -3 , more in debt, and there is a positive number in the arrow, because you took money away." After this task, he was able to solve all subtraction tasks; therefore, I wanted him to try and generalize what he saw in terms of the subtraction a positive subtrahend and negative subtrahend.

The first connection he made was between the movement of the direction and how it affected the minuend depending on whether the sign of the minuend was positive or negative.

When you subtract a negative, you get a positive, and when you subtract a positive you get a negative. Like see, here we subtracted a negative and you got 9 , so that would be more than that... so like if you did it to the -3 , which is more [than 6] so it's 9 . But if you went over to the -1 , and you subtracted a positive, then you would get a negative number. 
He gave the example of $6--3=9$. He had correctly modeled and solved this task, and used it to show that if he had a positive minuend and had to subtract a negative subtrahend, he would have to move right; thus he would increase the value of the minuend and get a positive difference. He also used the example $-1-3$ to emphasize his other point - that if you had a negative minuend and subtracted a positive subtrahend, you would obtain a negative difference. While all of these ideas were true, I wanted to encourage him to make a larger discovery. I asked him to tell me anything else he noticed. He said, "If you subtract a negative number you go right and you get bigger, and if you subtract a positive number, you do the opposite, you go left, and make the number [the minuend] smaller." He further explained, "I think the lower the negative or positive number you're taking away, the closer it is going to be to your first number - the one you start off with the blue stick." Even though he said the lower the value of number one has for the subtrahend, the closer the difference will be to the minuend, he really meant to say the lower the absolute value of the subtrahend the closer the difference between the minuend and subtrahend. While he said this, he moved the orange arrow to the right of the blue stick 3 spaces and to the left and then swung the orange arrow to the left of the blue stick 3 spaces. Thus, he essentially showed me the symmetry of the distance around the blue stick. He also identified the blue stick with the minuend because of the model. I think the symmetry was evident because he could physically move the arrow and see the distance between the tip of the arrow and the blue stick, as well as the symmetry in the expressions (see Figure 3).
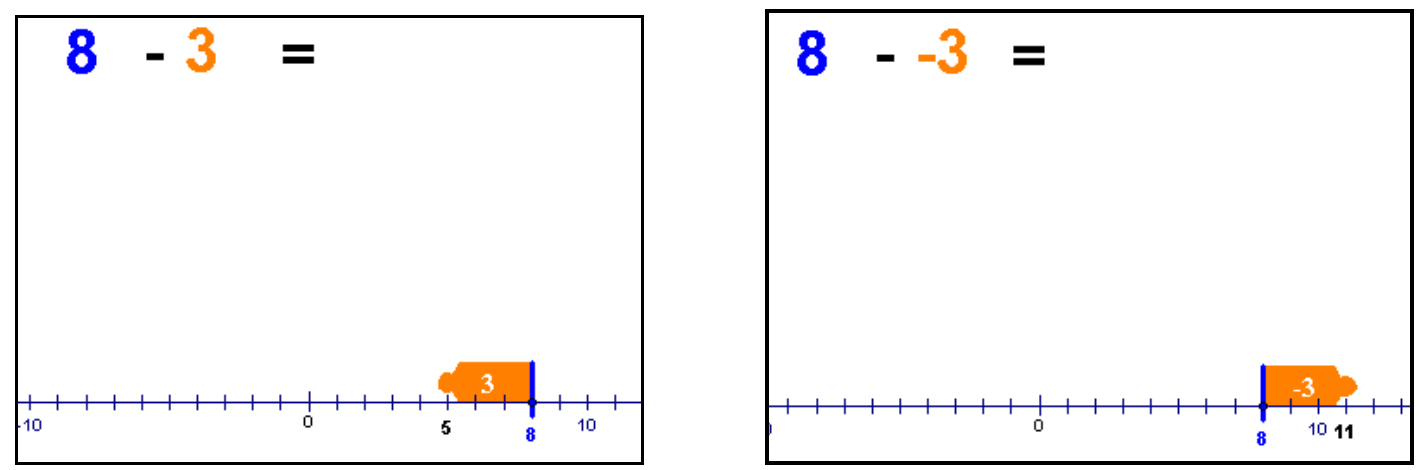

Figure 3. Symmetry shown around blue stick

After prompting him to consider the expressions and the answer to the expressions $3--5,6--3$, and $8--1$, he noticed, "You can add each one and get the answer. So $8+1=9,6+3=9$, and $3+5=8$. It's like making it into a positive, like if we subtracted a negative it's the same thing as adding it." When I asked him if he thought that he would always be able to add the second number if he was subtracting a negative number, he said, “well, yeah, I think so, but it might not always be positive.” Then he proceeded to verify his prediction by moving the blue stick to -4 and dragging the orange arrow to land on the -5 , and 
then moving the arrow to the right of -4 and landing on the -3 .

I was thinking about a negative. I mean how to get a negative [when the minuend was negative and subtrahend was positive], but that's always adding. So like trying to get it into the negatives. I think it depends on how much you're adding.

He was testing whether adding always meant that the difference had to be a positive number. He found that it did not, but subtracting a negative still was like adding. He used the minuend -4 to test hypothesis. He showed me that when he subtracted one, he landed on the -5 , which was consistent with his "moving left makes the difference negative" but when he subtracted -1 , he moved right and his difference was higher than -4 , but it was not a positive difference. Yet, despite not being a positive difference it was still similar to adding since $-4--1=-4+1$. He realized that the only way the difference would be positive would be if the absolute value of the subtrahend was larger than the absolute value of the minuend in tasks that had a negative minuend and subtrahend.

Given that he had recognized patterns and apply them, he was able to solve all subtraction tasks with and without the SAA. Furthermore, when I asked him to solve the tasks without the SAA, he asked if he "could check his answer on the computer." Moreover, when he justified his answers to the subtraction tasks, he used a number line to show me which direction he moved by using the method in the SAA. For example, when I asked him if he wanted to keep the answer of -10 to the task $-4--6$, what he had answered in his interview, he told me that he wanted to change it.

It would be -4 plus 6 . So it would be 2 . I would change my answer to $2 \ldots$ since subtract negative six, then I would go to the right 6 times. So it would be here and I would go right 6 , yeah six is right from -4 .

He drew a dot on -4 and then counted 6 steps to the right and landed on the 2 (see Figure 4 ).

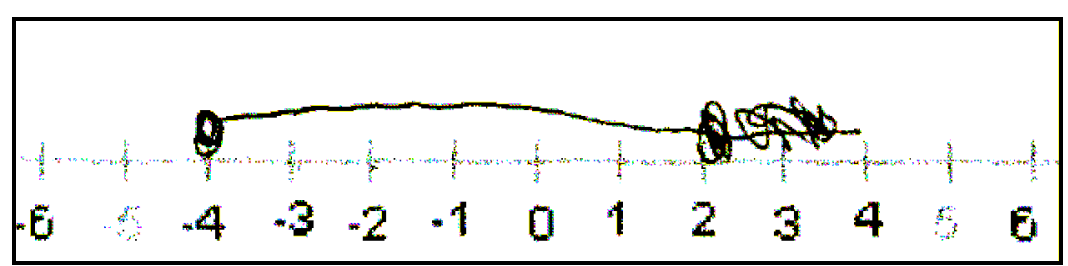

Figure 4. Using a number line to emulate the SAA

About a week later, I met with Jake again. He had arrived to our session very irate because a student had stolen his yearbook, and then waved it around in his class and the teacher confiscated it. It took him awhile to calm down and talk it through before he would turn his attention to math tasks. Once we started though, he was attentive.

I began by asking him to solve some subtraction tasks to see if he remembered anything from the last time we met. 
N: How would you solve $8--1$ ?

J: I don't like those negatives. So 8 minus -1 ?

$\mathrm{N}$ : yeah.

J: So it would be 9 .

$\mathrm{N}$ : How did you know that?

$\mathrm{J}$ : Cause I remembered from last time that when you have a negative, you have to go farther than what you have to begin with [to the right]

As he said his last sentence, he took a number line, positioned his pencil on the 8 and moved to the right 1 space. He had remembered and visualized the SAA and used this method to solve this subtraction task. I then asked some more subtraction tasks to make certain that he could answer all types, and he would often say, "You go farther than the initial number, then you go" a certain number to the right or left depending on the sign of the second number. Even though he frequently said this, he did not always reach for a number line, but rather waved his pencil in the air as if he was computing on an invisible number line in front of him. Another instance that is worth mentioning is that while he did this for all subtraction tasks, he also changed the signs when he was subtracting a negative subtrahend. Furthermore, for tasks in the form $-a-b$ where $a, b>0$, he used different procedure to justify his answer in addition to the number line SAA method. For example, when he solved the task $-3-7$ he justified his answer of -10 .

That would be -10 ...cause like, I did ...well, 3 plus 7 , that would be 10 , and since they are both negative, I put the negative sign. You could also use the number line and go left, because it's negative, yeah because you start at the -3 and because you're subtracting a positive number you got left, and if you subtract a negative number you go right.

Just like before, he used the distributive property and essentially solved $-a-b$ by doing $-(a+b)$. He was able to integrate both his idea of how to solve that task as well as the SAA number line strategy.

Because he was mainly solely using the SAA strategy when he operated on a number line, I also wanted to show him the SDA so he could use the distance method to solve subtraction tasks. I wanted him to compare these two methods especially seeing that in the beginning of the instructional section he had intermixed some of the ideas of these methods when he tried to solve subtraction tasks.

After briefly exploring the SDA and asking him to model two tasks, $-2--5$ and 5--1, which he did correctly, he told me told me that he preferred the SDA to the SAA in solving subtraction tasks.

Oh, it's [the difference] the number in between. I think I like this one better!...Because it's...kind of easier to show, cause like you put each dot on the each one and it shows you how you can get it in between. So kind of like that. It's just easier.

Jake believed that the SDA helped him to solve subtraction tasks easier because he could statically represent the minuend and subtrahend and then calculate the distance in between to obtain the difference.

The SAA was not as easy because he could not statically represent the subtrahend, for he had to use the subtrahend to move the arrow that many spaces away from the minuend. 
As I had him solve more tasks with the SDA he made a connection between the numbers in determining the sign of a task in the form $-a-b$. Note that he typically solved $-a-b$ by using the distributive property: adding $a$ and $b$ and then tacking on a negative sign. When I asked him why it was negative he said that the difference was negative because he considered both $a$ and $b$ to be negative and the operation to be addition. However, this time in solving the task $-4-7$, he moved the blue dot to the -4 and the orange dot to the 7 and told me the answer was "- 11 because I subtracted more than what I had to begin with." I asked him why he said this and he responded, "you can see the orange number is higher. I like this one a lot better!"

Because the SDA allowed Jake to represent the minuend and subtrahend as dots on a number line, it allowed him to visually compare the values of both. This affordance allowed him to see why the difference was negative instead of using rules to determine the sign of the difference. For tasks where the subtrahend was smaller than the minuend, he was also able to tell me that the difference was going to be positive because the minuend was larger for the same reason: seeing the blue dot was higher on the number line than the position of the orange dot.

At this point, since he was able to answer all subtraction tasks correctly without the help of the SDA, I moved onto asking him questions about equals. Before he left this session, I returned to subtraction to see if he could remember what we discussed. For each task, he correctly solved the abstract expression and then justified his answer using the SDA method. In fact, he would use a number line and place dots on the minuend and subtrahend and draw an arrow to show the direction he traveled in between the numbers (see Figure 5). After awhile, he stopped using the number line and just told me that he found the distance between the numbers and determined whether the sign was negative or positive depending upon whether the subtrahend was greater or less than the minuend. He said, "if you're subtracting more than the first one [minuend] it's a negative answer, and if you're not subtracting more than the first one, you're answer is positive." After solving these tasks, he reiterated that he "definitely liked the second computer thing better."

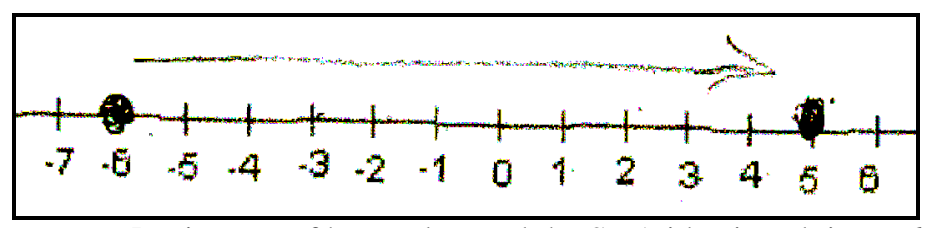

Figure 5. Diagram of how Jake used the SDA idea in solving - $6-5$

While he was able to solve abstract subtraction tasks correctly, I wanted to see how he would translate from a contextual representation to an abstract context. 
$\mathrm{N}$ : Suppose the high temperature was 32 degrees and the low temperature was 0 degrees. How J: 32 . many degrees did it change?

$\mathrm{N}$ : How did you find that out?

J: I just did, I took 32 minus 0 equals 32. I mean, it didn't change cause it kind of like, I don't know how to explain it, it just kind of uh, it subtracted itself out, cause you get back as much as it was worth....it came down as much as it came up.

Jake created the equation $32-0=32$ and thought about it on a number line as if the temperature rose to 32 on the number line from 0 degrees and then fell the same amount in order to get back to the low temperature of 0 degrees. While he did not use any prior methods to answer this task, he did show a previous strategy when he answered the next contextual task.

$\mathrm{N}$ : If Alaska's low temperature for yesterday was -17 degrees and it's high temperature was -6 degrees, how many degrees did it change?

$\mathrm{J}$ : Uh, positive eleven. I mean, actually, negative eleven.

$\mathrm{N}$ : Ok, and how did you get that?

J: Because I did 17 minus 6, which I just solved by looking at the 6 and 7, so I just knew that it had one more, so it would have been 11 . I knew that -6 was higher, so I knew it [the answer] was going to be a negative number, so it was kind of fast.

Jake knew that the distance between the numbers was 11 because 10 plus 6 was 16 and he needed 1 more to get to 17. Furthermore, he used the low temperature, the first number appearing in the context as the minuend and the second number appearing in the context, or the high temperature, as the subtrahend. Even though he did not use a number line, he visualized the placements of the dots as if he placed one on the minuend and subtrahend. Thus, since the subtrahend was larger than the minuend, he knew that the difference was going to be negative. He knew how to use the SDA idea to help him solve contextualized tasks.

\section{Summary of Jake's Interactions and Understanding with Technology}

- Jake had both a conceptual and procedural understanding of addition and subtraction of negative numbers.

- By manipulating multiple sequences of subtraction expressions, noticing patterns, using contextual problems, and noticing the connection between movement away from the minuend and the corresponding equation, Jake was able to see subtraction as both take-away and a distance. However, he preferred using the idea of distance and referenced the SDA in justifying his answers.

- Jake was able to create correct subtraction equations for tasks represented contextually. In creating these equations, he used the SDA method to justify his answers.

- Jake no longer believed that subtraction meant to move left in all cases, only in cases where he subtracted a positive subtrahend. In working with the number line, he noticed that when he subtracted a negative subtrahend, he in fact, moved to the right and justified this in terms of moving negatively past the minuend.

- Jake noticed that the subtraction of a negative number was the same as addition. 
- After working with SAA Jake noticed that subtracting a negative subtrahend from a positive minuend elicited a positive difference; subtracting a positive subtrahend from a negative minuend elicited a negative difference; when subtracting a negative subtrahend, the difference was larger than the minuend; when subtracting a positive subtrahend, the difference was smaller than the minuend; the lower the absolute value of a subtrahend will cause the difference to be in between the minuend and subtrahend; if the absolute value of a negative subtrahend was larger than the absolute value of a negative minuend, the difference was positive.

- The SDA allowed him to realize why the difference was negative or positive because he could see which of the subtrahend or minuend values were higher on the number line. He did not need to rely on rules to solve the expression.

- After working with the technology and tasks, Jake successfully attained Level 4 in both Peled's QD and NLD.

\section{Discussion}

Before working with the technology applets, Jake did not have a clear conceptual understanding of subtraction. He used the same, although incorrect, number line strategy, and various, not always correct abstract procedures when solving subtraction tasks. When using a number line, he always believed that a subtraction or a negative sign indicated that he had to move left even if he was subtracting a negative subtrahend. Furthermore, when he used the number line, he always moved to the left the amount represented by the subtrahend; thus, he viewed subtraction as take-away. Jake used a variety of abstract procedures depending on the form of the subtraction task. He solved tasks in the form $a-b$ where $0<b<a$ by transforming the expression into equation $b+?=a$ and using the number line to solve for the question mark. For tasks in the form $a-b$ where $0<a<b$, he was able to correctly answer and provide justification by using the idea of taking away more objects from his initial set of objects. For tasks in the form $-a-b$, he came up with the correct answer and viewed it as addition of the absolute value of the numbers and tacking on a negative sign. For tasks in the form $a--b$, he believed that they were initially equivalent to $a-b$ and then eventually used the strategy $a+?=b$ and tacked on a negative sign to the value in the question mark because $b$ was negative. For tasks in the form $-a--b$, he solved them by taking the absolute value of the difference between $a$ and $b$, and then tacking on a negative sign, i.e. solving $-|a-b|$. By using these rules and movements on a number line, he was able to answer correctly all subtraction tasks except those that subtracted a negative subtrahend, whose absolute value was higher than the absolute value of the minuend.

When Jake utilized the SAA, he was able to realize easily that the applet embodied subtraction by observing how his manipulation impacted the visual in terms of beginning and ending points and the value in between them. Furthermore, Jake noticed quickly that when he moved to the left, the arrow value 
increased and when he moved to the right, the arrow value decreased. Due to this observance and his idea of subtraction as take-away, he was able to see the correlation of subtraction and movement on the number line. Yet it was interesting in that he initially used the SAA as the SDA when he created his own abstract tasks, and thus used a distance method instead of the take-away method. It is important to note that he had never used the distance method before this. He could have used it now because the visual of the arrow value was prominent; thus, he could have believed that the arrow value was giving him the answer instead of being the value that was taken away, like he normally assumed. Yet, when I gave him tasks to solve with the SAA, he once again used his idea of take away to solve them.

While he used the SAA to solve subtraction tasks, the visualization and manipulation affordances allowed him to arrive at a few generalizations that he had not known about subtraction before such as:

$$
\begin{aligned}
& \text { If } a--b=c \text { for } a, b \in \mathrm{N} \text {, then } c>0 \\
& \text { If }-a-b=c \text { for } a, b \in \mathrm{N} \text {, then } c<0 \\
& \text { If } a--b=c \text { for } a \in Z \text { and } b \in \mathrm{N} \text {, then } c>a \\
& \text { If } a-b=c \text { and } a \in Z \text { and } b \in \mathrm{N} \text {, then } a>c \\
& \text { If }-a--b=c \text { and }|b|>|a| \text { for } a, b \in \mathrm{N} \text {, then } c>0 \\
& \text { If } a--b=c \text { for } a \in Z \text { and } b \in \mathrm{N} \text {, then } a+b=c
\end{aligned}
$$

He also made some generalizations in regards to the number line. He realized that subtraction did not always indicate for him to move to the left on a number line. In fact, now he knew that if he subtracted a negative subtrahend, he had to move to the right, and if he subtracted a positive subtrahend, he had to move to the left of the minuend. He was able to see this by moving the arrow to the left and right of the blue stick and seeing the effect it had on the difference. He also knew that the lower the absolute value of the subtrahend was, the closer the difference would be between the minuend and subtrahend. He was able to see this by observing the symmetry of the values inside the arrow when he moved it to the left and right of the blue stick.

After making these generalizations, he was able to solve all types of subtraction tasks. Furthermore, he remembered the visual of the SAA when he returned a week later and used that idea to justify his correct answers to subtraction tasks. Even though he could answer all subtraction tasks correctly using the SAA, he still liked the SDA better because it illustrated subtraction more clearly to him. After using the SDA he reaffirmed the generalizations he made with the SAA, and he was also able to interpret the sign in the answer more easily. Through the static representation of the minuend and subtrahend, he liked that he could see the difference as the distance between the two and knew if the sign of the difference was positive or 
negative depending upon whether the subtrahend was farther right than the minuend, or higher in value. He was easily able to see this because of the dots representing the minuend and subtrahend on the number line. Moreover, after the instructional section, he was able to easily solve contextual tasks and justified his answer using the ideas from the SDA.

\section{The Equal Sign}

\section{Prior Conceptions}

Prior to using technology, Jake had a relational (Seo \& Ginsburg, 2003) conception of the equal sign. Only his answer to Task 1 could be classified as operational. After I asked if he could point out the similarities and differences among $5+6,5+6=$, and $5+6=11$, he said, "They are all the same questions. I mean, 5 plus 6 equals 11, and 5 plus 6 equals 11. They are all 11. Is this a trick question?” As I assured him that this was not a trick question, told me, "Ok, but they are all the same numbers." When I asked him what the equal sign meant in the latter two, he looked at me quizzically and pointed to the equation $5+6=11$ and said, "That 5 and 6 equals 11." When I questioned him about $5+6=$, he said, "Well, 5 plus 6 equals 11 , so they're saying like, you can do 11 is equal to $5+6$. ." He proceeded to write the equation $11=5+6$ and told me that it was the same as $5+6=11$, but that when someone wrote $5+6=$, it meant that they wanted you to write the equation this way, with the 11 in the front. When I asked if it was ok to write the 11 first he said, "Yeah, I've seen it..., well, I don't remember where it was. I think it is algebraish, like kind of putting equations and stuff."

Just as Jake did not have an issue rewriting the task $5+6=11$ with its equivalent partner, he also did not take issue with the other non-canonical forms in Task 2 in Module 3. He told me that while he had seen 3 = 3 before, he "didn't get it. I didn't understand it. I guess 3 equals 3 . I mean, that's all - it's just it." When I specifically asked if it was ok to write the equal sign in between the 3's, he told me it was since they were the same value. I think he struggled with the idea of why would anyone want to write 3 equals 3 , when it was obvious that they were the same number. Similarly, he believed that it was ok to write $8=10-2$ since both sides were equal in value. Similarly, he believed that $2 \times 5=4+6$ was written correctly because each side had a value of 10

Because Jake had a relational view of the equal sign, he believed that the rest of Task 2 was written correctly because the equal sign just noted that one could "put it in different ways to show that they all equal into each other. So 12 inches goes in a foot, and 10 pennies go into one dime." He saw that the equal sign allowed one to express the same amount in a different contextual fashion. 
However, his relational conception of the equal sign ceased when I asked him what the equal sign meant in the task $3+5=?+2$. He responded that the equal sign meant, "to find the missing number." When I asked him what the missing number was, he responded, "it would be 6 , because $6+2$ equals 8 and 3 plus 5 equals $8 . "$

To make sure I was not misinterpreting his idea of the equal sign, I asked him again what he thought the equal sign meant in this equation. He reiterated that it meant to find the missing value. Even though, he knew both sides had to equal the same amount because of the equal sign, the equal sign in this task told him that he had to solve for the question mark. Thus, the equal sign told him to "do something" to find the answer. Similarly, he had the same view of the equal sign when a letter replaced the question mark. Even though he had a relational interpretation of the equal sign when the equation strictly used numerals, this relational interpretation did not continue when he worked with equations that contained variables.

When it came to solving 1-step equations, Jake used an unusual method that does not quite fit within the categories described by Knuth et al (2006).

$\mathrm{N}$ : So what does the equal sign mean in $x+5=3$ ?

$\mathrm{J}$ : That you're just trying to figure out what equals the 3 , which probably is a negative number. $\mathrm{N}$ : And why do you think it's a negative number?

$\mathrm{J}$ : Because if you have, if that number is positive, then you plus 5 then it's just going to be more than 3.

$\mathrm{N}$ : So do you know how to find the value of $x$ ?

$\mathrm{J}$ : Yeah, we've done it earlier this year. So I guess you would just do 3 minus 5. I think.

$\mathrm{N}$ : How did you know you were supposed to do 3 minus 5 ?

J: Cause uh, 5 minus 3 would give you a positive number, but you wanted to go further down, so you do like the less, so it would be 3 minus 5 cause 3 is less. So it would be -2 .

Once again, Jake did not believe that the equal sign showed the equivalence between two expressions, rather it indicated that he needed to solve for the variable in the equation. Even though he said that he had done this in his math class in the beginning of the year, he did not seem to be totally sure in how to solve this equation. While he reasoned correctly that $x$ had to be a negative number because when he added 5 to it, it had to equal 3, he only knew that he needed to combine the values together to get this negative number. Since he needed to get something smaller, he chose subtraction. Then he decided that since 5-3 was not negative, then he had to combine 3 and 5 by subtracting 5 from the 3 ; thus he obtained the value of -2 . While this is the correct answer, he happened upon it by using pieced together information: knowing that his answer had to be negative, and knowing that he had to combine the values in the equation to get the value of $x$. While he eventually did use an unwinding method simply because he subtracted 5 from 3 , this procedure does not clearly fit into any category. This is also contrary to Knuth and colleagues' study that students who had a relational understanding the equal sign typically solved equations algebraically by 
applying the same transformation to both sides of the equation.

When I asked him what the equal sign meant in $2 m+9=1$, he once again told me that it was what the m equaled and followed it up that he had seen these types of equations but "I haven't understood what that meant." When I asked him to solve the equation he said, "We've solved them before, but I can't remember how we did it." When I asked him to take a guess at it, he said, "Well, I really can't remember, you times two by something don't you?" His inability to use a method to solve the 2-step equation is not so surprising given that he had a random procedure for solving 1-step equations.

\section{Summary of Jake's Prior Conceptions}

- Jake exhibited a relational view of the equals sign for numerical equations and the comparison of numbers and units.

- Jake was familiar with non-canonical forms and was able to state why they made sense.

- Jake believed that the equal sign did not show the equivalence of two expressions when one of the expressions contained a variable. He believed that the equal sign meant that he had to solve for the value of the variable.

- Jim accurately solved 1-step equations using an unmethodical method of pieced together reasoning. Because his method was not methodical, he was unable to solve 2-step equations.

\section{Analysis of Interaction with the Equality Applet Jake's Interaction and Understanding with Technology}

As Jake played around with the Equal Balance Applet (EBA), he easily picked up on the fact that to maintain the equilibrium of the balance, he could "add negatives and positives to each side, like you can add -1 there and add -1 there. You just need to make them even." When I challenged him to find another technique to keep the pans balanced, he discovered that he could exchange weights and break them into single units that equaled the total weight. For instance, when he explained this strategy, he showed me that when he had two 5-weights, one on each pan, he could exchange one of the 5-weights for five 1-weights and the pans would stay balanced (see Figure 6).

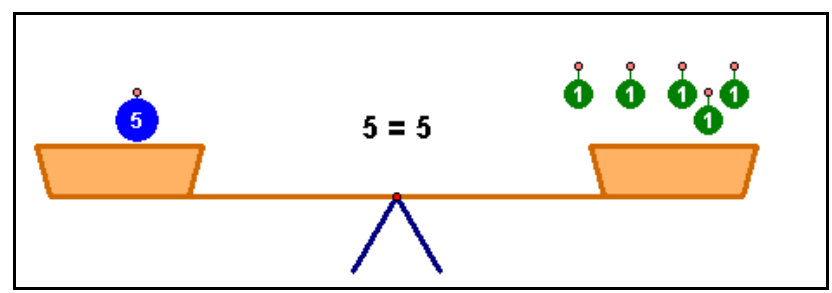

Figure 6. The exchange of weights that maintained the balance of the pans.

When I asked him to find another way to keep the pans balanced by only changing one side of the pan, he 
saw that he could place a zero pair on one side because the positive and negative values would cancel each other. After discovering the strategies of how to maintain equilibrium, Jake worked through the Tasks in Module 3.

When Jake modeled the task $3+5=?+2$ using the EBA, he first placed three 1-weights and one 5-weight on the left pan, and then placed two 1-weights on the right pan. He then said, "Well, so we'd have to add 6 so the right would be 6 plus 2." It seemed that he was able to solve this equation without placing the question mark on the pan as a placeholder because he could easily see what he needed to add to the right pan to make the left and right pans balance. While I let him place a 5-weight and 1-weight on the pan, I then asked him to remove them and use the question mark so it looked like the original equation. I asked him if he could manipulate the pans using his discovered strategies in order to figure out the value of the question mark. After placing the question mark on the right pan he said, "Well, this side is 8 and that is 6 , so we just have to do 8 minus 2 and that's 6." At first, I wondered if he was simply trying to combine the numbers on the equation, since the corresponding equation on the screen said $8=?+2$. I thought this was likely since he had done that in his interview: simply found different ways to combine the numbers in the equation to figure out the value of the variable. Yet, when I asked him how he knew that he said, "You can move these 2 off of here and there." He proceeded to remove two 1-weights off of the left and right side pans and saw that the question mark was equal to 6 . While he could have created an equation to fit the answer he knew to be correct, I also think that he was able to see why he had to subtract 2 because this time, he showed that he subtracted two from both sides, not simply from the 8. Furthermore, the EBA lent itself to seeing the visual representation and the need of keeping the pans balanced, and the only way to do that was if he subtracted 2 from the 8 was to subtract 2 from the right pan as well. He easily related the removing of a positive weight as the subtraction of that value.

After he gave me the answer, I asked him how he could tell that the question mark was equal to 6 . He said that he had gotten "6 on the left and zero on the right." When I asked him if he had anything remaining on the left pan, he said, "I can't remember," even though he was clearly looking at the screen, which showed the question mark in plain view. When I said, "What's this?" and pointed to the question mark, he said, "Oh, I thought you meant what number was left on the pan." This comment provided insight into his case and other students' responses when I had asked what was remaining on the pans. Most often during the initial tasks with the EBA, after they obtained the variable by itself, interviewees would state that they had nothing remaining on the pan containing the variable, or said that it was zero, when an $x$ weight, or question mark was clearly sitting in the pan. I could not understand this; yet, I am assuming that 
each student was looking for a number when I had asked this question. Since there was no number on the pan containing the variable, they said that the pan contained zero. This could mean that they did not view a variable as a number, but rather an entity that they had to solve for, whose value would appear on the opposite side of the equal sign.

After Jake removed 5 from both the left and right pans when modeling the task, $x+8=21$, using the EBA I asked him what he remained on each pan. He asked, "Do you mean number wise, or everything?" and went on to say, "Number wise, 15 is on the right side, and 2 is on the left. Oh, and then an $x$." This also points to the fact that the variable was an afterthought, and not considered to be a number until he found the value of it. He then told me what he needed to do to solve for $x$.

To get $x$ by itself, I need to subtract 2 more, so like, well, for this, I have to take 5 away and put 3 back. So I have $x$ plus 0 equals um, so the right pan is 13. So I guess $x$ is 13 too.

Jake realized that since the right pan had three 5-weights, when he subtracted two 1-weights from the right pan, he would have to take away the 5-weight and replace three 1-weights (see Figure 7).

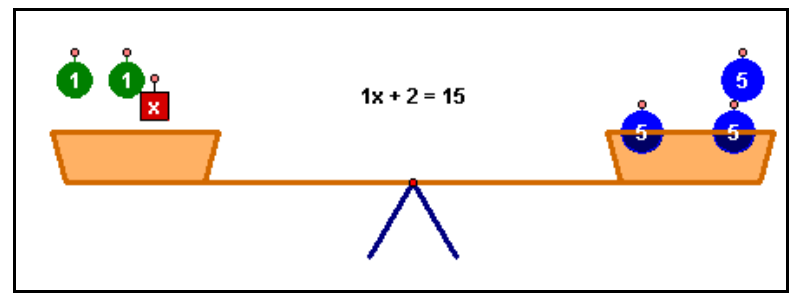

Figure 7. EBA showing $\mathrm{x}+8=21$ after removing 6 from each side.

He noticed this very quickly, unlike some other students. This may have occurred because he had seen how exchanging weights during the time he was playing with the EBA impacted the balance. It took him two steps to find the value of $x$ with the EBA, like all the other interviewees. This occurred because students easily saw that they could remove one 5-weight and one 1-weight from both sides since both sides had these weights, but had to do an exchange to remove two more 1-weights from both sides. Therefore, it was natural that the solution procedure required two steps using the EBA. Unfortunately, this may have hindered students understanding of algebraically solving equations. Due to this, I asked Jake if he could have done it faster by looking at his written work of subtracting 6 and the subtracting 2 more. He said, "Yeah, we could have subtracted all of... like you could have taken away 8 at first." After saying this, he re-wrote the equation and subtracted 8 instead so the solution procedure only showed one step instead of two (see Figure 8). When he glanced back at his work, he said, "Oh, I've done this before! Not with the computer, but this here with Miss Beecher. I'm not good with this, but I understand this." As he said this, he had pointed the algebraic representation of the task and then to the EBA. While he noticed the 
connection between what he was doing with the EBA and how he had solved it algebraically, he still had to state that he was not good at solving equations algebraically but rather he could do it with the EBA. This was probably due to the fact that when he had tried to solve 1-step equations before, he haphazardly figured out the answer instead of following a methodical procedure, which the EBA allowed him to figure out. Thus, the EBA made sense to him, while the abstract algebraic reasoning did not, until he could make the connection as to why he needed to subtract 8 from both sides.

\begin{tabular}{rl|}
\hline$x+8$ & $=21$ \\
-8 & $=-8$ \\
\hline$x$ & $=13$ \\
\hline
\end{tabular}

Figure 8. 1-step procedure in solving $\mathrm{x}+8=21$ algebraically

One interesting note is that when he subtracted 8 from 21 algebraically, he had asked for a number line, which I gave to him. He then started at the 21 and counted to the left 8 spaces. This is interesting because he had just learned how to use the SDA to solve subtraction tasks, yet reverted to using the take away method for a task having a positive minuend and subtrahend.

After he seemed to figure out how the EBA and solving algebraic equations connected, I gave him the task $x-6=-15$ to solve on paper. Yet, he was unable to do so. He said, " $x$ plus 6 and 15 , so you'd uh, wouldn't you, couldn't you just add? Add 16 plus 15. Uh, no. [long pause] Could you give me a hint? Could I use the computer?" Jake seemed to think that because there was a -6 and -15 , he had to add enough to get 16 on the one side and 15 on the other. This is what I think he meant when he said that he had to add the 6 and 15. Then he thought he might have had to add both of these numbers to get the value of $x$. This seems plausible, because when he had solved one-step equations in the interview, he had just tried to combine the numbers to get something that could potentially be the value of $x$. Yet, he knew this to not be quite right, so he asked if I could give him a hint or allow him to use the EBA to help him solve the equation. I let him use the EBA and he immediately said, "Oh, so take away the -6 and leave the $x$, so we have to take away -6 from here too." Because of the visual the EBA afforded, he could see objects on the pan with the $x$ and knew they had to be removed to figure out the value of $x$. He took away the balloons totaling -6 on the left side, and removed a -5 balloon from the right side. He paused and said, "Oh, I have to take a -1 , so I have to put back four of them [-1 balloons]." He had noticed that when he removed the -5 balloon from the right side after he had removed -6 value of balloons from the left, the pans were not balanced, this reminded him that he had not totally removed -6 worth from the right side, and prompted him to split up the remaining two -5 balloons into one -5 balloon and four -1 balloons. After moving the balloons, he wrote down his arithmetic and said, "So I took -6 from one side, so I took away minus, so 
that's a minus -6 from both sides. That's uh, that's uh, like a positive. But it's going to be negative... so it's going to be either -8 or -9 ." He grabbed a number line and started at the -15 and counted to the right and counted up to the -6 and said, " $-15,1,2,3,4,5,6,7,8$, 9, so -9." When I asked him how he knew he had to travel to the right, he said, "Because uh, it's like you are subtracting more than like -15." There are two things that are interesting about how he solved this task. First, as soon as he had the visual of the balance in front of him, he was able to solve this equation. Second, in subtracting -6 from -15 , he noticed that subtracting a negative was similar to a positive and he used his knowledge of the SDA to compute the value, for he began at the -15 and counted the number of spaces in between the -15 and -6 and knew that the answer had to be negative because he was subtracting a higher number from a smaller number. While he could find the answer and write the algebraic procedure afterward, he was still unable to solve this task without seeing the visual representation in the EBA. Yet after doing this task, he was able to solve all one step equations without the help of the EBA. One notable occurrence is that each time he solved the task, if the numbers were large and he had to subtract, he would use the SDA method and place a dot at the minuend and subtrahend and count the spaces in between to determine the answer.

Clearly the EBA helped Jake solve 1-step equations, yet I wanted to know what he had thought about the EBA.

J: It helped, but it kind of confused me a bit.

$\mathrm{N}$ : So how did it confuse you?

$\mathrm{J}$ : Well, it didn't confuse me too much, until we got to the $x$ and we had to take off and put back and count stuff.

$\mathrm{N}$ : Do you mean when you had to exchange the -5 for five $-1 \mathrm{~s}$ ?

$\mathrm{J}$ : Yeah. But finding out was $x$ was kind of easy to do.

Given that the EBA allowed him to see and manipulate objects that represented numbers and the equality and inequality represented by the balanced or unbalanced pans, Jake was able to figure out how to solve 1step equations. Furthermore, he was able to easily translate his movements into algebraic procedures, because removal intuitively represented subtraction and putting on objects represented addition.

He was able to apply this kind of thinking in solving 2-step equations. When I gave him $2 m+7=15$, he subtracted the 7 from both sides and then divided by 2 .

J: Well 15 minus 7 . So that would be uh, $8 \ldots$ so it would be $2 m$ plus 0 equals 8 right?

N: Ok. Now what will you do?

J: Don't you want to take the 2 out?

N: How would you do that?

J: Hmm. [He looked at the screen showing the equation] You would divide by 2.

$\mathrm{N}$ : How did you know you had to divide?

J: Because I kind of remember from Miss Beecher. Divide by 2 here, and then 8 divided by 2, that is 4 , so $m$ equals 4 . 
His comments are interesting because during the interview he was unable to solve 2-step equations, yet, after learning how to use the EBA to solve 1-step equations he was able to extrapolate the method to solve 2-step equations. Yet, he believed that he knew how because he finally remembered how his teacher taught him. I think he did remember that he had to divide, but I think the EBA prompted him to do so, since he looked at the screen containing the weights, and because he was unable to solve these types of tasks previously. Also it is important to note that when he talked about his procedures, he used algebraic transformations to solve the equation, rather than unwinding (see Figure 9).

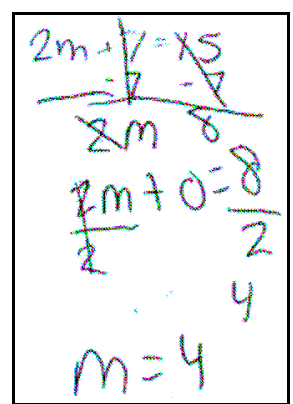

Figure 9. Jake uses algebraic transformation method to solve $2 m+7=15$

I asked him to solve a few more 2-step equations, which he did so correctly without looking or using the EBA. While he was able to solve for the variable, I wanted to know whether he understood why he had to perform the same operation to both sides.

Well, because, I can't remember why math-wise, but I just remember that whatever you do to this side, you have to do to this side to keep it balanced. So every time you subtract or divide you put it to the same thing to the other side.

It is interesting that he does not believe he is giving a mathematical answer, but rather one that he learned by using the EBA. He could visually see that he had to operate on both pans in order to keep the pans balanced and find $x$, but he did not believe this was a mathematical reason. While, he did not mention the fact that the equal sign required the pans to stay balanced, he at least realized that the expressions on either side of the equal sign contained the same amount of 'weight;' therefore, had to remain balanced. After he sat there for a few seconds, he went on to say more about it.

J: Well, I had to make it even out. Like if they are equal, then they are balanced.

$\mathrm{N}$ : So do you think that after each step the expression are equal?

$\mathrm{J}$ : Yeah, wait, say that again?

$\mathrm{N}$ : For instance, here in $2 m+9=3$, when you subtracted 9 from both sides, was $2 m+0$ equal to -6 ?

$\mathrm{J}$ : Well, no they aren't going to be equal.

$\mathrm{N}$ : What isn't going to be equal?

$\mathrm{J}$ : That's not going to be equal to that because that's going to be zero and that's going to be -6 .

$\mathrm{N}$ : True, 0 does not equal -6 . What about the whole thing, $2 m+0$, is that equal to -6 ?

$\mathrm{J}$ : Yeah, that is. 
Jake seemed to understand that the transformations made the expressions remain equal due to making the pans balance on the EBA, but he once again reverted to assuming that I was speaking solely of the numerals in the equation, instead of the expression containing a number and variable. Jake finally seemed to have a relational understanding of the equal sign when an equation involved a variable.

\section{Summary of Interaction and Understanding with Technology}

- Jake exhibited a relational view of the equals sign for numerical equations and the comparison of numbers and units as well as equivalence of two expressions when one of the expressions contained a variable. He no longer believed that the equal sign meant the value of the variable.

- Jake accurately solved 1-step and 2-step equations using the algebraic transformation method.

- Jake believed that the visual model and manipulation features allowed him to solve 1-step equations more easily than he was able to do before working with technology because it helped him to find the solution by intuitively seeing what he had to remove or add to the pans to keep them balanced, and making the connection between removal and addition of objects with the operations of subtraction and addition.

- Jake easily extrapolated his understanding of solving 1-step equations and to solving 2-step equations. In fact, he was able to do this without manipulating the EBA.

- Due to the visual, manipulation, and feedback characteristics of the applet, Jake's understanding the removal or subtraction of a negative number was the same as addition was reinforced.

- Jake used the visual aspect of the applet to intuitively tell him which operation to apply because he knew that he needed to get the variable weights by themselves. Unfortunately, the fact that he had to exchange some objects for smaller valued objects required more steps to solve the equation using the EBA than it did solving the equation on paper.

\section{Discussion}

Jake had a relational view of the equal sign for all equations without variables and in the comparison of values and units. Furthermore, he was able to justify non-canonical forms using this relational viewpoint. Yet, his relational viewpoint strictly included numerical equations, for when a variable entered the equation, he did not believe that the two expressions separated by the equal sign were the same value, but rather instead believed the equal sign signaled for him to solve the task referred to the value he obtained for the variable. Thus, the equal sign in the equation meant that the variable equaled a specific value.

Even though Jake had a relational view of the equal sign for numerical equations and comparison of units, he did not algebraically solve 1-step and 2-step equations, unlike the tendency shown in previous literature, rather he solved equations using some pieced together information that does not fit into a specific category described by Knuth and colleagues (2006). He was able to obtain a correct answer to 1-step 
equations, but could not solve 2-step equations.

When he began working with the applet, he visually understood that the equal sign in an equation meant that each expression had the same value as the other expression. Furthermore, he discovered many strategies to maintain the balance of the pans and the equality of the expressions. After discovering these strategies by manipulating the objects on the pans, he was able to model 1-step equations and solve them using the algebraic transformation strategy by removing or adding the same objects to both the right and left pans. Because the applet allowed him to drag different values and see the resulting effect of that manipulation on the pans and corresponding equation, Jake was able see a visual depiction of the equation and intuitively realize how to find the value of the variable without it algebraically. Furthermore, he was able notice the intuitive correspondence between his manipulations on the EBA and the mathematical operations, which allowed him to easily transform his solution procedure on the EBA to paper.

During the process of working with the technology applet, he discovered the relationship between the expressions by using the EBA, he also came to realize that there was a faster way to solve equations by looking at the pattern of his written work versus operating solely on the visual model of the equation. Although this method of solving on paper was faster than moving the objects on the applet, he liked working with the applet because it had helped him to use intuition of how to manipulate the objects to get the variable by itself. After discovering the procedure for solving 1-step equations on the EBA and its correlation to paper, Jake was able consistently solve both 1-step and 2-step equations algebraically, even though he was unable to solve 2-step equations prior to working with the EBA. 
CHAPTER 9

\section{CASE STUDY: JEN}

Jen's Background

During the time of the study, Jen was in eighth grade Standard Math. Standard Math mainly covers pre-algebra material. A student in Standard Math in eighth grade will take algebra in ninth grade. Data collection was at the end of the school year; thus, Jen had already covered pre-algebra material, such as creating and solving word problems using formulas and functions and solving equations containing one variable. In fact, Jen explained that she had solved equations towards the beginning of the year and that was the reason why she could not remember how to do them during the study.

Jen was not shy and never hesitated to answer my questions. In fact, sometimes she would anticipate the end of my question, jump the gun, and give a response that did not always answer my actual question. For example, on the fifth task, I gave her the number line with the dots on the numbers -6 and 5 . After I spoke the words, "Could you write," she jumped in and said, "Should I write the answer? Um, I think it would be -1 ." Then I completed my question: "Could you write a math problem using the dots on the numbers?" She answered this question as well, but her answer to my actual question was influenced by her thought process to the anticipated question. As will be discussed in her prior conceptions of addition section, she then tried to corroborate her movements on the number line, and subsequently the operation she used to relate the numbers, based on her answer of -1 .

Similarly when I gave her the task $x+5=3$ and asked, "Have you ever," she jumped in said, "I know how to solve this, you cross multiply! Wait, yeah, you do hmm.” After she wrote down $\frac{x}{5}=\frac{3}{100}$, tried to figure it out, and eventually gave up, I asked her if she had ever seen these types of problems before, to which she told me that she had solved them earlier in the year, and could not remember how to do them.

Not only did she try to anticipate my questions, she also tried to answer my questions quickly, which sometimes caused her to talk into circles, get confused, and mumble, "I don't know. I don't know." For example, after I asked her to solve $-4--1$, to which she obtained the answer of -3 , I asked her to show me how she could use a number line to answer that question. She began by putting a dot on -4 and a 
dot on -1 , but could not figure out how to move to get to her answer of -3 .

I can't get to -3 . No, hold on. Four, no, cause if you minus -1 it would be -2 , and if you minus -4 it would be -8 , so you can't, except put a dot there and say it is the answer. Starting at -1 and minus a -1 , you get -2 . Ok, like, I don't know. I don't know. I guess if you minus -1 from 4 it would be $-2 \ldots$ I don't know what I'm saying!

She said this excerpt very fast and almost under her breath to try to convince herself as well as tried to explain her reasoning to me. Her explanation became so convoluted that I am not sure she knew what question she was answering. Furthermore, she knew she was confusing herself and resorted to giving the answer of "I don't know." During these types of instances, she would often shut down, drop her pencil on the table, and slouch in her chair as if she had been totally defeated. She did not have the attitude of being defiant, but as truly frustrated and wanting to succeed, but feeling that she did not have the power to help herself. At this point, I would try to ask her questions so she could work through her reasoning, which would cause one of two sets of reactions. One set was her perking up because she could work through the task and explain her reasoning out loud to herself and to me, and realize what she understood in the task and what she did not understand before and get excited about her understanding. The second set was that it would cause her to sit there and wait as if she expected me to answer my own questions or give her a different task, because she was clearly done with the task before her. If she reacted in the latter manner, I tried to find a question that she could answer and build up my questions from that point. Due to her frustration, sometimes we simply moved on to the next task if it was during the task-based interview.

Unlike most participants, Jen never stated that she used rules that someone had given to her to answer the tasks. Instead, she would try to describe why she used a certain method, but never stated that she was taught that way. It was almost as if she had internalized the rules, used them, and believed they were correct, even if they did not make complete sense to her. After working with the technology, she was able to discover patterns that led her to using procedures based on how she manipulated the technology and saw how it visually displayed the concepts.

It is important to note that Jen had only used the computer in math class to aid in the study for the SOLs. Like other participants who took standard mathematics, Jen's teacher used Study Island to get her students ready for the state tests.

\section{Negatives and Operations on Integers}

\section{Prior Conceptions on Addition}

Jen had some procedural and conceptual understanding of addition of integers. At first when I gave her the three addition tasks to solve, $10+3,10+-3$, and $-5+-7$ she told me the answer to the first 
was 13, but said that she did not know the answers to the latter two. She placed her pencil on her paper and just sat there awaiting more help or instructions. Instead of providing her the answer, I asked her how she thought about the first task. She said, "I just added the 3 to the 10 and got 13." By asking her similar questions to the latter two tasks, she eventually figured out that she did, in fact, know how to add integers.

$\mathrm{N}$ : What about this one, $10+-3$ ?

$\mathrm{J}$ : I don't know. I guess it's negative something.

$\mathrm{N}$ : How did you know it was negative?

J: Cause you gotta minus 3 from 10 and anything you subtract has to be negative.

$\mathrm{N}$ : Do you think the number line would help you figure it out?

J: Um, I guess so. Wait, I think it's positive 7 or negative 7.

$\mathrm{N}$ : Ok, so why might it be positive 7 ?

J: Because it's 10 and you subtract 3, wait, oh, yeah, subtract 3 so it would be 7 .

$\mathrm{N}$ : Why did you decide to subtract 3 ?

$\mathrm{J}$ : Because it's negative.

$\mathrm{N}$ : What about the answer maybe being -7 ?

J: I think it's positive 7 now, not negative 7 .

Jen's thought process is interesting. Without prompting, she would have left the task blank without reasoning and finding an answer. Instead, by simply asking her questions about her thought process, she was able to conjecture answer possibilities, and then eliminate incorrect possibilities through reasoning. Because she encountered a negative addend, she assumed that her sum was going to be negative. Even though she did not explicitly state that she used a rule, this assumption might have been made due to hearing the rule that multiplication involving one negative factor produces a negative product, and she assimilated this rule to addition with a negative addend. It is unclear as to what prompted her to determine the value of the task. She did not look at the number line before saying that she believed the answer to be -7 or 7. Furthermore, it is unclear as to why she did not assume it was -7 based on her previous statement that the answer should be negative due to the fact that she had a negative addend. It could have been that in stating that she had to subtract 3 from 10 triggered her memory, which prompted her to now believe that the answer had to be a magnitude of 7. When I asked her whether she believed the answer to be positive or negative 7 , she decided that the answer had to be positive 7 because she realized that even though the 3 was negative and she was adding her numbers, it was similar to subtraction. Furthermore, she confirmed this answer by using the number line. She started at the 10 and moved back 3 spaces to land at the 7 . Thus, she was able to connect the computational arithmetic representation and the number line representation.

Immediately after answering $10+-3$, she wrote -12 next to the task $-5+-7$. I inquired how she knew the answer so quickly after she had told me that she was not sure before. She said, "I just added 5 and 7 and then put the negative sign in front of it." When I asked her why she did that, she said, "because they are both negative. Yeah, it's that." When I asked her to explain more, she said, "It's just that." Even though 
she did not state that she subtracted 7 from -5 or used the number line and to start at -5 and move 7 spaces to the left to land on -12 , obviously the previous answer and thought process impacted her ability to do this task. If she used either of those two strategies, she would have gotten -12. Instead, she added the absolute values of the addends and placed a negative sign in front of the sum because both of the addends were negative. Notice that she explicitly said that she only did this because both, not one addend, was negative. She was able to distinguish the difference between the second and third task and why one sum was negative and the other was not.

Jen seemed to have both a unary and binary (Kieran, 2007) understanding of addition of negative numbers depending on the type of expression presented. For addition tasks involving a negative and positive addend, Jen knew that she had to operate as if the task illustrated subtraction, a unary view. For addition tasks involving two negative addends, she did not necessarily view it as subtraction; rather she viewed it as addition and then tacking on a negative sign to the sum, depicting a binary view. Furthermore, these views could have been influenced through questioning and reasoning through the tasks together, as well as having her connect the computational arithmetic representation and graphical representation.

Since Jen connected her arithmetic representation to a number line representation, I wondered if she could move from a number line representation to an abstract representation. When I asked her to write a math statement using the information on the number line (see Figure 1), she instead told me that the answer was -1 . She then wrote $-6+5=-1$. I asked her to explain how she thought about this task.

J: I went to 6, then I minused 5, wait no, HOLD ON [pause]...OR you can go to 5 and minus 6. And it would be -1 . [pause] yeah.

$\mathrm{N}$ : How did you know what operation to choose?

$\mathrm{J}$ : I just picked one.

$\mathrm{N}$ : Ok. How did you get the answer of -1? I saw you counting. How did you know where to start?

J: Go to, well, it doesn't really matter. Like you can go to 5 and count back 6 ...or you could go to the -6 and subtract 5 and that would bring you to -1 .

$\mathrm{N}$ : So when you subtracted 5 you went to the right?

$\mathrm{J}$ : Yeah, or you could subtract 6 and go to the left.

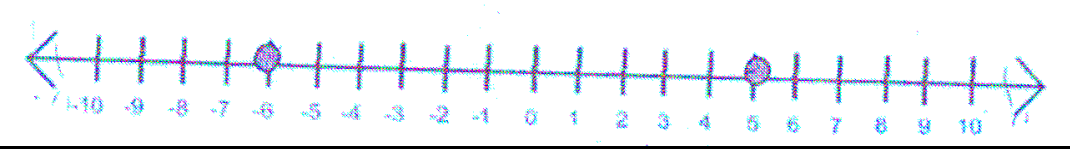

Figure 1. Moving from a number line to arithmetic representation

Even though she wrote $-6+5$, she verbally described her abstract representation as equivalent to $6-5$; thus, she believed that $-6+5=6-5$. She realized something was incorrect because she knew that $6-5=1$ and not -1 . Hence, she changed her decision and said $-6+5$ was equivalent to $5-6$. It is interesting that she wrote her abstract representation using the operation of addition, yet when she verbally described the 
equivalent expression she used the operation of subtraction. This is why I asked her how she knew which operation to use. Much like she stated, it seemed as if she just randomly chose one. It is as if she believed that as long as she kept one negative sign or a subtraction sign in the expression, and a 5 and a 6 , it did not matter the order in which they were placed, for they would produce the same answer.

She described her movement procedure on a number line by trying to connect her belief that order in the expression did not matter, so the order of movements did not matter. Therefore, she said that one could start at the 5 and count to the left 6 spaces to land on the -1 or one could start at the -6 and move to the right 5 spaces to land on the -1 . While these movements will produce the correct answer, she used the incorrect operation to verbally describe the second action. One will notice that she said that when she started at the -6 , she moved to the right because she subtracted 5, yet this is not true, for in order to move to the right, she would have to add 5 to -6 . Thus, when it came to subtraction, it was difficult for her to connect the verbal, graphical, and abstract representations. In fact, it seemed as if she viewed the operation of subtraction as heading towards the direction of zero. When she said that she subtracted 5 from the -6 , she moved towards the right since zero is to the right of -6 on the number line. Similarly, when she subtracted 6 from five, she moved to the left 6 spaces because zero is to the left of 5 on the number line. However, she could have also done this because 5 and -6 were on opposite sides of zero.

Another interesting point about the way Jen proceeded with this task was the fact that she never utilized the distance between the dots. She never once incorporated the 11 spaces between the -6 and 5 . Thus, she never created a relationship between the dots on the number line and the distance between the dots.

Even though Jen was able to move from an abstract representation to a number line representation, and on some levels, a number line representation to an abstract representation, she was unable to create a verbal representation for a task expressed arithmetically. After providing the answer of -12 to the task $-5+-7$, I asked her to write a story about her equation $-5+-7=-12$. She simply sat there and looked at me. When I asked if she had ever done word problems in class, she shook her head no. Then I asked if she had seen negative numbers anywhere in school or outside of school and she shook her head no. I offered up the idea of temperature and perhaps negative temperatures, but she said that she had never heard of them. I did not push this any farther because she seemed to have been having a "shut down" moment and I did not want her to stop talking during the session. Yet later in the session, when I had her move from a word problem to an arithmetic representation, she did not say that she had not seen them before. Therefore, I had asked her if she could make one up. She said she could not and put her pencil down, so I moved on to 
the next task. Given that word problems tend to be a sore spot for students, I did not want to push her to create a word problem that could potentially cause a discord with our interactions.

\section{Prior Conceptions on Subtraction}

Jen's conceptions of subtraction would most likely be categorized as take-away rather than distance, for she often took away the amount in the subtrahend from the minuend instead of viewing the difference as the distance between the minuend and subtrahend.

Jen used three different strategies to solve subtraction tasks, which were based on the form of the task. These strategies allowed her to calculate the correct answers for tasks in the form $a-b$ and $-a-b$, where $a, b>0$ and $-a--b$, where $a>b>0$. Unfortunately, she was unable to correctly calculate tasks in the form $-a--b$, where $b>a>0$, and in the form $a--b$ where $a, b>0$.

When she solved tasks in the form $a-b$ and $-a-b$, where $a, b>0$, she used the number line to justify her answer. She started at the minuend and moved to the left the absolute value of the subtrahend. For instance, when she solved 5-2 I noticed her looking on the number line and asked her what she was doing to get the answer of 3, and she said, "I went to the 5 and then I went back 2." Not only did she use this strategy for $-a-b$, but she also justified her answer these types of tasks using an arithmetic rule, which most likely she had picked up from adding negative numbers. She would sum the absolute value of the minuend and subtrahend, and then tack on a negative sign since "both numbers were negative." What is interesting is that she tried to extend the number line strategy as well as a variant of the arithmetic strategy to tasks in the forms $a--b$ and $-a--b$, although unsuccessfully.

For tasks in the form $a--b$, Jen first used her strategy of moving left because subtraction was used as the operation. However, when I asked if she was subtracting a positive amount or negative amount, she acknowledged the difference and the possible implications it could have on an answer, yet this did not sway her decision of how to solve the task.

$\mathrm{N}$ : Tell me how you solved $5--12$.

$\mathrm{J}$ : I went to the positive 5 and then minused 12 and I got -7 .

$\mathrm{N}$ : Is that the same as 5 minus -12 ?

J: No, yeah, I don't know. No.

$\mathrm{N}$ : So what's different about them?

$\mathrm{J}$ : One is negative and one is positive.

N: So would that make a difference do you think?

$\mathrm{J}$ : It just makes the number a negative or a positive. Like I guess, like the number answer is right, but it might have to be positive.

$\mathrm{N}$ : So which number might be positive or negative?

$\mathrm{J}$ : The 7 . But it's a -7 here because the twelve has a negative sign. 
Before I asked Jen what the difference between the expressions was, I had written the expression 5 - 12 directly underneath the expression $5--12$. She noticed that the difference in the expressions was in that the former expression she was supposed to subtract a positive twelve, whereas in the latter expression, she was to subtract a negative twelve. At this point, she laughed a little bit as if she thought she was telling me something so obvious that I should not have asked her this question. She acknowledged that the twelve had a different sign, but all that could potentially mean was that the answer might have a different sign. Yet, in this case because the 12 had a negative sign, it meant that she was sure the seven was negative.

Jen could correctly solve tasks in which both the subtrahend and minuend were negative, and the minuend was smaller, yet she could not do so when the minuend was larger than the subtrahend. This could have been due to the fact that the absolute value of the minuend was larger than the absolute value of the subtrahend, which triggered her memory of solving tasks with positive minuends and subtrahends, and thus influencing her decisions. In fact, it will be seen that she had a difficult time in determining the correct answer for tasks with a negative minuend larger than the negative subtrahend.

When Jen solved the task $-4--6$, she could not decide if the answer was $-2,2$, or -10 . In fact, she admitted up front that she had been having difficulties with this task.

J: I just don't know about that one.

$\mathrm{N}$ : So what are you not sure about in this task?

J: If you go to -4 and then minus 6 or if you go to -6 and minus 4 .

$\mathrm{N}$ : Ok, so let's try them both and see what happens.

J: So go -4 and subtract 6 .

At this point she started at the -4 on the number line and moved to the left 6 spaces and landed on the -10 . I asked her if she subtracted 6 or -6 , to which she responded, "I subtracted -6." Therefore, she believed that subtracting 6 and -6 were the same movement on the number line, which confirmed her answer to the task $5--12$ of being -7 just as $5-12$ was -7 . Thus, she believed that $-4--6=-4-6$. She then decided to subtract -4 from -6 .

J: Go to -6 and then minus -4

$\mathrm{N}$ : And what would happen then?

J: It's still minus 10. No, [pause] it would be minus 2, I don't know! [pause] It would be 2!

When Jen exclaimed that she did not know, she threw up her hand, dropped her pencil, and slumped back into her chair. She exuded this attitude of 'Why is she asking me these things?' combined with the frustration of not knowing the answer quickly enough, and yet an eagerness to try to reason it out for herself. She eventually decided that the answer was 2 and stated this boldly. To calm her down, I tried to work through each answer individually. Since I saw that she got the answer of -10 by starting at the -6 , moving to the left 4 spaces, and landing on the -10 , I asked her how she got the answer of -2 . She said, "I 
counted 4 to 6 , and that was 2 and I put the negative sign because they were both negative." As she explained this to me, she put her pencil tip on the 4 and then on the 6 , and noticed that there was a difference of 2 spaces between the 4 and 6 . Because both the minuend and subtrahend were negative, she believed that the answer needed to have a negative sign. What was interesting about this approach was that this was the first time she had considered distance in determining a difference. I then asked about her answer of 2 .

$\mathrm{J}$ : I went 6 then subtracted 4 and you get 2 .

$\mathrm{N}$ : So how did you know which way to go on the number line?

J: I don't know, I was just going. I think it would be 2 cause like if you make them positive, you know, and you change that to adding, it gives 10. If you subtract it gives 2.

She changed all the negative signs in the task, $-4--6$, to positive signs; thus, she created the new expression $4+6$, which she knew to be equal to 10 . She then decided that since this task, $-4--6$, required her to subtract, and not add, that her answer had to be of magnitude 2. Yet, it was still unexplained as to why it had to be 2 and not -2 .

$\mathrm{N}$ : Ok, so why did you think at one time the answer was -10 ?

$\mathrm{J}$ : Because then you put the negatives in front of them, so you get all negatives, so -10 .

$\mathrm{N}$ : So what about this one, $-4+-6$ ?

$\mathrm{J}$ : It's also -10 .

$\mathrm{N}$ : Ok, so this is -10 as well.

J: I don't know. Well, yeah, no, well, that will equal, naw, that won't equal -10 . Well, yeah, it would equal -10 . Yeah it will be negative because adding negatives. Negative ten!

$\mathrm{N}$ : Ok, how does this [pointing to $-4+-6$ ] differ from $-4--6$ ?

J: [pause] So you're going to go the other way. So like you go this way for 4 , and like you go this way for 6 . So $1,2,3,4,5,6 \ldots$ so 2 .

Note that for awhile she had thought that $-4-6=-4--6$, much like she did with the expressions $5--12$ and 5-12. Yet in this instance, when I asked her to note the difference between the expressions, she decided to change her answer and move the opposite direction on the number line, unlike what she did with the expression $5--12$. It is important to know her actions during her last remarks. When she referred to going different directions for the 4 and 6 , she meant that for the task $-4--6$ she would start at -4 and move to the right 6 spaces, whereas for the task $-6--4$, she would start at the -6 and move to the left 4 spaces to land on the -10 . This idea helped her work through the issues of whether $-4-6$ was equal to -4 --6 , whether $-4--6$ was equal to $-6--4$, and whether or not the answer to $-4--6$ was -10 or 2 . She found that $2=-4--6 \neq-6--4=-10$ and that $-4--6 \neq-4+-6$. It seems as if she used her answer to the arithmetic expression and her belief that some expressions were not equal to determine her movements on the number line.

While she had difficulty with expressions in the form $-a--b$ when $b>a>0$, she seemed to 
have no difficulty with solving expressions of the form $-a--b$ when $a>b>0$.

$\mathrm{N}$ : So what about- $4--1$ ?

J: You're going to -4 and then subtracting 1 , well negative 1 .

$\mathrm{N}$ : So how did you think about that one?

J: It's -3 because you go to your -4 and go back 1 .

$\mathrm{N}$ : Ok, how did you know to go to the right?

J: I don't know. I just did. I got the difference between the 1 and 4, and since it's negative you have -3 .

Jen mentally rewrote the task such that the minuend and subtrahend were both positive and used this equation to help her solve the task with the negative minuend and subtrahend. Thus, she used the equation $4-1=3$ to help her solve $-4--1$. She believed that since both numbers in the former expression were positive and elicited a positive answer, that the latter expression would have the same magnitude as the answer, but would be negative because the minuend and subtrahend were both negative. She did not use this strategy on the task $-4--6$ because it differs in that when the negative signs are removed, the expression becomes $4-6$, which elicits a negative number and not a positive number. So when she tacks on the negative signs to the 4 and 6 , she was unsure as to what to do with the negative sign in the answer. Therefore, she resorted to trying to use the number line to justify her answer.

Even though she had been using a number line to verbally discuss her reasoning for her answers to subtraction tasks, I specifically asked her to illustrate on a number line how she would find the answer for $-4--1$. She asked, "Do I just put the dots on it?" To which I responded, "Sure, however you want to think about it." At this point, she just put a dot on the -4 , a dot on the -1 and then a dot on the -3 , such that they were isolated points on the number line with no connection to one another. When I asked if she could describe a relationship between the dots she said, "No, well, hold on. Four, no, cause if you minus -1 it would be -2 , and if you minus -4 it would be -8 , so you can't, except put a dot there and say it is the answer."

N: Could you talk more about how you're getting those numbers?

$\mathrm{J}$ : Starting at -1 and minus a -1 , you get -2 .

$\mathrm{N}$ : So if you start from -1 and then move to the left 1 , you get -2 . What if you started at -4 ?

$\mathrm{J}$ : If you move this way, wait, so if you subtract -4 you move this way, you get -8 .

$\mathrm{N}$ : So if you start at -4 , you have to subtract 4 ? Could you start at -4 and subtract -1 ?

J: OH, YEAH! Here, go there, and that's the answer.

For some unexplained reason, she thought that if she started at the -4 , she would have to subtract a positive 4 , and if she started at the -1 , she would have to subtract 1 . This was how she was getting the answers of -8 and -2 respectively. Yet, it seemed as if she was still a little confused as to how the number line fit by her ongoing explanation. 
$\mathrm{N}$ : Oh, ok. So you moved to the right by 1 this time from -4 .

$\mathrm{J}$ : Yes, cause, yes, cause if you make these positives, well, says that these are all negatives, and you, then instead of doing it, you'd make this positive, and you'd add it, so you add 4 and go to the right. Wait no, yeah. Make that positive, I don't know what I'm saying!

She was trying to use another expression with which she was more familiar to help her answer this task, yet, she could not make her answer, found arithmetically, jive with her graphical representation. She had tried to say that she moved to the right because it was similar to adding in that when she removed all the negative signs in the task $-4--1$ it transformed into $4+1$, which she would add together, and hence move to the right. Yet, when she actually added these numbers, she obtained 5, which did not have the same magnitude as the magnitude of her answer -3 . Thus, she was unable to justify her movement to the right on the number line to show that $-4--1$ was equal to- 3 , yet she did not change her arithmetic answer. I tried to ask her more questions to see if she could determine why she should move to the right.

$\mathrm{N}$ : How would you solve $-4-1$ ?

$\mathrm{J}$ : You'd go that way [to the left]. If you're minusing it [subtracting a -1], you'd go this way [to the right]. This one you get a -5 , so you go that way [left] but for this one you will get a position [answer]

$\mathrm{N}$ : What did you say the answer to $-4--1$ was?

J: -3 .

$\mathrm{N}$ : So this had negative numbers, but you moved to the right and got a negative answer.

J: Yeah, but don't you go positive since it's [the subtrahend] a positive number, or would you have to go negative, like this way [left]?

$\mathrm{N}$ : Hmm. So what was telling you that you should go to the right for this task?

$\mathrm{J}$ : My answer.

This task proved to be difficult, because she had not really used a number line to help her solve this expression, rather she solely used her arithmetic strategy and determined her movement on the number line based on her answer to the task. Yet, by asking her to explain her understanding of how she subtracted positive and negative numbers on a number line, not only helped me to understand that she usually determined the direction to move based on the sign of the subtrahend, but also created a cognitive conflict for her, which could have allowed her to become more open to seeing patterns with the technology during our instructional session.

I then returned to contextual tasks. Since she was unwilling to generate a contextual task given an arithmetic expression, I wondered if she could go from a contextual task to an arithmetic expression. Even though before she said that she had never heard of negative temperature, she answered the temperature tasks accurately.

$\mathrm{N}$ : So let's try some temperature tasks. If the lowest temperature in Alaska was 0 degrees and the highest temperature was 32 degrees, how many degrees did it drop to get to the low temperature?

$\mathrm{J}$ : 32, minus, because you do it number line sort of, you go to 32, and then if you dropping it, you have to go to positive, so you have to go to zero... so it's just like minus 32 . 
When I asked her how she would write the expression, she wrote $32-0=32$, even though she said that the answer was -32 . I was hoping she would use this same strategy to answer the next temperature task.

$\mathrm{N}$ : Alaska's high temperature for December $16^{\text {th }}$ was $-6^{\circ} \mathrm{F}$. The day's low temperature was $-17^{\circ} \mathrm{F}$. How many degrees did it drop from the high to the low temperature?

$\mathrm{J}:-10$, no, yeah -10 .

$\mathrm{N}$ : So, I saw you doing something on the number line, what were you doing there?

$\mathrm{J}$ : I put a dot at the -17 because the low temperature was -17 .

$\mathrm{N}$ : And the -6 for the same reason?

$\mathrm{J}$ : Yeah,

$\mathrm{N}$ : And then I saw you doing something, what was that?

$\mathrm{J}$ : I counted the distance in between.

$\mathrm{N}$ : Oh, and how did you know to count the distance?

J: I don't know, I just knew how to do it.

$\mathrm{N}$ : Would you be able to write an equation to show what you did?

$\mathrm{J}$ : Yeah, I guess. [She writes $-17--6=-10$ ]

$\mathrm{N}$ : How did you choose those?

$\mathrm{J}$ : Because the -17 , and -6

$\mathrm{N}$ : And why did you write subtraction?

$\mathrm{J}$ : Because it dropped.

This task proved interesting for many reasons. One reason is that this is the first time she referred to subtraction as a distance. A second reason is that she made the connection among her movements on a number line, her equation, and the context (see Figure 2). Moreover, she could relate the points on her number line by the distance. A third reason is that even though she said she that she counted the numbers in between, she only came up with 10 instead of a 11. A fourth reason is that in this instance, unlike the last, she believed the answer was -10 degrees, and showed an equation that expressed the answer as a negative instead of positive. Another interesting fact is that if she were to solve the expression arithmetically, she would have gotten -11 by using her method of changing the -17 to positive 17 , the -6 to positive 6 , subtracting, and then adding on a negative sign to the difference.

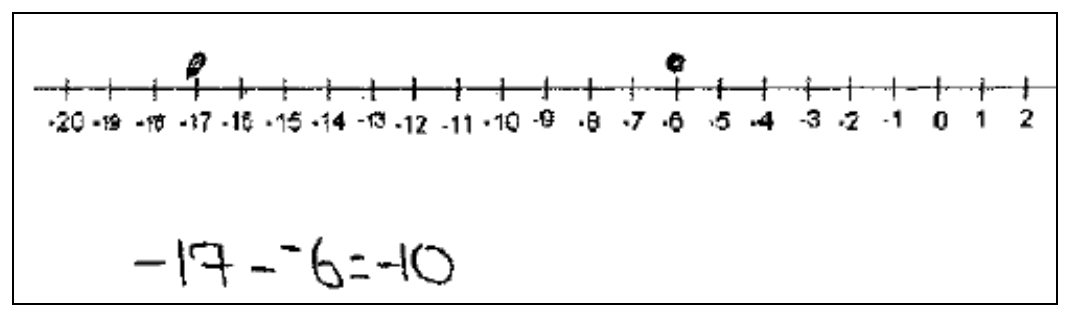

Figure 2. Solving a temperature task on a number line and writing the corresponding equation

\section{Discussion on Prior Conceptions}

In the beginning of the task based interview, Jen did not seem to be able to solve tasks with negative addends, yet as we talked about her confusion, I realized that Jen could correctly compute addition 
tasks involving negative numbers arithmetically. She understood that adding a negative to a positive addend was similar to subtraction, but did not view that adding two negative addends was also subtraction; instead, she viewed it as addition of absolute values with the tacking on of a negative sign to their sum. Furthermore, Jen could connect her arithmetical computation with movement on a number line when one addend was negative, but did not use the number line to show me how she obtained her answer when she added two negative addends.

Jen had a difficult time connecting her representations when she moved from a number line context to an arithmetical context. Much like Bruno and Martinon (1996) described, Jen used the dots as part of her equation, but did not incorporate a relationship between the two numbers such that it could be conceived on a number line. She used the numbers to create an equation in her head, which she solved, but her answer did not relate to the distance between the numbers represented on the number line. Furthermore, when she tried to use the number line to explain her equation, the back and forth movement influenced her belief of arithmetical properties of her equation. For example, she saw that she could start at -6 and move to the right 5 spaces, or start at 5 and move to the left 6 spaces. This seemed to indicate to her that in whatever expression she came up with, she would be able to commute the numbers. Fortunately, she came up with the expressions $-6+5$ and $5-6$, yet if she had chosen subtraction I think she still would have believed that commutivity held. In fact, when she verbalized her expression, she did choose subtraction, and said as much. Furthermore, once we looked at subtraction tasks, she believed that commutivity held. This belief that commutivity held for subtraction was also seen in Murray's study (1985).

In agreement with Murray's (1985) findings, Jen was able to solve addition tasks more accurately than she was able to solve subtraction tasks. Jen was able to solve arithmetically subtraction tasks that contained a positive subtrahend and tasks that contained both a negative minuend and subtrahend such that the minuend was smaller. This was not found in previous studies, but it does relate to the idea of using an analogy (Murray 1985) to solve an expression. Murray found that students would often switch the signs on the minuend and subtrahend, find the answer, and then switch the sign. Yet, Jen would only switch the sign of the answer if it were not already negative.

Jen's methods for solutions differed depending on the task. For tasks involving a positive subtrahend, Jen used the number line to justify her answer by starting at the position of the minuend and moving to the left the absolute value of the subtrahend. This was seen in the findings of Bell (1982). For tasks that involved a negative minuend and positive subtrahend, Jen also justified her answer by saying that 
she changed the numbers to positive, added them, and then tacked on a negative sign to their sum. This procedure was not described in previous literature.

For tasks with a positive minuend and negative subtrahend, Jen noticed the difference between $a--b$ and $a-b$, in that the first was subtracting negative $b$ and the second was subtracting $b$, but solved them in the same manner and believed they had the same answer. This is consistent with the findings of Murray (1985). Murray believed that since students were unfamiliar with the notation in these types of tasks, they transformed them into tasks with which they were familiar and believed they were equal.

For tasks with both a negative minuend and subtrahend, Jen transformed the task by changing the signs of the minuend and subtrahend to positive, subtracting, and then making sure that the answer had a negative sign. This procedure worked for tasks in which the negative minuend was smaller than the negative subtrahend, but not vice versa. She used the strategy of using a similar task to help her solve the present task, yet did not conceptually understand how the two tasks related. A similar strategy was seen in Murray's findings (1985) and was classified as using an analogy, yet, the student knew that since he switched the signs on the minuend and subtrahend, he had to switch the sign on the answer. Yet, Jen believed that since the minuend and subtrahend were negative, the answer had to be negative. Therefore, when she subtracted a smaller negative subtrahend from a larger negative minuend, the answer to her transformed task was already negative; thus she did not adjust the sign.

During the interview, Jen also showed me that she believed that subtraction of a $b$ was the same movement as subtraction of $-b$. In order to move on the number line, she simply moved left in both cases. Thus, she believed that $-a--b=-a-b$. As she worked through the tasks, she also showed that she believed that $-a--b=a-b \neq-b--a=-b+-a$ when $b>a>0$. Another instance during the interview, it was seen that even though she typically believed that subtracting a negative subtrahend or subtracting a positive subtrahend meant to move left on the number line, she would abandon that thought if her answer to the task indicated the other direction on the number line. For instance, since she could obtain the correct answer to tasks such that the negative minuend was smaller than the negative subtrahend, she would move to the right the number of spaces indicated by taking the absolute value of the subtrahend. Yet, she only did this so her number line movement would agree with her computational answer.

Jen had no issues crossing over zero; therefore, she had a continuous view of the number line (Peled et al, 1989). Jen achieved Level 4 in the Number Line Dimension (NLD) with respect to addition. It is unclear as to whether she quantitatively thought about addition of negative numbers because she did not complete the contextual portions of the interview. Yet, she could add negative numbers in all types of tasks, 
but did not contextualize the task or her answer; thus, she attained Level 2* in the Quantitative Dimension (QD). An asterisk in the QD indicates that Jen was able to solve higher-level tasks than her number level indicates, but she did not put the answer or task into context. With respect to subtraction, she only used the number line to solve tasks in the form $a-b$ and $-a-b$, where $a, b>0$. This would imply that she attained Level 2 in the NLD with respect to subtraction and parts of Level 3 and 4. I rated her as earning a score of L3* because she could solve higher-level tasks than her number level indicates but could not do all same or all opposite signed numbers. With respect to the QD in subtraction, Jen attained Level 1+ because she was able to go beyond that level but not fully attain Level 2. She did not put her tasks or answers into context and did not speak of taking away objects.

\section{Summary of Jen's Prior Conceptions}

- Jen had some conceptual and procedural understanding of addition of negative numbers. She saw the relationship between the addition of a negative and subtraction. She was also able to move between a number line representation and a computational arithmetic representation. She was unwilling to generate a contextual representation given an arithmetic representation.

- Based on her movement on the number line, Jen believed that subtraction was commutative.

- Jen was able to correctly solve tasks in the form $a-b$ and $-a-b$, where $a, b>0$ and $-a--b$, where $a>b>0$. For the first two types, she used a number line to justify her answer by starting at the minuend and moving left $b$ spaces. For the second type, she also used a computational procedure by adding the absolute values of the numbers, and tacking on a negative sign to the sum. For the third task, Jen changed the signs of the minuend and subtrahend to positive and subtracted to calculate the answer. To this answer, she added a negative sign and believed that this was the answer to the original task.

- Jen tried to extend her strategies for solving tasks in the form $-a--b$, where $b>a>0$, and in the form $a--b$ where $a, b>0$. Yet, she was unsuccessful. For tasks in the form $-a--b$, where $b>a>0$, she performed the exact same strategy as she did to solve $-a--b$, where $a>b>0$, only she kept the answer negative because the subtrahend and minuend were negative. For tasks with a positive minuend and negative subtrahend, Jen believed that $a--b=a-b$.

- Jen believed that subtraction or a negative sign meant to move to the left. Only when found an answer computationally that could not be gotten according to this movement did she move contrary to this belief.

- When $b>a>0$, Jen believed that $-(b-a)=-a--b \neq-b--a=-b+-a$.

- Jen attained Level 4 in Peled's NLD and Level 1+ in the QD for addition.

- Jen attained Level 3* in Peled's NLD and Level 1+ in the QD with respect to subtraction.

- Jen exhibited a combination of unary and binary understanding of negative numbers. The way she viewed negative numbers depended on the form of the task. 


\section{Analysis of Interaction with Subtraction Applets}

Because Jen could accurately solve addition tasks with and without a number line, she did not use the Addition Arrow Applet (AAA). Because she had used the take-away method when she used the number line on subtraction tasks, I used the Subtraction Arrow Applet (SAA) with her first. Even in doing so, she ended up using the SAA in the manner of the Subtraction Dot Applet (SDA) because the SAA had helped her visualize subtraction as the distance between two numbers. Moreover, when I asked her which method she preferred to use, viewing the distance between the numbers as the difference, or using the arrow to represent the amount taken away by the subtrahend, she said that she preferred the former.

\section{Jen's Interaction and Understanding with Technology}

When Jen used the SAA, I did not have the hot-linked equation displayed because I wanted to see how the sole illustration of the arrow value appearing inside the arrow impacted her method of solving subtraction tasks. After manipulating the SAA without attempting to solve any tasks, Jen was able tell me the significance of the blue stick, where the arrow began, where the arrow ended, and the displayed arrow value in terms of subtraction. As she was exploring the SAA, she had moved the blue stick to the 8 , moved the end of the arrow to the 5, pressed the show arrow value button, and said, " 3 is the distance between them." Furthermore, she noticed that each time that she moved the end of the arrow to the right by one, the value inside the arrow decremented by 1 , and even went negative if she went to the right of the blue stick (see Figure 3). To see if she could apply her discoveries regarding the arrow value and the relationship between the value and the subtrahend of an expression, I asked her to model some abstract subtraction tasks.
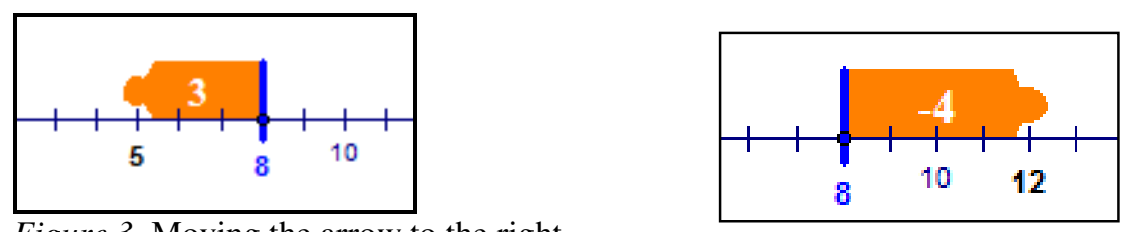

Figure 3. Moving the arrow to the right

When I asked her to model 5-2 on the SAA, she moved the blue stick to the 5 and moved the orange arrow to the left 3 spaces. She said, "I put the endpoint on 2, like that." I asked if she could tell me the answer to the expression based on the visual depicted on the screen. She said, "Yes, cause if you count like the spaces in between you get 3, in the orange part" (see Figure 4). I had not turned on the visual display of the equation. If I had done so, it would have displayed 5-3, since I had intended this applet to illustrate subtraction as take away and not distance. Because she seemed to be using the SAA to illustrate distance rather than take-away, I asked her to model 5-3 to see if she consistently use the applet in that 
manner. She said, "It would be 2" and moved the arrow to the right by one.

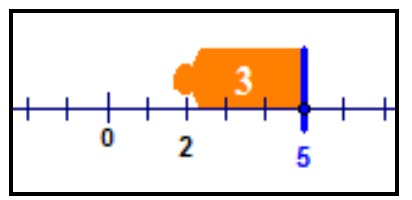

Figure 4. Using the SAA to model distance for the task 5-2

To make sure I understood that she was, in fact, using the SAA to illustrate subtraction as a distance, I asked her how she knew where to end the arrow. She said, "You put the end of the arrow on the second number." I then asked her where she noticed the answer, and she pointed to inside the arrow and said, "it is "counting it [the spaces in between] for you." She continued to use this method to model 5-4 and 5-1, yet when it came for her to model 5-0, she answered the task before actually modeling it. She said, "It's zero. Wait, yeah, wait, no 5, hold on." At this point, she moved the arrow so that it landed on the 5 and then moved it again so that it landed on the 0 and had a 5 inside the arrow value, and sang the answer in a high pitch, "FIVE" (see Figure 5).

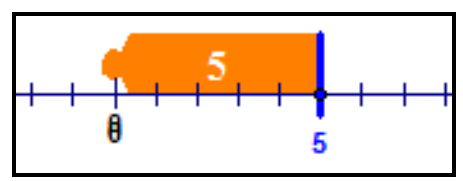

Figure 5. Final destination of the arrow for 5-0

At this point, I asked her to use the SAA and model her thinking and what confused her. She showed me that at first she thought to use the arrow so that it landed on 5, yet changed her mind and moved the arrow so that it landed on the 0 and the 5 showed inside of the arrow. I asked her if she noticed anything similar she used this alternate method to illustrate 5-3 and 5-2, and she said, "One way the answer is in the arrow and the other the answer is at the bottom." When I asked her which way she liked better, she had told me that she liked, "the answer in the arrow." While she preferred the distance method while using the applet, she had realized that there was a relationship between both methods in regards to the placements of the subtrahend and answer.

To make sure she truly understood the method that she had chosen, I asked her to model the task 5-5. She answered this without moving the applet and said, "It would be zero." When I asked her to show it to me with the applet she moved the arrow so it was placed directly on top of the blue stick (see Figure 6).

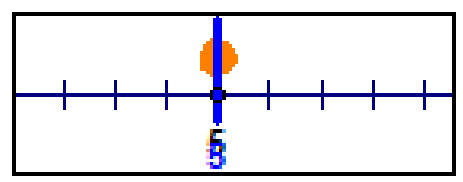

Figure 6. Jen illustrating 5-5 using distance method on the SAA

Spontaneously, she moved the arrow to the right by one. I asked her what equation this represented, to 
which she responded, "Five minus 6, which would be -1 ." She had taken a number line representation and had constructed a correct equation that related all the represented values. She had not been able to do this in the interview. Given that she had modeled 5-2, 5-3, 5-4, ..5-6, I asked her to predict the answer to 5-7 without moving the applet. She said, "It would be negative 2." When I asked her to explain, she moved the arrow to the right by one and said, "Cause that's what it says. Like if you look at this one [pointed to equation 5-6 on her paper] it's -1 , and your just putting another 1 on top of it, so it would be -2." She noticed that she had increased the value of the subtrahend by 1 so that it went from 6 to 7 ; therefore, she knew the size of her arrow would increase by 1 unit as well, which would make it show -2 inside (see Figure 7).
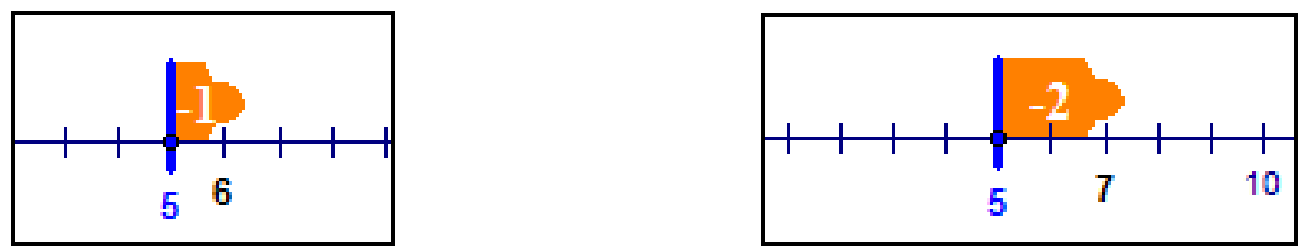

Figure 7. Modeling 5-6 and 5-7

Prior to working with the SAA, Jen had been able to correctly solve computational arithmetic tasks with a positive and negative minuend. However, she had never used the distance idea to model them on a number line. Furthermore, she had always said that she needed to move to the left, whereas, in using this model, she had moved to the right. Thus, she had expanded her view of subtraction to incorporate both distance and the potential for moving to the right on the number line.

Given that she had easily solved these types of tasks, I wanted to see if she was able to notice any patterns as she had modeled them on the number line.

$\mathrm{N}$ : What do you notice when the arrow value is negative?

$\mathrm{J}$ : The answer is negative.

$\mathrm{N}$ : Yeah. And when the answer is negative?

$\mathrm{J}$ : Hmm. [She drags the arrow back and forth on the number line] When you drag it in the positive direction past the blue stick.

$\mathrm{N}$ : Um, hmm. And what about when you drag it in the negative direction past the blue stick?

$\mathrm{J}$ : It would be a positive answer. I think it's all the way. [She drags the arrow farther and farther to the left of the blue stick] Yeah, all the way down.

The SAA allowed Jen to explore how the movement on the number line impacted the answer to the task, as well as how the signs and magnitude of the minuend and subtrahend determined the sign of the answer. She noticed that when she subtracted a number that was to the right of the original number on the number line, she knew the answer was going to be negative. When she subtracted a number lying to the left of the minuend on the number line, she knew the answer was going to be positive. At this point in time, I did not think she quite understood why that was true, but she had seen the pattern through exploration. 
I then asked her to use the model to solve tasks with a positive minuend and negative subtrahend, and with the SAA, she was able to solve them correctly. For her first task, I asked her to solve $5--1$, before moving the SAA, she immediately said, "Well, I know it's [the answer] positive." When I asked if she could guess what the number might be, she paused and did not say anything. Then I said, "You can go ahead and model it." She placed the blue stick on the five, and dragged the end of the arrow so that it landed on the 1, and said, "Wait no." and moved it so that it landed on the -1 , and said, "6." She had paid attention to the sign of the subtrahend and knew that the task was not 5-1 but rather 5--1, and this required her to move the end of the arrow to -1 and not 1 . I had her complete the task $5--4$ by using the SAA and she correctly modeled and answered the task. Then I asked her to predict what the answer would be to $5--6$. She said, "Hmm, 11 I'm thinking." When I asked her why she thought it might be 11, she said, "Cause I added the 2 to the 4 and I got 6 , so I added 2 to the 9 and got 11." She really seemed to like to use previous tasks to help her answer the present task, much like she did in her interview. I then asked if she had thought about it in any other way. She said, "Well, you could think it's like putting the point down on -6 and having 11 in the middle." As she said this, she moved the end of the arrow to land on -6 and pointed to the 11 displaying inside the arrow. Thus, due to the SAA, she had an alternative strategy to solve these subtraction tasks.

I asked her to solve the task $7--1$ without the applet. At first she told me that the answer was 5. When I asked her to explain to me how she obtained that answer.

$\mathrm{J}$ : I went to the 7, like I put the blue line on the 7, and then the orange arrow, you do...no wait, I messed up.

$\mathrm{N}$ : You can draw on this number line if it helps.

$\mathrm{J}$ : It does. So this dot is the blue line, and then an orange dot on the -1 , then count the spaces in between. So 8 .

$\mathrm{N}$ : So how do you know it's positive 8 and not -8 ?

$\mathrm{J}$ : Because I moved to the left, so it would be positive.

I am unsure as to how Jen obtained the initial answer of 5. Yet, when I had her explain her reasoning, she used the method of the SAA to help her visualize the procedure (see Figure 8). In fact, she noted that drawing the same type of picture on the number line aided her in determining the difference.

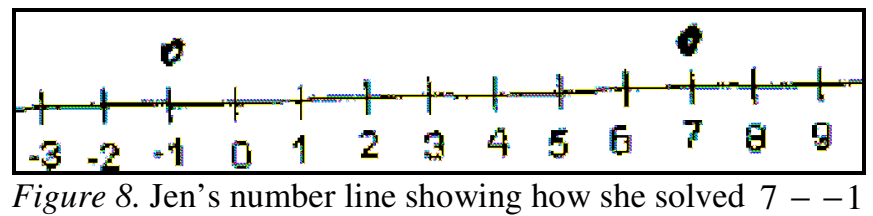

While the applet helped Jen solve these types of tasks, I wanted her to know why moving to the left from the minuend caused her to obtain a positive difference and moving to the right of the minuend 
caused her answer to be a negative difference. Thus, I asked her different types of subtraction tasks in hopes she could articulate the meaning behind the sign in the difference. She came to the conclusion that moving a positive direction from minuend would elicit a negative difference because she was doing opposite the sign indicated on the number line.

$\mathrm{N}$ : Why does it make sense that when you move to the right of the first number, you get a negative answer?

$\mathrm{J}$ : Because you're like changing like the positives and the negatives like on a top line. Like say there was another number line on top, and this side would be positive [pointed to the left of zero] and this side would be negative [pointed to the right of zero]

$\mathrm{N}$ : Ok. So why do you think the number line is reversed?

J: I don't know.

Jen was able to visualize a second number line lying above the original number line to help her with the subtraction tasks, most likely because when the arrow would travel to the right on the original number line, it would show a negative value inside of it, and when it would travel to the left of the minuend on the original number line, it would show a positive value inside of it, located above the original number line (see Figure 11).
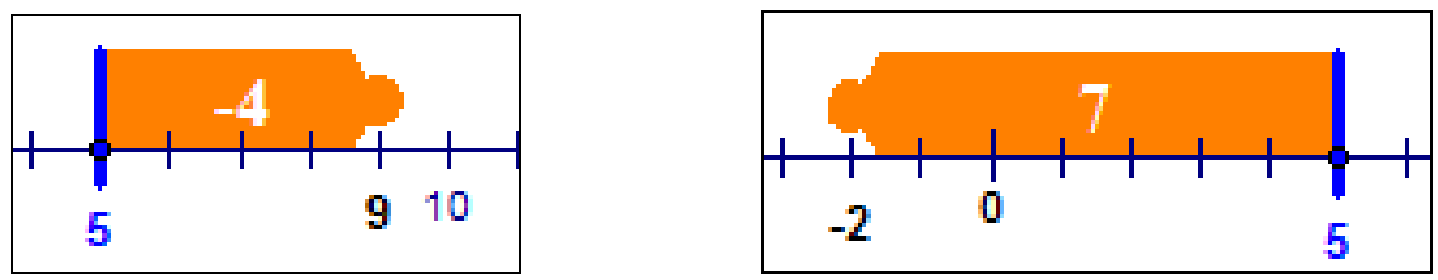

Figure 9. Visualizing a second number line above the original blue number line

Yet, Jen did not fully understand why there had to exist this second number line. Due to this uncertainty, I had her try to generalize the pattern of answers to tasks with a positive minuend and negative subtrahend.

$\mathrm{N}$ : So what was your answer to $5--1$ ?

J: 6 .

$\mathrm{N}$ : Is there another way we can think about using a 5 and 1 and be able to get 6 ?

$\mathrm{J}$ : Umm [pause] changing the minus to adding and changing the negative to a positive... 5 plus 1.

$\mathrm{N}$ : Right. What about this one, $5--4$, what did you get?

J: 9 . It would be like 5 plus 4 .

$\mathrm{N}$ : What about the next ones?

J: 5 plus 6 , and then 4 plus 2 .

$\mathrm{N}$ : So what do you think subtracting a negative is really doing?

$\mathrm{J}$ : Making it positive, well, adding. I don't know.

She was making very quick progress and then the last question made her slow down to think, so she started to shut down until I said, "You're doing good. Tell me again what you're thinking?" This time, she stopped, looked at the paper, and said slowly, "It's making the answer positive." And then Jen exclaimed, “OH! You're adding them! So that's why you're going right! I get that!" I then asked why 6 - 4 was a positive 2, and she said, "because you're moving to the left. If the higher number is second it will be like, 
but if the higher number is first it will be positive." As she said this, she moved the SAA so that the blue stick was on 6 and she moved the end of the orange arrow to the 8 , showing that when she subtracted a higher value from the 6 , it would be negative, and then when she moved the end of the arrow to the 4 , the answer was positive. By looking across her tasks and answers, she was able to connect the visual representation with the arithmetic representation of subtraction tasks. Furthermore, by experimenting with many tasks, she was able to come to this conclusion. Had she not had the technology, she would not have been able to do all the tasks as quickly.

In hopes that she would see the same connection for tasks in the form $-a--b$ where $a, b>0$, I asked her to solve $-5--1$. When I gave her this task, she sighed heavily and tried to think of the answer in her head. At this point, I told her that she could use the SAA to help if she wanted. She gave a sigh of relief, grabbed the mouse, moved the blue stick to the -5 , placed the end of the arrow on the -1 , and said, "Negative four." When I asked her why it was -4 she told me that there were 4 spaces in between -5 and -1 . When I asked why it was negative, she said, "Because you're subtracting two negatives. I don't know." It was strange that she just finished telling me that she knew the answer was going to be negative when she moved to the right, yet in this instance, she referred back to her previous method of when she subtracted two negative numbers, the difference had to be negative. When I asked her which way she traveled on the number line she said, "Left, oh, I mean right. That's why it's negative." Then I asked her if she thought that since she went to the right it was like adding the 1 to the -5 . She said, "Yeah, because if you make them positives, like if you change all the signs and change that to adding, then... wait no. No, no it's not like adding." She knew that moving to the right was like addition, yet she could not connect this idea with her arithmetic representation. She believed that the quick way of subtracting a negative subtrahend was to change all the signs in the task and then add the numbers, rather than changing the subtraction sign and negative sign of the subtrahend. However, she did realize that $-5--1 \neq 5+1$.

Jen was not the only student who believed that she could change the negative signs only for the tasks that had a positive minuend and negative subtrahend. Due to this view, I asked her to tell me what specific signs she changed in $5--6$.

$\mathrm{J}$ : The negatives.

$\mathrm{N}$ : Well, did you change the sign of the 5, or did it stay positive?

$\mathrm{J}$ : Oh, it stayed.

$\mathrm{N}$ : So what signs do you think you might change in this task?

$\mathrm{J}$ : The second sign, and like the subtraction sign.

$\mathrm{N}$ : Ok, so change those. What do you get?

J: -5 plus 1. Yeah! That would be um, negative, oh wait, that would be -4 !

Jen picked up on patterns pretty quickly, she simply needed someone to ask her more directed questions for 
her to take notice of what was consistent with her previous computations and movements. Although, right after this task, she solved the task $-5--6$ on paper, without the aid of the SAA, and she obtained the answer of -11 .

$\mathrm{N}$ : How did you get your answer?

$\mathrm{J}$ : Well, I just added the 5 and 6 and got 11 . Yeah, you add 5 and 6 cause you change all the signs. $\mathrm{N}$ : Did you change all the signs up here?

J: Well, no.

$\mathrm{N}$ : What signs did you change?

J: I changed these. So I have to change that to adding and that to positive [pointing to the subtraction sign and negative sign on the -6 , respectively]

$\mathrm{N}:$ Oh.

$\mathrm{J}$ : So it would be positive 1 .

Jen did not seem to remember that she only needed to change the subtraction sign and sign on the subtrahend until I called her attention to it. It seemed that the word "adding" brought to mind the idea of adding only positive addends. Yet, when questioned, she always adjusted her answer. To check her answer, I had her model the task with the SAA, which she did so appropriately.

I then allowed her to choose the method she wanted to solve the task $-4--1$. She immediately said that the answer as -3 and told me that since "one was 4 and the other was 1 " she "subtracted 1". When I asked her why it was negative, she said, "Because 4 and 1 are negative." When I asked her to model it using the SAA, she modeled it correctly and said, "Negative 3." Thus, she reverted back to her old strategies of solving subtraction tasks with a negative minuend and subtrahend and did utilize her generalizations or visualizations, perhaps because she saw no need to do so since she obtained the correct answer.

Since I had noticed that her method worked for tasks in which the value of the minuend was smaller than the value of the subtrahend, I asked her to solve the task $-4--6$. Her responses were interesting.

J: Negative, no positive 10 , no negative 10 because you gotta change those two signs.

$\mathrm{N}$ : So what are you going to change in the problem?

$\mathrm{J}$ : I'm going to change the problem to -4 plus positive 6 .

$\mathrm{N}$ : And what's that?

$\mathrm{J}: 2,10$ ?

$\mathrm{N}$ : So how did you get those?

J: If you start at -4 and then you go to positive 6 , and then you count the spaces in between, you get 10 .

Her immediate reaction was to resort to her idea of the equality of $-4--6$ and $-4-6$, which would have given her the answer of -10 , yet upon recalling what we discussed, she realized that she had to change some of the negative signs. This made her believe that the answer was most likely positive valued since she would no longer have two negative numbers in the expression. As I asked her to work through the problem, 
she changed her initial reactions. She remembered, correctly, that she had to change the subtraction sign to an addition sign and the negative sign on the 6 to a positive sign. Most likely what happened next was that she computed $-4+6$ and obtained the answer of 2 . Yet, remembering the SAA, she believed that she had to begin at -4 and travel to the positive 6 , which would leave 10 spaces in between (see Figure 12). Even though the SAA would show -10 inside the arrow when she would do this, she already ruled out the possibility of the answer being negative.

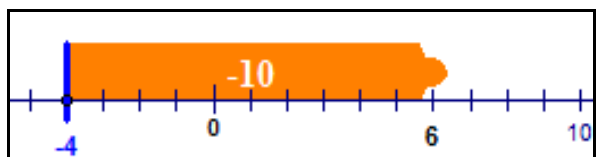

Figure 10. Jen visualizing $-4-6$ on the SAA

Jen tried to combine her arithmetic strategy of changing the subtraction sign and negative sign in a subtraction tasks with a negative subtrahend, as well as the method utilized on the SAA. This idea may have emerged from the fact that because she had used the SAA to come up with the quick sign changing method, she thought she could integrate the two. I then had her model the original task using the SAA.

$\mathrm{N}$ : So where would you put the blue stick?

$\mathrm{J}$ : At -4 .

$\mathrm{N}$ : And how would you move?

$\mathrm{J}$ : End up at the -6 . So it would be 2. Maybe I should just stick with this way.

At this point, she seemed to like the visualization of a number line to compute theses types of tasks versus switching the signs. After this task, she used the method of the SAA on a number line to solve tasks when it involved a negative minuend and a negative subtrahend. She said, "Sometimes it's easier to do the changing signs and sometimes doing the computer. It's [changing the signs] more hard when the negative number is in front." Despite the difficulty, she was able to accurately compute all the subtraction tasks that I gave to her.

At the next session, I gave her a variety of subtraction tasks to solve without using the applets. She solved all of them correctly. For tasks that had a positive minuend and subtrahend, she explained her reasoning as moving left on the number line. For tasks that had a positive minuend and negative subtrahend, she explained the answer using her changing signs strategy and addition. For tasks involving a negative minuend, she used the method of the SAA, by starting at the minuend on the number line and moving to the number represented by the subtrahend on the number line, counting the spaces in between, and determining the sign based on the direction she traveled. After correctly solving each task, I asked her if there was a way to predict whether the answer was going to positive or negative.

Well, if there's a negative number in front and a positive, then it will be negative. If there is a positive in front and a negative then it will be a positive answer. And then positive and positive 
equals...um a negative, or positive...then a negative minus a negative will be a positive, or a negative.

She concluded that if she had a negative minuend and positive subtrahend, the answer would be negative. If she had a positive minuend and negative subtrahend, the answer would be negative. She also recognized that she was not able to tell if the answer would be positive or negative if both the minuend and subtrahend had the same sign. When I asked her how she made this overall conclusion she said, "Oh, well, I don't know. Cause I looked at these" and pointed to the tasks that she had written down. She seemed to have a very procedural understanding of subtraction since she based on her answer on placement of the negative numbers.

Since she was unsure about how to determine the sign of the answer for a same-signed subtrahend and minuend, I tried to get her to think about the possibilities. I asked her, "If you start out with a negative number, where would the blue stick go?" She pointed to the negative side of the number line.

$\mathrm{N}$ : If you subtracted a positive number, how would you do that?

J: I'd go to the right, so I'd get a positive answer.

$\mathrm{N}$ : If you started out with a positive number and subtracted a negative number what would you do?

J: I'd have to go to the left, and get a positive.

$\mathrm{N}$ : Ok, so now the harder part. If you started with a positive number...

J: It could be either! Cause like you could start at 10 and subtract 20 and it would be negative, or you could start at like 20 and subtract 10 and it would be positive.

$\mathrm{N}$ : Great. So now what about if start at a negative?

J: It would be the same thing. It would be negative, positive, or zero.

$\mathrm{N}$ : Could you ever find out whether the answer would be positive or negative if you had a positive minus a positive or a negative minus a negative?

$\mathrm{J}$ : The higher number is in front, it will be positive, and if the lower number is in front, it will be negative.

$\mathrm{N}$ : Great. How did you know that?

J: Cause I can see it.

$\mathrm{N}$ : So did this computer file help?

$\mathrm{J}$ : Yeah.

$\mathrm{N}$ : How did it help?

$\mathrm{J}$ : Like the stick and the arrow and seeing the number of spaces in between.

The SAA allowed Jen to enact many possibilities from which to generalize the rules of subtraction. It also allowed her to see subtraction in action, which influenced her ability to determine the sign of the answer and reason through why the answer was negative or positive. Furthermore, after working with the applet, she was able to correctly answer every subtraction task that I gave her to solve, and did so without the use of the applet. While she preferred her old methods for tasks with a positive minuend and subtrahend and tasks with a negative minuend and positive subtrahend, she used the changing sign method for tasks with a positive minuend and negative subtrahend, and the SAA movement method for tasks with a negative minuend and negative subtrahend. 
Summary of Jen's Interactions and Understanding with Technology

- Jen had an increased conceptual and procedural understanding of addition and subtraction of negative numbers. Moreover, she was eventually able to solve all types of subtraction tasks, even those she could not solve prior to working with the applet.

- For tasks that had a positive minuend and subtrahend, Jen explained her reasoning as moving left on the number line. For tasks that had a positive minuend and negative subtrahend, she explained the answer using her changing signs strategy and addition. For tasks involving a negative minuend, Jen started at the minuend on the number line and moved to the number represented by the subtrahend, counted the spaces in between, and determined the sign based on the direction she traveled.

- Jen could now use the number line to help her solve all subtraction tasks. Not only had she been able to move from the arithmetic representation to the number line representation, but also she was able to move in the opposite direction, which she was unable to do prior to working with the SAA.

- By manipulating multiple sequences of subtraction expressions, noticing patterns, using contextual problems, and noticing the relationship between the placement of the subtrahend and difference. Jen was able to see subtraction as both take-away and a distance. However, she preferred using the idea of distance. Prior to working with the SAA, she had not been able to see subtraction as a distance.

- The applet allowed Jen to pay attention to the signs of the subtrahend and tell the difference between $a--b$ and $a-b$, and no longer thought they were equal. Furthermore, she was able to understand why $a--b$ was equivalent to $a+b$ based on the movement and corresponding visual on the SAA.

- After manipulating the SAA and seeing the arrow value, Jen saw how the movement on the number line impacted the answer to the task, as well as how the signs and magnitude of the minuend and subtrahend determined the sign of the answer. She noticed that when she subtracted a number that was to the right of the original number on the number line, she knew the answer was going to be negative. When she subtracted a number lying to the left of the minuend on the number line, she knew the answer was going to be positive.

- Eventually, Jen was able to obtain the correct answer to tasks in the form $-a--b$ and preferred to explain her reasoning using the SAA method, and not using the idea of adding $b$ to $-a$. Jen had difficulty viewing that subtracting a negative subtrahend from a negative minuend was addition, for she believed that addition meant that every negative sign needed to change to a positive sign before adding the resulting numbers.

- Jen was able to generalize across her solved tasks and conclude how to determine the sign of the difference. By manipulating the SAA, she noticed that when the minuend was greater than the subtrahend, the difference was going to be positive and when the subtrahend was greater than the minuend, the difference was going to be negative.

- After working with the SAA and accompanying tasks, Jen successfully attained Level 4 in both Peled's QD and NLD for subtraction and addition. 


\section{Discussion}

Prior to working with the SAA, Jen viewed subtraction as taking away the amount in the subtrahend. She had rarely referred to subtraction as a distance between the minuend and subtrahend, however after working with the SAA, she often used the idea of distance to solve subtraction tasks.

Also prior to working with the SAA, Jen did not have clear conceptual understanding of subtraction. Jen used three different strategies to solve subtraction tasks, which were based on the form of the task, and could not make a connection among these strategies. The strategies she used allowed her to calculate the correct answers for tasks in the form $a-b$ and $-a-b$, where $a, b>0$ and $-a--b$, where $a>b>0$. Unfortunately, she was unable to correctly calculate tasks in the form $-a--b$, where $b>a>0$, and in the form $a--b$ where $a, b>0$. For tasks involving a positive subtrahend, Jen used the number line to justify her answer by starting at the position of the minuend and moving to the left the absolute value of the subtrahend. For tasks that involving a negative minuend and positive subtrahend, Jen also justified her answer by saying that she changed the numbers to positive, added them, and then tacked on a negative sign to their sum. She tried to extend these strategies to help her solve tasks in the form $a--b$ and $-a--b$, although unsuccessfully.

For tasks with a positive minuend and negative subtrahend, Jen noticed the difference between $a--b$ and $a-b$, in that the first was subtracting negative $b$ and the second was subtracting $b$, but solved them in the same manner, by taking away $b$ from $a$; thus she believed they had the same answer. For tasks with both a negative minuend and subtrahend, Jen transformed the task by changing the signs of the minuend and subtrahend to positive, subtracting, and then making sure that the answer had a negative sign. Jen believed that since the minuend and subtrahend were negative, the answer had to be negative. Thus, Jen obtained the correct answer for tasks in which both the subtrahend and minuend were negative, and the minuend was smaller, but not vice versa.

Prior to working with the SAA, Jen had a difficult time connecting the representations when she moved from a number line context to an arithmetical context. Jen used the dots as part of her equation, but did not incorporate a relationship between the two numbers such that it could be conceived on a number line. Moreover, she typically believed that when she subtracted, she should move to the left on the number line. Only once did she move to the right of the minuend, and this was due to her computed answer.

After exploring the SAA, Jen realized how the applet embodied subtraction by observing how her manipulation impacted the visual in terms of the beginning, ending, and direction of the arrow, and the value in between the blue stick and tip of the arrow. She observed how the arrow value changed as she 
moved the tip of the arrow from left to right. This fostered the connection between the values of the minuend, subtrahend, and difference, and the relationship among the numbers, operations and movement on the number line. In fact, the arrow value seemed to make greatest impact on Jen. She knew which way to move because of the value shown inside the arrow, and this allowed her to see subtraction as a distance between numbers. Furthermore, it allowed her to connect the ideas of subtraction as a distance and takeaway. Moreover, it allowed her to compute all forms of subtraction tasks accurately and provided her with more strategies to use to solve the tasks on paper.

Through observation, manipulation, visual feedback, and sequencing of tasks, she was able to see many patterns that helped her develop strategies to solve subtraction tasks. Jen was able to understand the connection of subtracting a negative subtrahend from a positive minuend as being the same as addition, especially by witnessing the movement to the right, which is the direction she traveled most often when she "added." However, she did not necessarily fully understand the impact this had with a negative minuend and negative subtrahend. Instead of changing the subtraction sign to an addition sign, and the negative sign of the subtrahend to a positive sign, she preferred to visualize the movement of the SAA to help her solve tasks in the form $-a--b$. Through exploration of the SAA, Jen also noticed the relationship between the sign and values of the minuend and subtrahend and how they determined the sign of the difference. She noticed that when she had to travel to the right of the minuend, i.e. subtract a higher value from a lower value, the difference was negative. When Jen had to travel to the left of the minuend, i.e. subtract a lower value from a higher value, the difference was positive.

Jen was able to correctly solve all subtraction tasks after working with the SAA, even though she did not necessarily justify her answers using the SAA method. However, Jen did note that the SAA helped her understand subtraction because it allowed her to easily see what was happening.

\section{The Equal Sign \\ Prior Conceptions}

By her responses on the interview tasks, Jen seemed to understand the relational view of the equal sign, yet couched her description using operational ideas (Seo \& Ginsburg, 2003). This could mean that she had an operational understanding of the equal sign, but the way she worked with the equal sign in tasks seemed to indicate differently.

When I asked her what was different and the same among the expression and equations 5+6, $5+6=$, and $5+6=11$, Jen said, "That one doesn't have an equal sign, this one has the equal sign, and that 
one has an equal sign and the answer." Moreover, when I asked her the significance of the equal sign in the latter two equations, she said, "That says what it's equal to, like what's coming up, and that one says it equals the answer." Jen believed that the equal sign in the equation $5+6=$ suggested an upcoming answer, and the equal sign in $5+6=11$ denoted that 11 was the answer to the task $5+6$. When children gave these types of comments, they were categorized as having an operational view of the equal sign according to Seo and Ginsburg (2003). Unlike children classified as having an operational view of the equal sign, Jen seemed comfortable with non-canonical forms and finding the missing value, which would indicate that Jen had some relational ideas about the equal sign.

Jen was familiar with non-canonical forms such as $3=3$ and $8=10-2$. Jen told me that she had seen 3 = 3 when she had learned about absolute value. She said, "I forget what it's called. Like, um, where there is a negative number and it equals a positive. The one where like a negative and a line here and a line here where like it's equal." As she said this, she drew I-3lon her paper and told me that was equal to 3. When I asked if she had seen it anywhere else, she said, "Well, like in a problem. Like solving equations." I then asked if it was ok to use the equal sign in this manner, and she said, "There's nothing wrong really, it's just different." It was interesting that while she did not feel that using the equal sign in this manner was inappropriate, she still felt as if it is unorthodox. Similarly, she felt the same way about $8=10-2$. She noted that while it was not incorrect to write the task this way the equal sign meant, "It's really like ten minus two equals eight." For neither task did Jen mention the words "same as" when describing the equal sign, until she encountered the rest of Task 2 . These types of tasks she had seen in class when she did conversions. She said, "I've seen these in problems before, like um, you have them with 2 feet equals how many inches, so you have to do inches." She was referring to using the equal sign to help her convert an amount of one unit into an amount of another unit.

She also used the word "the same" when describing the relationship between the expressions $2 \times 5$ and $4+6$. While she did not say that the equal sign signified that the expressions were the same, she did say that it was fine to place an equal sign in between the expression because they "equaled the same number." While she had difficulty solving for the question mark in the task $3+5=$ ? +2 , she eventually used the words "the same number" to justify her solution.

$\mathrm{N}$ : Could you tell me what the question mark is in this task $3+5=?+2$ ?

$\mathrm{J}$ : Well, 4.

$\mathrm{N}$ : How did you...

J: Cause, I don't know. [long pause] Yeah, 4 cause like, that...I don't know. I don't know.

$\mathrm{N}$ : So tell me what you're thinking about.

$\mathrm{J}$ : Like, that and doing like half or ... like adding them, but getting to the same number. 
She was pointing to the paper as she was describing how she got to her answer. It looked as if she was adding the 3 and 5 to get 8 , and then dividing it by 2 to get the value of 4 . However, this did not quite fit with her verbal description of them being equal to the same number. Another way she could have thought about it was looking at the task as a pattern and the addition signs and equal sign as comma separators. Hence, she could have seen the pattern 3,5,?,2 and believed the question mark to be equal to 4 because 3 and 5 had a difference of 2 , and 2 and 4 had a difference of 2 . I tried to make sure I could get a better idea of what she did, although, in asking my questions, I may have influenced her reasoning.

$\mathrm{N}$ : So let me see, you said you added something, what did you add?

$\mathrm{J}$ : I added 3 and 5 and got 8 .

$\mathrm{N}$ : So then what did you do? Add these numbers [pointing to the ? and 2]

$\mathrm{J}$ : Yeah, to get 8.

$\mathrm{N}$ : So when you put your 4 into the question mark, what did you get?

$\mathrm{J}$ : Oh, you would get 6 , so you have to put a 6 in there to get 8 .

$\mathrm{N}$ : Can you talk a little more about that?

$\mathrm{J}$ : Cause like 8 equals 8 , and like you do that and you get 8 [pointing to the addition sign in the left expression] and you have to do that and get 8 [pointing to the addition sign in the right expression].

Through our interactions, Jen was able to determine the value of the question mark. One will notice that Jen used a guess and test strategy (Knuth et all, 2006). She first thought the answer was 4, but upon checking this answer in the equation, it did not work. Therefore, she saw that she was off by a value of 2 so increased her answer from 4 to 6 and checked this answer.

While Jen could solve for the value of an unknown in an equation when the unknown was represented by a question mark, she was unable to do so when the unknown was a letter. For instance, immediately after solving for the question mark, I asked her to solve the task $x+5=3$. If she had solved this task using her guess and test method, she would have been able to solve for $x$, even though it would require to add a negative number to a positive number. This should not have been difficult for her since she could add positive and negative integers. Yet, she abandoned this strategy for this task, which may point to the fact that she did not thoroughly understand addition and subtraction of negative numbers. The strategy that she chose was surprising.

J: You cross multiply! Wait, yeah, you do hmm.

$\mathrm{N}$ : If you need to write something down go ahead.

$\mathrm{J}$ : Ok, so you do cross multiply, so you do x over something, equals over 5, equals 3 over 100. I'm guessing it's... [she wrote $\frac{x}{5}=\frac{3}{100}$ ]

$\mathrm{N}$ : Ok, and then how would you find $x$ ?

$\mathrm{J}$ : The $x$ is 2 , so there, 3 times 5 is 15 , then...well, no.

$\mathrm{N}$ : So how did you figure out $x$ was equal to 2 ?

J: I did 5 minus 3. So what I gotta do is if it's positive, subtract the two numbers, if it's multiplication, add the two numbers, I think. So if that was minus, wait, no, I don't know.

$\mathrm{N}$ : It's ok, keep on going. Tell me what you're thinking. 
J: I guess I'm thinking that if it was minus, you would add these two numbers, and if it's plus, then you would subtract these two numbers.

$\mathrm{N}$ : So you're saying that if it was $x-5=3$ then you would $\ldots$

$\mathrm{J}$ : Then you would add those two numbers, so it would be 8 .

$\mathrm{N}$ : Ok, so for this one, $x+5=3$ ?

J: I subtracted 3.

N: How did you know to subtract the 3 ?

$\mathrm{J}$ : Because it was the low number.

$\mathrm{N}$ : Can you check your answer of 2 ?

$\mathrm{J}$ : It would be 7, if you put the 2 right there [pointed to the $x$ ] because you have to add the 2 .

$\mathrm{N}$ : Is that what we want it to equal?

J: Huh?

$\mathrm{N}$ : So you got 7, but the equation says it has to equal 3.

J: I don't know then. I guess I wouldn't add them [the 2 and 5] then.

Jen was not consistent in the method she chose. At first she thought that she had to find the answer through cross multiplication. She may have thought about doing this for a couple of reasons. One reason is that there was one value on the right side of the equal sign, the 3 , and one number immediately preceding the equal sign, the 5. She could have just learned cross multiplication in her math class, which would have triggered her to believe that since she had an equal sign separating one value from another, she was to use cross multiplication. Another reason is that the task asking her to describe what the equal sign meant in 1 dime $=10$ pennies and 12 inches $=1$ foot could have sparked her thoughts about cross multiplication in order to convert values. Even though she said that she would use cross multiplication to solve this task, and wrote $\frac{x}{5}=\frac{3}{100}$, she did not use cross multiplication to find the value of $x$. Instead, she subtracted 3 from 5 to get a value of 2 for the $x$. While the idea of subtraction was correct, she switched the values of the subtrahend and minuend. In fact, she did this because she believed that she had to subtract the lower value from the higher value. This points to the fact that she did not have a thorough conceptual understanding of subtraction of negative numbers. At first she did not check her value for $\mathrm{x}$, for she believed that she had obtained the correct solution. However, once I asked to, she found that when she added 2 to 5 , she obtained 7, yet this did not seem to both her, until I specifically pointed out the fact that the expression had to equal 3. While she recognized that this did not fit the equation, she did not know how to use another method or adjust her thinking. She simply just stopped. When I asked her to solve it a different way, she kept saying, "I don't know." Therefore, I did not ask her to solve a 2-step equation since she was unable to solve 1-step equations. However, I did ask her whether she had done both types of tasks in math class, to which she responded, "Yeah, we did them at the beginning of the year. That's why I don't remember."

\section{Summary of Jen's Prior Conceptions}

- Jen exhibited a mixture of relational and operational views of the equal sign. She typically 
described her understanding in operational terms, but seemed to be able to work with the equal sign in a relational aspect. When it came to discussing the equal sign in an equation with a missing value, she was eventually able to reason through a task that had the missing value represented by a question mark whose value was positive based on the equivalence of expressions. However, she could not figure out how to solve a 1-step equation involving $x$, whose value was negative.

- Jen was familiar with non-canonical forms but often described the acceptability of the forms based on its similarity to canonical forms using the equal sign, which often typified an operational description.

- Jen believed that the equal sign showed the equivalence of two expressions in the case where the missing value was a positive number represented by a question mark, yet this did not direct her to use an algebraic method to solve equations. However, it allowed her to use a guess and check strategy to find the correct solution.

- Jen could not accurately solve a 1-step equation with the variable $x$, which represented a negative number. She tried to use cross multiplication and her own method of using an inverse operation, but to no avail.

\section{Analysis of Interaction with the Equality Applet}

\section{Jen's Interaction and Understanding with Technology}

As Jen explored the Equal Balance Applet (EBA), she noticed a few of the features that allowed her to discover the two rules that maintained the balance equilibrium. When the pans were not balanced, an inequality appeared above the fulcrum of the balance, and the pan that was up in the air contained a lighter, or negative valued objects. After Jen had dragged a 5-weight to the right pan and a -5 balloon to the left pan, I asked her what she noticed.

The positive 5 ...is heavier, so they are not equal. Like if you do a positive and a negative, like whatever sides the positives are on, that's the side's going to be heavier, and the negatives is going to be lighter.

After noticing the pans were not balanced, and hence the values not equal, I asked her to try and make the pans balanced. At first she said, "I'd put a 5 there, I think," but saw that the pans were uneven and remarked, “No, guess not.” After a short pause, she saw the inequality displayed above the fulcrum and said, "Oh, it's zero, so you have to add another 5 to that side" (see Figure 13).

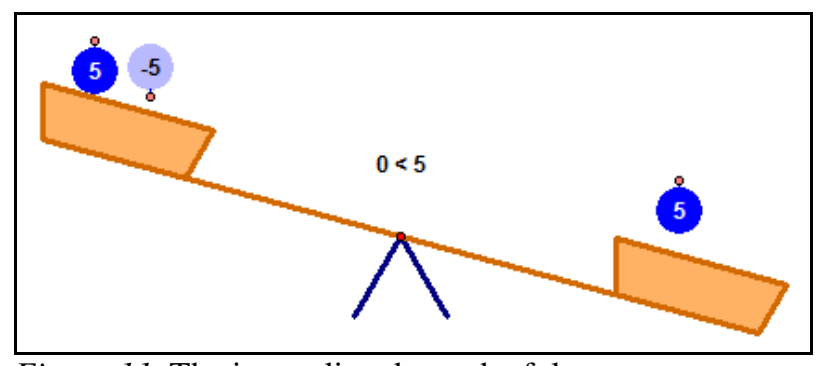

Figure 11. The inequality above the fulcrum

After she added another 5 to the left pan, I asked her to remove the objects, except for the 5 on the left pan, 
and manipulate the applet to see how she could get the pans to balance. Immediately she saw that she could add a 5-weight to the right pan. Jen said, "We have a positive 5 here and then you have to do a positive 5 over there." Thus, she discovered that she could add the same object to both sides to maintain the equilibrium of the pans. I asked her to remove all the objects, and asked if she saw another way to adjust only one pan so that the pans remained balanced. She said, "putting a 1 and a -1 on the left... If you do like a positive and negative." Thus, she discovered that she could maintain the balance by adding a positive and negative of the same magnitude to one side.

As Jen modeled the equation $3+5=?+2$, she remarked, "I wouldn't use any negative weights...for this problem, since there are no negatives." After she placed the three single weights and one 5-weight onto the left pan, she paused before completing the right pan. I asked her, "Ok, so what would you do for the right pan?" She dragged two 1-weights onto the right pan, but did not utilize the question mark on the screen (Figure 14).

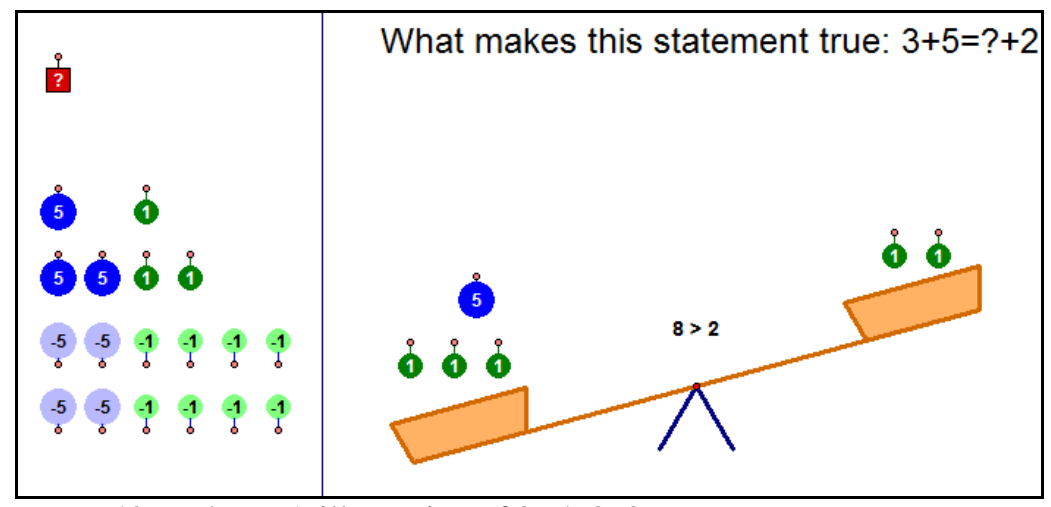

Figure 12. Jen's EBA illustration of $3+5=?+2$

I asked her how she would find the value of the question mark.

J: You'd take two away from here [points to the left pan with her mouse arrow] and see how many, and you'd have 5 and 1.

$\mathrm{N}$ : Can explain a little more about that?

$\mathrm{J}$ : Ok, like there's two over here [waves the mouse arrow onto the right pan] so I like, [pointing to the left pan] see those two, so then you see what's left and then put back over there.

She then dragged a 5-weight and 1-weight from the storage unit to the right pan. Without using the question mark, she seemed to solve this equation using some features of the applet, but not all of them. Because she saw two green 1-weights on the right side and three green 1-weights on the left side, she knew that if she had to make the pans balanced, she had to add the remaining amount on the left pan minus 2 to the right pan. While she did place the question mark on the right pan and use the balance aspect of the applet, she did this task using the visual of the equation and figuring out what she needed to add to the right pan to make the pans balance. 
I pointed out the question mark to her on the screen and asked her if she could model the equation exactly as it was written. I then asked her if there was a way to figure out what the question mark was equal to by having the pans remain balanced. Jen said, "Take out these two and then take out these two." She removed two 1-weights from the left pan and two 1-weights from the right pan. I asked her what taking away two of the 1-weights was like in writing an equation and she said, "like subtracting 2." Furthermore, she noticed the new equation by referring to the applet.

J: Now it shows 6 equals question mark.

$\mathrm{N}$ : So now let's look at this [referring to the algebra representation on her paper]. You told me that you had 3 plus 5 and took away 2 and got 6 on this left pan. On the right pan, you told me that we had a question mark plus 2 and you subtracted 2 and got the question mark.

J: So you would get 4 , no 6 ?

I was not sure what she was answering by her last uncertain statement. I was trying to point out the correspondence between the algebraic representation she wrote and the applet's representation. Yet, she did not seem to understand what I was doing. In fact, it seemed as if she knew that when she removed 2 from the right pan, the question mark weight remained; yet this did not seem correct with her algebraic representation. It seemed that when she subtracted 2 from the right hand side of the equation, she believed that she should subtract 2 from both the question mark value, which she had determined to be 6 , and the number 2 . This is why she wavered between the answers 4 and 6 . I tried to get her to talk about her apparent confusion.

$\mathrm{N}$ : Does that make sense?

J: Not really.

$\mathrm{N}$ : What doesn't make sense?

$\mathrm{J}$ : This makes sense, this does [points to the computer screen] but this doesn't [points to the algebraic representation on paper]

$\mathrm{N}$ : Ok, so on the balance, tell me what you did in this equation to solve for the question mark.

$\mathrm{J}$ : I took away 2 from this side and 2 from that side.

$\mathrm{N}$ : And what operation did you say that was like?

J: Subtraction.

N: So you basically subtracted 2 from over here and subtracted 2 from over here?

J: Yeah.

$\mathrm{N}$ : So what are you left with?

J: 6 .

$\mathrm{N}$ : Ok, so when we subtracted 2 from the left pan, you got 6 . What happened when you subtracted 2 from the right pan?

$\mathrm{J}$ : Zero.

$\mathrm{N}$ : Well, is there something else there?

$\mathrm{J}$ : Oh yeah, the question mark.

$\mathrm{N}$ : Does that make more sense?

$\mathrm{J}$ : Yeah. The zero thing was confusing.

Much like other interviewees, Jen had difficulty interpreting the remaining question mark on the right pan (see Figure 15). It would seem as if she believed that once she solved for the variable, it disappeared and 
she was only to pay attention to the remaining numbers. Since there was no number value on the right pan, she believed that the value of zero, even though it clearly contained an object. This would indicate that Jen might not have viewed the variable as a number, but rather an entity to be solved for, whose value followed immediately after the equal sign.

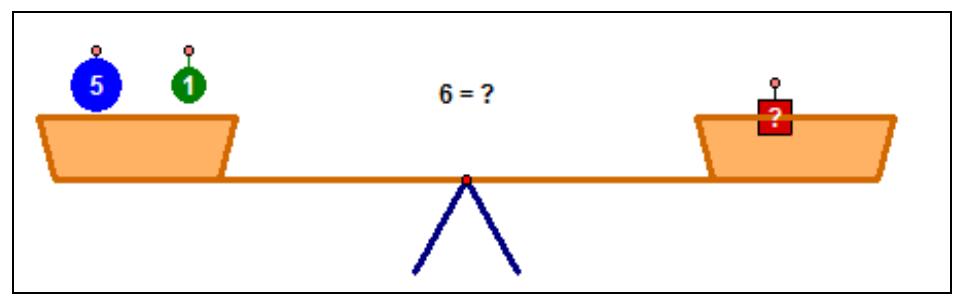

Figure 13. After removing two 1-weights from both sides

Jen had some difficulty extending this method of the EBA to 1-step equations that involved letters, however, this could have been to due to adopting a defeatist attitude. Sometimes when she became frustrated, she would simply give up and sit in her chair and wait, almost as if she wanted to see if I would do the task for her. Since she had had difficulty with solving 1-step equations in the interview, I was not too surprised that she wanted to shut down during these tasks. It took a lot scaffolding questions to get her to use the EBA and understand how it connected with solving equations on paper.

$\mathrm{N}$ : So how about $x-20=-13$ How would you figure out what the $x$ is?

$\mathrm{J}$ : I dunno. Um [very long pause]

$\mathrm{N}$ : So is there anything you could add or remove from the pans?

$\mathrm{J}$ : Um, a -5 ? (whispers as if unsure)

I encouraged her to try her idea, especially since she would see that her idea was correct. After she removed a -5 balloon from the left pan and began to move a -5 balloon from the right pan, she said, "Oops, I guess not." As she moved the -5 balloon from the right pan to the storage unit, she noticed that once she passed the fulcrum, the left pan popped up into the air (see Figure 16). This happened because the -5 registered as being placed on the left pan since she had not moved it totally to the storage unit. I told her to keep on going, and she seemed relived that she was correct in her thinking. 


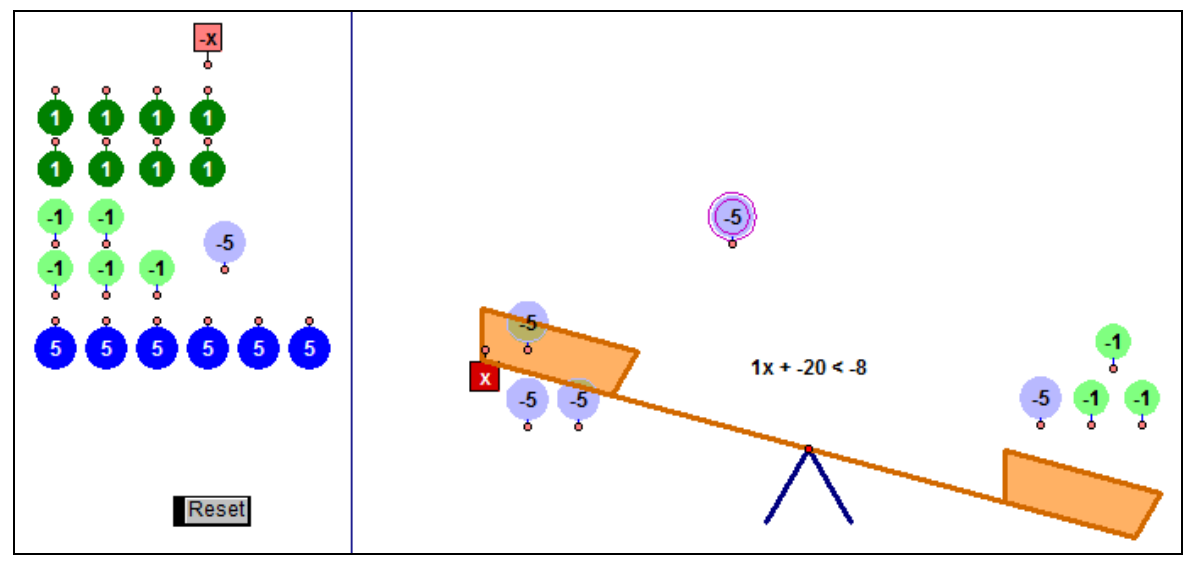

Figure 14. Moving a -5 balloon from the right pan to the storage unit

$\mathrm{N}$ : Ok, is there anything else you can do to help solve for $x$ ?

$\mathrm{J}$ : Do another -5 .

$\mathrm{N}$ : Ok, so how much did you take off altogether?

$\mathrm{J}$ : two negative fives, so negative 10 .

$\mathrm{N}$ : What is our new equation?

$\mathrm{J}: x$ minus 10 equals negative three [waves mouse over the fulcrum where the equation is displayed]

$\mathrm{N}$ : So can you do anything else to find $x$ ?

J: Take off the negative fives. Well, take off the negative fives, I think, or I guess we could take off $x$ to get it by itself.

Jen knew that in order to solve for $\mathrm{x}$, she had to get $\mathrm{x}$ by itself on the left pan. By looking at the visual, she thought of two ways to do this: take off the two -5 balloons, or take of the $x$ weight (see Figure 17).

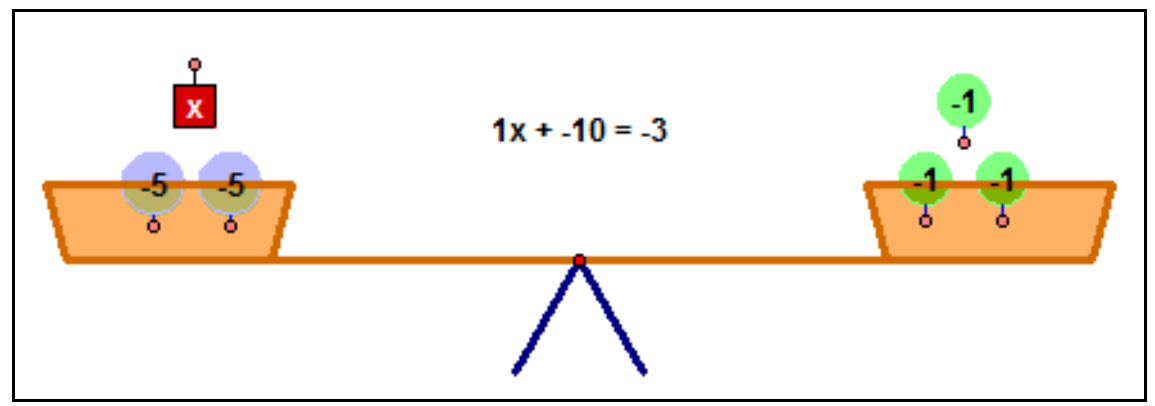

Figure 15. After subtracting -10 from both sides in the equation $\mathrm{x}-20=-13$

She forgot about the rules she came up with to keep the pans balanced, so I had to remind her.

$\mathrm{N}$ : Ok, so if you took off the negative fives, could you do the same to the right pan?

J: No. Oh. What about add two negative fives to that side [right pan] and then take them away over here [left pan]. No, I don't know.

$\mathrm{N}$ : Go ahead and try what you're thinking.

The EBA allowed Jen to experiment and test her hypotheses. She put two negative fives on the right pan, and removed two negative fives from the left pan. While she was able to get $x$ by itself on the left pan, she noticed the pans were not balanced, especially since she pointed to the displayed inequality that read $x+0>-13$ and said, "No, it doesn't work." After replacing two negative fives on the left pan and 
removing two negative fives on the right pan, I encouraged her and told that that was ok, that we could just try something else to see if it worked. I asked her if she could add anything to both pans that would get the $x$ by itself. She said, "Um, two positive fives?" So she dragged two 5-weights from the storage unit to the left pan and then to the right pan. She noticed that the pans balanced, but did not say anything; rather she just sat there and stared at the equation $x+0=7$.

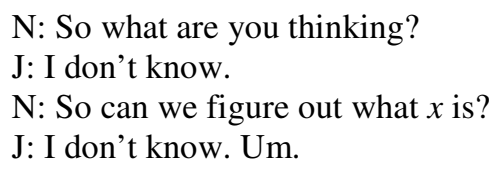

What seemed to be causing a conflict with figuring out $x$ was the equation versus the visual display on the EBA. She saw that the equation showed the value of $x$, but the pans did not seem to appear equal to the equation (see Figure 18). The visual depiction of this scenario seemed to overwhelm her.

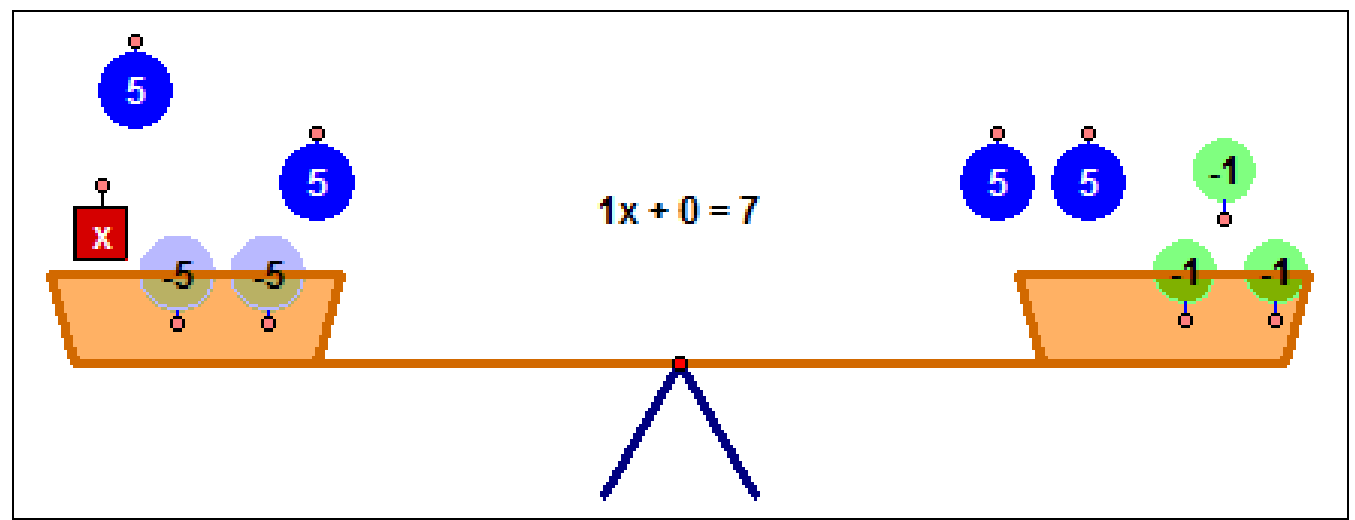

Figure 16. After adding two 5-weights to both sides of the equation $\mathrm{x}-10=-3$

$\mathrm{N}$ : Are we finished?

J: I don't know.

$\mathrm{N}$ : Well, can we figure out the answer?

J: I don't know. Um.

$\mathrm{N}$ : Well, when we add up the numbers on the left pan, what does it come to?

J: Zero.

$\mathrm{N}$ : So can we place them in the storage unit without altering the balance?

$\mathrm{J}$ : Yeah, I mean, no, I mean, Yeah we can!

As she did this, she said, "Oh, $x$ equals 7." When I asked her to explain how she knew that she said,

"Because you add the 5 plus the 5 and that's 10 and then you minus, well, add negative 3." It seemed that Jen had difficulty with having so many objects on the left pan with $x$ even though they summed to zero. She had to physically remove them from the pan to clearly see the value of $x$. In fact, she needed to do this despite the fact that her equivalent equation said $x+0=7$ when the objects remained on the pan. Thus, it would seem that the visual influenced her more than the algebraic equation when solving 1-step equations.

Next, I had her solve the 1-step equation that she could not solve in the interview, $x+5=3$, by 
using the EBA.

$\mathrm{N}$ : So how would you go about finding the value of $x$ in $x+5=3$ ?

J: Hmm. [Stretches in her chair] Wouldn't you, well, you could add 5 to this one and 5 to that one.

$\mathrm{N}$ : Ok, go ahead and try that.

After she dragged a 5-weight to the right and left pans, she made a slightly disgusted smirk towards the computer screen. When I asked her what she was thinking, she noted that the pans were balanced, but what she did did not help her solve the task.

$\mathrm{N}$ : Did that make it harder to easier to solve?

J: Harder! Way harder! Haha [chuckles]

$\mathrm{N}$ : So you can hit reset and go to the original task and try again.

She hit the reset button and looked at the screen and said, "Oh, would you do a -5 ?" I encouraged her to try what she was thinking. She dragged a -5 balloon to both sides to get the resulting equation $x=-2$. She did not state that $x$ was equal to -2 . I think she again became confused because $x$ was still not by itself on the left pan. I asked her what she could do with the 5 and -5 on the left pan. She said, "Take them out," and moved them to the storage unit. I asked her what she was left with on the pans. She said, "2, I mean -2. Wait, yeah -2 because there is a -5 and 3" (see Figure 18). Prior to working with the EBA, Jen had believed the answer to be 2 . This is most likely why she initially said $x$-value was 2 . However, upon inspection of the right pan, Jen noticed that the answer had to be -2 because she had to add the -5 to the positive 3 . I asked her if this $x$-value answered her original equation to which she responded, "Yeah, because you just gotta minus the 5 from the 3 , not minus the 3 from the 5." This time she realized in what order to subtract to find the $\mathrm{x}$-value, most likely due to the manipulation and visual depiction of the equation on the EBA.

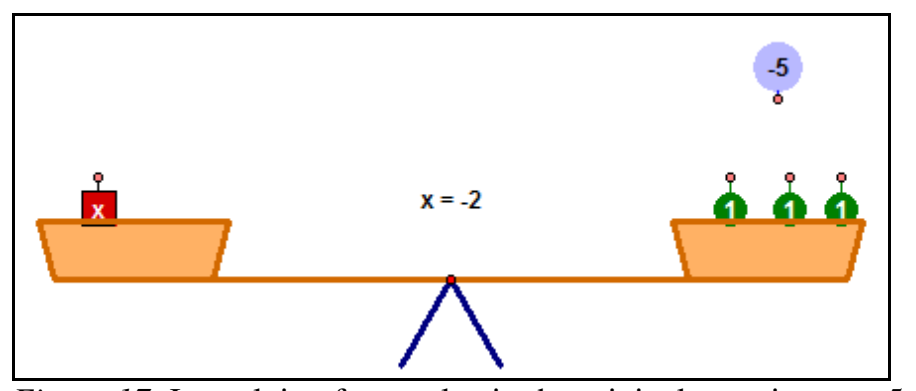

Figure 17. Jen solving for $\mathrm{x}$ value in the original equation $\mathrm{x}+5=3$

The more Jen worked with the EBA, the more familiar she became with the method of solution and could model it accurately on paper. Her major breakthrough in realizing this method came as she solved the next task, $x+8=21$. She immediately removed a 1-weight and 5-weight from the left pan and right pans. When I asked her to tell me what she did she said, "I took off 6 from both sides...like minusing 6." I asked her what remained and she said, "Well 8 minus 6 is 2, so $x$ plus 2," and pointed to the left pan. I asked if why that made sense to do that and she said, "Cause you just gotta put the $x$ in its place, and then 
you minus those two numbers." What she is referring to is the fact that when she first used the EBA, she did not know if she had to subtract the amount she removed from both the $x$ and the number, or just the number. Now she told me that she knows that she only had to subtract the value of 6 from the number and leave the $x$ remaining without having 6 being subtracted from it. She went on to say, "Then it would be 21 minus 6 which is 15," and pointed to the right pan.

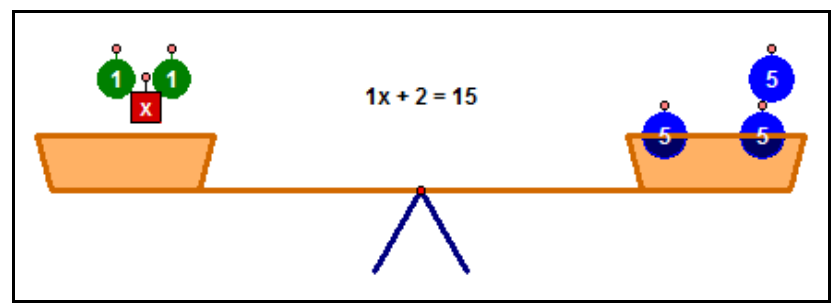

Figure 18. After removing 6 from both sides in the equation $\mathrm{x}+8=21$

It is also important to note that she wrote the appropriate algebraic steps in solving the equation on paper and ended up with the equation $x+2=15$. When I asked her what she would do next, she said, "Can you trade those fives? Like 1 five for five ones? Then you could take those two from here and to from over there.” As she made the exchange, I asked her what she was going to write on her paper. She took away 2 from both sides and said, "It would be zero, and then 13." I asked if she would have an empty left pan and she said, "No, so it would be x plus zero, then 13, or equal to 13." After she made the correct notation on paper and compared it to her visual, she said, "Wow, 13!" I asked her if the EBA helped her solve equations and she said, "Yes!"

Every other 1-step equation I gave to her, she solved correctly, both with the EBA and without the EBA. It is interesting that when she had to remove a negative, she immediately knew this was like adding a positive value in her algebraic equation. The visual and her knowledge of working with the SAA helped her interpret doing the inverse operation to solve equations. For instance when she solved the equation $x-6=-15$, she said, "I need to subtract -6 , well add 6 , but you could subtract -6 ... because that's a -6 over there, so you'd have to subtract -6 to take it away to leave $x$, but that is the same as adding 6 , cause you're removing a negative." She noticed that simply using the paper method was faster because she did not have to do any exchanges or take time to manipulate the objects.

I then gave Jen a 2 -step equation to solve, $2 m+7=15$. I asked her if she would like to solve this task using the paper or the applet. She quickly said, "The computer, please." I asked her what she would do first. Jen said, "Would take this 5 off; then take this 5 off." She removed a 5 from the left and right pan, wrote that she subtracted 5 from both sides on her paper, and said, "So it would be $2 m$ plus 2 equals 10 ." At this point, the applet required her to make an exchange, which she did so without any difficulty. "Take off 
these two and five over there and put back 3." She removed two 1-weights from the left side, removed a 5weight from the right pan and then placed three 1-weights on the right pan. As she wrote this on her paper, she said, "Oh, we could have just subtracted 7 to begin with... it's 8 !" I asked her what was equal to 8 and she said, "the right pan." She then told me she had $2 m$ on the left pan, so I asked her what she thought $m$ was equal to. She said, "so, um 4...I took half of 8." She realized that this was the same as dividing and said, "Oh, we've been doing opposites to find $m$." She noticed that since she could subtract 7 at first, she was doing the opposite of adding, and then when she divided by 2 , she was doing the opposite of the multiplication by 2 that was in the original equation.

I gave her four more 2-step equations to do on paper, which she correctly solved and did so algebraically. I then asked if working with the EBA had helped her to solve equations.

Yeah, it just shows like you stuff. Like you can do it on paper, but this shows you what's happening. It helps you see it. It helped [me] know what to do at first. Like what I had to take off and to get to where you needed to be.

Before working with the EBA, Jen could not remember how to solve 1-step equations. By the end of the session, she was able to correctly solve 1-step and 2-step equations using algebraic transformations. Her ability to do this was influenced by the visual and manipulation aspects of the EBA because it allowed her to intuitively understand why she needed to apply inverse operations to solve for the variable.

\section{Summary of Interaction and Understanding with Technology}

- Jen exhibited a relational view of the equals sign for the equivalence of two expressions when one of the expressions contained a variable.

- Jen accurately solved 1-step and 2-step equations using the algebraic transformation method.

- Jen believed that the visual model and manipulation features allowed her to solve 1-step equations more easily than she was able to do before working with technology because it helped her to find the solution by intuitively seeing what she had to remove or add to the pans to keep them balanced.

- Jen easily extended her understanding of solving 1-step equations and to solving 2-step equations.

- Due to the visual, manipulation, and feedback characteristics of the applet, Jen was able to try different strategies for solving for a variable. The applet allowed her to reason through her misconceptions and try another strategy to check her understanding. Jen had a little difficulty at first correlating the algebraic equation with the visual of the applet, but by the end of the session, she could move between the representations easily.

- Jen did not encounter difficulty when she had to exchange some objects for smaller valued objects on the EBA, even though it required more steps to solve the equation. Due to not having any difficulty, it seemed that it took her a little more time to realize that she could solve the same equation on paper using only 1 step rather than multiple steps. 


\section{Discussion}

Jen exhibited both a relational and operational view of the equal sign. While sometimes described the equal sign as meaning "the same as" she also described it as indicating "the answer." Jen was able to use her relational view to help her solve the 1 -step equation $3+5=?+2$, but could not do the same for the 1 step equation $x+5=3$. In fact, she tried to rely on her memory of procedures of how to solve for the variable, which included cross multiplication and subtracting the lower number from a higher number disregarding on which side the variable was.

Knuth and colleagues' (2006) believed that students who did not exhibit a relational view of the equal sign did not solve equations by algebraic transformations. This seemed to hold in Jen's case when she solved for the variable when the value was negative. She completely dismissed the meaning behind the equal sign and resorted to using procedures. However, when she solved the equation $3+5=?+2$, she did use the meaning of the equal sign, but used a guess and check strategy to find the value.

When Jen began working with the EBA, her relational understanding of the equal sign was reinforced through the visual picture of the balance. By exploring the EBA and how it used the weights and balloons to represent equations, she discovered strategies that would keep the pan balanced, and hence maintain the equivalence of equations. After making these discoveries, specifically adding or subtracting the same amount to both sides, and adding and subtracting the same amount to one side, she was about to see how to use the applet to help her solve 1-step equations. In the first couple of instances, she had difficulty understanding the connection between the algebraic expression and the expression in the pan that contained the variable, specifically when she subtracted an amount from that side of the equation. She was not sure if she had to subtract the value from the unknown value and subtract the value from the number added to the unknown value. She eventually came to understand this connection, which led her to be able to solve 1-step equations algebraically. She also had a little difficulty in understanding that when she added a $\mathrm{y}$-weight to the a side of an equation that contained $-\mathrm{y}$ balloon, that she could remove this pair without effecting the balance of the pans. When she did this a few times and saw the pans remained balanced, she understood that it was due to objects summing to zero. She did not have any difficulty using the EBA and exchanging weights, as some interviewees did. In fact, since she was allowed to drag and manipulate weights and balloons as often as she had ideas, she was able to see how she was transforming the equation. Moreover, she was able to intuitively realize how to find the value of the variable and model this using algebraic operations. Furthermore, she was able to extend the EBA to solve 2-step equations. 
During the process of working with the applet, Jen discovered the relationship between the displayed equation, objects on the pans, and solving equations algebraically. She enjoyed the visual of the applet because it allowed her to "see" how to solve the equations first and then figure out the pattern of applying inverse operations on paper. Furthermore, she was able to consistently solve both 1- and 2-step equations algebraically, even though she could not do this prior to working with the EBA. 
CHAPTER 10

\section{CASE STUDY: HOPE}

\section{Hope's Background}

Hope is in sixth grade Advanced Mathematics. While Hope seems to enjoy doing mathematics, she often becomes easily distracted and continually loses her focus when solving mathematical tasks. Due to her inability to stay focused, she takes a long time to complete a task. Furthermore, it is difficult for her to recognize patterns because of the length of time it takes for her to complete a task and her inability to stay focused within one task. For instance, I asked her to compare her answers to subtraction tasks in order to generalize why subtraction of a negative was like adding. First she focused on the type of tasks with a positive number subtracting a negative number (Type A tasks) and stated that it was like adding. When I asked if this held true for a negative subtracting a negative (Type B tasks) she tried to generalize among these types of tasks, but then forgot what she had discovered for the Type A tasks, on which she had to return to generalize. Writing down what she found had helped her a bit, but then she sometimes wondered how she got those answers in the first place and questioned if they were correct. During one of these instances when she was trying to remember what she did, she reassured me that her confusion was normal and that it usually took her a long time to solve math problems. She said, "Oh gosh, I'm crazy. I do not know what is going on. I'm usually at this for hours. I usually do my math homework for a long time. But I'll get it!"

She was often overly optimistic in her ability to do mathematics; yet she was cognizant of the different kinds of subtraction tasks and was able to categorize which ones she found easy and difficult. During some of the tasks she commented, "Oh, come on! Give me something harder." or "You got to be kidding me!" for items she found very easy. She commented on the more difficult ones by saying comments such as "Ok, I was doing fine... and now...it's kind of hard." or "These are the kinds I have trouble with."

Sometimes she would shut down when I would ask her to explain her answer, even if it was correct and she had explained a correct reasoning to me a few minutes prior. Usually this behavior was accompanied by a yawn. For instance, she would often yawn, and then say, "I don't know," followed by either "all I know is that I'm tired," or "honestly I don't remember." Sometimes she would say non sequitur 
statements afterwards such as “Um, I don't know. I did know (pause). We have a bad case of ants at our house!" Either she did not obtain enough sleep and this was impacting her memory, or her thoughts were occupied. Another possibility was that she was exhausted doing addition and subtraction tasks, for at one point, she told me that she did not "want to do subtraction anymore" and asked if could she "move on to the next thing." While the reason she could not stay focused was not clear, this behavior caused our sessions to move at a slower pace.

For each of our sessions, she arrived significantly late. One time she had forgotten that she had to meet with me and subsequently I had to go find her in the school. For our two other sessions, her parent dropped her off to tutoring late. Coupled with her inability to stay focused and her arriving late to our sessions, our time together was not as efficient as it could have been. Thus, she only completed tasks in Module 1,2, and 3, and the instructional section in Module 2 and began the technology section in Module 3 but did not finish it.

\section{Negatives and Operations on Integers}

\section{Prior Conceptions on Addition}

Hope seemed to have inconsistent procedural and conceptual knowledge of addition of integers. Although she did not abstractly solve all the addition tasks correctly on the first try, she was able to solve them correctly when she used a number line. Three types of addition tasks: one involving positive addends, one involving a positive and negative addend, and one involving two negative addends, seemed to become progressively harder for her to solve, respectively. She easily solved $10+3$ and told me she was "very sure" about that answer. However, when she looked at $10+-3$, she paused, wrote the answer 7 , and hesitantly commented, "Seven, I'm pretty sure." When I asked to explain how she got that she said, "Well, it's like subtraction even though it's addition because of the negative sign. Like, if you switched it around to get $10-3$, that would equal 7." After explaining how she got her answer, she was "positively sure" that it was the correct answer. This explanation shows that Hope had a unary (Kieran, 2007) understanding of negative numbers in addition, for she saw the connection between addition of a negative and subtraction. Yet, this understanding was called in to question when she solved the third task: $-5+-7$. Her initial answer to this task was 2 . She explained, "Well, since it is -5 and -7 , then there's 2 to get to -7 , so I just did -5 and then took away the negative on the seven, and then I just put the negative back (on the seven)." Since she believed that adding a negative was like subtraction, she needed to find the difference between the two numbers, however the two numbers she used were -5 and -7 , instead of -5 and 7 . Yet, because of 
her latter comment that she "took away the negative on the seven" and had to "put the negative back" I wondered if she solved the problem $-5+7$. I wrote the task $-5+7$ directly underneath the task $-5+-7$ and asked if she had solved $-5+7$ to help her find the answer of $-5+-7$. She responded that she did not, but by asking her this question she experienced a cognitive conflict and realize the correct answer.

$\mathrm{N}$ : Oh, so if you did solve $-5+7$, what would you get?

$\mathrm{H}$ : Negative five plus seven would also be 2. I think, yeah!

$\mathrm{N}$ : So they both have answers of 2 then?

H: Yeah. [long pause as she looks from one task to the other] It might be 12 or 2. Well, it might be -12 .

$\mathrm{N}$ : Which one is -12 .

$\mathrm{H}$ : The top one $[-5+-7]$

$\mathrm{N}$ : Why did you decide to change your mind?

H: When I looked at it, I don't know. Since there are two negatives in the top one [ $-5+-7]$ then it [the answer] will be a negative number. But the one down here [pointing to $-5+7$ ] there is only one negative, then it will be 2 . Not sure how I got 2, but that's just how I really do it. So.

Since she was able to tell the difference between the two tasks and give me the correct answer, I was under the impression that she understood how to solve these problems, but was unable to articulate it very well. I believed that if she could use a number line to show me, this might help her to explain it better.

N: Do you think you could show me on a number line how you thought about these tasks?

$\mathrm{H}$ : Maybe. So we start at the -5 and go down 7 , since 5 plus a 7 would equal right here [pointed to 12]. You start at -5 and go up 7 to get to the 2 [for $-5+7$ ]. But before [for task $-5+-7$ ] you add these two [numbers] and get back here.

$\mathrm{N}$ : So you're saying that if both 5 and 7 were positive then...

$\mathrm{H}$ : then they go up to the 12 , but since they are both negative, then you do -5 plus -7 , you go back to the -12 .

As she was saying this, she showed me how she was thinking about these tasks. For the task $-5+7$, she started at -5 and traveled up 7 because the number was positive, and in doing so, she landed on the 2 , which represented the answer. For the task $-5+-7$, she reasoned that if both numbers were positive, she would start at the 5 and travel up 7 to arrive at the 12; however, since both numbers were negative, she did the analog in the negative numbers. Thus, she started at -5 and traveled down 7 to the -12 .

It seemed that tasks involving the addition of a negative and positive addend were easier for her to solve that tasks involving two negative addends. When asked to use a number line to solve the task, she started at the first addend and then used the sign of the second addend to tell her the direction to travel and the magnitude of the second number to tell her how many spaces to travel. Since tasks involving two negative addends were harder to solve than tasks involving the addition of a negative and positive, she used a different strategy. She first stripped away the negative signs on both of the addends to create an analogous task involving the addition of two positive addends, used the number line to solve this analogous problem by starting at the first addend and traveling up the number of spaces represented by the magnitude of the 
second addend, and then applied this reasoning to the negative number side of the number line. Thus, she indirectly used the signs of the addends to tell her which way to travel since she first had to decide which way she would travel by changing the signs of the addends.

Since she could accurately translate an abstract addition task into movement on a number line, I wanted to see if she could translate an abstract task into a verbal context. I asked her if she could create a story about the task $-5+-7$.

$\mathrm{N}$ : Could you tell make up a story that used the numbers -5 and -7 and added them?

H: I'm not sure. What do you mean?

$\mathrm{N}$ : Well, like a word problem. Do you do word problems in math class?

H: No.

$\mathrm{N}$ : Have you ever seen negative numbers anywhere - inside or outside of school?

H: Yeah, on my dad's stuff. He's a mechanic. When I go into his office...when he writes stuff down, he uses the negative. I don't know what he uses it for. I've just seen it.

$\mathrm{N}$ : Have you ever seen negative numbers in temperature or dealing with money?

$\mathrm{H}$ : In temperature but not in money.

$\mathrm{N}$ : How could we use the -5 and -7 in a temperature problem?

H: I don't know.

At this point, she yawned and looked around the room. I tried to point back to the paper and guide her in coming up with a temperature problem, but she resisted and said, "I don't want to do this. I'm not good at it." I asked her why she thought she was not good at it and she said, "I've never done this before. Can we do something else?" Since she seemed to be bothered by this task, and her focus kept shifting, I let this task go and moved on to see if she could translate from a number line context to an abstract context.

I asked her if she could write a math story using the dots on the number line depicted in Figure 1. She wrote, " $-6+5=$ " and then she proceeded to tell me how to get the answer.

H: If you want the answer, you have to add this. You start here at 5, and then you count: 1, 2, 3, 4, 5,6 , and you get -1 .

$\mathrm{N}$ : So this dot [pointing to the dot on the -6] tells you how much you are adding?

H: um hmm.

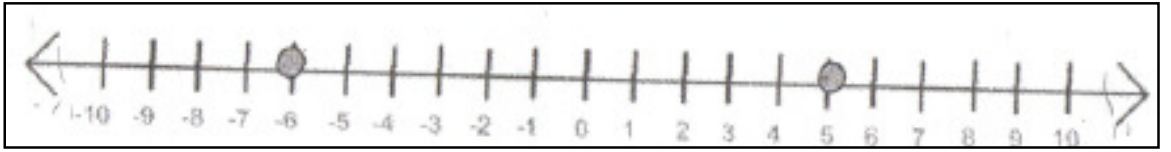

Figure 1. Number line task

It is interesting that when she solved abstract addition problems by using the number line, she used the first addend to signify where to start on the number line and the sign and magnitude of the second addend to determine the direction and number of spaces to count. However, in this instance, after she wrote her abstract expression represented by the dots on the number line, she used the second number in her expression as the starting point on the number line and then counted down 6 spaces because the first 
addend was -6 . I conjecture that she wrote her expression this way because -6 was the first number she came to on the number line with a dot on it when reading from left to right. However this may be untrue given that she used a different strategy when I asked if she could write another task using these same numbers. After my question, she paused for awhile and then wrote, " $5--6$ " and explained, "Five subtract six, and this would be, think it would be. I'm not really sure. It would be 0 or -1 ." Since she did not articulate this very well, I asked her if she could tell me more about how she got those possible answers. She responded, "Cause I subtracted -6 from right here [pointed to the 5] so I subtract 6 from here, and I land here [pointed to the -1 ]". As she said this she started at the -6 , counted 11 to the right, and landed on 5. After this movement, she moved to the left 6 spaces and obtained the answer of -1 . What confused her was that she did not know if she was supposed to go back these 6 spaces or to go back 5 spaces, which would have put her on 0 . In essence she did two tasks on the number line: first she completed the task $-6+11=5$, and then she did not know whether to model the equation $5-6=-1$ or $5-5=0$.

I tried to get her to tell me what she was thinking about as she moved along the number line in hopes to figure out why she was confused.

H: I'm not sure what I'm doing.

$\mathrm{N}$ : Well, let's talk about what you did with your pencil. You started at -6 and counted up 11 to get to the 5 , and then did you go back 6 spaces?

$\mathrm{H}$ : Yeah, because it is -6 .

N: So why did you go up to the 5 ?

H: I'm not sure. This is kind of hard to do. I can do $-6-5$, that would be easy. $\mathrm{N}$ : Ok, let's try $-6-5$ then.

$\mathrm{H}$ : Negative six minus five is...that would be -11 because you go to the left to get -11 from -6 .

I believe that she wrote $5--6$ because she knew that she could use the numbers not only in an addition task, but also in a subtraction task. She knew she wanted to use the numbers 5 and -6 , and since she used addition before, she decided to use subtraction and thus wrote $5--6$. However, because she was unfamiliar with how to solve this type of task, at least on a number line, as will be seen in the next section, she made up a procedure to fit with the tasks that she knew how to solve, which was based on the signs of the numbers. I believe that she counted up 11 simply because she wanted to start at the -6 and get to the 5 on the number line. Based on her mode of operation from previous tasks, she had to start at one of the dots and then move the amount and direction represented by the other dot. Because she did not do this yet, only simply counted the spaces in between the dots, she felt that she had to use the -6 or 5 again in her procedure. This led her to the confusion with moving backwards 5 spaces versus moving backwards 6 spaces. I think she chose to move back 6 spaces because there was a negative sign attached to the 6 , which is more prominent that the implicitly understood positive sign on the 5. After realizing that she did not 
know how to answer this, she acknowledged that if she had written $-6-5$ instead, she could have solved that type of task by starting at the -6 and going backwards 5 to land on the -11 . This gave me a glimpse into how she solved subtraction tasks before we specifically looked at the subtraction tasks.

\section{Prior Conceptions on Subtraction}

Before we looked at the subtraction tasks, she told me that she had seen the negative sign on a number not only used to represent a negative whole number but to also "subtract a whole number." However, she specified that when writing a subtraction task with a negative subtrahend, it was to be written so that "you have ... the negative higher so you can tell which is subtraction and which is the negative." She showed me by writing - - 3 to signify that she was subtracting a negative three. Because she did not write the negative sign higher when she wrote the expression for the previous task $5--6$, I asked her if it was ok if I presented tasks that did not have a higher negative sign. She told me that it would be ok and that she would know what it meant if I kept the negative and subtraction sign at the same level.

Because she interpreted the subtraction sign as moving to the left on the number line for the amount of the subtrahend, Hope had a "take-away" notion of subtraction. In addition to this abstract takeaway notion of understanding, she used the number line to help her explain her answers to subtraction tasks. She believed that if the number had a negative sign in front of it, or if it had a subtraction sign in front of it, she had to move to the left on the number line. Thus, she could solve subtraction tasks involving a positive minuend and subtrahend and subtraction tasks involving a negative minuend and positive subtrahend. She had difficulty when she encountered tasks involving a subtraction sign followed by a negative number. Because of this difficulty, she created alternative procedures to answers these types of tasks.

In subtraction tasks involving a positive minuend and subtrahend, she would abstractly figure out the answer, and then ask for a number line to show me how she got the answer. She would place the tip of her pencil on the number represented in the minuend, and then move left the amount of spaces of the subtrahend. Similarly she solved subtraction tasks involving a negative minuend and positive subtrahend.

When she attempted to solve 5--12 she could not answer this without trying to use a number line first, unlike the types of subtraction tasks mentioned in the previous paragraph. She determined the answer to be -7 .

$\mathrm{N}$ : I saw you using the number line to answer this one. Tell me how you did that.

H: I went to 5 and I subtracted 12 from 5.

$\mathrm{N}$ : What did you subtract from 5 ?

$\mathrm{H}$ : Negative twelve from five. 
$\mathrm{N}$ : And how did you do that?

$\mathrm{H}$ : I went to the 5 and subtracted 12 , I went back 12. I went from the 5 and then $1,2,3 \ldots .12$, and landed right here. So I subtracted 12 from a negative number.

Hope started at the 5, and counted to the left 12 spaces and landed on the number -7 on the number line. It is interesting that throughout this dialogue, Hope verbally refers to the task $5--12$ as the task $5-12$, for she frequently says that she is subtracting 12 from 5 instead of -12 . Yet, when I brought attention to this by asking her again what she subtracted from 5 , she acknowledged that it was a -12 . This led me to wonder whether she was not attending to the subtraction or negative sign in the task, or if she was simply forgetting to say the negative sign when she explained how she solved the task. So, I wrote the task 5-12 directly below the task $5--12$ and asked her to tell me which task she solved.

H: Both

$\mathrm{N}$ : You solved both of them?

$\mathrm{H}$ : Um hmm. They are both -7 .

$\mathrm{N}$ : Is there anything different about the way these two problems look?

H: Yeah. $5--12$ has a negative sign in front of the 12. At first, it looked like two subtraction signs.

$\mathrm{N}$ : Oh, because you usually see the negative sign as higher than the subtraction sign?

$\mathrm{H}$ : Yeah, but I knew what it meant.

$\mathrm{N}$ : Is there a difference between subtracting a positive 12 from 5 versus subtracting a negative 12 from 5 ?

$\mathrm{H}$ : No, it's just a negative number there. It has the same answer.

It is interesting that she described the task $5--12$ as having two subtraction signs. This may signify that she thought it since it had two subtraction signs, it was redundant, and therefore she only had to really subtract 12 once. However, because she could tell me the difference between $5--12$ and $5-12$, even though she solved them in the same manner, I think she believed that the negative number in this situation meant nothing in terms of a solution, but rather was simply just a reflection of the positive number on the negative side of the number line.

Based on her initial method of solving $-4--6$, it seemed that she used the same procedure to solve a subtraction task involving a negative subtrahend as she does involving a positive subtrahend: by moving to the left the magnitude of the subtrahend from the minuend. Yet, after explaining how she solved $-4--6$ in this fashion, she came up with another method.

H: Negative 4 minus negative 6 is $1,2,3,4,5,6 \ldots$ it's -10 .

$\mathrm{N}$ : So how did you know to go left on the number line?

$\mathrm{H}$ : Since you are subtracting and not adding, you are going go left, this way. [pause] This is confusing. Uh, I don't know. This is kind of confusing me.

$\mathrm{N}$ : Which part is confusing you?

$\mathrm{H}$ : The negative minus the negative number?

$\mathrm{N}$ : And why is that confusing to you?

$\mathrm{H}$ : Because when you're subtracting, you are going down. But since this is a negative, you are going down too. Maybe you go up? 
$\mathrm{N}$ : Why do you think you should go up?

$\mathrm{H}$ : Because they are opposites. Like if you fold the number line, like this. So say they weren't negative [numbers] then you'd start it back here, like this was the front and go down. Now if you were subtracting 6 minus 4, so you'd go up if you were subtracting on the other side. This means on the other side you're going up. So you're going up for this problem.

Hope figured that because she normally went to the left when she subtracted, she should move to the left when she subtracted -6 from -4 . However, she became confused because a negative number, similar to a subtraction sign, also meant to move left. Instead of reasoning that the movement was simply redundant, she believed that this might imply that she had to do the opposite of what she would do to solve $-4-6$ on the number line. Since she would solve $-4-6$ by starting at -4 and moving left 6 , she conjectured that she might have to move right on the number line from -4 to solve $-4--6$ because -6 was on the opposite direction of the number line than 6 . She then came up with another method to reinforce this idea using the number line. She took her number line, folded it at zero so that the numbers were on the outside and held it so the positive numbers were showing toward her and the negative numbers were showing away from her (see Figure 2). Then she changed the task by making the numbers positive and switching the order so that she was solving the task $6-4$. She then put her right thumb at the 6 , and wrapped her right index finger around the number line so it was touching the -6 on the other side. She slid her thumb and finger from the 6 to the 4 and said that this was "subtraction" because she was "going down." At this point, she flipped the number line to show the negative numbers and suggested that if someone were watching her perform that action, he would see that she was traveling down and solving the problem $-6--4$. So, to solve $-4--6$, she would slide from the -4 to the -6 , and this means she would be traveling up from 4 to 6 on the positive side, which meant she would be going up 2.

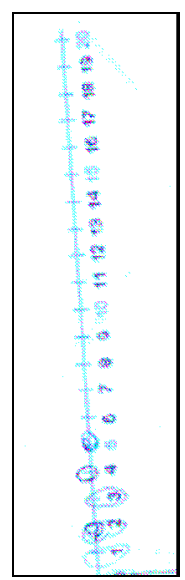

Figure 2. Hope's folded number line

When I asked her how she figured this out she exclaimed, "I do not know! I just figured it out somehow!" I then asked her to reiterate how she found this answer. She said, "When you subtract on the 
positive side, you go down... so if you subtract on the negative side you go up." She did not make reference to the fact that she went up on the negative side because she had to switch the order of the numbers, so I wondered if she simply did not say this, or that she was incorrectly generalizing.

After coming to this realization with the relationship between addition and subtraction of positive and negative numbers, she immediately said, "I think I want to change my answer to 5 - - 12. I asked her what she wanted to change it to and she replied, “ $1,2,3, \ldots 12$. Hmm. Oh gosh, I'm crazy. I don't know what is going on." As she tried to use her folded number line, she started at the 5 and tried to go down 12 spaces. However, in using the folded number line, she ran out of spaces after 5 and did not know how to cross over zero. Her folded number line method only seemed to work when both of the numbers in the subtraction task were positive or both were negative.

When I asked her what was confusing her, she responded, "I don't know which way to go now." I tried to refer back to the direction she traveled in the other tasks such as $5-2$ and $-4--6$ to see if she could figure out exactly what was stumping her.

H: Well I know $5-2=3$. That's kind of easy. And here I counted up [referring to $-4--6$ ]. $\mathrm{N}$ : So why are you ok with these two tasks, but feel that this one is confusing?

$\mathrm{H}$ : Since I' $m$ taking away a whole number, I mean a positive number, I mean, since I' $m$ taking away a negative number away from a positive number. And I'm not very good at it.

She was referring to the fact that in the prior tasks, the signs of the minuend and subtrahend matched, but in this task one sign was negative and one sign was positive and it was in the form that a positive number subtracted a negative number. Because she seemed thoroughly stymied, I asked her if she could tell me how she had gotten the initial answer to the task $-4--1$, which she had originally thought was -5 . She had solved this task before she had realized her folded number line strategy. Thus, she had solved it abstractly by not attending to the subtraction sign and focused on combining the negative four and the negative 1 to obtain the answer of -5 . However, at this point in time, she hastily erased her past answer and wrote a -3 beside the expression. When I asked her why she changed it she said, "That one is easy! Since they are both negative we go down." She subsequently showed me using the folded number line by moving her thumb from 4 to 1 on the positive side and thus her index finger moved from -4 to -1 on the negative side.

\section{Discussion of Prior Conceptions}

Hope seemed to have a unary (Kieran, 2007) understanding of negative numbers in addition tasks with one negative addend. She used different strategies to solve the three different kinds of tasks: positive addends, mixed signed addends, and negative addends. For the positive addends she automatically knew 
how to solve them due to memorization. For mixed-signed addends, she knew that addition of a negative number was similar to subtraction. For two negative addends, she eventually knew that the sum was a negative number, but had to use the number line to obtain the answer. In using the number line for all addition problems, she started at the number represented by the first number and moved in the direction and amount of spaces represented in the sign and magnitude of the second addend. Thus, she moved to the left if the sign was negative and to the right if the sign was positive. This is the same procedure described by Bell (1982). However for tasks that involved two negative addends, she first translated the task into a task involving two positive numbers, used her procedure on the number line to answer the translated problem, and then applied that idea to the negative numbers. By translating the addition task of two negative addends to an addition task of two positive addends, Hope was demonstrating what Murray (1985) described as the strategy of using analogies of operations on positive numbers to help the her answer the same operation on negative numbers. Also in agreement with Murray's findings, Hope was able to solve addition tasks better than she was able to solve subtraction tasks.

Because she felt more confident in solving addition tasks, she used addition prior to subtraction in determining the abstract equation that was represented by dots on a number line. In solving this task, she used the dots as the addends, but had to create the answer on the number line since it was not shown. Hope also came up with the correct subtraction equation that was modeled in this task, however, she could not explain or show how she did so, and could not obtain the correct answer to her subtraction expression. This implied that she simply placed the numbers together represented by the dots with a subtraction symbol in between them. Bruno and Martinon (1996) found that students had difficulty moving from the number line context to an abstract context because they could not formulate operations in the abstract setting, which can be seen in Hope's case.

Hope correctly solved subtraction tasks that contained a positive subtrahend without the use of a number line. She seemed to be able to do this abstractly because she saw the equivalence between the problems $a-b$ and $a+-b$. Since she believed that both the subtraction sign and negative sign meant to move left, she illustrated how she could obtain the answer using a number line by starting at $a$ and moving left $b$ spaces. However, she was initially unable to adjust her subtraction procedures when it came to tasks involving a negative subtrahend. She eventually came up with the idea of folding the number line and using an analogous expression to help her solve subtraction tasks involving a negative minuend and subtrahend. While using an analogous expression containing positive numbers to help her solve a task involving negative numbers was shown in literature (Murray, 1985), I did not come across her strategy of folding the 
number line and generalizing the movement resulting from the subtraction involving positive numbers to the movement resulting from the subtraction involving negative numbers. She could not use this strategy when she had a positive minuend and negative subtrahend because she could not cross over zero, since her number line was folded with the lowest point being at zero. She never figured out a method to solve these; thus, she succumbed to the belief that the answer to $a--b$ was the same as the answer to $a-b$, even though she understood that one task required the subtraction of a negative number where the other required the subtraction of a positive number. This method of solving tasks in the form $a--b$ by translating them to $a-b$ was noted in Peled and colleagues (1989).

With respect to addition, Hope attained Level 1+ in Peled's (1991) Quantitative Dimension (QD) and Level 3+ in the Number Line Dimension (NLD). Hope could accurately solve addition tasks with one negative addend, yet had slight difficulty solving tasks with two negative addends. She consistently used a number line to solve addition tasks; therefore, she earned a L3+ in NLD. In the QD, Hope knew that positive and negative numbers were opposite in quality characteristic, but she never contextualized the task or answer; therefore, she attained L1+.

Because Hope understood that addition meant to step right and subtraction meant to step left, even if a larger number was subtracted from a smaller number, and she was able to recognize that addition and subtraction were opposite in direction and extend this so that she could solve tasks involving same-signed numbers, she at least reached Peled's (1991) Number Line Dimension (NLD) Level 3 with respect to subtraction. Furthermore, she was able to solve tasks with a negative minuend and positive subtrahend using her addition understanding. It was not until she discovered her folded number line strategy that she consistently answered tasks with a negative minuend and subtrahend. Because she viewed the number line as divided instead of continuous, she could not use this strategy to solve tasks with a negative subtrahend and positive minuend. Peled and colleagues (1989) also observed students struggling with subtraction tasks containing a negative subtrahend and positive minuend. They explained that since students were unable to model these expressions due to the consecutive negative signs, students either ignored the negative sign and changed the expression to $a-b$ or they moved one of the negative signs to the minuend and changed the expression to $-a-b$. Hope performed the former strategy. She attained Level 4 - in the NLD with respect to subtraction.

In terms of her level in Peled's (1991) Quantitative Dimension (QD) with respect to subtraction, Hope could accurately solve tasks with same-signed numbers and tasks with a negative minuend and positive subtrahend; thus she could be categorized as operating on L4-. While Hope mentioned that she had 
heard of negative numbers in terms of temperature and money, she did not detail this understanding so that she could be categorized as definitely obtaining Level 1, since students operating on this level view negative numbers as the counterparts to their positive value in terms of being opposite in quality. However, Hope was able to subtract a larger natural number from a smaller natural number, which clearly placed her on Level 2. Since she did not contextualize her answer, but did mention taking away items, she attained Level 2* in the QD with respect to subtraction.

\section{Summary of Hope's Prior Conceptions}

- Hope showed inconsistent conceptual and procedural understanding of addition of negative numbers. She used different procedures to solve different types of addition tasks. When she used the number line to explain her answer, she started at the number represented by the first addend and moved in the direction and amount of spaces represented in the sign and magnitude of the second addend.

- Hope was unable to correctly solve tasks of the form $a--b$. She mentally transformed these tasks into $a-b$, and applied her additive procedures to solve the task even though she was aware that the task specified subtraction. She believed that addition and subtraction tasks containing the same numbers elicited the same answer.

- Hope preferred using addition rather than subtraction to create corresponding equations for problems illustrated on a number line. While she was able to create the correct subtraction task for the number line, she was unable to solve it, which brings into question whether she understood that it was the correct subtraction task.

- Hope believed that subtraction meant take away rather than distance. This is evident in the way she subtracted using a number line. She used the minuend to represent the initial starting place and the subtrahend to represent how far she needed to travel to end up on the difference.

- In working with a number line for both addition and subtraction, Hope believed that the signs of the numbers told her which way to travel. In all cases, negative and subtraction both meant to move to the left, and positive and addition both meant to move to the right.

- Hope attained Level 1+ in the QD and Level 3+ in the NLD with respect to addition.

- Hope attained Level 2* in the QD for subtraction, and Level 4- in Peled's NLD. For tasks involving a positive subtrahend, she viewed the number line as continuous, but for subtraction tasks that involved a negative minuend and subtrahend, she operated on the number line as if it were divided due to her folded number line strategy.

- Hope exhibited a combination of unary and binary understanding of negative numbers. The way she viewed negative numbers depended on the form of the task.

- Hope algebraically solved addition tasks involving negatives and justified the procedure using number lines. She used the same procedure (using the signs of the numbers to tell the direction on the number line) for subtraction tasks involving a positive subtrahend. She used her folded number line strategy for tasks involving a negative subtrahend and minuend. She was unable to correctly solve subtraction tasks involving a positive minuend and negative subtrahend. 
Analysis of Interaction with Subtraction Applets

Because Hope seemed to have an adequate understanding of addition with negative numbers, she did not use the technology instruction in Module 1. Since she had difficulty in understanding subtraction, Hope used the subtraction applets in Module 2. Hope worked with both the Subtraction Arrow Applet (SAA) and the Subtraction Dot Applet (SDA) during her technology instruction sessions. At first she was introduced to the SAA because I wanted to use her notion of take away and build upon it so she could solve subtraction tasks involving a negative subtrahend in the same manner she solved subtraction tasks with positive subtrahends; thus, she would not have to switch strategies for different types of tasks. However, in doing so, she became confused and naturally gravitated to the distance method, so I switched her to the SDA.

\section{Hope's Interaction and Understanding with Technology}

Since Hope used the idea of take-away for subtraction tasks involving a positive subtrahend, she started using the SAA first. After Hope explored how the SAA operated by moving dots and pressing the buttons, I asked Hope to model the task 5 - 2. I wanted to start with a task she already solved using the take-away method and have her generalize how this could be applied to other subtraction tasks. She placed the blue stick on the 5 and then pulled the arrow down two spaces so that the ending dot appeared on the 3 . When I asked her to explain this model she said, "Well, this is 5 (pointing to the 5 in the expression and the blue stick), and this is 2 (pointing to the 2 in the expression) and you go down 2 like this (points to the arrow), and you end up here (points to the 3)."

To extend her thought process about the way she was moving the arrow, I had her model the task $5-0$. At first she pointed to the zero on the number line and asked, "This zero?" Then she left the blue stick on the five, and pulled the arrow so that it landed on the 0 instead of moving the arrow so that the two dots were on top of each other; thus, she was exhibiting the method that would be shown in the SDA. She mumbled, "go to zero?" paused, looked at the screen where the hot-linked expression showed 5-5, and said, "Oh, five minus five." She moved the arrow so that the black dot was above the blue 5 and said, "This is 5 minus 0. I'm right on top of it." When I asked her why she changed her mind, she said that she just got confused for a second but looked at the expression and realized what she needed to do. The fact that their was a hot-linked expression to the number line model was advantageous because it provided her with instantaneous feedback to her actions, which allowed her to change the way she modeled the expression on the number line. I then asked her to model 5--1 in hopes that she would notice the pattern concerning the 
direction to move if the subtrahend was positive, zero, or negative. Before she moved the arrow, she pointed the arrow of the mouse to -1 on the number line and hovered there for a brief second, almost like she wanted to move the arrow so that it would land there - enacting the method of the SDA. After these few moments, she moved the arrow down 6 spaces and landed on the -1 . She looked at the expression, which displayed 5-6, paused and said, "Um..." and moved the arrow so that it landed at 4. The expression showing on the SAA was " $5-1=$ ". She said, "I would say that," but seemed unsure. I asked her if she was confused and she said that she was. This action provided more information of how she viewed a subtraction task involving a positive minuend and negative subtrahend. Since she believed that a negative sign and subtraction sign signified to move left on the number line, she only moved left once and thus believed that $5-1=5--1$. However, she also struggled in determining how she should use the applet to model this type of problem. She seemed to want to use the method employed in the SDA instead of the SAA. Since she seemed confused but could not tell me exactly why she was confused, I assumed that she did not completely know herself and switched applets to allow her to explore the SDA.

After figuring out how the SDA worked, she easily modeled the task $5-2$ by placing the blue dot on the 5 and the orange dot on the 2. When she clicked the "show arrow value" button, I asked her what the 3 represented, to which she responded, "How much you're taking away (pause). The distance between them." As she said this, she used the mouse and circled the distance between the 5 and the 2 . This applet seemed to model her internalized view of subtraction, even though she used the word "take-away" when describing the action of subtraction. Next, she modeled $5-0$. This seemed to cause her great difficulty at first, most likely because she was unsure when she used the SAA for this task. She started out by placing the blue and orange dot on 5 , but then she noticed that the equation said 5-5, and promptly moved the orange dot to the 0 . She told me, "The answer is 5 because you're taking away nothing." Yet, even though she stated this, she also noted that there were "five in between" and that was also the reason why the answer was five. I then asked her to model the expression $5--1$. While she placed the orange dot at -1 , she told me the answer was "four because you're taking away 1, well, pretty much." It is interesting that she moved the dot correctly, and explained to me before that the answer was the value in between the minuend and subtrahend, yet for this task, she reverted back to her old methods of viewing $5--1$ as $5-1$. When I asked her why she thought it was four, she said that it just was. She then pressed the "Show Answer" button and saw that the answer was 6 and said, "Naw, it's six. Oh, because it's in between." To make sure she understood what the distance in between meant, I asked her to predict the answer for $5--2$. She moved the dot to the 2 and answered "seven" without pressing the button that showed the answer to the 
task. I asked her to predict the value of 5--12. She said, "Well, first of all, you would have to move it here (she moved the orange dot to the -12 ), so then the answer would be $1,2,3,4 \ldots 17$ because you have 17 in the middle." When I tried to get her to generalize on the tasks involving a positive minuend and negative minuend to determine what the sign of the difference would be she said, "I don't know. All I know is that I'm tired." I tried to get her to focus on the chart that she had made using these tasks. She was unfocused for awhile but finally said, "Wait a minute! It's like you're going down one. Like that's adding 2 because you're going down 2." Instead of noticing that subtracting a negative subtrahend was like adding, she noticed the differences in the subtrahend and how that affected the differences in the answer. For instance, she saw that when she subtracted 2 from 5 , she received an answer that was smaller than the answer she received when she subtracted 0 from 5 . After seeing this, she determined that when she subtracted-2 from 5 , she would get an answer two greater than the answer she received when she subtracted 0 from 5 . She used the SDA to model this and said, "It's much easier using the this." She liked using the SDA to explain and show the modeling of the problem to find the answer rather than doing it in her head.

H: Well, you're going into the negatives. I don't know. It's seven. Well, since that's 5, you have to go to the -2 , you had to add 7."

$\mathrm{N}$ : Can you tell me a little bit more about that?

H: (Yawn) I'm really tired. I didn't get a lot of sleep last night. Like going 2 to 5, yeah, I'm not really sure.

$\mathrm{N}$ : Well you pointed to something on the screen up here and you said, "Oh, I'm adding"

$\mathrm{H}$ : You're adding two from here. This is 5 right here, plus the 0 to the -2 , so you get 7 . It's how many spaces in between.

While she sometimes would lose focus and state that she did not know certain things, I think she needed something to draw her attention back to the problem. By pointing to the applet and showing me her movements as she explained, this allowed her to concentrate more on the reasoning of how she found the answer. During her explanation, she was able to articulate that subtracting a negative subtrahend from a positive minuend was like adding because she physically saw the distance between the positive minuend and zero and the distance from the negative subtrahend and zero, and noticed that the total distance between the minuend and subtrahend required the values to be added. At one point, when I asked her to find the answer to $7--10$ she said, "You count how many in between." When I asked her if she wanted to count them she said, “No, I don't need to count them, I already know the answer." I asked her how she knew the answer and she said, "It's just 7 plus 10." When I asked how it was 7 plus 10 when the expression used subtraction, she said, "Well, you count in between, but they are both adding." I asked her then if $7--10$ was the same as $7+10$, she replied that they were both equal to 17 . She then used this fact to solve other subtraction tasks with a positive minuend and negative subtrahend and always justified her answer by 
using addition abstractly without using the SDA to solve for or explain her answers.

After using the strategy that subtraction of a negative subtrahend from a positive minuend was similar to addition, I wanted to see if she could apply this same idea when the minuend was negative. I asked her to model $-4--1$ on the SDA. She moved the blue dot to the -4 and the orange dot to the -1 and said, "Negative 4, and then-1. It's negative 3. Because you're taking away -3." It is important to note that she said that it was taking away -3 after she clicked on the "Show Arrow Value" button and saw the -3 appear in the arrow. I think she interpreted the arrow as taking away the value inside of it, which is fine to do, but before she had always said that she "took away" the subtrahend value instead of the answer. I then asked if she could explain that further. She said, "Well, you can really do it either way. Negative 4 minus -1 is -3 or -4 minus -3 is -1 ." As she told me the latter scenario, she moved the orange dot to the -3 and pointed to the -1 in the arrow. Thus, she noticed the equivalence between the tasks $-a--b=-c$ and $-a--c=-b$ where $a, b, c>0$ by noticing the relationship between the expression and answer in the SDA with their graphic representation of the dots and arrow. She may have easily figured this out due to the fact that she could already solve these types of tasks using a different strategy (her folded number line), which allowed her to focus on the relationships rather than solely trying to figure out the answer. While she saw the equivalence of forms, I was unsure that she saw that when she subtracted a negative subtrahend, regardless of the sign in the minuend, it was similar to addition, so I asked her to solve $-4--6$. She modeled this correctly on the SDA and said, "The answer is 2." This time she did not show the arrow value, but simply placed the dots on the appropriate minuend and subtrahend. I asked her how she got her answer.

It's positive because it's a smaller adding a bigger number. Like if you went to the positive 6 . So 4 less 6 , that would be -10 . (pause) I don't know why though. Oh wait, because it would be like adding because you have a 4 and a 6 and they are both negative. But if you want to get a positive, you have to go waaaaay back down here.

While her wording is not totally clear, her actions helped interpret her verbiage. When she said, "smaller adding a bigger number" she pointed to the -4 and then to the -6 . Instead of considering the value of the numbers, she instead used the words bigger and smaller to indicate the absolute value of the -4 and -6 , and thus -4 was smaller in comparison to -6 . She knew she had to add the 6 because the expression was subtracting a negative subtrahend. She compared this expression to the expression $-4-6$ whose answer was -10 . In using this comparison, she realized that $-4--6$ was liked adding and $-4-6$ was like subtracting. While she said "if you want to get a positive," she meant that if she was to subtract a positive that she would end up down the number line on the negative side, for when she said this, she slid the orange dot into the negatives and pointed the expression $-4-6$. To make sure this was exactly what she meant I 
asked her to explain how she knew the sign of the answer. She said, "When the orange dot is on this side [right] of the blue dot, it's positive. When it's on this side [left], it's negative." As she said this she pressed the "Show Answer" button and moved the orange dot to the right and left of the blue dot respectively to show me the sign in the hot-linked difference, while the blue dot remained on the -4 . I asked if she thought this would happen when the blue number was positive, and she said, "I'm not sure, but it happens if the blue is negative, yeah." What is interesting, is that she had done many subtraction tasks involving a positive minuend and negative subtrahend in which she could tell me the way she moved to get the correct answer before attempting these past tasks with a negative minuend, yet she did not remember her strategy.

Therefore, to jog her memory, I asked her to place the blue dot on a positive number. She chose 5. Then I asked her to model the task $5-7$. She slide the orange dot to the 7 and said, "It's negative, weeee, whoops now it's 8." She slid the orange dot down the positive side and then back to 5 and then back to 8 instead of the 7. She seemed to enjoy sliding it because she kept making noises and smiling as she moved it back and forth and watched the answer adjust accordingly. She finally settled on the 7. I asked her why the difference was negative.

Cause you're going...cause you're subtracting seven. This is seven, but if you subtract seven, you go the -2 . So taking it back to 7 , you should get a positive 2 . Wait, but you get a negative 2 . Oh wait, I know why, because you went up. Huh? [pause] Take 7 away from 5, take 7 away from 5, then you get right there see 7 and 7 , and -2 and -2 , and 5 and 5 .

What happened during this discourse was that she started off with the blue dot on the 5 and the orange dot on the 7 and deemed that the answer was -2 . However, when it came to justifying this answer, she used the SDA as the SAA and counted 7 spaces to the left and landed on -2 . At this point, she wondered why she had started at the 7 to begin with, and therefore, brought the orange dot back up to the 7 . In doing this, she thought that since she was going to opposite direction the answer should be 2, but then she clicked the "Show Answer" button to show me she was right, but the answer showed a -2. For a brief moment, I think she remembered that when using the SDA and going to the right of the blue dot or "up" caused the answer to be negative. Yet at this point, she stopped and was not sure what she was doing anymore. Finally she placed the orange dot back onto the -2 and clicked the "Show Arrow Value" button and showed me that the value in the arrow said 7 . She then wrote on her paper $5-7=-2$ and pointed to the minuend and then to the blue dot, the subtrahend and then to the value in the arrow, and the difference and then the orange dot. I asked her whether the equation displaying was the same as the original equation she started with and she said, "kind of, well sort of." While she knew the answer to 5-7, she did not use the SDA as intended to help her explain the answer. She seemed to prefer using the take-away method on these tasks even though 
she knew how to use the SDA to model and answer the task.

Given that she had eventually solved correctly all types of subtraction tasks, I wanted her to come up with a rule about the sign of the answer if the minuend and subtrahend had the same sign. When she tried to answer this question, she realized the difference between the tasks in the forms $a--b$ and $a-b$ where $a, b>0$.

$\mathrm{N}$ : So if these numbers have the same sign, what do you think the sign in the answer will be?

$\mathrm{H}$ : Like if you're taking away 2 from 5? Like they are both positive. Five minus 3, wait no. There we go. It would be 5 minus 2 is 3 . So that would be different.

$\mathrm{N}$ : How do you mean different?

H: You're not taking away; you're not taking um, -2 away from 5, like we just did before, instead you're taking 2 away from 5 , which is 3 .

$\mathrm{N}$ : How did you know they were different?

$\mathrm{H}$ : Because you count the numbers in between. Here it's 4, 3, 2 .

What happened was that she began modeling her created task of $5-2$ by putting the blue dot on the 5 and the orange dot on the 3 so that the arrow would show that she took 2 away. However, the screen showed the corresponding expression $5-3$. She seemed confused at this first, and then moved the orange dot to 2 so the equation read $5-2$. When asked how these two expressions were different, she instead told me the difference between the expressions $5--2$ and $5-2$. She modeled the difference in these two expressions by moving the orange dot to the -2 at first and showed me the answer was 7 when she counted in between, and then she moved the orange dot to 2 and counted down from 5 the numbers in between 5 and 2. I think she compared these two tasks because she remembered doing $5-7$ prior to this task. Despite why she showed me the difference between the two expressions, the fact was that now she knew there was a difference in the answers between the two expressions, something she did not know before because she believed that $5--2$ and $5-2$ elicited the same answer.

I asked her to solve some tasks for me without the use of the computer. While she correctly answered each task, she used different explanations for different types of tasks. When she solved tasks in the form positive minus a negative, she explained that she added the two numbers. When asked if she could explain it another way, she used the number line and put her pencil tip on the minuend and her left index finger on the subtrahend, and counted the spaces in between with her pencil tip. When she solved a task with a positive minus a positive, she explained that she started with the number represented by minuend on a number line and moved to the left the number of spaces represented in the subtrahend. However, when I asked if she could explain it another way, she said, "Well, we can put a dot at 4 and a dot at 8 , but it would still be -4 because you'd still have 4 in between to get to 8 ." This was her second explanation to the task $4-8$. When she solved tasks involving a negative minus another negative, she used the folded number line 
and did not specify another way to solve it because "since the two numbers are negative, I can use this way." When she solved tasks depicting a negative subtracting a positive, for example, $-6-2$, she paused and at first said 4, but then reneged and said, “well, no, no it's not, 6 minus 2 is 4, but this is a negative number" referring to the 6 . After a few moments, she asked if she could use the SDA to help her figure out the answer, but before I could answer her, she grabbed the computer and mouse and started modeling it. "It would be -8 ! See, you're subtracting 2 from the -6 ! Wee, this is cool!" As she said this, she slid the dots back and forth in the SDA. Then she went on to explain why she was confused at first.

Well, I thought that if you were subtracting 2 it would be like this, because you were subtracting 2 from -6 . I just figured it would be -4 . But it's -8 because, because this needs to be here, because it makes more sense if it is here. Actually, it's kind of like adding.

Instead of putting the orange dot at 2 , she instead tried to solve the task in her head and came up with the answer -4 . She placed the orange dot at -4 and the equation displayed $-6--4=-2$. Then she said that she realized that she needed to place the orange dot on 2 because it made more sense to place the dot on the subtrahend value, and in doing so, she got the answer of -8 . I think she believed this process was like addition because she moved to the right on the number line; furthermore, she added the absolute values and placed a negative on the answer. When I asked her to explain exactly what she meant by adding, she said the following:

Because -6 plus, I mean minus 2, it's like -6 minus 2 , so you have -6 then you take 2 away, well, you would go this way and that's -8 . So like if it's a negative minus a positive you have to count this way because here, we went up 6 to get to zero, and then we have to go up two more to get to 2. So for a negative minus a positive, you have to count right.

At first she placed the blue dot on the -6 and the orange dot on the 2 and waved the mouse around the distance between the middle. Then she used the words "take 2 away" and all of a sudden, counted from the -6 down by 2 to land on the -8 . She realized that she could solve it both ways and still get -8 . She reverted back to what was showing on the SDA and explained it in terms of how she modeled it using the SDA as well. At this point our time had expired.

The next time we met, I wanted to see if Hope remembered what she had worked on from the first session. I asked Hope to solve the following tasks: $6-2,6-9,-4-8,6--10$, and $-4--1$. Unfortunately, Hope could not accurately solve the tasks that did not involve a positive minuend and subtrahend. What is interesting is that in diagnosing her conceptions in the first section of Module 2, she was also able to solve tasks involving a negative minuend and positive subtrahend, but not this time. For the other tasks, she seemed to revert back to her conceptions prior to working with technology. Her explanations for the first task did not change, for she believed the answer to $6-2$ was 4 because she "took 
two away from six." However, what is interesting is her adjusted explanation for why $6-9$ equaled -3 .

Before she would have said that she took 9 away from 6 so she had to go into the negative numbers because she did not have enough, however this time she said, "Since you can't take 9 away from 6, I just went down into the negative numbers and got 3 . I started here, then $1,2,3,4 \ldots 9$, so I went down into the negative numbers." This time, she believed that she could not subtract 9 from 6 , so she had to use a number line in order to get the answer. She then showed me how she counted on the number line: she started at 6 and went to the left 9 spaces to land on the -3 .

At first she believed that the answer to $-4-8$ was 4 , based on the notion that she was adding these numbers and using her folded number line idea.

I got 4. If you had -4 take away 8 you would go up the chart. Like so if you folded it in half. So it's supposed to go this direction, wait no, this direction, so subtraction and addition. So 4, yeah, you add up and subtract down. So since adding is going up on the positives, then when you turn the number line around to the other side, then adding is going up on the negative side.

As she said this, she folded the number line at zero with the positive side showing towards her, placed her thumb on the 4 , and wrapped her index finger around so that it was touching the -4 on the other side. Then she slid her thumb and index finger up the number line to the 8 . She said that the answer was 4 because she moved 4 spaces from the 4 to the 8 . Since she thought this was what she was doing on the other side moving from the -4 to the -8 , she thought the answer would be the same. I think she initially transformed the task $-4-8$ to $-4+-8$, and that is why she was performing addition on her folded number line. I think she may have been remembering the SDA and that is why she started at the 4 and moved to the 8 (on the positive side, and -4 and -8 on the negative side respectively) and counted the spaces in between to get the difference. Yet, once she changed the task to addition, this procedure did not work. She was combining her notions of the folded number line and the SDA to try to help her find the answer. When I asked her to clarify whether she was performing addition or subtraction, she told me that she was doing subtraction and went on to explain herself again.

See, if we took -4 and then subtracted 8 , I go up, wait [pause]. Did I do that backwards? So if negative four subtraction 8 , I'm subtracting this one...hmmm... - 4 subtract 8 [mumbles]. How did I do that? I didn't get the same answer as earlier. How did I do that? I didn't get what I did. I always do that! Now I'm getting -12 , but I'm not sure.

As she said this, she fiddled around with her folded number line sliding her fingers up and down and flipping it from one side to the other side. Finally, she put the folded number line down, and wrote the task $-4-8$ on her paper and wrote -12 . Since she knew how to solve this type of task abstractly before working with the SAA and SDA, I think she simply needed to focus on the task without the distractions of trying to explain it on the number line. When she did this, she found the correct answer. I think she mentally 
transformed this task into $-4+-8$, so she could mentally solve it. However, since she could not explain it using a number line, she confused herself with trying to employ different methods.

For the task $6--10$ she got -4 for the difference because she used the number line and started at 6 and moved to the left 10 spaces, similar to how she would solve $6-10$. For the task $-4--1$, she said that she was not sure if the answer was -5 or -3 , but she would not explain how she got those two answers.

To help her move past her confusion, I asked her if she wanted to use the technology applets to help her figure out the answers. She responded, "Yeah, I like them! They're cool! We can do neat stuff with them." When I asked her to explain what she meant by neat stuff, she said, "Like play around on them to do subtraction." However, she also noted that she sometimes got confused using them. When I asked her what was confusing, she said, "It's only kind of confusing, but it's also kind of easy. You know where to put the dots, and to count the numbers in between, that is subtraction, but I'm not sure why it's positive or negative." She knew that she had to place the dots on the minuend and subtrahend and count the spaces in between to find the magnitude of the difference, but she did not understand (or at least did not remember since she was able to tell me before) when the sign of the difference would be positive or negative.

Because she had difficulty with determining the sign of the difference, I encouraged her to use the SAA because it agreed with her prior knowledge of take-away. In using this prior knowledge, I hoped to get her to see that when she took away a positive number, she would move left, and therefore, had to go the opposite direction when she took away a negative number since the negative numbers were essentially opposite of positive numbers. She modeled the $6-2$ correctly using the SAA and told me that she knew to go left since "I'm subtracting, so you have to!" Similarly she modeled the task $6-9$ and landed on -3 . As she solved this she said, "I'm counting how many are in the middle." What she did was count 9 to the left of 6 and thus there were 9 between 6 and -3 . I then asked her to model $-4-8$ in hopes that she would use the same strategy: start at the minuend and move to the left the amount of spaces of the subtrahend. Instead, she placed the blue stick on the -4 and dragged the arrow right so that it landed on the 8 . After this action she said, "Woah...um." She paused and then dragged the arrow so that it landed on the 4, so that there were 8 in between. At this point, there was no equation showing on the SAA. I asked her to explain why she moved right this time.

H: Because you have a negative number. Well those are positive numbers, so you go left. Now we started with a negative number, so we go right.

$\mathrm{N}$ : So for $6-2$ and $6-9$ you went left. But for this one $-4-8$ you went to the right?

$\mathrm{H}$ : Yeah, because it was subtraction. [long pause] Oh my gosh! I did it again! I don't know what I'm doing. I'm confused!

$\mathrm{N}$ : What are you confused about?

$\mathrm{H}$ : The direction I'm going? 
She seemed to believe that if the subtraction task had a negative minuend, then she needed to move to the right, however, she never believed this before. At this point in time, I asked her to press the "Show Expression" button on the SAA. It displayed $-4--8=4$. At first she said, "See it's 4." However, she noticed that the expression was not the original expression. Yet, she disregarded this and tried to explain it to me using her folded number line strategy.

H: I was subtracting. So here you have a positive number (points to the 8) so you should go right on that. Yeah, subtract. You subtract 8 from -4 , so you go right. Because you go this and this and down to zero, which is both subtraction. And from here to here is addition.

$\mathrm{N}$ : Can you go over that with me again?

H: If you go from the -4 to the 0 and the 4 to the 0 , you get 4 , and then you're adding them because you did both, because you're going each way.

When Hope said, "because you go this and this and down to zero" she placed her right index finger on the 4 and her left index finger on the -4 and slid them simultaneously toward zero. Since she was going toward zero, she believed this movement embodied subtraction since it was down. As she did this, she noted that there were 4 spaces between 4 and 0 and -4 and 0 ; thus, when the number of spaces were summed, it was a total of 8 spaces in between. Thus, she believed that she was subtracting 8 from -4 . It is important to note that she had never used her folded number line strategy when the signs of the minuend and subtrahend differed, because her folded number line strategy had to be modify in order to do so. Thus, she invented this new idea to deal with subtraction of numbers with differing signs.

Since she did not seem to agree that the expression and the number line model was linked in the SAA, and because she was not consistent in moving to the left when she subtracted a positive subtrahend, I let her use a calculator to determine the answer for $-4-8$.

$\mathrm{H}$ : Grr. Negative 4 minus 8 is -12 .

$\mathrm{N}$ : So do you know why it is -12 ?

$\mathrm{H}$ : Because you're adding 8 to the -4 .

$\mathrm{N}$ : You're adding 8 ?

$\mathrm{H}$ : We move it to the left instead of having 8 in between the -4 and 4. It just doesn't make sense.

When she shouted, "Grr," she slammed the calculator down on the table with disgust. I think she was frustrated because she believed that the calculator gave her the right answer, but she was unable to understand why it worked. When she did describe why it worked, she used the word "adding" to explain. I think she was looking at the expression $-4-8$ and using the strategy that Bell (1982) saw in his students: they factored out the negative, added the 4 and 8 , and then tacked on the negative sign; thus, she added 8 to the -4 .

Because she was frustrated, I tried to relate her movements to the left with the other tasks 
containing a positive subtrahend. She caught on and said, "I just realized that's $-4-8$ instead of $-4+8$. Oh, I see what I did before, I added before instead of subtracted. So it is subtracting going to the left either way and addition THAT way!" When she said, "either" she pointed to the different signed minuends. At one point in time, she thought that she went to the right because she had a negative minuend, instead of following the same direction because the subtrahend was the same sign in all of the compared tasks. After giving her other tasks containing a positive subtrahend, she was able to solve them all using the SAA and number line, and eventually without a number line. Therefore we moved on to tasks involving a negative minuend and subtrahend.

I asked her to model the task $-4--1$ on the SAA. She placed the blue stick on the -4 and moved the arrow to left and said, "Because it says subtract a negative one, we go to -5 ." Since she had just told me that subtract meant move to the left, I think she was assuming that subtracting of any number meant she had to move to the left. So I asked her what she would do to solve the task $-4-1$ on the SAA. She started to repeat the same motions and said, "Wait a minute! Aaah. What am I doing?! Negative four minus negative one is negative three." When I asked her why she changed her answer she yawned and said, "Because it's subtracting a -1 instead of just a 1." By having the visual displaying of how she solved $-4--1$ on the SAA, and her repeated actions to solve the different task $-4-1$, she realized that she had made a mistake and changed her answer. She realized that since $-4-1$ and $-4--1$ were different tasks, she should manipulate the SAA differently to solve them; therefore, she resolved the task $-4--1$ by moving the arrow to the right one space. The fact that she had the display of her prior actions showing on the screen as she tried to solve the second task, allowed her to visually compare her movements. The fact that she had to enact the same movements also helped her realize that they were the exact same movements she used to solve $-4--1$. Thus, by using the visualization and manipulation of the applet, and the knowledge that $-4-1$ and $-4--1$ were different tasks, helped her realize the direction she had to travel to subtract a negative subtrahend. After coming to this realization, she was able to solve tasks involving a negative subtrahend and minuend without the SAA. In explaining her answer, she would take a bare number line, place her pencil on the minuend and travel to the right the value of the subtrahend and land on the answer.

She followed the identical process in solving tasks involving a positive minuend and negative subtrahend. At first she modeled $6--10$ as if she was modeling $6-10$. When I asked her to model $6-10$, she noticed what she had done and changed her answer to $6--10$ from -4 to 16 . She had told me it was "like adding" and when she solved similar tasks, she added the absolute value of the number together to 
obtain the answer. However, when asked to explain her reasoning, she would show me on a bare number line and move to the right because she was "subtracting a negative." In fact, in solving four more tasks without the applet, she told me that she had to move left on a number line when she subtracted a "positive second number" and move to the right when she subtracted a "negative second number." Thus, by enacting the movement of the subtraction tasks on the SAA, she understood that the sign of the subtrahend determined the direction of movement on the number line.

At the beginning of the second session, I had asked Hope to solve subtraction tasks without the applet because I wanted to see if she could still solve them correctly since she had done so at the end of the first session; however, she was unable to solve them all correctly. Thus, I wanted to check to see if she could still do addition tasks since she was able to do them in the first session. I asked her to solve $7+-2$, $7+-10,-4+8$, and $-6+-4$. After tapping her pencil and looking around the room, I finally got her to focus on these tasks. While she obtained the correct answer for all of these problems, she explained that the first two were "like subtracting," the third was like "adding," and the last she did not qualify as either, instead she told me which direction she had to travel on the number line, which was determined since both numbers were negative.

At our last session, I began by asking Hope to solve subtraction tasks for me to see if she remembered what she had learned from the previous two sessions. She easily solved tasks involving a positive subtrahend. However, when I asked her to solve tasks containing a negative subtrahend, she had difficulties. For instance, when I asked her how to solve $8--13$, she was unsure of the answer or how to solve it.

H: Hmm. Pretty sure that that would be -5 , but I'm not really sure.

$\mathrm{N}$ : Is there a way you figure it out?

$\mathrm{H}$ : I could use a number line. Well, subtracting is this way, so but hmm. And that's where -13 is too. But I'm confused.

$\mathrm{N}$ : Could you explain to me what you did there on the number line?

H: I stuck my finger on 5 and my other finger on -13 , but I need to count down by 13 , but the number is -13 , so I' $m$ not sure I'm right.

$\mathrm{N}$ : Hmm. What if you had the task $8-13$, how would you solve that?

$\mathrm{H}$ : Well, that would be -5 because you can't take away a bigger number away from a smaller number and get a positive number.

$\mathrm{N}$ : Ok, so what about $8--13$ ?

H: I'm subtracting -13 . I'm pretty sure they are the same though, but not quite sure.

$\mathrm{N}$ : Hmm. Well, I saw that you just put your finger on the 8 and your pinky on the -13 . What are you thinking?

H: I don't know. Hmm. Well, I was doing the 8 take away -13 , but then I don't know why I put my finger on the -13 . See that's my problem, taking away negative numbers away from whole numbers.

As depicted in the above discourse, Hope struggled between her ideas of subtraction as take-away and the 
memory of the SDA. She believed that she should start at 8 and move to the left 13 spaces because she was subtracting and -13 was located to the left of 8 on the number line. However, her gestures on the number line did not correspond with her take-away view of subtraction. She had placed a finger on the 8 and a finger on the -13 , much like she did with the SDA dots. It seemed that this process lurked in the back of her mind, but she could not reason why it was there or how to use it to solve this task. Instead, she resorted to reasoning through the task using her take-away notion of subtraction. This conflict led her to believe that her original answer of -5 was not right, but she could not articulate why. Yet, this time she was aware that even though she thought $8--13$ and $8-13$ might elicit the same answer, she was not completely sure that they did.

To try to incorporate her notion of take-away and have her realize the direction she needed to travel in solving this task, I allowed her to use the SAA. She moved the stick and arrow around with only the arrow value showing for a few seconds and talked her way through her reasoning.

Ah, yes, each time I move to the right, I am taking less away each time. See that's taking away 1, and now this is taking away 0 - you're taking away nothing. So if I go right one more time, I take away $-1 \ldots \mathrm{OH}$ ! The arrow tells you how much you are taking away. But if you look on a number line, the number line decreases going this way, and increases going this way, but the arrow doesn't....Since you're taking -1 away, then that means you're pretty much adding 1 .

By moving the arrow around and observing the change in the expression and answer, she realized that each time she moved the arrow to the right, she was subtracting one less and each time she moved it to the left, she was subtracting one more from the number represented by the blue stick (the minuend). She juxtaposed the movement of subtraction of positive and negative numbers with the left and right sides of the number line. I do not believe that she thoroughly understood this oppositeness - that subtraction of a positive number required her to move left and why the subtraction of a negative number required her to move to the right, even though the numbers left of zero were negative and the numbers to the right of zero were positive. Yet, she did notice that the subtraction of a negative was the same as addition. After this discovery, I asked her to solve $8--13$ without the use of the SAA. She looked at a bare number line, started at 8, and counted up 13 and said, "21! Because it's like adding." She could solve any task I gave her that had a positive minuend and negative subtrahend using her idea of adding without using a number line. Thus, I asked her to solve tasks involving a negative minuend and negative subtrahend. She easily solved this by moving to the right on a bare number line. After correctly answering a few she told me, "I want to show you on the computer how I did it." When I asked her why she would rather use the computer when she could correctly solve them without it, she told me "It's not as much complicated." I asked her to tell me how the SAA helped her solve these tasks. 
If you're subtracting negatives, you go up, if you're subtracting positives you go down. Say you had a 6 and you're taking 8 away, it would end up to be -2 , but if you were at 6 and taking away -2 , it would end up at 8 .

By using the SAA, she was able to see the equivalence between $a-b=c$ and $a-c=b$. During our sessions together, she seemed to like the manipulatability and colorfulness of the SAA in helping her solve the tasks. I think it also helped her focus and concentrate one why she was moving a specific direction and how the sign of the subtrahend, and hence the movement, determined the sign of the difference.

Because she seemed very comfortable using the SAA and describing why subtraction of a negative subtrahend was like addition using this applet, I wanted to make sure she had a connection between the SAA and the SDA, especially since she seemed to try to use the distance idea in solving $8--13$ at the beginning of the third session. After exploring the SDA, I asked her to model the task $-10--3$. She put the blue dot on the -10 and the orange dot on the -3 and told me the answer was " -7 because you're taking 7 away from -10." Yet, after she said this she shook her head and said that the expression and said, that it needed to be written $-3-7=-10$. When I asked her how she knew it was -7 , she said, "because I went that way. If I went the other way, then I would be adding 10, so it would then be at 7." Even though she modeled the expression using the distance method, she still understood the expression as take-away and tried to rearrange the expression to fit the SAA. Since she knew how to solve $-10--3$ and that the answer was -7 , she answered -7 , however, she did not use the SDA visual to explain it, and rather she rearranged the dots so that the SDA was using the SAA methods. She went on to try to explain it better to me.

Yeah, cause if you were at 30, say we're at 30, and you wanted to subtract, I don't know, maybe 50 or something, you would end up at -20 because 50 is bigger than 30 and 30 plus 20 would be 50 , so it would be at -20 . So that's 30 , and that's 20 , so you would do is add the 30 and the 20 to get 50 .

As she said this, she placed the blue dot on 30 and the orange dot on -20 and noted that she subtracted 50 from the 30 to get to the -20 . She referred back to her take-away method. When I tried to ask her if she could write an expression with the 30 and -20 and subtraction, she looked at me quizzically, and asked, "Change the signs?" I had her take notice to the equation that was hot-linked to her model, which showed $30--20$. She agreed that the answer to that task was 50, but that was not the task she was illustrating. When I asked her what she thought the blue dot, orange dot, and arrow represented, she said, "The first number...the second number... and the total in here." She knew that the 50 was represented in the distance between the 30 and -20 but did not view the visual on the number line as illustrating subtraction. In fact, she grabbed the mouse and opened the SAA and said, "This is subtraction, and the other way is the total 
value." As she said the latter few words, she maximized the SDA. When I asked her to explain further she said, "This is the in between total, and that's subtraction. See, 50 is in between here and that's subtracting 20." As she said this, she had the blue dot on the 30 and the orange dot on the -20 and waved her mouse to point at the 50 in between. Then she pointed to the blue dot at 30 and pointed to the right and counted up 20 to land on 50 to illustrate the subtracting of -20 . She then said, "Pretty much you're just switching the answer and second number."

To make sure that I understood what she was saying, I asked her to $1--2$ using a number line. At first she pointed to the 1 and then to the -2 and said, "That will be $-2,1$, take away, equals 3 . But if you were to do 1, it would be like that." To show me the second method, she put her pencil on 1 and went to the right 2 spaces and landed on 3 . When I asked if she could use either way to show subtraction she said, "Um $\mathrm{hmm}$. That's what you're taking way, and that's your answer. And then that's what your answer is and that's what you're taking way." As she said this, she pointed to the -2 on the number line and waved her pencil in between 1 and -2 to signify the difference, and then pointed to the 3 and the 2 spaces in between the 1 and the 3 to signify the amount taken away.

After realizing the connection between the two applets, she consistently used both explanations (without the applets) in conjunction with a paper number line to explain her answer to the 10 tasks that I gave to her during the remainder of the session. While she did not need a paper number line to solve the tasks, she did use it to explain her answer. In fact, at one point when I asked her how she obtained her answer she told me, "Cause if you're ADDING a negative number, then it's pretty much like subtracting a whole number, and if you're SUBTRACTING a negative number, it's like adding a whole number." She emphasized adding and subtracting in her sentence like she wanted to tell me once and for all how she solved these tasks. It is interesting that she classified tasks in the form $-a-b$ as adding a negative number even though there was no addition sign in the expression. This may be due to the fact that she viewed $-a-b$ as opposite of $-a--b$, which she viewed as subtracting a negative. After this statement, I asked her to solve one more task: $-9--11$.

H: 2. Cause you take 9 away from 11 you get 2 . so it's pretty much, 9 minus 11 is -2 . so -9 minus -11 is 2 . If you use a number line, -9 is right here, minus -11 , which is right here, so 2 , or $1,2,3 \ldots 11$. right there.

$\mathrm{N}$ : So how did you know to go to the right?

$\mathrm{H}$ : Because you're taking, your questions are hard,. Kind of like adding 11 to a negative number, because when you're subtracting a negative, you're adding, so you go right.

Hope now used multiple strategies to explain her answer. First, she made analogous tasks. She solved 11-9 and obtained 2. She used this equation to confirm that $9-11=-2$, which she used to explain the reason for 
$-9--11=2$. As if this explanation was not enough, she also showed me both methods using the number

line in the manner of the SDA and the SAA, respectively. She then connected the reason of why she moved to the right when she subtracted a negative number, because moving right was synonymous with addition.

\section{Summary of Hope's Interactions and Understanding with Technology}

- Hope had both improved conceptual and procedural understanding of addition and subtraction of negative numbers.

- By manipulating multiple sequences of subtraction expressions, noticing patterns, and noticing the connection between movement away from the minuend and the corresponding equation, Hope was able to see subtraction as both take-away and a distance. She was able to explain solution methods of different subtraction tasks using multiple strategies.

- In working with a number line for both addition and subtraction, Hope was able to reason why subtraction of a positive number meant to move left whereas subtraction of a negative number meant to move right. Due to seeing this connection, she was able to accurately solve subtraction of negative subtrahends by changing the signs as well as by moving to the right on the number line from the minuend.

- As Hope worked through the technology applets and tasks, she decreased her frequency of following a specific method of solving a specific type of subtraction task. In fact, she no longer needed to use her divided number line since she was able to apply both the distance and take-away notion to a number line. While she was able to solve all subtraction tasks, it is important to note that she seemed to be able to do this at the end of each session, but would not consistently remember for the next session. Yet, as she worked through the technology applets and saw visual representations and connections between the algebraic equation and movement on a number line, her memory of parts of understanding of procedures and concepts of subtraction improved each time.

- While Hope's attention seemed to wander during our sessions, when she manipulated and observed the visual on the technology applets, her attention was focused. This was not always true when she solved tasks abstractly or when she had to justify her solution methods without the computer. In fact, she wanted to use the technology applets to explain her solutions because she enjoyed manipulating the applets.

- After working with the technology and tasks, Hope successfully attained Level 4- in both Peled's QD and NLD.

\section{Discussion}

Because Hope did not have a clear conceptual understanding of subtraction prior to working with the technology applets, she used a variety of procedures to solve subtraction problems. She based the type of procedure she used on the type of task. She could accurately and abstractly solve tasks in the form $a-b$ and $-a-b$ where $a$ and $b$ were non-negative integers. While she could accurately solve tasks in the form $-a--b$, she could only do so by using a divided number line. Hope could not accurately solve tasks with a positive minuend and negative subtrahend. In solving these types of tasks $a--b$, she believed they were 
equivalent to the task $a-b$. Therefore, she solved them on the number line in the same manner: starting at $a$ and moving left $b$ spaces.

As Hope worked with the technology applets, she was able to solve all tasks abstractly since she saw that the subtraction of a negative number was addition of the absolute value of that number. She also was able to solve subtraction tasks using multiple strategies including the take-away method and distance method. Even though she was able to learn and develop strategies and insights into subtraction tasks, it took her awhile to thoroughly understand these ideas and remember them.

Even though Hope seemed to have a "take-away" notion of subtraction, the SAA did not improve her understanding of subtraction of a negative subtrahend. Even though at first the hot-linked expression helped her to decide how to model the task on the number line, she eventually ignored it when it came to modeling 5--1, for instead she modeled 5-1 and believed that task elicited the same, correct answer. Based on this conception, she used the SDA. She relied on the hot-linked expression to guide her direction in manipulating the dots on the number line, and even realized subtraction as a distance between the minuend and subtrahend. Furthermore, by seeing the distance between the minuend and subtrahend, and the amount measured from each number from zero, she was able to make the connection that it was similar to adding. However, she did not see this abstractly, i.e. that she had to change the subtraction and negative sign to positive signs when they followed one another, but rather specifically to the number line and summing the distance between the minuend and zero, and the distance between the subtrahend and zero.

Using the SDA she also saw the equivalence relationship between the tasks $-a--b=-c$ and $-a--c=-b$ where $a, b, c>0$, which she had not mentioned before. By using this applet she also saw the difference between $a--b$ and $a-b$, which she had previously thought were the same. During this session, after experiencing both the SAA and SDA, she did not consistently use one method to solve a task, even though when I asked her to use the method and a bare number line, she could was able to do so. When solving tasks in the form $a--b$, she added the numbers, and then showed me using the method of the SDA on a bare number line. When solving tasks in the form $a-b$ and $-a-b$, she was able to use both methods to explain the answer. When solving tasks in the form $-a--b$ she solely relied on her folded number line for an explanation.

After her first instructional session, Hope seemed to have a better understanding of subtraction with negative numbers, however when we met for our second session, she did not seem to remember what I had thought she had learned, for she could not accurately solve tasks that did not involve a positive minuend and subtrahend. I think her inability to solve some of these tasks was due to having too many 
strategies and not thoroughly understanding how to apply one across each type of subtraction task. When we met for the second session, she tried to combine her knowledge of the SDA and the folded number line to solve tasks in the form $-a-b$. By doing this, she inappropriately took pieces from each strategy and obtained the incorrect answer. Eventually, she disregarded the number line and could correctly solve this task abstractly. I think the strategies that she had learned interfered with her prior conceptions and strategies, so much so that it created more confusion. Throughout the session, it was found that the SDA had helped her realize that subtraction could be considered as a distance between the minuend and subtrahend, but she had not understood how to determine the sign of the difference. During the second session, we addressed this issue using both the SDA and SAA. However, she did not believe the hot-linked answer on the applets. Thus, it was necessary for her to use a calculator for her to realize that she solved the tasks incorrectly. After realizing the correct answer, this helped her reason through the movements on the number line using the SAA and determining the sign in the difference. She came to realize that she needed to move to the left when subtracting a positive subtrahend and move to the right when subtracting a negative subtrahend, and noted that this movement was similar to addition.

Because Hope made more connections among subtraction, movement on the number line, and how to solve tasks abstractly, and could do so for many problems prior to the ending of the second session, it seemed that her conceptual and procedural understanding improved. However, when she returned for her third session, Hope could still not correctly solve tasks with a negative subtrahend. Yet, she was aware, unlike the previous time that she did not feel as confident in her incorrect answer. In solving a task in the form $a--b$, she combined her knowledge of the SDA and SAA methods, but not appropriately. I think this was due to the fact that she seemed to like using the SDA method in solving tasks in the form $a--b$, but kept thinking of subtraction as "take-away." Unlike the previous sessions, it only took 1 example with the SAA to help her remember how to solve this task. Through the visualization and manipulation features, her memory of the method returned and she generalized how subtraction of a negative subtrahend was similar to addition. Furthermore, she could solve accurately every subtraction task I gave to her. Thus, I wanted to make sure she thoroughly understood the SDA. By using both of the applets, she was about to see the equivalence between $a-b=c$ and $a-c=b$ and was able to use both methods to explain how to solve different subtraction tasks. She also was able to visualize the connection of the SAA to the SDA and have a true unary understanding of the negative sign. Because she was inconsistent in finding the correct answers, if I had more time, I would interview her again to see if she could remember all that she discovered in our sessions. 


\section{The Equal Sign}

\section{Prior Conceptions}

Prior to technology, Hope viewed the equal sign as both operational and relational dependent upon the task. For Tasks 1 and 2a, her responses could be classified as operational, whereas her responses to Task $2 b, c$, and $d$ could be classified as relational. When asked what was similar and different in the expression/equations in Task 1, she answered in terms of superficial features.

The three 5+6's are the same, and the equal sign in the same in the last two... That's 5 plus 6 , that's 5 plus 6 , and that's 5 plus 6 . This has an equal sign, and this has an equal sign and the eleven. And that one's all by itself.

I then asked her what she thought the equal sign meant after viewing these three statements, primarily pointing to the second and third statement. She pointed to the second equation and said, "It equals something, but we don't know what it equals, well not quite." I asked her to explain that to me a little more.

H: Well, we don't know what it equals yet. Cause we're starting with this, [pointing to the $5+6$ ] and then we have to actually add them together to get the answer, but 5 plus 6 equals 11 .

Everybody knows that. Well, except maybe Irma Pope.

$\mathrm{N}$ : So what about this one, the $5+6=11$, what do you think equal sign means here?

$\mathrm{H}$ : Equals means, what 5 plus 6 equals.

She believed that equal sign meant that she had to add the terms in the left expression in order to obtain the answer on the right. This was further evident in how she answered Tasks $2 \mathrm{a}$ and $2 \mathrm{~b}$.

For Task 2a, she told me that she had never seen someone use an equal sign to separate the same number. In fact, she believed that is was not the correct to write it simply based on the fact that "it just look[ed] different." When I asked her what she thought the equal sign meant in this context, she told me that it meant "nothing," but when I asked her to guess why someone might write it that way, she responded, "Um, to say that 3 equals 3 plus something, I'm guessing. Like if we add 3 plus 0 .” Thus, she believed that the equal sign signaled for someone to apply an operation to two numbers on one side of the equation in order to obtain an answer on the other side. In fact, she more firmly believed that about $3=3$ after seeing Task $2 b$.

$\mathrm{N}$ : Ok, so what do you think the equal sign means in $8=10-2$ ?

$\mathrm{H}$ : Oh, I get it, back there it means that 3 equals 3 minus 0 . Because 8 equals 10 minus 2, except it's kind of backwards.

When I asked her to explain what she meant by backwards she pointed to the $10-2$ and said it should be "over there" where the 8 was and the 8 should have been where the $10-2$ was. Yet, I asked if it was ok to write this way, and she responded, "It's ok because $10-2$ is just in back and the 8 is in front. But it still equals...it doesn't matter...it means 8 equals 10 minus 2 which equals 8 . So 8 equals 8 . They're the same.” 
She used her knowledge of this task and retrospectively applied it to Task $2 \mathrm{a}$. By using Task $2 \mathrm{~b}$, she was able to integrate her ideas of the operation and relational notions of the equal sign, since she saw not only the operation and answer, but also the fact that both sides were the same amount. She used the relational aspect of the equal sign in answering Tasks $2 \mathrm{c}, 2 \mathrm{~d}$, and 3, for she believed that as long as both sides of the equation were the same value, the equal sign was used effectively. Similarly she used this strategy to solve for the value of the question mark in $3+5=?+2$.

$\mathrm{H}$ : What plus 2 is 8 ? [pause] Oh, 6 !

$\mathrm{N}$ : You got that answer quickly! Tell me how you thought about it?

$\mathrm{H}$ : Because 3 plus 5 is 8 , and 6 plus 2 is 8 .

$\mathrm{N}$ : So what does the equal sign mean here?

$\mathrm{H}$ : It means that they are both equal to each other. Which means if you add both of them together, like 5 plus 3 is 8 and then 2 plus 6 is 8 , well, then they would be equal to each other because they are both 8 .

Unlike previous relational responses Hope gave concerning the notion of the equal sign, she articulated a little differently what the equal sign meant in equations containing a variable. When I asked her what it meant in the equation $x+5=3$ she said, "Well, what it equals... I can solve it. What plus 5 is 3 . It's -2 are you kidding me?! It's simple! Come on!' I asked her why she thought it was so easy to solve and she said, "It was like what we were doing over here," as she pointed to the number line. She ruffled through the papers and showed me how to use the number line by placing her pencil on the 5 and her left index finger on the 3 and moved to the right 2 spaces, enacting the method in the SDA. She then said, "I knew it had to be a negative number because it went from 3 to 5 ." Thus, while she may have said that she solved it by trying to figure out what number need to be added to 5 (arithmetic method), she also was able to think about unwinding the equation so she could solve for $x$ using subtraction. Even though she seemed to understand the equal sign relationally, she did not solve 1-step equations algebraically as proposed by Knuth and colleagues (2006). Knuth and colleagues found that students who had a relational understanding the equal sign typically solved equations algebraically by applying the same transformation to both sides of the equation.

Even though Hope used two different methods to solve the 1-step equation, she solved the 2-step equation using an arithmetic strategy based on what she had learned in math class.

H: Two times $m$ plus 9 equals 1 . We've done this in class before.

$\mathrm{N}$ : How did you do it in class?

$\mathrm{H}$ : Well, what we did was we wrote it out. Two times m equals blank plus 9 equals 1 . So it would have to be negative numbers.

$\mathrm{N}$ : What's the blank for?

$\mathrm{H}$ : It's the number that it's equal to $2 * \mathrm{~m}$.

$\mathrm{N}$ : So then what would you do after this?

H: You'd add 9 to get 1 . Ah, so this would be 5 . m equals 5 . 
N: How did you get that?

H: Well, 2 times 5 would be 10 plus 9 is 19 . Wait, what?

$\mathrm{N}$ : At one point you said you thought $\mathrm{m}$ was going to be negative. Why did you say that?

H: Well, I'm not sure. Well, I have to add 9, so it wouldn't go to 1 unless it was an odd number.

No, I mean.. I mean uh, a negative number.

$\mathrm{N}$ : So you know it's a negative, but do you know how to find $\mathrm{m}$ ?

H: Well, I think $m$ is 5. So 2 times 5 is 10 then plus 9 equals 1 . I don't know how that works, but it does.

As she described what she had learned in class, she wrote $2 * \mathrm{~m}=\ldots$ ? $+9=1$. The format of this equation may be why she viewed the equal sign in solving equations differently than she did equations with no variables. Since she wrote $2 m=$ , she thought that the equal sign indicated that she had to find the value of $m$ instead of signifying the relationship between two expressions. Furthermore, this strategy hindered her in finding the value of $m$. because she broke it into smaller pieces that masked the bigger picture. Initially she could see she had to find a negative value because when she multiplied the number by something and then added 9 , she had to get 1 . Yet, after she broke the equation down into $2 m$ and the addition of 9 , she concentrated on trying to find a way to solve for $m$ that did not take into account that the $m$ had to be a negative number. When I tried to bring her attention to the fact that her answer did not match her original idea that $m$ had to be a negative number, she acknowledged that $m$ was not negative, but the strategy had to work, even though she did not understand how it did. This experience showed me that she did not know how to integrate her conceptual and procedural knowledge of solving equations.

Unfortunately, our time expired after only initially allowing her to work with the EBA. She was able to discover that there were two strategies to balance the scale: "We can put the same [amount] on each side or put negative numbers on the one side to make it zero to keep it balanced." She seemed to enjoy manipulating the EBA and trying to figure out how to balance the scale, she said, "It was fun. I liked swinging the balls [weights and balloon] around.”

\section{Summary of Hope's Prior Conceptions}

- Hope exhibited a relational and operational view of the equals sign for numerical equations and the comparison of numbers and units.

- Hope was not familiar with non-canonical forms but was able to make sense of them by looking at the equal sign operationally.

- Hope believed that the equal sign did not show the equivalence of two expressions when one of the expressions contained a variable. She believed that the equal sign meant the value of the variable.

- Hope accurately solved 1-step equations using the unwinding and guess and test methods. She tried to use the guess and test method along with a strategy she learned in math class to solve 2step equations, but was not able to find the correct value for the variable. 


\section{CHAPTER 11 \\ CASE STUDY: DON \\ Don's Background}

Don is in sixth grade Standard Math, which covers average sixth grade material during the school year. A student in Standard Math in sixth grade will most likely take pre-algebra in eighth grade and algebra in ninth grade. At the time of the study, data collection was during the end of the year, and Don had told me that he had seen and learned how to solve both 1-step and 2-step equations in his math class.

Don seemed like an average middle school student. He craved attention, but also wanted me to know that he was a leader. He told me stories about how he had led his football team to victory and was one of the best players on the team. He also told me about how he takes care of his one brother and two sisters because they listen to him and not their mom, and he does this so his mom can get ready for a newborn baby girl. After he regaled me with stories of him as the hero, I also noticed that when he worked on tasks, this carried over into his confidence in his answers. When he responded, "Yes" to a yes or no question, he frequently extended the 's' sound and said it in a matter-of-fact voice. He always seemed confident in his answers and reasoning.

While Don was confident in his answers, he would infrequently ask me if his answer was incorrect. Even though he did not seem to believe his answer was wrong, he was very astute to verbal and non-verbal cues. Typically, when an interviewee would compute an incorrect answer, I would create another task that would lead the interviewee into a cognitive conflict in hopes that he would see his misconceptions and adjust accordingly. Don picked up on the fact that if he was given a similar but not exact task, he must have gotten some answer incorrect. This led him to ask me if he had gotten an answer wrong. Similarly, he noticed verbal cues. At the beginning of our second session, I had asked him if he wanted any lollipops. He responded, "I noticed that you said popS." I gave him a perplexed look since I had no idea where he was going with this, and he said, "Does that mean I can have more than one?" He was very attuned to what I said, and when I was working with him on a task in our second session, he stopped, looked at me, and said, "You're really into math, aren't you?!"

Because he was so perceptive to my words and actions, I often wondered if he purposefully stated 
incorrect answers to see my reaction. For instance, when I asked him if he had ever seen negative sign used as an operation, he said, "A multiplication sign?" He then tilted his head and peered at me from the corner of his eye. I looked at him a little bewildered, and he responded as if he had been slightly confused, "Naw, a subtraction sign!" and smiled slyly. He continued to act in this manner for addition tasks, but ceased in doing so for subtraction tasks.

While Don used arithmetical procedures to solve addition and subtraction tasks, he did not believe that using a number line could help him find the answer. Yet, after using the technology, Don was able to reference the number line he saw within the AAA, SAA, and SDA and used this in conjunction with a number line to help him solve addition and subtraction tasks.

\section{Negatives and Operations on Integers}

\section{Prior Conceptions on Addition}

When I asked Don if he had ever seen negative numbers, in particular, -3 , he responded, "Yess, on a number line thingy...like when it goes $-1,1,-2$ and 2, -3 and 3." Based on his answer, he seemed to view negative numbers as the opposite of their positive partners. He correctly answered the task $10+3$ to be 13 ; yet, when it came to $10+-3$ he seemed to have difficulty in answering and disregarded his duality notion of positive and negative numbers.

D: Seven. Oh, um, I did it wrong, I didn't see the plus sign. Can I redo that one?

N: Sure.

D: Um, it [the answer] still should just be 10, right?

$\mathrm{N}$ : Why do you think the answer is 10 ?

D: Uh, because it's plus negative three, and the negative number is just making it smaller, so that's why I still think it's -10 .

$\mathrm{N}$ : So the -3 is making the 10 smaller?

D: Yeah, well, um...I thought you said this would be easy? [smiles slyly]

N: So I noticed that you had 7 first and then you switched it to 10, so maybe tell me a little bit about why you thought it might be 7 at first?

D: Because I didn't see the plus sign, I thought it was 10 minus 3.

$\mathrm{N}$ : And then you said that the negative number makes it smaller?

D: Um, and also a negative number, I just think is zero, cause I just do [says hurriedly]

$\mathrm{N}$ : And why do you think a negative number is zero?

D: Because it's a negative number!

$\mathrm{N}$ : Do you think a number line could help solve this?

D: Naw.

I then told him that 7 was the correct answer to which he exclaimed, "AGWH!" I was not sure by his demeanor if he truly was surprised at this answer or if he was simply pretending to be. It was difficult for me to interpret Don's answers with respect to his mannerisms. In one sense it seemed as if he knew the correct answer, but changed it and made an attempt to provide a plausible reason to back up his incorrect answer. Initially he wrote and gave the correct answer, yet withdrew this because he claimed that he did not 
see the addition sign at first. While his answer of 10 is consistent with some previous literature findings in that students tend to believe that negative numbers are equivalent to zero, it is not consistent with his own understanding of the duality of the number line and the oppositeness of negative numbers and positive numbers. Furthermore, he stated that a negative number added to the 10 should make the 10 smaller in size, yet his answer of 10 does not take this into consideration. Moreover, the lopsided smile he flashed after stating that I had told him these tasks would be easy, made me think he was up to something. In addition to these actions, he jumped the gun and gave me his justification that he thought negative numbers were equivalent to zero prior to me even asking his reasoning behind his answer of 10. It was almost as if he did not want to have to deal with that question, so he gave an unexplainable and unchallengeable reason, of "I just do." He dismissed the idea of a number line helping him solve the problem even though he used the number line to describe negative numbers.

Contrary to what he believed about negative numbers contributing a zero amount when they were added, he subtracted the values when two negative addends were involved. Furthermore, he believed that tasks with two negative addends elicited a positive answer. For example, when he solved the task $-5+-7$, he came up with the answer of 2. I asked him how he came up with that answer, and he responded, "Because I know, uh, 5 minus 7 is 2, so yeah." After his statement, I wrote the task $5-7$ directly beneath the task $-5+-7$ and asked him if there was anything that was different about these two tasks. He simply reiterated his answer, "It's 2." However, when he said "two" he elongated the "eww" sound and went up in pitch. He then tilted his head to the side, smiled, and asked, "Am I wrong again?" As before, I was not sure if he did not understand or he was telling me incorrect answers on purpose to get a reaction out of me. I pointed out that the first expression had two negative numbers and an addition sign, whereas the second expression had two positive numbers and a subtraction sign. He commented, “Ah...I'm guessing like with negative numbers, if you're plussing them, all you have to do is just switch it and take the negative sign off of it." He believed that $-5+-7$ was equivalent to $5-7$ because he could transform the former equation by removing the negative signs and changing the addition sign to a subtraction sign. What is interesting is that not only did he believe that these expressions were transformationally equivalent, but that they also equaled 2 , which none of them did. Since he knew that $7-5$ was equal to 2 , so it is plausible that he thought $7-5=5-7=-5+-7$.

Given that he had addition difficulties, I had anticipated that he would have subtraction difficulties as well. Therefore, I had him work through addition tasks with technology instruction prior to assessing his subtraction understanding. It was only after he could obtain correct answers to addition tasks in the first 
session, that I asked him to solve subtraction tasks.

\section{Prior Conceptions on Subtraction}

Don could accurately solve subtraction tasks in the form $a-b$ where $a \geq b \geq 0$ arithmetically and using a number line by counting the spaces between $a$ and $b$. However, he could not accurately solve any other form of subtraction tasks. After he worked with the Addition Arrow Applet (AAA), I gave him a number line with a dot on the 5 and a dot on the -6 and asked him to write a math equation for it. He gave the expression $-6-5$, which was an appropriate response in and of itself, since the answer of -11 would have related the distance between the dots. Yet, when I asked him how he would find the answer to that expression, he said, "You would, uh, you would have the -6 , and would plus 5 to the -6 , and then you'd ...have a -1 ." I wrote the expression $-6+5$ underneath the expression $-6-5$, and asked him if these tasks were different since one added a five and the other subtracted a 5. He said, "It's basically the same thing." When I asked him to tell me more, he just looked at me as if it was obvious.

$\mathrm{N}$ : Would adding a number give a different answer than subtracting a number?

D: No.

N: Why not?

D: Cause, it doesn't.

Because he knew how to solve subtraction tasks with positive minuends and subtrahends such that the subtrahend was less than the minuend, I wanted him to compare the tasks $6+5$ and $6-5$ in order to understand my point.

$\mathrm{N}$ : So if we had $6+5$, what would that answer be?

D: 11 .

$\mathrm{N}$ : Uh huh. And if we did $6-5$, what would that answer be?

D: 1 .

$\mathrm{N}$ : So here we added a 5 and here we subtracted a 5 . Did we get different answers?

D: Yes, but it's not a negative number.

As he said the last statement, he pointed to the 6 . He believed that if the minuend was negative, the idea of getting different answers to addition and subtraction of the same subtrahend did not hold true anymore.

Thus, this example was irrelevant, since it had a positive minuend. He then followed up and said, "Negative six plus five is -1 and negative six minus five is -1 too."

When I asked him to solve $5--12$, he asked, "Is that a negative 12?" I said that it was and asked him how he typically wrote subtraction tasks. Even though he wrote $-6-5$ horizontally for the previous task, he retracted that idea and said that he usually saw subtraction tasks vertically and wrote five minus twelve (see Figure 1). 


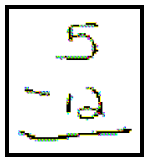

Figure 1. Five minus twelve vertically

$\mathrm{N}$ : How do you know if you are subtracting a negative twelve or a positive twelve?

D: Cause there is a negative sign in front of it.

$\mathrm{N}$ : So how would you write that?

D: Just like I did right here [refers to Figure 1]

N: How do you know that it's not 5 minus 12 instead of 5 minus negative 12 ?

D: Well, I could just write it with a negative number [see Figure 2]

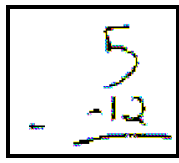

Figure 2. Five minus negative twelve vertically

Most likely, Don did not change his written expression until he was pressed into doing so because he thought that 5-12 was equivalent to 5--12; therefore, he saw no need to write it differently. In fact, he believed that both expressions were equal to -7 .

N: How did you get -7 ?

D: Cause the negative number is bigger than the positive number and you know that 5 plus 7

is $12 \ldots$ because I figure, subtract 12 and 5 and I get 7.

$\mathrm{N}$ : And it's negative seven because?

D: Cause 12 is bigger, because it's a 5 and a -12 , and 12 is a bigger number.

$\mathrm{N}$ : So if you did $-12-5$, what would that be?

D: It would still be -7 .

$\mathrm{N}$ : What about $-5-12$ ?

D: -7 .

$\mathrm{N}$ : Why are they -7 ?

$\mathrm{D}$ : Cause 12 is a negative number and it's bigger.

$\mathrm{N}$ : So it doesn't matter which way we write it, whether it's -12 and -5 , or 5 and -12 ?

D: Yeah.

It is important to note that after working with the AAA, Don noticed that when he added a positive and negative number, it was the same as subtraction and the answer took on the sign of the higher absolutevalued addend. Furthermore, he could interchange the number in addition tasks. He over-generalized these discoveries and applied them to subtraction tasks. Thus, to solve subtraction tasks with negative numbers, he took the difference of the absolute values of the minuend and subtrahend, and used the sign of the higher absolute-valued number. He did this for all forms of subtraction tasks.

$\mathrm{N}$ : What about this one, $-4--6$ ?

D: -2 .

N: How did you get that?

D: Uh, I did 4 minus 6 and I got 2.

$\mathrm{N}$ : Why did you say -2 ?

D: Because both numbers are negative, so the higher number is negative. 
While I was unsure if he was being truthful in his answers to addition tasks, I was sure that he was truthful in his answers to subtraction tasks, mainly because he was consistent in his reasoning and did not behave in a conspicuous manner that led me to believe otherwise.

\section{Discussion on Prior Conceptions}

Based on Don's conceptions of negative numbers as opposing partners of positive numbers on the number line, he had a symmetric view of negative numbers (Kieran, 2007). He did not seem to understand them in terms of the opposite quality, only in terms of lying to the left of the number line in a symmetrical fashion around zero. In fact, he believed that adding a negative to a positive number was sometimes the same as adding the value of zero to the positive number, which was also found in the study by Peled and colleagues (1989). Yet, the students in their study believed there were no such things as negative numbers, and therefore, negative numbers were equivalent to zero. While Don did not believe that addition always made numbers larger, he did believe that his answer would always be positive after applying the operation of addition.

Prior to working with the AAA, he did not have a unary understanding of negative numbers. Even though he described his knowledge of negative numbers in regards to the number line, he did not believe that the number line could help him solve addition tasks with negative numbers. He also seemed to apply different justifications to different forms of addition tasks. While he believed that adding a negative number to a positive number did not impact the answer, he used subtraction when adding two negative numbers, although not in an appropriate manner. To solve tasks in the form $-a+-b$ and $-b+-a$ such that $a \geq b \geq 0$, he solved the task $b-a$, and believed it was equivalent to the previous tasks. Thus, he obtained a positive answer for all addition tasks despite the signs and magnitudes of the addends. This idea and procedure were not noted in any prior research.

Don relied solely on his ideas of negative numbers and prior knowledge of arithmetic to answer addition tasks. He did not believe that a number line could help him solve tasks with negative numbers until after working with the AAA. Only after working with the AAA, was he able to attempt to translate from a number line to abstract arithmetical representation, yet, he was unable to extend this knowledge correctly for subtraction tasks. In moving from a number line task to a subtraction abstract task, he used the numbers represented on the number line as independent entities and did not make the connection between the distance between the numbers and subtraction. This approach was also found in the study conducted by Bruno and Martinon (1996).

Contrary to Murray's findings (1985), Don could not solve addition tasks more accurately than he 
could solve subtraction tasks. After working with the AAA, Don did not attempt to adjust his number line knowledge to subtraction, rather he used the discoveries he made from the AAA and directly applied them to subtraction tasks and assumed that they would hold even though the operation differed. He solved all subtraction tasks using the following procedure: subtract the lower absolute-valued number from the higher absolute-valued number and apply the sign of the highest absolute-valued number to the resulting difference. Thus he believed that subtraction was commutative, which was consistent with Murray's findings (1995). He also believed in the equivalence of the following forms: $a-b,-a-b,-b-a$, and $-a--b$ for all whole numbers. Hence, subtraction elicited only two types of answers: $|a-b|$ if the higher absolute-valued number was positive, or $-|a-b|$ if the higher absolute-valued number was negative. This was similar but not exact to results reported in previous literature. Researchers (Murray, 1985; Peled et al, 1989) saw that first grade students did not believe that a larger number could be subtracted from a smaller number; therefore, they switched the positive minuend and subtrahend to obtain a positive answer. Don did this for positive minuends and subtrahends as well. However, he also exhibited a strategy seen in Murray's work (1985) regarding the sign of the difference. Don used the sign of the higher absolute-valued number in the expression as his the sign for his difference.

Prior to working with the applets, Don could not use the number line to help him solve addition and subtraction tasks. Because he noticed that the negative numbers could be partnered with positive numbers located on the opposite side of the number line, he had a divided number line view of the number line (Peled et al, 1989). Don achieved Level 1 in the Number Line Dimension (NLD) (Peled, 1991) for addition because he could not correctly compute tasks with a negative number. He did not view negative numbers as opposite in quality of positive numbers; therefore, Don did not attain any level on the Quantitative Dimension (QD) with respect to addition. With respect to subtraction, he also attained Level 1 in the NLD and fell below Level 1 in the QD because he was unable to obtain the correct answer to all tasks except in the forms $a-b$ where $a \geq b \geq 0$ and $-b--a$ where $b \geq a \geq 0$. Even though he could obtain the correct answer to $-b--a$, which was a requirement for Level 3 , he was only able to do this because his procedure happened to fit this type of form, but he did not conceptually understand why it did so.

\section{Summary of Don's Prior Conceptions}

- Don did not have a conceptual or procedural understanding of addition of negative numbers, and was inconsistent in his reasons for solving addition tasks. When he attempted to add a negative number to a positive number, he believed that negative numbers were equivalent to zero; thus, they contributed nothing to the sum. When he attempted to add two negative addends, he 
subtracted the smaller absolute valued addend from the higher absolute valued addend.

- Don was unable to move between an abstract and number line representation with respect to addition and subtraction.

- After working with the AAA, Don did not correctly extend and adjust his number line movement to subtraction tasks. He over-generalized his understanding and procedure for determining the sign for addition tasks to determining the sign for subtraction tasks, for he believed the sign of the difference would be the same sign as the higher absolute-valued number in the subtraction expression. Don also over-generalized commutivity with respect to subtraction.

- Don's procedure for subtraction was to subtract the lowest absolute-valued number from the highest absolute-valued number to find the difference, and then he applied the sign of the highest absolute-valued number to that difference. This allowed him to obtain the correct answer to tasks in the form $a-b$ where $a \geq b \geq 0$ and $-b--a$ where $b \geq a \geq 0$ but for no other forms. Due to this procedure, he believed in the equivalence of subtraction tasks containing the same numbers; thus, he only ever obtained two types of answers to subtraction tasks: $|a-b|$ if the higher absolute-valued number was positive, or $-|a-b|$ if the higher absolute-valued number was negative.

- Don attained Level 1 in Peled's NLD and fell below Level 1 in the QD for addition.

- Don attained Level 1 in Peled's NLD and fell below Level 1 in the QD for subtraction.

- Don had a symmetric view of negative numbers since he believed they were opposite partners of the positive numbers on a number line.

\section{Analysis of Interaction with Addition Applet \\ Don's Interaction and Understanding with Technology}

When Don used the Addition Arrow Applet (AAA), I did not have the hot-linked equation

representing the visual on the number line because I wanted to restrict his visual field of information so he would not be overwhelmed with the information. Furthermore, I wanted to see how the illustration of the arrow values inside of the arrows impacted his interpretation of addition before he saw the direct impact on the expression. I asked him to move the blue arrow slider to the left and to the right to get an idea of what was occurring. Initially, he moved it slowly and increased his speed right and left so the numbers zoomed to positive and negative and eventually became a blur. He stopped the arrow on 9.

D: That's my lucky number.

$\mathrm{N}$ : Why is that your lucky number?

D: Cause, well, it just is.

$\mathrm{N}$ : You had to have picked your lucky number for some reason.

D: Uh...

N: Do you play sports or something?

D: Well, yes, it was my first number when I was on a team and we made the championships and I made the winning touchdown.

$\mathrm{N}$ : Wow, that's great!

This interaction was important for two reasons. One reason is that it was odd that he did not know why 9 
was his lucky number and seemed to make up his justification on the spot. Hence, his conspicuous mannerisms and attitude kept me wondering about what he was thinking. The second reason is that his understanding of football came into play later during our instructional session.

After zooming the blue (top) arrow back and forth on the number line, Don noticed that the absolute value of the number inside of the blue arrow represented the length of the arrow and the sign corresponded to the direction the arrow pointed. If the arrow pointed to the right, the number was positive; if the arrow pointed to the left, the value was negative.

When I introduced the orange arrow (bottom) into the applet, how the number related to the orange arrow was not clear for Don. He had moved the blue arrow to the 10 and had just clicked the 'Show Orange Arrow' button so the orange arrow started at 10 and went to the left 16 spaces (see Figure 3).

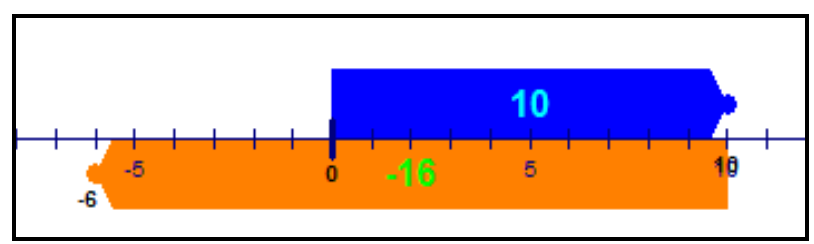

Figure 3. Determining the starting point of the bottom arrow

$\mathrm{N}$ : Where does the orange arrow start?

D: At the -16 .

$\mathrm{N}$ : It starts at the -16 ?

D: Oh, wait, the 10 .

$\mathrm{N}$ : Right. So if you moved this blue arrow, tell me what happens to the orange arrow? Where does it start?

D: It starts 6 more after.

N: So if you move the blue arrow to the 18 , where does the orange arrow start?

D: Uh, at the 24, no, uh, at the 18 [see Figure 4]

$\mathrm{N}$ : Good. Where does the orange arrow end?

D: -6 .

$\mathrm{N}$ : Good, so how long is the orange arrow?

D: -24 .

$\mathrm{N}$ : Why do you think the value inside the orange arrow is negative?

D: Because it's at the -6 .

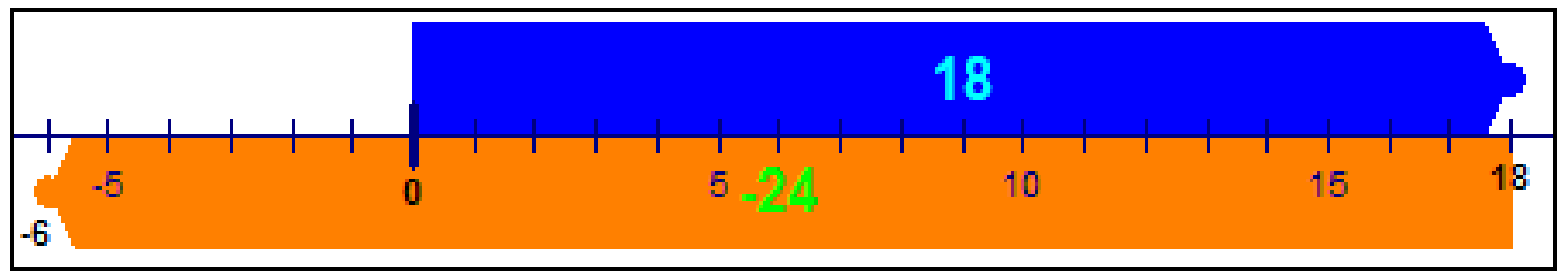

Figure 4. Finding the starting position of the bottom arrow

Initially, Don had a difficult time determining whether the arrow began where the blue arrow ended, or if the arrow began at the value showing inside the orange arrow. Since the value inside the orange arrow was more prominent, he most likely chose the -16 as his first answer. Only after examining the arrows more 
closely, did he see that the orange arrow always began where the blue arrow ended and that absolute value inside the orange arrow represented the spaces in between where it started and ended. After Don figured out how to determine where the arrow began and the length of the arrow, he still did not understand the sign of the value inside the orange arrow. At this point, I had him try different values for the orange arrow and he noticed that when he slid the orange arrow to the left of the ending point of the blue arrow (i.e. orange arrow pointed left), the value inside the orange arrow was negative. Similarly, if he slid the orange arrow so that it pointed to the right, the value inside the orange arrow would be positive. The AAA allowed him to easily determine this since he could simply slide the arrow multiple times to see a pattern and then conjecture why it worked. He said, "Oh, it's negative because it's pointing toward the negative numbers... it's positive because it's pointing to the positive numbers...this is cool!" He believed that since the numbers to the right of zero were positive, then when the arrow pointed to the right, it was pointing in the positive number direction, which made the arrow value become positive and vice versa for negative values.

Because Don had difficulty computing the task $10+-3$, I wanted him to model this task on the AAA. I asked him to place the blue arrow so that it landed on the 10, place the orange arrow so that it had a -3 inside of it, and asked him where the orange arrow landed. He said, "On the 7." I asked him what he thought 7 meant, and he responded, "The answer?" Since he had an interrogative response instead of declarative response, I did not think he made the conceptual connection between the visual on the applet and the equation $10+-3=7$. Therefore, I asked him to model $10+3$ on the AAA. He correctly modeled this and showed me that the answer was where the orange arrow ended (see Figure 5). At this point, I believed that he had made the connection of moving on the number line and the addition of negative numbers.

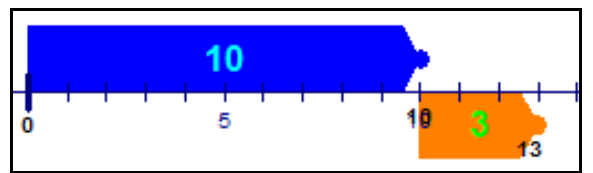

Figure 5. Don modeling $10+3$ on the AAA

He modeled all tasks that had positive addends, or with one negative addend correctly on the AAA, without prompting. Furthermore, he was able to see why the answer was negative or positive based on the lengths of the arrows for these types of tasks. When he modeled $-5+6$, he told me that he had moved to blue arrow to the left since the first addend was negative, moved the orange arrow to the right since 6 was positive, and got the answer of 1, since that was where he landed.

$\mathrm{N}$ : How could you figure out if the answer was going to positive or negative answer by just looking at the arrows?

D: The orange is longer then it is positive. The blue is longer it is negative. 
While this justification fits this specific case, he generalized this and noted that "the longer is the answer" meaning that the sign of the value in the longer arrow was also the sign in the answer. Yet, when I introduced two negative addends, he reverted to his previous misconceptions and could not easily correlate the number line movements and the expression without contextual help. When I asked him to do $-5+-7$, he moved the blue arrow to the -5 and the orange arrow so that the value inside the orange arrow showed -7. He said, "-12. It has to be 2 though." At this point, he moved the orange arrow to the right so that it landed on the 2, and showed the value of positive 7 inside of it (see Figure 6). I asked him how he would write the expression for this model, which he knew how to do since he had been doing these types of tasks before tasks with two negative addends.

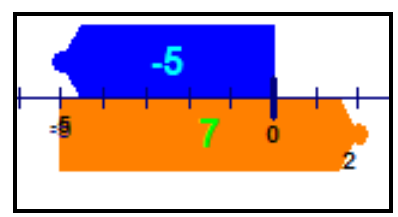

Figure 6. The AAA showing $-5+7$

D: Negative five plus seven.

$\mathrm{N}$ : That's right, so $-5+7$ would be 2 , but not $-5+-7$.

D: Really?

$\mathrm{N}$ : Am I confusing you?

D: No.

Based on his incredulity, I thought I might have been confusing him, so I decided to use context to see if that representation helped him interpret the others. I went back to expressions with a positive and negative addend since he knew how to model those on the AAA and felt comfortable with the answer elicited by the AAA, and put this type of expression in football terms.

N: How about we think about it in terms of football? Say that you lost 8 yards on the first play. D: But my team would never lose 8 yards.

N: Ok, so maybe I should say the other team lost 8 yards on the first play. Then they gained 4 yards on the next play. So where did they end up?

D: -4 .

$\mathrm{N}$ : Why is it -4 ?

D: Cause they have 4 yards to go.

N: Right, to get back to where they began. Does this make more sense?

D: Yeah, but there's 10 yards in football.

$\mathrm{N}$ : Oh, but I thought they had 4 downs to make those 10 yards from where they began?

D: Yeah, they'll never make it.

As I said that the team lost 8 yards, Don moved the blue arrow to the -8 , and when I said that they gained 4 yards on the next play, he moved the orange arrow 4 spaces to the right. The context of football and his understanding of the AAA seemed to dovetail nicely. He then was able to interpret where the orange arrow landed as the answer and put that answer into football context. After only one example using football, we 
returned to the task $-5+-7$. He moved the blue arrow to the -5 and I said, "So they lost 5 yards." Then he moved the orange arrow to the left 7 spaces.

$\mathrm{N}$ : So then they lost another 7 yards. So where did they end up on the field?

D: -12 . They have 12 more yards to go to get to the beginning.

$\mathrm{N}$ : Right, they have 12 more yards to get to where they started.

D: Coool! That helped!

Given the football context, he was able to model, answer, and interpret all the contextual expressions that I gave to him. I wanted to see if he could maneuver from an abstract expression to context representation and find the answer. He was able to do this very well for all types of addition tasks, including tasks with two negative addends.

N: So if I asked you to solve this problem, $-2+-7$, how would you do that?

D: It's -9 .

$\mathrm{N}$ : Good, and how did you get that?

D: Uh, they lost 2 yards, and then they lost 7 more, so 2 plus 7 is 9 .

$\mathrm{N}$ : You said it was -9 , why -9 ?

D: Because they lost yards, so it's negative. They have to get 9 yards to get to the start.

After working with the AAA and the contextual representation of adding integers in terms of football, I wanted to see if he could generalize across his answers to determine a computational method to ascertain the sign of the answer. After looking at the equations he had solved, he said, "If the two numbers that are negative, then the answer will be negative, and if the two numbers are positive, the answer is going to be positive." I then asked him to look at the expressions that had different signed addends. He looked at the following expressions:

$$
\begin{aligned}
& 10+-3=7 \\
& -5+6=1 \\
& -8+4=-4 \\
& -4+6=2 \\
& 7+-4=3
\end{aligned}
$$

$\mathrm{N}$ : What do you notice if the problems have different signs?

D: Then they'll be positive, also.

$\mathrm{N}$ : Well, $-8+4$ has a negative answer, he's not positive.

D: Well, he's different.

N: Hmm. Why is he different? We have to figure out what makes him special.

D: Cause 8 is half; I mean 4 is half of 8 .

$\mathrm{N}$ : Which number, the 4 or the 8 , is bigger?

D: The 8 . OH! So if the negative number is bigger, it's going to be negative...if the positive number is bigger it's going to be positive...like that computer thing.

Not only did he realize that the higher absolute valued-number determine the sign of the answer, he also connected the idea of "bigger" to the length of the arrows in AAA. He remembered that the longer arrow determined the sign of the answer in the applet. I gave him a variety of addition tasks and he always told 
me the sign first, based on his latest discovery, and then provided me answer based on directional movements on the number line. Afterwards I told him that I was impressed with how quickly he picked this concept up and asked him if he had ever done this before. He shook his head no, but went on to explain that he had added positive and negative numbers.

D: Well, I have added negative numbers, but I've never did it this way.

$\mathrm{N}$ : How did you do it before?

D: I just did it. I have no clue.

$\mathrm{N}$ : Which way do you like the best?

D: This way, [pause] with the computer.

$\mathrm{N}$ : Why?

D: It just made me see it better.

Don seemed to enjoy the visual aspect of using the AAA and his ability to see how his movements in real time on the number line resembled addition, which allowed him to get a sense of addition of positive and negative numbers. Since he could provide me with correct answers to all addition tasks, we moved on to subtraction tasks in Module 2. We ended our first session after he worked with the subtraction arrow applet (SAA).

At the next session, I wanted to see if he remembered how to add integers. After I gave him the task $3+-8$, he droned, then sang in a higher pitched voice "number line please." Even though he had not used the subtraction dot applet (SDA), he placed dots on the number line, which he had never done before. He said, "I put a dot on the 3 and a dot on the -8 , and I counted these spaces there. I knew 8 was a bigger number and it was negative, so I knew it was -11 ” (see Figure 6).

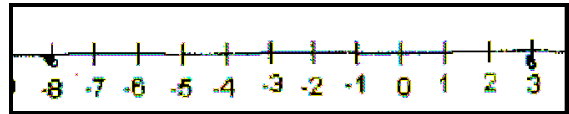

Figure 7. Dots on 3 and -8 to help solve $3+-8$

When using the SAA the previous session, he had discovered that subtraction meant the distance between the numbers, and he noticed that he had to count the spaces in between the numbers represented in the task to compute the difference. Even though the AAA and SAA used arrows to depict addition and subtraction, respectively, he used dots to represent his addends. One reason he did this may be because he viewed the tips of the arrows as the dots, since they resemble dots. He was using the distance discovery from subtraction and integrating it with the idea he found for addition: that the sign of the sum was the same sign of the larger addend. In hopes to get him to remember the generalizations he had made with each operation, I asked him to solve a corresponding subtraction task.

$\mathrm{N}$ : What would you do if I asked you to solve $3--8$ ?

D: Uh, the same thing, -11 .

$\mathrm{N}$ : Does it matter that this one is adding -8 and this one is subtracting -8 ? 
D: Sometimes it does.

$\mathrm{N}$ : Do you remember which operation we counted the spaces in between for?

D: Uh, addition, no subtraction, subtraction. [pause] Final answer is subtraction.

$\mathrm{N}$ : So which problem here is subtraction?

D: Oh, it's 11. Cause 3 plus 8, that's just plain 11 .

$\mathrm{N}$ : So you pointed to $3+-8$ and said that's just plain 11 . Then what is $3--8$ ?

D: -11 .

$\mathrm{N}$ : Why is this -11 ?

D: Cause it just is.

Don remembered that the SAA encouraged him to count the spaces in between the numbers, yet he did not relate subtraction with the task $3--8$, but rather $3+-8$. Since there were 11 spaces in between 3 and -8 on the number line, he believed that since the task $3+-8$, had an addition sign, it must be positive 11 , whereas, since the task $3--8$ had an extra negative sign, the answer must be negative. To see if he could figure out the correct answer, I asked him to explain the task in terms of football.

D: Uh, they gained 3 yards, then lost 8 yards. So it's negative.

$\mathrm{N}$ : Why is it negative?

D: Because they lost more yards.

N: Right, so where would they be?

D: 3 minus 8 , so 5 .

At this point, I reopened the AAA, to see if he remembered how to use it to solve addition tasks. I also wanted him to realize the differences in the visualizations of addition and subtraction. I asked him to solve the task $4+-6$. He moved the blue arrow so that it landed on the 4 , and then said, "They lost 6 " and moved the orange arrow so that it landed on the -6 instead of traveling to the left 6 spaces (see Figure 7). I asked him, "Last time when a team lost six yards, where did you put the arrow?" He said, "Right there, at 6." I asked him to think about what it would look like if he was on a football field and a team lost 6 yards. He just stared at me. I finally asked him what the number inside the orange arrow meant. He moved the arrow so that it said -6 inside the arrow, and he said, "Oh, -2 , they have to gain 2 yards to get back to where they were." I had him display the equation that corresponded with the movements on the number line so he could see the relationship.

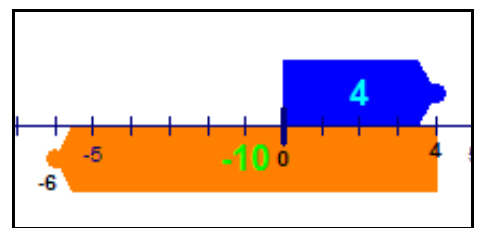

Figure 8. Don's initial model of $4+-6$ on the AAA

The fact that he became confused as to the number inside the orange arrow versus where the orange arrow landed is not too surprising. He had been confused between what these numbers meant the first time he worked with the AAA. Furthermore, the SAA that he had worked with last session reinforced his second 
number being represented by the tip of an arrow.

With the expression displaying on the applet, I asked him to solve contextual football tasks. He would correctly transform them into abstract tasks and model them on the applet. Then I had him hide the expression and answer abstract addition tasks. He was able to solve and model all of these tasks correctly using the AAA. I asked him, "So if we have a positive number and then add another positive number, what happens?" He manipulated the AAA so that both arrows were pointing to the right and said, "That means we're gaining yards." I then asked if he could tell me about the answer. He said, "It's positive....because they both move to the right...towards positive numbers." By putting the numbers into the idea of gaining yards in football and getting visual feedback from the AAA after manipulating the arrows to show addition, he was able to state that the only type of answer to be obtained by adding two positive numbers was a positive answer. I asked, "What if we have a positive plus a negative number, what will happen?" He moved the blue arrow to the 2 and then moved the orange arrow so that a -4 was inside of it, and said, "Then it will go negative." As he said this, he kept moving the blue and orange arrows around. I asked if he thought it would always be negative if he had a positive and negative number and added them together. $\mathrm{He}$ slid the orange arrow farther and farther left past -4 , and started to say "yeah" but stopped half way through, when he positioned the orange arrow so that it landed on the 1 . He noticed that inside the orange arrow the number was negative, but the answer, where it landed, was positive. He said, "Um, you can't tell." He then slid the blue arrow to -2 and left the orange arrow to remain on the 1 . He noticed that this was also a negative number plus a positive number. He tried a few more examples and looked at me as if there were no way to tell the sign of the answer, since it could be either positive or negative. Then he stopped the applet when it displayed $5+-4$ (see Figure 8) and I tried to draw his attention to the length of the arrows as in the first session.

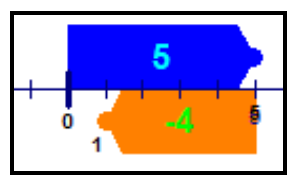

Figure 9. $5+-4$ depicted on the AAA

N: So what do you notice about the number inside the orange arrow?

D: If the answer is 1, it's always going to be one less. It's going to be a negative number that is less than the positive number, one less than the positive number. [points to the blue then the orange arrow value]

$\mathrm{N}$ : Right. How far would you have to travel with the orange arrow to get an answer of a negative number?

D: 2 more.

$\mathrm{N}$ : So how big does the number have to be inside the orange arrow so that we get a negative answer?

D: Uh, [moves the arrow around] -6 . 
$\mathrm{N}$ : So do you notice anything about the values in the orange and blue arrows that happen to get a negative answer?

D: Well, do you mean when it will be negative?

$\mathrm{N}$ : Yeah.

D: When the negative number is bigger than the positive number.

$\mathrm{N}$ : Can you explain more about that?

D: Like 6 is bigger than 5, so when the negative number is bigger the answer will be negative. If the positive number was bigger than the negative number, then it will be positive...so basically, whichever one is bigger; um it's going to be that [sign].

At first he noticed that the answer was positive 1 because his orange arrow value was 1 less than the blue positive value. After I asked him to move the orange arrow until he obtained a negative answer, he noticed that when the "bigger," or higher absolute-valued number had a negative sign, the answer was negative and when the higher absolute-valued number had a positive sign, the answer was positive because the higher absolute-valued arrow was longer and overcame the other arrow's length. By moving the arrows around, and testing many different numbers to see when he could get a negative or positive answer and the relationship between the arrow movements and the numbers inside the arrows that represented the addends, Don was able to discover the relationship between the magnitudes and sign of the answers.

I then asked him to figure out what a negative plus a negative would be. Instantly he said, "that's going to be a positive too. Wait, no it's not, it's going to be negative." When I asked him to justify he explained, "Because it's two negatives, and one number has to be bigger than the other, but still it's just going to be a negative." This response was interesting because he did not contextualize the numbers in terms of losing yards in football; therefore, needing to make up those yards, but rather, he made the connection to his previous discovery: the higher absolute valued-number determined the sign of the answer. Since both numbers were negative, the higher absolute valued- number was negative, so naturally the sign would be negative.

I wanted to see how he would compute addition tasks without the AAA and see if he would incorporate his new generalizations. I asked him to solve $-8+-2$.

D: It's going to be negative.

N: How would get the answer?

D: Can I show you on the computer?

$\mathrm{N}$ : How about you show me without the computer first?

D: Awe, computers are so much more fun. They actually show you the answer! [pause] Can I have a number line?

At first he started to place the dots on -8 and -2 and counted the spaces in between them, stopped, erased the dots, and said, "There would be arrows, can I draw them instead of dots?" While I am not sure why he asked me if he could do that since I had not restricted him before, I said, "You can draw anything you want to help you figure out the problem." He exclaimed, “OK!" and then mumbled to himself, "Do I start at 
zero, oh, yes." He drew an arrow beginning at zero and down to -8 and then another arrow beginning at -8 going to the -10 . Not only did he draw these arrows, he drew them so that one was on top of the number line and the second one was below the number line, so that it exactly simulated the AAA (see Figure 9).

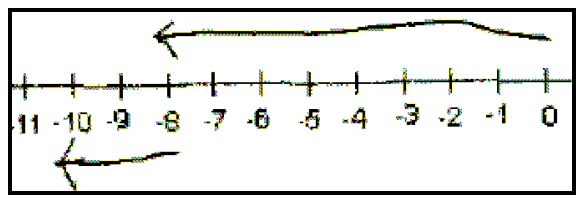

Figure 10. Don's drawing of how he solved $-8+-2$ on the number line

I asked him why he moved that way on the number line. He said, "Because it was losing yards basically." I wanted to see if he could put the task into contextual form.

$\mathrm{N}$ : Could you tell me a story about this problem?

D: A long one or a short one?

$\mathrm{N}$ : A short one.

D: Ok, cause I was going to tell you a super long one and all that. Ok, uh, the Dallas Cowboys lost 8 yards against the New England Patriots, then they lost 2 more yards, and now they have 10 yards to go.

N: Great.

As I gave him more tasks to solve, he did not seem to view addition of two negative numbers as the same as subtraction, but he did notice that the addition of a positive and negative number was the same as subtraction. When I asked him to solve the task $5+-7$ without the applet, he did it without the number line.

D: I can do this in my head. -2 .

N: Great! How did you think about that?

D: Well, I knew it was going to be a negative number, because 7 is a negative number and it's larger. And then I just did 5 minus 7, and I got 2.

$\mathrm{N}$ : How did you know to do minus?

D: Cause I saw a pattern of what we did the other day.

$\mathrm{N}$ : Oh? What pattern did you see?

D: I saw when we were doing, uh, when we were adding a negative number and a positive number, um, you can just subtract those two numbers, and then that's the answer. And if you want to know if it's positive or not, you just look at which number is bigger.

$\mathrm{N}$ : Where did you notice that?

D: Like I said, I notice every small detail. [pause] When we were doing it the other day with the sheet thingy [chart] I just noticed there was a pattern about how I could do it.

This was the first time Don had ever said anything about how adding a negative and a positive number was the same as subtraction. Before, he would compute the correct answer and justify it in terms of the movement of the arrows, but this time, he used his discoveries about the sign of the answer and his generalization of the chart he created after doing so many addition tasks with the AAA, that he was able to have a unary (Kieran, 2007) perspective of addition of negative numbers. After working with the AAA, he used various procedures to perform addition. He knew how to use the number line to help him obtain the 
answer. Abstractly, he computed addition tasks in the form $-a+-b$ where $a, b>0$, by adding the absolute values of $a$ and $b$ and then adding on a negative sign since the highest value was negative; thus, showing the equivalence of $-a+-b$ and $-(a+b)$. For tasks in the form $-a+b$ and $b+-a$ for $a>b \geq 0$ he subtracted $b$ from $a$, and then tacked on a negative sign. For tasks in the form $-b+a$ and $a+-b$ for $a>b \geq 0$, he subtracted $b$ from $a$ and left the difference positive valued.

\section{Summary of Don's Interactions and Understanding with Technology}

- Don believed that the addition of a negative addend to a positive addend did not affect the sum. He now had a unary view of addition of negative numbers, because he was able to see that the addition of positive and negative addends was the same as subtraction.

- In the AAA, Don easily understood what the directionality and length of the blue arrow represented. He had a more difficult time understanding what the directionality and length of the orange arrow represented but was able to overcome this confusion.

- Don had a clearer conceptual and procedural understanding of addition of negative numbers. It is not certain that he totally understood addition of integers, but he was able to solve all addition tasks by the end of the first and second sessions and make generalizations and connections among representations.

- Don could now use the number line to help him solve all addition tasks. In fact, by the end of the second session, he used it more often to solve the tasks than he did the football context or abstract rules. He believed that addition was commutative, but he no longer believed that $a+-b=a$ and $-a+-b=a-b$ for $a \geq b \geq 0$. Thus, he no longer believed that addition gave a sum that was greater than or equal to each addend.

- $\quad$ By manipulating multiple sequences of addition expressions, noticing patterns, using contextual problems, and noticing the connection between movement of the arrows and corresponding equation, Don was able to see how addition worked. He preferred using the idea of football in the first session, but preferred using the idea of the AAA to justify his answers in the second session.

- After working with the AAA and accompanying tasks, Don attained Level 2- in the NLD and Level 1 in the QD with respect to addition.

\section{Discussion}

Before working with the AAA, Don did not have a clear or consistent conceptual and procedural understanding of addition. Don had inconsistent ideas of negative numbers and how the operation of addition affected them. Don described his understanding of negative numbers in terms being on the opposite side of the number line to their positive counterparts, a symmetric view; however, he did not provide any quality characteristic to this idea. Even though he described negative numbers in terms of the number line, he did not feel that a number line could help him solve addition tasks.

Prior to working with the AAA, Don accurately solved all addition tasks with two positive 
addends, but could not explain how he obtained the answer. Don could not accurately solve any addition tasks that involved a negative addend. Don believed that when he added a positive and negative addend, he obtained the positive addend as sum since the negative addend put forth a zero amount to the sum, i.e. $a+-b=a$. Since he believed that addition was commutative, this also was the answer for $-b+a$.

Don did not believe that when he added two negative addends that he obtained a value of zero. Instead, for tasks involving two negative addends, Don subtracted the lower absolute-valued addend from the higher absolute-valued addend. Thus, his addition procedures always gave him a positive sum that was greater or equal to the value of each addend: for two positive addends, his sum was greater than both the addends; for a positive and negative addend, his sum was equal to the positive addend; for two negative addends, his sum was greater than both addends.

After exploring the AAA, Don understood the directionality and length of the top and bottom arrow, however, initially he confused where the bottom arrow landed and the value inside the arrow as representing amount of spaces moved and the answer, respectively. However, the more he manipulated the arrows and obtained instantaneous feedback from the applet, he understood how he could tell when the sign in the answer would be positive or negative based on the lengths of the arrows representing the addends. Once he tried to use this knowledge to solve addition with two negative addends, he reverted to his incorrect procedure and did not seem to believe the applet at first. However, upon providing him with a football context to the abstract equation, and allowing him to see the football-like movements with the applet, he reasoned through his misconception and was able to maneuver among the contextual, abstract, and number line representations of addition. By analyzing many tasks with the aid of the AAA, he was able to make the generalization that the higher absolute-valued addend determined the sign of the answer. By the end of the first session, he was able to compute addition tasks by adding the absolute values of the addends for two positive and two negative addend tasks, and subtracting when one addend was positive and one was negative, and then applying the appropriate sign. Moreover he was able to check and justify his answer by simulating the method shown in the AAA.

To see if Don could remember what he had done in his first session with respect to addition, I had him solve some addition tasks without the AAA in his second session. He incorrectly completed these tasks, most likely due to his inappropriate integration of his understanding of the AAA and the SAA in our previous session. To solve these tasks, he used the distance between the addends for the magnitude of the sum, and the sign of the largest absolute-valued addend for the sign of the sum. When I tried to create a cognitive conflict, he adjusted his idea of the sign rather than the notion of distance between the addends 
with respect to the sum. In order to get him back on track, we had to move to the contextual representation of football. After phrasing addition tasks in terms of football, Don was able to maneuver among the number line, abstract, and contextual representations with ease. He seemed to just need that reminder of how addition was similar to gaining and losing yards in football. Moreover, it seemed that the applet allowed him to manipulate and visualize the movements he was imagining on the football field. In fact, after working with the context of football and the AAA, Don was able to integrate the idea of gaining and losing yards and the lengths of the arrows and why one cannot instantly tell the sign of the sum if one adds a positive and negative addend. Furthermore, he saw how the higher absolute-valued number overcame the smaller absolute-number both on the applet and in terms of yardage, which allowed him to predict the sign of sum for all addition tasks. After awhile, he did not need to use the football context to answer tasks and instead drew arrows on a number line, simulating the AAA, to justify his answers to addition tasks. By the end of the session, he had a unary view of negative numbers with respect to addition because he saw that in computing the magnitude for an addition task with a positive and negative number, he had to subtract.

Don attained Level 1 in the QD with respect to addition because he knew that negative numbers were opposite in quality of context. He was able to solve all addition tasks using context at the end of his second session, but it was very bumpy transition. I am not certain that he would remember how to do so at this point in time. Don attained Level 2- in the NLD with respect to addition because through his session he discovered that addition meant to step right and subtraction meant to step left on the number line (a criteria of Level 2). Yet, I do not think that he would remember how to solve all addition tasks at this point in time without some guidance.

Analysis of Interaction with Subtraction Applets

Don's Interaction and Understanding with Technology

Because Don previously worked successfully with the AAA, which embodied addition by having the lengths of the arrows represent the magnitude of the addends, I had Don first use the subtraction arrow applet (SAA) because it also had an arrow whose length was represented by the second number in the expression. Both applets also used the sign of the second number to determine the direction the arrow traveled. Thus, it seemed a natural progression for Don to use the SAA prior to using the subtraction dot applet (SDA).

When using the SAA, I did not have the hot-linked equation representing the visual on the number line because I wanted to see how the sole illustration of the arrow value appearing inside the arrow 
impacted his method of solving subtraction tasks. As Don was manipulating the arrows on the SAA, he stopped so that the arrow started at -7 and ended on the zero (see Figure 10).

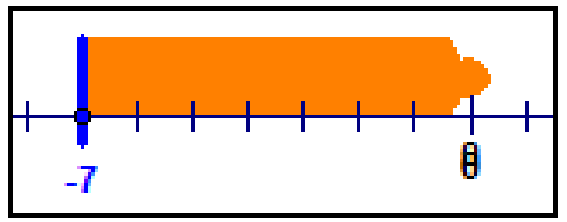

Figure 11. SAA depicting $-7--0$

I asked him where the arrow started.

D: The zero.

N: Does it start at zero or end at zero?

D: Oh, well, where it starts.

Similarly to the AAA, he was getting confused with regards to the beginning and ending position of the orange arrow. This confusion could have been for a couple of reasons. One reason is that the tip, or point, of the arrow was not extremely tapered, so it may not be obvious that the arrow was pointing to the left, and hence began at -7 . Another possible reason for his belief that the arrow started at 0 rather than ended at 0 was because this time the orange arrow was on top of the number line, and the arrow on top of the number line in the AAA always began at zero (see Figure 8). After we agreed that the tip was showing the ending placement of the arrow, he constantly answered correctly where the arrow began and ended from that point forward.

I asked him to move the tip of the arrow to the right in increments of one unit and describe what was happening to the value inside of the arrow. He said, "It's getting smaller... each time 1 smaller." He even noticed that this continued once he moved to the right of the blue stick so that the arrow displayed negative numbers. After realizing how the applet operated, I asked him to use the applet to model the expression $5-2$. He moved the blue stick to the 5 and moved the orange arrow to the left three spaces so that the tip landed on the 2 (see Figure 11).

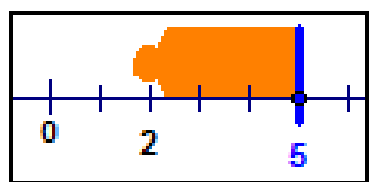

Figure 12. Don modeling $5-2$ on the SAA

N: So how did you know where to put the blue stick?

D: Because it says 5 .

N: How did you know where to drag the orange arrow to?

D: Because it says to subtract 2.

$\mathrm{N}$ : What is the answer?

D: 3 .

$\mathrm{N}$ : What do you think would be inside the arrow?

D: The 3 . 
$\mathrm{N}$ : So why is it 3 ?

D: Because 2 minus, well, because 3 is in the middle. Because I just went 1, 2, 3 .

It is important to note that I had not had him show the arrow value for this task. He knew that the answer would be inside the arrow value by the way he modeled the task, since he represented the minuend with the blue stick and the subtrahend with the tip of the arrow. He used the idea of distance, while using the SAA, which was intended to illustrate subtraction as take-away. What is interesting is that when he explained why 3 was inside the arrow, he pointed to the 5 and subsequently moved his finger to the left by 1 unit when he counted each of " $1,2,3$ " to land on the 2 , which would resemble the idea of take-away. It was as if he used the applet to model the task as it was given, computed the answer by counting the spaces in between the minuend and subtrahend, and then explained his answer in terms of taking away the distance from the minuend to show he obtained the subtrahend. Not only could he translate the abstract expression to a number line representation, he could also translate from a number line representation back to an abstract expression. Furthermore, he was able to see the relationship between the minuend and subtrahend on the number line instead of viewing them as independent points.

To see if he would be consistent in his procedures, I asked him to model $5-4$. He left the blue stick on the 5, and, at first, moved the tip of the orange arrow to the 1 , looked at the resulting image on the computer screen, and then moved the orange tip to the 4 (see Figure 12). I asked him why he decided to move the arrow so that it landed on the 4 .

D: Uh, cause 4 is in the problem.

$\mathrm{N}$ : What do you think the answer is?

D: 1 .

$\mathrm{N}$ : Why?

D: Because 4 minus 5 is 1 .

$\mathrm{N}$ : Does anything else on the screen show you the answer?

D: It's just one space in between them.

One thing to note in the above excerpt is that Don incorrectly verbalized subtraction tasks. He referred to tasks in the form $a-b$ as ' $b$ minus $a$.' It is possible that he said this because he believed subtraction was commutative, but later on, one will notice that he realized that subtraction was not commutative by computing the correct answers accordingly, but on occasion he referred the task $a-b$ as ' $b$ minus $a$.'
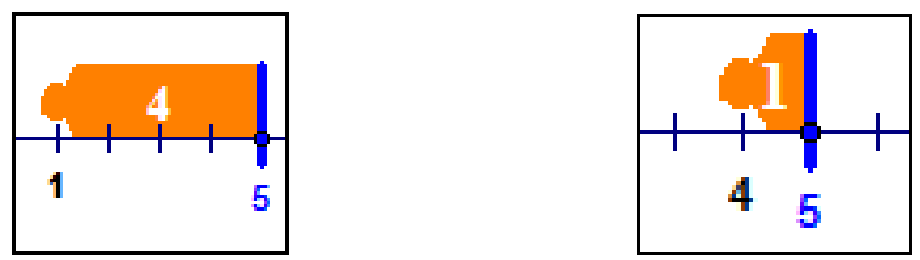

Figure 13. Don modeling $5-4$ on the SAA

Based on Don's manipulation and explanation of subtraction with the SAA, he seemed to understand that 
he could model the same task in two different ways: distance or take-away. However, he preferred to use the distance method. It is important to note that the hot-linked expression was still hidden, and he was making these connections to subtraction all by himself. Since the task 5-5 gave many interviewees trouble in determining which way they wanted to model the task, I wanted to have Don model this task to make sure that Don was consistent in his modeling method so that he would be comfortable in modeling subtraction tasks before introducing negative numbers. He placed the orange arrow directly on top of the blue stick so that no distance showed between them (see Figure 13) and said, "It's zero." When I asked him to explain why, he told me, "Because there is no space between them." Thus, it seemed that Don used the SAA, not as it was designed to be used, but to show distance between the minuend and subtrahend.

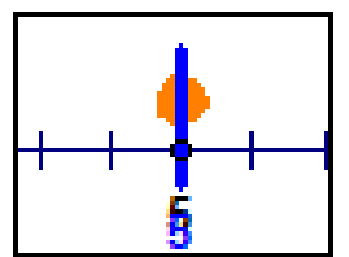

Figure 14. Don modeling 5 -5 on the SAA

Prior to manipulating and exploring with the SAA, Don believed that $5-6$ was positive 1 . However, when I asked him to show me how he would solve 5- 6 using the SAA, without hesitation, he moved the arrow to the right so that it landed on the 6 and said, "Five minus six, you end up with -1 ." When I asked him to explain why he thought -1 was the answer, he said, "Cause you're going to the right now...it's 1 space in between them." Through the manipulation and visual feedback of the applet and the sequencing of accompanying tasks, Don saw how he could use the number line to represent the minuend and subtrahend, and then subsequently figure out the answer. He also saw the need to for traveling to the left and right depending on where he needed to land for the subtrahend, and how it affected the sign of the difference.

Without any intermediate tasks containing a negative subtrahend, I asked him to model $5--12$, which he did correctly and got the answer of 17 . When I asked him why the answer was 17, he said, "Cause I'm going to the left and it's not a negative number [in the arrow], and uh, 12 plus 5 is 17 ." I asked him why he added the 5 and 12, and he said, "Uh, cause I just did. I have no clue." I did not think he had made the connection to the subtraction of a negative subtrahend as the same as addition, rather I believed that he saw the possibility of obtaining 17 from adding 5 and 12 as well as subtracting -12 from 5 . I asked him what he thought the answer of 17 meant, and he responded, "It's the number of spaces in between the 5 and $-12 . "$

Since he was able to use the SAA to correctly obtain answers to all forms of subtraction tasks, I 
asked him to predict the answers instead of modeling the task right away. In doing this, I saw that he reverted back to his old habits of computing the answer until I asked him for how he got his answer. At that point, he would try to put it in terms of the SAA, and when he did so, he realized he had obtained the incorrect answer and corrected himself.

$\mathrm{N}$ : So tell me, what do you think the answer would be for $-1-2$ ?

D: It's 1 .

N: How did you get 1 ?

D: I started at the -1 and moved...oh, wait, -3 .

$\mathrm{N}$ : How did you get -3 ?

D: Because I'm going to the right and there's 3 spaces in between them.

Initially, he solved $-1-2$ by subtracting the smaller absolute-valued number from the higher absolutevalued number and applying the sign of the higher absolute valued- number, therefore, getting an answer of 1. Yet, upon discussing his reasoning, he realized that he was incorrect. It is important to note, that he did not use the applet or a number line when he discussed this task. He visualized both the movement and number line in his head to compute the correct answer. He operated in this fashion for many more tasks after this one. He would quickly come up with an answer, most likely gotten from his previous incorrect method, which he did not state, but upon supplying a justification for his answer, he would realize that he obtained the incorrect answer and supply the correct answer by visualizing and talking about the movement on a number line. As he made adjustments, I noticed he was beginning to say that he added the numbers when the minuend was positive and the subtrahend was negative. I do not think he totally thought through the idea of subtracting a negative as being addition, rather he noticed that he could obtain the same value as when he added the positive numbers. For instance, when I asked him to solve the task $3--2$, he initially thought the answer was 1, but then changed his mind to the answer of 5 .

D: It's going to be a 1.

$\mathrm{N}$ : Why did you think it's going to be a 1 ?

D: Cause, uh, naw, it's 5.

$\mathrm{N}$ : Why did you change your mind?

D: Cause we go to the left and it's 5 spaces in between, and 3 plus 2 is 5 .

N: Ok, so talk more about why you decided to add the 3 and 2 to get 5 .

D: Cause I just know to add them.

$\mathrm{N}$ : How did you know to add the 3 and 2 when the problem uses a subtraction sign -2 from 3 ?

D: I don't know.

While he frequently said he could "add the numbers" for tasks involving a positive minuend and negative subtrahend, he only did so for individual tasks and did not seem to make the broader connection to all tasks in this form. Thus, I had him look at some of the tasks that had a positive minuend and negative subtrahend to see if he could generalize across the tasks: $3--2,5--12,4--1$, and $4--8$. He said, "So you are subtracting a negative...if you are subtracting a positive and negative number, you'll add." When I asked 
him if he could write these tasks another way, he tapped his index finger on the subtraction sign and negative sign and said, "Change these signs to plus."

After he made the discovery with subtraction of a negative subtrahend from a positive minuend was similar to addition, I asked if he thought it would be the same for tasks with a negative minuend. I had him look at the following tasks: $-4--6,-4--3,-1--2$, and $-1--5$.

N: So here we have $-1--2$ and you got 1 . Then for $-1--5$ you got 4 . Do you think if we subtract a negative number with these types of tasks, it would still be like adding?

D: No.

$\mathrm{N}$ : Why not?

D: Because as soon as you just take off the negative sign, and I mean, uh, subtract it by that, and then that's your answer.

$\mathrm{N}$ : Can you tell me more about that?

D: If you take, like this, if you just take off the negative sign and just do it like that, you can still get your answer.

At this point, he covered up the negative signs from both the subtrahend and minuend from the expression $-1--2$ with his fingers to show me $1-2$. He thought that in order for this expression to resemble addition, he had to change all the negative signs in the expression, so that he would get $1+2$, but he knew this would give him the value of 3 and not the value of 1 ; therefore, the expression $-1--2$ could not be the same as addition. Furthermore, under his old way of operating with subtraction, he believed the expression 1-2 to be 1 , which initially reinforced the idea that this expression showed subtraction since he had to keep the subtraction sign in order to get the value of 1 . While he initially thought this expression equaled 1 , he changed his mind and said that $1-2$ was equal to -1 .

$\mathrm{N}$ : So $1-2$ equals 1 ?

D: Yeah, well, no, -1 .

$\mathrm{N}$ : So is $-1--2$ the same as $1-2$ ?

D: No. [pause] Yes, in a way.

$\mathrm{N}$ : How?

D: It's the same numbers, but different.

N: So what about $-1+2$ ? What's the answer to that?

D: Uh, 1.

$\mathrm{N}$ : Is that the same as what we got here, for $-1--2$ ?

D: Yeah.

$\mathrm{N}$ : What about $-1+5$ ?

D: 4. Oh, so subtraction is still changing it to a positive!

$\mathrm{N}$ : Do you think it's easier to do this way or harder?

D: Easier.

Don saw how he needed to change the signs in order to obtain the correct answer. The fact that he was able to add negative numbers allowed him to see this connection. I gave him tasks that had negative minuends and negative subtrahends and then tasks with positive minuends and negative subtrahends. He solved all of these tasks by changing the subtraction sign to an addition sign and the negative sign of the subtrahend to a 
positive sign. Yet when I reverted back to asking him to solve subtraction tasks with negative minuends and subtrahends, he all of sudden abandoned his strategy. I asked him how he would solve $-4--1$, and he wrote $-1+4=5$.

N: So how did you get 5 ?

D: Um.... I don't know.

$\mathrm{N}$ : Could you use anything to help you?

D: A number line... $-4,-1$, it's 3 .

$\mathrm{N}$ : Why is it 3 ?

D: -3 , because there are 3 spaces in between and they are both negative numbers.

When he believed that a number line would help him find the answer, he grabbed a number line, drew a dot on the -4 and a dot on the -1 , and then counted the spaces from the -4 to the -1 (see Figure 14). After he counted the spaces to determine the magnitude of the answer, he determined the sign using his addition rules. The reason he did this may be because we had just talked about how subtracting a negative subtrahend was similar to addition, and how he could change a subtraction to an addition task. Therefore, he tried to combine his knowledge of subtraction to determine the magnitude, i.e. the distance in between the numbers, and his knowledge of addition to determine the sign of the answer.

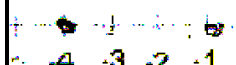

Figure 15. Don's number line visual to help him solve $-4--1$

In hopes to rectify his misconception, I gave him the task $-4--5$, and allowed him to solve it by moving the SAA. He moved the applet to correctly model this task.

D: 1 .

$\mathrm{N}$ : Is it positive or negative 1 ?

D: Positive [in a droning voice]

$\mathrm{N}$ : Why is it positive?

D: Uh, because it's two negative numbers.

$\mathrm{N}$ : So up here for $-4--1$, you said the answer was negative because it was two negative numbers, and the answer for this one is positive because you have two negative numbers?

At this point, Don whispered to himself while he dragged the arrow to the left and right of the -4 and kept doing this but stayed below zero. After a few seconds he said, "Oh! Because the 5 is bigger than the 4 and the 4 is first." He saw that when he dragged the arrow to the left of the -4 as he would do to subtract a number that was positive and had a larger absolute value, he would obtain a negative answer. When he dragged the arrow to the right of -4 , as he would do to subtract a negative number that had a smaller absolute value, he would obtain a positive answer. He had made this discovery through pure manipulation of the applet. To make sure I understood what he was saying, I asked him to solve the task - 5 - -4 . Without moving the applet, he quickly said, "-1." When I asked him how he got that answer so quickly, he 
said, "Because they are both negative numbers... and the 5 is first, so, and the 4 is smaller...so negative and one space between." He finally saw that subtraction was not commutative, and could compute all tasks with negative minuends and subtrahends. Eventually, he started adapting this strategy by noticing that he could strip away the negative signs, subtract the values, and determine the sign of the difference based on the position of the highest absolute-valued number. If the minuend was the higher absolute-valued number, the sign would be negative, if the subtrahend was the higher-absolute valued number, the sign would be positive. For example, when I asked him how he got the answer of 3 to the task $-3--6$, he said, "Because 6 is bigger than the 3, and the 3 is first, and I just took these [negative signs] off and I did 6 minus 3."

By the end of the first session, Don had three different methods for solving subtraction tasks, and the method that he used depended upon the form of the task. For subtraction tasks involving a negative minuend and subtrahend, he subtracted the absolute values of these numbers and determined the sign based on the placement of the higher absolute valued number. For tasks with a positive minuend and negative subtrahend, Don changed the subtraction sign to an addition sign and the negative sign of the subtrahend to a positive sign and added the resulting values. For tasks with a positive minuend and subtrahend and tasks with a negative minuend and positive subtrahend, Don used the number line and placed dots on the number line to represent the minuend and subtrahend. He counted the spaces between these dots and determined the sign of the answer based on which direction he traveled: if he went to the right, the sign was negative; if he went to the left, the sign was positive.

At our next session, after we had reviewed addition tasks, I asked him to draw how he would solve the task $8-3$ on a number line. He said, "I would go to 8 , then minus 3." He drew an arrow on top of the number line from the zero extending to the 8 , and then started a bottom arrow from the 8 and went back 3 spaces (see Figure 15).

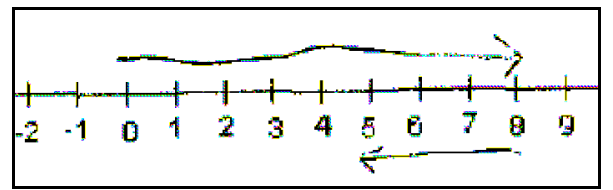

Figure 16. Don's drawing of how he solved $8-3$ using a number line

After working with the SAA last time, even though the applet had shown one arrow, Don had used dots to represent his minuend and subtrahend and counted the spaces in between them to determine the magnitude of the difference, much like the SDA, which he had never seen. However, this time, before working with the SAA, but after working with the AAA, he chose to use arrows, much like how they were displayed on the AAA. Another noticeable difference in method of using the number line was that he used the idea of "take-away" for this task. He moved to the left 3 spaces, which was represented by the subtrahend, and 
landed on his answer of 5. This method was the idea behind the SAA, yet the applet only showed the bottom arrow unlike Don's diagram. While Don had seen the SAA in his previous session, he had not used the SAA in this manner before.

To see if he would continue to operate in this manner, I asked him to solve the task $8--2$. He asked, "Can I have another number line? [pause] Should I still be using the arrows for this one?" He must have noticed something different in his method of operation, since he asked the latter question, but I told him he could use whatever he wanted to solve the task.

D: I don't think I should use the arrows for this; it's only for some problems.

$\mathrm{N}$ : How so?

D: Cause -2 is way over there. Well, I'm still going to use the arrows, I'm just going to count, I'm just going to count how many spaces are between this arrow.

$\mathrm{N}$ : Ok, how many spaces would that be?

D: $1,2,3, \ldots 10.10$.

$\mathrm{N}$ : Why is it positive?

D: Eight's a positive number and 8 is bigger so it's a positive number.

$\mathrm{N}$ : Could you tell me what directions you went?

D: Well, I draw the arrow to which number is first from 0 , and then I put the cap on, then from 8 to -2 , I just knew 8 to -2 .

$\mathrm{N}$ : Do you think the direction you drew the arrows affected whether the answer was positive or negative?

D: Maybe.

Don drew an arrow on top of the number line that started at the 0 and ended at the 8 . Then he started at the 8 and drew an arrow so that it ended at the -2 (see Figure 16). Based on Don's questioning of whether he should use arrows, I think he decided to abandon his strategy of taking away the amount in the subtrahend since the subtrahend this time was -2 . He did not know which direction to move in order to "take away" a negative value. I think he briefly remembered the idea of using dots and counting the spaces in between, but since he had just been using arrows in the AAA, he continued to use these types of arrows, but integrated the idea of counting the spaces in between the beginning and end of the bottom arrow. He also tried to integrate the discovery he made from addition in determining the sign of the answer. He really held on to the belief that the sign of the answer was strictly based on the sign accompanying the larger absolutevalued number, and forgot that last time he noticed that it depended on the placement of the larger absolutevalued number in the expression.

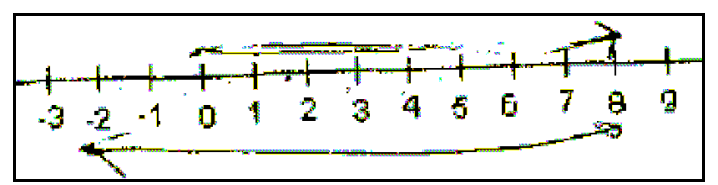

Figure 17. Don's drawing of how he solved $8--2$ using a number line

To see if he could come up with another reason of why the difference was positive, I asked him to solve the 
task $8--12$. He drew a similar picture on the number line as he did for the task $8--2$, only extended the bottom arrow so that it would land on the -12 .

D: 8 and -12 . Well, I can also just do 12 plus 8 . That gives me 20, so it's -20 .

$\mathrm{N}$ : So how did you know to do plus?

D: Cause I noticed a pattern when I was doing this.

$\mathrm{N}$ : When you were drawing the arrows?

D: Yes, because with this one, it, the answer was also the same thing as 2 plus 8 , which is 10 . So it's a pattern.

As Don said this, he pointed to the top arrow in the task $8--2$ and showed me that there were 8 spaces in between the beginning and ending of the arrow. Then he pointed to the bottom arrow and showed me that there were two spaces from the 0 to the -2 that he had to add on to get the total amount of spaces between the 8 and the -2 , so he added. He used the same logic to obtain the number of spaces between the 8 and 12. While he could understand where the magnitude of the difference came from and why he had to "add" the values, he still was confused on the sign of the difference.

$\mathrm{N}$ : So for this task [8--2], you got a positive, but for this task [8--12] you got a negative? Why is that?

D: Because 8 is bigger than 2, but 12 is bigger than 8 .

I asked him to check his answers with a calculator. As he pressed the buttons to enter the expressions, he said, “Am I wrong? I don't think I'm wrong." At this point, he said, "See 10!" after he entered in $8--2$. Then after entering the other expression he said, "It's 20. Is it a negative 20 or a positive 20?" I said, "Does it show a negative sign in front of the answer?" He responded, "No, so just regular 20." At this point, he gave me a defeated look. Since he seemed to notice patterns quickly, I tried to cheer him up and said, "So you're good at noticing patterns, so let's figure out why these are both positive."

Given that he knew that $8--2$ was positive 10 , I had him start with the task $8--3,8--4$, and $8--5$ and asked him to predict what the answer would be, then asked him to model them on the SAA. He gave the correct answers for all of them. Then he jumped in and shouted, "What about 27?!" I said, "Good one, what would $8--27$ be?" He responded, "Oh, why did I have to say that?" and smiled playfully. He dragged the arrow so that it landed on the -27 and said, "35." Each time he moved the arrow to the left by one, he noticed that the difference kept increasing and remained positive. I asked him to write down the following expressions and compute the answer, without the SAA, to each of them: $8--2,8--3 \ldots$, $8--12$. As he did this in order, he answered all correctly and said, "You just add one more...you keep adding a number until you get to 20." I said, "So you said 'adding' what do you mean by that?" He responded, "Oh, I see, whenever you're minusing a positive number and a negative number, it's always going to be a positive." He seemed to understand that when he had a task in the form $a--b$, he would 
count the spaces in between and make sure it was positive. However, he did not say specifically this format, instead by his description, he also could conceive that $-a-b$ would be positive. To test theory, I asked him to solve the task $-4-1$ without the SAA.

D: Can I have a calculator?

\section{$\mathrm{N}$ : How about we try this one without a calculator first?}

D: Well, on some parts of the test I can have a calculator.

$\mathrm{N}$ : Well, you don't want to get the other parts of the test wrong, so how about we see if we can find some more patterns?

D: Can I have a number line?

I gave him a number line and he drew a top and bottom arrow (see Figure 17), even after just working with the SAA, which depicted only one arrow.

D: 5

$\mathrm{N}$ : How did you get 5 ?

D: I went to the -4 , then I went from the -4 to 1 , then I counted how many spaces in between them and there were 5 . Also if you do it the shorter way, you can do 4 plus 1, which is 5 .

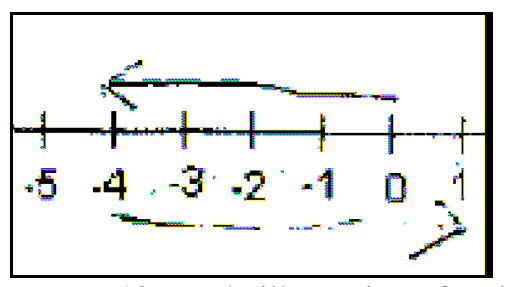

Figure 18. Don's illustration of $-4-1$

As I had supposed, Don believed that anytime he had a subtraction task with a positive and negative number, despite their order, he believed that the difference was positive. I called his attention to direction he traveled for this task versus the other previous tasks.

D: Oh, so it's -5 !

$\mathrm{N}$ : Why did you decide to change your mind?

D: Because first, it went to the left, and that's toward the negative numbers. If you don't know [the sign of the answer] it's whichever the way the first arrow is going towards. That determines whether it's going to be a negative or a positive. If the arrow is pointing to the left, it's going to be a negative, and if it's pointing to the right, it's going to be a positive. It's the first arrow.

Don certainly was very good at noticing similarities and differences in his drawings and movements on the number line. Unfortunately, I had not intended to give a task that led him down this incorrect path of believing that it was the first arrows' direction that determined the sign. Yet, this showed that he had not made the connection that the arrow depicted in the SAA was the same as the second arrow he drew. Instead of having him analyze his drawings and the applet, I had him solve the task $-4--6$. He asked for another number line, drew his arrows, and then said, "Negative two." I asked him to check his answer on the calculator and he said, "It's positive 2." He paused for a second and then said, "Ok, so it's the bottom 
arrow. The opposite one of the bottom arrow is pointing towards is determining it going to be negative or positive." I asked him to explain this to me a little more and he said, "It's the opposite. If it points left, it will be positive. If it points right, it will be negative." I then asked him why he thought that would be true. He looked at me for awhile and tilted his head. Then I said, "So normally you said we get a negative when we go left, when do we get a negative?" He sprung to life and said, "Oh, it's the opposite of addition." Based on his movements on the number line he finally realized how the sign of the difference connected not only with the direction he traveled, but also how subtraction related to addition and why the direction he traveled mattered in determining the sign.

To test whether he truly understood what he said, I gave him a variety of subtraction tasks. For each task, he counted the spaces between the minuend and subtrahend, and justified the sign based on the direction of the second arrow. It was interesting to note his first illustration immediately after he discovered the oppositeness of subtraction and addition. I asked him to solve $-7--10$. He not only drew two arrows, one above the number line and one below the number line, but he also placed dots on the beginning of the bottom arrow and the end of the bottom arrow (see Figure 18). This could have been his attempt to integrate the dots and arrows for subtraction tasks, or it could have been to help him focus on the second arrow's direction. Also, he brought into play the oppositeness of sign. He said, "It's 3 because there are 3 spaces between -7 and -10 and the arrow goes left and the opposite of left is right, so it's positive." In explaining the sign of the difference, he usually referenced the opposite of the direction he traveled in drawing the bottom arrow.

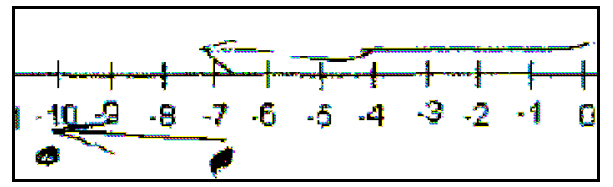

Figure 19. Don's integration of both the dots and arrows for $-7--10$

Since he was able to answer all subtraction tasks, I wanted to test whether he could remember how to solve a mixture of addition amongst subtraction tasks. He easily solved addition tasks by drawing both the arrows on the number line, as done in the AAA, used football context to justify his answer, and used the rules he found for addition: addition of a negative and positive number was the same as subtraction of the absolute values and the sign was determined by the sign attached the higher absolute valued addend, and addition of the same signed numbers was similar to adding the absolute values of the addends and attaching the sign of the addends. Upon returning to subtraction tasks, he had no difficulty solving tasks with a negative subtrahend or tasks with a positive minuend and subtrahend, but he had difficulty with solving tasks with a negative minuend and positive subtrahend. For tasks with a negative subtrahend, he simply changed the 
subtraction sign and negative sign into an addition sign and positive sign respectively, and applied his rules of addition. For tasks with a positive minuend and subtrahend, he subtracted the smaller value from the higher value and attached a negative sign if the subtrahend was larger than the minuend. For tasks with a negative minuend and positive subtrahend, he transformed the subtraction task into an addition task for no apparent reason and solved the resulting addition task. For example, when asked to solve the task $-6-7$, he got the value of 1 .

$\mathrm{N}$ : How did you get 1 ?

D: I added them.

N: So what would you get for this $-6+7$ ?

D: The same thing.

$\mathrm{N}$ : Are they the same? This one is adding 7 and this one is subtracting 7.

D: The signs are different, but not really.

He did not use the number line to help him solve this task. Therefore, I thought by comparing some of the subtraction tasks that he had done correctly with his movements on the number line would help him reason through his answer and see that addition was not the same as subtraction.

$\mathrm{N}$ : So for this problem [-7--10], you started at the -7 and ended at the -10 and counted the amount of spaces in between. Here we have $-6-7$. Where would you start?

D: At the -6 .

N: Right, and where would you end?

D: The -7 .

$\mathrm{N}$ : Well, here you ended at the -10 , where would you end here?

D: Oh, the 7. 1, 2, 3..13.

$\mathrm{N}$ : Is it positive or negative 13 ?

D: It's going right, so opposite of left, so negative thirteen.

By showing him how he had used the number line before to solve subtraction tasks with a negative subtrahend and minuend, he easily extended this knowledge to subtraction tasks with a negative minuend and positive subtrahend. Since he initially answered that he ended at the -7 instead of the 7 , he could have easily thought that $-6-7$ was 1 since he visualized the incorrect positioning of the subtrahend on the number line.

Since he had a little difficulty with subtracting a positive subtrahend from a negative minuend, I decided to show him the subtraction dot applet (SDA) to see if that could help him visualize subtraction better. As he worked with the SDA, he noticed that when the arrow pointed to the left, he was subtracting a smaller number from a larger number and when his arrow pointed to the right, he was subtracting a larger number from a smaller number. Using this idea and the idea that right was the opposite of left, which typically signified negative numbers such that moving to the right indicated a negative answer, he was able to see that when he subtracted a larger number from a smaller number, he obtained a negative difference. I think it was easier for him to make this connection with the SDA because he could tell when the blue dot 
was above or below the orange dot and he knew that the blue dot represented the first number and the orange dot represented the second number and the farther to the right on the number line, the higher value of the number. Thus, when the orange dot was to the right of the blue dot, he was subtracting a higher number from a lower number. For example, when I gave him the task $-7-5$, he said, “- 12 because the opposite is, because the arrow is pointing that way, right, and that's the positive numbers, so the opposite of right is left, and the opposite of positive is negative, so it's going to be positive." When I asked he noticed anything else, he expounded on his justification.

Because 5 is bigger than -7 , and whenever you're subtracting a larger number, it's going to be negative, or smaller number than it's going to be positive. Whenever, like say it's 5-7, then whenever you have .... a smaller number that you're trying to subtract ... a larger number, then it's going to be a negative number, -2 .

He had made the connection of moving to the right and being negative with the idea of subtracting a larger number from a smaller number. In fact, he was able to make up his own task to show me how he understood it. He created the task 5-7 and showed me using his index finger than when he started at 5 and moved to the right to subtract 7 , he was subtracting something bigger, which meant that the answer had to be negative.

I asked him a mixture of addition and subtraction tasks without the applets, to see if he would remember what he had learned. He answered and justified them all correctly. For the addition tasks, he used his abstract rules, and for subtraction tasks he used the number line and the SDA method to justify his answers. For example, when I asked him to solve $-6+2$ he got the answer of -4 because he subtracted 2 from 6 and knew to apply the sign of the higher absolute-valued addend.

N: If I gave you $-6+2$, how would you do that?

D: I would do 6 minus 2 , which his 4.

$\mathrm{N}$ : So this is positive 4 for the answer?

D: No, negative 4.

$\mathrm{N}$ : Why?

D: Because 6 is bigger than 2 .

After I stopped asking him to solve tasks, he asked me, "Do I get a worksheet now?" I was a little taken aback that he asked this and asked, "Why do you think you get a worksheet?" He said, "That's just what most teachers do. If we get something, they give us a worksheet and say 'Do it!'” I chuckled, and said, "Well, how about if you solve some temperature problems for me?"

I wanted to see how Don would work though a contextual representation of a subtraction task. I asked him a few temperature tasks that included the high and low values of the day and asked the change between them.

$\mathrm{N}$ : So if the high temperature was 32 degrees, and the low temperature of the same day was 0 
degrees, how many degrees did it change?

D: Can you hand me a number line? Oh, never mind. It dropped 32 degrees.

He wrote $32-0=32$ when I asked for an equation. He did not need to use the number line to solve this task, however, he did use the number line to solve the next task.

$\mathrm{N}$ : So if the high temperature was -6 degrees, and the low temperature of the same day was -17 degrees, how many degrees did it change?

D: -11 !

$\mathrm{N}$ : How did you get -11 ?

D: Because there are 11 spaces between them, and the arrow is pointing towards the positive side and the opposite of positive is negative.

Instead of using the dots as he had many times before, he instead used his two arrows, one above the number line and one below the number line to compute this task. His top arrow started from the zero and traveled left and ended at the -17 . His bottom arrow started at the -17 and traveled to the right and landed on the -6 . When I asked him to write an equation, he said, "Voila" and wrote $-17--6=-11$. When I asked him why he chose subtraction, he said, "I just did. I just knew it would be." What is interesting about his two expressions is that for his first expression, he subtracted the lower temperature from the higher temperature, yet in this instance, he subtracted the higher temperature from the lower temperature. I asked him why he did this, and he said, "I don't know. Are we done yet?" Since he had answered all the subtraction tasks and addition correctly, and seemed to be getting annoyed about continuing to answer the same type of tasks, I began asking him equal sign questions.

\section{Summary of Don's Interactions and Understanding with Technology}

- Don had a clearer conceptual and procedural understanding of subtraction of negative numbers.

- Don could now use the number line to help him solve all subtraction tasks by visualizing the arrows he had seen in the applets and then drawing them on the number line. In fact, he used it to justify all of his answers. When he initially obtained an incorrect answer using his previous methods, he would come to the correct solution when he visualized the movements on the number line during the justification of his answer. Don no longer believed that there were only two types of answers for subtraction tasks: $|a-b|$ if the higher absolute-valued number was positive, or $-|a-b|$ if the higher absolute-valued number was negative.

- Don no longer believed that addition and subtraction tasks involving the same numbers elicited the same answer. Furthermore, he saw that addition was the inverse operation of subtraction.

- By manipulating multiple sequences of subtraction expressions, noticing patterns, using contextual problems, and noticing the connection between movement away from the minuend and the corresponding equation, Don was able to see subtraction as both take-away and a distance. He preferred using the idea of distance.

- Don was able to create correct subtraction equations for tasks represented contextually. In creating the equation that contained two negative numbers, he used the distance idea by drawing two arrows on the number line and counting spaces represented by the length of the second arrow. 
- Don noticed that the subtraction of a negative number was the same as addition. He easily saw this for tasks with a positive minuend and negative minuend, but initially had difficulty understanding how to change the signs for tasks with a negative minuend and subtrahend.

- In predicting an answer to a subtraction task before reasoning through the task, Don often combined his knowledge of addition and subtraction rules. He typically used the idea of distance between the numbers for the magnitude of the answer, but determined the sign based on the higher absolute-valued number. Yet, upon reasoning through the task, he realized his incorrect answer and would adjust accordingly by using the idea behind the SAA and SDA.

- By manipulating the SAA and SDA and observing the changing values inside the arrow, Don realized why the difference was negative or positive based on the direction of the arrow. He could see that he had to travel left of the minuend if the subtrahend was positive, and to the right of the minuend if the subtrahend was negative. He also realized why the difference was positive or negative depending upon whether he subtracted a larger number from a smaller number or vice versa.

- After working with the SAA, SDA and accompanying tasks, Don could intermittently solve talks in Level 1* in both Peled's QD and NLD, yet it was not a completely smooth transition.

\section{Discussion}

Before working with the subtraction applets, Don did not have a clear conceptual understanding of subtraction, yet was consistent in his procedural methods of subtraction. Don used his own crafted rules and procedures to solve and justify his answers to subtraction tasks. He correctly solved tasks in the form $a-b$ where $a \geq b \geq 0$ arithmetically and using a number line by counting the spaces between $a$ and $b$. For every other form, he did not believe a number line could help him solve subtraction tasks, and applied the same arithmetical procedure for each form: subtract the lowest absolute-valued number from the highest absolute-valued number in the expression to find the magnitude of the answer, and apply the sign of the highest absolute-valued number to that magnitude. By using this procedure, he also obtained the correct answer to tasks in the form $-b--a$ where $b \geq a \geq 0$, but for no other forms. He believed that subtraction tasks containing the same numbers, despite the order, elicited the same answer. In fact, there were only two types of answers to the entire set of subtraction tasks: $|a-b|$ if the higher absolute-valued number was positive, or $-|a-b|$ if the higher absolute-valued number was negative. Furthermore, he believed that answers to a subtraction task and addition task with the same numbers did not necessarily elicit a different answer.

After overcoming the initial obstacle of determining the end and beginning of the orange arrow in the SAA, Don understood how he could use the position of the blue stick and the length and directionality of the orange arrow to model subtraction tasks. Don observed how the arrow value changed as he moved the tip of the arrow from left to right. This influenced his understanding of the connection between the 
values of the minuend, subtrahend, and difference, and the relationship among the numbers, operations, and movement on the number line. Don knew which way to move because of the value shown inside the arrow, and this allowed him to see subtraction as take away as well as a distance between numbers, while he preferred the distance method. In fact, he typically used the SAA to see the distance between the subtrahend and minuend rather than using it to show how much he took away from the minuend. Not only could he translate the abstract expression to a number line representation, he could also translate from a number line representation back to an abstract expression. Furthermore, he was able to see the relationship between the minuend and subtrahend on the number line instead of viewing them as independent points.

By using the SAA, he obtained the correct answer to all forms of subtraction tasks, yet upon asking him to predict the answer to a subtraction task prior to manipulating the SAA, he reverted back to his old habits of computing the answer until I asked him for his justification. As he detailed his justification by visualizing both the movement and number line in his head, he always realized that he had obtained the incorrect answer and provided me with the correct answer.

Upon examining his answers, Don easily saw how a positive minus a negative number was the same as subtraction and changed the signs accordingly to compute the answer. However, he had more difficulty seeing that a negative subtracting a negative was the same as addition. He eventually noted that he could correctly change the subtraction sign to an addition sign and negative sign of the minuend to a positive sign, yet never fully viewed this as addition. After discovering this, problems developed for he started integrating rules from addition and subtraction. Yet, as he tested his incorrect conjectures on the SAA, he finally realized his incorrect procedure, and understood that subtraction was not commutative, and the sign of the difference depended on the position of the largest absolute-valued number. By the end of the first session, Don had three different correct procedures for solving subtraction tasks, and the procedure that he used depended upon the form of the task.

When asked to solve subtraction tasks in our next session, Don used arrows similar to the AAA on a number line. He also combined his subtraction and addition rules to find the answer by using the distance between the minuend and subtrahend for the magnitude and attached the highest absolute valued-number sign to this magnitude. Thus, he obtained incorrect answers for some subtraction tasks. After checking his answers with a calculator, he came to realize that sign was dependent upon the direction of the bottom arrow in his drawing. After discovering this idea, he correctly solved all subtraction tasks by using this idea, which was similar to the SAA. Yet, after giving him a mixture of addition and subtraction tasks to solve, he applied incorrect procedures. At this point, he began working with the SDA and began to 
understand where the sign of the difference came from for he was able to see that when he subtracted a larger number from a smaller number, by witnessing how the placement of the orange dot with respect to the blue dot affected the sign in the answer. After working with the SDA and understanding that subtraction was the inverse operation of addition, he was able to correctly answer a mixture of addition and subtraction tasks without becoming confused or reverting to incorrect procedures. Furthermore, he did not revert to using procedures to solve these tasks; rather he relied on the visualization of the movement on a number line.

\section{The Equal Sign}

\section{Prior Conceptions}

By his responses to the interview tasks, Don had primarily a relational view (Seo \& Ginsburg, 2003) of the equal sign with non-lettered tasks. Only his response to Task 1 could be classified as operational. Don pointed out that the only difference between the tasks $5+6,5+6=$, and $5+6=11$ was that "one has no equal sign, one has an equal sign, and one has an equal sign plus the answer." I went on to ask him what he thought the equal sign meant in each of these forms. He pointed to $5+6=$ then $5+6=11$ and said, "This hasn't been solved yet, and this has been solved." Thus, the equal sign indicated whether the expression had an answer following it, or whether he needed to use a procedure to find the answer to the expression.

Even though Don described the equal sign in Task 1 as operational, he described the equal sign relationally in all the tasks except the last two tasks. He could not tell me where he had seen non-canonical forms such as $3=3$ and $8=10-2$, but assured me that it was acceptable to write them in this manner because the values were equivalent. Similarly, he described that the equal sign in the dime/penny, and feet/inches task indicated that one unit was comprised of another amount of a smaller unit. For instance, he said, "One foot is 12 inches because it's how many inches are in one foot." and that the equal sign in 10 pennies $=1$ dime "tells you that it takes 10 pennies to equal 1 dime." Don believed in the acceptability of writing the equal sign between the expressions $2 \times 5$ and $4+6$ and between $3+5$ and $?+2$ because he had seen those types of tasks in his math book and it indicated that both expressions were equivalent.

$\mathrm{N}$ : Where have you seen these problems?

D: In my math book.

$\mathrm{N}$ : What does the equal sign mean there?

D: That it's the same answer.

$\mathrm{N}$ : What's the same answer?

D: The question mark plus 2 equals the same thing as 3 plus 5 .

$\mathrm{N}$ : So could you find out what the question mark is?

D: You do 3 plus 5 then 2 minus whatever 3 plus 5 is.

$\mathrm{N}$ : What is that? 
D: 6, just plain 6, no negative.

Don used the unwinding strategy (Knuth et al, 2006) to solve $3+5=?+2$. He summed the left side of the equation, and subtracted 2 from that sum to obtain the answer of 6 . Since we had just worked with positive and negative numbers, he confirmed that the numerical answer he gave was positive six and not a negative six.

Even though Don had a relational idea of the equal sign in the above task and seemed to understand the equivalence relation between two expressions, he did not apply the same unwinding strategy to the 1 -step equation $x+5=3$. However, he did become aware that $x$ had to be a negative value in order for the expressions to be equivalent.

$\mathrm{N}$ : What does the equal sign mean in $x+5=3$ ?

D: No.

$\mathrm{N}$ : What do you mean by, 'No'? What does the equal sign mean?

D: You can't do that. You can't do that. It has to be subtraction because 5 is already bigger than 3.

$\mathrm{N}$ : Oh, so you're saying that I can't write an equal sign here?

D: Well, [pause], well, if it's a plus sign you can't. It would have to be a subtraction sign because 5 is bigger than 3 already, so it has to be subtraction.

$\mathrm{N}$ : Oh, ok, so this isn't true that $x$ plus 5 is equal to 3 ?

D: Well, [pause], I guess it can be if it's a negative number.

$\mathrm{N}$ : Can you talk more about that?

D: It can be a negative number. It's possible to be a negative number.

$\mathrm{N}$ : So could you find that number for me?

D: Uh, -8 .

N: How did you get -8 ?

D: Cause I know that 5 plus 3 is 8 .

At first, Don believed that the equal sign could not be put between the two expressions since he felt that they would never be equivalent since he was only considering positive values for $x$. Yet, when he realized that he could replace $x$ with a negative number, he believed that the statement could be true, but he was unable to solve for the correct value. Instead, he added the numbers on either side of the equal sign, and stuck a negative sign in front of that value because he knew it had to be negative. This procedure is interesting because in the previous task, he had subtracted the value on the same side of the variable but here he added. Furthermore, he did not check his answer. At this point in our session, since he had worked with the AAA and understood addition of integers, had he checked his answer, he would have known that 8 did not work make this equation true.

This task seemed harder for Don to solve than $3+5=?+2$. It is interesting that he solved this task differently because both tasks are 1-step linear equations. The differences between the two equations are that $3+5=?+2$ has an operation on both sides of the equation, whereas $x+5=3$ only has one operation on one side, and the solution of $3+5=?+2$ is a positive number, where the solution of $x+5=3$ 
is a negative number. The fact that the value of the variable was negative could have impacted his solution method.

When I asked Don if he had seen equations like $2 m+9=1$ before, he said, "Yes, in math class." I asked him if he knew what the equal sign meant here, and he responded by verbalizing the operations in the equation.

D: Sure. It's 2 times some number equals 10 .

$\mathrm{N}$ : Where did the 10 come from?

D: Yeah, well, adding 9 and 1.

$\mathrm{N}$ : Could you solve for $m$ ?

D: 5 .

$\mathrm{N}$ : Does that work?

D: No, because it has to equal 1. So 2 times some number plus 9 equals 1 , which that can't work. I don't think it can.

$\mathrm{N}$ : Do you think you can find what $m$ is?

D: Well, $m$ could be zero, but I don't know.

$\mathrm{N}$ : How did you get that?

D: Just pick numbers and see if they work.

Even though Don had solved 2-step equations before, he could not remember the procedure for doing so.

He decided that he should combine like terms, the constants, even though they were on opposite sides of

the equal sign, just as he did in the 1-step equation. Then he divided this number by 2 and obtained 5 . Upon prompting him to check his answer, he realized that the value of $m$ could not be 5 because the product of 2

and 5 was 10 and 10 plus 9 is 19 and not 1 , which he knew had to be the resulting answer. Thus, he resorted to guess and test strategy (Knuth et al, 2006), but could not find any values to satisfy the equation in the remaining time of our session.

\section{Summary of Don's Prior Conceptions}

- Don exhibited a relational view of the equals sign for numerical equations and the comparison of numbers and units. Furthermore, it seemed that he also had a relational view for algebraic equations even though he could not solve for the variable.

- Don was familiar with non-canonical forms and was able to state why they made sense.

- Don believed that the equal sign showed the equivalence of two expressions, yet this did not direct his to use an algebraic method to solve equations.

- Don accurately solved a 1-step equation that had an operation on both sides of the equal sign with the variable represented by a question mark by using the unwinding strategy. Don could not accurately solve 1-step and 2-step equations and attempted to use the guess and test method, but it did not lead him to obtain the correct answer. 


\section{CHAPTER 12}

\section{CROSS CASE ANALYSIS}

The preceding case-study chapters present a detailed description of each participant's understanding of addition and subtraction of integers and the notion of equality in the form of initial and subsequent strategies used after working with the applets as well as their interaction while using the applets. In this chapter, I account how the findings provide answers to the research questions.

1. How do non-accelerated middle school students initially understand pre-algebraic concepts such as addition and subtraction of integers and the notion of equality?

2. How do non-accelerated middle school students, after receiving technologically enhanced prealgebraic instruction, understand addition and subtraction of integers and the notion of equality?

3. How do non-accelerated middle school students, who received technologically enhanced prealgebraic instruction, interact with the dynamic applets created in GSP, specifically in regards to analyzing, generalizing, communicating, and justifying their responses to algebraic tasks?

I will answer these research questions by discussing several assertions that are apparent upon cross-case analysis of the participants' initial interview responses and instructional observation and responses. Crosscase summary tables will be presented with the data contained in previous chapters and will aid in the warranting of assertions. Throughout the chapter, findings will be related to previous literature.

\section{Research Question 1}

Eleven assertions concerning participants' initial understanding of adding and subtracting integers are presented: one concerning Peled's rating in addition and subtraction, four concerning addition, two concerning subtraction, and four concerning the relationship between addition and subtraction. Evidence supporting each assertion is presented in eight summary tables, which were generated by reflecting on each participant's responses to the tasks. 


\section{Participants' Initial Understanding of Addition and Subtraction of Integers}

To begin this section, I present a summary table describing Peled's (1991) Quantitative Dimension (QD) levels and Number Line Dimension (NLD) levels. I then discuss how these levels fit with my participants' understanding of addition and subtraction with integers.

Four assertions will be presented concerning the participants' initial understanding. These assertions detail which types of addition tasks participants were able to correctly solve and how they were able to do so using multiple representations, what level they attained within Peled's integer framework, and how their attained level relates to their solution procedure.

Assertion 1. Most students had difficulty with subtraction of opposite and same-signed numbers, but could solve addition tasks with opposite and same-signed numbers. Therefore, most students have differing understanding levels with respect to addition and subtraction. Furthermore, some students do not fit precisely in one level even after separating the categories with respect to addition and subtraction. Therefore, Peled's categorization of students' understanding of integers did not fit cleanly with my data.

Peled's level categorization in the QD and NLD are briefly described in Table 1 (see page 30 for more detail).

Table 1

Peled's Description of Dimensions

Levels Quantitative Dimension $\quad$ Number Line Dimension

Level 1 Believes negative numbers are opposite in quality as positive numbers

Can subtract larger whole numbers

Level 2 from smaller whole numbers and put answer into context

Can add same signed numbers; can subtract same signed numbers such

Level 3 that the absolute value of the minuend is larger than the absolute value of the subtrahend

Can add and subtract opposite signed Level 4 numbers; can determine the sign of the operation and signs of the numbers determine sign in the answer
Believes number line is either continuous or divided; farther to the right ones goes, the larger the number

Believes that add means step right and subtract means to step left on the number line; can add and subtract all whole numbers

Believes that addition and subtraction are opposite in direction and can extend this to work with all same signed numbers

Can operate with different signed numbers; knows that the sign of the operation and second number determines movement on the number line 
A student operating in the QD views both positive and negative numbers as amount of items that exist, whereas a student operating in the NLD views positive and negative numbers as situated on a number line and operations as indicating movements on that number line.

Table 2

Initial Participant Levels in Quantitative and Number Line Dimensions

\begin{tabular}{|l|l|l|l|l|l|l|l|l|l|l|l|l|l}
\hline Participant & Don & Carla & Abby & Jen & Jim & Beth & Jake \\
\hline
\end{tabular}

\begin{tabular}{|l|l|l|l|l|l|l|l}
\hline Add QD & Below L1 L4 & L1+ & L1+ L1+ & L4 & L4 & L4- \\
\hline
\end{tabular}

\begin{tabular}{|l|l|l|l|l|l|l|l|l|}
\hline Add NLD & L1 & L1 & L4 & L4 & L3+ & L4 & L4 & L4 \\
\hline
\end{tabular}

\begin{tabular}{l|l|l|l|l|l|l|l|l} 
Sub QD & Below L1 L2* & L2* & L1+ L2* & L2* & L2* & L2* \\
\hline
\end{tabular}

\begin{tabular}{l|l|l|l|lll|l|l|l|l} 
Sub NLD & L1 & L1 & L3* & L3* & L4- & L3* & L3* & L3*/L4- \\
\hline
\end{tabular}

All participants attained different levels for addition and subtraction in the NLD and QD (see

Table 2). Except for Don, every other participant did not fit cleanly within a level within every dimension for addition or subtraction.

With respect to addition, a plus sign following the level number (see Table 2) means that the participant was able go beyond that level, but could not completely attain the subsequent level. A minus sign indicates that participant attained some of that level, but could not completely attain it. For description of an asterisk, see next page. For instance, Abby noted that positive and negative numbers were opposite in quality, and could solve all types of addition tasks, but she never put her answer into a context (a requirement of Level 2). She knew that $5+-12$ was equivalent to $5-12$, and when I asked her to solve it, she said, "You're going to get a negative number if you subtract 12 away from 5 because you don't have enough...so -7." She never situated it into context; in fact, she declined to do so when I asked her.

Jake was able to situate his answers in terms of context and solve all types of addition tasks, but he did not mention that the sign of the addends determined the sign of the answer. When I asked him to give a story about the equation $-5+-7$, he said, "It's negative five degrees outside...then it dropped seven degrees more, so it would be negative twelve [degrees]."

With respect to addition in the NLD, Hope initially faltered in working with two negative addends, but came into a cognitive conflict by way of task presentation and rectified her own misconception. She then used positive addend tasks to help her figure out which way to move on the number line for a negative addend task. Eventually she was able to solve all types of addition tasks, after a minor setback. She initially stated that $-5+-7$ was equal to 2 , but after giving her the task $-5+7$, she realized that $-5+7$ was 2 and $-5+-7$ was -12 . 
Negative five plus seven would be two.... Since there are two negatives in the top one [ $-5+-7]$ then it [the answer] will be a negative number, -12 , but the one down here $[-5+7]$ there is only one negative, then it will be 2 .

With respect to subtraction in the QD, no participant ever put a subtraction task into context. At most they talked about taking away items when they had to operate on natural numbers. Those students who mentioned taking away items for tasks with a positive minuend and subtrahend such that the subtrahend was larger than the minuend, obtained a L2*. The asterisk represents the fact that they were able to solve higher-level subtraction tasks than their level number indicates, but did not put the answer into context. Students who obtained an L1+ never mentioned any idea of taking away items, yet knew that positive and negative numbers were opposite in quality, and they could subtract a larger whole number from smaller whole number.

With regards to the NLD in subtraction, a score of a L3* indicates that the student was able to subtract with natural numbers, know that plus and minus signs are opposite in direction, but cannot do all same-signed subtractions, yet can solve some opposite-signed subtraction tasks.

Jake obtained a L3*/L4- because he initially could not solve all same signed subtraction tasks, but eventually could solve all of them and some of the opposite signed subtraction tasks. Hope obtained a score of L4- because she could not cross over zero to solve subtraction tasks in the form $a--b$, but could solve all same-signed tasks and tasks in the form $-a-b$.

Peled (1991) postulated that there were only two dimensions for integral operations: Quantitative Dimension and Number Line Dimension. Each dimension combined addition and subtraction knowledge and contained four levels that were hierarchical, with the exception of Quantity Level 1 and 2, which could be reversed. Yet, based on my participant population, I found the necessity for three alterations to his categorical framework: addition and subtraction needed to be separated, levels were not hierarchical, and another dimension needed to be added to fully capture students' understanding.

Addition and subtraction levels within the QD and NLD needed to be separated, for students tended to do better on addition tasks than subtraction tasks; therefore they obtained a higher level in addition than subtraction. The fact that students correctly solved addition tasks more often than subtraction is consistent with previous research (Bell, 1982; Human \& Murray, 1987; Murray, 1985).

Another inconsistent finding with Peled's categorization is that the levels were not strictly hierarchical. With respect to subtraction, Abby, Jim, Carla, and Beth could solve some opposite signed numbered tasks correctly (Level 4), yet not solve all same signed numbered tasks correctly (Level 3). 
Furthermore, Jake could accurately solve all opposite signed tasks correctly (Level 4) but not all samesigned numbered tasks correctly (Level 3).

Peled's dimensions for his categorical framework use a number line and context to describe how students solve addition and subtraction tasks, but I do not believe this adequately captures the knowledge of my participants' understanding of integral addition and subtraction. I believe that there should be another dimension: Memorized facts. Memorized facts would be used to describe the students who operated with abstract number procedures to solve tasks. Every participant used memorized facts to solve some addition or subtraction task, except for Beth who always used the number line (see Table 3).

Table 3

Initial Dimension Levels and How Students Operated for Addition Tasks

\begin{tabular}{|l|l|l|l|l|l|l|l|l|}
\hline Participant & Don & Carla & Abby & Jen & Hope & Jim & Beth & Jake \\
\hline Grade & 6 & 8 & 6 & 7 & 6 & 7 & 8 & 7 \\
\hline Add QD & Below L1 & L4 & L1+ & L1+ & L1+ & L4 & L4 & L4- \\
\hline Add NLD & L1 & L1 & L4 & L4 & L3+ & L4 & L4 & L4 \\
\hline Operated & $\begin{array}{l}\text { Memorized } \\
\text { facts }\end{array}$ & $\begin{array}{l}\text { Memorized } \\
\text { facts }\end{array}$ & $\begin{array}{l}\text { Memorized } \\
\text { facts then } \\
\text { Number } \\
\text { Line }\end{array}$ & $\begin{array}{l}\text { Memorized } \\
\text { facts then } \\
\text { Number } \\
\text { Line }\end{array}$ & $\begin{array}{l}\text { Number } \\
\text { Line }\end{array}$ & $\begin{array}{l}\text { Memorized } \\
\text { facts then } \\
\text { Number } \\
\text { Line }\end{array}$ & $\begin{array}{l}\text { Number } \\
\text { Line }\end{array}$ & $\begin{array}{l}\text { Memorized } \\
\text { facts then } \\
\text { Number Line }\end{array}$ \\
\hline Context & Unable & Debt & Refrained & Refrained & Unable & Refrained & Debt & Temperature \\
\hline
\end{tabular}

Assertion 2. After several tries, except Don, every participant was able to correctly solve all addition tasks.

Those participants, who could correctly solve addition tasks, obtained a high level in at least one of Peled's dimensions with respect to addition, with three participants scoring high levels in both dimensions.

Moreover, participants, who

a) had a low NLD score, solely relied on memorized facts methods to solve addition tasks

b) had a high NLD score, were able to use a number line and sometimes memorized facts to solve addition tasks

c) were able to use both a number line and memorized facts to solve addition tasks, preferred using memorized facts first and justify their answer by using the number line.

Every participant who had a high score in QD or NLD, also had at least one strategy to solve addition tasks, either by using context or by using the number line (see Table 3). By having at least one strategy and being able to use it well, students could accurately solve all addition tasks. Both Don and Carla had low NLD scores and primarily used memorized facts to solve addition tasks, yet Carla knew how to put addition tasks into context, which most likely allowed her to reason through her answer by use of another representation. For instance, after she computational solved the task $-5+-7$ accurately to obtain -12 , I 
asked her if she could write a story about it. She wrote, "Sarah was in debt of $\$ 5$. She borrowed $\$ 7$ more dollars from her sister. So now Sarah is in debt of \$12."

Most students who obtained a high NLD score (Abby, Jen, Jake, Jim, Hope, and Beth) were also able to use memorized facts to solve addition tasks (Abby, Jen, Jake, and Jim), and frequently did so but used the number line to justify their answer (see Tables $3 \& 4$ ).

Table 4

Using Memorized Facts and a Number Line for Addition Task Justification

\begin{tabular}{|l|l|l|}
\hline Participant & $\begin{array}{l}\text { Memorized } \\
\text { Facts Solution }\end{array}$ & Number Line Justification \\
\hline Jen & $-6+5=-1$ & "Go to 5 and count back 6 and that would bring you to the $-1 . "$ \\
\hline Abby & $-5+-7=-12$ & "You go, -5 , plus 7, so one, two, three, four, five, six, seven, and you're at $-12 . "$ \\
\hline Jake & $-6+11=5$ & $\begin{array}{l}\text { "Because I did the lowest, which is }-6, \text { cause like I want to go farther into the } \\
\text { positives. So I did -6 and I...counted the spaces, which was 11, which would be } \\
-6 \text { plus 11, would equal...5. }\end{array}$ \\
\hline Jim & $-6+11=5$ & $\begin{array}{l}\text { "If it's negative, you count down, and if it's positive then you [count right] like } \\
-6+11=5 . "\end{array}$ \\
\hline
\end{tabular}

All students who solved addition tasks correctly could use at least two representations to help them obtain the answer: context and number line, context and memorized facts, memorized facts and number line (see Table 3). It seems that students who could answer addition tasks by using two different representation, solved tasks more accurately. This supports NCTM's (2000) recommendation of using multiple representations in the classroom to facilitate mathematical learning.

Assertion 3. There seems to be two relationships including a high QD: one between a high QD level and $N L D$ level, and one between a high QD level and grade level. Unlike relationships with a high level in $Q D$, there does not seem to be a relationship between a high score in the NLD and grade level. Moreover, students earned a higher score on the NLD than on the $Q D$.

a. None of the $6^{\text {th }}$ grade participants earned a high score in the $Q D$.

b. There seems to be a correspondence between having a high score in the $Q D$ and having a high score in the NLD, except with regards to Carla, who was unable to use the number line to solve addition tasks.

c. There does not seem to be a correspondence between having a high score in the $Q D$ and using memorized facts, a number line, or both to correctly solve addition tasks.

None of the sixth grade participants earned a high score in the QD (see Table 3). This may be due to not experiencing contextual tasks until their later math classes. In fact, Don and Hope said that they had 
never done word problems before, and most seventh and eighth grade participants earned a high score in the QD and eighth grader participants situated some of their answers in terms of debt.

If a participant earned a high score in the QD, they also earned a high score in the NLD, except for Carla who did not know how to use the number line(see Table 3). This would imply that using a number line is either easier to use to solve addition tasks than context, or is more often shown how to be used to solve addition tasks before context is. Past research (Bruno \& Martinon, 1996) has shown that students have an easier time moving from the abstract representation to a number line representation than from an abstract representation to a contextual representation. This is supported by the way my participants operated on addition tasks.

Among the participants who obtained L4- or L4 in the QD (Beth, Carla, Jim, and Jake), there does not seem to be a consensus of one specific operating method to solve addition tasks (see Table 3). Beth consistently used a number line, while Carla used memorized facts, and Jim and Jake used both memorized facts and a number line to solve addition tasks correctly.

Assertion 4. Participants, who refrained from giving a context for Task 4 in Module 1, could solve addition tasks using both memorized facts and a number line. Jake was the only participant who was able to solve addition tasks using memorized facts, a number line, and context.

Participants, who refrained from giving a context for the task $-5+-7$ after they had given the correct answer of -12 , were Abby, Jen, and Jim (see Table 3).

- Abby ignored my request for her to write a story about $-5+-7$ and upon asking a second time, showed me how she computed the value of -12 on the number line.

- Jen said, "I just added the 5 and 7 and put a negative in front of it." She then proceeded to show me how to solve it using a number line."

- Jim said, "It was pretty easy. I just see it the way it is...If there are two negatives, and you add them together... and as soon as you do it, you ...put a negative sign on there."

It seemed that as long as they could justify their answers using two representations, the number line and memorized facts, they felt no need to give a context. It was almost as if having one more representation would not be beneficial to solving the task, and require more work to get an answer they already knew to be correct. What is interesting is that Jake, who also typically used memorized facts and justified his answer by using a number line, did not refrain from giving a contextual representation of the this task. In fact, he situated the task in terms of temperature. Those participants, who did refrain, often used both memorized facts and a number line to solve addition tasks (see Table 3). 
Assertion 5. Dependent upon the task, a variety of memorized facts and number line strategies to solve addition tasks were used across participants. Yet, most individual participants consistently used the same strategy despite the task form.

a) For addition tasks involving two positive addends, five participants could not describe how they obtained their answer their answer. The participants who could describe their procedure earned a high score in the NLD. Participants who could not describe their procedure earned a high or low score in the NLD.

b) For addition tasks involving one negative addend, three participants used memorized facts and justified their answer with a number line; three solved by using only a number line, and two only used memorized facts.

c) For addition tasks involving two negative addends, three participants used memorized facts and justified their answer with a number line; two participants used a number line; and three participants solely used memorized facts.

Table 5

How Participants Initially Operated on Specific Forms of Addition Tasks

\begin{tabular}{|l|l|l|l|l|l|l|l|l|}
\hline Student & Jim & Jake & Abby & Hope & Beth & Jen & Carla & Don \\
\hline Grade & 7 & 7 & 6 & 6 & 8 & 7 & 8 & 6 \\
\hline Level & ST & adv & adv & adv & ST & ST & ST & ST \\
\hline Add NLD & L4 & L4 & L4 & L3+ & L4 & L4 & L1 & L1 \\
\hline$a+b$ & NL & Can't describe & $\begin{array}{l}\text { Can't } \\
\text { describe }\end{array}$ & NL & Counts on & $\begin{array}{l}\text { Can't } \\
\text { describe }\end{array}$ & $\begin{array}{l}\text { Can't } \\
\text { describe }\end{array}$ & $\begin{array}{l}\text { Can't } \\
\text { describe }\end{array}$ \\
\hline \multirow{2}{*}{$\begin{array}{l}a+-b \\
\text { Memorized } \\
\text { facts then NL }\end{array}$} & $\begin{array}{l}\text { Memorized } \\
\text { facts then NL }\end{array}$ & $\begin{array}{l}\text { Memorized } \\
\text { facts then NL }\end{array}$ & NL & NL & $\begin{array}{l}\text { Memorized } \\
\text { facts then NL }\end{array}$ & $\begin{array}{l}\text { Memorized } \\
\text { facts }\end{array}$ & $\begin{array}{l}\text { Memorized } \\
\text { facts }\end{array}$ \\
\hline & Unary & Unary & Unary & Unary & Binary & Unary & Unary & Binary \\
\hline$-a+-b$ & $\begin{array}{l}\text { Memorized } \\
\text { facts then NL }\end{array}$ & $\begin{array}{l}\text { Memorized } \\
\text { facts then NL }\end{array}$ & $\begin{array}{l}\text { Memorized } \\
\text { facts then NL }\end{array}$ & NL & NL & $\begin{array}{l}\text { Memorized } \\
\text { facts }\end{array}$ & $\begin{array}{l}\text { Memorized } \\
\text { facts }\end{array}$ & $\begin{array}{l}\text { Memorized } \\
\text { facts }\end{array}$ \\
\cline { 2 - 8 } & Unary & Binary & Binary & Binary & Binary & Binary & Binary & Binary \\
\hline
\end{tabular}

Jake, Abby, Jen, Carla, and Don could not describe how they solved an addition task containing two positive addends(see Table 5). Most of them said something to the effect of just knowing the answer intuitively. The students who tried to describe or justify their answer, showed me by starting at the first positive addend and moving the amount of the second addend toward the right on the number line. While Beth did not specifically point to a number line, she verbalized her counting as if she was taking 1increment steps from the first positive addend.

Jim, Jake, Jen, and Abby used memorized facts to find their answer to addition tasks with one positive and one negative addend, and justified their answer by showing me how to obtain the same answer on the number line (see Table 5). Jim, Jake, and Abby started at the first addend and moved to the right if 
the second addend was positive or moved to the left if the second addend was negative. Hope similarly moved on the number line, but Jen and Beth chose to start at the first negative addend they came to reading from left to right and move right the amount specified by the other addend. Therefore, they never had to move to the left on the number line for tasks containing only one negative addend.

Carla and Don never used a number line to justify or solve these tasks, for they only used memorized factual procedures (see Table 5). Carla's procedure was similar to Jim, Jake, Jen, and Abby, who saw the equivalence of $a+-b$ and $a-b$ and hence, operated as if the task was subtraction. Don, however, used an incorrect procedure for he believed that a negative number was worth a zero amount and therefore, the sum of a positive addend and negative addend was simply the positive addend.

Again, Jim, Jake, and Abby consistently solved all tasks with negative addends by using memorized facts to find the sum and then justified their answer by using the number line (see Table 5). They operated by using the distributive property and transforming $-a+-b$ into $-(a+b)$. While they did not explicitly state they were operating by using the distributive property, they added the absolute value of the addends together and tacked on a negative sign at the end (see Table 6).

Table 6

Description of $-5+-7$

\begin{tabular}{|l|l|l|}
\hline Participant & Task & Memorized facts Description of $-a+-b$ \\
\hline Jake & $-5+-7$ & "I just added those two which equaled twelve...so $-12 "$ \\
\hline Abby & $-5+-7$ & $\begin{array}{l}\text { "I added five plus seven, and the yare both negative, so I got a negative } \\
\text { number, }-12 . "\end{array}$ \\
\hline Carla & $-5+-7$ & "I just added five and seven and then put the negative sign in front of it." \\
\hline Jim & $-5+-7$ & "Two negatives together would equal a negative, so $-12 . "$ \\
\hline
\end{tabular}

In justifying their answer, they started at $-a$ on the number line and moved to the left $b$ spaces. Both Hope and Beth used the exact same movement on the number line.

Jen, Carla, and Don never used a number line to justify or solve these tasks, for they only used memorized factual procedures. Jen and Carla's procedure was similar to procedure used by Jim, Jake, and Abby; therefore, they obtained the correct answer. Don, however, used an incorrect procedure for he subtracted the smaller absolute-valued addend from the larger absolute-valued addend (see Table 5).

All participants consistently used the same method of operating for addition tasks containing at least one negative addend, except for Jen, who used both memorized facts and a number line to solve tasks with one negative addend, and only used memorized facts to solve tasks containing two negative addends. 
It is interesting to note that participants, who used both memorized facts and the number line to correctly solve addition tasks containing negative addends, were sixth and seventh graders. One would think that the older students would have had more experience with different strategies; and therefore use more strategies to solve addition tasks containing negative numbers.

Another interesting observation is that while all the participants, except Don and Carla, viewed addition tasks with opposite-signed addends as subtraction, only Jim, who was in seventh grade standard math, commented that addition tasks with two negative addends was also similar to subtraction (see Table 7).

Table 7

Participants' Reasoning of Addition with a Negative Addend

\begin{tabular}{|l|l|l|}
\hline Participant & Task & Justification \\
\hline Jake & $10+-3$ & "You put 10, and then you plus, but since it's negative, you subtract." \\
\hline Abby & $10+-3$ & $\begin{array}{l}\text { "Cause you have a negative and a positive number, and so even though it says } \\
\text { to add them, you subtract them." }\end{array}$ \\
\hline Jen & $10+-3$ & "You gotta minus three from 10..and ...subtract." \\
\hline Carla & $10+-3$ & "If you're adding a negative to a positive, then you're basically subtracting it." \\
\hline \multirow{2}{*}{ Jim } & $10+-3$ & "It's just subtracting if it's plus with a negative [number]." \\
\cline { 2 - 3 } & $-5+-7$ & $\begin{array}{l}\text { "You're adding them together and, you know how that's like } \\
\text { subtracting... which makes it go lower." }\end{array}$ \\
\hline
\end{tabular}

The next two assertions deal with participant understanding of subtraction. These assertions detail which types of subtraction tasks participants were able to correctly solve and how they were able to do so using multiple representations, what level they attained within Peled's integer framework, and how their attained level relates to their solution procedure.

Assertion 6. All of the participants earned a low level in $Q D$ for subtraction, while most of the participants earned a medium to high level in the NLD. Participants, who earned the highest level in the NLD, created their own strategy for working with negative subtrahends, which previous literature did not depict.

All participants, except Don and Jen, mentioned take-away in their tasks containing a positive minuend and subtrahend and could take away a larger subtrahend from a smaller minuend (see Table 8). Don had one method of operating for subtraction tasks: la-bl and would apply the sign of the higher absolute-valued number. Jen never mentioned any idea of taking away items, yet knew that positive and negative numbers were opposite in quality, and she could subtract a larger whole number from smaller whole number.

All participants, except Don and Carla, attained a Level 3 or higher in the NLD with respect to subtraction (see Table 8). Don and Carla never used a number line to solve subtraction tasks and instead 
relied on computational procedures. The rest of the participants could subtract natural numbers, knew that plus and minus signs indicate opposite directional movement, could solve some opposite signed subtraction tasks, but cannot do all same-signed subtractions. Both Jake and Hope attained the highest NLD level, L4-, because they could not solve only one type of subtraction task form (see Table 8). Jake could not solve subtraction tasks of the form $-a--b$ for $a<b$, and Hope could not solve subtraction tasks of the form $a--b$. However, they were able to solve more subtraction tasks than the rest of the participants, and I believe that it was due to their creative strategies.

Table 8

Participant Initial Subtraction Understanding

\begin{tabular}{|c|c|c|c|c|c|c|c|c|}
\hline Student & Don & Carla & Jen & Abby & $\mathrm{Jim}$ & Beth & Jake & Hope \\
\hline Grade & 6 & 8 & 7 & 6 & 7 & 8 & 7 & 6 \\
\hline Level & St & $\mathrm{St}$ & $\mathrm{St}$ & Adv & $\mathrm{St}$ & $\mathrm{St}$ & Adv & Adv \\
\hline Sub QD & Below L1 & L2* & L1+ & L2* & L2* & L2* & L2* & L2* \\
\hline Sub NLD & L1 & L1 & L3* & L3* & L3* & L3* & L3* / L4- & L4- \\
\hline Operated & $\begin{array}{l}\text { Memorized } \\
\text { facts }\end{array}$ & $\begin{array}{l}\text { Memorized } \\
\text { facts }\end{array}$ & $\begin{array}{l}\text { Depends } \\
\text { on Task }\end{array}$ & $\begin{array}{l}\text { Memorized } \\
\text { facts and } \\
\text { NL }\end{array}$ & \begin{tabular}{|l|} 
Memorized \\
facts and \\
NL
\end{tabular} & $\mathrm{NL}$ & $\begin{array}{l}\text { Memorized } \\
\text { facts and } \\
\text { NL }\end{array}$ & $\begin{array}{l}\text { Depends } \\
\text { on Task }\end{array}$ \\
\hline$a-b$ & $\begin{array}{l}\text { Some } \\
\text { correct }\end{array}$ & All correct & $\begin{array}{l}\text { All } \\
\text { correct }\end{array}$ & All correct & All correct & $\begin{array}{l}\text { All } \\
\text { correct }\end{array}$ & All correct & $\begin{array}{l}\text { All } \\
\text { correct }\end{array}$ \\
\hline$-a-b$ & $\begin{array}{l}\text { None } \\
\text { correct }\end{array}$ & All correct & $\begin{array}{l}\text { All } \\
\text { correct }\end{array}$ & All correct & All correct & $\begin{array}{l}\text { All } \\
\text { correct }\end{array}$ & All correct & $\begin{array}{l}\text { All } \\
\text { correct }\end{array}$ \\
\hline$a--b$ & $\begin{array}{l}\text { None } \\
\text { correct }\end{array}$ & $\begin{array}{l}\text { None } \\
\text { correct }\end{array}$ & $\begin{array}{l}\text { None } \\
\text { correct }\end{array}$ & $\begin{array}{l}\text { None } \\
\text { correct }\end{array}$ & $\begin{array}{l}\text { None } \\
\text { correct }\end{array}$ & $\begin{array}{l}\text { None } \\
\text { correct }\end{array}$ & All correct & $\begin{array}{l}\text { None } \\
\text { correct }\end{array}$ \\
\hline$-a--b$ & $\begin{array}{l}\text { Some } \\
\text { correct }\end{array}$ & $\begin{array}{l}\text { None } \\
\text { correct }\end{array}$ & $\begin{array}{l}\text { Some } \\
\text { correct }\end{array}$ & $\begin{array}{l}\text { None } \\
\text { correct }\end{array}$ & $\begin{array}{l}\text { None } \\
\text { correct }\end{array}$ & $\begin{array}{l}\text { None } \\
\text { correct }\end{array}$ & $\begin{array}{l}\text { Some } \\
\text { correct }\end{array}$ & $\begin{array}{l}\text { All } \\
\text { correct }\end{array}$ \\
\hline
\end{tabular}

Jake was the only participant to correctly solve tasks in the form $a--b$. To do this, he transformed this task into $-b+?=a$. He mentally solved this task and then justified his answer on a number line by starting at $-b$ and counting the spaces he needed to move to reach $a$. The number of spaces was his answer to the task. Previous literature (Bell, 1982; Bruno \& Martinon, 1996; Human \& Murray, 1987; Murray, 1985; Peled, 1991; Peled et al, 1989) had not mentioned this strategy.

Hope was the only participant to correctly solve all tasks in the form $-a--b$. To do this, she took her number line, folded it at zero so that the numbers were on the outside. She held it so the positive numbers were showing toward her and the negative numbers were showing away from her. Then she changed the task $-a--b$ by making the numbers positive and switching the order if $b>a$ so that she was solving a subtraction task where the positive minuend was greater than the positive subtrahend, let us all this new task $c-d$, such that $c$ is $b$ if $b>a$ or $c$ is $a$ if $a>b$. She then put her right thumb on $c$, and wrapped 
her right index finger around the number line so it was touching $-c$. She slid her thumb and finger from the $c$ to $d$. She then flipped the number line to show the negative numbers and suggested that if someone were watching her perform that action, he would see that she was traveling such that she was solving the task $-c$ $--d$. If $c=b$, she would slide from $-d$ to the $-c$, and this means she would be traveling up from $d$ to $c$ on the positive side, which meant she would be going up the value of the difference, so the difference would be positive. If $c=a$, she would slide from $-c$ to $-d$, so she would be traveling down from $c$ to $d$ on the positive side, which meant she would be going down the value of the difference, so the difference would be negative. Previous literature (Bell, 1982; Bruno \& Martinon, 1996; Human \& Murray, 1987; Murray, 1985; Peled, 1991; Peled et al, 1989) had not mentioned this strategy.

Assertion 7: Except Don, everyone could accurately solve tasks with a positive subtrahend; most did so by transforming it into an addition task and using corresponding additive memorized facts and a number line. Most of the participants could not solve tasks with a negative subtrahend. In fact, the most difficult task seemed to be those with a positive minuend and negative subtrahend.

Abby, Hope, Jim, Jen, Jake and Carla used transformed the task $-a-b$ into an addition task and used their understanding of addition to solve it. The first five participants listed transformed the task to $-a+-b$, while Carla transformed it into $-(a+b)$. Carla was the only one who did not use a number line to justify her answer, but those who did started at $-a$ and moved left $b$ spaces. Even though Beth did not transform this task, she too operated in this manner on the number line, which was how she operated on the number line to solve addition tasks in the form $-a+-b$.

Given that every participant operated on subtraction tasks containing a positive subtrahend by using a similar additive strategy, it makes sense that Don was unable to correctly solve all of these types of subtraction tasks, for he did not conceptually or procedurally understand addition of negative numbers. It seems that in order to do well in subtraction with negative numbers, one must first understand addition with negative numbers. This also supports the idea that Peled's categories should be separated into two sets: addition and subtraction.

Another interesting occurrence that is not consistent with Peled's categorical interpretations is students were able to do some opposite-signed subtraction tasks before being able to correctly solve all same-signed subtraction tasks. This would go against the levels being hierarchical. Everyone but Don was able to correctly solve $-a-b$, but only Hope was able to correctly solve all subtraction tasks with samesigned numbers. This does not agree with previous literature. As with Peled, Murray (1985) found that subtraction tasks with opposite signed numbers were more difficult for middle school students to solve than 
tasks with similar signed numbers. Table 9 provides types of subtraction tasks and lists the rating in increasing difficulty according to Murray and my own study.

Table 9

Ratings of Initial Difficulty of Subtraction Tasks

\begin{tabular}{|c|c|c|}
\hline Task & $\begin{array}{l}\text { Murray } \\
\text { Rating }\end{array}$ & My Rating \\
\hline$\overline{a-b}$ & $a>b 1$ & 1 \\
\hline$a-b$ & $b>a 3$ & 2 \\
\hline$-a-b$ & 5 & 3 \\
\hline$-a--b$ & $a>b 2$ & 4 \\
\hline$-a--b$ & $b>a 4$ & 5 \\
\hline$a--b$ & 6 & 5 \\
\hline
\end{tabular}

By far, the most difficult types of subtraction tasks were those with a negative subtrahend, the harder of the two types being a positive minuend minus a negative subtrahend (see Tables $8 \& 9$ ). This is consistent with previous research (Murray, 1985; Peled, 1991; Peled et al, 1989).

For tasks in the form $-a--b$, half the students correctly solved for at least half the these types of subtraction tasks, where only one participant was able to correctly solve for at least half of subtraction tasks in the form $a--b$. Only Hope could correctly solve tasks in the form $-a--b$ because she made up her own number line strategy, using the folded number line idea. Three people, Don, Jen, and Jake, got half of the subtraction tasks in the form $-a--b$, and the ones they got right were of the same structure: $-a--b$ with $a>b$. This is consistent with the findings of Murray (1985). He found that students were able to solve $-a--b$ with $a>b$. correctly more often than they were able to solve $-a--b$ with $b>a$. Not only were all three able to correctly solve that specific structure, but also they all did so using memorized facts. Jen, Jake, and Don operated by subtracting $b$ from $a$ and applying a negative sign to that difference. The four participants who were unable to get these types of subtraction tasks correct typically transformed the task $-a--b$ into $-a-b$ and/or moved left $b$ spaces from $-a$ on the number line, this is exactly how the students in Murray's study operated in transforming the task, and Bell (1982) described students using the same method on the number line to answer this type of task.

It is interesting to note that using a number line did not seem to impact the correctness of the solution. Hope solely used a number line, Jen and Don solely used memorized facts, and Jake used both. Of the participants who obtained the incorrect answers, Carla used memorized facts, Beth solely used a number line, and Abby and Jim used both.

Another observation is that of the students who got only half correct (Don, Jen, and Jake) did not operate in the same manner, nor get the same answer, for tasks in the form $-a--b$ with $b>a$. Don and Jen 
subtracted $a$ from $b$ and applied a negative sign to that difference, while Jake subtracted $b$ from $-a$, and then moved from left $b$ spaces from $-a$ on the number line.

Interesting enough, of the participants who got tasks in the form $-a--b$ partially or all correct, were sixth and seventh graders (see Table 8).

Only Jake could correctly solve tasks in the form $a--b$ because he made up his own procedure to correctly solve this type of task by solving $b+?=a$ and counting up from $-b$ to $a$ on the number line. Most of the other participants transformed this task into $a-b$ and/or operated on the number line by not attending to all of the negative signs. Operating in this manner is also consistent with Murray's findings (1985), for the most incorrect answer was gotten by ignoring one of the negative signs.

Participants who used only memorized facts to solve subtraction tasks, Don and Carla, did not use the same memorized facts procedure as those who used both a number line and memorized factual procedure. Don subtracted the lower absolute valued-number from the higher absolute valued-number and attached the sign corresponding with the higher absolute valued number. Carla transformed $a--b$ into $-a-b$, and then solved it as if it were addition of two negative addends (see Table 18).

Participants who used both strategies (Abby, Jen, Jim, Jake, and Hope) operated in the same manner: mentally solving $a-b$ and moving left $b$ spaces from $a$ on the number line (see Table 18). Hope typically used both strategies for solving and justifying answers to subtraction tasks, yet for these forms, she did not justify her reasoning by using a number line.

Beth, who only used a number line to solve subtraction tasks, operated on the number line corresponding to Carla's memorized facts method. Beth always started on the number line at the first negative number she came in the task when reading it from left to right; therefore, she started at $-b$. She then moved to the left if the operation was subtraction or the other addend was negative; thus, she moved left $a$ spaces from $-b$. This allowed her to get the same answers as Carla did.

The following four assertions relate findings of participant understanding of both addition and subtraction. These assertions compare and detail the attained levels of addition and subtraction and how this relates to participants' use of multiple representations to solve operations on integers.

\section{Assertion 8. Overall, participants had more difficulty solving subtraction tasks than addition tasks} containing negative numbers.

Everyone, but Don, could correctly solve all addition tasks, yet no participant could solve all subtraction tasks. This can be explained in some part by the fact that they used their addition knowledge and strategies to help them solve subtraction tasks, sometime successfully, sometimes unsuccessfully. The 
fact that almost all of the participants did better in addition than subtraction is not too surprising given that other researchers (Bell, 1982; Human \& Murray, 1987; Murray, 1985) also found that students had a more difficult time with subtraction and negative numbers than they did with addition and negative numbers. However, Human and Murray found that students had more difficulty in solving tasks of the form $a+-b$ than tasks of the form $-a--b$ with $a>b$, which is inconsistent with my data. Disregarding Don, every student could solve all types of addition tasks, yet only Jen and Jake could solve tasks in the form $-a--b$ with $a>b$.

Assertion 9. If Participant $A$ obtained a higher level than Participant $B$ in the $Q D$ with respect to addition, Participant A also obtained at least the same level than Participant B in the $Q D$ with respect to subtraction. Moreover, participants with scores of

a) L1 or below in the $Q D$ in addition earned at least the same $Q D$ score with respect to subtraction.

b) L4- and higher in the $Q D$ in addition, earned at most the same $Q D$ score with respect to subtraction.

In comparing QD scores in addition, students who earned at least an L4- earned L2* in the QD with respect to subtraction (Carla, Jim, Beth, and Jake) (see Table 10). Hope and Abby also attained L2* in the QD with respect to subtraction, but only attained L1+ in the QD with respect to addition. Since L2* was the highest level attained in the QD with respect to subtraction, anyone who obtained a higher level in the QD with respect to addition attained at least the same level or higher than another participant did in the QD with respect to subtraction.

Table 10

Participant Addition and Subtraction Initial Understanding

\begin{tabular}{|c|c|c|c|c|c|c|c|c|}
\hline Student & Don & Carla & Jen & Abby & Hope & $\mathrm{Jim}$ & Beth & Jake \\
\hline Add QD & Below L1 & L4 & L1+ & $\mathrm{L} 1+$ & L1+ & L4 & L4 & L4- \\
\hline Add NL & L1 & L1 & $\mathrm{L} 4$ & L4 & L4- & $\mathrm{L} 4$ & $\mathrm{~L} 4$ & $\mathrm{~L} 4$ \\
\hline $\begin{array}{l}\text { Add } \\
\text { Operated }\end{array}$ & Memorized facts & semorized facts & $\begin{array}{l}\text { Memorized } \\
\text { facts and NL }\end{array}$ & $\begin{array}{l}\text { Memorized } \\
\text { facts and NL }\end{array}$ & $\begin{array}{l}\text { Number } \\
\text { Line }\end{array}$ & $\begin{array}{l}\text { Memorized } \\
\text { facts and NL }\end{array}$ & $\begin{array}{l}\text { Number } \\
\text { Line }\end{array}$ & $\begin{array}{l}\text { Memorized } \\
\text { facts and NL }\end{array}$ \\
\hline Sub QD & Below L1 & L2* & L1+ & L2* & $\mathrm{L} 2 *$ & L2* & $\mathrm{L} 2 *$ & $\mathrm{~L} 2 *$ \\
\hline Sub NLD & L1 & L1 & L3* & L3* & L4- & L3* & L3* & $\mathrm{L} 3 * / \mathrm{L} 4-$ \\
\hline $\begin{array}{l}\text { Subtract } \\
\text { Operated }\end{array}$ & Memorized facts & Memorized facts & $\begin{array}{l}\text { Depends on } \\
\text { Task }\end{array}$ & $\begin{array}{l}\text { Memorized } \\
\text { facts and NL }\end{array}$ & $\begin{array}{l}\text { Depends } \\
\text { on Task }\end{array}$ & $\begin{array}{l}\text { Memorized } \\
\text { facts and NL }\end{array}$ & $\mathrm{NL}$ & $\begin{array}{l}\text { Memorized } \\
\text { facts and NL }\end{array}$ \\
\hline Sum Level & Below 4 & 8* & $9+*$ & $10+*$ & $11-*$ & $13 *$ & $13 *$ & $14-*$ \\
\hline
\end{tabular}

In regards to within one participant, if the participant scored L1 or below in the QD of addition (Don, Jen, Abby, and Hope), attained at least that level in the QD with respect to subtraction (see Table 10). This seems to go against the idea that students typically do better with negative numbers in addition 
tasks than subtraction tasks. However, that is not the case, but rather the definitions of the categories with in the QD. The participants who scored lower in the QD with respect to addition than they did with respect to subtraction were Abby and Hope. They earned a L2* for subtraction which meant that mentioned taking away items for tasks with a positive minuend and subtrahend such that the subtrahend was larger than the minuend, even though they did not situate the answer or task into specific context) and were able to solve higher level subtraction tasks, but not in the hierarchical fashion and did not put them into context. Both Abby and Hope earned a L1+ in the QD of addition because they were able to solve all types of addition tasks, but they never situated their task or answer into context. Abby and Hope were able to solve all addition tasks, but not all subtraction tasks. The main reason that they scored a higher number in the QD with respect to subtraction than addition was they had mentioned the idea of "take away items" when they subtracted. These instances support the idea of creating another dimension to the categories so researchers can fully qualify students' understanding and operating with addition and subtraction of integers.

What is interesting is that those participants who attained L4- or higher in the QD with respect to addition (Jake, Jim, Beth, and Carla) earned at most the same level in the QD with respect to subtraction. This is the opposite of participants who earned lower level scores in the QD with respect to addition. The highest scoring participants in the QD with respect to addition were also in the set of participants who scored the highest in the QD with respect to subtraction.

Assertion 10. Unlike the $Q D$, scores in the NLD with respect to addition and subtraction directly related.

If one assumes that Levels 1 and 2 are considered the lower end of the dimension, and Levels 3 and 4 are the higher end of the dimension, one will notice that participants who scored in the lower levels of the NLD in addition also scored in the lower levels in the NLD with respect to subtraction (Don and Carla). Participants who scored in the higher levels of the NLD in addition also scored in the higher levels in the NLD with respect to subtraction (Jen, Abby, Hope, Jim, Beth, and Jake). Furthermore, if a participant attained some of Level 4 with respect to subtraction, they also attained some of Level 4 with respect to addition (see Table 10). It would seem that how well one used the number line to solve subtraction tasks indicated how well he would use a number line to solve addition tasks. Moreover it could also be postulated that the necessity of working with the number line well to solve addition tasks was a prerequisite of using it well to solve subtraction tasks.

By adding the numerical amount of the levels attained in the QD and NLD for both addition and subtraction, I calculated what I call the Sum Levels score. 
Assertion 11. Sum Level scores correlate with the type of representations they used to solve addition and subtraction tasks.

Participants who completed all addition and subtraction tasks solely using memorized facts obtained the lowest Sum Level score (Don and Carla) (see Table 10). A representational difference between Carla and Don is that Carla was able to give context to tasks when asked (see Table 3). Thus, one might say she had two representations with which to work. Furthermore, the participant who earned the highest Sum Level score, Jake, typically used memorized facts and the number line to complete addition and subtraction tasks, and was able to contextualize tasks when asked. Participants having a Sum Level score between Carla $\left(8^{*}\right)$ and Jake (14-*) used at most two representations, typically using a number line and memorized facts, except Beth who typically used a number line and could situate tasks into the context of debt.

\section{Participants' Initial Understanding of Equality}

The next four assertions concern participants' initial understanding of equality. These assertions detail how participants described the meaning of the equal sign and how they solved for variables in 1-step and 2-step equations.

Assertion 1. For statements not containing a missing value, all participants had a relational understanding of the equal sign, even though they could be also categorized as having an operational understanding for the first task.

Participants who viewed the equal sign operationally tended to say that the equal sign meant to find the answer of an expression, or "do something" to complete the statement. All participants answered in an operational manner to Task 1 (see Table 11), yet given the task, it is not surprising, given that it is natural and correct to do so.

Most participants focused on superficial features of the task or described the equal sign as indicating them to operate on the expression. This task by itself would not appropriately classify students' understanding of the equal sign; therefore, one must take into consideration participants' responses to other tasks without a missing value. Even the participants were not sure how to answer this task. In fact for this task, Jake asked, "Is this a trick question?" For the rest of the tasks without a missing value, all participants gave responses such that they could be seen to have a relational understanding of the equal sign. Most of them said that the answers were the same for tasks that used arithmetic, and for tasks that compared items and required no arithmetic, participants used words such as "the same" or "are in" to describe the equal 
sign. These answers are consistent with the findings of Seo and Ginsburg (2003), who postulated that students' understanding of the equal sign is not well integrated.

Table 11

Participants' Responses to Task 1, Module 3

Participant Response to 1st Task

Don $\quad$ "This hasn't been solved yet, and this has been solved."

Carla "That's already solved for you, and that's just to solve the problem."

Abby $\quad$ "This is just 5 plus 6, here they have an equal sign, and here they have the answer."

Hope $\quad$ "It equals something, but we don't know what it equals [5+6 =]"

Jim "This one has the answer, this one is going towards the answer, and this one, well they haven't finished it yet."

Jake $\quad$ They are all the same question...[5+6=11] that's 5 plus 6 equals 11 . [5+6=] they're saying like, you can do 11"

Jen "That says what it's equal to, like what's coming up, and that one says

Beth "It's giving you the answer."

One point worth mentioning, is that half of the participants [Jen, Abby, Hope and Beth], mentioned something to the effect that $8=10-2$ was written backwards, yet all believed that equality held since both sides of the equation had the same answer.

When it came to tasks with a missing value, half of the participants [Don, Hope, Jim, and Beth] mentioned the equivalence of expressions for the task $3+5=?+2$, yet no participant mentioned equivalent expressions for tasks with a variable depicted as a letter. In fact, Abby, Jake, and Jim stated something to the effect that the equal sign meant to solve for the missing variable, while the rest of the participants could not describe what the equal sign meant in those tasks and reiterated that it "just meant equal."

Assertion 2. Eventually, all participants obtained the correct answer for the missing question mark in task $3+5=?+2$. Most of the participants answered this task using an arithmetical method of operating.

All participants, except Jen and Abby, quickly obtained the value of the question mark in the task $3+5=?+2$ (see Table 12). Initially Jen obtained the answer of 4 , while Abby initially obtained the answer of 8 . Jen's procedure was to add 3 and 5 and then divide by 2 . Abby's procedure was to add the 3 and 5 and place the answer directly to the right of the equal sign. They both realized that because there was an equal sign, the expressions had to have the same answer. After realizing this, they both gave the correct answer of 6 . Every participant justified their answer of 6 by stating that the expressions had to be "equal" or have the same answer. 
Table 12

Six Participants' Initial Understanding of Solving Equations

\begin{tabular}{|c|c|c|c|c|c|c|}
\hline & Jen & Abby & Jake & Jim & Beth & Carla \\
\hline $3+5=?+2$ & arithmetical & arithmetical & arithmetical & arithmetical & arithmetical & algebraic \\
\hline answer & 4 then 6 & 8 then 6 & 6 & 6 & 6 & 6 \\
\hline$x+5=3$ & $\begin{array}{l}\text { cross } \\
\text { multiplication, } \\
\text { numerical } \\
\text { manipulation }\end{array}$ & $\begin{array}{l}\text { combination } \\
\text { of } \\
\text { arithmetic, } \\
\text { unwinding } \\
\text { and } \\
\text { algebraic }\end{array}$ & $\begin{array}{l}\text { quantitative } \\
\text { reasoning, } \\
\text { number } \\
\text { combinations }\end{array}$ & $\begin{array}{l}\text { quantitative } \\
\text { reasoning, } \\
\text { guess and } \\
\text { test }\end{array}$ & algebraic & $\begin{array}{l}\text { quantitative } \\
\text { reasoning, } \\
\text { number line }\end{array}$ \\
\hline answer & 7 & -2 & -2 & -2 & -2 & -2 \\
\hline $2 m+1=9$ & declined & PEMDAS & $\begin{array}{l}\text { can't } \\
\text { remember }\end{array}$ & $\begin{array}{l}\text { quantitative } \\
\text { reasoning, } \\
\text { guess and } \\
\text { test }\end{array}$ & $\begin{array}{l}\text { misapplication } \\
\text { of class } \\
\text { procedure }\end{array}$ & $\begin{array}{l}\text { misapplication } \\
\text { of class } \\
\text { procedure }\end{array}$ \\
\hline answer & none & 7.5 or 5.5 & none & -5 & 7 or -1 & 4.5 \\
\hline
\end{tabular}

According Boulton-Lewis et al (2000), this meant that students had an arithmetical understanding or pre-

algebraic understanding of the equal sign, depending on how they solved for the variable. An arithmetical understanding requires students to believe that the equal sign denotes the same answer on each side of the symbol, and students understand the use of arithmetical operations to solve for a missing value. A prealgebraic understanding requires students to not only view the equal sign as symbolizing the same answer to each expression, but also requires students to be able to solve for the missing value by using inverse procedures. Everyone but Don solved the task arithmetically (Knuth et al, 2006) since they computed the left expression and thought about what number when added to two would give them the sum of the left side. Don solved the task using the unwinding method since he subtracted 2 from the sum of 3 and 5. Carla, even though she did not state that both expressions were equivalent, solved the task algebraically by rewriting the expression in a step-by-step manner and applying the same transformations on each side. Knuth and colleagues would say that she operated algebraically, but Boulton and colleagues believed that in order for a response to be algebraic, the student needed to view the equal sign as denoting equivalence, which Carla did not explicitly state. Even though she did not state equivalence of expressions, I categorized her operating method as algebraic.

Students tended to have a more difficult time solving equations with a letter representing the variable (see Table12). In fact, two participants, Don and Jen, could not compute the correct value for $x$ in the task $x+5=3$.

Assertion 3. All participants who used some form of logic to complete $x+5=3$ obtained the correct answer. To solve this task, everyone used a different strategy than they had used to solve $3+5=?+2$. 
In solving 1-step equations, only Hope, Abby, and Beth used strategies explicitly described by Knuth and colleagues (2006) (see Table 12). Hope used a combination of arithmetic and unwinding strategies; Abby used a combination of arithmetic, unwinding, and algebraic; Beth used the algebraic strategy. The rest of the participants did not use a strategy specifically categorized by Knuth and colleagues:

- Don guessed but did not check his answer

- Jen used cross multiplication and numerical manipulation

- Jake used quantitative reasoning and number combinations

- Jim used quantitative reasoning and the guess and test strategy

- Carla used quantitative reasoning and a number line

By using quantitative reasoning, I mean that participants reasoned that the value of $x$ had to be negative since they had to add 5 to their answer to obtain 3 . Don and Jen were the only participants to obtain an incorrect answer, although not the same incorrect answer, and they both did not use a logical pattern to obtain their answer. Don guessed that the answer would be -8 because he combined the values of 5 and 3 and attached a negative sign, for no apparent reason. Jen believed she needed to use cross multiplication and set up the proportion $\frac{x}{5}=\frac{3}{100}$, but did not follow through in solving for $x$. Instead, she subtracted 3 from 5 and got 2 and then added 2 and 5 together to obtain the answer of 7 . While Jen knew that her answer of 7 was incorrect since she put it back into the equation to see if it worked, but Don did not check his answer, even after prompting him to do so; therefore he did not know his answer was incorrect.

Jake and Carla's method of operating was not described by previous research, yet they both obtained the correct answer. Jake reasoned that the value of $x$ would be negative. In doing so, he tried to combine the numbers in the task (5 and 3) using basic operations to obtain a negative value. By subtracting 5 from 3 , he got the answer of -2 . He checked this answer and saw that it made the expressions have the same answer; thus, he believed that $x=-2$. Carla, on the other hand, used a number line to solve this task, which is fascinating in and of itself because she had never used a number line to solve tasks until after she used the integer applets. She guessed at a value on the number line, then traveled to the right 5 spaces and noted where she landed. When she started on -2 , she saw that after she traveled 5 spaces to the right, she landed on the 3 . Thus, she believed that $x=-2$.

Every participant used a different strategy from the strategy they used to solve the previous task $3+5=?+2$ (see Table 12). While it is unclear as to why they did so, it may be due to the following possibilities: 
- $3+5=?+2$ has an operation on both sides of the equal sign where $x+5=3$ does not

- $3+5=?+2$ has a question mark where $x+5=3$ has a letter denoting the variable

- The value of variable in $3+5=?+2$ is positive, where the value of $x+5=3$ is negative.

If students believed that the equal sign meant to do something, they had to "do something" on both sides of the equal sign for the question mark task, where there was nothing "to do" on the right side of the equal sign in the letter task. According to previous literature (Wagner, 1983), students have a more difficult time when variables are in lettered form due to the duplicity of letters in language and math. One has also seen, through this data and previous literature, that students have a more difficult time with negative numbers than positive numbers. Therefore, all possibilities are likely to have affected students solution methods and answers to this task.

Assertion 4. None of the participants obtained the correct answer for a two-step equation. Most of them tried to rely on a procedure from their math class to answer this task and did not use the idea of equivalent expressions. Only Hope and Jim reasoned that the value of the variable had to be negative.

Every participant used a different strategy to solve this task than they used to solve $3+5=?+2$ and $x+5=3$, except for Jim and Don (see Table 12). Jim once again reasoned that the value of $x$ had to be negative and tried to use that knowledge to guess an answer; yet, he could never find one that worked in the equation. While Don guessed at answer for the 1-step lettered equation, he guessed and actually checked his answer to the 2-step lettered equation, even though none of his answers fit the equation. Most of the participants tried to remember and use a procedure they had learned in class. No one correctly remembered it (see Table 13).

Table 13

Responses to Finding the Value of a Variable in an Equation

Participant Responses to Finding the Value of a Variable in an Equation

Carla "You have to... divide by 2 on both sides, then cross off the 2, and then 9 divided by 2 is 4.5 , so $\mathrm{m}$ is $4.5 "$

Abby "You have to divide by 2 [first]..cause if you do it be PEMDAS, you're supposed to do multiplication and dividing first."

Hope $\quad$ "We've done this in class before... what we did was write it out. Two times blank plus 9 equals $1 . . . m$ is 5 . I don't know how that works, but it does."

Jake "We've solved them before, but I can't remember how we did it...you times two by something don't you?"

Beth "Subtract 2... so the variable can be by itself, so you have to cross it out. And you have to do the same thing on the other side."

Because Jen knew she could not solve the 1-step equation, she declined to attempt the 2-step equation. 
I think two factors hindered participants ability to solve this equation. Every participant had noted that they had solved two-step equations in math class. Most of the participants alluded to the fact that they had learned a specific procedure to solve them. One factor is that participants may have believed that they needed the procedure because only the procedure would help them find the answer to the problem. Without that procedure, there was no way to find the answer. If this was the case, the reason they may have believed the exact procedure to be necessary was because they did not have a good understanding of the equal sign. The second factor is that without a good understanding of the equal sign and knowing that it denotes the expressions to be equivalent, one would have a very difficult time knowing how to use reason to find the missing value.

\section{Research Question 2}

Five assertions concerning participants' final understanding operation on integers and solving equations are presented: two concerning Peled's rating in addition and subtraction, one concerning the reason for incorrect solution methods, one concerning how the applets impacted participant understanding of operations on integers, and one concerning how the EBA impacted participant understanding of solving equations.

\section{Participants' Final Understanding of Addition and Subtraction of Integers}

In determining participants' final understanding, I relied on how they performed on tasks that I gave to them to solve when they arrived for their last session. I did not allow them to use the applets or a calculator to determine their answer; however, I supplied them with a paper number line if they asked for it.

Abby, Jim, and Hope had met with me for three sessions, and the rest of the participants only met with me for two sessions. Table 14 gives each type of subtraction task, how they operated when they began the session without the applet, and how they operated at the end of the session. Gray boxes represent that the participant did not use that specific strategy. If a participant has SDA or SAA in the number line column (NL), it means they either used a paper number line and emulated the visual depicted on the SDA or SAA, respectively, or they imagined the number line in their head and talked about using it in the fashion of the SDA or SAA respectively. Participants who have "move left $b$ " under the number line, means they started at the minuend and moved left $b$ spaces on the number line. Hope has "Folded NL" in her number line column. This refers to her creative use of a folded number line. 
Table 14

How Participants, Who Had Three Sessions, Operated After Using Subtraction Applets

\begin{tabular}{|c|c|c|c|c|c|c|c|c|}
\hline & \multicolumn{2}{|l|}{ Abby } & \multicolumn{2}{|c|}{ Jim } & \multicolumn{2}{|c|}{ Hope } \\
\hline & & & Memorized facts & NL & Memorized facts & NL & Memorized facts & NL \\
\hline \multirow{5}{*}{$a-b$} & 1st Session & End & $a+-b$ & SDA & & move left $b$ & & SDA \\
\hline & \multirow{2}{*}{ 2nd Session } & Begin & $a+-b$ & SDA & & move left $b$ & & move left $b$ \\
\hline & & End & $a+-b$ & SDA & & move left $b$ & & move left $b$ \\
\hline & \multirow{2}{*}{ 3rd Session } & Begin & $a+-b$ & SDA & & move left $b$ & & move left $b$ \\
\hline & & End & $a+-b$ & SDA & & move left $b$ & & move left $b$ \\
\hline \multirow{5}{*}{$-a-b$} & 1st Session & End & $-a+-b$ & SDA & $-a+-b$ & move left $b$ & $-(a+b)$ & SDA \\
\hline & \multirow{2}{*}{ 2nd Session } & Begin & $-a+-b$ & SDA & $-a+-b$ & move left $b$ & & combine \\
\hline & & End & $-a+-b$ & SDA & $-a+-b$ & move left $b$ & $-(a+b)$ & SAA \\
\hline & \multirow{2}{*}{ 3rd Session } & Begin & $-a+-b$ & SDA & $-a+-b$ & move left $b$ & $-(a+b)$ & \\
\hline & & End & $-a+-b$ & SDA & $-a+-b$ & move left $b$ & $-(a+b)$ & SAA \& SDA \\
\hline \multirow{5}{*}{$a--b$} & 1st Session & End & & SDA & $a+b$ & & $a+b$ & SDA \\
\hline & \multirow{2}{*}{ 2nd Session } & Begin & $a+b$ & SDA & $a-b$ & move left $b$ & $a-b$ & move left $b$ \\
\hline & & End & $a+b$ & SDA & $a+b$ & & $a+b$ & SAA \\
\hline & \multirow{2}{*}{ 3rd Session } & Begin & $a+b$ & SDA & $a-b$ & & & move left $b$ \\
\hline & & End & $a+b$ & SDA & $a+b$ & & $a+b$ & SAA \& SDA \\
\hline \multirow{5}{*}{$-a--b$} & 1st Session & End & & SDA & $-a+b$ & & & Folded NL \\
\hline & \multirow{2}{*}{ 2nd Session } & Begin & $-a+b$ & $-a+b$ & $-a-b$ & move left $b$ & & Folded NL \\
\hline & & End & $-a+b$ & $-a+b$ & $-a+b$ & & & SAA \\
\hline & \multirow{2}{*}{ 3rd Session } & Begin & $-a+b$ & SDA & $-a+b$ & & & Folded NL \\
\hline & & End & $-a+b$ & SDA & $-a+b$ & & & SAA, SDA \\
\hline \multicolumn{3}{|c|}{ Final QD } & \multicolumn{2}{|l|}{$\mathrm{L} 4$} & \multicolumn{2}{|c|}{ L4- } & \multicolumn{2}{|c|}{ L4- } \\
\hline \multicolumn{3}{|c|}{ Final NLD } & \multicolumn{2}{|l|}{$\mathrm{L} 4$} & \multicolumn{2}{|c|}{ L4- } & \multicolumn{2}{|c|}{ L4- } \\
\hline
\end{tabular}

Table 15 depicts how the rest of the participants operated to solve subtraction tasks, for they only met with me for two sessions. Gray boxes represent that the participant did not use that specific strategy. If a participant has SDA or SAA in the number line column (NL), it means they either used a paper number line and used the applet idea depicted on the SDA or SAA, respectively, or they imagined the number line in their head and talked about using it in the fashion of the SDA or SAA respectively. Participants who have "move left $b$ " under the number line, means they started at the minuend and moved left $b$ spaces on the number line. Don has "combine" located in his columns. This means that he used a combination of strategies in an incorrect manner: the AAA and SDA. 
Table 15

How Participants, Who Had Two Sessions, Operated After Using Subtraction Applets

\begin{tabular}{|c|c|c|c|c|c|c|c|c|c|c|c|c|}
\hline & & & Jake & & Don & & Carla & & Beth & & Jen & \\
\hline & & & $\begin{array}{l}\text { Memorized } \\
\text { facts }\end{array}$ & NL & $\begin{array}{l}\text { Memorized } \\
\text { facts }\end{array}$ & NL & $\begin{array}{l}\text { Memorized } \\
\text { facts }\end{array}$ & NL & $\begin{array}{l}\text { Memorized } \\
\text { facts }\end{array}$ & NL & $\begin{array}{l}\text { Memorized } \\
\text { facts }\end{array}$ & NL \\
\hline \multirow{3}{*}{$a-b$} & $\begin{array}{l}1 \text { st } \\
\text { Session }\end{array}$ & End & $a-b$ & $\begin{array}{l}\text { move } \\
\text { left } b\end{array}$ & & SDA & & SAA & & $\begin{array}{l}\text { move } \\
\text { left } b\end{array}$ & & SDA \\
\hline & \multirow{2}{*}{$\begin{array}{l}\text { 2nd } \\
\text { Session }\end{array}$} & Begin & & $\begin{array}{l}\text { move } \\
\text { left } b\end{array}$ & & combine & & SAA & & $\begin{array}{l}\text { move } \\
\text { left } b\end{array}$ & & $\begin{array}{l}\text { move } \\
\text { left } b\end{array}$ \\
\hline & & End & & $\begin{array}{l}\text { move } \\
\text { left } b\end{array}$ & & SDA & & SAA & & $\begin{array}{l}\text { move } \\
\text { left } b\end{array}$ & & $\begin{array}{l}\text { move } \\
\text { left } b\end{array}$ \\
\hline \multirow{3}{*}{$-a-b$} & $\begin{array}{l}1 \text { st } \\
\text { Session }\end{array}$ & End & $-a+-b$ & $\begin{array}{l}\text { move } \\
\text { left } b\end{array}$ & & SDA & & SAA & & $\begin{array}{l}\text { move } \\
\text { left } b\end{array}$ & $-(a+b)$ & SDA \\
\hline & \multirow{2}{*}{$\begin{array}{l}\text { 2nd } \\
\text { Session }\end{array}$} & Begin & $-a+-b$ & $\begin{array}{l}\text { move } \\
\text { left } b\end{array}$ & combine & combine & & SAA & & $\begin{array}{l}\text { move } \\
\text { left } b\end{array}$ & $-(a+b)$ & $\begin{array}{l}\text { move } \\
\text { left } b\end{array}$ \\
\hline & & End & $-a+-b$ & $\begin{array}{l}\text { move } \\
\text { left } b\end{array}$ & & SDA & & SAA & & $\begin{array}{l}\text { move } \\
\text { left } b\end{array}$ & $-(a+b)$ & $\begin{array}{l}\text { move } \\
\text { left } b\end{array}$ \\
\hline \multirow[t]{3}{*}{$a--b$} & $\begin{array}{l}1 \text { st } \\
\text { Session }\end{array}$ & End & $a+b$ & SAA & $a+b$ & & $\begin{array}{l}\text { analogous } \\
\text { task } a-b \\
\text { then } \\
\text { determine } \\
\text { sign }\end{array}$ & SAA & $a+b$ & SAA & $a+b$ & SDA \\
\hline & \multirow{2}{*}{$\begin{array}{l}\text { 2nd } \\
\text { Session }\end{array}$} & Begin & & SAA & combine & combine & & SAA & $a+b$ & SAA & $a+b$ & \\
\hline & & End & & SDA & & SDA & & SAA & $a+b$ & SAA & $a+b$ & \\
\hline \multirow[t]{3}{*}{$-a--b$} & $\begin{array}{l}1 \text { st } \\
\text { Session }\end{array}$ & End & $-a+b$ & SAA & $\begin{array}{l}a-b \text { then } \\
\text { sign based on } \\
\text { position }\end{array}$ & & & SAA & & SAA & & SDA \\
\hline & \multirow{2}{*}{$\begin{array}{l}\text { 2nd } \\
\text { Session }\end{array}$} & Begin & & SAA & combine & combine & & SAA & $-a+b$ & SAA & & SDA \\
\hline & & End & & SDA & & SDA & & SAA & $-a+b$ & SAA & & SDA \\
\hline \multicolumn{3}{|l|}{ Final QD } & \multicolumn{2}{|l|}{ L4 } & \multicolumn{2}{|l|}{ L1 } & \multicolumn{2}{|l|}{ L4 } & \multicolumn{2}{|l|}{ L4 } & \multicolumn{2}{|l|}{ L4 } \\
\hline \multicolumn{3}{|l|}{ Final NL } & \multicolumn{2}{|l|}{ L4 } & \multicolumn{2}{|l|}{ L1* } & \multicolumn{2}{|l|}{ L4 } & \multicolumn{2}{|l|}{ L4 } & \multicolumn{2}{|l|}{ L4 } \\
\hline
\end{tabular}

Assertion 1. Not all participants fit cleanly into Peled's NLD and QD with respect to subtraction and addition upon the beginning of their last session.

While over half of the participants attained the highest level in the NLD and QD with respect to subtraction, three of them did not: Hope, Jim, and Don (see Tables 14 \& 15). Hope and Jim received a score L4- because they were able to accurately solve all subtraction tasks except in the form $a--b$ by the beginning of their third session. They both had only a minor setback in solving this type of task, and realized their mistake. Hope used the SAA, without the equation displaying, and figured out her mistake and changed her answer to that task. For all subsequent tasks in the form $a--b$, she used the expression $a+b$ and justified her answer by visualizing an number line and explaining her answer in terms of movement based upon the SDA and SAA. Jim realized his mistake after he compared the task $a-b$ to $a--b$. After which, he changed his original answer to $a--b$ and changed the expressions $a--b$ into $a+b$ for subsequent tasks. On the other hand, Don obtained a score of $\mathrm{L} 1 *$ because he faltered on each 
type of task at the beginning of the last session. Upon working with the SDA during his last session, Don solved all subtraction tasks by visualizing the number line in his head, and describing the movement on the SDA (see Table 15).

One will notice that the NLD levels and QD levels are the same for each participant who attained higher than a Level 3 (see Table 15). Participants only supplied a context of the task and answer when asked. Thus, given that they could give a context for tasks they correctly solved, they earned the same level. Only Don could not give a context for subtraction tasks, yet knew that negative and positive numbers were opposite in quality characteristic; therefore, he obtained Level 1.

Don was the only participant to use the Addition Arrow Applet (AAA). Don was able to solve all addition tasks with positive addends both before and after the instructional session. Table 16 describes how Don attempted to solve subtraction tasks with a negative addend. Don attained a final score of L2- in the NLD because through his session, he discovered that addition meant to step right and subtraction meant to step left. However, he was unable to solve opposite or same-signed addend tasks. He was able to determine the sign of the sum by using the same sign as the sign of the highest absolute-valued addend. Don attained a final score of L1 in the QD because he knew that negative and positive numbers were opposite in quality context. Furthermore, during the sessions, when I asked him to situate his answers into context, he could do so.

Table 16

How Don Operated on Addition Tasks

\begin{tabular}{|c|c|c|c|c|}
\hline & \multicolumn{2}{|l|}{ 1st Session } & \multicolumn{2}{|l|}{ 2nd session } \\
\hline & Before Applet & After Applet & Beginning & End \\
\hline $\begin{array}{l}a+-b \\
-a+b\end{array}$ & $\begin{array}{l}a+-b=a \\
-a+b=b\end{array}$ & $\begin{array}{l}\text { Sign = highest } \\
\text { absolute valued } \\
\text { addend; used } \\
\text { AAA method } \\
\text { on NL }\end{array}$ & $\begin{array}{l}\text { Sign = highest } \\
\text { absolute } \\
\text { valued addend; } \\
\text { SDA to find } \\
\text { distance }\end{array}$ & $\begin{array}{l}\text { Sign }=\text { highest } \\
\text { absolute valued } \\
\text {; addend; } \\
\text { magnitude }=\mid a \\
-b \mid\end{array}$ \\
\hline$-a+-b$ & $|a-b|$ & $\begin{array}{l}\text { Sign of highest } \\
\text { absolute valued } \\
\text { addend; used } \\
\text { AAA method } \\
\text { on NL }\end{array}$ & $\begin{array}{l}\text { Sign = highest } \\
\text { absolute } \\
\text { valued addend; } \\
\text { SDA to find } \\
\text { distance }\end{array}$ & $-(a+b)$ \\
\hline Add NLD & L1 & & L2- & \\
\hline Add QD & Below L1 & & L1 & \\
\hline
\end{tabular}


Assertion 2. All participants increased in their understanding and Peled level with respect to subtraction after using the SAA and SDA. Don increased his understanding and Peled level with respect to addition after using the $A A A$.

Table 17

Participant $Q D$ and NLD Levels Before and After Using Subtraction Applets

Don Carla Jen Jim Abby Beth Jake Hope

NLD QD NLD QD NLD QD NLD QD NLD QD NLD QD NLD QD NLD QD

Prior L1 Below L1 L1 L2* L3* $^{2}$ L1+ L3* L2* L3* L2* L3* $^{2}$ L2 L3*/L4- L2* L4- L2*

\begin{tabular}{llllllllllllll} 
After L1* L1* & L4 & L4 & L4 & L4 & L4- & L4- L4 & L4 & L4 & L4 & L4 & L4 & L4- & L4- \\
\hline
\end{tabular}

By looking at Table 17, one can easily see that each participant remained the same or increased in his or her Peled level score in at least one dimension. All participants, except Hope, increased in both dimensions. Hope remained at the same high level in the NLD before and after working with the SAA and SDA. During the last session, she was still unable to accurately solve tasks in the form $a--b$, until she worked with the SDA during her last session (see Table 14).

By looking at Table 16, one notices that Don's understanding of addition of negative addends increased slightly. While Don was unable to determine the sign of the sum with at least one negative addend, he could find the magnitude. By the end of our last session, he could answer all addition tasks correctly.

It is noteworthy that participants' increased understanding was due to the discoveries they made while using the applets and/or the direct visual impact of the applets (see Table 18). Participants solved the difficult types of tasks (those with a negative subtrahend) correctly by the end of their last session by using what they learned while working with the applets. For instance, Jim used the generalization that subtraction of a negative number was like addition to solve tasks with negative subtrahends. Everyone else, enacted either the method of the SDA or the SAA on the number line.

Assertion 3. Even though each participant increased overall in his or her understanding of subtraction of negative numbers, some had difficulty remembering how to solve specific tasks from one session to the next. Similarly, Don had difficulty remembering how to solve addition tasks with negative addends from his first session to second session. Most incorrect answers were due to participants reverting back to old solution methods, or trying to integrate new knowledge of subtraction (or addition) to previous knowledge of operating on integers. 
For addition tasks in the beginning of the second session, Don used only one method of operating: combining his discoveries from using the AAA and SDA (see Table15). He determined the magnitude of the answer by finding the distance between the addends or minuend and subtrahend, and applied the sign of the highest absolute valued number in the task to this value. Therefore, Don obtained incorrect answers to the tasks in the forms: $a+-b,-a+b,-a+-b$ with $a, b>0$, due to having the incorrect magnitude. For all subtraction tasks in the beginning of the second session, Don used only one method of operating: combining his discoveries from using the AAA and the SAA. He determined the magnitude by starting at $-a$ and traveling to the left $b$ spaces if $b>0$ and traveling to the right $b$ spaces if $b<0$. Then he applied the sign of the highest absolute valued number in the task to this value. Therefore, Don obtained incorrect answers to the tasks in the forms $a-b,-a-b, a--b$, and $-a--b$ for $b>a>0$, due to the incorrect sign (see Table 15).

With regards to subtraction, everyone who obtained the correct answer for subtraction tasks with positive subtrahends in the interview section, also did so during the beginning of the last session of working with them (see Tables $14 \& 15$ ). Only Hope, faltered with one of these types of tasks: $-a-b$ for $a, b>0$. In the beginning of the second session, Hope used a combination of her folded number line strategy and the SDA to this type of task. Since she knew that $-a-b$ was equivalent to $-a+-b$, she decided to use the latter expression. She determined the magnitude of the answer by finding the distance between $-a$ and $-b$. In doing so, she folded her number line, and traveled from $a$ to $b$. She determined the sign of the magnitude based on the direction she traveled. If she traveled up from $a$ to $b$, the sign was positive; if she traveled down from $a$ to $b$, the sign was negative. Her use of this method was not too surprising given that she enjoyed using the SDA in her first session, and enjoyed using her folded number line, yet could never do so with opposite signed numbers. After working with the SAA during our second session, she came into the third session know how to accurately solve subtraction tasks in the form $-a-b$ for $a, b>0$.

Much like the initial interview section, the hardest subtraction tasks were the ones with negative subtrahends, with the hardest type being of the form $a--b$ (see Tables $14 \& 15$ ). Abby, Jim, and Hope faltered on this task during the beginning of their second to last session; Jim, Hope, and Don faltered on this type during their last session. Only Jim faltered in his second to last session on tasks in the form $-a--b$, and only Don person faltered in his last session.

Abby, Jim, and Hope incorrectly solved tasks in the form $a--b$ in the beginning of their second to last session (see Table 14). Out of those three, only Abby correctly solved this type of task in the beginning of her last session. There was a difference in how Abby, Jim, and Hope operated. Jim and Hope 
reverted to solving this type of task using their previous methods: transforming it into $a-b$ and solving this expression instead, and justifying this answer by starting at $a$ on the number line and moving $b$ spaces to the left. On the other hand, Abby solved this task by transforming it into $a+b$ and using the SDA to justify this answer but believed that there was a possibility that some rule said that her answer should have been calculated as $a-b$. When Jim and Hope returned, Jim solely used memorized facts to solve these types of tasks and transformed $a--b$ into $a-b$, yet upon giving him the task $a+-b$, he recognized his mistake. At the beginning of Hope's third session, she solely used the number line and moved left $b$ spaces from $a$. She did not whole-heartedly believe this was the correct answer, but she finally settled on it. At least this time, she was aware that she could be incorrect.

Jim incorrectly solved the tasks in the form $-a--b$ at the beginning of his second session. He reverted back to his old strategies of transforming $-a--b$ into $-a-b$ and solving for the latter expression. They he justified this solution by starting at $-a$ on the number line and moving left $b$ spaces. Upon showing him the SAA, he remembered that he had to travel right $b$ spaces from $-a$, and adjusted his answer accordingly.

\section{Assertion 4. The applets impacted how participants viewed the operation of subtraction and solved} subtraction tasks. Don's interaction with the AAA impacted how he viewed the operation of addition and solved addition tasks.

Except for Don, who never explicitly specified how he viewed subtraction, all participants had an initial idea of taking away items. By the end of the last session, all participants saw subtraction as both take away and distance. Half of the participants changed their method of solving subtraction tasks because they preferred distance instead of take away. Participants, who preferred viewing subtraction as distance, also preferred the SDA rather than the SAA.

All participants had a unary understanding of subtracting a negative from a positive minuend by the time the session was over and could transform the task $a--b$ into $a+b$. While participants saw that $-a--b$ had the same answer as $-a+b$, only Jim and Beth truly saw it as addition due to the movements on the SAA and their belief that addition means to "move right" on the number line and subtraction means to "move left".

It is interesting to note that even though all participants, except Don, could correctly answer tasks in the form $a-b$ and $-a-b$ before using the applet, some participants abandoned their old strategies in favor for visualizing the applets in their head or using discoveries they found after working with the applets 
(see Table 18). Some participants abandoned their strategies completely, some abandoned their strategies between sessions, and some did not waver in their operating procedures (see Tables 14, 15, and 18).

Abby, Hope, Don, and Carla completely abandoned their strategies for solving tasks in the form $a-b$ (not Hope) and $-a-b$ in favor for discoveries they learned working with the applets. Before using the applets, Abby justified her answers to $a-b$ and $-a-b$ by starting at $a$ and moving left $b$ spaces on a number line. After the first time she used the applets until the end of the third session, she justified her answer to those tasks by visualizing the number line in her head and using an explanation of movement as the SDA. Before using the applets, Hope justified her answer to $-a-b$ by starting at $-a$ and moving left $b$ spaces on a number line. After using the applets, she justified her answer to those tasks by visualizing the number line in her head and using an explanation of movement in the SDA and SAA. Before using the applets, Don solved $a-b$ and $-a-b$ by taking the difference of $a$ and $b$ and attaching the sign of the highest absolute valued number. After the first time he used the applets until the end of the third session, he justified his answer either by visualizing the number line in his head and using an explanation of movement as the SDA, or using an explanation combining the AAA and SAA. Before using the applets, Carla solved $a-b$ and $-a-b$ by transforming them into $a+-b$ and $-a+-b$ and solving these new expressions. She did not use any movement on the number line to justify her answers. Yet, after working with the SAA in her first session, she never used memorized facts and only solved and justified her answers by visualizing the number line in her head and using an explanation of movement as the SAA.

Jen abandoned her strategy of solving $a-b$ and $-a-b$ immediately after she worked with the applet in the first session, but reverted to previous strategies for the entire second session. Before using the applets, Jen justified her answers to tasks with a positive subtrahend by starting at $a$ and moving left $b$ spaces on a number line. At the end of her first session, after she had worked with the SDA, she did not move this way on the number line to justify her answers, instead she used the distance method by visualizing the movements on the SDA.

Hope abandoned her strategy of solving $a-b$ immediately after she worked with the applet in the first session, but reverted to her previous strategy for the entire second and third session. Before using the applets, Hope solved and justified her answer tasks in the form $a-b$ by starting at $a$ and moving left $b$ spaces on a number line. At the end of her first session, after she had worked with the SDA, she did not move this way on the number line to justify her answers, instead she used the distance method by visualizing the movements on the SDA. 
Table 18

How Participants Operated Beginning of First Interview and End of Last Session

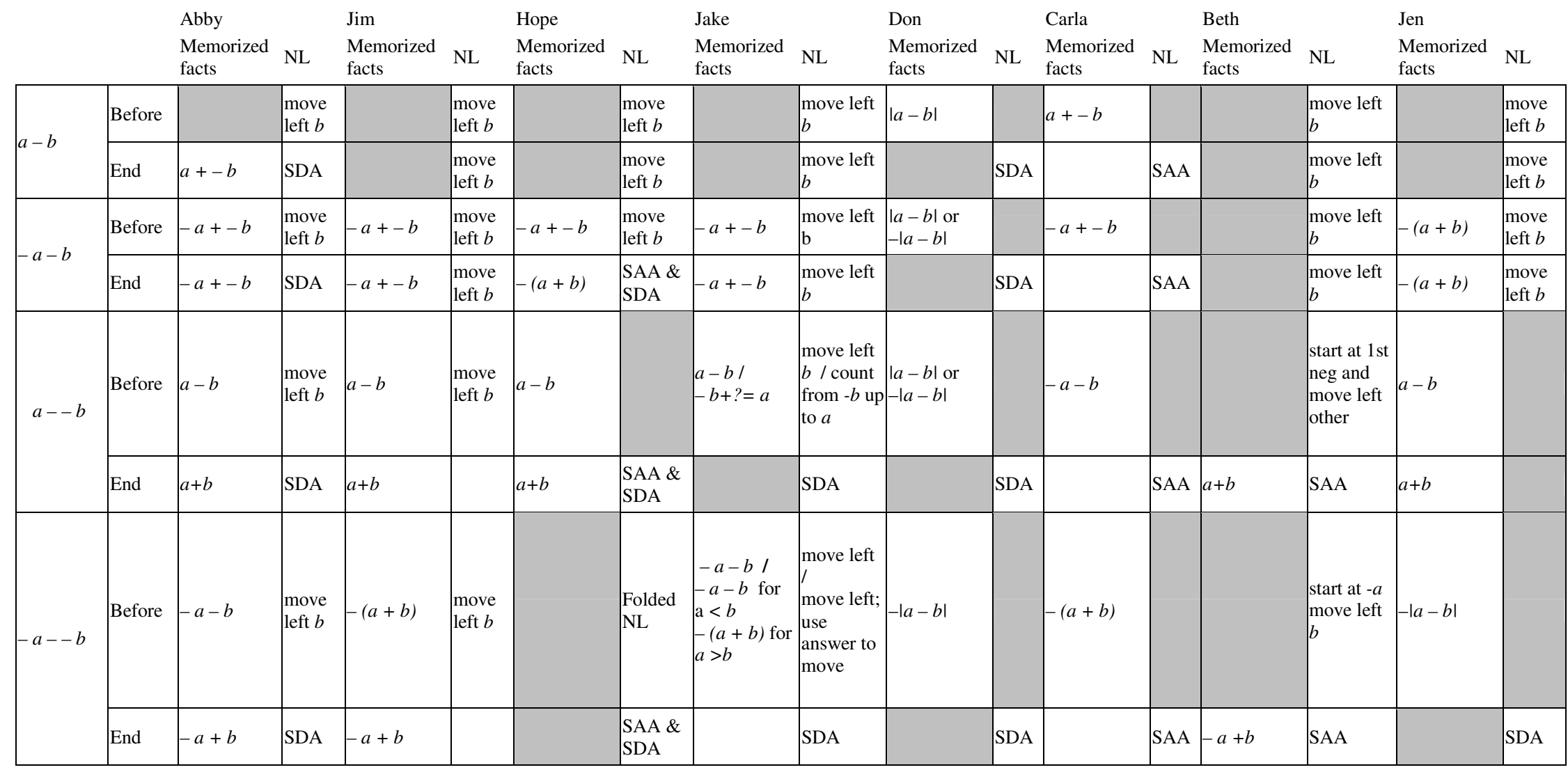


Jim, Jake, and Beth never changed their procedure for solving tasks with positive subtrahend even after working with the applets. They may have felt that since they obtained the correct answer, that they did not need to adjust their method and use the strategy proposed by the applets. Jim, Jake, and Beth all used the number line by starting at $a$ and moving left $b$ spaces to find or justify their answers. Jim and Jake also transformed the expression $a-b$ into $a+-b$ and $-a--b$ into $-a+-b$ and solved these latter expression computationally.

Tasks with a negative subtrahend were harder than tasks with a positive subtrahend. Participants, who could correctly solve at least some tasks with negative subtrahends (Jake, Don, Jen, and Hope) before using the applets, abandoned their old strategies in favor for visualizing the applets in their head or using discoveries they found after working with the applets (see Table 18). In fact, even those participants who obtained an incorrect answer before using the applets did the same. Most participants abandoned their strategies completely, and two abandoned their initial strategies between sessions. Ultimately, at the end of the last session, every participant used a discovery from using the applets to answer subtract tasks with negative subtrahends (see Tables 14, 15, and 18).

Abby, Jake, Don, Carla, Beth, and Jen completely abandoned their strategies for solving tasks in the form $a--b$ and $-a--b$ in favor for discoveries they learned working with the applets (see Table 18).

Before using the applets, Abby and Jen transformed $a--b$ into $a-b$ and justified their answer by moving left $b$ spaces on the number line. Afterwards, Abby and Jen transformed $a--b$ into $a+b$, and Abby justified her answer by visualizing the number line and enacting the movements of the SDA. Don and Carla used memorized rules to solve $a--b$, while Beth solely used a number line. Don transformed $a--b$ into $|a-b|$ or $-|a-b|$ depending on the sign of the highest absolute valued number, and Carla transformed $a--b$ into $-a-b$. After using the applets, Don referenced the SDA, SAA, AAA, and transformed $a--b$ into $a+b$. Carla referenced the SAA and noted the opposite movement on the number line for the tasks $a--b$ and $a-b$. Jake correctly answered tasks in the form $a--b$ before using the applets, yet upon using the applets by transforming the task $a--b$ into $-b+?=a$ and starting on the number line at $-b$ and counting the spaces until he reached $a$. He never referred to this strategy after working with the applets; instead transformed the expression into $a+b$ and referenced the SAA and SDA.

Before using the applets, Jake, Jen and Don obtained the correct answers for tasks in the form $-a--b$ with $a>b$, by transforming the task into $-(a-b)$. After using the applets, Jake used the expression $-a+b$ and referenced the SAA and SDA; Jen referenced the SDA; Don mentioned used the 
expression $a-b$ and determined the sign based on the position of the largest absolute valued number and referenced the AAA, SAA, and SDA. Carla, Beth and Abby all had different ways of completing tasks in the form $-a--b$ before working with the applets, however, afterwards, all referenced either the SAA or SDA. Before working with the applets, Carla transformed $-a--b$ into $-(a+b)$; Beth started at $-a$ on the number line and moved left $b$ spaces; Abby transformed $-a--b$ into $-a-b$ and started at $a$ and moved left $b$ spaces on a number line. After working with the applets, Carla immediately and consistently visualized the number line and explained the movements of the SAA; Beth did the same as Carla but also transformed the expression into $-a+b$; Abby transformed the expression into $-a+b$ and visualized the number line and explained the movements of the SDA.

Those participants who reverted to their previous operating methods for $a--b$ and $-a--b$ for some portion of a session were Jim and Hope (see Tables 14 \& 15). Both Jim and Hope transformed the expression $a--b$ into $a-b$, and Jim justified his answer by moving left $b$ spaces on the number line from $a$, prior to working with the applets. At the beginning of the second session, both Jim and Hope operated in the same manner, yet at the end, Jim and Hope correctly transformed the expression to $a+b$ and Hope justified her answer by moving according to the SAA. At the beginning of the last session, Jim transformed the expression into $a-b$ and Hope moved left $b$ spaces on the number line from $a$, yet by the end of the third session, Jim and Hope correctly transformed the expression to $a+b$ and Hope justified her answer by moving according to the SAA and SDA.

For tasks in the form $-a--b$, Jim transformed the expression into $-(a+b)$ and justified his answer by moving left $b$ spaces on the number line from $a$, prior to working with the applets. Hope could correctly answer these types of tasks by using her folded number line strategy. At the end of the first session, Jim transformed the expression into $-a+b$, and Hope continued to use her folded number line strategy. At the beginning of the second session, both Jim and Hope used their initial strategies, yet at the end of the second session, Jim once again transformed the expression into $-a+b$, and Hope solved the tasks visualizing the number line and using the method of the SAA. At the beginning of the third session and end of the third session, Jim consistently transformed the expression into $-a+b$. Hope began the third session by using her folded number line strategy, yet ended it by visualizing the number line and using the methods of the SAA and SDA.

Overall, by the end of the last session each participant solved tasks with a negative minuend either by transforming the task into the correct expression $a+b$ and $-a+b$, which they learned using the applets, or visualized the number line and described their imagined movements that corresponded to the 
SAA or SDA. Abby and Beth consistently used both the new expression and applets; Hope used the new expression and applet for $a--b$ and only the expression for $-a--b$; Jake, Don, Carla, and Jen consistently used the applets; Jim consistently used the new expressions. Overall, five participants used $a$ $+b$, four used the idea behind the SDA, and three used the idea behind the SAA to solve the hardest task, $a$ $--b$. Three participants used $-a+b$, five used the idea behind the SDA, and three used the idea behind the SAA to solve the task $-a--b$.

Don was the only one to use the AAA, and he completely abandoned his initial strategy for solving tasks in the form $a+-b,-a+b$ and $-a+-b$ in favor for discoveries he learned working with the AAA and SDA (see Table 18). Don solved addition tasks with one negative addend by giving the value of the positive addend, however after working with the applet in his first session, he visualized the number line and described movements of the AAA to obtain the magnitude of the sum. He determined the sign of the sum by using the sign of the highest absolute valued addend. These strategies he discovered by using the AAA. At the beginning of his second session, he obtained the sign of the sum by using the sign of the highest absolute valued addend, but obtained the magnitude by visualizing the number line and describing the movements of the SDA. While this method was incorrect, the applets still impacted his operating methods; so much so that he abandoned his initial operating strategy. By the end of the second session, Don obtained the sign of the sum by using the sign of the highest absolute valued addend, and obtained the magnitude by taking the difference of $a$ and $b$, which he discovered by using the AAA.

For tasks with two negative addends, Don initially absolute value of the difference of $a$ and $b$. After working with the AAA, he visualized the number line and described movements of the AAA to obtain the magnitude of the sum by the end of the first session. At the beginning of the second session, he obtained the sign of the sum by using the sign of the highest absolute valued addend, but obtained the magnitude by visualizing the number line and describing the movements of the SDA. While this method was incorrect, the applets still impacted his operating methods; so much so that he abandoned his initial operating strategy. By the end of the second session, Don added the absolute values of the addends and attached a negative sign.

The applets also made an impact on the type of representations participants used by the end of their last session (see Table 19). The row labeled "Before" indicates what representation each participant used in the initial interview to solve that specific task. The row labeled "End" indicates what representation each participant used at then end of the last session. 
Table 19

Type of Representations Used By Participants

\begin{tabular}{|c|c|c|c|c|c|c|c|c|c|}
\hline & & Don & Carla & Jen & Hope & Jake & $\mathrm{Jim}$ & Abby & Beth \\
\hline \multirow{2}{*}{$a-b$} & Before & $\begin{array}{l}\text { Memorized } \\
\text { facts }\end{array}$ & $\begin{array}{l}\text { Memorized } \\
\text { facts }\end{array}$ & $\begin{array}{l}\text { Number } \\
\text { line }\end{array}$ & $\begin{array}{l}\text { Number } \\
\text { line }\end{array}$ & $\begin{array}{l}\text { Number } \\
\text { line }\end{array}$ & $\begin{array}{l}\text { Number } \\
\text { line }\end{array}$ & $\begin{array}{l}\text { Number } \\
\text { line }\end{array}$ & $\begin{array}{l}\text { Number } \\
\text { line }\end{array}$ \\
\hline & End & $\begin{array}{l}\text { Number } \\
\text { line }\end{array}$ & $\begin{array}{l}\text { Number } \\
\text { line }\end{array}$ & $\begin{array}{l}\text { Number } \\
\text { line }\end{array}$ & $\begin{array}{l}\text { Number } \\
\text { line }\end{array}$ & $\begin{array}{l}\text { Number } \\
\text { line }\end{array}$ & $\begin{array}{l}\text { Number } \\
\text { line }\end{array}$ & Both & $\begin{array}{l}\text { Number } \\
\text { line }\end{array}$ \\
\hline \multirow{2}{*}{$-a-b$} & Before & $\begin{array}{l}\text { Memorized } \\
\text { facts }\end{array}$ & $\begin{array}{l}\text { Memorized } \\
\text { facts }\end{array}$ & Both & Both & Both & Both & Both & $\begin{array}{l}\text { Number } \\
\text { line }\end{array}$ \\
\hline & End & $\begin{array}{l}\text { Number } \\
\text { line }\end{array}$ & $\begin{array}{l}\text { Number } \\
\text { line }\end{array}$ & Both & Both & Both & Both & Both & $\begin{array}{l}\text { Number } \\
\text { line }\end{array}$ \\
\hline \multirow{2}{*}{$a--b$} & Before & $\begin{array}{l}\text { Memorized } \\
\text { facts }\end{array}$ & $\begin{array}{l}\text { Memorized } \\
\text { facts }\end{array}$ & $\begin{array}{l}\text { Memorized } \\
\text { facts }\end{array}$ & $\begin{array}{l}\text { Memorized } \\
\text { facts }\end{array}$ & Both & Both & Both & $\begin{array}{l}\text { Number } \\
\text { line }\end{array}$ \\
\hline & End & $\begin{array}{l}\text { Number } \\
\text { line }\end{array}$ & $\begin{array}{l}\text { Number } \\
\text { line }\end{array}$ & $\begin{array}{l}\text { Memorized } \\
\text { facts }\end{array}$ & Both & $\begin{array}{l}\text { Number } \\
\text { line }\end{array}$ & $\begin{array}{l}\text { Memorized } \\
\text { facts }\end{array}$ & Both & Both \\
\hline \multirow{2}{*}{$-a--b$} & Before & $\begin{array}{l}\text { Memorized } \\
\text { facts }\end{array}$ & $\begin{array}{l}\text { Memorized } \\
\text { facts }\end{array}$ & $\begin{array}{l}\text { Memorized } \\
\text { facts }\end{array}$ & $\begin{array}{l}\text { Number } \\
\text { line }\end{array}$ & Both & Both & Both & $\begin{array}{l}\text { Number } \\
\text { line }\end{array}$ \\
\hline & End & $\begin{array}{l}\text { Number } \\
\text { line }\end{array}$ & $\begin{array}{l}\text { Number } \\
\text { line }\end{array}$ & $\begin{array}{l}\text { Number } \\
\text { line }\end{array}$ & $\begin{array}{l}\text { Number } \\
\text { line }\end{array}$ & $\begin{array}{l}\text { Number } \\
\text { line }\end{array}$ & $\begin{array}{l}\text { Memorized } \\
\text { facts }\end{array}$ & Both & Both \\
\hline \multirow{2}{*}{$\begin{array}{l}a+-b \\
-a+b\end{array}$} & Before & $\begin{array}{l}\text { Memorized } \\
\text { facts }\end{array}$ & & & & & & & \\
\hline & End & $\begin{array}{l}\text { Memorized } \\
\text { facts }\end{array}$ & & & & & & & \\
\hline \multirow{2}{*}{$-a+-b$} & Before & $\begin{array}{l}\text { Memorized } \\
\text { facts }\end{array}$ & & & & & & & \\
\hline & End & $\begin{array}{l}\text { Memorized } \\
\text { facts }\end{array}$ & & & & & & & \\
\hline
\end{tabular}

For subtraction tasks in the form $a-b$, every participant solely used a number line to solve this task after working with the SAA or SDA. This meant that three participants changed from representations they initially used. Don and Carla switched representations and went from using memorized facts to using a number line, and Abby integrated her understanding of using a number line as well as using memorized facts.

For subtraction tasks in the form $-a-b$, every participant used a number line either with or without the accompaniment of memorized facts; the majority (5) using both; thus, everyone used a number line and no one used only memorized facts to answer these types of tasks. Half of the participants who used a number line used it in the fashion of either the SDA or SAA. This meant that two participants changed from representations they initially used. Don and Carla went from using only memorized facts to solely using a number line representation.

For subtraction tasks in the form $a--b$, two participants used only memorized facts, three used only the number line, and three used both; thus, six out of the eight participants used a number line, all in the fashion of either the SAA or SDA. This meant that six participants changed from representations they initially used. Don and Carla switched from solely using memorized facts to solely using a number line; 
Hope integrated the number line to her memorized facts, and Beth integrated memorized facts to her number line; Jim and Jake disintegrated from using both representations to solely using memorized facts and a number line, respectively. Yet, the memorized facts and number line strategy they used they discovered from using the applets.

For subtraction tasks in the form $-a--b$, one participant used only memorized facts, five participants used only a number line, and two used both; thus, seven of the eight participants used a number line, all in the fashion of either the SAA or SDA. This meant that six participants changed from representations they initially used. Don, Carla, and Jen switched from solely using memorized facts to solely using a number line; Beth integrated memorized facts to her number line; Jim and Jake disintegrated from using both representations to solely using memorized facts and a number line, respectively. Yet, the memorized facts and number line strategy they used they discovered from using the applets.

There are a couple of interesting features about the representations that participants used. First, it seems as if using a number line in the in the fashion of the SAA or SDA is the most often preferred way to solve tasks with a negative subtrahend. Second, Don and Carla are the participants who changed the most often. In fact, they went from using memorized facts as their only method to using a number line as their only method. Furthermore, they used memorized facts to obtain incorrect answers to most subtraction tasks with a negative subtrahend, to using a number line in the SAA or SDA fashion to obtaining the correct answers to these tasks.

Third, Jen, Abby, and Hope changed the least in their usage of representations. Jen switched only one time and in doing so she changed from using symbolic representation to incorrectly solve $-a--b$ to using a number line in the fashion of the SDA and solving it correctly. Abby integrated only one time and in doing so she incorporated using a number line in the fashion of the SDA to solve tasks in the form $a-b$. Hope integrated only one time and in doing so she incorporated the use of a number line in the fashion of the SDA and SAA to solve tasks in the form $a--b$. Fourth, even though those participants who did not change representations they used to solve tasks with a negative subtrahend, changed their specific operating procedure to using some discovery they made with the applets. Jen and Abby both transformed the task $a$ $--b$ into $a+b$ and Abby visualized using a number line in the manner of SDA. Hope and Abby visualized using a number line in the manner of the SDA. Hope also used the number line in the manner of the SAA. Abby accompanied her visualized number line with the representation of memorized facts: she transformed $-a--b$ into $-a+b$. 


\section{Participants' Final Understanding of Equality}

One assertion will be presented concerning the participants' final understanding of they equal sign and solving 1-step and 2-step equations. In determining participants' final understanding, I relied on how they performed on tasks, after working with the EBA. I did not allow them to use the EBA to answer these tasks, however, I did supply them with a number line if they asked for it.

Two participants, Don and Hope, did not have enough time to complete the instructional module using the EBA. While they have been included in describing participants' prior conceptions, they are not included in the description of participants' final understanding. When I mention all participants in this section, I am referring to the six participants who worked with the EBA.

Assertion 5. All participants were able to solve one and two-step equations algebraically by visualizing the EBA, or using equation solving methods they learned from using the EBA. All participants saw the importance of applying the same transformation to each side in order to keep the equation balanced.

Before working with the EBA, participants had a plethora of strategies to 1-step and 2-step equations, with and without a letter symbolizing the variable. In fact, some of the strategies were not described by literature. Moreover, most participants were not consistent in their solution strategy from one type of equation to the next (see Table 20).

After working with the EBA, all of the participants could solve 1-step and 2-step equations by applying algebraic transformations to both sides of the equation. Based on the visual of the EBA, participants knew why they had to apply the same transformation to both sides - to keep the expressions equal (see Table 21). Based on the visual, manipulation, and instantaneous feedback afforded by the EBA, participants were able to easily understand the order of operations that needed to be applied, and no longer had a hazy idea of some class procedure (see Table 22). 
Table 20

Six Participants' Initial Understanding of Solving Equations

\begin{tabular}{|c|c|c|c|c|c|c|}
\hline & Jen & Abby & Jake & Jim & Beth & Carla \\
\hline $3+5=?+2$ & arithmetical & arithmetical & arithmetical & arithmetical & arithmetical & algebraic \\
\hline answer & 4 then 6 & 8 then 6 & 6 & 6 & 6 & 6 \\
\hline$x+5=3$ & $\begin{array}{l}\text { cross } \\
\text { multiplication, } \\
\text { numerical } \\
\text { manipulation }\end{array}$ & $\begin{array}{l}\text { combination } \\
\text { of } \\
\text { arithmetic, } \\
\text { unwinding } \\
\text { and } \\
\text { algebraic }\end{array}$ & $\begin{array}{l}\text { quantitative } \\
\text { reasoning, } \\
\text { number } \\
\text { combinations }\end{array}$ & $\begin{array}{l}\text { quantitative } \\
\text { reasoning, } \\
\text { guess and } \\
\text { test }\end{array}$ & algebraic & $\begin{array}{l}\text { quantitative } \\
\text { reasoning, } \\
\text { number line }\end{array}$ \\
\hline answer & 7 & -2 & -2 & -2 & -2 & -2 \\
\hline $2 m+9=1$ & declined & PEMDAS & $\begin{array}{l}\text { can't } \\
\text { remember }\end{array}$ & $\begin{array}{l}\text { quantitative } \\
\text { reasoning, } \\
\text { guess and } \\
\text { test }\end{array}$ & $\begin{array}{l}\text { misapplication } \\
\text { of class } \\
\text { procedure }\end{array}$ & $\begin{array}{l}\text { misapplication } \\
\text { of class } \\
\text { procedure }\end{array}$ \\
\hline answer & none & 7.5 or 5.5 & none & -5 & 7 or -1 & 4.5 \\
\hline
\end{tabular}

Table 21

Participants' Understanding of Applying the Transformations

Student Task Quote

Jim $3 m+2=14$ Cause they are the same thing...they balance....need to add

Beth $3+5=?+2$ Subtract 2 from that side too

Abby $x+8=21$ If you want to figure out what $x$ is, then you subtract 8 there,

Jake $3+5=?+2$ You can move these 2 off of here and there

Carla $3+5=?+2$ They're balanced because they are equal...I could take off a

Jen $3+5=?+2$ Take out these two and then take out these two

Table 22

Participants' Understanding of Order of Operations to Solve Equations

\begin{tabular}{|c|c|c|}
\hline Student & Task & Quote \\
\hline Jim & $2 m+7=15$ & Subtract first... it makes it easier to get to [the variable term to equal] zero \\
\hline Beth & $2 m+7=15$ & Take a 5 off...take a 2 off \\
\hline Abby & $2 x-16=-6$ & So we're undoing it...so we did the opposites \\
\hline Jake & $2 m+7=15$ & Minus 7 first, so 15 minus 7 is 8 ..so it would be $2 \mathrm{~m}$ plus 0 is 8 \\
\hline Carla & $2 m+7=15$ & $\begin{array}{l}\text { We have to make a zero pair with the } 7 \text {. The variable, you hold that of for } \\
\text { last...you divide because...the opposite of multiplication is division }\end{array}$ \\
\hline Jen & $2 m+7=15$ & Oh, we're just doing the opposites to find $m$. \\
\hline
\end{tabular}

Participants enjoyed using the EBA because it could help them see what needed to be done to solve the equation (see Table 23). Based on their manipulations of the EBA, they also saw how to transform their actions into symbolic operations (see Table 24). By the end of their last session, each participant was able to apply and write the correct algebraic transformations on paper to solve an equation. 
Table 23

Participants' View of the EBA

\begin{tabular}{ll}
\hline Student & Quote \\
\hline Jim & $\begin{array}{l}\text { Because you find out how, as long as it gets to, if you can get it to zero, it's } \\
\text { easier...it shows you what the m is. It was harder to see what the m was } \\
\text { before. This is an easier way for me to do it. }\end{array}$ \\
Beth & $\begin{array}{l}\text { Like it gave you a visual to know what you had to subtract or add or multiply } \\
\text { Abby }\end{array} \quad \begin{array}{l}\text { It's better than paper. I don't have to use my brain really...I knew what to do } \\
\text { from that thingy }\end{array}$ \\
Jake & $\begin{array}{l}\text { I'm not good at this [solving on paper], but I understand this [solving on } \\
\text { computer]...it was kind of easy to do }\end{array}$ \\
Carla & $\quad \begin{array}{l}\text { It helped because you got to see it...you could see if it was equal or not } \\
\text { Jen }\end{array}$
\end{tabular}

Table 24

Participants' Movement Translation on the EBA to Symbolic Operations

\begin{tabular}{|c|c|c|}
\hline Participant & Task & Quote \\
\hline $\mathrm{Jim}$ & $x+2=15$ & Added two negative ones, or take away $2 \ldots$ subtract 2 \\
\hline Beth & $2 m+7=15$ & Take off two ones ...subtract 2 \\
\hline Abby & $2 m+7=15$ & $\begin{array}{l}\text { Get rid of } 2 \ldots \text { and } 5 \text { so } 7 \text {, subtract } 7 \ldots \text { because there [are] two of } \\
\text { them, well, half of } 8 \text { would be } 4\end{array}$ \\
\hline Jake & $x-6=-15$ & $\begin{array}{l}\text { So I took away }-6 \ldots \text { so that's minus }-6 \text { from both sides, that's uh, } \\
\text { like [adding] a positive }\end{array}$ \\
\hline Carla & $x-6=-15$ & $\begin{array}{l}\text { Take off }-6 \ldots \text { so you subtract }-6 \text { from both sides, so you're really } \\
\text { adding it }\end{array}$ \\
\hline Jen & $x+8=21$ & I took off 6 from both sides...like minusing 6 \\
\hline
\end{tabular}

Research Question 3

\section{Participants' Interaction with the Applets}

Five assertions concerning participants' interactions with the applets are presented in this section. They depict the impact of the applets' visual and dynamic nature on the participants' view of operation on integers and solving equations.

Assertion 1. During the initial interactions with the applets, participants became confused due to some of the visual features, yet these were overcome and some of the features proved useful in their understanding.

There were three main interfacial attributes that caused some confusion when participants operated on integers: (1) where the arrows began and ended, (2) the numbers located inside the arrows, (3) the veracity of the hot-linked equation to the arrow movements on the number line. Don had a particularly hard time with both the AAA and SAA in determining the beginning and ending points of the arrows. Since this 
is primarily key in understanding how the applets depict addition and subtraction, this feature is crucial. This confusion could be because the tips of the arrows in the applets are not extremely tapered. Once I told the beginning and ending of the arrows, he could operate accordingly.

The numbers located inside the arrows presented two issues. One issue deals with the influence it had on interpreting what the number inside each arrow represented, and the other issue deals with the verbal interpretation of the direction of the arrow and sign of the number inside the arrow. In the beginning, participants did not always operate consistently with their interpretation of the numbers inside the arrows. Sometimes they would view it as representing the subtrahend, while other times they would view it a representing the difference. While this caused a problem at first in getting them to notice patterns, it ultimately allowed them to see the connection between the expressions $a-b=c$ and $a-c=b$ for integers $a, b$, and $c$. The value inside the arrow and the direction that it faced presented an inaccurate verbal description of subtraction of a negative subtrahend. Carla had modeled the task - 3-- 2 using the SAA. In doing so, the arrow pointed to the right, which Carla had discovered was similar to addition. The number inside the arrow was a negative two, which helped her figure out that when she subtracted a negative number, she had to move to the right. Yet, she combined these discoveries and said she "added a negative" when the fact was she was subtracting a negative which was the same as adding.

Abby, Hope and Jim had trust issues with the hot-linked equation depicting their movements on the number line. They knew that the arrows corresponded with the numbers in the expression, but they did not believe the direction of the arrows necessarily had anything to do with subtraction. In regards to the SDA, Jim stated, "It doesn't matter how far apart they are, at least not all the time. It depends on the question." They believed that in some instances the expression and movements on the number line elicited the same answer as subtraction tasks, but not all the time. This allowed them hold on to their prior misconceptions. In fact, when I asked Jim to explore at the SAA instead, he did not believe that he needed to move to the right of the minuend when he subtracted a negative subtrahend and maintained, "left is anything that is negative or subtraction." Yet, the hot-linked expression proved useful for some participants because it allowed them to see how the direction and magnitude of the arrows represented subtraction. In fact, because of this visual, all participants were able to solve any subtraction task.

There were five main interfacial features of the EBA that caused some confusion when participants used the EBA: (1) the size in value of the objects, (2) the size of the pans, (3) automatic combining similar terms in a pan, (4) automatic registering of a value before final placement on the pans or storage area, and (5) the meaning of the question mark. The size of the values of the objects required 
students to convert bigger values for smaller values, which required extra steps in participants' solution procedure. For instance, when participants solved $x+8=21$, they could remove a 5-weight and 1-weight from both sides, but after that, they had to exchange a 5-weight on the right side for five 1-weights. This meant that their written procedure looked like the left system of equations rather than the right system.

$$
\begin{array}{cr}
x+8=21 & x+8=21 \\
-6=-6 & -8=-8 \\
x+2=5 & x=13 \\
-2=-2 & \\
x=13 &
\end{array}
$$

Because the solution process was elongated, it was more difficult to see how much one subtracted from the expression containing the variable. Once I encouraged them to see if they could do the solution process more quickly on paper, they were able to see that they were to subtract the amount that would "get $x$ by itself."

The pan size was small compared to the objects that needed to be placed in the pans. When Carla was solving $3+5=?+2$, she did not notice that there were more objects hidden behind other weights. They were stacked upon each other because there was not enough room to place all of them on the pan. Luckily, she took note of the equation displayed above the fulcrum of the balance, which made her pause because it did not look like the objects in the pans and the equation correlated. This encouraged her to shuffle through the objects to see that there were more objects there than she had once thought.

Two other features of the applet interrupted Jen's understanding of solving equations on the EBA. The first occurred when she was trying to figure out what she needed to add to both sides to simplify the equation $x-20=-13$. She thought that she might need to add a -5 balloon to balance the pans. After she moved it to the right pan, she noticed that the pans did not balance. Upon dragging the balloon back to the storage unit, it registered on the left pan because it crossed the fulcrum but was not in the storage unit yet. This led her to believe that she could not get the equations to balance for the original equation, which caused her to become frustrated.

The other feature that unnecessarily prolonged Jen's confusion of solving equations was that the EBA combined like terms for the students. For instance, if there was a 5-weight and a 1-weight on the same pan, the expression would shows +6 . Jen's issue came when she applied so many objects to the pans that the objects did not seem to correspond to the equation because there seemed to be more objects than values displayed. When Jen solved the equation $x-20=-13$, she first removed two -5 balloons from both sides; then she added two 5-weights to both the left and right pans. The hot-linked equation showed $x+0=7$, but 
she had numerical-valued objects in the left pan, even though the equation portrayed that she had zero (see Figure 1).

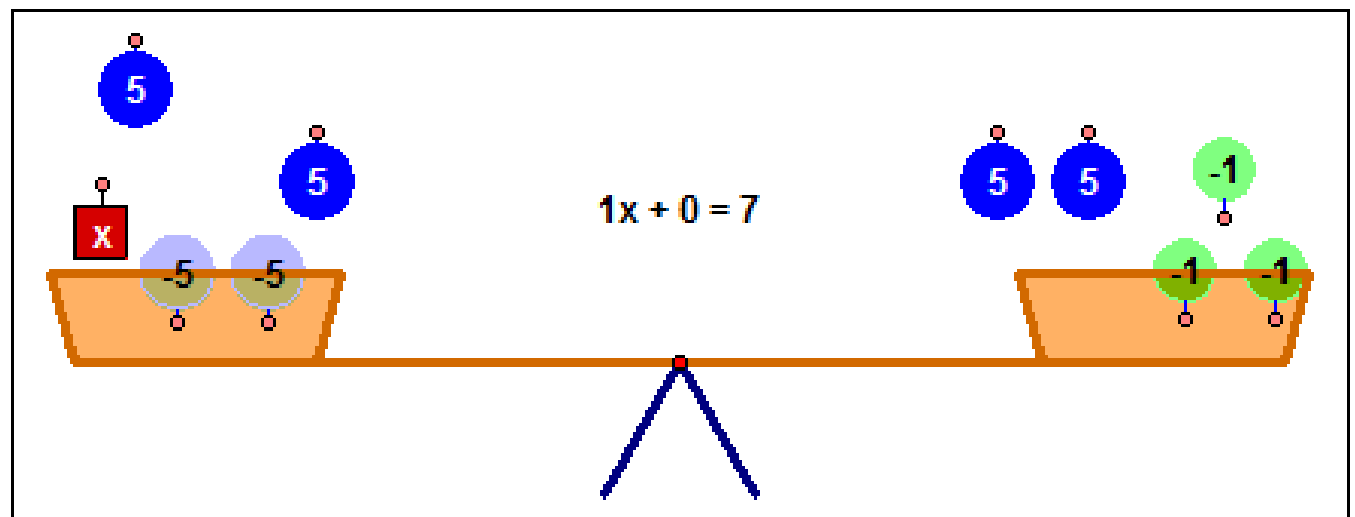

Figure 1. After adding two 5-weights to both sides of the equation $\mathrm{x}-10=-3$

There were two other difficult ideas that related to the visual depiction on the EBA, but were not due to programmed features. One dealt with an inherent attribute of using a balance to depict equality, and the other dealt with students understanding of variable. Before testing her theory, naturally believed that the absence of objects had to be lighter than the presence of objects. Yet, upon testing this theory, she realized that if she had balloons on one pan, and nothing on the other pan, the pan containing no objects would register as heavier. After they obtained the variable by itself on a pan, Jake, Carla, and Jen would state that nothing remained on that pan or said that it was equal to zero. The numerical amount was equal to zero, but the pan contained a variable of worth. After first, I did not know why participants overlooked this object in the pan, but at one point, I asked Jake to tell me what remained on the pans, and he asked, "Do you mean number wise, or everything?" This led me to believe that participants may not have viewed a variable as a number, but rather an entity for which they had to solve that would appear on the opposite side of the equal sign. Moreover, this same idea may have caused the confusion of using the question mark on the pans as a variable to create equality in the task $3+5=?+2$.

Even though some features increased the length of time before participants' came to a complete understanding of operations with integers and solving equations, these same features, once understood, helped participants to intuitively see how to operate and why they needed to operate in a specific manner.

Assertion 2. The interfacial features allowed participants to intuitively understand how they depicted addition and subtraction of integers and how solving equations related to a balancing of expression pans.

As was mentioned in Assertion 1, the numbers located inside the arrows and veracity of the hotlinked equation to the arrow movements were interfacial attributes that caused confusion in operating on 
integers. Yet, it was these exact same features that allowed participants to understand addition and subtraction of negative numbers intuitively.

The numbers inside the arrows helped in both the addition and subtraction applets. Don easily saw that the sign of the number inside the arrow correlated with the direction the arrow pointed, and the magnitude inside the arrow depicted its length. He said, "Oh, it's negative because it's pointing toward the negative numbers...it's positive because it's pointing to the positive numbers...this is cool!" Similarly, participants noted that the sign of the numbers inside the arrows of the SAA correlated with the direction the arrow pointed, and the magnitude inside the arrow depicted its length. Because of these numbers, participants easily maneuvered between addition and subtraction expressions that they could solve correctly and its representation on a number line. One point worth mentioning is that Carla never used the number line to solve subtraction tasks and only used a number line to show answers as specific points. Yet when she used the SDA, she was able to show the relationship between the dots and difference. In fact, students saw how subtraction could be seen as take away through the SAA, even when using negative numbers, and how subtraction could be seen as distance through the SDA.

Another affordance of the numbers inside the arrows, was that most participants, except Jim, instinctively knew they had to travel right when they subtracted a negative number when using the SAA. Because they saw the negative number inside the arrow when they traveled to the right, and because they knew that addition typically required them to move to the right, they were able to discover that subtracting a negative was the same as adding a positive number. Carla said, "it helped me see which way to go." and Jen said, "It helped show me which way to go."

The hot-linked equation also enforced this idea because if participants ever became bogged down with looking at the numbers inside the arrows and not remembering the operation that they were enacting, they could look at the hot-linked equation to do so. Furthermore, the equation came in handy when participants started to confuse themselves into which method they preferred - distance or take away. While turning the equation off allowed them to interchange these methods and see how the methods related, the displayed equation allowed them to consistently perform the same idea in order to come to some generalizations. Moreover, it provided a link between two representations. Since two representations were hot linked, students were able to act on one representation and see an immediate reaction in all connected representations. This allowed them to receive immediate feedback and aided in their understanding of symbolic notation. 
Only one feature that caused some prolongation to participant understanding, allowed participants to operate intuitively with the EBA: the automatic combining similar terms in a pan. This feature was displayed in the equation located above the fulcrum. Because the equation automatically combined similar terms, participants were quickly able to see if how they adjusted the objects on the pans created a more difficult or simplified equation to solve. In fact, Abby frequently declared, "Let me try something really fast...I'm going to try something different...don't ask me why I'm trying this, but it just might work." After determining if the equation was simplified, they could choose whether to continue to solve the equation, or hit the reset button to try a new strategy. The reset button was an advantageous feature of the applet, because it allowed students to work at their own pace, using their own ideas and strategies to solve equations.

Another advantage of the displayed equation is that it supported the idea of equal meaning equivalence and the reason behind applying the same transformation to each side of the equation. Students intuitively saw why and how they needed to keep the pans balanced, and only had two rules to do so: applying the same operation to both sides, or applying inverse operations to one side. The correlation of the inequalities and notion of heavier and lighter helped participants to realize what they needed to do to the pans.

Not all students used the EBA as intended. Carla, Abby, Beth, and Jim were able to see that if they kept the pans balanced, that they could obtain equivalent equations. Therefore, they were able to determine the value of a variable by placing a single variable on one side and an amount of numerical values on the other. While this was not the intent of the applet, it allowed them to check their initial prediction as well as check their final answer.

The ability to use the EBA through their own intuitions allowed them to see how to solve 1-step equations, and in doing so, extended this idea to 2-step equations. Every participant who used the EBA figured out that they had to remove or add numerical values to both sides of the pans in order to get the variable on one side of the equation by itself, and only after this could they use division. Thus, they saw the reason through the visual of why they had to use addition and subtraction, in any order, prior to using multiplication and division. What is noteworthy, is that Jake, Carla, Jen, and Jim were able to do this right away without any faltering.

Assertion 3. Through dynamic interaction, the applets allowed participants to predict, manipulate, observe, and interpret those actions and results, which promoted discovery and generalization. 
Some participants, Don, Jake, and Hope, were not able to completely and accurately solve all subtraction tasks by the beginning of their last session, while each participant made many connections while using the applets that improved their procedural and conceptual understanding. These connections were made due to the following features of the applets: manipulation, visualization, and instantaneous immediate feedback.

By allowing the participants to freely manipulate the applets and watch the corresponding hotlinked equation and numbers in the arrows adjust accordingly, they were able to make two discoveries: the difference between the expressions $a-b$ and $a--b$, and the equivalence of the equations $a-b=c$ and $a-c=b$ for all integers.

After manipulating the SAA in particular, participants noticed that if they put the blue stick on the minuend and dragged the arrow to the left $b$ spaces, they were subtracting $b$. They also noted that when the went $b$ spaces in the opposite direction they were in essence subtracting a negative of that same value, $a--b$. They also noted the symmetry of this action. This enforced the idea that subtracting a positive number required movement of the same magnitude in the opposite direction of subtracting a negative number. It seemed only natural, except for Jim, that if one subtracted a positive value one should travel left, but if one subtracted a negative of the same value, the only direction to go is right.

By manipulating the SDA and SAA, participants easily saw the equivalence of equations $a-b=c$ and $a-c=b$. Abby said, "Well it was helpful on those where like you had a choice on like which way to go. Sometimes you could move it [the orange dot] to get the answer and sometimes how to get the answer by putting it on both numbers. So sometimes it depends on where you put it [the orange dot]." She noticed that when she moved the amount of the subtrahend, the difference was where she landed. When she moved to the subtrahend on the number line, the distance between the minuend and subtrahend was the difference. Participants realized that they could solve the same task in different ways.

After manipulating the SAA, many participants noted that when they had a negative subtrahend, it was the same as adding the positive magnitude of that subtrahend. They easily came to this conclusion because when they had a negative subtrahend, they had to move the right that many spaces on the SAA. They knew that addition of positive numbers required them to move right on the number line. They assimilated this knowledge with the movement on the SAA to realize that subtraction of a negative is the same as a adding a positive. Even though this came very easy by looking at the SAA, when participants solved tasks in the form $-a--b$, most of them changed the expression to $-a+b$, but did not consider this as addition because they did not add the absolute value of the numbers; rather they subtracted the absolute 
value of the numbers. It is interesting to note that Abby mentioned, almost as if she was surprised, that when she subtracted a negative number from a positive number, the difference was always bigger. I assume that since she was surprised at this fact, she had assumed that subtraction had always made numbers smaller. Another interesting fact is that while Beth and Carla knew that they could change the signs, they preferred to use a number line to enact the movements of the applet instead. Beth stated, "I don't really like the changing signs, they get me confused...it's better to use the number line." By working with the applets, she had a choice of which representation to use to help her solve subtraction tasks.

Instead of seeing addition at first, Jake saw that a positive minuend and negative subtrahend had to result in a positive difference because of the way he operated on the SDA. He had made this conjecture and "proven" it to himself. He reasoned that if one placed the blue dot at positive number and had to place the orange dot at a negative number, the only way to do this was to travel to the left, and traveling to the left meant that the answer was always positive. Furthermore, Jake, Abby, Jen, and Hope expounded on that idea and noted that when the blue dot was higher than the orange dot on the number line, the difference always had to be positive. They easily reasoned that if one subtracted a lower number from a higher number, the difference had to be positive. Similarly if one subtracted a higher number from a lower number, the answer had to be negative.

While using the SDA, Jake also made the discovery that when he subtracted a lower absolute value from the minuend versus a higher absolute value from the minuend, the difference was closer to the minuend. He saw this through the movement of the dots. He noted this based on his movements of the orange dot in relation to the blue dot and the corresponding distances. For instance, suppose we construct four tasks such that the minuend is 100 and the subtrahend is $94,3,-3$, and -100 respectively and compare the following:

$\begin{array}{lll}100-94=6 & 100-3=97 & 3<94 \text { and } 97 \text { is closer to } 100 \text { than } 6 \text { is } \\ 100-3=97 & 100--3=103 & |3|=|-3| \text { and } 97 \text { is the same distance away from } 100 \text { as } 103 \text { is } \\ 100--3=103 & 100--100=200 & |-3|<|-100| \text { and } 103 \text { is closer to } 100 \text { than } 200 \text { is }\end{array}$

This was a dynamite discovery.

Carla constructed the relationship between the equations $-a--b=c$ and $a-b=-c$ for integers $a, b$, and $c$. She noted that she could solve the latter task easier, so she stripped away the negative signs, found the answer, and then multiplied her answer by negative one. This was what Human and Murray (1987) referred to as "oppositeness".

Besides using analogous symbolic expressions, participants were also able to maneuver between symbolic, number line, and contextual representations. Most of them did so in the context of temperature. 
The participant who really made the connection between the three was Don using the addition applet and using the context of football.

In regards to the EBA, everyone was able to conclude the two rules that maintained the equilibrium of the balance: adding or subtracting the same amount to both sides, or adding and subtracting the same amount from one side. They discovered these rules simply by predicting what would happen when they dragged weights and balloons to the pans, did it, saw the result, and altered their conjecture. Since they were able to do many trials in a short amount of time, these discoveries did not take a long time and were remembered by most of the participants.

Also by manipulating and obtaining visual feedback based on their manipulations, participants often stated how they saw that removing a negative amount was the same as subtraction of a negative which was the same as adding a positive amount to the pans, and the removal of a positive amount was the same as adding a negative. Thus, their addition discoveries were reinforced by the EBA.

Not only did the applets allow participants to discover relationships and generalize upon them, but also they made a consequential impact on participants' memory.

\section{Assertion 4. Students' imagery of the applets made an impact on how students remembered operating on} integers and solving equations from one session to the next.

After the first session, participants referenced the visual image they remembered from the applets when solving operations on integers. Sometimes they did not remember it correctly, and sometimes they did. Don was one participant who became confused with all the imagery he had seen in the first session. He had combined the idea of the AAA and the SDA to devise his own procedure for operating on addition and subtraction tasks: Count the distance between the numbers in the expression for the magnitude, and use the sign of the highest absolute-valued number for the answer. In fact, at the next session, he used a number line to solve the task $3+-8$ and drew the exact same image he had seen using the SDA in the previous session (see Figure 2).

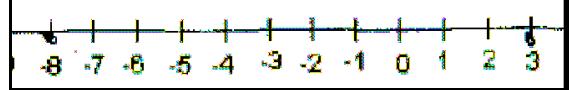

Figure 2. Solving $3+-8$ using a combination of the SDA

Other participants, Abby, Carla, Jen, Jake, and Beth remembered the applets and used their visual to help them solve subtraction tasks correctly when they returned after their first session. In solving $8--1$, Jake visualized the number line in his head and waved his pencil in the air by starting at the imaginary 8 and moving to the right 1 space to land on the 9, enacting the SAA. He said, "I remembered from the last 
time, that when you have a negative, you have to go farther than what you have to begin with." Similarly, when Carla solved $8--1$, she referenced the visual of the SAA and said, "because you have 8 right here, and you'd have to move, like on the computer, you show that if it was a negative, it would be going to the right - subtracting that many. So I got 9 for it." Both Beth and Jen solved $-6--4$ by referencing or enacting the SAA. Beth said, "I remember from last time, when it was a negative number, we always go to the right." Jen used a number line, started at the -6 and moved to the right 4 spaces. Only Abby talked about the SDA when she returned two weeks after our previous session. In solving $8--3$, Abby placed her right pinky finger on the 8 and left pinky finger on the -3 on the number line and said, "Well, that would be there and that would be here, and it would be...1,2, $3 \ldots 11$, because that's what we did before and it worked." Furthermore, she told me that she had remembered the SDA and went on to say, "I've been thinking about that... I just kind of missed it... it's just something you get used to, and then it's just gone and it sticks in your head. It's just like there...when I wake up sometimes I just think about it.“

All six participants worked with the EBA during their last session; therefore, I am unable to say how much they remembered from one session to the next. However, within one session, both Beth and Jim needed to be reminded of the two rules of maintaining the equilibrium of the balance. This would seem to indicate that if they could not remember within even one session, they most likely would not remember a week later.

Beth and Jim had easily discovered the rules of maintaining equilibrium by using positive and negative valued objects, yet when they started to solve equations they could no longer figure out how to keep the pans balanced. It took a couple times prompting them with "How do we keep the pans balanced?" before they consistently remembered and solved the equation. This could have been due to a couple of reasons. The first reason is that when a variable was introduced they did not believe the rules still held. Another reason is that when the variable was introduced, there were more objects located on the pans. Either way, the visual was more convoluted with objects.

Assertion 5. Participants professed that the applets made operations on integers and solving equations easy and enjoyable.

Many participants found working with the applets and enjoyable and meaningful learning experience. Some of the participants compared the SDA to the SAA and mentioned why they preferred one or the other. Most said that the differences between the SDA and SAA in their mind related to determining the magnitude and sign of the difference. Half of the participants seemed to prefer the SDA based on their usage of it versus the SAA. Participants who preferred the SDA said similar statements as Jake and Beth 
did. Jake said, "I like this one [SDA] better because it's kind of easier to show cause like you put each dot on each one and it shows you how you can get it in between... It's just easier." Beth said, "Like you could put the dot on one and the dot on the other one and just count the spaces there. But the other one [SAA], you kind of like have to figure out the problem. Like figure which way to go. I think the negatives, you can just see the answer" better with the SDA. The SDA seemed to easily show the magnitude of the difference by illustrating subtraction as distance. Both Jake and Beth believed that it was easier because they statically represented the minuend and subtrahend and then calculated the distance in between them to obtain the difference. Whereas, the SAA did not allow them to statically represent the subtrahend; therefore, they had to use the subtrahend to determine the movements of the arrow. However, the SAA allowed students to easily determine the sign of the difference. Once they determined which way to travel on the SAA, they did not have to think about the sign of the difference because they knew wherever the tip of the arrow landed, was the difference.

Other comments that were made about AAA, SAA, and SDA were the following:

- 'It made it [subtraction] easier...because it's showing me how it's actually working. Like the computer showed the difference between them [the numbers], and which way it's going. Then on the paper, we worked it out."

- "It's much easier using this"

- "Wee, this is cool!"

- 'Yeah, I like them! They're cool! We can do neat stuff with them... like play around on them to do subtraction. You know where to put the dots, and to count the numbers in between them."

- "It's just not as complicated [as doing it on paper]"

- "It... kind of helped me on my SOLs. Some of it I knew, but other stuff...it helped me feel confident in my answers. It reminded me to look at the signs."

- "This is cool!"

- "Well, I have added negative numbers before, but I've never did it this way! I just did it. I have no clue [how]! I like it this way, with the computer...it just made me see it!"

- "Computers are so much more fun. They actually show you the answer."

Not only did they profess that they enjoyed working with the applets and how it made understanding subtraction and solving equations easier, I also noticed that it helped Abby and Hope focus on tasks in order to discover patterns, and helped Hope articulate her understanding in terms of explanation. By visualizing the applets, she was able to say exactly what she meant in communicating her understanding. 
Each participant, who worked with the Algebalance, commented on how it made it easier to understand how to solve equations (see Table 25). Overall, they seemed to enjoy working with the computer applets and improved in their understanding of addition and subtraction of integers and solving 1 and 2-step equations.

Table 25

Participant Description of EBA

Participant Description of EBA

Oh, I've done this before, not with the computer, but with Miss

Jake Beecher. I'm not good with this [solving equations on paper], but I understand this [EBA]; it helped ... finding out what $x$ was easy to do.

Yes, I like it because you had to move the numbers from the

Carla balance beam and watch them to see if they be equal to each other.. it helped because you got to see it...like it was in your face and you could see if it was equal or not.

Abby Oh cool! Its better than paper, I don't have to use my brain really... I just knew from that thingy [EBA].

Beth Yeah, like it gave you like a visual to know what you had to subtract or add or multiply.

Yeah, it just shows like you stuff. Like you can do it on paper,

Jen but this shows you what's happening. It helps you see it. It helped [me] know what to do at first. Like what I had to take off and to get to where you needed to be.

You find out how [to solve it]. It shows you what it [the

Jim variable value] is. It was harder to see what it [the variable value] was before. This is an easier way for me to do it.

Implications from the Research

The major finding of this study is that while middle school students hold some misconceptions about how to solve addition and subtraction tasks with negative numbers and solve one and two-step equations, dynamic applications of these topics can significantly deepen conceptual understanding.

There has been a lot of work focusing on students' difficulties with pre-algebraic and algebraic notions and the transition from arithmetic to algebra, but new work was needed to focus on pre-algebraic understanding obtained from dynamic environments using multiple representations at the middle school level. Kieran (2007) recommended that future research concentrate on how students become (a) "aware of structure and in using symbols to express structure", (b) see "relations between graphical representations and letter-symbolic forms", and (c) make "connections between verbal problem-solving activities and generating equations" (p. 729). I was unable to find any research on the use of a dynamic geometry 
package used to aid in the understanding of operations on integers or solving equations. Therefore, in hopes to combine Kieran's recommendation and the lack of research on using geometry software to mediate middle school students' misconceptions, I created three and modified one GSP applets. These applets allowed participants to become aware of symbolic structure of integral expression and equations, see the relationship among the abstract, tabular, and visual representation, and make connections between contextual problems and symbolic expressions and equations.

My study provided a detailed analysis of participant misconceptions and interactions so that the dynamic applets might be used most effectively with students. The findings of this study have implications for middle school teachers, teacher educators, and future researchers.

There are benefits for using dynamic applets to mediate understanding of addition and subtraction on integers and the solving of equation. Participants, who had a good grounding in addition knowledge improved their understanding of subtraction of integers and made many procedural and conceptual connections, especially with regard to multiple representations. The participant, who was not as strong in addition, was able to make connections in understanding of addition as well as subtraction, although not at a deeper level. However, by the end of the study, all participants were able to correctly add and subtract integers. Participants who had the opportunity to work with the EBA were able to make many procedural and conceptual connections, especially with regard to multiple representations. Moreover, participants were able to make these connections, solve addition and subtraction tasks, and solve equations in a short amount of time compared to some students who had been exposed to these concepts for three years, (those participants in $8^{\text {th }}$ grade).

Not only did the dynamic applets allow students to deepen their understanding of pre-algebraic concepts, but also the participants enjoyed doing so. All the participants stated how much fun they had working with the applets and how the applets made a visual impact in their remembering how to operate. This helped participants be able to focus on the task and take an active stance in their learning.

Some final recommendations are worth noting. Because students may not believe that the applets are depicting what they say they are depicting, the facilitator must be prepared to use something that they trust will give them the correct answer. In this case, most students trusted a calculator. Also, because some misconceptions are resistant to change, types of examples need to be addressed more than once or twice. The facilitator must also be prepared to quickly analyze the student's misconceptions and quickly provide a task that requires the student to question his or her conception. 
Suggestions for Future Research

There are a plethora of opportunities for future research. While studies documenting students' misconceptions of negative numbers, the equal sign, and solving equations are numerous, I have been unable to find any research on the use of a dynamic geometry packed used to aid in the understanding of operations on integers or solving equations. A couple of focused suggestions follow.

The longest time between participant sessions was two weeks. It would be interesting to see if participants retained their knowledge negative numbers and solving equations after working with the applets or if they reverted back to their previous misconception after an extended period elapsed time between sessions.

Another idea for future research regards the time spent on instruction. In this study, after about two to three hours of instruction, with each session lasting about an hour, participants who were unable to subtract negative subtrahends and unable to solve two-step equations, did so by the end of our sessions. With this in mind, a natural question is: If more time was sent with the dynamic applets, would the participants, who reverted back to their previous misconceptions and strategies, do so?

Given the success of the dynamic applets in a one-to-one setting with students who were already familiar with operation on negative numbers and solving equations, one would like to know the impact of the dynamic applets in the more typical one-to-many setting with students who are seeing the definitions for the first time. A randomized design could be implemented across a large district of middle schools. A randomly selected group of teachers would be introduced to the applets and asked to use them in their instruction. One could observe these classes, and administer a written exam with follow up interviews to compare the answers of students who received the technology to those who did not.

\section{Final Conclusion}

This research study provides an in depth analysis of how dynamic applets enhance students' understanding of addition and subtraction of integers, the idea of the equal sign, and the solving of equations. This study capitalized on the recommendations provided by Wright (1991) and Kieran (2007).

Wright (1991) recommended that algebra learning should "provide opportunities for constructing relationships between quantities and concrete pictoral models to construct meaning” (p. 291). The applets allowed participants to construct generalizations about numeric expressions and equations by providing manipulatable, visual, digital objects. In doing so, participants were able to overcome some of their misconceptions and improved in their understanding of operations on integers and solving equations. 
Kieran (2007) noted that students gather algebraic meaning from four areas: “(a) meaning from algebraic structure itself, involving the letter-symbolic form; (b) meaning from other mathematical representations, including multiple representations; (c) meaning from the problem context; and (d) meaning derived from that which is exterior to the mathematics/problem context" (p. 711). This is affirmed in my study. Participants gained deeper understanding of operations on negative numbers and solving equations. They did this by viewing how the hot-linked expression or equation changed as they manipulated digital objects. By using the applets, they were also able to see how one representation connected to another. They saw and used the connection between symbolic forms and the number line. Some students, especially Don, gained understanding of operations on integers through the use of context; in his case, it was football. Participants also gained knowledge through the exterior ideas of the tasks. Participants used the mental pictures of the applets to solve equations and operations on integers; therefore, the applets became part of their concept image (Vinner \& Dreyfus, 1989).

With regards to improvement on operations with negative numbers, my study was consistent with other researchers' findings (Bruno \& Martinon, 1996; Human \& Murray, 1987; Janvier, 1988; Murray, 1985). Bruno and Martinon also believed that Peled's (1991) dimensions should be split so that the QD had two portions: abstract and contextual. My research supports this idea, for I see a need for the category, Memorized Facts. Yet, this is somewhat different than Bruno and Martinon proposed. They used their abstract category in the sense as it was another representation on which to operated and noted how students moved among the representations. However, I use memorized facts as a category to show how the students operated on the abstract representation. My category is more of an operating procedure while theirs is a representation.

Murray (1985) found that even with instruction, students still had more difficulty with subtraction tasks than addition tasks. My study confirmed that finding, for even though all participants improved in their understanding, some tasks still remained difficult, namely subtraction tasks with negative subtrahends. Furthermore, Human and Murray (1987) noted that between instructional sessions, students decrease their usage of instructional methods of solving addition and subtraction tasks and revert back to their previous operating methods. I also saw that in between sessions with my participants.

My research agrees with Janvier (1988) in that he found that students who were exposed to visual images that embodied subtraction and addition tasks were able to use those visual pictures to correct any mistakes that they made. Similarly, when my participants checked their answers and realized they had a mistake, they used the SDA or SAA method to find the correct solution. 
This study provides evidence that that the dynamic applets can enhance understanding for all ages of middle school students. This finding agrees with NCTM's (2000) Technology Principle: "Technology is essential in teaching and learning mathematics; it influences the mathematics that is taught and enhances students learning."

Students need an in depth understanding of pre-algebraic concepts in order to succeed in their algebra class, which can open the door to many opportunities. Instruction incorporating dynamic applets can better prepare students. 


\section{REFERENCES}

Abramovich, S., \& Brantlinger, A. (1998). Tool kit approach to using spreadsheets in secondary mathematics teacher education. In S. McNeil, J.D. Price, S. Boger-Mehall, B. Robin, J. Willis (Eds.), Technology and teacher education annual, 1998. Charlottesville, VA: Association for the Advancement of Computing in Education.

Ainley, J. (1996). Purposeful contexts for formal notation in a spreadsheet environment. Journal of Mathematical Behavior, 15, 405-422.

Ainley, J., Bills, L., \& Wilson, K. (2004). Constructing meanings and utilities within algebraic tasks. Proceedings of the Twenty-Eighth Conference of the International Group for the Psychology of Mathematics, Bergen, Norway, 2, 1-8.

Ainley, J., Bills, L., \& Wilson, K. (2005). Designing spreadsheet-based tasks for purposeful algebra. International Journal of Computers for Mathematical Learning, 10, 191-215.

Ainsworth, S. (1999). The functions of multiple representations. Computers \& Education, 33, 131-152.

Ainsworth, S., Bibby, P., \& Wood, D. (1997). Information technology and multiple representations: New opportunities - new problems. Journal of Information Technology, 6(1), 93-105.

Ainsworth, S., Bibby, P., \& Wood, D. (2002). Examining the effects of different multiple representational systems in learning primary mathematics. The Journal of the Learning, 11(1), 25-61.

Akerhielm, K., Berger, J., Hooker, M., \& Wise, D. (1998). Factors related to college enrollment. Final report. Washington, D.C. (ERIC Document Reproduction Service No. ED421053). Retrieved January 6, 2007 from http://nces.ed.gov/pubsearch/pubsinfo.asp?pubid=2007312 database.

Alberts, B. (1998). Preface. The nature and role of algebra in the K-12 curriculum: Proceedings of a national symposium (pp. xi). Washington, D.C.: National Academy Press.

Anderson, W. (1976). The development and evaluation of a unit of instruction designed to teach second graders the concept of numerical equality. Unpublished doctoral dissertation, Florida State University.

Arcavi, A. (1995). Teaching and learning algebra: Past, present and future. Journal of Mathematical Behavior, 14(1), 145-162.

Artigue, M. (2002). Learning mathematics in a CAS environment: The genesis of a reflection about instrumentation and the dialectics between technical and conceptual work. International Journal of Computers for Mathematical Learning, 7(3), 245-274.

Atanda, R. (1999). Do gatekeeper courses expand education options? Education Statistics Quarterly, 1(1). Retrieved October 5, 2007, from http://nces.ed.gov/pubsearch/pubsinfo.asp?pubid=1999303

Balacheff, N., \& Kaput, J. (1996). Computer-based learning environments in mathematics. In A. J. Bishop, 
K. Clements, C. Keitel, J. Kilpatrick \& C. Laborde (Eds.), International handbook of mathematics education (pp. 469-501). Dordrecht, The Netherlands: Kluwer.

Ballew, J. H. (1965). A study of the effect of discovery learning upon critical thinking abilities of high school algebra students. (Doctoral dissertation, The University of North Carolina at Chapel Hill).

Barnes, W. E., \& Asher, J. W. (1962). Predicting students' success in first-year algebra. Mathematics Teacher, 55(8), 651-654.

Baroody, A. J., \& Coslick, R. T. (1998). Fostering children's mathematical power : An investigative approach to K-8 mathematics instruction. Mahwah, NJ: Lawrence Erlbaum Associates.

Baroody, A. J., \& Ginsburg, H. P. (1983). The effects of instruction on children's understanding of the "equals" sign. The Elementary School Journal, 84, 199-212.

Bass, H. (1998). Algebra with integrity and reality. The nature and role of algebra in the K-12 curriculum: Proceedings of a national symposium (pp. 9-15). Washington, D.C.: National Academy Press.

Bastable, V., \& Schifter, D. (2007). Classroom stories: Examples of elementary students engaged in early algebra. In J. Kaput, D. Carraher \& M. Blanton (Eds.), Algebra in the early grades. Mahwah, NJ: Erlbaum.

Battista, M., \& Van Auken Borrow, C. (1998). Using spreadsheets to promote algebraic thinking. Teaching Children Mathematics, 4(8), 470-478.

Bednarz, N., \& Janvier, B. (1996). Emergence and development of algebra as a problem-solving tool: Continuities and discontinuities with arithmetic. In N. Bednarz, C. Kieran \& L. Lee (Eds.), Approaches to algebra: Perspectives for research and teaching (pp. 115-136). Dordrecht, The Netherlands: Kluwer.

Behr, M., Erlwanger, S., \& Nichols, E. (1980). How children view the equals sign. Mathematics Teaching, 92, 13-15.

Bell, A. (1982). Looking at children and directed numbers. Mathematics Teaching, 100, 66-72.

Bell, A. (1995). Purpose in school algebra. Journal of Mathematical Behavior, 14, 41-73.

Bell, A. (1996). Problem-solving approaches to algebra: Two aspects. In N. Bednarz, C. Kieran \& L. Lee (Eds.), Approaches to algebra: Perspectives for research and teaching (pp. 167-185). Dordrecht, The Netherlands: Kluwer.

Belli, G., \& Gatewood, T. (1987). Readiness for formal learning in the middle grades: A study of predictors for success in eight grade algebra. Journal of Early Adolescence, 7(4), 441-451.

Bodanskii, F. (1991). The formation of an algebraic method of problem solving in primary school. In V. V. Davydov (Ed.), Psychological abilities of primary school children in learning mathematics (pp. 275338). Reston, VA: National Council of Teachers of Mathematics.

Boers-van Oosterum, M. A. M. (1990). Understanding of variables and their uses acquired by students in tradiation and computer-intensive algebra. (Doctoral dissertation, University of Maryland).

Booth, L. R. (1988). Children's difficulties in beginning algebra. In A. F. Coxford, \& A. P. Shulte (Eds.), 
The ideas of algebra K-12 (pp. 20-32). Reston, VA: The National Council of Teachers of Mathematics.

Booth, L. R. (1989). A question of structure. In S. Wagner, \& C. Kieran (Eds.), Research issues in the learning and teaching of algebra. (pp. 57-59). Hillsdale, NJ: Lawrence Erlbaum Associates.

Borwein, J. (2005). The experimental mathematician: The pleasure of discovery and the role of proof. International Journal of Computers for Mathematical Learning, 10, 75-108.

Boulton-Lewis, G. M., Cooper, T. J., Atweh, B., Pillay, H., \& Wilss, L. (2000). Readiness for algebra. Proceedings of the 24th Conference of the International Group for the Psychology of Mathematics Education, 2, 89-96. Hiroshima, Japan.

Brenner, M., Mayer, R., Mosely, B., Brar, T., Durán, R., Reed, B., et al. (1997). Learning by understanding: The role of multiple representations in the learning of algebra. American Educational Research Journal, 34(4), 663-689.

Breslich, E. R. (1921). Problems in teaching secondary mathematics. Chicago: University of Chicago Press.

Brownell, W. A. (1935). Psychological considerations in the learning and the teaching of arithmetic. In W. D. Reeve (Ed.), The teachings of arithmetic. (tenth yearbook of the national council of teachers of mathematics) (pp. 1-31). New York: Bureau of Publications, Teachers College, Columbia University.

Bruno, A., \& Martinon, A. (1996). Beginning learning negative numbers. Proceedings of the 20th Conference of the International Group for the Psychology of Mathematics Education, Valencia, Spain, 2, 161-168.

Byers, V., \& Herscovics, N. (1977). Understanding school mathematics. Mathematics Teaching, 81, 24-27.

Carpenter, T. C., Franke, M. L., \& Levi, L. (2003). Thinking mathematically: Integrating arithmetic and algebra in elementary school. Portsmouth, NH: Heinemann.

Carpenter, T. P., Hiebert, J., Fennema, E., Fuson, K., Wearne, D., Murray, H., et al. (1997). Making sense: Teaching and learning mathematics with understanding. . Portsmouth, NH: Heinemann.

Carpenter, T. P., \& Lehrer, R. (1996). Teaching and learning mathematics with understanding. University of Wisconsin, Madison, WI: National Center for Research in Mathematical Sciences Education.

Carraher, D., Brizuela, B., \& Earnest, D. (2001). The reification of additive differences in early algebra: Viva la difference! The Future of the Teaching and Learning of Algebra. Proceedings of the 12th ICMI Study Conference. The University of Melbourne, Australia, 1, 163-170.

Carraher, D., \& Schliemann, A. (2007). Early algebra and algebraic reasoning. In F. K. Lester (Ed.), Second handbook of research on mathematics teaching and learning (pp. 669-705). Charlotte, NC: Information Age Pub Inc.

Center for Public Education. (2005). Math scores improve on 2005 national assessment. Retrieved November 7, 2007, from http://www.centerforpubliceducation.org/site/c.kjJXJ5MPIwE/b.1533115/k.B44D/Math_scores_impr ove_on_2005_national_assessment.htm

Chiu, M. M., Kessel, C., Moschkovich, J., \& Muñoz-Nuñez, A. (2001). Learning to graph linear functions: 
A case study of conceptual change. Cognition and Instruction, 19(2), 215-252.

Clements, D. H., \& Battista, M. T. (1990). Constructivist learning and teaching. Arithmetic Teacher, 38, 34-35.

Confrey, J. (1998). What do we know about K-14 students' learning of algebra? The nature and role of algebra in the K-14 curriculum: Proceedings of a national symposium (pp. 37-40). Washington, D.C.: National Academy Press.

Cooper, T. J., Boulton-Lewis, G. M., Atweh, B., Pillay, H., Wilss, L., \& Mutch, S. (1997). The transition from arithmetic to algebra: Initial understanding of equals, operations and variable. Proceedings of the 21 st Conference of the International Group for the Psychology of Mathematics Education, 2, 8996. University of Helsinkim, Lahti Research and Training Center, Finland.

Dalton, B., Ingels, S., Downing, J., \& Bozick, R. (2007). Advanced mathematics and science coursetaking in the spring high school senior classes of 1982, 1992, and 2004.Washington, D.C. (ERIC Document Reproduction Service No. ED497754).

Davis, B. M. (1998). Middle school algebra: Variables for success. (ERIC Document Reproduction Service No. ED436363)

Davis, R. B. (1975). Cognitive processes involved in solving simple algebraic equations. Journal of Mathematical Behavior, 1(3), 7-35.

Davis, R. B. (1992). Understanding "understanding". Journal of Mathematical Behavior, 11(4), 225-241.

de Vise, D. (2005, November 21). Middle schools in Maryland find advanced math is right formula. More students taking courses and succeeding. [Electronic version] The Washington Post, p. B01. Retrieved October 5, 2007, from http://www.nctm.org/news/content.aspx?id=592

Demana, F., \& Leitzel, J. (1988). Establishing fundamental concepts through numerical problem solving. In A. F. Coxford, \& A. P. Shulte (Eds.), The ideas of algebra, K-12 (1988 yearbook) (pp. 61-69). Reston, VA: National Council of Teachers of Mathematics.

Denmark, T., Barco, E., \& Voran, J. (1976). Final report: A teaching experiment on equality. (PMDC Technical Report No. 6). Tallahassee, Florida: Florida State University.

Department of Education. (1997). Mathematics equals opportunity: Middle school: Getting on the road to challenging mathematics and science courses. Washington, DC: Department of Education. (ERIC Document Reproduction Service No. ED415119)

Dick, T. (2007). Keeping the faith: Fidelity in technological tools for mathematics education. In G. W. Blume, \& K. Heid (Eds.), Research on technology and the teaching and learning of mathematics: Syntheses, cases, and perspectives (pp. 333-339). Greenwich, CT: Information Age.

Dickson, P. (1985). Thought-provoking software: Juxtaposing symbol systems. Educational Researcher, 14, 30-38.

Drier, H. (2001). Teaching and learning mathematics with interactive spreadsheets. School Science and Mathematics, 101(4), 170-179.

Dugdale, S., Thompson, P., Harvey, W., Demana, F., Waits, B., Kieran, C., et al. (1995). Technology and algebra curriculum reform: Current issues, potential directions, and research questions. Journal of 
Computers in Mathematics and Science Teaching, 14(3), 325-357.

Erbas, A. (2004). Teachers' knowledge of student thinking and their instructional practices in algebra. (Doctoral dissertation, University of Georgia).

Erikson, F. (1986). Qualitative methods in research on teaching. In M. C. Wittcock (Ed.), Handbook of research on teaching (pp. 119-161). New York: Macmillan.

Evers, W. M., \& Clopton, P. (2003, October 6). California's algebra crisis. [Op-Ed \& Commentary]. The Daily Report. Retrieved October 5, 2007, from http://www.hoover.org/pubaffairs/dailyreport/archive/2829516.html

Falkner, K. P., Levi, L., \& Carpenter, T. P. (1999). Children's understanding of equality: A foundation for algebra. Teaching Children Mathematics, 6(4), 232-236.

Fey, J. (1989). School algebra in the year 2000. In S. Wagner, \& C. Kieran (Eds.), Research issues in the learning and teaching of algebra (pp. 199-213). Reston, VA: Lawrence Erlbaum Associates.

Filloy, E., Rojano, T., \& \& Rubio, G. (2001). Propositions concerning the resolution of arithmeticalalgebraic problems. In R. Sutherland, T. Rojano, A. Bell \& R. Lins (Eds.), Perspectives on school algebra. (pp. 155-176). Dordrecht, The Netherlands: Kluwer.

Flanders, H. (1988). Computer software for algebra: What should it be? In A. F. Coxford, \& A. P. Shulte (Eds.), The ideas of algebra, K-12 (pp. 149-151). Reston, VA: The National Council of Teachers of Mathematics.

Flexer, B. K. (1984). Predicting eight-grade algebra achievement. Journal for Research in Mathematics Education, 15(5), 352-360.

Foley, G. (1998). The role of algebraic structure in the mathematics curriculum of grades 11-14. The nature and role of algebra in the K-12 curriculum: Proceedings of a national symposium (pp. 87-88). Washington, D.C.: National Academy Press.

French, D. (2002). Teaching and learning of algebra. London: Continuum.

Friedlander, A., \& Tabach, M. (2001). Developing a curriculum of beginning algebra in a spreadshet environment. The Future of the Teaching and Learning of Algebra. Proceedings of the 12th ICMI Study Conference, The University of Melbourne, Australia, 1, 252-257.

Fujii, T. (2003). Probing students' understanding of variables through cognitive conflict problems: Is the concept of a variable so difficult for students to understand? In Proceedings of the 27th Conference of the International Group for the Psychology of Mathematics Education (PME), Honolulu, HI, 1, 4965.

Fujii, T., \& Stephens, M. (2001). Fostering an understanding of algebraic generalization through numerical expressions: The role of quasi-variables. The Future of the Teaching and Learning of Algebra. Proceedings of the 12th ICMI Study Conference, The University of Melbourne, Australia, 1, 258-264.

Gallardo, A. (1996). Qualitative analysis in the study of negative numbers. Proceedings of the 20th Conference of the International Group for the Psychology of Mathematics Education. Valencia, Spain, 2, 377-384.

Gallardo, A. (2000). Historical-epistemological analysis in mathematics education: Two works in didactics 
of algebra. In R. Sutherland, T. Rojano, A. Bell \& R. Lins (Eds.), Perspectives on school algebra (pp. 121-140). Dordrecht, The Netherlands: Kluwer Academic Publishers.

Gallardo, A. (2002). The extension of the natural-number domain to the integers in the transition from arithmetic to algebra. Educational Studies in Mathematics, 49, 171-192.

Gelman, R., \& Gallistel, C. R. (1978). The child's understanding of number. Cambridge: Harvard University Press.

Gluck, K. (2000). Eye movements and algebra tutoring. (Doctoral dissertation, Carnegie Mellon University).

Goldenberg, E. P. (1988). Mathematics, metaphors, and human factors: Mathematical, technical, and pedagogical challenges in the educational use of graphical representations. Journal of Mathematical Behavior, 7, 135-173.

Goldin, G. (2002). Representation in mathematical learning and problem solving. In L. English (Ed.), Handbook of international research in mathematics education (pp. 197-218). Mahwah, NJ: Lawrence Erlbaum Associates.

Heibert, J., \& Carpenter, T. P. (1992). Learning and teaching with understanding. In D. A. Grouws (Ed.), Handbook of research on mathematics teaching and learning (pp. 65-97). Reston, VA: National Council of Teachers of Mathematics.

Heibert, J., \& Lefevre, P. (1986). Conceptual and procedural knowledge in mathematics: An introductory analysis. In J. Hiebert (Ed.), Conceptual and procedural knowledge: The case of mathematics (pp. 127). Hillsdale, NJ: Lawrence Erlbaum Associates.

Heid, K. (1997). The technological revolution and the reform of school mathematics. American Journal of Education, 106(1), 5-61.

Heid, M. K. (1990). Uses of technology in prealgebra and beginning algebra. Mathematics Teacher, 83(3), 194-198.

Herscovics, N. (1989). Cognitive studies of algebra problem solving and learning. In S. Wagner, \& C. Kieran (Eds.), Research issues in the learning and teaching of algebra (pp. 93-114). Reston, VA: Lawrence Erlbaum Associates.

Herscovics, N., \& Kieran, C. (1980). Constructing meaning for the concept of equation. The Mathematics Teacher, 73, 572-581.

Herscovics, N., \& Linchevski, L. (1994). A cognitive gap between arithmetic and algebra. Educational Studies in Mathematics, 27(1), 59-78.

Horne, M. (1999). The development of a framework of growth points to monitor students' comprehension of algebra in grades 7-9. Making the Difference. Proceedings of the 22nd Annual Conference of the Mathematical Education Research Group of Australasia. Adelaide, South Australia, 261-268.

House, P. A. (1988). Reshaping school algebra: Why and how? In A. F. Coxford, \& A. P. Shulte (Eds.), The ideas of algebra, K-12 (pp. 1-7). Reston, VA: The National Council of Teachers of Mathematics.

Hoyles, C., Noss, R., \& Kent, P. (2004). On the integration of digital technologies into mathematics classrooms. Journal of Computers in Mathematics and Science Teaching, 9(3), 309-326. 
Hubbard, R. (2003). An investigation into the modeling of word problems leading to algebraic equations. Paper presented at the The Mathematics Education into the 21st Century Project: Proceedings of the International Conference: The Decidable and the Undecidable in Mathematics Education, Brno, Czech Republic, 119-123.

Hughes, M. (1986). Children and numbers. New York: Blackwell.

Human, P., \& Murray, H. (1987). Non-concrete approaches to integer arithmetic. Proceedings of the Eleventh Conference of the International Group for the Psychology of Mathematics Education, Montreal: University of Montreal, 437-443.

Huntley, M. A., Rasmussen, C., Villarubi, R., Sangton, J., \& Fey, J. (2000). Effects of standards-based mathematics education: A study of the Core-Plus Mathematics Project Algebra/Functions Strand. Journal for Research in Mathematics Education, 31, 328-361.

Janvier, C. (1988). Comparison of models aimed at teaching signed integers. Proceedings of the Annual Conference of the International Group for the Psychology of Mathematics Education, Noordwijkerhout, The Netherlands, 1, 135-140.

Jensen, R., \& Williams, B. (1993). Research ideas for the classroom: Middle grades mathematics. In D. Owens (Ed.), Research ideas for the classroom: Middle grades mathematics (pp. 225-243). New York: Macmillian Publishing Company.

Jiang, Z., \& McClintock, E. (2000). Multiple approaches to problem solving and the use of technology. Journal of Computers in Mathematics and Science Teaching, 19(1), 7-20.

Kaput, J. (1989a). Linking representations in the symbol systems of algebra. In S. Wagner, \& C. Kieran (Eds.), Research issues in the learning and teaching of algebra (pp. 167-194). Reston, VA: National Council of Teachers of Mathematics.

Kaput, J. (1989b). The role of information technology in the affective dimension of mathematical experience: Some preliminary notes. In D. B. McLeod, \& V. M. Adams (Eds.), Affect and mathematical problem solving: A new perspective. New York: Springer-Verlag.

Kaput, J. (1992). Technology and mathematics education. In D. Grouws (Ed.), Handbook of research on mathematics teaching and learning (pp. 515-556). New York: MacMillan.

Kieran, C. (1979). Children's operational thinking within the context of bracketing and the order of operations. The Proceedings of the Third International Conference for the Psychology of Mathematics Eeducation, University of Warwick, Warwick, England. 128-132.

Kieran, C. (1980, April). Constructing meaning for non-trivial equations. Paper presented at the annual meeting of American Educational Research Association, Boston, MA. (ERIC Document Reproduction Service No. ED184899)

Kieran, C. (1981). Concepts associated with the equality sign. Educational Studies in Mathematics, 12(3), 317-326.

Kieran, C. (1989). The early learning of algebra: A structural perspective. In S. Wagner, \& C. Kieran (Eds.), Research issues in the learning and teaching of algebra (pp. 33-56). Reston, VA: Lawrence Erlbaum Associates.

Kieran, C. (1990). Cognitive processes involved in learning school algebra. In P. Nesher, \& J. Kilpatrick 
(Eds.), Mathematics and cognition: A research synthesis by the international group for the psychology of mathematics education (pp. 96-112). New York: Cambridge University Press.

Kieran, C. (1992). The learning and teaching of school algebra. In D. Grouws (Ed.), Handbook of research on mathematics teaching and learning (pp. 390-419). New York: Macmillan Publishing Company.

Kieran, C. (1996). The changing face of school algebra. In C. Alsina, J. Alvarez, B. Hodgson, C. Labord \& A. Perez (Eds.), Eighth international congress on mathematical education: Selected lectures (pp. 271290). Sevilla, Spain: S.A.E.M. Thales.

Kieran, C. (2004). Algebraic thinking in the early grades: What is it? The Mathematics Educator, 8(1), 139151.

Kieran, C. (2007). Learning and teaching of algebra at the middle school through college levels: Building meaning for symbols and their manipulation. In F. K. Lester (Ed.), Second handbook of research on mathematics teaching and learning (pp. 707-762). Charlotte, NC: Information Age Pub Inc.

Kieran, C., \& Chalouh, L. (1993). Prealgebra: The transition from arithmetic to algebra. In D. T. Owens (Ed.), Research ideas for the classroom: Middle grades mathematics (pp. 179-198). New York: Macmillan.

Kieran, C., \& Saldanha, L. (2005). Computer algebra systems (CAS) as a tool for coaxing the emergence of reasoning about equivalence of algebraic expressions. Proceedings of the 29th Conference of the International Group for the Psychology of Mathematics Education, Melbourne, Australia, 3, 193-200.

Kilpatrick, J., \& Swafford, J. Findell, B. (Eds.). (2001). Adding it up. Washington, D.C.: National Academy Press.

Kleiman, G. (1998). Middle school algebra from a modeling perspective. The Nature and Role of Algebra in the K-14 Curriculum: Proceedings of a National Symposium, 63-66.

Klein, A., Beishuizen, M., \& Treffers, A. (1998). The empty number line in dutch second grades: Realistic versus gradual program design. Journal for Research in Mathematics Education, 29(4), 443-464.

Knuth, E. J., Stephens, A. C., McNeil, N., \& Alibali, M. W. (2006). Does understanding the equal sign matter? Evidence from solving equations. Journal for Research in Mathematics Education, 37(4), 297-312.

Koedinger, K. R. (2001). Cognitive tutors as modeling toll and instructional model. In K. D. Forbus, \& P. J. Feltovich (Eds.), Smart machines in education: The coming revolution in educational technology (pp. 145-168). Menlo Park: CA: AAAI/MIT Press.

Koedinger, K. R., \& Anderson, J. R. (1998). Illustrating principled design: The early evolution of cognitive tutor for algebra symbolization. Interactive Learning Environments, 5, 161-180.

Kohn, J. (1978). A physical model for operations with integers. Mathematics Teacher, 71(9), 734-736.

Kronholz, J. (1998, June 16). Low x-pectations: Students fear algebra, and then comes the ninth grade crunch. The Wall Street Journal. Retrieved August 4, 2007, from http://www.middleweb.com/backstories1.html

Kurz, T., Middleton, J., \& Yanik, H. (2005). A taxonomy of software for mathematics instruction. Contemporary Issues in Technology and Teacher Education, 5(2), 123-137. 
Lagrange, J. (1999). Complex calculators in the classroom: Theoretical and practical reflections on teaching precalculus. International Journal of Computers for Mathematical Learning, 4, 51-81.

Lawson, D. R. (1990). The problem, the issues that speak to change. In E. L. Edwards (Ed.), Algebra for everyone (pp. 1-6). Reston, VA: National Council of Teachers of Mathematics. (ERIC Document Reproduction Service No. ED325385).

Lee, J., Grigg, W., \& Dion, G. (2007). The nation's report card: Mathematics 2007. No. NCES 2007-494). Washington, D.C.: National Center for Education Statistics, Institute of Education Sciences, U.S. Department of Education.

Leinhardt, G., Zaslavsky, O., \& Stein, M. K. (1990). Functions, graphs, and graphing: Tasks, learning and teaching. Review of Educational Research, 60(1), 1-64.

Leitzel, J. (1989). Critical considerations for the future of algebra instruction. In S. Wagner, \& C. Kieran (Eds.), Research issues in the learning and teaching of algebra (pp. 25-32). Reston, VA: Lawrence Erlbaum Associates.

Lesh, R., Post, T., \& Behr, M. (1987). Representations and translations among representations in mathematics learning and problem solving. In C. Janvier (Ed.), Problems of representation in the teacher and learning of mathematics (pp. 33-40). Hillsdale, NJ: Lawrence Erlbaum Associates, Inc.

Linchevski, L., \& Herscovics, N. (1994). Cognitive obstacles in pre-algebra. Proceedings of the 18th Conference of the International Group for the Psychology of Mathematics Education, Lisbon, Portugal, 3, 176-182.

Linchevski, L., \& Livneh, D. (1999). Structure sense: The relationship between algebraic and numerical contexts. Educational Studies in Mathematics, 40(2), 173-196.

Lincoln, Y. S., \& Guba, E. G. (1985). Naturalistic inquiry. Newberry Park, CA: Sage Publications.

Lochhead, J., \& Mestre, J. (1988). From words to algebra: Mending misconceptions. In A. F. Coxford, \& A. P. Shulte (Eds.), Ideas of algebra, K-12 (pp. 127-135). Reston, VA: The National Council of Teachers of Mathematics.

Lodholz, R. (1990). The transition from arithmetic to algebra. In E. L. Edwards (Ed.), Algebra for everyone (pp. 24-33). Reston, VA: National Council of Teachers of Mathematics.

MacGregor, M., \& Stacey, K. (1997). Students' understanding of algebraic notation: 11-15. Educational Studies in Mathematics, 33(1), 1-19.

Masalski, W. J. (1999). How to use the spreadsheet as a tool in the secondary school mathematics classroom. (Windows and Macintosh Operating Systems Second ed.). Reston, VA: National Council of Teachers of Mathematics.

Mathematical Sciences Education Board, \& National Research Council. (1990). Reshaping school mathematics: A philosophy and framework for curriculum. Washington, DC: National Academy Press.

Mathematical Sciences Education Boards, Board on Mathematical Sciences, Committee on the Mathematical Sciences in the Year 2000, \& National Research Council. (1989). Everybody counts: A report to the nation on the future of mathematics education. Washington, D.C.: National Academies Press. 
Matz, M. (1980). Towards a computational theory of algebraic competence. Journal of Children's Mathematical Behavior, 3(1), 93-166.

Matz, M. (1982). Towards a process model for high school algebra errors. In D. Sleeman, \& J. S. Brown (Eds.), Intelligent tutoring systems (pp. 25-50). London: Academic Press.

McAuley, J. (1990). Please sir I didn't do nothin. Mathematics in School, 19(1), 45-47.

Mestre, J. P. (1987). Why should mathematics and science teachers be interested in cognitive research findings? Academic connections (pp. 3-5, 8-11). New York: The College Board.

Minsky, M., \& Papert, S. (1972). Research at the laboratory in vision, language, and other problems of intelligence. Cambridge, MA: Artificial Intelligence Laboratory, MIT.

Mitchell, J. H., Hawkins, E. F., Jakwerth, P. M., Stancavage, F. B., \& Dossey, J. A. (1999). Student work and teacher practices in mathematics. Washington, D.C. : U.S. Department of Education. Office of Educational Research and Improvement. National Center for Education Statistics. (NCES No. 1999453)

Mogull, R. G., \& Rosengarten, W. (1972). Predicting student success in elementary algebra. California Journal of Educational Research, 23, 104-107.

Morris, R. (Ed.). (1980). Studies in mathematics education. Paris: United Nations Educational, Scientific and Cultural Organization.

Moseley, B., \& Brenner, M. E. (1997). Using multiple representations for conceptual change in prealgebra: A comparison of variable usage with graphic and text based problems. (ERIC Document Reproduction Service No. ED413184)

Moses, R. P., \& Cobb, C. (2001). Radical equations: Math literacy and civil rights. Boston: Beacon Press.

Mullis, I. V. S., Martin, M. O., Gonzalez, E. J., \& Chrostowski, S. J. (2004). TIMSS 2003 international mathematics report: Findings from IEA's trends in international mathematics and science study at the eighth and fourth grades. MA: Boston: Chestnut Hill.

Murdock, J. (2007). Algebra: A matter of life and death?". Gallery Workshop at the National Council of Teachers of Mathematics 2007 Regional Conference, Richmond, VA. Gallery Workshop at NCTM 2007 Regional Conference, Richmond VA.

Murray, J. C. (1985). Children's informal conceptions of integer arithmetic. Proceedings of the Annual Conference of the International Group for the Psychology of Mathematics Education. Noordwijkerhout, The Netherlands, 1, 147-152.

National Council of Supervisors on Mathematics. (1989). Essential mathematics for the twenty-first century: Position of the NCSM. The Arithmetic Teacher, 37(1), 44-46.

National Council of Teacher of Mathematics. (1991). Professional standards for teaching mathematics. Reston, VA: NCTM.

National Council of Teacher of Mathematics. (2000). Principles and standards for school mathematics. Reston, VA.: The National Council of Teachers of Mathematics, Inc. 
National Council of Teachers of Mathematics. (1989). Curriculum and evaluation standards for school mathematics. Reston, VA: The National Council of Teachers of Mathematics, Inc.

National Council of Teachers of Mathematics, \& Mathematical Sciences Education Board. (1998). The nature and role of algebra in the K-14 curriculum: Proceedings of a national symposium. Washington, D.C.: National Academy Press.

National Research Council. (1991). For good measure. Principles and goals for mathematics assessment. Washington, DC: National Academy Press.

Neurath, R. A., \& Stephens, L. J. (2006). The effect of using microsoft excel in a high school algebra class. International Journal of Mathematical Education in Science \& Technology, 37(6), 721-726.

Noss, R. (1988). The computer as a cultural influence in mathematical learning. London: London Institute of Education.

Nunn, T. P. (1919). The teaching of algebra. London: Longmans.

Nussbaum, B. (1988, September 19). Needed: Human Capital--dwindling supply? The U.S. has lost much ground to competitors, and investing in people looks like the way to retake it. After years of neglect, the problem of human capital has become a crisis. BusinessWeek, p. 100.

Osgood, C. (1980). Lectures on languge performance. New York: Springer- Verlag.

Patton, M. Q. (2002). Qualitative research and evaluation methods (Third ed.). Thousand Oaks, CA: Sage Publications.

Payne, A., \& Watson, D. (1997, August 26). New standards higher math a new state rule calls for students to learn more advanced math earlier and to master algebra in order to graduate. [Electronic Version]. Virginian Pilot. Retrieved October 4, 2007, from http://scholar.lib.vt.edu/VA-news/VAPilot/issues/1997/vp970826/08260362.htm

Pea, R. (1986). Cognitive technologies for mathematics education. In A. Schoenfeld (Ed.), Cognitive science and mathematics education (pp. 89-122). Hillsdale, NJ: Lawrence Erlbaum.

Pelavin, S. H., \& Kane, M. B. (1988). Minority participation in higher education. Washington, DC.: Pelavin Associates.

Peled, I. (1991). Levels of knowledge about signed numbers: Effects of age and ability. Proceedings of the 15th Conference of the International Group for the Psychology of Mathematics Education, Assisi, Italy, 3, 145-152.

Peled, I. Nukhopadhyay, S., \& Resnick, L. (1989). Formal and informal sources of mental models for negative numbers. Proceedings of the 13th Annual Conference of the International Group for the Psychology of Mathematics Education, Paris, France, 3, 106-110.

Perks, P. (1995). Calculators and young children: A bridge to number? In L. Burton, \& B. Jaworski (Eds.), Technology in mathematics teaching (pp. 173-189). Sweden: Chartwell-Bratt.

Philipp, R. A. (1992). A study of algebraic variables: Beyond the student-professor problem. Journal of Mathematical Behavior, 11, 161-176. 
Piaget, J. (1964). Development and learning. In E. R. Ripple, \& V. N. Rockcastle (Eds.), Piaget rediscovered. Ithaca, NY: School of Education, Cornell University. (ERIC Document Reproduction Service No. ED001819).

Piaget, J., \& Inhelder, B. (1973). Memory and intelligence. New York: Basic Books.

Piccioto, H., \& Wah, A. (1993). A new algebra: Tools, themes, concepts. Journal of Mathematical Behavior, 12(1), 19-42. Retrieved October 12, 2007, from www.picciotto.org/math-ed/newalgebra/new-algebra.html.

Piez, C., \& Voxman, M. (1997). Multiple representations—using different perspectives to form a clearer picture. The Mathematics Teacher, 90, 164-166.

Pirie, S., \& Kieren, T. (1989). A recursive theory of mathematical understanding. For the Learning of Mathematics: An International Journal of Mathematics Education, 9(3), 7-11.

Posner, G. J., Strike, K. A., Hewson, P. W., \& Gertzog, W. A. (1982). Accomodation of a scientific conception: Toward a theory of conceptual change. Science Educaiton, 66(2), 211-227.

Richards, J. (1991). Mathematical discussions. In E. von Glasserfeld (Ed.), Radical constructivism in mathematics education (pp. 13-51). Dordrecht, The Netherlands: Kluwer.

Rojano, T. (1996). Developing algebraic aspects of problem solving within a spreadsheet environment. In N. Bednarz, C. Kieran \& L. Lee (Eds.), Approaches to algebra (pp. 137-145). Netherlands: Kluwer Academic Publishers.

Rosnick, P. (1981). Some misconceptions concerning the concept of variable. Mathematics Teacher, 74(6), 418-420,450.

Ruthven, K. (1996). Calculators in the mathematics curriculum: The scope of personal computing technology. In A. J. Bishop, K. Clements, C. Keitel, J. Kilpatrick \& C. Laborde (Eds.), International handbook of mathematics education (pp. 435-468). Dordrecht, The Netherlands: Kluwer.

Ruthven, K., \& Hennessy, S. (2002). A practitioner model of the use of computer-based tools and resources to support mathematics teaching and learning. Educational Studies in Mathematics, 49(1), 47-88.

Sargent, B. L. (1985). Variables that correlate with success in first year algebra. (Doctoral dissertation, Virginia Polytechnical Institute).

Schifter, D. (1999). Reasoning about operations: Early algebraic thinking in grades K-6. In L. V. Stiff, \& F. R. Curcio (Eds.), Developing mathematical reasoning in K-12 (pp. 62-81). Reston, VA: National Council of Teachers of Mathematics.

Schoenfeld, A., \& Arcavi, A. (1988). The meaning of variable. The Mathematics Teacher, 81(6), 420-427.

Seo, K. H., \& Ginsburg, H. P. (2003). You've got to carefully read the math sentence: Classroom context and children's interpretations of the equals sign. In A. J. Baroody, \& A. Dowker (Eds.), Development of arithmetic concepts and skills: Constructing adaptive expertise (pp. 161-189). Mahwah, NJ:

Lawrence Erlbaum Associates, Inc.

Sfard, A. (1995). The development of algebra: Confronting historical and psychological perspectives. Journal of Mathematical Behavior, 14, 15-39. 
Silva, C. M., \& Moses, R. P. (1990). The algebra project: Making middle school mathematics count. Journal of Negro Education, 59(3), 375.

Silver, E. (1979). Student perceptions of relatedness among mathematical verbal problems. Journal for Research in Mathematics Education, 10, 195-210.

Silver, E. (1986). Using conceptual and procedural knowledge: A focus on relationships. In J. Hiebert (Ed.), Conceptual and procedural knowledge: The case of mathematics (pp. 181-198). Hillsdale, NJ: Lawrence Erlbaum Associates.

Silver, E., \& Kenney, P. A. (2000). Results from the seventh mathematics assessment of the national assessment of educational progress. Reston, VA: National Council of Teachers of Mathematics.

Simon, M. A. (1995). Reconstructing mathematics pedagogy from a constructivist perspective. Journal for Research in Mathematics Education, 26(2), 114-145.

Skemp, R. (1978). Relational and instrumental understanding. Arithmetic Teacher, 26, 9-15.

Skemp, R. (1982). Symbolic understanding. Mathematics Teaching, 99, 59-61.

Skemp, R. (1987). The psychology of learning mathematics. Hillsdale, NJ: Lawrence Erlbaum Associates.

Sorrentino, F. M., \& Curcio, F. R. (Eds.). (1986). Soviet politics and education. Lanham, MD: University Press of America.

Sowder, J. (1989). Series forward. In S. Wagner, \& C. Kieran (Eds.), Research issues in the learning and teaching of algebra (pp. vii-x). Hillsdale, NJ: Lawrence Erlbaum Associates.

Stacey, K., \& MacGregor, M. (1997). Building foundations for algebra. Mathematics Teaching in the Middle School, 2(4), 253-260.

Stacey, K., \& MacGregor, M. (1997). Ideas about symbolism that students bring to algebra. Mathematics Teacher, 90(2), 110-113.

Stacey, K., \& MacGregor, M. (2002). Curriculum reform and approaches to algebra. In R. Sutherland, T. Rojano, A. Bell \& R. Lins (Eds.), Perspectives on school algebra (pp. 141-153). Hingham, MA: Kluwer Academic Publishers.

Steiner, M. (1975). Mathematical knowledge. Ithaca, NY: Cornell University Press.

Stevenson, H., Lee, S., \& Stigler, J. (1986). Mathematics achievement of Chinese, Japanese, and American children. Science, 231(3), 693-699.

Strauss, A., \& Corbin, J. (1998). Basics of qualitative research: Techniques and procedures for developing grounded theory (Second ed.). Thousand Oaks, CA: Sage Publications.

Sutherland, R. (1989). Providing a computer-based framework for algebraic thinking. Educational Studies in Mathematics, 20(3), 317-344.

Sutherland, R., \& Rojano, T. (1993). A spreadsheet approach to solving algebra problems. Journal of Mathematical Behavior, 12(4), 353-383. 
Swan, M. (2000). Making sense of algebra. Mathematics Teaching, 171, 16-19.

Thomas, M., \& Tall, D. (1986). The value of the computer in learning algebraic concepts. Proceedings of the Tenth International Conference on the Psychology of Mathematics Education, London, 313-318.

Thompson, P. (1992). Notations, conventions, and constraints: Contributions to effective uses of concrete materials in elementary mathematics. Journal for Research in Mathematics Education, 23(2), 123147.

Thorpe, J. (1989). Algebra: What should we teach and how should we teach it? In S. Wagner, \& C. Kieran (Eds.), Research issues in the learning and teaching of algebra (pp. 11-24). Reston, VA: Lawrence Erlbaum Associates.

U.S. Department of Education. (1991). America 2000: An education strategy. Washington, D.C. (ERIC Document Reproduction Service No. ED327985).

Usiskin, Z. (1988). Conceptions of school algebra and uses of variables. In A. F. Coxford (Ed.), The ideas of algebra, K-12 (1988 yearbook) (pp. 8-19). Reston, VA: National Council of Teachers of Mathematics.

Usiskin, Z. (1995). Why is algebra important to learn? American Educator, 19(1), 30-37.

Vinner, S. (1997). The pseudo-conceptual and the pseudo-analytical thought processes in mathematics learning. Educational Studies in Mathematics, 34(2), 97-129.

Vinner, S., \& Dreyfus, T. (1989). Images and definitions for the concept of function. Journal for Research in Mathematics Education, 20(4), 356-366.

Wagner, S. (1983). What are these things called variables? Mathematics Teacher, 76(7), 474-479.

Wagner, S., \& Kieran, C. (1989). An agenda for research on the learning and teaching of algebra. In S. Wagner, \& C. Kieran (Eds.), Research issues in the learning and teaching of algebra (pp. 220-237). Reston, VA: Lawrence Erlbaum Associates.

Wagner, S., \& Parker, S. (1993). Advancing algebra. In P. S. Wilson (Ed.), Research ideas for the classroom: High school mathematics (pp. 117-139). New York: Macmillan Publishing Company.

Warren, E. (2004). Generalising arithmetic: Supporting the process in early years. Proceedings of the 28th Conference of the International Group for the Psychology of Mathematics Education, Bergen, Norway, 4, 417-424.

Wren, F. L. (1935). A survey of research in teaching of secondary algebra. Journal of Educational Research, 28(8), 597-610.

Wright, P. I. (1991). A study of relationships between specific algebra and arithmetic content. (Doctoral dissertation, University of Virginia).

Zbiek, R. (1998). How might technology enhance algebraic reasoning? The nature and role of algebra in the K-14 curriculum: Proceedings of a national symposium (pp. 35-36). Washington, D.C.: National Research Council.

Zbiek, R., Heid, M., Blume, G., \& Dick, T. (2007). Research on technology in mathematics education. In F. 
K. Lester (Ed.), Second handbook of research on mathematics teaching and Learning (pp. 1169-1207). Charlotte, NC: Information Age Pub Inc.

Zuckerbrod, N. (2007, December 4). US students do worse in science and math. [Electronic version]. Associated Press, Retrieved December 4, 2007 from http://hosted.ap.org/dynamic/stories/M/MATH_SCIENCE_SCORES?SITE=DCTMS\&SECTION=U S\&TEMPLATE=DEFAULT 


\section{APPENDIX A}

Module 1: Addition Tasks

1. Have you ever seen a number like this -3 ? Where?

2. Do you know what this is called -3 ?

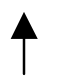

Probes: Do you know what it means?

Have you seen it anywhere else?

Maybe in a math problem like $5-3$ ?

3. Evaluate the following: $10+3,10+-3$, and $-5+-7$ ?

Probes: How did you get that answer?

What is your sign in the answer? Why?

4. Write a story for $-5+-7$.

Probe: Can you tell me your story and how it relates to $-5+-7$ ?

5. Write a math problem using the information in the number line

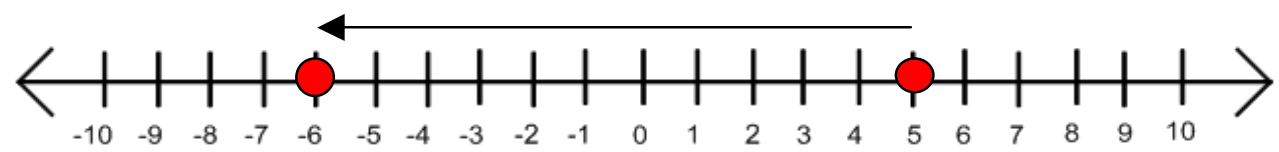

Probes: Can you explain to me what you wrote and why you wrote it that way? Can you write it another way? How? Why?

\section{Module 1: Addition Instruction}

Use the sliders to help you answer the following questions.

1. $10+-3$; Josh rushed for 10 yards on the first play. Then he was thrown for a loss of 3 yards on the next play. What was the total yardage gained for those two plays?

\section{Guiding Questions}

a. Use the blue slider to show the 10 yards that Josh lost. Use the orange slider to show the loss of 3 on the next play.

b. What direction does your blue slider point? Why? What is the relationship between the way the arrow points and the sign of the number?

c. What is the number inside your blue slider? What does it mean? How does this relate to the blue number in the expression?

d. What direction does your orange slider point? Why? What is the relationship between the way the arrow points and the sign of the number?

e. What is the number inside your orange slider? What does it mean? How does this relate to the orange number in the expression?

f. What number does the orange slider point to? Why? 
g. Predict what you think the total yardage gained from these two plays.

h. Click the Show Answer button to find out.

i. Write down your expression in the chart

2. $-8+4$; Drew lost seven yards on the first play. Then he gained ten yards on the next play. What was the total yardage gained for those two plays? (Guiding questions as above)

3. $-5+-7$; Joey lost five yards on his first run and 7 more yards on the next play. What was Joey's total yardage for those two downs?

Extra Tasks: $2+5,3+-8,-6+11,-12+-2$

\begin{tabular}{|rl|}
\hline Blue Number + Orange Number & $=$ Answer \\
\hline+ & $=$ \\
\hline+ & $=$ \\
\hline+ & $=$ \\
\hline+ & $=$ \\
\hline
\end{tabular}

\section{Generalizing Questions}

1. If the signs of the numbers are the same, what do you notice about the sum? What about if they are different?

2. When adding two integers with the same sign, what direction(s) do the sliders point? What about when adding two integers with different signs?

3. Is it possible to add two negative numbers and get a positive sum? What about two positive numbers to get a negative sum?

4. If you add a positive and a negative number, how do you know what the sign of the sum will be?

5. What do you notice about adding numbers with opposite signs?

Module 2: Subtraction Tasks

1. Evaluate the following $5-2,5--12,-4--6$, and $-4--1$

Probes: How did you get that answer?

What is your sign in the answer? Why?

2. Can you show $-4--1$ on a number line?

Probes: Why did you move that direction on the number line?

Does this match your answer?

3. On February $14^{\text {th }}$ the high temperature was $32^{\circ} \mathrm{F}$, and the low temperature was $0^{\circ} \mathrm{F}$. How many degrees did it change?

Probes: How did you get that answer?

Which is warmer $32^{\circ} \mathrm{F}$ or $0^{\circ} \mathrm{F}$ ?

4. If the high temperature in Alaska was $-6^{\circ} \mathrm{F}$ and the day's low temperature was $-17^{\circ} \mathrm{F}$, how many degrees it change? Show this on a number line.

Probes: Why did you move that direction on the number line?

Which is warmer $-6^{\circ} \mathrm{F}$ or $-17^{\circ} \mathrm{F}$ ? 
Module 2: Subtraction Instruction

Use the sliders to help you answer the following questions.

Evaluate the following: $5-2,5-0,5--2 ;-4--1,-4--5,-4--4$

$S D A$

Guiding Questions

a. Use the blue dot to show the 5 .

b. Click Show Second Dot button. Use the orange dot to show the 2 .

c. Guess how many numbers this dropped?

d. Click on Show Arrow button. What does this arrow show about the dots?

e. What direction does the arrow point? Why? What is the relationship between the way the arrow points and the sign of the orange number?

f. Predict the answer.

g. What is the number above the arrow mean? How does this relate to the answer? If you're not sure, press Show Answer button.

h. Write down your expression in the chart

\begin{tabular}{|cc|}
\hline Blue Number - Orange Number & $=$ Answer \\
\hline & $=$ \\
\hline
\end{tabular}

$S A A$

Guiding Questions

a. Use the blue stick to show the first number.

b. Click on the Show Arrow button. Drag the arrow to show how to subtract the second number. Which direction did you go? Why? How far did you move? Why?

c. Click the Show Arrow Value. What does this value mean?

d. Click Show Expression. Does the expression match our problem? Why or why not?If not, can you get it so that it does?

e. Predict the answer to this expression. Click Show Answer. How does the answer and the tip o the arrow relate?

f. Write down your expression in the chart

\begin{tabular}{|ccc|}
\hline Blue Number & $-\begin{array}{c}\text { Orange Arrow } \\
\text { Value }\end{array}$ & $=$ \\
& Answer \\
\hline & & $=$ \\
\hline
\end{tabular}

Generalizing Questions

1. If the signs of the numbers are the same, what do you notice about the sign of the difference? What about if they are different?

2. Is it possible to subtract a negative number from a positive number and a positive difference? What about a negative difference?

3. Is it possible to subtract a positive number from a negative number and a positive difference? What about a negative difference?

4. If you subtract a positive number from another positive number, how do you know what the sign of the difference will be?

5. If you subtract a negative number from another negative number, how do you know what the sign of the difference will be? 
Module 3: Equal Sign Tasks

1. a. What are the differences between the following: $5+6,5+=, 5+6=11$

b. What does the equal sign mean in $5+6=$ and $5+6=11$ ? Can it mean anything else?

2. What does the equal sign mean in the following and is it ok to use it this way?
a. $3=3$
b. $8=10-2$
c. 1 foot $=12$ inches
d. 1 dime $=10$ pennies

Probes: Why does it mean that?

Can it mean anything else?

3. a. What does the equal sign mean in a problem like this: $2 \times 5=4+6$ ?

b. Is it ok to write it like this? Why or why not?

4. a. Have you seen a problem like this: $3+5=?+2$

b. Is it ok to write it like this? Why or why not?

c. What is the value of the question mark? How did you get the answer?

5. What does the equal sign mean in $x+5=3$ ? Can you figure out what $x$ is?

6. What does the equal sign mean in $2 m+9=1$ ? Can you figure out what $x$ is?

\section{Module 3: Equal Sign Instruction}

1. Which objects weigh a pan down, and which ones pull it up?

2. Find a combination of weights and balloons that balances the two pans. List the objects you put on each pan.

3. Write down the algebraic equation that corresponds to your arrangement of weights and balloons. Press the Show Formula button to check your answer.

4. Try each of these operations and write down how it affects the balance. Before each operation, make sure the pans are balanced and that each pan contains enough items to carry out that operation. In your answer, state whether the operation makes the left pan heavier, makes the right pan heavier, or leaves the two pans in balance.

a. Drag a 1 from the storage area onto each pan.

b. Drag a 1 from the right pan to the left pan.

c. Drag a 1 and a -1 together from the left pan to the right pan.

d. Drag a 1 from the storage area onto the left pan and a -1 onto the right pan.

e. Drag a -5 onto each pan.

5. Write down at least two rules to describe things you can do that will keep the pans balanced. For each rule, write down which parts of 4 illustrate the rule.

6. Use the balance to help you find out what should go in the box for: $3+5=?+2$.

7. Use the balance to help you solve for $x: x+5=3$.

8. Use the balance to help you solve for $x: x+8=21$. 
9. Use the balance to help you solve for $x: x-6=-15$.

10. Use the balance to help you solve for $x: x+7=-10$.

11. Use the balance to help you solve for $x: x-20=-13$.

12. Use the balance to help you solve for $m: 2 m+9=3$

13. Use the balance to help you solve for $m: 2 m+7=15$

14. Use the balance to help you solve for $m: 2 m-16=-6$ 